\title{
Copper-Catalyzed Difluoroalkylation of Alkene/Nitrile Insertion/Cyclization Tandem Sequences: Construction of Difluorinated Bicyclic Amidines
}

\footnotetext{
Zheng Li, ${ }^{\text {a }}$ Yu-Heng Wu, ${ }^{\text {a }}$ Ji-Ming Xi, ${ }^{a}$ Zhong-Lin Wei, ${ }^{a}$ Wei-Wei Liao ${ }^{\text {a, b* }}$

${ }^{a}$ Department of Organic Chemistry, College of Chemistry, Jilin University, 2699 Qianjin Street, Changchun 130012, P R China.

${ }^{b}$ State Key Laboratory of Organometallic Chemistry, Shanghai Institute of Organic Chemistry, Chinese Academy of Sciences, Shanghai 200032, P R China

E-mail: wliao@jlu.edu.cn
}

Table of Contents

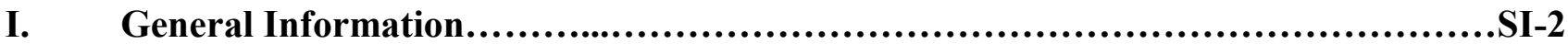

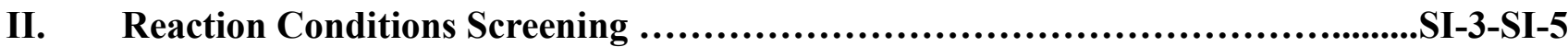

III. Preparation of Substrates.....................................................

IV. General Procedure and Experimental Details..............................SI-16-SI-30

V. Other Unsuccessful Substrates.....................................................

VI. Scale-up Reaction and Synthetic Transformations...........................SI-31-SI-33

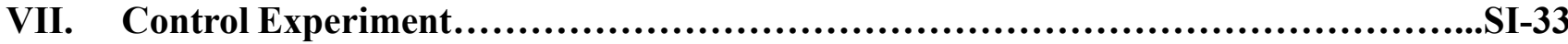

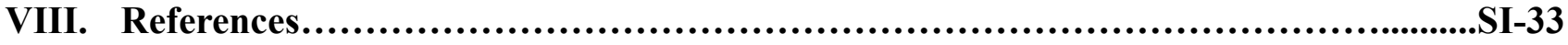

IX. Crystal Data and Structure Refinement for Compound 3ca....................SI-34-SI-39

X. $\quad{ }^{1} \mathrm{H}$ and ${ }^{13} \mathrm{C}$ NMR Spectral Copies............................................ 


\section{General Information}

All reactions were carried out under inert atmospheric condition unless otherwise noted, and solvents were dried according to established procedures. Reactions were monitored by thin layer chromatography (TLC) visualizing with ultraviolet light (UV), $\mathrm{KMnO}_{4}, p$-anisaldehyde stain, and phosphomolybdic acid (PMA) stain; column chromatography purifications were carried out using silica gel. For reactions that require heating, oil bath was used. Proton nuclear magnetic resonance ( ${ }^{1} \mathrm{H}$ NMR) spectra were recorded on a 300, 400 or $500 \mathrm{MHz}$ spectrometer in $\mathrm{CDCl}_{3}$ or DMSO, fluorine nuclear magnetic resonance $\left({ }^{19} \mathrm{~F}\right.$ NMR) spectra were recorded on a 376 or $470 \mathrm{MHz}$ spectrometer in $\mathrm{CDCl}_{3}$ or DMSO, and carbon nuclear magnetic resonance $\left({ }^{13} \mathrm{C} \mathrm{NMR}\right)$ spectra were recorded on 125 or $100 \mathrm{MHz}$ spectrometer in $\mathrm{CDCl}_{3}$ or DMSO unless otherwise noted. Chemical shifts for protons are reported in parts per million downfield from tetramethylsilane (TMS) and are referenced to residual protium in the NMR solvent $\left(\mathrm{CHCl}_{3}=\delta 7.26 \mathrm{ppm}, \mathrm{DMSO}=\delta 2.50 \mathrm{ppm}\right)$. Chemical shifts for carbon are reported in parts per million downfield from tetramethylsilane (TMS) and are referenced to the carbon resonances of the solvent residual peak $\left(\mathrm{CDCl}_{3}=\delta\right.$ $77.16 \mathrm{ppm}, \mathrm{DMSO}=\delta 39.52 \mathrm{ppm})$. NMR data are presented as follows: chemical shift $(\delta \mathrm{ppm})$, multiplicity $(\mathrm{s}=$ singlet, $\mathrm{d}=$ doublet, $\mathrm{t}=$ triplet, $\mathrm{q}=$ quartet, $\mathrm{m}=$ multiplet, $\mathrm{br}=$ broad), coupling constant in Hertz $(\mathrm{Hz})$, integration . Mass spectra were recorded on the Bruker MicrOTOF Q II. 


\section{Reaction Conditions Screening}

\section{Optimization of Reaction Conditions}

Table S1. Optimization Reaction Conditions: Additives ${ }^{a}$

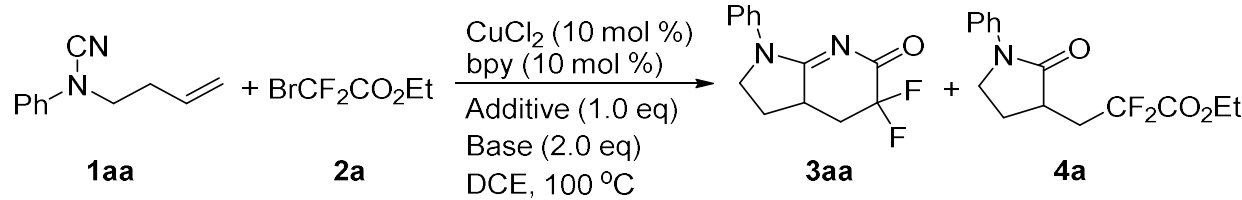

\begin{tabular}{ccccccc} 
entry & additive & base & $\mathrm{t}(\mathrm{h})$ & yield of 3aa $(\%)^{b}$ & yield of $\mathbf{4 a}(\%)^{b}$ & conv $(\%)^{c}$ \\
1 & - & - & 12 & nd & nd & $<5$ \\
2 & - & $\mathrm{NaOAc}$ & 12 & trace & trace & 25 \\
3 & $B_{2}$ pin $_{2}$ & $\mathrm{NaOAc}$ & 12 & 60 & 5 & 72 \\
4 & $\mathrm{Na}_{2} \mathrm{~S}_{2} \mathrm{O}_{5}$ & - & 12 & 6 & trace & 36 \\
5 & $\mathrm{~K}_{2} \mathrm{~S}_{2} \mathrm{O}_{5}$ & - & 12 & 11 & trace & 49 \\
6 & $\mathrm{~K}_{2} \mathrm{~S}_{2} \mathrm{O}_{5}$ & $\mathrm{NaOAc}$ & 12 & 38 & 38 & 85 \\
7 & $\mathrm{Et}_{3} \mathrm{SiH}$ & - & 12 & 0 & 0 & $\mathrm{nr}$ \\
8 & $\mathrm{~B}_{2} \mathrm{pin}_{2}$ & $\mathrm{NaHCO}_{3}$ & 24 & 27 & trace & 47 \\
9 & $\mathrm{~B}_{2} \mathrm{pin}_{2}$ & $\mathrm{Na}_{2} \mathrm{CO}_{3}$ & 24 & 22 & 5 & 57 \\
10 & $\mathrm{~B}_{2}$ pin $_{2}$ & $\mathrm{Cs}_{2} \mathrm{CO}_{3}$ & 24 & 26 & 7 & 45 \\
11 & $\mathrm{~B}_{2}$ pin $_{2}$ & $\mathrm{KF}_{12}$ & 12 & 11 & trace & 32 \\
12 & $\mathrm{~B}_{2}$ pin $_{2}$ & $\mathrm{~K}_{2} \mathrm{CO}_{3}$ & 12 & 7 & trace & 13 \\
$13^{d}$ & $\mathrm{~B}_{2}$ pin $_{2}$ & $\mathrm{NaOAc}(1.0 \mathrm{eq})$ & 12 & 53 & 8 & 87 \\
$14^{e}$ & $\mathrm{~B}_{2}$ pin $_{2}$ & $\mathrm{NaOAc}(0.2 \mathrm{eq})$ & 12 & 11 & trace & 14 \\
\hline
\end{tabular}

${ }^{a}$ Reaction conditions: 1 aa $(0.2 \mathrm{mmol}), \mathbf{2 a}(0.4 \mathrm{mmol}), \mathrm{CuCl}_{2}(10 \mathrm{~mol} \%)$, bpy (10 mol \%), additive (1.0 eq), and base (2.0 eq) in DCE $(1.5 \mathrm{~mL})$ at $100{ }^{\circ} \mathrm{C}$ under $\mathrm{N}_{2} .{ }^{b}$ Isolated yield. ${ }^{c}$ Calculation based on the recovery of 1 aa. ${ }^{d}$ Base $\left(1.0\right.$ eq).${ }^{e}$ Base $(0.2$ eq).

Table S2. Optimization Reaction Conditions: Copper Catalysts ${ }^{a}$

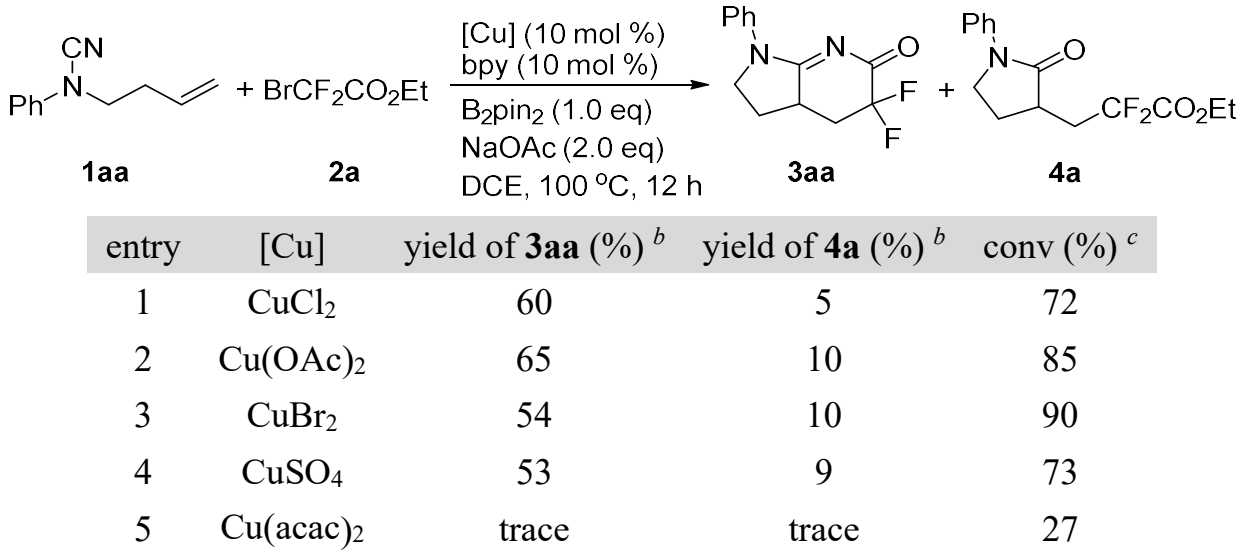




\begin{tabular}{ccccc}
6 & $\mathrm{CuI}$ & 44 & trace & 63 \\
7 & $\mathrm{CuBr}$ & 62 & 10 & 84 \\
8 & $\mathrm{CuCl}$ & 65 & 11 & 76 \\
9 & $\mathrm{Cu}_{2} \mathrm{O}$ & 73 & 9 & 85 \\
10 & $\mathrm{Cu}(\mathrm{TC})$ & 60 & 12 & 75 \\
\hline
\end{tabular}

${ }^{a}$ Reaction conditions: 1aa $(0.2 \mathrm{mmol}), 2 \mathrm{a}(0.4 \mathrm{mmol}), \mathrm{Cu}$ catalyst $(10 \mathrm{~mol} \%)$, bpy (10 mol \%), B2pinz $(0.2 \mathrm{mmol})$ and $\mathrm{NaOAc}(0.4$ mmol) in DCM $(1.5 \mathrm{~mL})$ at $100{ }^{\circ} \mathrm{C}$ under $\mathrm{N}_{2} .{ }^{b}$ Isolated yield. ${ }^{c}$ Calculation based on the recovery of 1 aa.

Table S3. Optimization Reaction Conditions: Ligands ${ }^{a}$

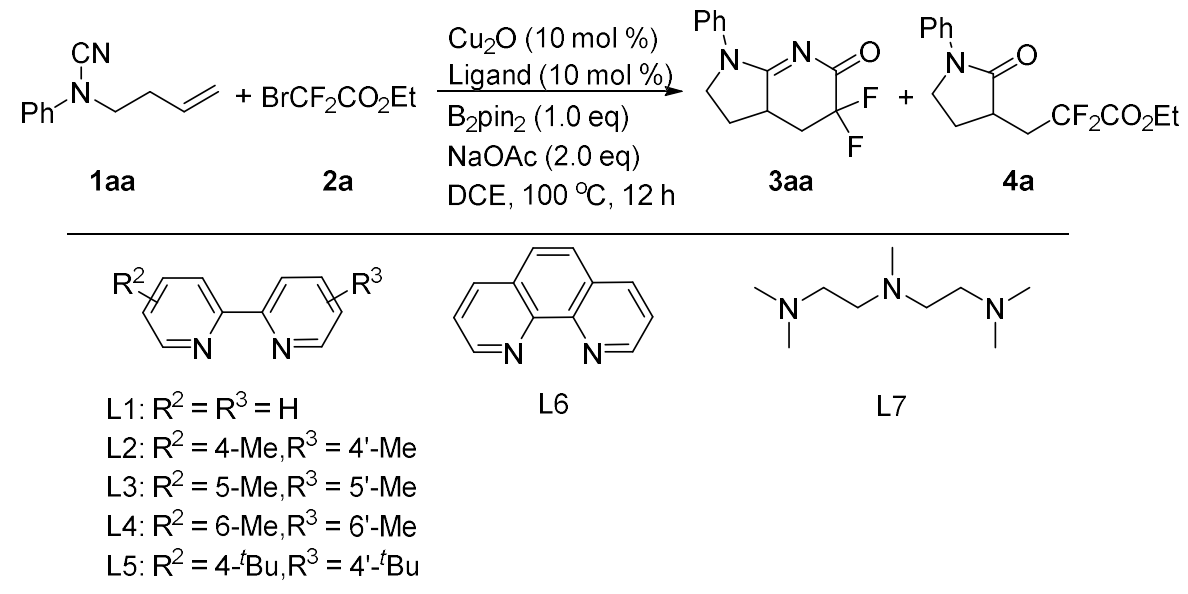

$\begin{array}{cccccc}\text { entry } & \text { ligand } & \mathrm{t}(\mathrm{h}) & {\text { yield of 3aa }(\%)^{b}}^{b} & {\text { yield of } \mathbf{4 a}(\%)^{b}}^{b} & \text { conv }(\%)^{c} \\ 1 & \text { L1 } & 12 & 73 & 9 & 85 \\ 2 & \text { L2 } & 12 & 71 & 11 & 100 \\ 3 & \text { L3 } & 12 & 61 & 13 & 100 \\ 4 & \text { L4 } & 12 & 19 & \text { trace } & 42 \\ 5 & \text { L5 } & 12 & 81 & 6 & 100 \\ 6 & \text { L6 } & 12 & 17 & 6 & 49 \\ 7 & \text { L7 } & 12 & 35 & 12 & 52 \\ 8 & \text { L5 } & 12 & 37 & 8 & 80\end{array}$

${ }^{a}$ Reaction conditions: 1aa $(0.2 \mathrm{mmol}), \mathbf{2 a}(0.4 \mathrm{mmol}), \mathrm{Cu}_{2} \mathrm{O}(10 \mathrm{~mol} \%)$, ligand $(10 \mathrm{~mol} \%), \mathrm{B}_{2} \mathrm{pin}_{2}(0.2 \mathrm{mmol})$ and $\mathrm{NaOAc}(0.4 \mathrm{mmol})$ in DCM $(1.5 \mathrm{~mL})$ at $100{ }^{\circ} \mathrm{C}$ under $\mathrm{N}_{2} .{ }^{b}$ Isolated yield. ${ }^{c}$ Calculation based on the recovery of 1 aa.

Table S4. Optimization reaction conditions: solvents ${ }^{a}$

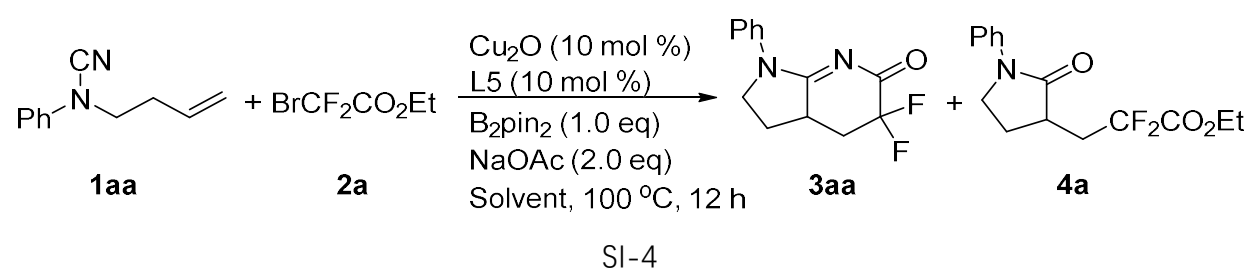




\begin{tabular}{ccccc} 
entry & solvent & yield of $\mathbf{3 a a}(\%)^{b}$ & yield of $\mathbf{4 a}(\%)^{b}$ & conv $(\%)^{c}$ \\
1 & $D C E$ & 81 & 6 & 100 \\
2 & 1,4-dioxane & 55 & trace & 80 \\
3 & $\mathrm{CH}_{3} \mathrm{CN}$ & 0 & trace & 28 \\
4 & DMSO & trace & trace & 58 \\
5 & DMF & 0 & trace & 33 \\
6 & Toluene & 50 & 5 & 85 \\
7 & THF & 57 & $>5$ & 68 \\
8 & Acetone & 53 & 7 & 73 \\
9 & Trifluorotoluene & 54 & trace & 96 \\
\hline
\end{tabular}

${ }^{a}$ Reaction conditions: 1aa $(0.2 \mathrm{mmol}), \mathbf{2 a}(0.4 \mathrm{mmol}), \mathrm{Cu}_{2} \mathrm{O}(10 \mathrm{~mol} \%), \mathrm{L} 5(10 \mathrm{~mol} \%), \mathrm{B}_{2} \mathrm{pin}_{2}(0.2 \mathrm{mmol})$ and $\mathrm{NaOAc}(0.4 \mathrm{mmol})$ in $\operatorname{DCM}(1.5 \mathrm{~mL})$ at $100{ }^{\circ} \mathrm{C}$ under $\mathrm{N}_{2} .{ }^{b}$ Isolated yield. ${ }^{c}$ Calculation based on the recovery of $\mathbf{1 a a}$.

Table S5. Optimization reaction conditions: other parameters ${ }^{a}$

\begin{tabular}{|c|c|c|c|c|c|c|c|}
\hline & ${ }_{1 \text { aa }}^{C N}$ & & $2 a$ & $\begin{array}{l}\mathrm{Cu}_{2} \\
\mathrm{Et} \\
\frac{\mathrm{L} 5(}{\mathrm{B}_{2} \mathrm{pi}} \\
\mathrm{NaC} \\
\mathrm{DCE}\end{array}$ & $\begin{array}{l}(10 \mathrm{~mol} \%) \\
0 \mathrm{~mol} \%) \\
\mathrm{p}_{2}(\mathrm{x} \text { eq }) \\
\begin{array}{l}\mathrm{Ac}(\mathrm{y} \text { eq }) \\
100^{\circ} \mathrm{C}: 12 \mathrm{~h}\end{array}\end{array}$ & $Y_{F}+{ }^{\mathrm{N}} Y^{\mathrm{O}}$ & \\
\hline entry & {$[\mathrm{Cu}] / \mathrm{L}$} & $\mathrm{x}$ & $\mathrm{y}$ & $\mathrm{T}\left({ }^{\circ} \mathrm{C}\right)$ & yield of $3 \mathbf{a a}(\%)^{b}$ & yield of $4 a(\%)^{b}$ & $\operatorname{conv}(\%)^{c}$ \\
\hline 1 & $\mathrm{Cu}_{2} \mathrm{O} / \mathrm{L} 5$ & 1.0 & 2.0 & 100 & 81 & 6 & 100 \\
\hline 2 & $\mathrm{Cu}_{2} \mathrm{O} / \mathrm{L} 5$ & 0.5 & 2.0 & 100 & 39 & 3 & 67 \\
\hline 3 & $\mathrm{Cu}_{2} \mathrm{O} / \mathrm{L} 5$ & 1.0 & 1.0 & 100 & 37 & 8 & 80 \\
\hline 4 & $\mathrm{Cu}_{2} \mathrm{O} / \mathrm{L} 5$ & 1.2 & 2.0 & 100 & 41 & 10 & 100 \\
\hline 5 & $\mathrm{Cu}_{2} \mathrm{O} / \mathrm{L} 5$ & 1.0 & 2.0 & 80 & 36 & 6 & 64 \\
\hline $6^{d}$ & $\mathrm{Cu}_{2} \mathrm{O} / \mathrm{L} 5$ & 1.0 & 2.0 & 100 & 76 & 6 & 100 \\
\hline $7^{e}$ & $\mathrm{Cu}_{2} \mathrm{O} / \mathrm{L} 5$ & 1.0 & 2.0 & 100 & 68 & 4 & 94 \\
\hline $8^{f}$ & $\mathrm{Cu}_{2} \mathrm{O} / \mathrm{L} 5$ & 1.0 & 2.0 & 100 & 40 & 13 & 68 \\
\hline $9^{g}$ & $\mathrm{Cu}_{2} \mathrm{O} / \mathrm{L} 5$ & 1.0 & 2.0 & 100 & 77 & 10 & 100 \\
\hline $10^{\mathrm{h}}$ & $\mathrm{Cu}_{2} \mathrm{O} / \mathrm{L} 5$ & 1.0 & 2.0 & 100 & 52 & 7 & 100 \\
\hline $11^{i}$ & $\mathrm{Cu}_{2} \mathrm{O} / \mathrm{L} 5$ & 1.0 & 2.0 & 100 & 69 & trace & 100 \\
\hline 12 & $\mathrm{Cu}_{2} \mathrm{O} /-$ & 1.0 & 2.0 & 100 & trace & nd & 29 \\
\hline 13 & $-/ \mathrm{L} 5$ & 1.0 & 2.0 & 100 & - & - & $\mathrm{nr}$ \\
\hline $14^{j}$ & $\mathrm{Cu}_{2} \mathrm{O} / \mathrm{L} 5$ & 1.0 & 2.0 & 100 & 69 & trace & 90 \\
\hline
\end{tabular}

${ }^{a}$ Reaction conditions: 1 aa $(0.2 \mathrm{mmol}), \mathbf{2 a}(0.4 \mathrm{mmol}), \mathrm{Cu}_{2} \mathrm{O}(10 \mathrm{~mol} \%), \mathrm{L} 5(10 \mathrm{~mol} \%), \mathrm{B}_{2} \mathrm{pin}_{2}(0.2 \mathrm{mmol})$ and $\mathrm{NaOAc}(0.4 \mathrm{mmol})$ in $\operatorname{DCM}(1.5 \mathrm{~mL})$ at $100{ }^{\circ} \mathrm{C}$ under $\mathrm{N}_{2} .{ }^{b}$ Isolated yield. ${ }^{c}$ Calculation based on the recovery of 1 aa. ${ }^{d} 4 \AA$ MS. ${ }^{e} \mathbf{2 a}\left(0.3 \mathrm{mmol}^{\prime} .{ }^{f} \mathrm{ICF}_{2} \mathrm{CO}_{2} \mathrm{Et}\right.$ 2b $(0.4 \mathrm{mmol}) .{ }^{g} \mathrm{DCE}(1.0 \mathrm{~mL}) \cdot{ }^{h} \mathrm{DCE}(2.0 \mathrm{~mL}) \cdot{ }^{i} \mathrm{~L} 5(20 \mathrm{~mol} \%) \cdot{ }^{j} \mathrm{Cu}_{2} \mathrm{O}(5 \mathrm{~mol} \%)$ and $\mathrm{L} 5(5 \mathrm{~mol} \%)$. 


\section{Preparation of Substrates}

1) Compounds 1aa-1sa, 1ad-1ag and 5g were prepared according to the known procedures, ${ }^{1,2}$ and the NMR spectra data of compounds1aa-1ha, 1 ja-1 ma, 1ad-1ag and $5 \mathrm{~g}$ were in accordance with the reported data of the known literatures. ${ }^{1,2}$ Compound 1ah was prepared according to the known procedure, ${ }^{3}$ and the NMR spectra data of compound 1ah were in accordance with the reported data of the known literature. ${ }^{3}$ Compound $2 \mathbf{c}$ was prepared according to the known literature, and the NMR spectra data were in accordance with the reported data of the known literature. ${ }^{4}$

2) Preparation of substrates 1 ta and $\mathbf{8}^{5,6}$

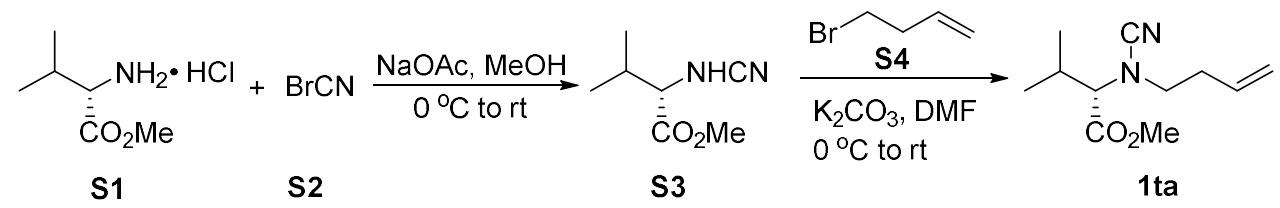

To a stirring solution of $\mathbf{S 1}$ (2 mmol, 1.0 equiv.) and $\mathrm{NaOAc}$ (3.0 equiv.) in $\mathrm{MeOH}(6.7 \mathrm{~mL}, 0.3 \mathrm{M})$ at $0{ }^{\circ} \mathrm{C}, \mathrm{BrCN}$ (1.2 equiv.) was added. Then the reaction was warmed to room temperature and stirred for $12 \mathrm{~h}$. Upon completion, the solvent was removed under reduced pressure. To the residue was added water and extracted with ethyl acetate, and the combined organic layers were washed by brine and dried over $\mathrm{Na}_{2} \mathrm{SO}_{4}$, then filtered and concentrated under reduced pressure. The residue was purified by flash column chromatography (silica gel, EtOAc/Petroleum ether $\left.\left(60-90^{\circ} \mathrm{C}\right)\right)$ to give compound $\mathbf{S 3}$.

Under a $\mathrm{N}_{2}$ atmosphere, to a two neck round bottom flask was added $\mathbf{S 3}$ (1 mmol, 1.0 equiv.), $\mathrm{K}_{2} \mathrm{CO}_{3}$ (2.0 equiv.) and DMF ( $3.3 \mathrm{~mL}, 0.3 \mathrm{M}$ ) at $0{ }^{\circ} \mathrm{C}$. The reaction mixture was stirred for half an hour. Then, bromide $\mathbf{S 4}$ (1.1 equiv.) was added dropwise at $0{ }^{\circ} \mathrm{C}$, and the resulting reaction mixture was warmed to room temperature and stirred for 14 h. Upon completion, $\mathrm{H}_{2} \mathrm{O}$ was added and the mixture was extracted with EtOAc, and the combined organic layers were washed by brine and dried over $\mathrm{Na}_{2} \mathrm{SO}_{4}$, then filtered and concentrated under reduced pressure. The residue was purified by flash column chromatography (silica gel, EtOAc/Petroleum ether $\left(60-90^{\circ} \mathrm{C}\right)$ ) to give compound 1 ta.

Compound $\mathbf{8}$ was prepared according to the same procedure.

3) Preparation of substrates 1ua-1va

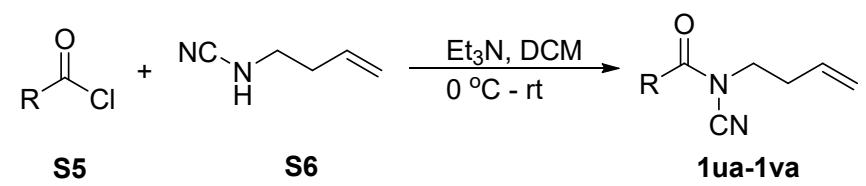

To a solution of $\mathbf{S 6}$ (1.0 mmol, 1.0 equiv.) and $\mathrm{Et}_{3} \mathrm{~N}$ (1.2 equiv.) in $\mathrm{DCM}(3.3 \mathrm{~mL}, 0.3 \mathrm{M})$ at $0{ }^{\circ} \mathrm{C}, \mathbf{S 5}$ (1.2 equiv.) was added. Then, the reaction was allowed to warm up to room temperature and stirred for 20-24 h. Upon completion, the resulting mixture was washed with $1 \mathrm{M} \mathrm{HCl}$, and extracted with DCM. The combined organic layers were washed by brine and dried over $\mathrm{Na}_{2} \mathrm{SO}_{4}$, then filtered and concentrated under reduced pressure. The residue was purified by flash column chromatography (silica gel, EtOAc/Petroleum ether $\left(60-90{ }^{\circ} \mathrm{C}\right)$ ) to give the desired compounds (1ua1va).

4) Preparation of substrates $\mathbf{1 a b - 1 a c , ~} \mathbf{5 a - 5 \mathbf { f } ^ { 7 }}$ 


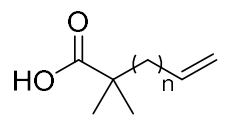

S7
1) $(\mathrm{COCl})_{2}, \mathrm{DMF}$ ( cat.), DCM, rt,

2) $\mathrm{Et}_{3} \mathrm{~N}, \mathrm{DCM}, \mathrm{O}^{\circ} \mathrm{C}-$

3) R-NHCN (S8), THF, $\mathrm{O}^{\circ} \mathrm{C}-\mathrm{rt}$

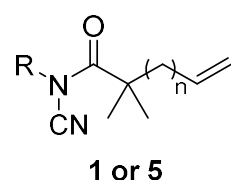

To a suspension of acid $\mathbf{S 7}$ (1.2 equiv.) in DCM (1 mL, 1.2 M) was added DMF (2 drops), followed by dropwise addition of $(\mathrm{COCl})_{2}(1.1$ equiv.) at room temperature. The mixture was stirred for 15-20 minutes, whereupon gas evolution ceased and all acid dissolved. The reaction flask was cooled to $-12{ }^{\circ} \mathrm{C}$ and a solution of $\mathrm{Et}_{3} \mathrm{~N}(2.4$ equiv.) in DCM (3.3 mL, 0.3 M) was dropwise added. Thereafter, a solution of cyanamide $\mathbf{S 8}$ (1.0 mmol, 1.0 equiv.) in $\mathrm{CH}_{2} \mathrm{Cl}_{2}$ /THF $(3 / 1,1.2 \mathrm{M}$ ) was slowly added at the same temperature. The resulting mixture was allowed to warm up to room temperature and stirred for 20-24 h. Upon completion, $\mathrm{Et}_{2} \mathrm{O}(30 \mathrm{~mL})$ was added to allow precipitation of triethylamine hydrochloride, which was filtrated through a short Celite column. Without further treatment, the filtrate was concentrated under reduced pressure, and the resulting oily residue was purified by flash column chromatography (silica gel, EtOAc/Petroleum ether $\left(60-90{ }^{\circ} \mathrm{C}\right)$ ) to afford the desired compounds (1ab-1ac, 5a-5c and 5e-5f).

Under slightly modified reaction condition, compound $\mathbf{5 d}$ was prepared. To a solution of $\mathbf{S 8}$ (1.3 equiv.), Et ${ }_{3} \mathrm{~N}$ (3.0 equiv.) and DMAP (0.005 equiv.) in anhydrous DCM $(5 \mathrm{~mL}, 0.2 \mathrm{M})$ was added the corresponding acyl chloride (1 mmol, 1.0 equiv.) (prepared from the aforementioned procedure) under a $\mathrm{N}_{2}$ atmosphere. Then the reaction mixture was stirred for $19 \mathrm{~h}$. The reaction was quenched with saturated aqueous $\mathrm{NaHCO}_{3}$ and the mixture was extracted with DCM. The combined organic layers were washed by brine and dried over $\mathrm{Na}_{2} \mathrm{SO}_{4}$, then filtered and concentrated under reduced pressure. The residue was purified by flash column chromatography (silica gel, EtOAc/Petroleum ether $\left(60-90^{\circ} \mathrm{C}\right)$ ) to give compound $\mathbf{5 d}$.

The NMR spectra data of compounds 1 ab were in accordance with the reported data. ${ }^{1}$<smiles>C=CCCN(C#N)c1ccccc1</smiles>

Purified by column chromatography (silica gel, EtOAc/Petroleum ether: 1/12) to afford the title compound (89\% yield, $153 \mathrm{mg}$ ) as a colorless oil. ${ }^{1} \mathrm{H}$ NMR $\left(500 \mathrm{MHz}, \mathrm{CDCl}_{3}\right) \delta 7.42-7.35(\mathrm{~m}, 2 \mathrm{H}), 7.17-7.09(\mathrm{~m}, 3 \mathrm{H}), 5.93-5.81$ $(\mathrm{m}, 1 \mathrm{H}), 5.26-5.14(\mathrm{~m}, 2 \mathrm{H}), 3.67(\mathrm{t}, J=7.3 \mathrm{~Hz}, 2 \mathrm{H}), 2.59$ (q, $J=7.0 \mathrm{~Hz}, 2 \mathrm{H}) .{ }^{13} \mathrm{C}$ NMR $\left(100 \mathrm{MHz}, \mathrm{CDCl}_{3}\right) \delta 139.8$, 133.0, 129.7, 123.6, 118.6, 116.0, 113.5, 48.8, 31.6.<smiles>C=CCCN(C#N)c1ccc(Cl)cc1</smiles>

Purified by column chromatography (silica gel, EtOAc/Petroleum ether: 1/12) to afford the title compound (85\% yield, $176 \mathrm{mg})$ as a colorless oil. ${ }^{1} \mathrm{H}$ NMR $\left(400 \mathrm{MHz}, \mathrm{CDCl}_{3}\right) \delta 7.36-7.30(\mathrm{~m}, 2 \mathrm{H}), 7.08-7.02(\mathrm{~m}, 2 \mathrm{H}), 5.88-5.76$ $(\mathrm{m}, 1 \mathrm{H}), 5.22-5.15(\mathrm{~m}, 2 \mathrm{H}), 3.63(\mathrm{t}, J=7.2 \mathrm{~Hz}, 2 \mathrm{H}), 2.56(\mathrm{q}, J=7.0 \mathrm{~Hz}, 2 \mathrm{H}) .{ }^{13} \mathrm{C}$ NMR $\left(100 \mathrm{MHz}, \mathrm{CDCl}_{3}\right) \delta 138.5$, 132.8, 129.7, 129.0, 118.8, 117.3, 113.0, 49.0, 31.6. 
1ca

Purified by column chromatography (silica gel, EtOAc/Petroleum ether: 1/12) to afford the title compound (90\% yield, $226 \mathrm{mg}$ ) as a colorless oil. ${ }^{1} \mathrm{H}$ NMR $\left(400 \mathrm{MHz}, \mathrm{CDCl}_{3}\right) \delta 7.54-7.42(\mathrm{~m}, 2 \mathrm{H}), 7.07-6.91(\mathrm{~m}, 2 \mathrm{H}), 5.91-5.73$ $(\mathrm{m}, 1 \mathrm{H}), 5.25-5.09(\mathrm{~m}, 2 \mathrm{H}), 3.63(\mathrm{t}, J=7.2 \mathrm{~Hz}, 2 \mathrm{H}), 2.56(\mathrm{q}, J=7.0 \mathrm{~Hz}, 2 \mathrm{H}) .{ }^{13} \mathrm{C} \mathrm{NMR}\left(100 \mathrm{MHz}, \mathrm{CDCl}_{3}\right) \delta 139.1$, $132.8,132.6,118.9,117.6,116.4,112.9,49.0,31.6$.<smiles>C=CCCN(C#N)c1ccc(OC)cc1</smiles>

Purified by column chromatography (silica gel, EtOAc/Petroleum ether: 1/12) to afford the title compound (90\% yield, $182 \mathrm{mg}$ ) as a colorless oil. ${ }^{1} \mathrm{H}$ NMR $\left(400 \mathrm{MHz}, \mathrm{CDCl}_{3}\right) \delta 7.11-7.02(\mathrm{~m}, 2 \mathrm{H}), 6.97-6.80(\mathrm{~m}, 2 \mathrm{H}), 5.90-5.74$ $(\mathrm{m}, 1 \mathrm{H}), 5.22-5.11(\mathrm{~m}, 2 \mathrm{H}), 3.79(\mathrm{~s}, 3 \mathrm{H}), 3.59(\mathrm{t}, J=7.3 \mathrm{~Hz}, 2 \mathrm{H}), 2.53(\mathrm{q}, J=7.0 \mathrm{~Hz}, 2 \mathrm{H}) .{ }^{13} \mathrm{C} \mathrm{NMR}(100 \mathrm{MHz}$, $\left.\mathrm{CDCl}_{3}\right) \delta 156.4,133.2,133.2,118.5,118.4,114.9,114.5,55.6,49.8,31.8$.<smiles>C=CCCN(C#N)c1ccc(C)cc1</smiles>

Purified by column chromatography (silica gel, EtOAc/Petroleum ether: 1/15) to afford the title compound (88\% yield, $164 \mathrm{mg})$ as a colorless oil. ${ }^{1} \mathrm{H}$ NMR $\left(400 \mathrm{MHz}, \mathrm{CDCl}_{3}\right) \delta 7.19-7.12(\mathrm{~m}, 2 \mathrm{H}), 7.06-6.96(\mathrm{~m}, 2 \mathrm{H}), 5.83(\mathrm{~m}$, $1 \mathrm{H}), 5.26-5.08(\mathrm{~m}, 2 \mathrm{H}), 3.62(\mathrm{t}, J=7.2 \mathrm{~Hz}, 2 \mathrm{H}), 2.60-2.49(\mathrm{~m}, 2 \mathrm{H}), 2.32(\mathrm{~s}, 3 \mathrm{H}) .{ }^{13} \mathrm{C} \mathrm{NMR}\left(100 \mathrm{MHz}, \mathrm{CDCl}_{3}\right) \delta$ $137.4,133.4,133.2,130.2,118.5,116.2,113.9,49.0,31.7,20.6$.<smiles>C=CCCN(C#N)c1ccc(C(=O)OCC)cc1</smiles>

Purified by column chromatography (silica gel, EtOAc/Petroleum ether: $1 / 5$ ) to afford the title compound (75\% yield, $183 \mathrm{mg}$ ) as a colorless oil. ${ }^{1} \mathrm{H}$ NMR $\left(500 \mathrm{MHz}, \mathrm{CDCl}_{3}\right) \delta 8.06(\mathrm{~d}, J=8.9 \mathrm{~Hz}, 2 \mathrm{H}), 7.15(\mathrm{~d}, J=8.9 \mathrm{~Hz}, 1 \mathrm{H}), 5.84$ (ddt, $J=17.1,10.3,6.8 \mathrm{~Hz}, 1 \mathrm{H}), 5.25-5.15(\mathrm{~m}, 2 \mathrm{H}), 4.37$ (q, $J=7.1 \mathrm{~Hz}, 2 \mathrm{H}), 3.72(\mathrm{t}, J=7.2 \mathrm{~Hz}, 2 \mathrm{H}), 2.60$ (q, $J=7.0$ $\mathrm{Hz}, 1 \mathrm{H}), 1.39(\mathrm{t}, J=7.1 \mathrm{~Hz}, 3 \mathrm{H}) .{ }^{13} \mathrm{C}$ NMR $\left(125 \mathrm{MHz}, \mathrm{CDCl}_{3}\right) \delta 165.8,143.7,132.7,131.4,125.7,119.0,115.1$, $112.4,61.0,48.7,31.6,14.3$.<smiles>C=CCCN(C)c1cccc(C)c1</smiles>

Purified by column chromatography (silica gel, EtOAc/Petroleum ether: 1/12) to afford the title compound (87\% yield, $162 \mathrm{mg})$ as a colorless oil. ${ }^{1} \mathrm{H}$ NMR $\left(400 \mathrm{MHz}, \mathrm{CDCl}_{3}\right) \delta 7.25(\mathrm{t}, J=7.9 \mathrm{~Hz}, 1 \mathrm{H}), 6.98-6.86(\mathrm{~m}, 3 \mathrm{H}), 5.90-$ 
$5.78(\mathrm{~m}, 1 \mathrm{H}), 5.23-5.13(\mathrm{~m}, 2 \mathrm{H}), 3.64(\mathrm{t}, J=7.0 \mathrm{~Hz}, 2 \mathrm{H}), 2.62-2.52(\mathrm{~m}, 2 \mathrm{H}), 2.36(\mathrm{~s}, 3 \mathrm{H}) .{ }^{13} \mathrm{C}$ NMR $(100 \mathrm{MHz}$, $\left.\mathrm{CDCl}_{3}\right) \delta 139.9,139.8,133.1,129.5,124.5,118.5,116.8,113.7,113.0,48.8,31.7,21.6$.<smiles>C=CCCN(C)c1ccccc1C</smiles>

Purified by column chromatography (silica gel, EtOAc/Petroleum ether: 1/12) to afford the title compound (90\% yield, $168 \mathrm{mg}$ ) as a colorless oil. ${ }^{1} \mathrm{H}$ NMR $\left(400 \mathrm{MHz}, \mathrm{CDCl}_{3}\right) \delta 7.26-7.16(\mathrm{~m}, 4 \mathrm{H}), 5.88-5.70(\mathrm{~m}, 1 \mathrm{H}), 5.24-5.10$ (m, 2H), 3.49 (t, $J=7.3 \mathrm{~Hz}, 2 \mathrm{H}), 2.56-2.44(\mathrm{~m}, 2 \mathrm{H}), 2.39$ (s, 3H). ${ }^{13} \mathrm{C}$ NMR $\left(100 \mathrm{MHz}, \mathrm{CDCl}_{3}\right) \delta 139.1,134.0$, $133.5,131.8,127.7,127.2,124.7,118.2,115.5,53.1,32.1,17.9$.<smiles>C=CCCN(C#N)c1cc(C)cc(C)c1</smiles>

Purified by column chromatography (silica gel, EtOAc/Petroleum ether: $1 / 10$ ) to afford the title compound ( $83 \%$ yield, $166 \mathrm{mg})$ as a colorless oil. ${ }^{1} \mathrm{H}$ NMR $\left(500 \mathrm{MHz}, \mathrm{CDCl}_{3}\right) \delta 6.73(\mathrm{~s}, 3 \mathrm{H}), 5.93-5.65(\mathrm{~m}, 1 \mathrm{H}), 5.29-5.14(\mathrm{~m}$, 2H), $3.61(\mathrm{t}, J=7.3 \mathrm{~Hz}, 2 \mathrm{H}), 2.68-2.45(\mathrm{~m}, 2 \mathrm{H}), 2.31(\mathrm{~s}, 6 \mathrm{H}) .{ }^{13} \mathrm{C} \mathrm{NMR}\left(125 \mathrm{MHz}, \mathrm{CDCl}_{3}\right) \delta 139.8,139.7,133.3$, 125.5, 118.5, 113.9, 48.8, 31.8, 21.5. IR (KBr): 2920, 2360, 2216, 1598, 1181, 832, $669 \mathrm{~cm}^{-1}$. HRMS (ESI) m/z: [M $+\mathrm{H}]^{+}$Calcd for $\mathrm{C}_{13} \mathrm{H}_{17} \mathrm{~N}_{2} 201.1386$; Found 201.1387.<smiles>C=CCCN(C#N)c1ccc(C)c(C)c1</smiles>

1 ja

Purified by column chromatography (silica gel, EtOAc/Petroleum ether: $1 / 10)$ to afford the title compound ( $80 \%$ yield, $160 \mathrm{mg}$ ) as a colorless oil. ${ }^{1} \mathrm{H}$ NMR $\left(500 \mathrm{MHz}, \mathrm{CDCl}_{3}\right) \delta 7.11(\mathrm{~d}, J=8.2 \mathrm{~Hz}, 1 \mathrm{H}), 6.93(\mathrm{~d}, J=2.7 \mathrm{~Hz}, 1 \mathrm{H})$, $6.82(\mathrm{dd}, J=8.2,2.7 \mathrm{~Hz}, 1 \mathrm{H}), 5.91-5.71(\mathrm{~m}, 1 \mathrm{H}), 5.39-4.92(\mathrm{~m}, 2 \mathrm{H}), 3.61(\mathrm{t}, J=7.3 \mathrm{~Hz}, 2 \mathrm{H}), 2.62-2.46(\mathrm{~m}, 2 \mathrm{H})$, $2.27(\mathrm{~s}, 3 \mathrm{H}), 2.22(\mathrm{~s}, 3 \mathrm{H}) .{ }^{13} \mathrm{C} \mathrm{NMR}\left(100 \mathrm{MHz}, \mathrm{CDCl}_{3}\right) \delta 138.2,137.6,133.2,132.1,130.6,118.4,117.7,114.1$, $113.4,49.0,31.7,20.0,19.0$.<smiles>C=CCCN(C#N)c1cccc2ccccc12</smiles>

Purified by column chromatography (silica gel, EtOAc/Petroleum ether: 1/12) to afford the title compound (81\% yield, $180 \mathrm{mg})$ as a colorless oil. ${ }^{1} \mathrm{H}$ NMR $\left(400 \mathrm{MHz}, \mathrm{CDCl}_{3}\right) \delta 7.88-7.70(\mathrm{~m}, 3 \mathrm{H}), 7.54-7.34(\mathrm{~m}, 4 \mathrm{H}), 5.96-5.80$ $(\mathrm{m}, 1 \mathrm{H}), 5.25-5.15(\mathrm{~m}, 2 \mathrm{H}), 3.81-3.71(\mathrm{~m}, 2 \mathrm{H}), 2.63(\mathrm{q}, J=7.0 \mathrm{~Hz}, 2 \mathrm{H}) \cdot{ }^{13} \mathrm{C}$ NMR $\left(100 \mathrm{MHz}, \mathrm{CDCl}_{3}\right) \delta 137.4$, 133.8, 133.1, 130.1, 130.0, 127.8, 127.2, 127.2, 125.3, 118.7, 116.5, 113.7, 112.2, 49.0, 31.7. 
<smiles>C=CCCN(C#N)c1ccc2c(ccn2[13CH3])c1</smiles>

Purified by column chromatography (silica gel, EtOAc/Petroleum ether: $1 / 8$ ) to afford the title compound (75\% yield, $274 \mathrm{mg}$ ) as a yellow oil. ${ }^{1} \mathrm{H}$ NMR $\left(500 \mathrm{MHz}, \mathrm{CDCl}_{3}\right) \delta 7.96(\mathrm{~d}, J=9.0 \mathrm{~Hz}, 1 \mathrm{H}), 7.74(\mathrm{~d}, J=8.4 \mathrm{~Hz}, 2 \mathrm{H}), 7.58(\mathrm{~d}, J$ $=3.6 \mathrm{~Hz}, 1 \mathrm{H}), 7.25-7.21(\mathrm{~m}, 3 \mathrm{H}), 7.13(\mathrm{dd}, \mathrm{J}=9.0,2.4 \mathrm{~Hz}, 1 \mathrm{H}), 6.61(\mathrm{~d}, J=3.6 \mathrm{~Hz}, 1 \mathrm{H}), 5.94-5.72(\mathrm{~m}, 1 \mathrm{H}), 5.29$ $-5.06(\mathrm{~m}, 2 \mathrm{H}), 3.65(\mathrm{t}, J=7.3 \mathrm{~Hz}, 2 \mathrm{H}), 2.55(\mathrm{~m}, 2 \mathrm{H}), 2.34(\mathrm{~s}, 3 \mathrm{H}) .{ }^{13} \mathrm{C}$ NMR $\left(125 \mathrm{MHz}, \mathrm{CDCl}_{3}\right) \delta 145.2,136.2$, 135.0, 133.1, 131.7, 131.5, 130.0, 128.0, 126.8, 118.6, 114.6, 114.4, 114.1, 108.9, 108.8, 49.7, 31.7, 21.6.

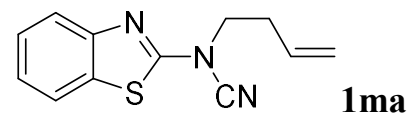

Purified by column chromatography (silica gel, EtOAc/Petroleum ether: $1 / 8$ ) to afford the title compound (64\% yield, $147 \mathrm{mg}$ ) as a yellow solid. mp: $44-45^{\circ} \mathrm{C} .{ }^{1} \mathrm{H}$ NMR $\left(300 \mathrm{MHz}, \mathrm{CDCl}_{3}\right) \delta 7.80-7.68(\mathrm{~m}, 2 \mathrm{H}), 7.49-7.39(\mathrm{~m}, 1 \mathrm{H})$, $7.33-7.27(\mathrm{~m}, 1 \mathrm{H}), 5.94-5.77(\mathrm{~m}, 1 \mathrm{H}), 5.29-5.14(\mathrm{~m}, 2 \mathrm{H}), 4.04(\mathrm{t}, J=7.1 \mathrm{~Hz}, 2 \mathrm{H}), 2.67(\mathrm{q}, J=7.0 \mathrm{~Hz}, 2 \mathrm{H}) .{ }^{13} \mathrm{C}$ NMR (125 MHz, $\left.\mathrm{CDCl}_{3}\right) \delta 161.5,151.1,132.7,132.3,126.7,124.4,121.7,121.5,119.1,111.3,50.1,31.8$.

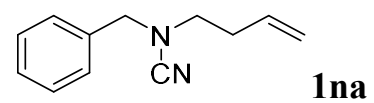

Purified by column chromatography (silica gel, EtOAc/Petroleum ether: 1/10) to afford the title compound (84\% yield, $156 \mathrm{mg}$ ) as a colorless oil. ${ }^{1} \mathrm{H}$ NMR $\left(300 \mathrm{MHz}, \mathrm{CDCl}_{3}\right) \delta 7.44-7.29(\mathrm{~m}, 5 \mathrm{H}), 5.88-5.64(\mathrm{~m}, 1 \mathrm{H}), 5.21-5.07$ (m, 2H), 4.20 (s, 2H), 3.01 (t, $J=7.2 \mathrm{~Hz}, 2 \mathrm{H}), 2.49-2.33(\mathrm{~m}, 2 \mathrm{H}) .{ }^{13} \mathrm{C}$ NMR $\left(125 \mathrm{MHz}, \mathrm{CDCl}_{3}\right) \delta 134.8,133.8$, 129.0, 128.718, 128.5, 118.1, 117.9, 56.0, 49.8, 32.0. IR (KBr): 2932, 2875, 2208, 1641, 1455, 920, 728, $699 \mathrm{~cm}^{-1}$. HRMS (ESI) m/z: [M + H] Calcd for $\mathrm{C}_{12} \mathrm{H}_{15} \mathrm{~N}_{2}$ 187.1230; Found 187.1225.<smiles>CN(CCCC#N)CCc1ccccc1</smiles>

Purified by column chromatography (silica gel, EtOAc/Petroleum ether: $1 / 10)$ to afford the title compound (77\% yield, $154 \mathrm{mg}$ ) as a colorless oil. ${ }^{1} \mathrm{H}$ NMR $\left(500 \mathrm{MHz}, \mathrm{CDCl}_{3}\right) \delta 7.37-7.29(\mathrm{~m}, 2 \mathrm{H}), 7.28-7.15(\mathrm{~m}, 3 \mathrm{H}), 5.72$ (ddt, $J=17.1,10.2,6.8 \mathrm{~Hz}, 1 \mathrm{H}), 5.21-4.92(\mathrm{~m}, 2 \mathrm{H}), 3.29-3.17(\mathrm{~m}, 2 \mathrm{H}), 3.01(\mathrm{t}, J=7.2 \mathrm{~Hz}, 2 \mathrm{H}), 2.95(\mathrm{t}, J=7.2 \mathrm{~Hz}$, $2 \mathrm{H}), 2.44-2.20(\mathrm{~m}, 2 \mathrm{H}) .{ }^{13} \mathrm{C} \mathrm{NMR}\left(100 \mathrm{MHz}, \mathrm{CDCl}_{3}\right) \delta 137.7,133.8,128.8,128.8,126.9,118.0,117.4,53.2,51.5$, 34.4, 32.2. IR (KBr): 2933, 2873, 2206, 1454, 920, $700 \mathrm{~cm}^{-1}$. HRMS (ESI) m/z: $[\mathrm{M}+\mathrm{H}]^{+}$Calcd for $\mathrm{C}_{13} \mathrm{H}_{17} \mathrm{~N}_{2}$ 201.1386; Found 201.1380.<smiles>C=CCCN(C)[C@@H](C)c1ccccc1</smiles>

Purified by column chromatography (silica gel, EtOAc/Petroleum ether: $1 / 5$ ) to afford the title compound ( $84 \%$ yield, $123 \mathrm{mg})$ as a colorless oil. ${ }^{1} \mathrm{H}$ NMR $\left(500 \mathrm{MHz}, \mathrm{CDCl}_{3}\right) \delta 7.41-7.35(\mathrm{~m}, 2 \mathrm{H}), 7.35-7.30(\mathrm{~m}, 3 \mathrm{H}), 5.83-5.56(\mathrm{~m}$, 
1H), $5.15-4.94(\mathrm{~m}, 2 \mathrm{H}), 4.10(\mathrm{q}, J=6.9 \mathrm{~Hz}, 1 \mathrm{H}), 3.01-2.92(\mathrm{~m}, 1 \mathrm{H}), 2.91-2.74(\mathrm{~m}, 1 \mathrm{H}), 2.47-2.22(\mathrm{~m}, 2 \mathrm{H})$, $1.64(\mathrm{~d}, J=7.0 \mathrm{~Hz}, 3 \mathrm{H}) .{ }^{13} \mathrm{C} \mathrm{NMR}\left(125 \mathrm{MHz}, \mathrm{CDCl}_{3}\right) \delta 140.8,133.9,129.0,128.4,126.6,117.9,116.5,59.7,49.6$, 32.0, 21.3. IR (KBr): 2979, 2932, 2205, 1494, 920, $701 \mathrm{~cm}^{-1}$. HRMS (ESI) m/z: $[\mathrm{M}+\mathrm{H}]^{+}$Calcd for $\mathrm{C}_{13} \mathrm{H}_{17} \mathrm{~N}_{2}$ 201.1386; Found: 201.1390.

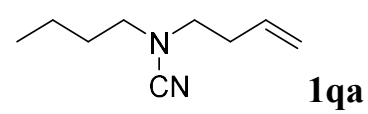

Purified by column chromatography (silica gel, EtOAc/Petroleum ether: 1/10) to afford the title compound (44\% yield, $67 \mathrm{mg})$ as a colorless oil. ${ }^{1} \mathrm{H}$ NMR $\left(300 \mathrm{MHz}, \mathrm{CDCl}_{3}\right) \delta 5.90-5.67(\mathrm{~m}, 1 \mathrm{H}), 5.34-5.01(\mathrm{~m}, 2 \mathrm{H}), 3.06(\mathrm{t}, J=$ $7.2 \mathrm{~Hz}, 2 \mathrm{H}), 3.00(\mathrm{t}, J=6.0 \mathrm{~Hz}, 2 \mathrm{H}), 2.50-2.33(\mathrm{~m}, 2 \mathrm{H}), 1.75-1.54(\mathrm{~m}, 2 \mathrm{H}), 1.51-1.29(\mathrm{~m}, 2 \mathrm{H}), 0.95(\mathrm{t}, J=7.3$ $\mathrm{Hz}, 3 \mathrm{H}) .{ }^{13} \mathrm{C}$ NMR $\left(125 \mathrm{MHz}, \mathrm{CDCl}_{3}\right) \delta 133.8,117.8,117.6,51.3,51.0,32.1,29.7,19.7,13.6$. IR (KBr): 2961, 2934, 2207, 1642, 1460, 1099, $919 \mathrm{~cm}^{-1}$. HRMS (ESI) m/z: [M+ H] ${ }^{+}$Calcd for $\mathrm{C}_{9} \mathrm{H}_{17} \mathrm{~N}_{2}$ 153.1386; Found: 153.1380 .<smiles>CC=CCCN(C#N)C(C)(C)C</smiles>

Purified by column chromatography (silica gel, EtOAc/Petroleum ether: $1 / 10)$ to afford the title compound (83\% yield, $126 \mathrm{mg})$ as a colorless oil. ${ }^{1} \mathrm{H}$ NMR $\left(500 \mathrm{MHz}, \mathrm{CDCl}_{3}\right) \delta 5.91-5.57(\mathrm{~m}, 1 \mathrm{H}), 5.31-4.84(\mathrm{~m}, 2 \mathrm{H}), 3.00(\mathrm{t}, J$ $=7.4 \mathrm{~Hz}, 2 \mathrm{H}), 2.75-2.30(\mathrm{~m}, 2 \mathrm{H}), 1.30(\mathrm{~s}, 9 \mathrm{H}) .{ }^{13} \mathrm{C} \mathrm{NMR}\left(125 \mathrm{MHz}, \mathrm{CDCl}_{3}\right) \delta 134.1,117.6,116.4,56.0,44.9,33.0$, 27.6. IR (KBr): 2979, 2201, 1578, 1370, 1192, $918 \mathrm{~cm}^{-1}$. HRMS (ESI) m/z: $[\mathrm{M}+\mathrm{H}]^{+}$Calcd for $\mathrm{C}_{9} \mathrm{H}_{17} \mathrm{~N}_{2} 153.1386$; Found: 153.1388 .<smiles>N#CC1CCCCC1</smiles>

Purified by column chromatography (silica gel, EtOAc/Petroleum ether: $1 / 10)$ to afford the title compound (75\% yield, $134 \mathrm{mg})$ as a colorless oil. ${ }^{1} \mathrm{H}$ NMR $\left(300 \mathrm{MHz}, \mathrm{CDCl}_{3}\right) \delta 5.95-5.65(\mathrm{~m}, 1 \mathrm{H}), 5.26-4.95(\mathrm{~m}, 2 \mathrm{H}), 3.08(\mathrm{t}, J$ $=7.3 \mathrm{~Hz}, 2 \mathrm{H}), 2.80-2.62(\mathrm{~m}, 1 \mathrm{H}), 2.49-2.32(\mathrm{~m}, 2 \mathrm{H}), 2.04-1.90(\mathrm{~m}, 2 \mathrm{H}), 1.90-1.76(\mathrm{~m}, 2 \mathrm{H}), 1.69-1.62(\mathrm{~m}$, $1 \mathrm{H}), 1.56-1.32(\mathrm{~m}, 2 \mathrm{H}), 1.32-1.11(\mathrm{~m}, 3 \mathrm{H}) .{ }^{13} \mathrm{C} \mathrm{NMR}\left(125 \mathrm{MHz}, \mathrm{CDCl}_{3}\right) \delta 134.0,117.7,116.5,59.4,49.4,32.6$, 31.1, 25.2, 25.1. IR (KBr): 2934, 2858, 2203, 1451, 1098, $917 \mathrm{~cm}^{-1}$. HRMS (ESI) m/z: $[\mathrm{M}+\mathrm{H}]^{+} \mathrm{Calcd}$ for $\mathrm{C}_{11} \mathrm{H}_{19} \mathrm{~N}_{2}$ 179.1543; Found 179.1540.

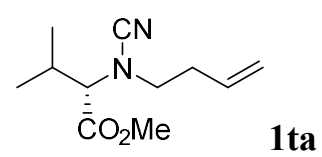

Purified by column chromatography (silica gel, EtOAc/Petroleum ether: $1 / 10)$ to afford the title compound (70\% yield, $147 \mathrm{mg})$ as a colorless oil. $\mathrm{H} \mathrm{NMR}\left(300 \mathrm{MHz}, \mathrm{CDCl}_{3}\right) \delta 5.85-5.61(\mathrm{~m}, 1 \mathrm{H}), 5.28-5.02(\mathrm{~m}, 2 \mathrm{H}), 3.80(\mathrm{~s}$, $3 \mathrm{H}), 3.25-3.11(\mathrm{~m}, 1 \mathrm{H}), 3.09-2.94(\mathrm{~m}, 2 \mathrm{H}), 2.52-2.33(\mathrm{~m}, 2 \mathrm{H}), 2.31-2.14(\mathrm{~m}, 1 \mathrm{H}), 1.11(\mathrm{~d}, J=6.6 \mathrm{~Hz}, 3 \mathrm{H})$, $0.97(\mathrm{~d}, J=6.7 \mathrm{~Hz}, 3 \mathrm{H}) .{ }^{13} \mathrm{C} \mathrm{NMR}\left(125 \mathrm{MHz}, \mathrm{CDCl}_{3}\right) \delta 170.8,133.4,118.1,115.1,69.5,52.3,51.7,32.0,29.5,19.8$, 19.0. IR (KBr): 2961, 2919, 2361, 2209, 1741, 1578, $1195 \mathrm{~cm}^{-1}$. HRMS (ESI) m/z: [M+ H] Calcd for $\mathrm{C}_{11} \mathrm{H}_{19} \mathrm{~N}_{2} \mathrm{O}_{2}$ 211.1441; Found 211.1433. 
<smiles>C=CCCN(C#N)C(C)=O</smiles>

Purified by column chromatography (silica gel, EtOAc/Petroleum ether: 1/20) to afford the title compound (75\% yield, $104 \mathrm{mg})$ as a colorless oil. ${ }^{1} \mathrm{H}$ NMR $\left(500 \mathrm{MHz}, \mathrm{CDCl}_{3}\right) \delta 5.83-5.56(\mathrm{~m}, 1 \mathrm{H}), 5.31-4.96(\mathrm{~m}, 2 \mathrm{H}), 3.64(\mathrm{t}, J$ $=7.0 \mathrm{~Hz}, 2 \mathrm{H}), 2.48-2.40(\mathrm{~m}, 2 \mathrm{H}), 2.39(\mathrm{~s}, 3 \mathrm{H}) .{ }^{13} \mathrm{C} \mathrm{NMR}\left(125 \mathrm{MHz}, \mathrm{CDCl}_{3}\right) \delta 169.3,132.9,118.8,110.9,45.3$, 32.0, 22.2. IR (KBr): 2234, 1729, 1246, 997, $925 \mathrm{~cm}^{-1}$. HRMS (ESI) m/z: $[\mathrm{M}+\mathrm{H}]^{+}$Calcd for $\mathrm{C}_{7} \mathrm{H}_{11} \mathrm{~N}_{2} \mathrm{O} 139.0866$; Found 139.0864 .<smiles>CC=CCCN(C#N)C(=O)C(C)(C)C</smiles>

Purified by column chromatography (silica gel, EtOAc/Petroleum ether: 1/20) to afford the title compound (77\% yield, $139 \mathrm{mg})$ as a colorless oil. ${ }^{1} \mathrm{H}$ NMR $\left(500 \mathrm{MHz}, \mathrm{CDCl}_{3}\right) \delta 5.98-5.63(\mathrm{~m}, 1 \mathrm{H}), 5.36-4.95(\mathrm{~m}, 2 \mathrm{H}), 3.68(\mathrm{t}, J$ $=6.9 \mathrm{~Hz}, 2 \mathrm{H}), 2.62-2.24(\mathrm{~m}, 2 \mathrm{H}), 1.40(\mathrm{~s}, 9 \mathrm{H}) .{ }^{13} \mathrm{C} \mathrm{NMR}\left(125 \mathrm{MHz}, \mathrm{CDCl}_{3}\right) \delta 176.9,133.1,118.7,111.6,47.6$, 41.2, 32.1, 26.8. IR (KBr): 2981, 2939, 2231, 1713, 1172, $923 \mathrm{~cm}^{-1}$. HRMS (ESI) m/z: [M+ H] $]^{+}$Calcd for $\mathrm{C}_{10} \mathrm{H}_{17} \mathrm{~N}_{2} \mathrm{O}$ 181.1335; Found 181.1330.<smiles>C=CC(C)(C)C(=O)N(C)c1ccccc1</smiles>

Purified by column chromatography (silica gel, EtOAc/Petroleum ether: $1 / 20)$ to afford the title compound (61\% yield, $131 \mathrm{mg}$ ) as a colorless oil. ${ }^{1} \mathrm{H}$ NMR $\left(300 \mathrm{MHz}, \mathrm{CDCl}_{3}\right) \delta 7.54-7.35(\mathrm{~m}, 3 \mathrm{H}), 7.34-7.27(\mathrm{~m}, 2 \mathrm{H}), 6.19$ (dd, $J=17.5,10.7 \mathrm{~Hz}, 1 \mathrm{H}), 5.40(\mathrm{~d}, J=10.6 \mathrm{~Hz}, 1 \mathrm{H}), 5.32(\mathrm{~d}, J=17.5 \mathrm{~Hz}, 1 \mathrm{H}), 1.57(\mathrm{~s}, 6 \mathrm{H}) .{ }^{13} \mathrm{C} \mathrm{NMR}\left(125 \mathrm{MHz}, \mathrm{CDCl}_{3}\right)$ $\delta$ 175.0, 140.5, 136.2, 129.8, 129.2, 126.3, 117.0, 110.4, 47.3, 25.9. IR (KBr): 2994, 2981, 2226, 1736, 1492, 1184, 1094, 766, $697 \mathrm{~cm}^{-1}$. HRMS (ESI) m/z: $[\mathrm{M}+\mathrm{H}]^{+}$Calcd for $\mathrm{C}_{13} \mathrm{H}_{15} \mathrm{~N}_{2} \mathrm{O}\left([\mathrm{M}+\mathrm{H}]^{+}\right): 215.1179$; Found: 215.1181.<smiles>C=CC(C)(C)C(=O)N(C)Cc1ccccc1</smiles>

Purified by column chromatography (silica gel, EtOAc/Petroleum ether: 1/20) to afford the title compound (63\% yield, $144 \mathrm{mg}$ ) as a colorless oil. ${ }^{1} \mathrm{H}$ NMR $\left(500 \mathrm{MHz}, \mathrm{CDCl}_{3}\right) \delta 7.41-7.29(\mathrm{~m}, 5 \mathrm{H}), 6.03(\mathrm{dd}, J=17.5,10.6 \mathrm{~Hz}, 1 \mathrm{H})$, $5.26(\mathrm{~d}, J=10.6 \mathrm{~Hz}, 1 \mathrm{H}), 5.12(\mathrm{~d}, J=17.5 \mathrm{~Hz}, 1 \mathrm{H}), 4.72(\mathrm{~s}, 2 \mathrm{H}), 1.45(\mathrm{~s}, 6 \mathrm{H}) .{ }^{13} \mathrm{C} \mathrm{NMR}\left(125 \mathrm{MHz}, \mathrm{CDCl}_{3}\right) \delta 175.1$, 140.3, 134.3, 129.0, 128.9, 128.7, 116.8, 111.0, 52.0, 46.9, 25.6. IR (KBr): 2981, 2934, 2361, 2229, 1709, 1137, 699 $\mathrm{cm}^{-1}$. HRMS (ESI) m/z: $[\mathrm{M}+\mathrm{H}]^{+}$Calcd for $\mathrm{C}_{14} \mathrm{H}_{17} \mathrm{~N}_{2} \mathrm{O}$ 229.1335; Found: 229.1320 .<smiles>C=CCC(c1ccccc1)N(C#N)c1ccccc1</smiles> 
Purified by column chromatography (silica gel, EtOAc/Petroleum ether: 1/10) to afford the title compound (77\% yield, $191 \mathrm{mg})$ as a colorless oil. ${ }^{1} \mathrm{H}$ NMR $\left(500 \mathrm{MHz}, \mathrm{CDCl}_{3}\right) \delta 7.43-7.34(\mathrm{~m}, 4 \mathrm{H}), 7.32-7.26(\mathrm{~m}, 3 \mathrm{H}), 7.12(\mathrm{~d}, J$ $=8.5 \mathrm{~Hz}, 2 \mathrm{H}), 7.05(\mathrm{t}, J=7.4 \mathrm{~Hz}, 1 \mathrm{H}), \delta 5.95-5.75(\mathrm{~m}, 1 \mathrm{H}), 5.27(\mathrm{~d}, J=16.8,1 \mathrm{H}), 5.17(\mathrm{~d}, J=10.2,1 \mathrm{H}), 4.69(\mathrm{dd}$, $J=9.3,6.0 \mathrm{~Hz}, 1 \mathrm{H}), 3.13-2.97(\mathrm{~m}, 1 \mathrm{H}), 2.85-2.69(\mathrm{~m}, 1 \mathrm{H}) .{ }^{13} \mathrm{C} \mathrm{NMR}\left(125 \mathrm{MHz}, \mathrm{CDCl}_{3}\right) \delta 140.5,139.3,133.1$, $129.7,129.1,128.5,126.4,124.1,119.2,117.3,112.7,62.5,39.9$.<smiles>C=C(C)CCN(C#N)c1ccccc1</smiles>

Purified by column chromatography (silica gel, EtOAc/Petroleum ether: 1/12) to afford the title compound (79\% yield, $147 \mathrm{mg}$ ) as a colorless oil. ${ }^{1} \mathrm{H}$ NMR $\left(400 \mathrm{MHz}, \mathrm{CDCl}_{3}\right) \delta 7.41-7.33(\mathrm{~m}, 2 \mathrm{H}), 7.15-7.07(\mathrm{~m}, 3 \mathrm{H}), 4.92-4.87$ $(\mathrm{m}, 1 \mathrm{H}), 4.79(\mathrm{~s}, 1 \mathrm{H}), 3.71(\mathrm{t}, J=7.7 \mathrm{~Hz}, 2 \mathrm{H}), 2.51(\mathrm{t}, J=7.5 \mathrm{~Hz}, 2 \mathrm{H}), 1.81(\mathrm{~s}, 3 \mathrm{H}) .{ }^{13} \mathrm{C} \mathrm{NMR}\left(100 \mathrm{MHz}, \mathrm{CDCl}_{3}\right) \delta$ $140.8,139.8,129.7,123.6,116.0,113.5,113.3,47.8,35.1,22.5$.<smiles>C=C(CCN(C#N)c1ccccc1)PC</smiles>

Purified by column chromatography (silica gel, EtOAc/Petroleum ether: $1 / 15)$ to afford the title compound (80\% yield, $199 \mathrm{mg}$ ) as colorless oil. ${ }^{1} \mathrm{H}$ NMR $\left(400 \mathrm{MHz}, \mathrm{CDCl}_{3}\right) \delta 7.43-7.28(\mathrm{~m}, 7 \mathrm{H}), 7.11-7.04(\mathrm{~m}, 3 \mathrm{H}), 5.41$ (brs, $\left.1 \mathrm{H}\right)$, 5.20-5.19 (m, 1H), 3.70-3.68 (m, 2H), $3.02(\mathrm{t}, J=7.4,2 \mathrm{H}) .{ }^{13} \mathrm{C} \mathrm{NMR}\left(125 \mathrm{MHz}, \mathrm{CDCl}_{3}\right) \delta 143.9,139.8,129.7,128.7$, $128.0,126.2,123.6,116.0,115.7,113.5,48.3,33.4$.<smiles>N#CN(CCC1=CC[14CH2]C1)c1ccccc1</smiles>

Purified by column chromatography (silica gel, EtOAc/Petroleum ether: $1 / 15)$ to afford the title compound ( $82 \%$ yield, $174 \mathrm{mg}$ ) as a colorless oil. ${ }^{1} \mathrm{H}$ NMR $\left(500 \mathrm{MHz}, \mathrm{CDCl}_{3}\right) \delta 7.40-7.33(\mathrm{~m}, 2 \mathrm{H}), 7.21-6.92(\mathrm{~m}, 3 \mathrm{H}), 5.50-5.45$ $(\mathrm{m}, 1 \mathrm{H}), 3.71(\mathrm{t}, J=7.3 \mathrm{~Hz}, 2 \mathrm{H}), 2.58(\mathrm{t}, J=7.3 \mathrm{~Hz}, 2 \mathrm{H}), 2.40-2.17(\mathrm{~m}, 4 \mathrm{H}), 1.95-1.82(\mathrm{~m}, 2 \mathrm{H}) .{ }^{13} \mathrm{C} \mathrm{NMR}(125$ $\left.\mathrm{MHz}, \mathrm{CDCl}_{3}\right) \delta 140.0,139.4,129.7,127.0,123.6,116.0,113.6,48.0,35.2,32.7,29.0,23.4$.<smiles>C=CCCN(C#N)C(=O)c1ccccc1</smiles>

Purified by column chromatography (silica gel, EtOAc/Petroleum ether: $1 / 3$ ) to afford the title compound (85\% yield, $170 \mathrm{mg})$ as a colorless oil. ${ }^{1} \mathrm{H}$ NMR $\left(500 \mathrm{MHz}, \mathrm{CDCl}_{3}\right) \delta 7.84-7.70(\mathrm{~m}, 2 \mathrm{H}), 7.59(\mathrm{t}, J=7.5 \mathrm{~Hz}, 1 \mathrm{H}), 7.48(\mathrm{t}, J=$ $7.8 \mathrm{~Hz}, 2 \mathrm{H}), 6.06-5.72(\mathrm{~m}, 1 \mathrm{H}), 5.29-5.21(\mathrm{~m}, 1 \mathrm{H}), 5.22-5.13(\mathrm{~m}, 1 \mathrm{H}), 3.85(\mathrm{t}, J=7.0 \mathrm{~Hz}, 2 \mathrm{H}), 2.70-2.45(\mathrm{~m}$, $2 \mathrm{H}) .{ }^{13} \mathrm{C}$ NMR $\left(125 \mathrm{MHz}, \mathrm{CDCl}_{3}\right) \delta 168.6,133.2,133.1,131.2,128.7,128.6,119.0,111.1,46.9,32.1$.<smiles>C=CCC(C)(C)C(=O)N(C)c1ccccc1</smiles> 
Purified by column chromatography (silica gel, EtOAc/Petroleum ether: 1/20) to afford the title compound (69\% yield, $158 \mathrm{mg}$ ) as a colorless oil. ${ }^{1} \mathrm{H}$ NMR $\left(400 \mathrm{MHz}, \mathrm{CDCl}_{3}\right) \delta 7.53-7.36(\mathrm{~m}, 3 \mathrm{H}), 7.33-7.22(\mathrm{~m}, 2 \mathrm{H}), 5.92-5.76$ $(\mathrm{m}, 1 \mathrm{H}), 5.39-5.27(\mathrm{~m}, 1 \mathrm{H}), 5.25-5.14(\mathrm{~m}, 1 \mathrm{H}), 2.71(\mathrm{dt}, J=7.4,1.2 \mathrm{~Hz}, 2 \mathrm{H}), 1.51(\mathrm{~s}, 6 \mathrm{H}) .{ }^{13} \mathrm{C} \mathrm{NMR}(125 \mathrm{MHz}$, $\left.\mathrm{CDCl}_{3}\right) \delta 175.6,136.3,133.0,129.8,129.3,126.6,119.2,110.9,45.3,43.6,25.1$. IR (KBr): 2980, 2938, 2229, 1725, 1491, 1172, 760, $694 \mathrm{~cm}^{-1}$. HRMS (ESI) m/z: $[\mathrm{M}+\mathrm{H}]^{+}$Calcd for $\mathrm{C}_{14} \mathrm{H}_{17} \mathrm{~N}_{2} \mathrm{O} 229.1335$; Found: 229.1338 .<smiles>C=CCC(C)(C)C(=O)N(C)c1ccc(OC)cc1</smiles>

Purified by column chromatography (silica gel, EtOAc/Petroleum ether: 1/30) to afford the title compound (69\% yield, $178 \mathrm{mg}$ ) as a colorless oil. ${ }^{1} \mathrm{H}$ NMR $\left(300 \mathrm{MHz}, \mathrm{CDCl}_{3}\right) \delta 7.20-7.14(\mathrm{~m}, 2 \mathrm{H}), 6.99-6.90(\mathrm{~m}, 2 \mathrm{H}), 5.92-5.73$ $(\mathrm{m}, 1 \mathrm{H}), 5.36-5.24(\mathrm{~m}, 1 \mathrm{H}), 5.23-5.15(\mathrm{~m}, 1 \mathrm{H}), 3.82(\mathrm{~s}, 3 \mathrm{H}), 2.69(\mathrm{dt}, J=7.4,1.2 \mathrm{~Hz}, 2 \mathrm{H}), 1.49(\mathrm{~s}, 6 \mathrm{H}) .{ }^{13} \mathrm{C} \mathrm{NMR}$ $\left(125 \mathrm{MHz}, \mathrm{CDCl}_{3}\right) \delta 175.9,160.1,133.1,128.9,128.0,119.1,115.0,111.1,55.7,45.2,43.7,25.2 . \mathrm{IR}(\mathrm{KBr}): 2979$, 2933, 2227, 1724, 1508, 1247, 1175, $832 \mathrm{~cm}^{-1}$. HRMS (ESI) m/z: $[\mathrm{M}+\mathrm{H}]^{+}$Calcd for $\mathrm{C}_{15} \mathrm{H}_{19} \mathrm{~N}_{2} \mathrm{O}_{2} 259.1441$; Found: 259.1450 .<smiles>C=CCC(C)(C)C(=O)N(C)c1ccc(Br)cc1</smiles>

Purified by column chromatography (silica gel, EtOAc/Petroleum ether: 1/80) to afford the title compound (44\% yield, $135 \mathrm{mg}$ ) as a colorless oil. ${ }^{1} \mathrm{H}$ NMR $\left(300 \mathrm{MHz}, \mathrm{CDCl}_{3}\right) \delta 7.58(\mathrm{~d}, J=8.9 \mathrm{~Hz}, 2 \mathrm{H}), 7.15(\mathrm{~d}, J=8.9 \mathrm{~Hz}, 2 \mathrm{H})$, $5.97-5.68(\mathrm{~m}, 1 \mathrm{H}), 5.39-5.05(\mathrm{~m}, 2 \mathrm{H}), 2.69(\mathrm{~d}, J=7.4 \mathrm{~Hz}, 2 \mathrm{H}), 1.50(\mathrm{~s}, 6 \mathrm{H}) .{ }^{13} \mathrm{C} \mathrm{NMR}\left(125 \mathrm{MHz}, \mathrm{CDCl}_{3}\right) \delta 175.4$, 135.3, 133.0, 133.0, 128.2, 123.2, 119.3, 110.5, 45.4, 43.6, 25.1. IR (KBr): 2971, 2918, 1660, 1519, 1391, 917, 822, $506 \mathrm{~cm}^{-1}$. HRMS (ESI) m/z: [M + H] ${ }^{+}$Calcd for $\mathrm{C}_{14} \mathrm{H}_{16} \mathrm{BrN}_{2} \mathrm{O}$ 307.0441; Found: 307.0436 .<smiles>C=CCC(C)(C)C(=O)N(C)CCCC</smiles>

Purified by column chromatography (silica gel, EtOAc/Petroleum ether: 1/30) to afford the title compound (56\% yield, $117 \mathrm{mg})$ as a colorless oil. ${ }^{1} \mathrm{H}$ NMR $\left(500 \mathrm{MHz}, \mathrm{CDCl}_{3}\right) \delta 5.85-5.62(\mathrm{~m}, 1 \mathrm{H}), 5.18(\mathrm{~d}, J=17.0 \mathrm{~Hz}, 1 \mathrm{H}), 5.11$ $(\mathrm{d}, J=10.1 \mathrm{~Hz}, 1 \mathrm{H}), 3.59$ (t, $J=7.3 \mathrm{~Hz}, 2 \mathrm{H}), 2.57$ (d, $J=7.3 \mathrm{~Hz}, 2 \mathrm{H}), 1.86-1.57$ (m, 2H), $1.39(\mathrm{~s}, 6 \mathrm{H}), 1.39-1.33$ (m, 2H), 0.95 (t, $J=7.4 \mathrm{~Hz}, 3 \mathrm{H}) .{ }^{13} \mathrm{C}$ NMR $\left(125 \mathrm{MHz}, \mathrm{CDCl}_{3}\right) \delta 175.9,133.0,119.0,111.7,48.5,45.0,43.6,29.6$, 25.0, 19.6, 13.7. IR (KBr): 2963, 2935, 2229, 1712, 1468, 1238, $1085 \mathrm{~cm}^{-1}$. HRMS (ESI) m/z: $[\mathrm{M}+\mathrm{H}]^{+}$Calcd for $\mathrm{C}_{12} \mathrm{H}_{21} \mathrm{~N}_{2} \mathrm{O} 209.1648$; Found: 209.1634.

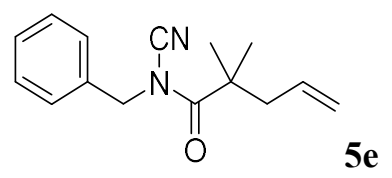

Purified by column chromatography (silica gel, EtOAc/Petroleum ether: $1 / 30$ ) to afford the title compound (72\% yield, $174 \mathrm{mg})$ as a colorless oil. ${ }^{1} \mathrm{H}$ NMR $\left(500 \mathrm{MHz}, \mathrm{CDCl}_{3}\right) \delta 7.47-7.31(\mathrm{~m}, 5 \mathrm{H}), 5.71-5.56(\mathrm{~m}, 1 \mathrm{H}), 5.16-4.94$ $(\mathrm{m}, 2 \mathrm{H}), 4.71(\mathrm{~s}, 2 \mathrm{H}), 2.54(\mathrm{~d}, J=7.4 \mathrm{~Hz}, 2 \mathrm{H}), 1.38(\mathrm{~s}, 6 \mathrm{H}) .{ }^{13} \mathrm{C} \mathrm{NMR}\left(125 \mathrm{MHz}, \mathrm{CDCl}_{3}\right) \delta 175.6,134.3,132.7$, 
129.0, 128.9, 119.1, 111.5, 52.2, 44.9, 43.5, 25.0. IR (KBr): 2928, 2976, 2360, 2341, 2207, 1454, 728, $698 \mathrm{~cm}^{-1}$. HRMS(ESI): calcd. for $\mathrm{C}_{15} \mathrm{H}_{19} \mathrm{~N}_{2} \mathrm{O}\left([\mathrm{M}+\mathrm{H}]^{+}\right): 243.1492$, found: 243.1489 .<smiles>C=CCC(C)(C)C(=O)N(C)Cc1ccccc1Br</smiles>

Purified by column chromatography (silica gel, EtOAc/Petroleum ether: $1 / 30)$ to afford the title compound (78\% yield, $250 \mathrm{mg}$ ) as a colorless oil. ${ }^{1} \mathrm{H}$ NMR $\left(500 \mathrm{MHz}, \mathrm{CDCl}_{3}\right) \delta 7.60(\mathrm{~d}, J=7.9 \mathrm{~Hz}, 1 \mathrm{H}), 7.40-7.28(\mathrm{~m}, 2 \mathrm{H}), 7.26$ $-7.19(\mathrm{~m}, 1 \mathrm{H}), 5.81-5.67(\mathrm{~m}, 1 \mathrm{H}), 5.21-5.13(\mathrm{~m}, 1 \mathrm{H}), 5.13-5.07(\mathrm{~m}, 1 \mathrm{H}), 4.83(\mathrm{~s}, 2 \mathrm{H}), 2.59(\mathrm{~d}, J=7.3 \mathrm{~Hz}, 2 \mathrm{H})$, $1.42(\mathrm{~s}, 6 \mathrm{H}) .{ }^{13} \mathrm{C}$ NMR $\left(125 \mathrm{MHz}, \mathrm{CDCl}_{3}\right) \delta 175.7,133.4,133.0,132.9,131.0,130.5,127.9,124.2,119.2,110.9$, 51.9, 45.0, 43.6, 24.9. IR (KBr): 2978, 2923, 2361, 2228, 1710, 1143, 998, $745 \mathrm{~cm}^{-1}$. HRMS (ESI) m/z: [M + H] Calcd for $\mathrm{C}_{15} \mathrm{H}_{18} \mathrm{BrN}_{2} \mathrm{O} 321.0597$; Found: 321.0590 .<smiles>C=CCCCN(C)c1ccccc1</smiles>

Purified by column chromatography (silica gel, EtOAc/Petroleum ether: $1 / 8$ ) to afford the title compound (82\% yield, $153 \mathrm{mg}$ ) as a colorless oil. ${ }^{1} \mathrm{H}$ NMR $\left(500 \mathrm{MHz}, \mathrm{CDCl}_{3}\right) \delta 7.45-7.31(\mathrm{~m}, 2 \mathrm{H}), 7.21-7.02(\mathrm{~m}, 3 \mathrm{H}), 5.80$ (ddt, $J=$ $16.9,10.1,6.6 \mathrm{~Hz}, 1 \mathrm{H}), 5.28-4.93(\mathrm{~m}, 2 \mathrm{H}), 3.62-3.57(\mathrm{~m}, 2 \mathrm{H}), 2.22(\mathrm{q}, J=7.1 \mathrm{~Hz}, 2 \mathrm{H}), 1.96-1.91(\mathrm{~m}, 2 \mathrm{H}) .{ }^{13} \mathrm{C}$ NMR $\left(125 \mathrm{MHz}, \mathrm{CDCl}_{3}\right) \delta 140.1,136.7,129.8,123.7,116.4,116.0,113.7,48.8,30.5,26.6$.<smiles>C=CCCN(C#N)CC(C)(C)C(=O)OC</smiles>

Purified by column chromatography (silica gel, EtOAc/Petroleum ether: $1 / 3$ ) to afford the title compound (72\% yield, $151 \mathrm{mg})$ as a colorless oil. ${ }^{1} \mathrm{H}$ NMR $\left(500 \mathrm{MHz}, \mathrm{CDCl}_{3}\right) \delta 5.84-5.59(\mathrm{~m}, 1 \mathrm{H}), 5.35-4.86(\mathrm{~m}, 2 \mathrm{H}), 3.72(\mathrm{~s}, 3 \mathrm{H}), 3.20$ (s, 2H), 3.09 (t, $J=7.3 \mathrm{~Hz}, 2 \mathrm{H}), 2.40(\mathrm{q}, J=7.1 \mathrm{~Hz}, 2 \mathrm{H}), 1.27(\mathrm{~s}, 6 \mathrm{H}) .{ }^{13} \mathrm{C}$ NMR $\left(125 \mathrm{MHz}, \mathrm{CDCl}_{3}\right) \delta 176.3,133.6$, 118.2, 118.0, 60.8, 52.9, 52.3, 44.4, 32.3, 23.3. IR (KBr): 2980, 2952, 2209, 1734, 1475, 1267, 1155, $919 \mathrm{~cm}^{-1}$. HRMS (ESI) $\mathrm{m} / \mathrm{z}:[\mathrm{M}+\mathrm{H}]^{+}$Calcd for $\mathrm{C}_{11} \mathrm{H}_{19} \mathrm{~N}_{2} \mathrm{O}_{2} 211.1441$; Found 211.1447.<smiles>C=CCCN(C)C(=O)C(C)C</smiles>

Purified by column chromatography (silica gel, EtOAc/Petroleum ether: $1 / 30$ ) to afford the title compound (72\% yield, $120 \mathrm{mg}$ ) as a colorless oil. ${ }^{1} \mathrm{H}$ NMR $\left(400 \mathrm{MHz}, \mathrm{CDCl}_{3}\right) \delta 5.95-5.58(\mathrm{~m}, 1 \mathrm{H}), 5.32-4.95(\mathrm{~m}, 2 \mathrm{H}), 3.69(\mathrm{t}, J$ $=6.9 \mathrm{~Hz}, 2 \mathrm{H}), 3.25-2.99(\mathrm{~m}, 1 \mathrm{H}), 2.64-2.27(\mathrm{~m}, 2 \mathrm{H}), 1.26(\mathrm{~d}, J=6.8 \mathrm{~Hz}, 6 \mathrm{H}) .{ }^{13} \mathrm{C} \mathrm{NMR}\left(125 \mathrm{MHz}, \mathrm{CDCl}_{3}\right) \delta$ 176.4, 133.0, 118.7, 110.7, 45.3, 33.5, 32.1, 19.0. IR (KBr): 2979, 2938, 2233, 1727, 1206, 1096, $923 \mathrm{~cm}^{-1}$. HRMS (ESI) $\mathrm{m} / \mathrm{z}:[\mathrm{M}+\mathrm{H}]^{+}$Calcd for $\mathrm{C}_{9} \mathrm{H}_{15} \mathrm{~N}_{2} \mathrm{O}$ 167.1179; Found 167.1176 . 
<smiles>C=CCCN(C#N)C(=O)Cc1ccccc1</smiles>

Purified by column chromatography (silica gel, EtOAc/Petroleum ether: $1 / 20)$ to afford the title compound (75\% yield, $160 \mathrm{mg}$ ) as colorless oil. ${ }^{1} \mathrm{H}$ NMR $\left(500 \mathrm{MHz}, \mathrm{CDCl}_{3}\right) \delta 7.38-7.33(\mathrm{~m}, 2 \mathrm{H}), 7.33-7.28(\mathrm{~m}, 3 \mathrm{H}), 5.75-5.62$ $(\mathrm{m}, 1 \mathrm{H}), 5.16-5.04(\mathrm{~m}, 2 \mathrm{H}), 3.97(\mathrm{~s}, 2 \mathrm{H}), 3.65$ (t, $J=7.0 \mathrm{~Hz}, 2 \mathrm{H}), 2.48-2.37(\mathrm{~m}, 2 \mathrm{H}) .{ }^{13} \mathrm{C} \mathrm{NMR}\left(125 \mathrm{MHz}, \mathrm{CDCl}_{3}\right)$ $\delta 170.2,132.7,132.1,129.4,128.9,127.8,118.8,110.7,45.7,41.3,31.9 . \mathrm{IR}(\mathrm{KBr}): 2364,2343,2233,1724,1110$, 924, 729, $697 \mathrm{~cm}^{-1}$. HRMS(ESI) m/z: [M+H] $]^{+}$Calcd. for $\mathrm{C}_{13} \mathrm{H}_{15} \mathrm{~N}_{2} \mathrm{O} 215.1179$; Found 215.1172.<smiles>C=CCCN(C#N)C(=O)OC(C)(C)C</smiles>

Purified by column chromatography (silica gel, EtOAc/Petroleum ether: $1 / 20$ ) to afford the title compound (77\% yield, $151 \mathrm{mg}$ ) as colorless oil. ${ }^{1} \mathrm{H}$ NMR $\left(400 \mathrm{MHz}, \mathrm{CDCl}_{3}\right) \delta 5.97-5.60(\mathrm{~m}, 1 \mathrm{H}), 5.45-4.86(\mathrm{~m}, 2 \mathrm{H}), 3.55(\mathrm{t}, J=$ $7.0 \mathrm{~Hz}, 2 \mathrm{H}), 2.92-2.07$ (m, 2H), 1.52 (s, 9H). ${ }^{13} \mathrm{C} \mathrm{NMR}\left(100 \mathrm{MHz}, \mathrm{CDCl}_{3}\right) \delta$ 151.0, 133.0, 118.8, 109.7, 85.6, 47.0, 32.3, 27.9. IR (KBr): 2982, 2936, 2242, 1748, 1395, 1290, 1151, $847 \mathrm{~cm}^{-1}$. HRMS(ESI) m/z: $[\mathrm{M}+\mathrm{H}]^{+}$Calcd. for $\mathrm{C}_{10} \mathrm{H}_{17} \mathrm{~N}_{2} \mathrm{O}_{2}$ 197.1285; Found 197.1275.<smiles>[B]N([B])CCC=C[AsH3]</smiles>

Purified by column chromatography (silica gel, EtOAc/Petroleum ether: $1 / 3$ ) to afford the title compound (90\% yield, $225 \mathrm{mg}$ ) as a white solid. mp: $55-60{ }^{\circ} \mathrm{C} .{ }^{1} \mathrm{H}$ NMR $\left(300 \mathrm{MHz}, \mathrm{CDCl}_{3}\right) \delta 7.83(\mathrm{~d}, J=8.3 \mathrm{~Hz}, 2 \mathrm{H}), 7.42(\mathrm{~d}, J=8.0$ $\mathrm{Hz}, 2 \mathrm{H}), 5.91-5.46(\mathrm{~m}, 1 \mathrm{H}), 5.37-4.92(\mathrm{~m}, 2 \mathrm{H}), 3.45$ (t, $J=7.2 \mathrm{~Hz}, 2 \mathrm{H}), 2.49$ (s, 3H), 2.40 (q, $J=7.1 \mathrm{~Hz}, 2 \mathrm{H})$. ${ }^{13} \mathrm{C}$ NMR $\left(125 \mathrm{MHz}, \mathrm{CDCl}_{3}\right) \delta 146.6,133.8,132.1,130.5,128.0,119.3,108.6,49.5,32.2,21.9 . \mathrm{IR}(\mathrm{KBr}): 2905$, 2229, 1371, 1174, 914, 691, $546 \mathrm{~cm}^{-1}$. HRMS (ESI) m/z: $[\mathrm{M}+\mathrm{Na}]^{+}$Calcd for $\mathrm{C}_{12} \mathrm{H}_{14} \mathrm{~N}_{2} \mathrm{NaO}_{2} \mathrm{~S} 273.0668$; Found: 273.0669 .<smiles>C=CCCN(C)CCC(=O)OC</smiles>

Purified by column chromatography (silica gel, EtOAc/Petroleum ether: $1 / 3$ ) to afford the title compound ( $31 \%$ yield, $57 \mathrm{mg})$ as a colorless oil. ${ }^{1} \mathrm{H} \mathrm{NMR}\left(500 \mathrm{MHz}, \mathrm{CDCl}_{3}\right) \delta 5.88-5.72(\mathrm{~m}, 1 \mathrm{H}), 5.32-4.98(\mathrm{~m}, 2 \mathrm{H}), 3.73(\mathrm{~s}, 3 \mathrm{H}), 3.34$ $(\mathrm{t}, J=6.9 \mathrm{~Hz}, 2 \mathrm{H}), 3.11(\mathrm{t}, J=7.2 \mathrm{~Hz}, 2 \mathrm{H}), 2.68(\mathrm{t}, J=6.8 \mathrm{~Hz}, 2 \mathrm{H}), 2.53-2.29(\mathrm{~m}, 2 \mathrm{H}) .{ }^{13} \mathrm{C}$ NMR $(100 \mathrm{MHz}$, $\left.\mathrm{CDCl}_{3}\right) \delta 171.2,133.7,118.2,116.8,52.2,51.5,47.3,32.8,32.2$. IR (KBr): 2954, 2360, 2342, 2212, 1737, 1438 , $1204 \mathrm{~cm}^{-1}$. HRMS (ESI) m/z: [M+ H] $]^{+}$Calcd for $\mathrm{C}_{9} \mathrm{H}_{15} \mathrm{~N}_{2} \mathrm{O}_{2}$ 183.1128; Found: 183.1122 .

\section{General Procedure and Experimental Details}

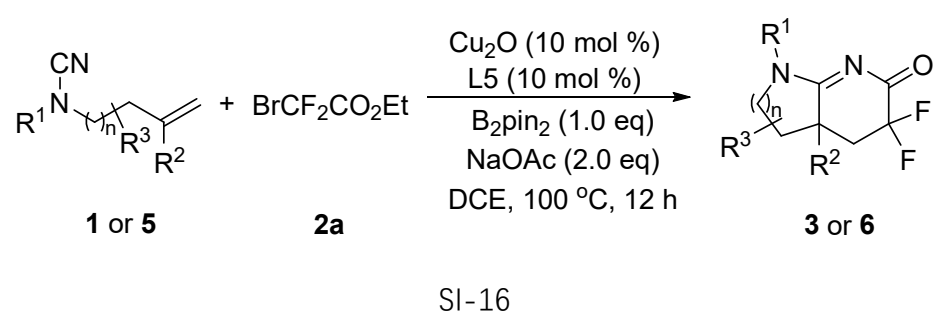


Substrate 1 or 5 (0.2 mmol, 1.0 equiv.), 2a (2.0 equiv.), bis(pinacolato)diboron (1.0 equiv.), $\mathrm{Cu}_{2} \mathrm{O}$ (10 mol \%), 4,4'-ditertbutyl-2,2'-bipyridine (L5, $10 \mathrm{~mol} \%), \mathrm{NaOAc}(2.0$ equiv.) and DCE $(1.5 \mathrm{~mL})$ were placed in a sealed tube under nitrogen atmosphere. The mixture was stirred at $100{ }^{\circ} \mathrm{C}$ for the desired time. Upon completion, the reaction was cooled to room temperature and diluted with water $(5 \mathrm{~mL})$. The aqueous phase was extracted with DCM $(3 \times 10$ $\mathrm{mL}$ ). The combined organic layers were washed with brine, dried over anhydrous $\mathrm{Na}_{2} \mathrm{SO}_{4}$. The organic layer was then concentrated under vacuum, the residue was purified by flash column chromatography (silica gel, EtOAc/Petroleum ether $\left.\left(60-90^{\circ} \mathrm{C}\right)\right)$ to give the desired products 3 or $\mathbf{6}$.

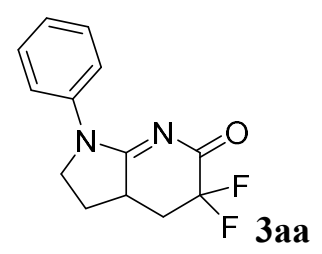

Purified by column chromatography (silica gel, EtOAc/Petroleum ether: $1 / 1)$ to afford the title compound (81\% yield, $41 \mathrm{mg})$ as a white solid. mp: $150-151^{\circ} \mathrm{C} .{ }^{1} \mathrm{H}$ NMR $\left(300 \mathrm{MHz}, \mathrm{CDCl}_{3}\right) \delta 7.71-7.60(\mathrm{~m}, 2 \mathrm{H}), 7.49-7.35(\mathrm{~m}, 2 \mathrm{H})$, $7.33-7.22(\mathrm{~m}, 1 \mathrm{H}), 4.24-4.12(\mathrm{~m}, 1 \mathrm{H}), 4.10-3.93(\mathrm{~m}, 1 \mathrm{H}), 3.46-3.19(\mathrm{~m}, 1 \mathrm{H}), 2.79(\mathrm{tdd}, J=13.9,5.5,2.7 \mathrm{~Hz}$, $1 \mathrm{H}), 2.66-2.40(\mathrm{~m}, 1 \mathrm{H}), 2.41-2.09(\mathrm{~m}, 1 \mathrm{H}), 2.09-1.92(\mathrm{~m}, 1 \mathrm{H}) .{ }^{13} \mathrm{C} \mathrm{NMR}\left(125 \mathrm{MHz}, \mathrm{CDCl}_{3}\right) \delta 174.9,171.0(\mathrm{t}$, $J=25.6 \mathrm{~Hz}), 137.9,129.2,127.2,123.0,110.9(\mathrm{dd}, J=245.7,244.4 \mathrm{~Hz}), 52.8,38.0(\mathrm{~d}, J=8.1 \mathrm{~Hz}), 35.6(\mathrm{t}, J=23.8$ $\mathrm{Hz}), 26.7 .{ }^{19} \mathrm{~F}$ NMR $\left(376 \mathrm{MHz}, \mathrm{CDCl}_{3}\right) \delta-101.44(\mathrm{~d}, J=282.3 \mathrm{~Hz}),-106.83(\mathrm{~d}, J=282.3 \mathrm{~Hz}) . \mathrm{IR}(\mathrm{KBr}): 1697$, 1536, 1306, 1272, 1198, $769 \mathrm{~cm}^{-1}$. HRMS (ESI) m/z: [M + H] ${ }^{+}$Calcd for $\mathrm{C}_{13} \mathrm{H}_{13} \mathrm{~F}_{2} \mathrm{~N}_{2} \mathrm{O}$ 251.0990; Found: 251.0999 .

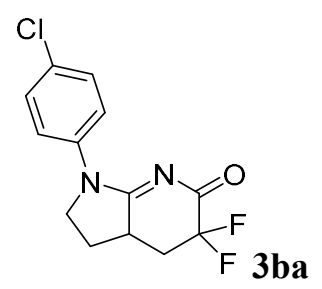

Purified by column chromatography (silica gel, EtOAc/Petroleum ether: $1 / 1$ ) to afford the title compound (70\% yield, $40 \mathrm{mg}$ ) as a white solid. mp: $158-159^{\circ} \mathrm{C} .{ }^{1} \mathrm{H}$ NMR $\left(300 \mathrm{MHz}, \mathrm{CDCl}_{3}\right) \delta 7.66(\mathrm{~d}, J=8.9 \mathrm{~Hz}, 2 \mathrm{H}), 7.37(\mathrm{~d}, J=8.9$ $\mathrm{Hz}, 2 \mathrm{H}), 4.16(\mathrm{td}, J=10.9,6.1 \mathrm{~Hz}, 1 \mathrm{H}), 4.02(\mathrm{t}, J=9.7 \mathrm{~Hz}, 1 \mathrm{H}), 3.44-3.24(\mathrm{~m}, 1 \mathrm{H}), 2.79$ (tdd, $J=14.0,5.5,2.7$ $\mathrm{Hz}, 1 \mathrm{H}), 2.65-2.45(\mathrm{~m}, 1 \mathrm{H}), 2.37-2.11(\mathrm{~m}, 1 \mathrm{H}), 2.12-1.88(\mathrm{~m}, 1 \mathrm{H}) .{ }^{13} \mathrm{C} \mathrm{NMR}\left(100 \mathrm{MHz}, \mathrm{CDCl}_{3}\right) \delta 175.1,170.9$ (t, $J=25.3 \mathrm{~Hz}), 136.5,132.4,129.2,124.2,110.8(\mathrm{dd}, J=245.4,246.5 \mathrm{~Hz}), 52.6,37.9(\mathrm{~d}, J=7.9 \mathrm{~Hz}), 35.8(\mathrm{t}, J=$ $23.2 \mathrm{~Hz}), 26.5 .{ }^{19} \mathrm{~F}$ NMR $\left(376 \mathrm{MHz}, \mathrm{CDCl}_{3}\right) \delta-101.40(\mathrm{~d}, J=282.3 \mathrm{~Hz}),-106.74(\mathrm{~d}, J=282.3 \mathrm{~Hz}) . \mathrm{IR}(\mathrm{KBr}): 1698$, 1540, 1306, 1270, 1197, 832, $537 \mathrm{~cm}^{-1}$. HRMS (ESI) m/z: $[\mathrm{M}+\mathrm{H}]^{+}$Calcd for $\mathrm{C}_{13} \mathrm{H}_{12} \mathrm{ClF}_{2} \mathrm{~N}_{2} \mathrm{O} 285.0601$; Found:285.0610.

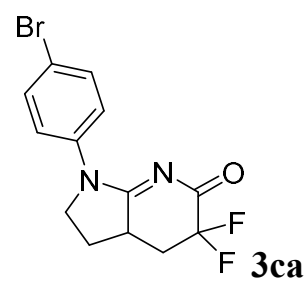


Purified by column chromatography (silica gel, EtOAc/Petroleum ether: $1 / 1)$ to afford the title compound (71\% yield, $47 \mathrm{mg}$ ) as a white solid. mp: $157-159{ }^{\circ} \mathrm{C} .{ }^{1} \mathrm{H}$ NMR $\left(500 \mathrm{MHz}, \mathrm{CDCl}_{3}\right) \delta 7.60(\mathrm{~d}, J=8.9 \mathrm{~Hz}, 2 \mathrm{H}), 7.52(\mathrm{~d}, J=8.9$ $\mathrm{Hz}, 2 \mathrm{H}), 4.20-4.11(\mathrm{~m}, 1 \mathrm{H}), 4.05-3.96(\mathrm{~m}, 1 \mathrm{H}), 3.43-3.25(\mathrm{~m}, 1 \mathrm{H}), 2.79(\mathrm{tdd}, J=13.9,5.6,2.6 \mathrm{~Hz}, 1 \mathrm{H}), 2.60-$ $2.48(\mathrm{~m}, 1 \mathrm{H}), 2.30-2.12(\mathrm{~m}, 1 \mathrm{H}), 2.09-1.95(\mathrm{~m}, 1 \mathrm{H}) .{ }^{13} \mathrm{C} \mathrm{NMR}\left(100 \mathrm{MHz}, \mathrm{CDCl}_{3}\right) \delta 175.1,170.9(\mathrm{t}, J=25.9 \mathrm{~Hz})$, 137.0, 132.2, 124.4, 120.3, $110.8(\mathrm{dd}, J=256.5,245.4 \mathrm{~Hz}), 52.5,38.0$ (d, $J=7.9 \mathrm{~Hz}), 35.8(\mathrm{t}, J=24.2 \mathrm{~Hz}), 26.5 .{ }^{19} \mathrm{~F}$ $\operatorname{NMR}\left(376 \mathrm{MHz}, \mathrm{CDCl}_{3}\right) \delta-101.39(\mathrm{~d}, J=282.2 \mathrm{~Hz}),-106.74(\mathrm{~d}, J=282.0 \mathrm{~Hz}) . \mathrm{IR}(\mathrm{KBr}): 1698,1535,1330,1181$, 1039, 842, $539 \mathrm{~cm}^{-1}$. HRMS (ESI) m/z: [M + H] $]^{+}$Calcd for $\mathrm{C}_{13} \mathrm{H}_{12} \mathrm{BrF}_{2} \mathrm{~N}_{2} \mathrm{O}$ 329.0096; Found: 329.0105 .

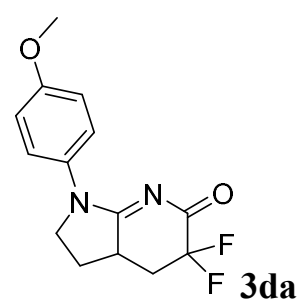

Purified by column chromatography (silica gel, EtOAc/Petroleum ether: $1 / 1)$ to afford the title compound ( $68 \%$ yield, $39 \mathrm{mg})$ as a white solid. mp: $167-169^{\circ} \mathrm{C} .{ }^{1} \mathrm{H}$ NMR $\left(400 \mathrm{MHz}, \mathrm{CDCl}_{3}\right) \delta 7.51(\mathrm{~d}, J=8.6 \mathrm{~Hz}, 2 \mathrm{H}), 7.18(\mathrm{~d}, J=8.3$ $\mathrm{Hz}, 2 \mathrm{H}), 4.13(\mathrm{td}, J=11.0,6.1 \mathrm{~Hz}, 1 \mathrm{H}), 4.04-3.93(\mathrm{~m}, 1 \mathrm{H}), 3.38-3.14(\mathrm{~m}, 1 \mathrm{H}), 2.75(\mathrm{tdd}, J=13.9,5.6,2.6 \mathrm{~Hz}$, $1 \mathrm{H}), 2.58-2.40(\mathrm{~m}, 1 \mathrm{H}), 2.33(\mathrm{~s}, 3 \mathrm{H}), 2.31-2.04(\mathrm{~m}, 1 \mathrm{H}), 2.08-1.77(\mathrm{~m}, 1 \mathrm{H}) .{ }^{13} \mathrm{C} \mathrm{NMR}\left(100 \mathrm{MHz}, \mathrm{CDCl}_{3}\right) \delta$ 174.7, $171.0(\mathrm{t}, J=25.3 \mathrm{~Hz}), 137.1,135.4,129.7,122.9,110.9(\mathrm{dd}, J=256.1,245.2 \mathrm{~Hz}), 52.8,37.9(\mathrm{~d}, J=7.9 \mathrm{~Hz})$, $35.9(\mathrm{t}, J=23.8 \mathrm{~Hz}), 26.6,21.1 .{ }^{19} \mathrm{~F} \mathrm{NMR}\left(376 \mathrm{MHz}, \mathrm{CDCl}_{3}\right) \delta-101.24(\mathrm{~d}, J=281.6 \mathrm{~Hz}),-106.60(\mathrm{~d}, J=281.6 \mathrm{~Hz})$. IR (KBr): 2925, 1698, 1553, 1511, 1248, 1021, $832 \mathrm{~cm}^{-1}$. HRMS (ESI) m/z: [M + H] Calcd for $\mathrm{C}_{14} \mathrm{H}_{15} \mathrm{~F}_{2} \mathrm{~N}_{2} \mathrm{O}_{2}$ 281.1096; Found: 281.1114.

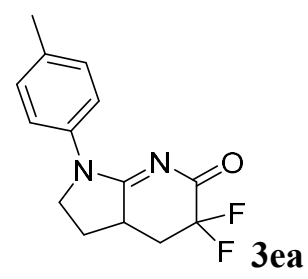

Purified by column chromatography (silica gel, EtOAc/Petroleum ether: $1 / 1$ ) to afford the title compound (74\% yield, $40 \mathrm{mg}$ ) as a white solid. mp: $168-170{ }^{\circ} \mathrm{C} .{ }^{1} \mathrm{H} \mathrm{NMR}\left(400 \mathrm{MHz}, \mathrm{CDCl}_{3}\right) \delta 7.51(\mathrm{~d}, J=8.6 \mathrm{~Hz}, 2 \mathrm{H}), 7.18(\mathrm{~d}, J=8.3$ $\mathrm{Hz}, 2 \mathrm{H}), 4.13(\mathrm{td}, J=11.0,6.1 \mathrm{~Hz}, 1 \mathrm{H}), 4.04-3.93(\mathrm{~m}, 1 \mathrm{H}), 3.38-3.14(\mathrm{~m}, 1 \mathrm{H}), 2.75(\mathrm{tdd}, J=13.9,5.6,2.6 \mathrm{~Hz}$, $1 \mathrm{H}), 2.58-2.40(\mathrm{~m}, 1 \mathrm{H}), 2.33(\mathrm{~s}, 3 \mathrm{H}), 2.31-2.04(\mathrm{~m}, 1 \mathrm{H}), 2.08-1.77(\mathrm{~m}, 1 \mathrm{H}) .{ }^{13} \mathrm{C} \mathrm{NMR}\left(100 \mathrm{MHz}, \mathrm{CDCl}_{3}\right) \delta$ 174.7, 171.0 (t, $J=25.3 \mathrm{~Hz}), 137.1,135.4,129.7,122.9,110.9$ (dd, $J=256.1,245.2 \mathrm{~Hz}), 52.8,37.9$ (d, $J=7.9 \mathrm{~Hz})$, $35.9(\mathrm{t}, J=23.8 \mathrm{~Hz}), 26.6,21.1 .{ }^{19} \mathrm{~F}$ NMR $\left(376 \mathrm{MHz}, \mathrm{CDCl}_{3}\right) \delta-101.24(\mathrm{~d}, J=281.6 \mathrm{~Hz}),-106.60(\mathrm{~d}, J=281.6 \mathrm{~Hz})$. IR (KBr): 2958, 2923, 1697, 1535, 1331, 1181, 846, $541 \mathrm{~cm}^{-1}$. HRMS (ESI) m/z: $[\mathrm{M}+\mathrm{H}]^{+}$Calcd for $\mathrm{C}_{14} \mathrm{H}_{15} \mathrm{~F}_{2} \mathrm{~N}_{2} \mathrm{O}$ 265.1147; Found:265.1145. 


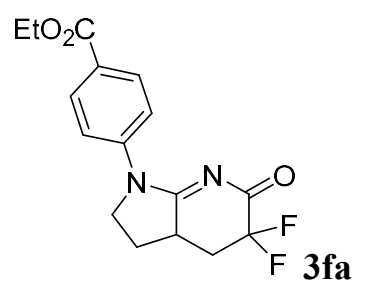

Purified by column chromatography (silica gel, EtOAc/Petroleum ether: $1 / 1)$ to afford the title compound (83\% yield, $54 \mathrm{mg}$ ) as a white solid. mp: $157-159{ }^{\circ} \mathrm{C} .{ }^{1} \mathrm{H}$ NMR $\left(300 \mathrm{MHz}, \mathrm{CDCl}_{3}\right) \delta 8.07(\mathrm{~d}, J=8.6 \mathrm{~Hz}, 2 \mathrm{H}), 7.83(\mathrm{~d}, J=8.8$ $\mathrm{Hz}, 2 \mathrm{H}), 4.38(\mathrm{q}, J=7.1 \mathrm{~Hz}, 2 \mathrm{H}), 4.28-4.13(\mathrm{~m}, 1 \mathrm{H}), 4.15-4.02(\mathrm{~m}, 1 \mathrm{H}), 3.47-3.26(\mathrm{~m}, 1 \mathrm{H}), 2.80(\mathrm{tdd}, J=14.0$, 5.7, $2.9 \mathrm{~Hz}, 1 \mathrm{H}), 2.64-2.50(\mathrm{~m}, 1 \mathrm{H}), 2.36-2.08(\mathrm{~m}, 1 \mathrm{H}), 2.11-1.92(\mathrm{~m}, 1 \mathrm{H}), 1.40(\mathrm{t}, J=7.1 \mathrm{~Hz}, 3 \mathrm{H}) .{ }^{13} \mathrm{C} \mathrm{NMR}$ $\left(100 \mathrm{MHz}, \mathrm{CDCl}_{3}\right) \delta 175.5,170.9(\mathrm{t}, J=26.0 \mathrm{~Hz}), 165.7,141.7,130.4,128.3,122.1,110.8(\mathrm{dd}, J=256.4,244.8 \mathrm{~Hz})$, 61.2, 52.3, 38.1 (d, $J=7.9 \mathrm{~Hz}), 35.7$ (t, $J=23.8 \mathrm{~Hz}), 26.4,14.3 .{ }^{19} \mathrm{~F}$ NMR $\left(376 \mathrm{MHz}, \mathrm{CDCl}_{3}\right) \delta-100.48--102.37$ (m), -106.07 - -107.18 (m). IR (KBr): 2963, 1701, 1540, 1367, 1275, 770, $706 \mathrm{~cm}^{-1}$. HRMS (ESI) m/z: [M + H] Calcd for $\mathrm{C}_{16} \mathrm{H}_{17} \mathrm{~F}_{2} \mathrm{~N}_{2} \mathrm{O}_{3}$ 323.1202; Found: 323.1209 .

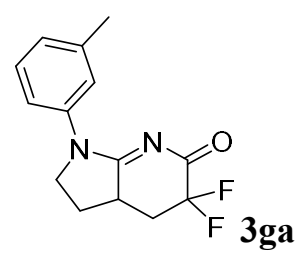

Purified by column chromatography (silica gel, EtOAc/Petroleum ether: $1 / 1)$ to afford the title compound (77\% yield, $41 \mathrm{mg}$ ) as a white solid. mp: $146-148{ }^{\circ} \mathrm{C} .{ }^{1} \mathrm{H} \mathrm{NMR}\left(500 \mathrm{MHz}, \mathrm{CDCl}_{3}\right) \delta 7.43(\mathrm{~d}, J=8.0 \mathrm{~Hz}, 1 \mathrm{H}), 7.37(\mathrm{~s}, 1 \mathrm{H}), 7.28$ (t, $J=7.8 \mathrm{~Hz}, 1 \mathrm{H}), 7.07$ (d, $J=7.6 \mathrm{~Hz}, 1 \mathrm{H}), 4.14(\mathrm{td}, J=11.0,6.1 \mathrm{~Hz}, 1 \mathrm{H}), 4.05-3.94(\mathrm{~m}, 1 \mathrm{H}), 3.39-3.25(\mathrm{~m}, 1 \mathrm{H})$, $2.76(\mathrm{tdd}, J=14.0,5.5,2.6 \mathrm{~Hz}, 1 \mathrm{H}), 2.58-2.47(\mathrm{~m}, 1 \mathrm{H}), 2.36(\mathrm{~s}, 3 \mathrm{H}), 2.27-2.09(\mathrm{~m}, 1 \mathrm{H}), 2.06-1.92(\mathrm{~m}, 1 \mathrm{H}) .{ }^{13} \mathrm{C}$ NMR $\left(100 \mathrm{MHz}, \mathrm{CDCl}_{3}\right) \delta 174.9,171.0(\mathrm{t}, J=25.8 \mathrm{~Hz}), 139.2,137.8,129.0,128.1,123.7,120.4,110.9(\mathrm{dd}, J=$ 256.0, 245.4 Hz), 53.0, $37.9(\mathrm{~d}, J=8.0 \mathrm{~Hz}), 35.9(\mathrm{t}, J=23.7 \mathrm{~Hz}), 26.7,21.6 .{ }^{19} \mathrm{~F} \mathrm{NMR}\left(376 \mathrm{MHz}, \mathrm{CDCl}_{3}\right) \delta-100.41$ - -101.93 (m), -105.81 - -107.89 (m). IR (KBr): 2961, 2937, 1694, 1537, 1331, 1119, 1043, $701 \mathrm{~cm}^{-1}$. HRMS (ESI) $\mathrm{m} / \mathrm{z}:[\mathrm{M}+\mathrm{H}]^{+}$Calcd for $\mathrm{C}_{14} \mathrm{H}_{15} \mathrm{~F}_{2} \mathrm{~N}_{2} \mathrm{O}$ 265.1147; Found: 265.1152.

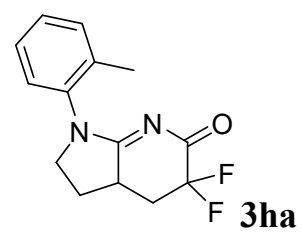

Purified by column chromatography (silica gel, EtOAc/Petroleum ether: $1 / 1)$ to afford the title compound (82\% yield, $44 \mathrm{mg}$ ) as a white solid. mp: $139-141{ }^{\circ} \mathrm{C} .{ }^{1} \mathrm{H}$ NMR $\left(500 \mathrm{MHz}, \mathrm{CDCl}_{3}\right) \delta 7.31-7.26(\mathrm{~m}, 2 \mathrm{H}), 7.27-7.21(\mathrm{~m}, 1 \mathrm{H})$, $7.13(\mathrm{~d}, J=7.6 \mathrm{~Hz}, 1 \mathrm{H}), 4.02(\mathrm{td}, J=11.1,6.0 \mathrm{~Hz}, 1 \mathrm{H}), 3.88-3.71(\mathrm{~m}, 1 \mathrm{H}), 3.45-3.27(\mathrm{~m}, 1 \mathrm{H}), 2.81(\mathrm{tdd}, J=13.8$, 5.6, $2.7 \mathrm{~Hz}, 1 \mathrm{H}), 2.64-2.46(\mathrm{~m}, 1 \mathrm{H}), 2.26-2.04(\mathrm{~m}, 2 \mathrm{H}), 2.20(\mathrm{~s}, 3 \mathrm{H}) .{ }^{13} \mathrm{C} \mathrm{NMR}\left(100 \mathrm{MHz}, \mathrm{CDCl}_{3}\right) \delta 176.0,170.9$ $(\mathrm{t}, J=26.3 \mathrm{~Hz}), 136.2,134.8,131.5,129.2,127.2,126.2,111.0(\mathrm{dd}, J=256.2,245.2 \mathrm{~Hz}), 54.0,37.0(\mathrm{~d}, J=8.1 \mathrm{~Hz})$, $35.9(\mathrm{t}, J=23.7 \mathrm{~Hz}), 27.6,17.9 .{ }^{19} \mathrm{~F}$ NMR $\left(470 \mathrm{MHz}, \mathrm{CDCl}_{3}\right) \delta-101.14(\mathrm{ddd}, J=282.1,32.6,14.1 \mathrm{~Hz}),-107.08(\mathrm{~d}$, $J=282.2 \mathrm{~Hz}$ ). IR (KBr): 2933, 2886, 1694, 1553, 1363, 1194, 1058, $724 \mathrm{~cm}^{-1}$. HRMS (ESI) m/z: $[\mathrm{M}+\mathrm{H}]^{+} \mathrm{Calcd}$ for $\mathrm{C}_{14} \mathrm{H}_{15} \mathrm{~F}_{2} \mathrm{~N}_{2} \mathrm{O}$ 265.1147; Found: 265.1155 . 


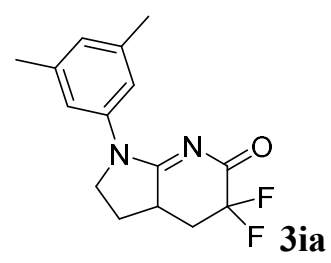

Purified by column chromatography (silica gel, EtOAc/Petroleum ether: $1 / 1)$ to afford the title compound (90\% yield, $50 \mathrm{mg}$ ) as a white solid. mp: $168-170{ }^{\circ} \mathrm{C} .{ }^{1} \mathrm{H} \mathrm{NMR}\left(400 \mathrm{MHz}, \mathrm{CDCl}_{3}\right) \delta 7.16(\mathrm{~s}, 2 \mathrm{H}), 6.90(\mathrm{~s}, 1 \mathrm{H}), 4.12(\mathrm{td}, J=$ 11.0, 6.1 Hz, 1H), $4.03-3.89(\mathrm{~m}, 1 \mathrm{H}), 3.36-3.23(\mathrm{~m}, 1 \mathrm{H}), 2.75(\mathrm{tdd}, J=14.3,5.6,2.6 \mathrm{~Hz}, 1 \mathrm{H}), 2.58-2.45(\mathrm{~m}$, $1 \mathrm{H}), 2.31(\mathrm{~s}, 6 \mathrm{H}), 2.27-2.06(\mathrm{~m}, 1 \mathrm{H}), 2.05-1.91(\mathrm{~m}, 1 \mathrm{H}) .{ }^{13} \mathrm{C} \mathrm{NMR}\left(100 \mathrm{MHz}, \mathrm{CDCl}_{3}\right) \delta 174.9,171.0(\mathrm{t}, J=25.3$ $\mathrm{Hz}), 138.9,137.6,129.1,121.2,111.0(\mathrm{dd}, J=256.1,245.2 \mathrm{~Hz}), 53.3,37.8(\mathrm{dd}, J=8.0,1.0 \mathrm{~Hz}), 35.9(\mathrm{t}, J=23.2$ $\mathrm{Hz}), 26.7,21.4 .{ }^{19} \mathrm{~F}$ NMR (376 MHz, $\left.\mathrm{CDCl}_{3}\right) \delta-99.15--102.84(\mathrm{~m}),-106.00--106.96(\mathrm{~m}) . \mathrm{IR}(\mathrm{KBr}): 2919,1685$, 1541, 1362, 1099, 1017, $695 \mathrm{~cm}^{-1}$. HRMS (ESI) m/z: [M+ H] Calcd for $\mathrm{C}_{15} \mathrm{H}_{17} \mathrm{~F}_{2} \mathrm{~N}_{2} \mathrm{O} 279.1303$; Found: 279.1312.

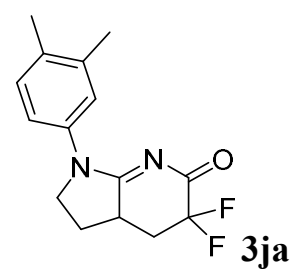

Purified by column chromatography (silica gel, EtOAc/Petroleum ether: 1/1) to afford the title compound (64\% yield, $36 \mathrm{mg}$ ) as a white solid. mp: $209-211{ }^{\circ} \mathrm{C} .{ }^{1} \mathrm{H} \mathrm{NMR}\left(400 \mathrm{MHz}, \mathrm{CDCl}_{3}\right) \delta 7.39-7.29(\mathrm{~m}, 2 \mathrm{H}), 7.14(\mathrm{~d}, J=8.0 \mathrm{~Hz}$, $1 \mathrm{H}), 4.12(\mathrm{td}, J=11.0,6.1 \mathrm{~Hz}, 1 \mathrm{H}), 4.03-3.92(\mathrm{~m}, 1 \mathrm{H}), 3.41-3.13(\mathrm{~m}, 1 \mathrm{H}), 2.76(\mathrm{tdd}, J=14.0,5.6,2.6 \mathrm{~Hz}, 1 \mathrm{H})$, $2.61-2.44(\mathrm{~m}, 1 \mathrm{H}), 2.26(\mathrm{~s}, 3 \mathrm{H}), 2.24(\mathrm{~s}, 3 \mathrm{H}), 2.19-2.08(\mathrm{~m}, 1 \mathrm{H}), 2.06-1.92(\mathrm{~m}, 1 \mathrm{H}) .{ }^{13} \mathrm{C} \mathrm{NMR}\left(100 \mathrm{MHz} \mathrm{CDCl}_{3}\right)$ $\delta 174.6,170.9(\mathrm{t}, J=25.3 \mathrm{~Hz}), 137.7,136.1,135.5,130.2,124.2,120.7,110.9(\mathrm{dd}, J=256.1,245.2 \mathrm{~Hz}), 53.1,37.9$ $(\mathrm{d}, J=7.9 \mathrm{~Hz}), 36.0(\mathrm{t}, J=23.8 \mathrm{~Hz}), 26.8,20.1,19.5 .{ }^{19} \mathrm{~F} \mathrm{NMR}\left(376 \mathrm{MHz}, \mathrm{CDCl}_{3}\right) \delta-101.25(\mathrm{~d}, J=282.2 \mathrm{~Hz}),-$ 106.67 (d, $J=281.8 \mathrm{~Hz}$ ). IR (KBr): 2937, 2923, 1692, 1539, 1361, 1090, 1022, $914 \mathrm{~cm}^{-1}$. HRMS (ESI) m/z: [M + $\mathrm{H}]^{+}$Calcd for $\mathrm{C}_{15} \mathrm{H}_{17} \mathrm{~F}_{2} \mathrm{~N}_{2} \mathrm{O} 279.1303$; Found: 279.1310 .

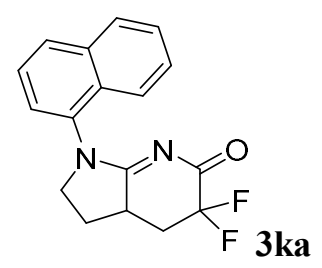

Purified by column chromatography (silica gel, EtOAc/Petroleum ether: $2 / 1)$ to afford the title compound (52\% yield, $31 \mathrm{mg}$ ) as a white solid. mp: $122-126{ }^{\circ} \mathrm{C} .{ }^{1} \mathrm{H}$ NMR $\left(300 \mathrm{MHz}, \mathrm{CDCl}_{3}\right) \delta 8.03-7.80(\mathrm{~m}, 2 \mathrm{H}), 7.59-7.46(\mathrm{~m}, 4 \mathrm{H})$, $7.45-7.34(\mathrm{~m}, 1 \mathrm{H}), 4.35-4.07(\mathrm{~m}, 1 \mathrm{H}), 4.06-3.90(\mathrm{~m}, 1 \mathrm{H}), 3.62-3.44(\mathrm{~m}, 1 \mathrm{H}), 3.05-2.78(\mathrm{~m}, 1 \mathrm{H}), 2.75-2.48$ (m, 1H), $2.50-2.08(\mathrm{~m}, 2 \mathrm{H}) .{ }^{13} \mathrm{C}$ NMR (125 MHz, DMSO) $\delta 178.1,169.9$ (t, $\left.J=25.9 \mathrm{~Hz}\right), 134.7,133.9,128.9$, 128.4, 127.2, 126.7, 125.7, 122.7, $111.9(\mathrm{dd}, J=253.7,244.5 \mathrm{~Hz}), 54.5,36.7,34.4(\mathrm{t}, J=21.8 \mathrm{~Hz}), 26.9 .{ }^{19} \mathrm{~F}$ NMR (470 MHz, DMSO) $\delta-99.18$ (ddd, $J=277.3,35.9,16.0 \mathrm{~Hz}$ ), -104.77 (dd, $J=276.8,6.2 \mathrm{~Hz}$ ). (Poor solubility in $\mathrm{CDCl}_{3}$ and limited solubility in $d$-DMSO). IR (KBr): 3152, 2959, 2228, 2595, 1493, 1252, 814, $488 \mathrm{~cm}^{-1}$. HRMS (ESI) $\mathrm{m} / \mathrm{z}:[\mathrm{M}+\mathrm{H}]^{+}$Calcd for $\mathrm{C}_{17} \mathrm{H}_{15} \mathrm{~F}_{2} \mathrm{~N}_{2} \mathrm{O} 301.1147$; Found: 301.1149 . 


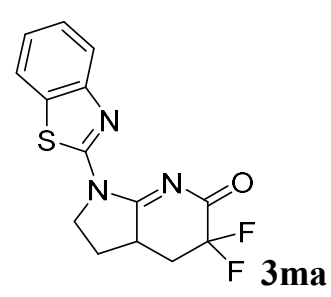

Purified by column chromatography (silica gel, EtOAc/Petroleum ether: $1 / 1$ with $5 \% \mathrm{Et}_{3} \mathrm{~N}$ ) to afford the title compound (49\% yield, $31 \mathrm{mg})$ as a white solid. mp: $185-192{ }^{\circ} \mathrm{C} .{ }^{1} \mathrm{H}$ NMR $\left(500 \mathrm{MHz}, \mathrm{CDCl}_{3}\right) \delta 7.91(\mathrm{~d}, J=8.1 \mathrm{~Hz}$, $1 \mathrm{H}), 7.87(\mathrm{~d}, J=7.9 \mathrm{~Hz}, 1 \mathrm{H}), 7.50(\mathrm{t}, J=7.7 \mathrm{~Hz}, 1 \mathrm{H}), 7.39(\mathrm{t}, J=7.6 \mathrm{~Hz}, 1 \mathrm{H}), 4.81(\mathrm{dd}, J=11.8,8.9 \mathrm{~Hz}, 1 \mathrm{H}), 4.31$ $(\mathrm{td}, J=11.6,6.2 \mathrm{~Hz}, 1 \mathrm{H}), 3.62-3.41(\mathrm{~m}, 1 \mathrm{H}), 2.91(\mathrm{tdd}, J=13.7,5.7,2.8 \mathrm{~Hz}, 1 \mathrm{H}), 2.74-2.62(\mathrm{~m}, 1 \mathrm{H}), 2.40-2.20$ (m, 1H), $2.19-2.04(\mathrm{~m}, 1 \mathrm{H}) .{ }^{13} \mathrm{C}$ NMR (125 MHz, DMSO) $\delta 176.4,169.1(\mathrm{t}, J=26.7 \mathrm{~Hz}), 155.8,147.9,133.5$, 126.5, 124.5, 122.2, 121.6, $112.0(\mathrm{dd}, J=244.4,244.4 \mathrm{~Hz}), 51.6,37.4(\mathrm{~d}, J=8.5 \mathrm{~Hz}), 33.9$ (t, $J=22.9 \mathrm{~Hz}), 25.6 .{ }^{19} \mathrm{~F}$ NMR (470 MHz, DMSO) $\delta-99.81$ (ddd, $J=277.6,36.5,15.5 \mathrm{~Hz}),-104.80$ (dd, $J=277.4,5.7 \mathrm{~Hz})$. IR (KBr): 2955 , 2922, 2849, 1716, 1571, 1196, $771 \mathrm{~cm}^{-1}$. HRMS (ESI) m/z: $[\mathrm{M}+\mathrm{H}]^{+}$Calcd for $\mathrm{C}_{14} \mathrm{H}_{12} \mathrm{~F}_{2} \mathrm{~N}_{3} \mathrm{OS}$ 308.0664; Found: 308.0674 .

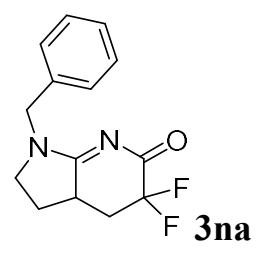

Purified by column chromatography (silica gel, EtOAc/Petroleum ether: $1 / 1)$ to afford the title compound (60\% yield, $32 \mathrm{mg}$ ) as a white solid. mp: $130-132{ }^{\circ} \mathrm{C} .{ }^{1} \mathrm{H}$ NMR $\left(400 \mathrm{MHz}, \mathrm{CDCl}_{3}\right) \delta 7.39-7.31(\mathrm{~m}, 3 \mathrm{H}), 7.30-7.26(\mathrm{~m}, 2 \mathrm{H})$, $4.87-4.57(\mathrm{~m}, 2 \mathrm{H}), 3.72-3.39(\mathrm{~m}, 2 \mathrm{H}), 3.29-3.10(\mathrm{~m}, 1 \mathrm{H}), 2.73$ (tdd, $J=13.8,5.6,2.4 \mathrm{~Hz}, 1 \mathrm{H}), 2.52-2.31(\mathrm{~m}$, 1H), $2.25-1.98(\mathrm{~m}, 1 \mathrm{H}), 1.95-1.72(\mathrm{~m}, 1 \mathrm{H}) .{ }^{13} \mathrm{C} \mathrm{NMR}\left(100 \mathrm{MHz}, \mathrm{CDCl}_{3}\right) \delta 175.6,170.9(\mathrm{t}, J=25.3 \mathrm{~Hz}), 134.4$, 129.1, 128.7, 128.5, 111.2 (dd, $J=246.4,245.4 \mathrm{~Hz}), 49.6,48.7,37.0$ (d, $J=7.9 \mathrm{~Hz}), 35.9(\mathrm{t}, J=24.3 \mathrm{~Hz}), 26.6 .{ }^{19} \mathrm{~F}$ $\operatorname{NMR}\left(376 \mathrm{MHz}, \mathrm{CDCl}_{3}\right) \delta-100.64(\mathrm{~d}, J=282.4 \mathrm{~Hz}),-106.64$ (d, $\left.J=282.4 \mathrm{~Hz}\right)$. IR (KBr): 2962, 1698, 1573, 1463 , 1340, 1267, 1116, $706 \mathrm{~cm}^{-1}$. HRMS (ESI) m/z: [M+ H] Calcd for $\mathrm{C}_{14} \mathrm{H}_{15} \mathrm{~F}_{2} \mathrm{~N}_{2} \mathrm{O}$ 265.1147; Found: 265.1149 .

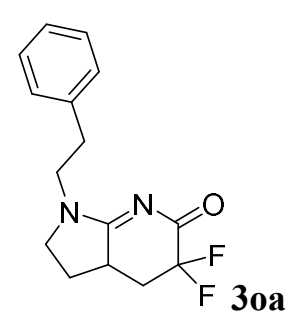

Purified by column chromatography (silica gel, EtOAc/Petroleum ether: $1 / 1)$ to afford the title compound (70\% yield, $39 \mathrm{mg}$ ) as a white solid. mp: $140-144{ }^{\circ} \mathrm{C} .{ }^{1} \mathrm{H}$ NMR $\left(400 \mathrm{MHz}, \mathrm{CDCl}_{3}\right) \delta 7.34-7.26(\mathrm{~m}, 2 \mathrm{H}), 7.27-7.16(\mathrm{~m}, 3 \mathrm{H})$, $3.91-3.74(\mathrm{~m}, 2 \mathrm{H}), 3.51-3.39(\mathrm{~m}, 1 \mathrm{H}), 3.39-3.27(\mathrm{~m}, 1 \mathrm{H}), 3.14-2.99(\mathrm{~m}, 1 \mathrm{H}), 2.95(\mathrm{t}, J=7.2 \mathrm{~Hz}, 2 \mathrm{H}), 2.75-$ $2.59(\mathrm{~m}, 1 \mathrm{H}), 2.40-2.22(\mathrm{~m}, 1 \mathrm{H}), 2.13-1.87(\mathrm{~m}, 1 \mathrm{H}), 1.75-1.64(\mathrm{~m}, 1 \mathrm{H}) .{ }^{13} \mathrm{C} \mathrm{NMR}\left(100 \mathrm{MHz}, \mathrm{CDCl}_{3}\right) \delta 175.5$, $170.7(\mathrm{t}, J=25.8 \mathrm{~Hz}), 137.7,128.8,128.8,127.0,111.1$ (dd, $J=256.2,245.3 \mathrm{~Hz}), 51.1,46.1,36.8(\mathrm{~d}, J=8.2 \mathrm{~Hz})$, $36.0(\mathrm{t}, J=24.2 \mathrm{~Hz}), 33.4,26.8 .{ }^{19} \mathrm{~F} \mathrm{NMR}\left(376 \mathrm{MHz}, \mathrm{CDCl}_{3}\right) \delta-100.70(\mathrm{~d}, J=282.3 \mathrm{~Hz}),-106.53(\mathrm{~d}, J=282.2 \mathrm{~Hz})$. 
IR (KBr): 2943, 2876, 1685, 1576, 1336, 1118, 758, $705 \mathrm{~cm}^{-1}$. HRMS (ESI) m/z: [M + H] ${ }^{+}$Calcd for $\mathrm{C}_{15} \mathrm{H}_{17} \mathrm{~F}_{2} \mathrm{~N}_{2} \mathrm{O}$ 279.1303; Found: 279.1310.

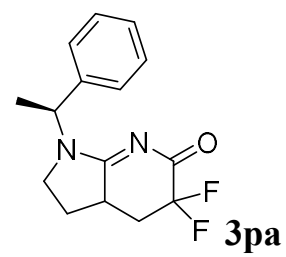

Purified by column chromatography (silica gel, EtOAc/Petroleum ether: 1/1) to afford the title compound (an inseparable mixture, $84 \%$ yield, $47 \mathrm{mg}, d r=1.2 / 1)$ as a white solid. The major isomer: ${ }^{1} \mathrm{H}$ NMR $\left(500 \mathrm{MHz}, \mathrm{CDCl}_{3}\right)$ $\delta 7.66-7.16(\mathrm{~m}, 5 \mathrm{H}), 6.22-5.83(\mathrm{~m}, 1 \mathrm{H}), 3.71-3.48(\mathrm{~m}, 1 \mathrm{H}), 3.34-3.25(\mathrm{~m}, 1 \mathrm{H}), 2.80-2.67(\mathrm{~m}, 1 \mathrm{H}), 2.49-$ $2.27(\mathrm{~m}, 1 \mathrm{H}), 2.20-1.98(\mathrm{~m}, 2 \mathrm{H}), 1.69-1.63(\mathrm{~m}, 1 \mathrm{H}), 1.59(\mathrm{~d}, J=7.2 \mathrm{~Hz}, 3 \mathrm{H})$. The minor isomer: ${ }^{1} \mathrm{H}$ NMR $(500$ $\left.\mathrm{MHz}, \mathrm{CDCl}_{3}\right) \delta 7.66-7.16(\mathrm{~m}, 5 \mathrm{H}), 6.22-5.83(\mathrm{~m}, 1 \mathrm{H}), 3.71-3.48(\mathrm{~m}, 1 \mathrm{H}), 3.26-3.17(\mathrm{~m}, 1 \mathrm{H}), 3.17-3.07(\mathrm{~m}$, $2 \mathrm{H}), 2.80-2.67(\mathrm{~m}, 1 \mathrm{H}), 2.49-2.27(\mathrm{~m}, 1 \mathrm{H}), 1.86-1.75(\mathrm{~m}, 1 \mathrm{H}), 1.61(\mathrm{~d}, J=7.3 \mathrm{~Hz}, 3 \mathrm{H}) .{ }^{13} \mathrm{C} \mathrm{NMR}(125 \mathrm{MHz}$, $\left.\mathrm{CDCl}_{3}\right) \delta 175.0,175.0,170.9(\mathrm{t}, J=26.5 \mathrm{~Hz}) 170.8(\mathrm{t}, J=26.5 \mathrm{~Hz}) 138.3,137.8,129.0,128.9,128.5,128.4,127.5$, 127.2, 111.3 (dd, $J=256.3,245.1 \mathrm{~Hz}), 51.6,51.4,45.4,45.2,37.3$ (d, $J=6.3 \mathrm{~Hz}), 37.2(\mathrm{~d}, J=6.3 \mathrm{~Hz}), 35.9$ (t, $J=$ $23.7 \mathrm{~Hz}), 26.7,26.5,16.0,15.9$. The major isomer: ${ }^{19} \mathrm{~F}$ NMR $\left(470 \mathrm{MHz}, \mathrm{CDCl}_{3}\right) \delta-100.42(\mathrm{ddd}, J=33.0,14.8,4.4$ $\mathrm{Hz}),-101.02(\mathrm{ddd}, J=32.9,14.5,4.1 \mathrm{~Hz}),-106.11,-106.71$. The major isomer: ${ }^{19} \mathrm{~F}$ NMR $\left(470 \mathrm{MHz}, \mathrm{CDCl}_{3}\right) \delta-$ 101.02 (ddd, $J=32.9,14.5,4.1 \mathrm{~Hz}),-106.11$. The minor isomer: ${ }^{19} \mathrm{~F}$ NMR $\left(470 \mathrm{MHz}, \mathrm{CDCl}_{3}\right) \delta-100.42(\mathrm{ddd}, J=$ 33.0, 14.8, 4.4 Hz), -106.71. IR (KBr): 2958, 1686, 1553, 1232, 1116, 908, 697, $642 \mathrm{~cm}^{-1}$. HRMS (ESI) m/z: [M + $\mathrm{H}]^{+}$Calcd for $\mathrm{C}_{15} \mathrm{H}_{17} \mathrm{~F}_{2} \mathrm{~N}_{2} \mathrm{O} 279.1303$; Found: 279.1302 .

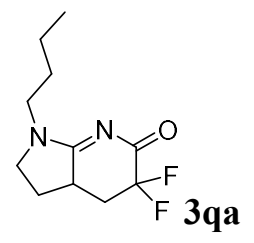

Purified by column chromatography (silica gel, EtOAc/Petroleum ether: $1 / 2$ ) to afford the title compound (86\% yield, $40 \mathrm{mg}$ ) as a white solid. mp: $150-152{ }^{\circ} \mathrm{C} .{ }^{1} \mathrm{H}$ NMR $\left(400 \mathrm{MHz}, \mathrm{CDCl}_{3}\right) \delta 3.78-3.56(\mathrm{~m}, 3 \mathrm{H}), 3.55-3.38(\mathrm{~m}, 1 \mathrm{H})$, $3.29-3.07(\mathrm{~m}, 1 \mathrm{H}), 2.85-2.57(\mathrm{~m}, 1 \mathrm{H}), 2.57-2.33(\mathrm{~m}, 1 \mathrm{H}), 2.24-1.95(\mathrm{~m}, 1 \mathrm{H}), 1.96-1.74(\mathrm{~m}, 1 \mathrm{H}), 1.69-1.50$ $(\mathrm{m}, 2 \mathrm{H}), 1.42-1.27(\mathrm{~m}, 2 \mathrm{H}), 0.94(\mathrm{t}, J=7.3 \mathrm{~Hz}, 3 \mathrm{H}) .{ }^{13} \mathrm{C} \mathrm{NMR}\left(100 \mathrm{MHz}, \mathrm{CDCl}_{3}\right) \delta$ 175.4, $170.7(\mathrm{t}, J=25.3 \mathrm{~Hz})$, $111.2(\mathrm{dd}, J=256.1,245.1 \mathrm{~Hz}), 50.3,44.7,37.0$ (d, $J=8.1 \mathrm{~Hz}), 35.9$ (t, $J=24.2 \mathrm{~Hz}), 29.1,26.7,20.0,13.8 .{ }^{19} \mathrm{~F}$ NMR $\left(376 \mathrm{MHz}, \mathrm{CDCl}_{3}\right) \delta-100.68$ (d, $\left.J=282.2 \mathrm{~Hz}\right),-106.60$ (d, $J=282.1 \mathrm{~Hz}$ ). IR (KBr): 2960, 2934, 1693, 1575, 1482, 1307, $1121 \mathrm{~cm}^{-1}$. HRMS (ESI) m/z: [M + H] $]^{+}$Calcd for $\mathrm{C}_{11} \mathrm{H}_{17} \mathrm{~F}_{2} \mathrm{~N}_{2} \mathrm{O}$ 231.1303; Found: 231.1308.

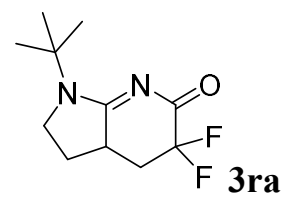

Purified by column chromatography (silica gel, EtOAc/Petroleum ether: $1 / 3$ ) to afford the title compound (49\% yield, $23 \mathrm{mg}$ ) as a white solid. mp: $155-158{ }^{\circ} \mathrm{C} .{ }^{1} \mathrm{H}$ NMR $\left(400 \mathrm{MHz}, \mathrm{CDCl}_{3}\right) \delta 3.86-3.78(\mathrm{~m}, 1 \mathrm{H}), 3.66(\mathrm{td}, J=11.1,6.1$ $\mathrm{Hz}, 1 \mathrm{H}), 3.15-2.94(\mathrm{~m}, 1 \mathrm{H}), 2.79-2.53(\mathrm{~m}, 1 \mathrm{H}), 2.46-2.18(\mathrm{~m}, 1 \mathrm{H}), 2.20-1.90(\mathrm{~m}, 1 \mathrm{H}), 1.86-1.65(\mathrm{~m}, 1 \mathrm{H})$, 
$1.54(\mathrm{~s}, 9 \mathrm{H}) .{ }^{13} \mathrm{C}$ NMR $\left(100 \mathrm{MHz}, \mathrm{CDCl}_{3}\right) \delta 175.3,170.0(\mathrm{t}, J=25.9 \mathrm{~Hz}), 110.9(\mathrm{dd}, J=255.2,244.9 \mathrm{~Hz}), 57.9,49.0$, $38.2(\mathrm{dd}, J=8.0,1.0 \mathrm{~Hz}), 36.1(\mathrm{t}, J=23.2 \mathrm{~Hz}), 27.9,26.4 .{ }^{19} \mathrm{~F}$ NMR $\left(376 \mathrm{MHz}, \mathrm{CDCl}_{3}\right) \delta-101.33(\mathrm{~d}, J=280.4 \mathrm{~Hz})$, -106.36 (d, $J=280.5 \mathrm{~Hz}$ ). IR (KBr): 2981, 2910, 1685, 1552, 1460, 1336, 1116, $1067 \mathrm{~cm}^{-1}$. HRMS (ESI) m/z: [M + $\mathrm{H}]^{+}$Calcd for $\mathrm{C}_{11} \mathrm{H}_{17} \mathrm{~F}_{2} \mathrm{~N}_{2} \mathrm{O} 231.1303$; Found: 231.1312 .

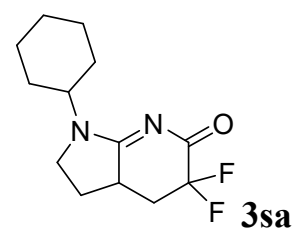

Purified by column chromatography (silica gel, EtOAc/Petroleum ether: $1 / 3$ ) to afford the title compound (72\% yield, $37 \mathrm{mg}$ ) as a white solid. mp: $167-170{ }^{\circ} \mathrm{C} .{ }^{1} \mathrm{H}$ NMR $\left(500 \mathrm{MHz}, \mathrm{CDCl}_{3}\right) \delta 4.44-4.35(\mathrm{~m}, 1 \mathrm{H}), 3.73-3.60(\mathrm{~m}, 1 \mathrm{H})$, $3.59-3.48(\mathrm{~m}, 1 \mathrm{H}), 3.24-3.05(\mathrm{~m}, 1 \mathrm{H}), 2.71(\mathrm{tdd}, J=14.0,5.6,2.3 \mathrm{~Hz}, 1 \mathrm{H}), 2.49-2.32(\mathrm{~m}, 1 \mathrm{H}), 2.20-1.95(\mathrm{~m}$, 1H), $1.89-1.73(\mathrm{~m}, 4 \mathrm{H}), 1.73-1.65(\mathrm{~m}, 2 \mathrm{H}), 1.49-1.33(\mathrm{~m}, 4 \mathrm{H}), 1.20-1.03(\mathrm{~m}, 1 \mathrm{H}) .{ }^{13} \mathrm{C}$ NMR $\left(100 \mathrm{MHz}, \mathrm{CDCl}_{3}\right)$ $\delta 174.6,170.8(\mathrm{t}, J=25.3 \mathrm{~Hz}), 111.4(\mathrm{dd}, J=255.9,245.1 \mathrm{~Hz}), 53.0,45.8,37.3(\mathrm{~d}, J=8.2 \mathrm{~Hz}), 35.9(\mathrm{t}, J=23.7 \mathrm{~Hz})$, 30.0, 29.9, 26.7, 25.2, 25.1, 25.0. ${ }^{19} \mathrm{~F}$ NMR $\left(376 \mathrm{MHz}, \mathrm{CDCl}_{3}\right) \delta-100.77(\mathrm{~d}, J=281.7 \mathrm{~Hz}),-104.51--108.10(\mathrm{~m})$. IR (KBr): 2925, 2853, 1684, 1558, 1466, 1319, 1010, $724 \mathrm{~cm}^{-1}$. HRMS (ESI) m/z: [M + H] $]^{+}$Calcd for $\mathrm{C}_{13} \mathrm{H}_{19} \mathrm{~F}_{2} \mathrm{~N}_{2} \mathrm{O}$ 257.1460; Found: 257.1458 .

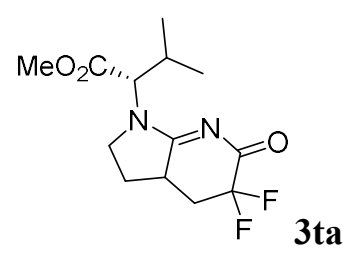

Purified by column chromatography (silica gel, EtOAc/Petroleum ether: 1/1) to afford the title compound (an inseparable mixture, $65 \%$ yield, $38 \mathrm{mg}, d r=1.2 / 1)$ as a yellow oil. The major isomer: ${ }^{1} \mathrm{H} \mathrm{NMR}\left(300 \mathrm{MHz}, \mathrm{CDCl}_{3}\right)$ $\delta 5.06(\mathrm{~d}, J=9.4 \mathrm{~Hz}, 1 \mathrm{H}), 4.12(\mathrm{dd}, J=10.8,8.8 \mathrm{~Hz}, 1 \mathrm{H}), 3.74(\mathrm{~s}, 3 \mathrm{H}), 3.66-3.54(\mathrm{~m}, 1 \mathrm{H}), 3.29-3.04(\mathrm{~m}, 1 \mathrm{H})$, $2.86-2.61(\mathrm{~m}, 1 \mathrm{H}), 2.54-2.39(\mathrm{~m}, 1 \mathrm{H}), 2.36-2.21(\mathrm{~m}, 1 \mathrm{H}), 2.17-1.97(\mathrm{~m}, 2 \mathrm{H}), 1.03(\mathrm{~d}, J=5.6 \mathrm{~Hz}, 3 \mathrm{H}), 0.96(\mathrm{~d}$, $J=6.8 \mathrm{~Hz}, 3 \mathrm{H})$. The minor isomer: ${ }^{1} \mathrm{H}$ NMR $\left(300 \mathrm{MHz}, \mathrm{CDCl}_{3}\right) \delta 4.99(\mathrm{~d}, J=10.3 \mathrm{~Hz}, 1 \mathrm{H}), 3.88-3.75(\mathrm{~m}, 1 \mathrm{H})$, $3.74(\mathrm{~s}, 3 \mathrm{H}), 3.29-3.04(\mathrm{~m}, 1 \mathrm{H}), 2.86-2.61(\mathrm{~m}, 1 \mathrm{H}), 2.54-2.39(\mathrm{~m}, 1 \mathrm{H}), 2.36-2.21(\mathrm{~m}, 1 \mathrm{H}), 1.96-1.73(\mathrm{~m}, 3 \mathrm{H})$, $1.01(\mathrm{~d}, J=5.7 \mathrm{~Hz}, 3 \mathrm{H}), 0.91(\mathrm{~d}, J=6.8 \mathrm{~Hz}, 3 \mathrm{H}) .{ }^{13} \mathrm{C} \mathrm{NMR}\left(100 \mathrm{MHz}, \mathrm{CDCl}_{3}\right) \delta 177.2,177.2,170.7(\mathrm{t}, J=25.9$ Hz), 170.7 (t, $J=26.3 \mathrm{~Hz}), 170.4,169.9,111.2(\mathrm{dd}, J=245.4,253.5 \mathrm{~Hz}), 111.1(\mathrm{dd}, J=254.5,246.4 \mathrm{~Hz}) 61.3,61.3$, $52.4,52.3,47.7,47.1,37.0$ (d, $J=8.1 \mathrm{~Hz}), 36.5$ (d, $J=8.2 \mathrm{~Hz}), 36.0$ (t, $J=24.2 \mathrm{~Hz}), 35.7$ (t, $J=23.3 \mathrm{~Hz}), 29.1,28.3$, 27.3, 27.2, 19.4, 19.2, 19.1, 19.0. The major isomer: ${ }^{19} \mathrm{~F}$ NMR $\left(470 \mathrm{MHz}, \mathrm{CDCl}_{3}\right) \delta-101.40$ (ddd, $J=32.9,27.4$, $14.1 \mathrm{~Hz}),-106.57(\mathrm{dd}, J=141.8,5.3 \mathrm{~Hz})$. The minor isomer: ${ }^{19} \mathrm{~F}$ NMR $\left(470 \mathrm{MHz}, \mathrm{CDCl}_{3}\right) \delta-100.80(\mathrm{ddd}, J=32.9$, 27.8, $14.2 \mathrm{~Hz}$ ), -107.17 (dd, $J=142.3,5.1 \mathrm{~Hz}$ ). IR (KBr): 2959, 2896, 1738, 1694, 1558, 1273, 1192, $1120 \mathrm{~cm}^{-1}$. HRMS (ESI) m/z: [M+ H] $]^{+}$Calcd for $\mathrm{C}_{13} \mathrm{H}_{19} \mathrm{~F}_{2} \mathrm{~N}_{2} \mathrm{O}_{3}$ 289.1358; Found: 289.1363. 


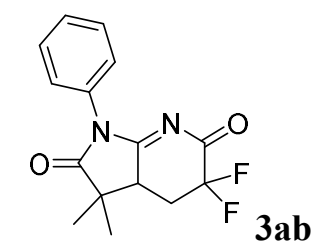

Purified by column chromatography (silica gel, EtOAc/Petroleum ether: $1 / 1)$ to afford the title compound (92\% yield, $54 \mathrm{mg}$ ) as a white solid. mp: $110-116^{\circ} \mathrm{C} .{ }^{1} \mathrm{H}$ NMR $\left(400 \mathrm{MHz}, \mathrm{CDCl}_{3}\right) \delta 7.54-7.37(\mathrm{~m}, 3 \mathrm{H}), 7.31-7.27(\mathrm{~m}, 2 \mathrm{H})$, 3.33 (ddd, $J=12.9,5.6,1.8 \mathrm{~Hz}, 1 \mathrm{H}), 2.67$ (tdd, $J=13.4,5.6,3.6 \mathrm{~Hz}, 1 \mathrm{H}), 2.48-2.19$ (m, 1H), 1.47 (s, 3H), 1.31 (s, $3 \mathrm{H}) .{ }^{13} \mathrm{C}$ NMR $\left(100 \mathrm{MHz}, \mathrm{CDCl}_{3}\right) \delta 180.0,179.0,170.0(\mathrm{t}, J=26.3 \mathrm{~Hz}), 131.4,129.4,126.8,111.7(\mathrm{dd}, J=247.5$, $246.4 \mathrm{~Hz}), 43.7(\mathrm{dd}, J=7.2,1.7 \mathrm{~Hz}), 43.4,30.3(\mathrm{dd}, J=24.9,22.8 \mathrm{~Hz}), 22.6,22.3 .{ }^{19} \mathrm{~F} \mathrm{NMR}\left(376 \mathrm{MHz}, \mathrm{CDCl}_{3}\right) \delta$ 102.77 (d, $J=280.8 \mathrm{~Hz}),-105.65$ (d, $J=280.9 \mathrm{~Hz}$ ). IR (KBr): 2977, 2937, 1713, 1638, 1577, 1379, 1305, 1148, 1076, $762 \mathrm{~cm}^{-1}$. HRMS (ESI) m/z: [M + H $]^{+}$Calcd for $\mathrm{C}_{15} \mathrm{H}_{15} \mathrm{~F}_{2} \mathrm{~N}_{2} \mathrm{O}_{2}$ 293.1096; Found: 293.1105.

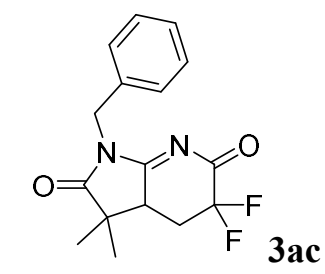

Purified by column chromatography (silica gel, EtOAc/Petroleum ether: $1 / 1)$ to afford the title compound (63\% yield, $39 \mathrm{mg}$ ) as a yellow oil. ${ }^{1} \mathrm{H} \mathrm{NMR}\left(400 \mathrm{MHz}, \mathrm{CDCl}_{3}\right) \delta 7.46-7.36(\mathrm{~m}, 2 \mathrm{H}), 7.34-7.28(\mathrm{~m}, 3 \mathrm{H}), 4.91-4.79(\mathrm{~m}, 2 \mathrm{H})$, 3.12 (ddd, $J=13.1,5.5,1.8 \mathrm{~Hz}, 1 \mathrm{H}), 2.61$ (tdd, $J=13.4,4.9,3.3 \mathrm{~Hz}, 1 \mathrm{H}), 2.32-2.10$ (m, 1H), 1.40 (s, 3H), 1.13 (s, 3H). ${ }^{13} \mathrm{C}$ NMR $\left(100 \mathrm{MHz}, \mathrm{CDCl}_{3}\right) \delta 180.7,179.1,170.0(\mathrm{t}, J=26.4 \mathrm{~Hz}), 134.7,129.1,128.1,128.5,111.9(\mathrm{dd}, J=$ $259.8,246.5 \mathrm{~Hz}), 44.0,43.6(\mathrm{~d}, J=7.4 \mathrm{~Hz}), 43.1,30.5(\mathrm{t}, J=23.9 \mathrm{~Hz}), 22.8,22.2 .{ }^{19} \mathrm{~F}$ NMR $\left(470 \mathrm{MHz}, \mathrm{CDCl}_{3}\right) \delta-$ $101.92--102.84(\mathrm{~m}),-105.32$ (d, $J=282.4 \mathrm{~Hz}$ ). IR (KBr): 2974, 2935, 1698, 1621, 1403, 1145, 732, $700 \mathrm{~cm}^{-1}$. HRMS (ESI) m/z: [M + H] $]^{+}$Calcd for $\mathrm{C}_{16} \mathrm{H}_{17} \mathrm{~F}_{2} \mathrm{~N}_{2} \mathrm{O}_{2}$ 307.1253; Found: 307.1249.

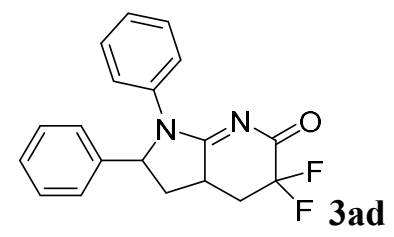

Purified by column chromatography (silica gel, EtOAc/Petroleum ether: $1 / 1$ with $0.5 \% \mathrm{Et}_{3} \mathrm{~N}$ ) to afford the title compound (an inseparable mixture, $81 \%$ yield, $53 \mathrm{mg}, d r=1.2 / 1$ ) as a white solid. The major isomer: ${ }^{1} \mathrm{H}$ NMR (500 $\left.\mathrm{MHz}, \mathrm{CDCl}_{3}\right) \delta 7.57(\mathrm{~d}, J=8.0 \mathrm{~Hz}, 2 \mathrm{H}), 7.41-7.36(\mathrm{~m}, 2 \mathrm{H}), 7.35-7.29(\mathrm{~m}, 3 \mathrm{H}), 7.24-7.17(\mathrm{~m}, 3 \mathrm{H}), 5.50(\mathrm{~d}, J=$ $8.5 \mathrm{~Hz}, 1 \mathrm{H}), 3.57-3.48(\mathrm{~m}, 1 \mathrm{H}), 2.73(\mathrm{tdd}, J=13.9,5.7,2.5 \mathrm{~Hz}, 1 \mathrm{H}), 2.58-2.49(\mathrm{~m}, 1 \mathrm{H}), 2.45-2.39(\mathrm{~m}, 1 \mathrm{H}), 2.38$ $-2.13(\mathrm{~m}, 1 \mathrm{H})$. The minor isomer: ${ }^{1} \mathrm{H}$ NMR $\left(500 \mathrm{MHz}, \mathrm{CDCl}_{3}\right) \delta 7.26-7.24(\mathrm{~m}, 4 \mathrm{H}), 7.24-7.17(\mathrm{~m}, 5 \mathrm{H}), 7.09(\mathrm{t}$, $J=7.3 \mathrm{~Hz}, 1 \mathrm{H}), 5.45$ (dd, $J=10.3,5.9 \mathrm{~Hz}, 1 \mathrm{H}), 3.47-3.38$ (m, 1H), $3.01-2.90$ (m, 1H), 2.82 (tdd, $J=14.0,5.5$, $2.5 \mathrm{~Hz}, 1 \mathrm{H}), 2.38-2.13(\mathrm{~m}, 1 \mathrm{H}), 1.96(\mathrm{q}, J=11.8 \mathrm{~Hz}, 1 \mathrm{H}) .{ }^{13} \mathrm{C}$ NMR (125 MHz, DMSO) $\delta$ 176.9, 176.6, 170.3 (t, $J=27.9 \mathrm{~Hz}), 170.1(\mathrm{t}, J=25.1 \mathrm{~Hz}), 139.2,138.4,137.0,136.6,128.9,128.8,128.4,128.3,128.2,127.9,127.0,126.9$, $126.2,126.0,124.9,112.1(\mathrm{dd}, J=254.2,244.0 \mathrm{~Hz}), 111.6(\mathrm{dd}, J=245.7,244.4 \mathrm{~Hz}), 67.3,66.9,36.6,36.4(\mathrm{~d}, J=$ $8.7 \mathrm{~Hz}), 35.1(\mathrm{~d}, J=8.5 \mathrm{~Hz}), 34.6,34.4(\mathrm{t}, J=22.3 \mathrm{~Hz}), 34.1(\mathrm{t}, J=22.7 \mathrm{~Hz})$. The major isomer: ${ }^{19} \mathrm{~F}$ NMR $(470 \mathrm{MHz}$, DMSO) $\delta-99.49(\mathrm{ddd}, J=277.0,35.5,15.8 \mathrm{~Hz}),-104.01(\mathrm{dd}, J=42.2,6.2 \mathrm{~Hz})$. The minor isomer: ${ }^{19} \mathrm{~F}$ NMR $(470$ 
MHz, DMSO) $\delta-99.25$ (ddd, $J=277.8,34.3,17.7 \mathrm{~Hz}),-104.60$ (dd, $J=41.1,6.6 \mathrm{~Hz})$. IR (KBr): 1686, 1531, 1328, 1279, 1196, $695 \mathrm{~cm}^{-1}$. HRMS (ESI) m/z: [M + H] ${ }^{+}$Calcd for $\mathrm{C}_{19} \mathrm{H}_{17} \mathrm{~F}_{2} \mathrm{~N}_{2} \mathrm{O}$ 327.1303; Found: 327.1297.

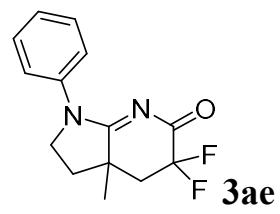

Purified by column chromatography (silica gel, EtOAc/Petroleum ether: $1 / 1$ ) to afford the title compound (41\% yield, $22 \mathrm{mg}$ ) as a white solid. mp: $133-137{ }^{\circ} \mathrm{C} .{ }^{1} \mathrm{H}$ NMR $\left(400 \mathrm{MHz}, \mathrm{CDCl}_{3}\right) \delta 7.73-7.60(\mathrm{~m}, 2 \mathrm{H}), 7.47-7.37(\mathrm{~m}, 2 \mathrm{H})$, $7.31-7.23(\mathrm{~m}, 1 \mathrm{H}), 4.25(\mathrm{td}, J=10.9,6.5 \mathrm{~Hz}, 1 \mathrm{H}), 4.08-3.95(\mathrm{~m}, 1 \mathrm{H}), 2.75-2.59(\mathrm{~m}, 1 \mathrm{H}), 2.43-2.27(\mathrm{~m}, 1 \mathrm{H})$, $2.25-2.09(\mathrm{~m}, 2 \mathrm{H}), 1.42(\mathrm{~d}, J=2.2 \mathrm{~Hz}, 3 \mathrm{H}) .{ }^{13} \mathrm{C} \mathrm{NMR}\left(100 \mathrm{MHz}, \mathrm{CDCl}_{3}\right) \delta 178.0,171.1$ (t, $\left.J=25.3 \mathrm{~Hz}\right), 138.0$, 129.2, 127.2, 122.9, $110.4(\mathrm{dd}, J=251.5,251.5 \mathrm{~Hz}), 51.1,43.1$ (dd, $J=24.2,22.0 \mathrm{~Hz}), 42.1(\mathrm{~d}, J=8.4 \mathrm{~Hz}), 35.1$, $22.7(\mathrm{~d}, J=3.2 \mathrm{~Hz}) .{ }^{19} \mathrm{~F}$ NMR $\left(376 \mathrm{MHz}, \mathrm{CDCl}_{3}\right) \delta-92.47$ (d, $\left.J=289.7 \mathrm{~Hz}\right),-97.19$ (d, $\left.J=289.7 \mathrm{~Hz}\right) . \mathrm{IR}(\mathrm{KBr}):$ 2979, 2934, 1696, 1545, 1314, 1154, 1140, $771 \mathrm{~cm}^{-1}$. HRMS (ESI) m/z: $[\mathrm{M}+\mathrm{H}]^{+}$Calcd for $\mathrm{C}_{14} \mathrm{H}_{15} \mathrm{~F}_{2} \mathrm{~N}_{2} \mathrm{O} 265.1147$; Found: 265.1155 .

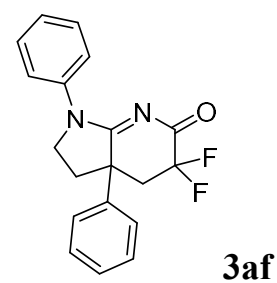

Purified by column chromatography (silica gel, EtOAc/Petroleum ether: $1 / 2$ ) to afford the title compound ( $81 \%$ yield, $53 \mathrm{mg}$ ) as a white solid. mp: $234-236^{\circ} \mathrm{C} .{ }^{1} \mathrm{H}$ NMR $\left(400 \mathrm{MHz}, \mathrm{CDCl}_{3}\right) \delta 7.71(\mathrm{~d}, J=7.8 \mathrm{~Hz}, 2 \mathrm{H}), 7.45$ (t, $J=8.0$ $\mathrm{Hz}, 2 \mathrm{H}), 7.39-7.25(\mathrm{~m}, 4 \mathrm{H}), 7.21(\mathrm{~m}, 2 \mathrm{H}), 3.96-3.81(\mathrm{~m}, 2 \mathrm{H}), 3.22(\mathrm{t}, J=14.9 \mathrm{~Hz}, 1 \mathrm{H}), 2.72-2.40(\mathrm{~m}, 3 \mathrm{H}) .{ }^{13} \mathrm{C}$ $\operatorname{NMR}\left(100 \mathrm{MHz}, \mathrm{CDCl}_{3}\right) \delta 175.1,171.2(\mathrm{t}, J=25.3 \mathrm{~Hz}), 138.8,137.9,129.5,129.3,128.2,127.3,126.3,123.0,109.8$ $(\mathrm{dd}, J=251.5,251.4 \mathrm{~Hz}), 51.4(\mathrm{~d}, J=8.5 \mathrm{~Hz}), 50.9,43.6(\mathrm{dd}, J=24.4,20.8 \mathrm{~Hz}), 38.2 .{ }^{19} \mathrm{~F} \mathrm{NMR}\left(376 \mathrm{MHz}, \mathrm{CDCl}_{3}\right)$ $\delta$-95.36 (ddd, $J=289.1,27.3,15.8 \mathrm{~Hz}$ ), -98.56 (dd, $J=289.2,11.3 \mathrm{~Hz}$ ). IR (KBr): 2923, 1686, 1554, 1094, 1043, $760,668,553 \mathrm{~cm}^{-1}$. HRMS (ESI) m/z: [M+ H] ${ }^{+}$Calcd for $\mathrm{C}_{19} \mathrm{H}_{17} \mathrm{~F}_{2} \mathrm{~N}_{2} \mathrm{O}$ 327.1303; Found: 327.1307 .

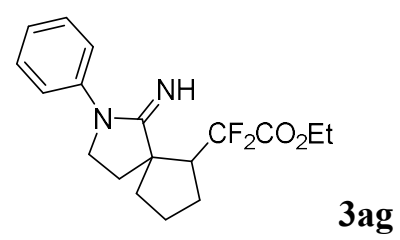

Purified by column chromatography (silica gel, $\mathrm{CH}_{2} \mathrm{Cl}_{2} / \mathrm{EtOH}$ : 20/1) to afford the title compound (45\% yield, $31 \mathrm{mg}$ ) as a yellow oil. ${ }^{1} \mathrm{H}$ NMR $\left(300 \mathrm{MHz}, \mathrm{CDCl}_{3}\right) \delta 7.65-7.46(\mathrm{~m}, 3 \mathrm{H}), 7.35-7.30(\mathrm{~m}, 2 \mathrm{H}), 4.34(\mathrm{q}, J=7.5,7.0 \mathrm{~Hz}, 2 \mathrm{H})$, $4.14-3.96(\mathrm{~m}, 2 \mathrm{H}), 3.96-3.78(\mathrm{~m}, 1 \mathrm{H}), 3.11-2.95(\mathrm{~m}, 1 \mathrm{H}), 2.90-2.69(\mathrm{~m}, 1 \mathrm{H}), 2.36-2.11(\mathrm{~m}, 2 \mathrm{H}), 2.10-1.98$ $(\mathrm{m}, 1 \mathrm{H}), 1.97-1.74(\mathrm{~m}, 3 \mathrm{H}), 1.38(\mathrm{t}, J=7.1 \mathrm{~Hz}, 3 \mathrm{H}) .{ }^{13} \mathrm{C}$ NMR $\left(125 \mathrm{MHz}, \mathrm{CDCl}_{3}\right) \delta 172.0,163.3(\mathrm{t}, J=33.1 \mathrm{~Hz})$, 135.3, 131.1, 130.4, 125.0, $116.8(\mathrm{t}, J=252.0 \mathrm{~Hz}), 63.8,56.3,54.9,49.7(\mathrm{t}, J=20.8 \mathrm{~Hz}), 40.4,29.1,24.0,21.6,14.1$. ${ }^{19} \mathrm{~F}$ NMR $\left(470 \mathrm{MHz}, \mathrm{CDCl}_{3}\right) \delta-105.57(\mathrm{dd}, J=255.7,7.7 \mathrm{~Hz}),-111.53(\mathrm{dd}, J=255.6,24.5 \mathrm{~Hz}) . \mathrm{IR}(\mathrm{KBr}): 2916$, 1754, 1625, 1694, 1314, 1074, 763, $694 \mathrm{~cm}^{-1}$. HRMS (ESI) m/z: $[\mathrm{M}+\mathrm{H}]^{+}$Calcd for $\mathrm{C}_{18} \mathrm{H}_{23} \mathrm{~F}_{2} \mathrm{~N}_{2} \mathrm{O}_{2}$ 337.1722; Found: 337.1730 . 


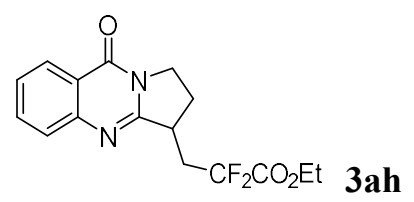

Purified by column chromatography (silica gel, EtOAc/Petroleum ether: $1 / 1)$ to afford the title compound (58\% yield, $36 \mathrm{mg})$ as a yellow oil. ${ }^{1} \mathrm{H}$ NMR $\left(300 \mathrm{MHz}, \mathrm{CDCl}_{3}\right) \delta 8.34-8.24(\mathrm{~m}, 1 \mathrm{H}), 7.79-7.65(\mathrm{~m}, 1 \mathrm{H}), 7.71-7.62(\mathrm{~m}, 1 \mathrm{H})$, $7.52-7.41(\mathrm{~m}, 1 \mathrm{H}), 4.50-4.30(\mathrm{~m}, 3 \mathrm{H}), 4.03-3.86(\mathrm{~m}, 1 \mathrm{H}), 3.69-3.47(\mathrm{~m}, 1 \mathrm{H}), 3.35-3.06(\mathrm{~m}, 1 \mathrm{H}), 2.78-2.61$ $(\mathrm{m}, 1 \mathrm{H}), 2.41-2.16(\mathrm{~m}, 1 \mathrm{H}), 2.14-1.91(\mathrm{~m}, 1 \mathrm{H}), 1.39(\mathrm{t}, J=7.2 \mathrm{~Hz}, 3 \mathrm{H}) .{ }^{13} \mathrm{C} \mathrm{NMR}\left(125 \mathrm{MHz}, \mathrm{CDCl}_{3}\right) \delta 163.9(\mathrm{t}$, $J=32.3 \mathrm{~Hz}), 161.0,159.4,149.1,134.4,127.2,126.7,126.6,121.0,115.7(\mathrm{t}, J=250.9 \mathrm{~Hz}), 63.4,44.9,38.2,36.6(\mathrm{t}$, $J=22.9 \mathrm{~Hz}), 28.0,14.1 .{ }^{19} \mathrm{~F}$ NMR $\left(376 \mathrm{MHz} \mathrm{CDCl}_{3}\right) \delta-103.41$ (ddd, $\left.J=260.9,25.1,9.7 \mathrm{~Hz}\right),-106.79$ (ddd, $J=$ 260.9, 22.3, 13.8 Hz). IR (KBr): 2925, 1765, 1680, 1582, 1121, 772, $695 \mathrm{~cm}^{-1}$. HRMS (ESI) m/z: $[\mathrm{M}+\mathrm{H}]^{+} \mathrm{Calcd}$ for $\mathrm{C}_{16} \mathrm{H}_{17} \mathrm{~F}_{2} \mathrm{~N}_{2} \mathrm{O}_{3}$ 323.1202; Found: 323.1206.<smiles>CCOC(=O)[C-]CC1CCN(c2ccccc2)C1=O</smiles>

Purified by column chromatography (silica gel, EtOAc/Petroleum ether: $1 / 5)$ to afford the title compound (6\% yield, $5 \mathrm{mg}$ ) as a yellow oil. ${ }^{1} \mathrm{H} \mathrm{NMR}\left(400 \mathrm{MHz}, \mathrm{CDCl}_{3}\right) \delta 7.66-7.55(\mathrm{~m}, 2 \mathrm{H}), 7.42-7.32(\mathrm{~m}, 2 \mathrm{H}), 7.20-7.11(\mathrm{~m}, 1 \mathrm{H})$, $4.35(\mathrm{q}, J=7.2 \mathrm{~Hz}, 2 \mathrm{H}), 3.90-3.71(\mathrm{~m}, 2 \mathrm{H}), 3.04-2.80(\mathrm{~m}, 2 \mathrm{H}), 2.62-2.41(\mathrm{~m}, 1 \mathrm{H}), 2.23-2.03(\mathrm{~m}, 1 \mathrm{H}), 2.00-$ $1.87(\mathrm{~m}, 1 \mathrm{H}), 1.36(\mathrm{t}, J=7.1 \mathrm{~Hz}, 3 \mathrm{H}) .{ }^{13} \mathrm{C} \mathrm{NMR}\left(100 \mathrm{MHz}, \mathrm{CDCl}_{3}\right) \delta 173.5,164.0(\mathrm{t}, J=32.5 \mathrm{~Hz}), 139.3,129.0$, $124.8,116.0(\mathrm{t}, J=251.5 \mathrm{~Hz}), 63.2,46.8,38.0,36.2(\mathrm{t}, J=23.1 \mathrm{~Hz}), 26.3,14.0 .{ }^{19} \mathrm{~F} \mathrm{NMR}\left(470 \mathrm{MHz} \mathrm{CDCl}_{3}\right) \delta-$ 103.52 (ddd, $J=259.2,24.6,10.0 \mathrm{~Hz}$ ), -107.06 (ddd, $J=259.2,22.1,14.8 \mathrm{~Hz}) . \mathrm{IR}$ (KBr): 2917, 2850, 2364, 2340, 1758, 1397, 1110, $758 \mathrm{~cm}^{-1}$. HRMS (ESI) m/z: $[\mathrm{M}+\mathrm{H}]^{+}$Calcd for $\mathrm{C}_{15} \mathrm{H}_{18} \mathrm{~F}_{2} \mathrm{NO}_{3}$ 298.1249; Found: 298.1256.<smiles>CC1(C)CC2=C(NC1=O)N(c1ccccc1)C(=O)C(F)(F)C2</smiles>

Purified by column chromatography (silica gel, EtOAc/Petroleum ether: $1 / 1)$ to afford the title compound (58\% yield, $36 \mathrm{mg}$ ) as a white solid. mp: $145-147{ }^{\circ} \mathrm{C} .{ }^{1} \mathrm{H}$ NMR $\left(400 \mathrm{MHz}, \mathrm{CDCl}_{3}\right) \delta 7.52-7.38(\mathrm{~m}, 3 \mathrm{H}), 7.19-7.11(\mathrm{~m}, 2 \mathrm{H})$, 6.58 (brs, 1H), 2.95 (tt, $J=16.5,1.5 \mathrm{~Hz}, 2 \mathrm{H}), 2.32$ (s, 2H), $1.29(\mathrm{~s}, 6 \mathrm{H}) .{ }^{13} \mathrm{C} \mathrm{NMR}\left(100 \mathrm{MHz}, \mathrm{CDCl}_{3}\right) \delta 174.5,159.7$ (t, $J=31.2 \mathrm{~Hz}), 134.4,130.2,129.4,128.9,127.4,111.7(\mathrm{t}, J=247.4 \mathrm{~Hz}), 87.4(\mathrm{t}, J=5.1 \mathrm{~Hz}), 38.4,37.8,35.6(\mathrm{t}, J$ $=24.8 \mathrm{~Hz}), 24.7 .{ }^{19} \mathrm{~F}$ NMR $\left(376 \mathrm{MHz}, \mathrm{CDCl}_{3}\right) \delta$-107.20. IR (KBr): 2980, 2933, 1730, 1687, 1331, 1115, 758, 693 $\mathrm{cm}^{-1}$. HRMS (ESI) m/z: [M+H] $]^{+}$Calcd for $\mathrm{C}_{16} \mathrm{H}_{17} \mathrm{~F}_{2} \mathrm{~N}_{2} \mathrm{O}_{2} 307.1253$; Found: 307.1259 . 
<smiles>COc1ccc(N2C(=O)C(C)(C)CC3=C2NC(=O)C3(F)F)cc1</smiles>

Purified by column chromatography (silica gel, EtOAc/Petroleum ether: $1 / 3$ ) to afford the title compound (54\% yield, $37 \mathrm{mg}$ ) as a yellow solid. mp: $170-173{ }^{\circ} \mathrm{C} .{ }^{1} \mathrm{H}$ NMR $\left(400 \mathrm{MHz}, \mathrm{CDCl}_{3}\right) \delta 7.07(\mathrm{~d}, J=9.0 \mathrm{~Hz}, 2 \mathrm{H}), 6.98(\mathrm{~d}, J=9.0$ Hz, 2H), 6.56 (brs, 1H), 3.85 (s, 3H), 2.96 (t, $J=16.6 \mathrm{~Hz}, 2 \mathrm{H}), 2.32$ (s, 2H), 1.29 (s, 6H). ${ }^{13} \mathrm{C}$ NMR (100 MHz, $\left.\mathrm{CDCl}_{3}\right) \delta 174.8,160.1,159.7(\mathrm{t}, J=31.0 \mathrm{~Hz}), 130.0,127.6,126.5,115.4,111.7(\mathrm{t}, J=247.3 \mathrm{~Hz}), 86.7(\mathrm{t}, J=5.1 \mathrm{~Hz})$, 55.7, 38.4, 37.8, 35.5 (t, $J=24.7 \mathrm{~Hz}), 24.8 .{ }^{19} \mathrm{~F}$ NMR (470 MHz, $\left.\mathrm{CDCl}_{3}\right) \delta-107.07$ (t, $\left.J=16.8 \mathrm{~Hz}\right)$. IR (KBr): 2957, 2926, 1703, 1685, 1513, 1250, 1198, $833 \mathrm{~cm}^{-1}$. HRMS (ESI) m/z: $[\mathrm{M}+\mathrm{H}]^{+}$Calcd for $\mathrm{C}_{17} \mathrm{H}_{19} \mathrm{~F}_{2} \mathrm{~N}_{2} \mathrm{O}_{3} 337.1358$; Found: 337.1360 .<smiles>CC1(C)CC2=C(NC(=O)C(F)(F)C2)N(c2ccc(Br)cc2)C1=O</smiles>

Purified by column chromatography (silica gel, EtOAc/Petroleum ether: $1 / 3$ ) to afford the title compound (32\% yield, $25 \mathrm{mg}$ ) as a white solid. mp: $160-165^{\circ} \mathrm{C} .{ }^{1} \mathrm{H}$ NMR $\left(400 \mathrm{MHz}, \mathrm{CDCl}_{3}\right) \delta 7.59(\mathrm{~d}, J=7.5 \mathrm{~Hz}, 2 \mathrm{H}), 7.03(\mathrm{~d}, J=8.0$ $\mathrm{Hz}, 2 \mathrm{H}), 6.77$ (brs, $1 \mathrm{H}), 2.95$ (t, $J=16.4 \mathrm{~Hz}, 2 \mathrm{H}), 2.31$ (s, 2H), 1.27 (s, 6H). ${ }^{13} \mathrm{C}$ NMR $\left(100 \mathrm{MHz}, \mathrm{CDCl}_{3}\right) \delta 174.5$, 159.9 (t, $J=31.3 \mathrm{~Hz}), 133.5,133.2,130.4,127.2,123.2,111.6$ (t, $J=247.6 \mathrm{~Hz}), 88.3,38.5,37.7,35.5(\mathrm{t}, J=24.8$ $\mathrm{Hz}), 24.7 .{ }^{19} \mathrm{~F}$ NMR $\left(376 \mathrm{MHz}, \mathrm{CDCl}_{3}\right) \delta-107.45$ (t, $\left.J=16.5 \mathrm{~Hz}\right) . \mathrm{IR}(\mathrm{KBr}): 3232,2959,2926,1729,1686,1488$, 1197, $1049 \mathrm{~cm}^{-1}$. HRMS (ESI) m/z: [M + H] Calcd for $\mathrm{C}_{16} \mathrm{H}_{16} \mathrm{BrF}_{2} \mathrm{~N}_{2} \mathrm{O}_{2}$ 385.0358; Found: 385.0349 .

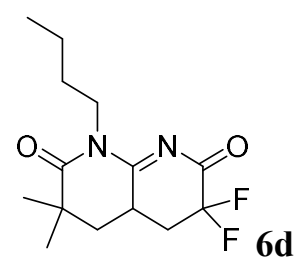

Purified by column chromatography (silica gel, EtOAc/Petroleum ether: $1 / 3)$ to afford the title compound (73\% yield, $42 \mathrm{mg}$ ) as a colorless oil. ${ }^{1} \mathrm{H}$ NMR $\left(400 \mathrm{MHz}, \mathrm{CDCl}_{3}\right) \delta 4.14-3.94(\mathrm{~m}, 2 \mathrm{H}), 3.30-3.10(\mathrm{~m}, 1 \mathrm{H}), 2.72-2.56(\mathrm{~m}$, $1 \mathrm{H}), 2.31-2.10(\mathrm{~m}, 1 \mathrm{H}), 1.93-1.82(\mathrm{~m}, 2 \mathrm{H}), 1.62-1.47(\mathrm{~m}, 2 \mathrm{H}), 1.39(\mathrm{~s}, 3 \mathrm{H}), 1.38(\mathrm{~s}, 3 \mathrm{H}), 1.36-1.27(\mathrm{~m}, 2 \mathrm{H})$, $0.95(\mathrm{t}, J=7.3 \mathrm{~Hz}, 3 \mathrm{H}) .{ }^{13} \mathrm{C}$ NMR $\left(125 \mathrm{MHz}, \mathrm{CDCl}_{3}\right) \delta 176.2,174.1,170.4(\mathrm{t}, J=25.2 \mathrm{~Hz}), 111.6(\mathrm{dd}, J=256.9$, $244.5 \mathrm{~Hz}), 42.7,39.1,38.1,36.2(\mathrm{t}, J=23.7 \mathrm{~Hz}), 31.2(\mathrm{dd}, J=7.0,2.0 \mathrm{~Hz}), 29.8,27.3,25.0,20.2,13.9 .{ }^{19} \mathrm{~F}$ NMR $\left(376 \mathrm{MHz}, \mathrm{CDCl}_{3}\right) \delta-106.59$ (d, $\left.J=274.2 \mathrm{~Hz}\right),-109.45$ (d, $J=274.2 \mathrm{~Hz}$ ). IR (KBr): 2960, 2927, 1711, 1536, 1391, 1122, 1078, $1055 \mathrm{~cm}^{-1}$. HRMS (ESI) m/z: [M + H] ${ }^{+}$Calcd for $\mathrm{C}_{14} \mathrm{H}_{21} \mathrm{~F}_{2} \mathrm{~N}_{2} \mathrm{O}_{2}$ 287.1566; Found: 287.1574 . 


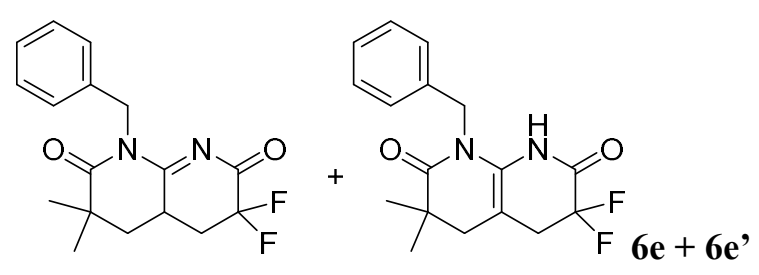

Purified by column chromatography (silica gel, EtOAc/Petroleum ether: 1/3) to afford the title compound (67\% yield, $\left.43 \mathrm{mg}, \mathbf{6 e} / 6 \mathbf{e}^{\prime}=10 / 1\right)$ as a colorless oil. 6e: ${ }^{1} \mathrm{H}$ NMR $\left(300 \mathrm{MHz}, \mathrm{CDCl}_{3}\right) \delta 7.40-7.34(\mathrm{~m}, 2 \mathrm{H}), 7.31-7.22(\mathrm{~m}, 3 \mathrm{H})$, $5.33-5.17(\mathrm{~m}, 2 \mathrm{H}), 3.30-3.13(\mathrm{~m}, 1 \mathrm{H}), 2.72-2.50(\mathrm{~m}, 1 \mathrm{H}), 2.31-2.02(\mathrm{~m}, 1 \mathrm{H}), 1.85(\mathrm{~d}, J=8.6 \mathrm{~Hz}, 2 \mathrm{H}), 1.34(\mathrm{~d}$, $J=1.8 \mathrm{~Hz}, 6 \mathrm{H}) .{ }^{13} \mathrm{C} \mathrm{NMR}\left(100 \mathrm{MHz}, \mathrm{CDCl}_{3}\right) \delta 176.3,174.1,170.1(\mathrm{t}, J=26.3 \mathrm{~Hz}), 136.4,128.9,128.6,127.8$, $111.6(\mathrm{dd}, J=256.9,244.6 \mathrm{~Hz}), 45.3,39.2,37.9,36.1(\mathrm{t}, J=23.6 \mathrm{~Hz}), 31.3(\mathrm{dd}, J=7.0,2.1 \mathrm{~Hz}), 27.2,25.0 .{ }^{19} \mathrm{~F} \mathrm{NMR}$ $\left(376 \mathrm{MHz}, \mathrm{CDCl}_{3}\right) \delta-106.41(\mathrm{~d}, J=274.6 \mathrm{~Hz}),-109.48(\mathrm{~d}, J=274.6 \mathrm{~Hz})$. HRMS (ESI) m/z: $[\mathrm{M}+\mathrm{H}]^{+} \mathrm{Calcd}$ for $\mathrm{C}_{17} \mathrm{H}_{19} \mathrm{~F}_{2} \mathrm{~N}_{2} \mathrm{O}_{2}$ 321.1409; Found: 321.1418 .
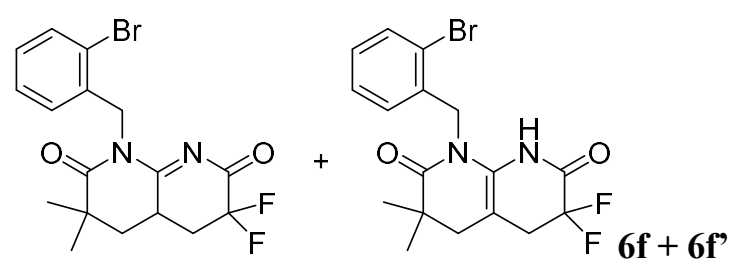

Purified by column chromatography (silica gel, EtOAc/Petroleum ether: 1/5) to afford the title compound (in separable mixture, $61 \%$ yield, $\left.49 \mathrm{mg}, \mathbf{6 f} / \mathbf{6 f} \mathbf{f}^{\prime}=5.6 / 1\right)$ as a colorless oil. $\mathbf{6 f}:{ }^{1} \mathrm{H} \mathrm{NMR}\left(400 \mathrm{MHz}, \mathrm{CDCl}_{3}\right) \delta 7.53(\mathrm{dd}, J$ $=7.9,1.1 \mathrm{~Hz}, 1 \mathrm{H}), 7.20(\mathrm{td}, J=7.6,1.1 \mathrm{~Hz}, 1 \mathrm{H}), 7.09(\mathrm{td}, J=7.8,1.5 \mathrm{~Hz}, 1 \mathrm{H}), 6.79(\mathrm{~d}, J=7.7 \mathrm{~Hz}, 1 \mathrm{H}), 5.33(\mathrm{~d}, J=$ $15.5 \mathrm{~Hz}, 1 \mathrm{H}), 5.23(\mathrm{dd}, J=15.5 \mathrm{~Hz}, 1 \mathrm{H}), 3.40-3.27(\mathrm{~m}, 1 \mathrm{H}), 2.72-2.58(\mathrm{~m}, 1 \mathrm{H}), 2.32-2.12(\mathrm{~m}, 1 \mathrm{H}), 2.02-1.86$ (m, 2H), 1.39 (s, 3H), 1.34 (s, 3H). 6f': ${ }^{1} \mathrm{H}$ NMR $\left(400 \mathrm{MHz}, \mathrm{CDCl}_{3}\right) \delta 7.57$ (dd, $\left.J=8.0,1.3 \mathrm{~Hz}, 1 \mathrm{H}\right), 7.25$ (td, $J=$ 7.6, 1.1 Hz, 1H), $7.17-7.13(\mathrm{~m}, 1 \mathrm{H}), 6.96(\mathrm{dd}, J=7.7,1.6 \mathrm{~Hz}, 1 \mathrm{H}), 4.91(\mathrm{~s}, 2 \mathrm{H}), 2.89$ (t, $J=16.3 \mathrm{~Hz}, 2 \mathrm{H}), 2.29-$ $2.24(\mathrm{~m}, 2 \mathrm{H}), 2.02-1.86(\mathrm{~m}, 1 \mathrm{H}), 1.23(\mathrm{~s}, 6 \mathrm{H}) .{ }^{13} \mathrm{C} \mathrm{NMR}\left(100 \mathrm{MHz}, \mathrm{CDCl}_{3}\right) \delta 176.0,174.9,174.4,170.0(\mathrm{t}, J=$ $26.3 \mathrm{~Hz}), 160.3(\mathrm{t}, J=31.3 \mathrm{~Hz}), 134.9,134.9,133.5,133.1,129.6,128.8,128.3,128.2,127.6,126.6,126.5,122.8$, 122.4, 111.6 (dd, $J=257.1,244.9 \mathrm{~Hz}), 111.5(\mathrm{t}, J=248.5 \mathrm{~Hz}), 88.7$ (t, $J=5.1 \mathrm{~Hz}), 46.2,45.1,39.3,38.3,38.0,37.4$, $35.9(\mathrm{t}, J=23.6 \mathrm{~Hz}), 35.3(\mathrm{t}, J=24.7 \mathrm{~Hz}), 31.4(\mathrm{dd}, J=6.9,2.1 \mathrm{~Hz}), 27.2,25.0,24.9,24.7 .6 \mathbf{f}:{ }^{19} \mathrm{~F} \mathrm{NMR}(376 \mathrm{MHz}$, $\left.\mathrm{CDCl}_{3}\right) \delta-106.57(\mathrm{ddd}, J=273.9,32.7,12.6 \mathrm{~Hz}),-109.49(\mathrm{dt}, J=273.9,5.2 \mathrm{~Hz}) .6 f^{\prime}:-110.23(\mathrm{t}, J=16.3 \mathrm{~Hz}) . \mathrm{HRMS}$ (ESI) $\mathrm{m} / \mathrm{z}:[\mathrm{M}+\mathrm{H}]^{+}$Calcd for $\mathrm{C}_{17} \mathrm{H}_{18} \mathrm{BrF}_{2} \mathrm{~N}_{2} \mathrm{O}_{2}$ 399.0514; Found: 399.0524.

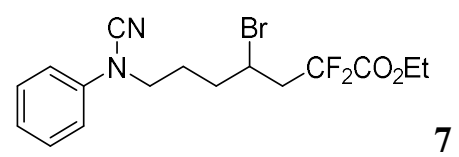

Purified by column chromatography (silica gel, EtOAc/Petroleum ether: $1 / 3$ ) to afford the title compound (58\% yield, $45 \mathrm{mg}$ ) as a yellow oil. ${ }^{1} \mathrm{H}$ NMR $\left(400 \mathrm{MHz}, \mathrm{CDCl}_{3}\right) \delta 7.46-7.31(\mathrm{~m}, 2 \mathrm{H}), 7.22-7.00(\mathrm{~m}, 3 \mathrm{H}), 4.34(\mathrm{q}, J=7.2 \mathrm{~Hz}$, $2 \mathrm{H}), 4.25-4.13(\mathrm{~m}, 1 \mathrm{H}), 3.84-3.45(\mathrm{~m}, 2 \mathrm{H}), 2.94-2.77(\mathrm{~m}, 1 \mathrm{H}), 2.75-2.56(\mathrm{~m}, 1 \mathrm{H}), 2.18-2.07(\mathrm{~m}, 2 \mathrm{H}), 2.05-$ $1.91(\mathrm{~m}, 2 \mathrm{H}), 1.36(\mathrm{t}, J=7.2 \mathrm{~Hz}, 3 \mathrm{H}) .{ }^{13} \mathrm{C} \mathrm{NMR}\left(100 \mathrm{MHz}, \mathrm{CDCl}_{3}\right) \delta 163.4(\mathrm{t}, J=32.1 \mathrm{~Hz}), 139.7,129.9,123.9$, 116.0, 114.7 (dd, $J=251.5,251.5 \mathrm{~Hz}), 113.5,63.4,48.7,45.0(\mathrm{t}, J=4.7 \mathrm{~Hz}), 43.6(\mathrm{t}, J=23.6 \mathrm{~Hz}), 35.8,25.5,14.0$. ${ }^{19} \mathrm{~F}$ NMR $\left(376 \mathrm{MHz}, \mathrm{CDCl}_{3}\right) \delta-101.71(\mathrm{~d}, J=265.1 \mathrm{~Hz}),-107.07$ (d, $\left.J=265.3 \mathrm{~Hz}\right)$. IR (KBr): 2984, 2939, 2218, 1766, 1599, 1498, 752, $690 \mathrm{~cm}^{-1}$. HRMS (ESI) m/z: $[\mathrm{M}+\mathrm{H}]^{+}$Calcd for $\mathrm{C}_{16} \mathrm{H}_{20} \mathrm{BrF}_{2} \mathrm{~N}_{2} \mathrm{O}_{2}$ 389.0671; Found: 389.0679 . 


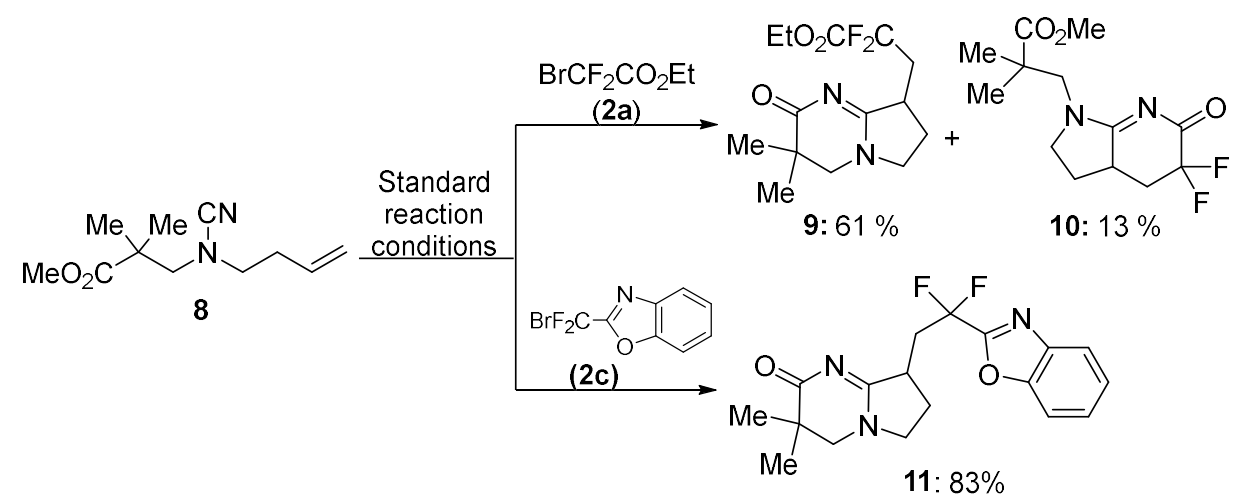

(1)

Purified by column chromatography (silica gel, EtOAc/Petroleum ether: $4 / 1$ with $5 \% \mathrm{Et}_{3} \mathrm{~N}$ ) to afford the title compound (61\% yield, $37 \mathrm{mg}$ ) as a colorless oil. ${ }^{1} \mathrm{H}$ NMR (300 MHz, $\left.\mathrm{CDCl}_{3}\right) \delta 4.33(\mathrm{q}, J=7.2 \mathrm{~Hz}, 2 \mathrm{H}), 3.65-3.43$ $(\mathrm{m}, 2 \mathrm{H}), 3.26(\mathrm{~s}, 2 \mathrm{H}), 3.21-2.92(\mathrm{~m}, 2 \mathrm{H}), 2.65-2.42(\mathrm{~m}, 1 \mathrm{H}), 2.25-1.91(\mathrm{~m}, 2 \mathrm{H}), 1.36(\mathrm{t}, J=7.1 \mathrm{~Hz}, 3 \mathrm{H}), 1.19$ $(\mathrm{s}, 3 \mathrm{H}), 1.18(\mathrm{~s}, 3 \mathrm{H}) .{ }^{13} \mathrm{C} \mathrm{NMR}\left(125 \mathrm{MHz}, \mathrm{CDCl}_{3}\right) \delta 184.0,172.6,163.7(\mathrm{t}, J=32.4 \mathrm{~Hz}), 115.6(\mathrm{t}, J=251.0 \mathrm{~Hz})$, 63.2, 54.4, 50.9, 38.1, 36.0 (t, $J=22.6 \mathrm{~Hz}), 35.4,27.3,24.1,23.6,13.9 .{ }^{19} \mathrm{~F}$ NMR $\left(470 \mathrm{MHz}, \mathrm{CDCl}_{3}\right) \delta-103.87$ (ddd, $J=258.0,26.6,8.7 \mathrm{~Hz}$ ), -107.31 (ddd, $J=258.1,23.4,13.1 \mathrm{~Hz}) . \mathrm{IR}(\mathrm{KBr}): 2967,2934,1764,1677,1583,1486$, $1120 \mathrm{~cm}^{-1}$. HRMS (ESI) m/z: [M + H $]^{+}$Calcd for $\mathrm{C}_{14} \mathrm{H}_{21} \mathrm{~F}_{2} \mathrm{~N}_{2} \mathrm{O}_{3}$ 303.1515; Found: 303.1513.

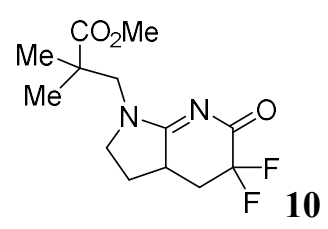

Purified by column chromatography (silica gel, EtOAc/Petroleum ether: $2 / 1$ with $5 \% \mathrm{Et}_{3} \mathrm{~N}$ ) to afford the title compound (13\% yield, $7 \mathrm{mg}$ ) as a colorless oil. ${ }^{1} \mathrm{H}$ NMR $\left(500 \mathrm{MHz}, \mathrm{CDCl}_{3}\right) \delta 3.98(\mathrm{~d}, J=13.7 \mathrm{~Hz}, 1 \mathrm{H}), 3.72(\mathrm{~s}, 3 \mathrm{H})$, $3.67(\mathrm{~d}, J=13.7 \mathrm{~Hz}, 1 \mathrm{H}), 3.62-3.47(\mathrm{~m}, 2 \mathrm{H}), 3.21-3.07(\mathrm{~m}, 1 \mathrm{H}), 2.72(\mathrm{tdd}, J=13.8,5.6,2.5 \mathrm{~Hz}, 1 \mathrm{H}), 2.47-2.28$ (m, $1 \mathrm{H}), 2.15-1.96(\mathrm{~m}, 1 \mathrm{H}), 1.91-1.78(\mathrm{~m}, 1 \mathrm{H}), 1.24(\mathrm{~s}, 3 \mathrm{H}), 1.22(\mathrm{~s}, 3 \mathrm{H}) .{ }^{13} \mathrm{C}$ NMR $\left(125 \mathrm{MHz}, \mathrm{CDCl}_{3}\right) \delta 177.1$, $170.7(\mathrm{t}, J=25.6 \mathrm{~Hz}), 111.1(\mathrm{dd}, J=256.7,245.2 \mathrm{~Hz}), 52.6,52.5,51.5,43.3,36.7$ (d, $J=8.2 \mathrm{~Hz}), 36.0(\mathrm{t}, J=23.8$ $\mathrm{Hz}$ ), 27.2, 24.2, 22.8. ${ }^{19} \mathrm{~F}$ NMR (470 MHz, $\left.\mathrm{CDCl}_{3}\right) \delta-101.10$ (ddd, $\left.J=282.0,33.0,14.1 \mathrm{~Hz}\right),-106.69--107.38(\mathrm{~m})$. IR (KBr): 2982, 2956, 1723, 1687, 1564, 1372, 1120, $771 \mathrm{~cm}^{-1}$. HRMS (ESI) m/z: [M + H] Calcd for $\mathrm{C}_{13} \mathrm{H}_{19} \mathrm{~F}_{2} \mathrm{~N}_{2} \mathrm{O}_{3}$ 289.1358; Found: 289.1357<smiles>CC1(C)CN2CCC(CC(F)(F)c3nc4ccccc4o3)C2=NC1=O</smiles>

Purified by column chromatography (silica gel, EtOAc/Petroleum ether: $2 / 1$ with $5 \% \mathrm{Et}_{3} \mathrm{~N}$ ) to afford the title compound ( $83 \%$ yield, $58 \mathrm{mg}$ ) as a colorless oil. ${ }^{1} \mathrm{H}$ NMR $\left(300 \mathrm{MHz}, \mathrm{CDCl}_{3}\right) \delta 7.86-7.72(\mathrm{~m}, 1 \mathrm{H}), 7.67-7.58(\mathrm{~m}$, 1H), $7.53-7.37$ (m, 2H), $3.67-3.39(\mathrm{~m}, 3 \mathrm{H}), 3.41-3.19(\mathrm{~m}, 3 \mathrm{H}), 2.73-2.33(\mathrm{~m}, 2 \mathrm{H}), 2.20-1.91(\mathrm{~m}, 1 \mathrm{H}), 1.19$ (s, 3H), 1.18 (s, 3H). ${ }^{13} \mathrm{C}$ NMR (100 MHz, $\left.\mathrm{CDCl}_{3}\right) \delta 184.0,172.5,157.3(\mathrm{t}, J=33.6 \mathrm{~Hz}), 150.5,139.8,127.0,125.4$, 
121.2, $116.5(\mathrm{t}, J=242.7 \mathrm{~Hz}), 111.4,54.4,50.9,38.4(\mathrm{t}, J=2.9 \mathrm{~Hz}), 37.7(\mathrm{t}, J=23.3 \mathrm{~Hz}), 35.3,27.4,24.1,23.5 .{ }^{19} \mathrm{~F}$ NMR (376 MHz, $\left.\mathrm{CDCl}_{3}\right) \delta-96.05$ (ddd, $\left.J=274.4,23.6,9.5 \mathrm{~Hz}\right),-98.45$ - -99.70 (m). IR (KBr): 2966, 2933, 2232, 1676, 1580, 1477, 1018, $730 \mathrm{~cm}^{-1}$. HRMS (ESI) m/z: $[\mathrm{M}+\mathrm{H}]^{+}$Calcd for $\mathrm{C}_{18} \mathrm{H}_{20} \mathrm{~F}_{2} \mathrm{~N}_{3} \mathrm{O}_{2} 348.1518$; Found: 348.1527 .

\section{Other Unsuccessful Substrates}<smiles>C=CCCN(C)C(=O)C(C)C</smiles>

s9<smiles>C=CCCN(C#N)C(=O)Cc1ccccc1</smiles>

S10<smiles>C=CCCN(C#N)C(=O)OC(C)(C)C</smiles>

S11<smiles>C=CCCN([13CH3])CCC(=O)OC</smiles>

$\mathrm{S} 12$

No desired products were obtained under the Optimized Reaction Conditions.

Scheme S1. Unsuccessful Substrates under the Optimized Reaction Conditions

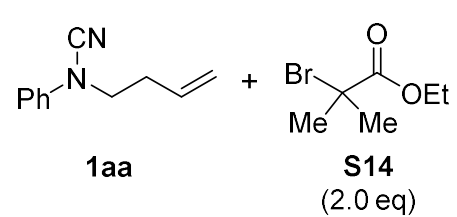<smiles>C=CCCN(C#N)c1ccccc1</smiles>

$\mathrm{Cu}_{2} \mathrm{O}(10 \mathrm{~mol} \%)$ $\mathrm{L} 5(10 \mathrm{~mol} \%)$ $\mathrm{B}_{2} \mathrm{pin}_{2}(1.0 \mathrm{eq})$ $\mathrm{NaOAc}(2.0 \mathrm{eq})$ DCE, $100^{\circ} \mathrm{C}, 12 \mathrm{~h}$

$\mathrm{Cu}_{2} \mathrm{O}(10 \mathrm{~mol} \%)$ $\mathrm{L} 5(10 \mathrm{~mol} \%)$, $\mathrm{B}_{2} \operatorname{pin}_{2}(1.0 \mathrm{eq})$ $\mathrm{NaOAc}(2.0 \mathrm{eq})$ DCE, $100^{\circ} \mathrm{C}, 12 \mathrm{~h}$

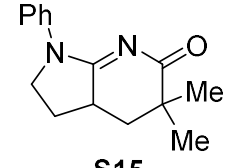

S15

nd<smiles>O=C1CCC2CCN(c3ccccc3)C2=N1</smiles>

nd

Scheme S2. Unsuccessful Examples with non-Fluorinated $\alpha$-Bromoesters under the Optimized Reaction Conditions 


\section{Scale-up Reaction and Synthetic Transformations}

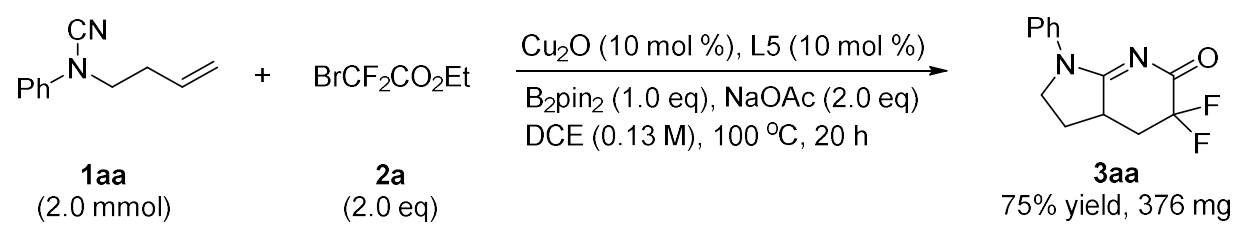

Compound 1 aa (2.0 mmol, 1.0 equiv.), 2a (2.0 equiv.), $\mathrm{B}_{2} \mathrm{pin}_{2}$ (1.0 equiv.), $\mathrm{Cu}_{2} \mathrm{O}$ (10 mol \%), L5 (10 mol \%), $\mathrm{NaOAc}(2.0$ equiv.) and DCE $(15 \mathrm{~mL})$ were placed in a sealed tube under nitrogen atmosphere. The mixture was stirred at $100{ }^{\circ} \mathrm{C}$ for the desired time. Upon completion, the reaction was cooled down to room temperature and diluted with water $(10 \mathrm{~mL})$. The aqueous phase was extracted with $\mathrm{CH}_{2} \mathrm{Cl}_{2}(3 \times 15 \mathrm{~mL})$. The combined organic layers were washed with brine, dried over anhydrous $\mathrm{Na}_{2} \mathrm{SO}_{4}$. The organic layer was then concentrated under vacuum, the residue was purified by flash column chromatography (silica gel, EtOAc/Petroleum ether $\left(60-90{ }^{\circ} \mathrm{C}\right)$ ) to give the desired product 3aa (376 mg, 75\%)

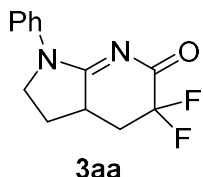

3aa

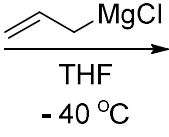

$91 \%$ yield, $d r=2.5 / 1$

To solution of $3 \mathbf{a a}(0.2 \mathrm{mmol}, 1.0$ equiv. $)$ in THF $(0.2 M)$ in a dried tube was added allylmagnesium chloride (1.2 equiv., $2 \mathrm{M}$ in THF) at $-40^{\circ} \mathrm{C}$. The mixture was stirred for $20 \mathrm{~min}$. Upon completion, the reaction was quenched with saturated ammonium chloride solution at $-40{ }^{\circ} \mathrm{C}$, and the mixture was extracted with DCM. The combined organic phases were washed with brine, dried over anhydrous $\mathrm{Na}_{2} \mathrm{SO}_{4}$. The organic layer was then concentrated under vacuum, and the titled compound 12 was obtained (91\% yield, $53 \mathrm{mg}, d r=2.5 / 1$, an inseparable mixture) as a yellow oil without the need for further purification (the product is sensitive to the silica gel). The major isomer: ${ }^{1} \mathrm{H}$ NMR (500 $\left.\mathrm{MHz}, \mathrm{CDCl}_{3}\right) \delta 7.86(\mathrm{~d}, J=8.0 \mathrm{~Hz}, 2 \mathrm{H}), 7.42-7.30(\mathrm{~m}, 2 \mathrm{H}), 7.12-7.00(\mathrm{~m}, 1 \mathrm{H}), 6.26-6.00(\mathrm{~m}, 1 \mathrm{H}), 5.44-5.21$ $(\mathrm{m}, 2 \mathrm{H}), 3.98-3.70(\mathrm{~m}, 3 \mathrm{H}), 3.08-2.93(\mathrm{~m}, 1 \mathrm{H}), 2.78-2.57(\mathrm{~m}, 2 \mathrm{H}), 2.43-2.21(\mathrm{~m}, 3 \mathrm{H}), 1.93-1.81(\mathrm{~m}, 1 \mathrm{H})$. The minor isomer: ${ }^{1} \mathrm{H}$ NMR $\left(500 \mathrm{MHz}, \mathrm{CDCl}_{3}\right) \delta 7.86(\mathrm{~d}, J=8.0 \mathrm{~Hz}, 2 \mathrm{H}), 7.42-7.30(\mathrm{~m}, 2 \mathrm{H}), 7.12-7.00(\mathrm{~m}, 1 \mathrm{H})$, $6.26-6.00(\mathrm{~m}, 1 \mathrm{H}), 5.44-5.21(\mathrm{~m}, 2 \mathrm{H}), 3.98-3.70(\mathrm{~m}, 1 \mathrm{H}), 3.26-3.13(\mathrm{~m}, 1 \mathrm{H}), 2.77-2.70(\mathrm{~m}, 2 \mathrm{H}), 2.43-2.21$ $(\mathrm{m}, 1 \mathrm{H}), 2.12-1.98(\mathrm{~m}, 2 \mathrm{H}), 1.93-1.81(\mathrm{~m}, 1 \mathrm{H}), 1.80-1.71(\mathrm{~m}, 2 \mathrm{H}) .{ }^{13} \mathrm{C} \mathrm{NMR}\left(125 \mathrm{MHz}, \mathrm{CDCl}_{3}\right) \delta 164.4,161.8$, 141.2, 133.4, 133.1, 122.9, 122.7, 121.4 (dd, $J=245.0,248.8 \mathrm{~Hz}), 121.3(\mathrm{dd}, J=255.5,247.1 \mathrm{~Hz}), 119.8,119.5$, 119.1, 118.8, 84.9 (dd, $J=27.0,22.2 \mathrm{~Hz}$ ), 84.0 (t, $J=26.3 \mathrm{~Hz}$ ), 49.3, 48.8, 41.8, 40.4, 39.2, 36.9, 33.9 (t, $J=25.2$ $\mathrm{Hz}), 31.7(\mathrm{t}, J=23.4 \mathrm{~Hz}), 28.4,27.0$. The major isomer: ${ }^{19} \mathrm{~F} \mathrm{NMR}\left(470 \mathrm{MHz}, \mathrm{CDCl}_{3}\right) \delta-111.86(\mathrm{dd}, J=25.2,9.5$ $\mathrm{Hz}),-111.99(\mathrm{t}, J=9.3 \mathrm{~Hz})$. The minor isomer: ${ }^{19} \mathrm{~F}$ NMR $\left(470 \mathrm{MHz}, \mathrm{CDCl}_{3}\right) \delta-104.05(\mathrm{dt}, J=242.7,18.7 \mathrm{~Hz}),-$ 109.67 (dt, $J=242.7,12.9 \mathrm{~Hz}$ ). IR (KBr): 3415, 2942, 2874, 1639, 1497, 1311, 1032, $756 \mathrm{~cm}^{-1}$. HRMS (ESI) m/z: $[\mathrm{M}+\mathrm{H}]^{+}$Calcd for $\mathrm{C}_{16} \mathrm{H}_{19} \mathrm{~F}_{2} \mathrm{~N}_{2} \mathrm{O}$ 293.1460; Found: 293.1470 .
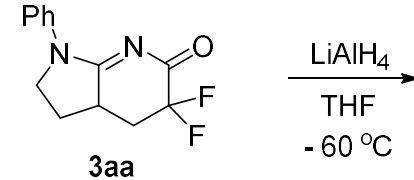

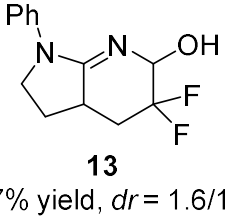


To solution of 3aa $(0.2 \mathrm{mmol}, 1.0$ equiv. $)$ in THF $(0.05 M)$ in a dried tube was added $\mathrm{LiAlH}_{4}(1.1$ equiv. $)$ at $-60{ }^{\circ} \mathrm{C}$. The mixture was stirred for $20 \mathrm{~min}$. Upon completion, the reaction was carefully quenched with water and the mixture was extracted with DCM. The combined organic phases were washed with brine before being dried over $\mathrm{Na}_{2} \mathrm{SO}_{4}$ and concentrated under reduced pressure. The residue was purified by column chromatography (silica gel, EtOAc/Petroleum ether: 1/1) to afford the title compound ( $77 \%$ yield, $39 \mathrm{mg}, d r=1.6 / 1$, an inseparable mixture) as a yellow oil. The major isomer: ${ }^{1} \mathrm{H}$ NMR $\left(300 \mathrm{MHz}, \mathrm{CDCl}_{3}\right) \delta 7.93-7.62(\mathrm{~m}, 2 \mathrm{H}), 7.44-7.28(\mathrm{~m}, 2 \mathrm{H}), 7.15-6.97$ (m, 1H), $5.02(\mathrm{dd}, J=7.8,4.0 \mathrm{~Hz}, 1 \mathrm{H}), 4.07-3.68(\mathrm{~m}, 2 \mathrm{H}), 3.14-3.08(\mathrm{~m}, 2 \mathrm{H}), 2.49-2.25(\mathrm{~m}, 2 \mathrm{H}), 2.20-2.04$ $(\mathrm{m}, 1 \mathrm{H}), 1.97-1.79(\mathrm{~m}, 1 \mathrm{H})$. The minor isomer: ${ }^{1} \mathrm{H}$ NMR $\left(300 \mathrm{MHz}, \mathrm{CDCl}_{3}\right) \delta 7.93-7.62(\mathrm{~m}, 2 \mathrm{H}), 7.44-7.28(\mathrm{~m}$, 2H), $7.15-6.97(\mathrm{~m}, 1 \mathrm{H}), 5.23(\mathrm{dd}, J=11.8,6.0 \mathrm{~Hz}, 1 \mathrm{H}), 3.14-3.08(\mathrm{~m}, 1 \mathrm{H}), 4.07-3.68(\mathrm{~m}, 2 \mathrm{H}), 3.06-2.90(\mathrm{~m}$, 1H), $2.70-2.52(\mathrm{~m}, 2 \mathrm{H}), 2.47-2.26(\mathrm{~m}, 1 \mathrm{H}), 1.97-1.79(\mathrm{~m}, 1 \mathrm{H}) .{ }^{13} \mathrm{C} \mathrm{NMR}\left(100 \mathrm{MHz}, \mathrm{CDCl}_{3}\right) \delta 163.9,163.1$, 140.6, 140.4, 128.9, 124.0, 123.7, 120.5 (dd, $J=254.6,240.1 \mathrm{~Hz}), 120.4,120.1,119.2$ (dd, $J=249.1,247.0 \mathrm{~Hz}$ ), $81.4(\mathrm{dd}, J=31.2,22.4 \mathrm{~Hz}), 80.1(\mathrm{dd}, J=37.7,22.0 \mathrm{~Hz}), 50.0,38.7(\mathrm{dd}, J=6.5,3.6 \mathrm{~Hz}), 37.6(\mathrm{t}, J=5.1 \mathrm{~Hz}), 34.0$ (t, $J=24.2 \mathrm{~Hz}), 31.7(\mathrm{t}, J=23.7 \mathrm{~Hz}), 27.7,27.3$. The major isomer: ${ }^{19} \mathrm{~F}$ NMR $\left(376 \mathrm{MHz}, \mathrm{CDCl}_{3}\right) \delta-105.71$ (dddd, $J$ $=249.0,27.6,11.1,7.9 \mathrm{~Hz}),-110.61--111.48(\mathrm{~m})$. The minor isomer: ${ }^{19} \mathrm{~F}$ NMR $\left(376 \mathrm{MHz}, \mathrm{CDCl}_{3}\right) \delta-104.82$ (dddd, $J=244.1,14.4,9.2,5.8 \mathrm{~Hz}$ ), -115.02 (ddt, $J=244.2,22.5,11.2 \mathrm{~Hz}$ ). IR (KBr): 3138, 2874, 1635, 1596, 1497, 1299 , 1045, $764 \mathrm{~cm}^{-1}$. HRMS (ESI) m/z: [M + H] $]^{+}$Calcd for $\mathrm{C}_{13} \mathrm{H}_{15} \mathrm{~F}_{2} \mathrm{~N}_{2} \mathrm{O}$ 253.1147; Found: 253.1154 .
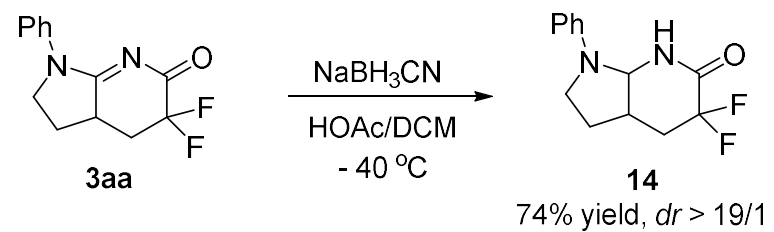

To solution of 3aa $(0.2 \mathrm{mmol}, 1.0$ equiv. $)$ in $\operatorname{HOAc} / \mathrm{DCM}(1 / 1,0.1 M)$ in a dried tube was added $\mathrm{NaBH}_{3} \mathrm{CN}(1.2$ equiv.) at $-40^{\circ} \mathrm{C}$. The mixture was stirred for 50 minutes. Upon completion, the reaction was quenched with saturated $\mathrm{NaHCO}_{3}$ at $-40^{\circ} \mathrm{C}$, and the mixture was extracted with DCM. The combined organic phases were washed with brine before being dried over $\mathrm{Na}_{2} \mathrm{SO}_{4}$ and concentrated under reduced pressure. The residue was purified by column chromatography (silica gel, EtOAc/Petroleum ether: 1/4) to afford the title compound $\mathbf{1 4}$ (74\% yield, $38 \mathrm{mg}, d r>$ 19/1) as a white solid. mp: $153-156{ }^{\circ} \mathrm{C} .{ }^{1} \mathrm{H}$ NMR $\left(500 \mathrm{MHz}, \mathrm{CDCl}_{3}\right) \delta 7.29(\mathrm{t}, J=7.8 \mathrm{~Hz}, 2 \mathrm{H}), 6.84(\mathrm{t}, J=7.3 \mathrm{~Hz}$, $1 \mathrm{H}), 6.64(\mathrm{~d}, J=8.0 \mathrm{~Hz}, 2 \mathrm{H}), 6.24$ (brs, $1 \mathrm{H}), 5.34(\mathrm{~d}, J=6.0 \mathrm{~Hz}, 1 \mathrm{H}), 3.54(\mathrm{td}, J=8.6,2.7 \mathrm{~Hz}, 1 \mathrm{H}), 3.41-3.16(\mathrm{~m}$, 1H), $2.85-2.74(\mathrm{~m}, 1 \mathrm{H}), 2.68-2.50(\mathrm{~m}, 1 \mathrm{H}), 2.41-2.23(\mathrm{~m}, 2 \mathrm{H}), 2.22-2.07(\mathrm{~m}, 1 \mathrm{H}) .{ }^{13} \mathrm{C} \mathrm{NMR}\left(125 \mathrm{MHz}, \mathrm{CDCl}_{3}\right)$ $\delta 162.0(\mathrm{t}, J=30.3 \mathrm{~Hz}), 144.8,130.0,118.8,112.4,112.2(\mathrm{t}, J=245.7 \mathrm{~Hz}), 69.4,47.2,35.2,32.5(\mathrm{t}, J=22.7 \mathrm{~Hz})$, 28.6. ${ }^{19} \mathrm{~F}$ NMR (470 MHz, $\left.\mathrm{CDCl}_{3}\right) \delta-102.04--102.57$ (m). IR (KBr): 3226, 2914, 2845, 1699, 1572, 1507, 1190, 978, $751 \mathrm{~cm}^{-1}$. HRMS (ESI) m/z: [M + H] ${ }^{+}$Calcd for $\mathrm{C}_{13} \mathrm{H}_{15} \mathrm{~F}_{2} \mathrm{~N}_{2} \mathrm{O}$ 253.1147; Found: 253.1157 .
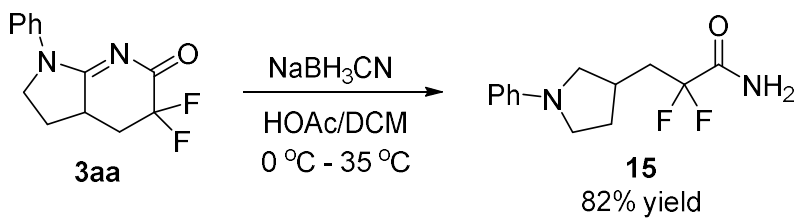

To solution of 3aa $\left(0.2 \mathrm{mmol}, 1.0\right.$ equiv.) in $\operatorname{HOAc} / \mathrm{DCM}(1 / 1,0.1 \mathrm{M})$ in a dried tube was added $\mathrm{NaBH}_{3} \mathrm{CN}(2.7$ equiv.) at $0{ }^{\circ} \mathrm{C}$. The mixture was warmed to $35^{\circ} \mathrm{C}$ and stirred for $4.5 \mathrm{~h}$. Upon completion, the reaction was quenched with saturated $\mathrm{NaHCO}_{3}$ and the mixture was extracted with DCM. The combined organic phases were washed with brine before being dried over $\mathrm{Na}_{2} \mathrm{SO}_{4}$ and concentrated under reduced pressure. The residue was purified by column 
chromatography (silica gel, EtOAc/Petroleum ether: 1/4) to afford the title compound $\mathbf{1 5}$ ( $82 \%$ yield, $42 \mathrm{mg}$ ) as a white solid. mp: $119-121^{\circ} \mathrm{C} .{ }^{1} \mathrm{H}$ NMR $\left(300 \mathrm{MHz}, \mathrm{CDCl}_{3}\right) \delta 7.28-7.17(\mathrm{~m}, 2 \mathrm{H}), 6.67(\mathrm{t}, J=7.3 \mathrm{~Hz}, 1 \mathrm{H}), 6.54(\mathrm{~d}$, $J=7.8 \mathrm{~Hz}, 2 \mathrm{H}), 6.30$ (brs, $1 \mathrm{H}), 5.85$ (brs, $1 \mathrm{H}), 3.55$ (t, $J=8.3 \mathrm{~Hz}, 1 \mathrm{H}), 3.44-3.22$ (m, 2H), 3.00 (t, $J=8.8 \mathrm{~Hz}, 1 \mathrm{H})$, $2.65-2.49(\mathrm{~m}, 1 \mathrm{H}), 2.43-2.15(\mathrm{~m}, 3 \mathrm{H}), 1.88-1.67(\mathrm{~m}, 1 \mathrm{H}) .{ }^{13} \mathrm{C} \mathrm{NMR}\left(125 \mathrm{MHz}, \mathrm{CDCl}_{3}\right) \delta 166.8(\mathrm{t}, J=27.6 \mathrm{~Hz})$, 147.6, 129.3, $117.8(\mathrm{t}, J=252.7 \mathrm{~Hz}), 115.9,111.6,53.4,47.2,37.3$ (t, $J=22.9 \mathrm{~Hz}), 32.6,32.2 .{ }^{19} \mathrm{~F}$ NMR $(470 \mathrm{MHz}$, $\left.\mathrm{CDCl}_{3}\right) \delta-104.23(\mathrm{dt}, J=258.4,23.3 \mathrm{~Hz}),-105.25(\mathrm{ddd}, J=258.3,20.3,20.2 \mathrm{~Hz})$. IR (KBr): 3396, 3186, 2848, 1681, 1508, 1379, 1186, $744 \mathrm{~cm}^{-1}$. HRMS (ESI) m/z: $[\mathrm{M}+\mathrm{H}]^{+}$Calcd for $\mathrm{C}_{13} \mathrm{H}_{17} \mathrm{~F}_{2} \mathrm{~N}_{2} \mathrm{O}$ 255.1303; Found: 255.1312 .

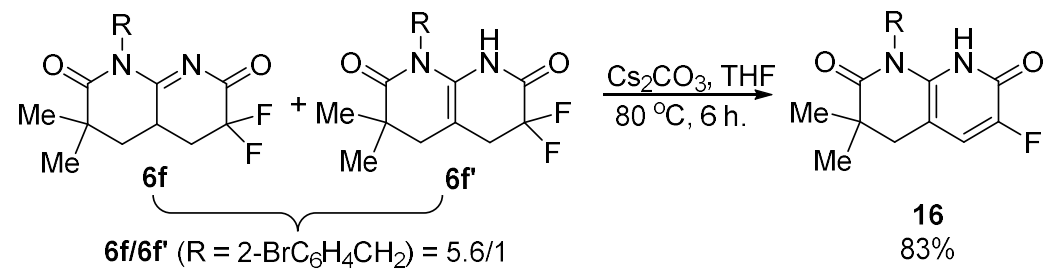

To solution of $\mathbf{6 f} / \mathbf{6} \mathbf{f}^{\prime}\left(\mathbf{6} \mathbf{6} / \mathbf{6} \mathbf{f}^{\prime}=5.6 / 1,0.2 \mathrm{mmol}, 1.0\right.$ equiv. $)$ in THF $(0.2 M)$ in a dried tube was added $\mathrm{Cs}_{2} \mathrm{CO}_{3}(5.0$ equiv.). The mixture was warmed to $80^{\circ} \mathrm{C}$ and stirred for $6 \mathrm{~h}$. Upon completion, water was added and the mixture was extracted with DCM, and the combined organic layers were washed by brine and dried over $\mathrm{Na}_{2} \mathrm{SO}_{4}$, then filtered and concentrated under reduced pressure. The residue was purified by flash column chromatography (silica gel, EtOAc/Petroleum ether: 1/5) to afford the title compound $\mathbf{1 6}(83 \%$ yield, $63 \mathrm{mg})$ as a white solid. $\mathrm{mp:} 110-114{ }^{\circ} \mathrm{C}$. ${ }^{1} \mathrm{H}$ NMR $\left(300 \mathrm{MHz}, \mathrm{CDCl}_{3}\right) \delta 7.53(\mathrm{dd}, J=7.8,1.4 \mathrm{~Hz}, 1 \mathrm{H}), 7.25(\mathrm{~d}, J=8.8 \mathrm{~Hz}, 1 \mathrm{H}), 7.15(\mathrm{td}, J=7.5,1.4 \mathrm{~Hz}, 1 \mathrm{H})$, $7.06(\mathrm{td}, J=7.7,1.8 \mathrm{~Hz}, 1 \mathrm{H}), 6.85$ (dd, $J=7.7,1.8 \mathrm{~Hz}, 1 \mathrm{H}), 6.32$ (brs, 1H), $5.22(\mathrm{~s}, 2 \mathrm{H}), 2.74(\mathrm{~s}, 2 \mathrm{H}), 1.25(\mathrm{~s}, 6 \mathrm{H})$. ${ }^{13} \mathrm{C}$ NMR $\left(125 \mathrm{MHz}, \mathrm{CDCl}_{3}\right) \delta 175.5,148.5(\mathrm{~d}, J=14.0 \mathrm{~Hz}), 143.7,141.8(\mathrm{~d}, J=250.4 \mathrm{~Hz}), 136.8,132.8,128.3$, $127.5,126.8,126.0(\mathrm{~d}, J=17.3 \mathrm{~Hz}), 122.9,112.0,45.4,37.8,37.6,24.9 .{ }^{19} \mathrm{~F}$ NMR $\left(470 \mathrm{MHz}, \mathrm{CDCl}_{3}\right) \delta-148.96(\mathrm{~d}$, $J=9.1 \mathrm{~Hz}$ ). IR (KBr): 3142, 2951, 2875, 1636, 1496, 1045, $764 \mathrm{~cm}^{-1}$. HRMS (ESI) m/z: $[\mathrm{M}+\mathrm{H}]^{+}$Calcd for $\mathrm{C}_{17} \mathrm{H}_{17} \mathrm{BrFN}_{2} \mathrm{O}_{2}$ 379.0452; Found: 379.0455 .

\section{Control Experiment}

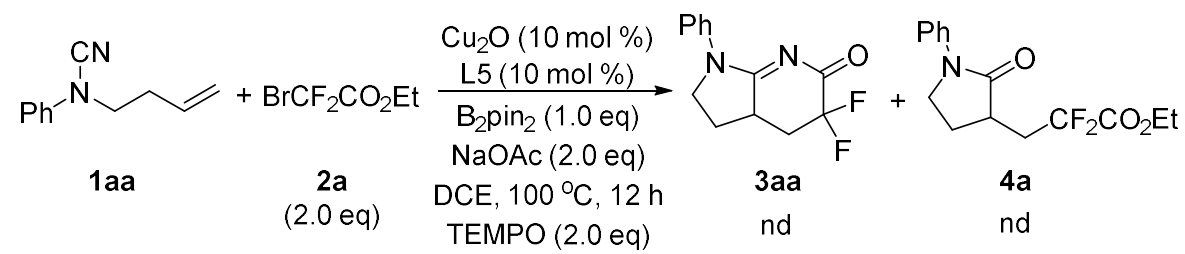

\section{References}

1. Li, Z.; Jiao, L.; Sun, Y.; He, Z.; Wei, Z.; Liao, W.-W. Angew. Chem. Int. Ed. 2020, 59, 7266-7270.

2. Chen, D.; Ji, M.; Zhu, C. Chem. Commun. 2019, 55, 7796-7799.

3. Zheng, J.; Deng, Z.; Zhang, Y.; Cui, S. Adv. Synth. Catal. 2016, 358, 746-751.

4. Li, Y.; Liu, J.; Zhao, S.; Du, X.; Guo, M.; Zhao, W.; Tang, X.; Wang, G. Org. Lett. 2018, 20, 917-920.

5. Miyazaki, Y.; Ohta, N.; Semba, K.; Nakao, Y. J. Am. Chem. Soc. 2014, 136, 3732-3735.

6. McCubbin, J. A.; Maddess, M. L.; Lautens, M. Org. Lett. 2006, 8, 2993-2996.

7. Pan, Z.; Wang, S.; Brethorst, J. T.; Douglas, C. J. J. Am. Chem. Soc. 2018, 140, 3331-3338. 


\section{Crystal Data and Structure Refinement for Compound 3ca}
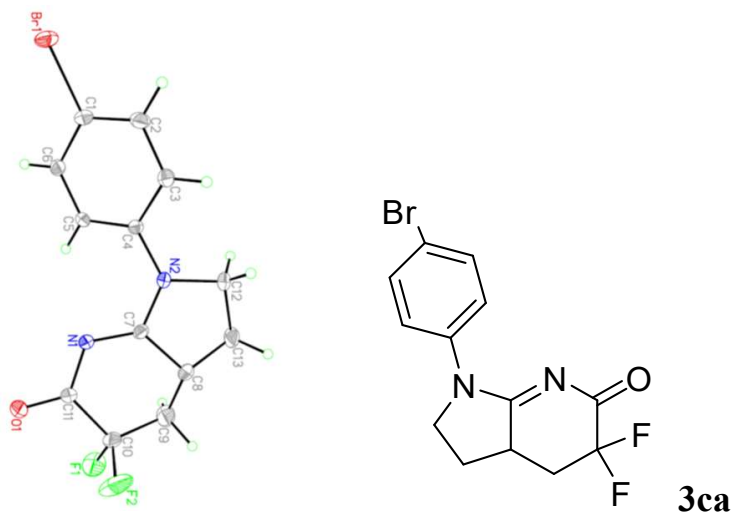

X-ray crystal structure of compound $\mathbf{3 c a}$. Single crystal of 3ca was obtained by slow evaporation of Petroleum ether /EtOAc solution. Crystal measurement of 3ca was measured on R-Axis RAPID of Rigaku Corporation plus $291.2 \mathrm{k}$. Thermal ellipsoids are set at $30 \%$ probability.

CCDC number: 2117718

Table S-crystal-1. Crystal data and structure refinement for $\mathbf{3 c a}$.

Identification code

Empirical formula

Formula weight

Temperature

Wavelength

Crystal system

Space group

Unit cell dimensions

Volume

Z

Density (calculated)

Absorption coefficient

$\mathrm{F}(000)$

Crystal size

Theta range for data collection

Index ranges

Reflections collected

Independent reflections

Completeness to theta $=25.149^{\circ}$ 3ca

C13 H10 Br F2 N2 O

328.13

293(2) K

$0.71073 \AA$

Monoclinic

$\mathrm{P} 2{ }_{1} / \mathrm{c}$

$\mathrm{a}=10.1587(3) \AA$ $\alpha=90^{\circ}$.

$\mathrm{b}=7.2397(2) \AA$ $\beta=106.560(2)^{\circ}$.

$\mathrm{c}=17.8833(5) \AA$ $\gamma=90^{\circ}$.

1260.69(6) $\AA^{3}$

4

$1.729 \mathrm{Mg} / \mathrm{m}^{3}$

$3.278 \mathrm{~mm}^{-1}$

652

$0.200 \times 0.180 \times 0.170 \mathrm{~mm}^{3}$

2.681 to $25.149^{\circ}$.

$-12<=\mathrm{h}<=12,-8<=\mathrm{k}<=8,-21<=\mathrm{l}<=21$

42535

$2248[\mathrm{R}(\mathrm{int})=0.0289]$

$99.4 \%$ 
Max. and min. transmission

Refinement method

Data / restraints / parameters

Goodness-of-fit on $\mathrm{F}^{2}$

Final $\mathrm{R}$ indices $[\mathrm{I}>2 \operatorname{sigma}(\mathrm{I})]$

$\mathrm{R}$ indices (all data)

Extinction coefficient

Largest diff. peak and hole
0.6057 and 0.5601

Full-matrix least-squares on $\mathrm{F}^{2}$

2248 / 3 / 172

1.037

$\mathrm{R} 1=0.0433, \mathrm{wR} 2=0.1054$

$\mathrm{R} 1=0.0464, \mathrm{wR} 2=0.1080$

$\mathrm{n} / \mathrm{a}$

0.773 and -0.838 e. $\AA^{-3}$

Table S-crystal-2. Atomic coordinates $\left(\right.$ x 104) and equivalent isotropic displacement parameters $\left(\AA^{2} \times 10^{3}\right)$ for $Y$. $U(e q)$ is defined as one third of the trace of the orthogonalized $U^{i j}$ tensor.

\begin{tabular}{|c|c|c|c|c|}
\hline & $\mathrm{x}$ & $\mathrm{y}$ & $\mathrm{z}$ & $\mathrm{U}(\mathrm{eq})$ \\
\hline $\operatorname{Br}(1)$ & $-1384(1)$ & $7316(1)$ & $5740(1)$ & $53(1)$ \\
\hline $\mathrm{F}(1)$ & $7083(4)$ & $3482(5)$ & $10297(2)$ & $86(1)$ \\
\hline $\mathrm{F}(2)$ & $7721(3)$ & $3193(6)$ & $9263(3)$ & $102(1)$ \\
\hline $\mathrm{O}(1)$ & $6104(3)$ & $6386(4)$ & $9401(2)$ & $76(1)$ \\
\hline $\mathrm{N}(2)$ & $3137(3)$ & $2435(4)$ & $7734(2)$ & $34(1)$ \\
\hline $\mathrm{C}(1)$ & $20(3)$ & $5811(5)$ & $6374(2)$ & $37(1)$ \\
\hline $\mathrm{C}(2)$ & $338(4)$ & $4169(6)$ & $6076(2)$ & $46(1)$ \\
\hline$C(3)$ & 1392(4) & $3092(6)$ & $6527(2)$ & $44(1)$ \\
\hline$C(4)$ & $2107(3)$ & $3633(5)$ & $7279(2)$ & $32(1)$ \\
\hline$C(5)$ & $1762(3)$ & $5277(5)$ & $7576(2)$ & $35(1)$ \\
\hline$C(6)$ & $719(3)$ & $6358(5)$ & $7124(2)$ & $38(1)$ \\
\hline$C(7)$ & $4275(3)$ & $2903(5)$ & $8296(2)$ & $34(1)$ \\
\hline $\mathrm{C}(8)$ & $5039(4)$ & $1176(6)$ & $8616(3)$ & $60(1)$ \\
\hline$C(9)$ & $5916(6)$ & $1401(7)$ & $9375(3)$ & $86(2)$ \\
\hline$C(10)$ & $6615(4)$ & $3205(6)$ & $9528(2)$ & $46(1)$ \\
\hline $\mathrm{C}(11)$ & $5736(4)$ & $4867(5)$ & $9142(2)$ & $43(1)$ \\
\hline $\mathrm{N}(1)$ & $4603(3)$ & $4601(4)$ & $8517(2)$ & $38(1)$ \\
\hline$C(12)$ & $2996(4)$ & $414(5)$ & $7631(3)$ & $50(1)$ \\
\hline$C(13)$ & $4072(5)$ & $-342(6)$ & $8318(3)$ & $60(1)$ \\
\hline
\end{tabular}

Table S-crystal-3. $\quad$ Bond lengths $[\AA]$ and angles $\left[{ }^{\circ}\right]$ for $\mathrm{Y}$. 


\begin{tabular}{|c|c|}
\hline $\operatorname{Br}(1)-C(1)$ & $1.893(3)$ \\
\hline $\mathrm{F}(1)-\mathrm{C}(10)$ & $1.335(5)$ \\
\hline $\mathrm{F}(2)-\mathrm{C}(10)$ & $1.339(5)$ \\
\hline $\mathrm{O}(1)-\mathrm{C}(11)$ & $1.210(5)$ \\
\hline $\mathrm{N}(2)-\mathrm{C}(7)$ & $1.341(5)$ \\
\hline $\mathrm{N}(2)-\mathrm{C}(4)$ & $1.423(4)$ \\
\hline $\mathrm{N}(2)-\mathrm{C}(12)$ & $1.476(4)$ \\
\hline$C(1)-C(2)$ & $1.378(5)$ \\
\hline$C(1)-C(6)$ & $1.385(5)$ \\
\hline$C(2)-C(3)$ & $1.383(5)$ \\
\hline $\mathrm{C}(2)-\mathrm{H}(2)$ & 0.9300 \\
\hline$C(3)-C(4)$ & $1.390(5)$ \\
\hline $\mathrm{C}(3)-\mathrm{H}(3)$ & 0.9300 \\
\hline$C(4)-C(5)$ & $1.389(5)$ \\
\hline$C(5)-C(6)$ & $1.379(5)$ \\
\hline $\mathrm{C}(5)-\mathrm{H}(5)$ & 0.9300 \\
\hline $\mathrm{C}(6)-\mathrm{H}(6)$ & 0.9300 \\
\hline$C(7)-N(1)$ & $1.306(5)$ \\
\hline$C(7)-C(8)$ & $1.497(5)$ \\
\hline $\mathrm{C}(8)-\mathrm{C}(9)$ & $1.404(7)$ \\
\hline$C(8)-C(13)$ & $1.469(6)$ \\
\hline$C(9)-C(10)$ & $1.475(7)$ \\
\hline $\mathrm{C}(9)-\mathrm{H}(9 \mathrm{~A})$ & 0.9700 \\
\hline $\mathrm{C}(9)-\mathrm{H}(9 \mathrm{~B})$ & 0.9700 \\
\hline$C(10)-C(11)$ & $1.540(5)$ \\
\hline $\mathrm{C}(11)-\mathrm{N}(1)$ & $1.370(5)$ \\
\hline $\mathrm{C}(12)-\mathrm{C}(13)$ & $1.496(6)$ \\
\hline $\mathrm{C}(12)-\mathrm{H}(12 \mathrm{~A})$ & 0.9700 \\
\hline $\mathrm{C}(12)-\mathrm{H}(12 \mathrm{~B})$ & 0.9700 \\
\hline $\mathrm{C}(13)-\mathrm{H}(13 \mathrm{~A})$ & 0.9700 \\
\hline $\mathrm{C}(13)-\mathrm{H}(13 \mathrm{~B})$ & 0.9700 \\
\hline $\mathrm{C}(7)-\mathrm{N}(2)-\mathrm{C}(4)$ & $127.7(3)$ \\
\hline $\mathrm{C}(7)-\mathrm{N}(2)-\mathrm{C}(12)$ & 112.1(3) \\
\hline $\mathrm{C}(4)-\mathrm{N}(2)-\mathrm{C}(12)$ & $120.2(3)$ \\
\hline$C(2)-C(1)-C(6)$ & $120.6(3)$ \\
\hline $\mathrm{C}(2)-\mathrm{C}(1)-\mathrm{Br}(1)$ & $119.0(3)$ \\
\hline
\end{tabular}




\begin{tabular}{|c|c|}
\hline $\mathrm{C}(6)-\mathrm{C}(1)-\mathrm{Br}(1)$ & $120.4(3)$ \\
\hline$C(1)-C(2)-C(3)$ & $119.3(3)$ \\
\hline $\mathrm{C}(1)-\mathrm{C}(2)-\mathrm{H}(2)$ & 120.4 \\
\hline $\mathrm{C}(3)-\mathrm{C}(2)-\mathrm{H}(2)$ & 120.4 \\
\hline$C(2)-C(3)-C(4)$ & $120.6(3)$ \\
\hline $\mathrm{C}(2)-\mathrm{C}(3)-\mathrm{H}(3)$ & 119.7 \\
\hline $\mathrm{C}(4)-\mathrm{C}(3)-\mathrm{H}(3)$ & 119.7 \\
\hline$C(5)-C(4)-C(3)$ & $119.5(3)$ \\
\hline $\mathrm{C}(5)-\mathrm{C}(4)-\mathrm{N}(2)$ & $121.9(3)$ \\
\hline $\mathrm{C}(3)-\mathrm{C}(4)-\mathrm{N}(2)$ & $118.5(3)$ \\
\hline$C(6)-C(5)-C(4)$ & $119.9(3)$ \\
\hline $\mathrm{C}(6)-\mathrm{C}(5)-\mathrm{H}(5)$ & 120.1 \\
\hline $\mathrm{C}(4)-\mathrm{C}(5)-\mathrm{H}(5)$ & 120.1 \\
\hline$C(5)-C(6)-C(1)$ & $120.1(3)$ \\
\hline $\mathrm{C}(5)-\mathrm{C}(6)-\mathrm{H}(6)$ & 119.9 \\
\hline $\mathrm{C}(1)-\mathrm{C}(6)-\mathrm{H}(6)$ & 119.9 \\
\hline $\mathrm{N}(1)-\mathrm{C}(7)-\mathrm{N}(2)$ & $123.8(3)$ \\
\hline $\mathrm{N}(1)-\mathrm{C}(7)-\mathrm{C}(8)$ & $127.6(3)$ \\
\hline $\mathrm{N}(2)-\mathrm{C}(7)-\mathrm{C}(8)$ & $108.6(3)$ \\
\hline$C(9)-C(8)-C(13)$ & $127.8(4)$ \\
\hline $\mathrm{C}(9)-\mathrm{C}(8)-\mathrm{C}(7)$ & $112.9(4)$ \\
\hline$C(13)-C(8)-C(7)$ & $105.4(3)$ \\
\hline$C(8)-C(9)-C(10)$ & $115.0(4)$ \\
\hline $\mathrm{C}(8)-\mathrm{C}(9)-\mathrm{H}(9 \mathrm{~A})$ & 108.5 \\
\hline $\mathrm{C}(10)-\mathrm{C}(9)-\mathrm{H}(9 \mathrm{~A})$ & 108.5 \\
\hline $\mathrm{C}(8)-\mathrm{C}(9)-\mathrm{H}(9 \mathrm{~B})$ & 108.5 \\
\hline $\mathrm{C}(10)-\mathrm{C}(9)-\mathrm{H}(9 \mathrm{~B})$ & 108.5 \\
\hline $\mathrm{H}(9 \mathrm{~A})-\mathrm{C}(9)-\mathrm{H}(9 \mathrm{~B})$ & 107.5 \\
\hline $\mathrm{F}(1)-\mathrm{C}(10)-\mathrm{F}(2)$ & $106.1(4)$ \\
\hline$F(1)-C(10)-C(9)$ & $109.6(4)$ \\
\hline$F(2)-C(10)-C(9)$ & $109.7(5)$ \\
\hline $\mathrm{F}(1)-\mathrm{C}(10)-\mathrm{C}(11)$ & 109.6(4) \\
\hline $\mathrm{F}(2)-\mathrm{C}(10)-\mathrm{C}(11)$ & $106.5(3)$ \\
\hline$C(9)-C(10)-C(11)$ & $115.0(3)$ \\
\hline $\mathrm{O}(1)-\mathrm{C}(11)-\mathrm{N}(1)$ & $122.4(3)$ \\
\hline $\mathrm{O}(1)-\mathrm{C}(11)-\mathrm{C}(10)$ & $117.5(3)$ \\
\hline $\mathrm{N}(1)-\mathrm{C}(11)-\mathrm{C}(10)$ & $120.1(3)$ \\
\hline
\end{tabular}




$\begin{array}{ll}\mathrm{C}(7)-\mathrm{N}(1)-\mathrm{C}(11) & 117.5(3) \\ \mathrm{N}(2)-\mathrm{C}(12)-\mathrm{C}(13) & 103.8(3) \\ \mathrm{N}(2)-\mathrm{C}(12)-\mathrm{H}(12 \mathrm{~A}) & 111.0 \\ \mathrm{C}(13)-\mathrm{C}(12)-\mathrm{H}(12 \mathrm{~A}) & 111.0 \\ \mathrm{~N}(2)-\mathrm{C}(12)-\mathrm{H}(12 \mathrm{~B}) & 111.0 \\ \mathrm{C}(13)-\mathrm{C}(12)-\mathrm{H}(12 \mathrm{~B}) & 111.0 \\ \mathrm{H}(12 \mathrm{~A})-\mathrm{C}(12)-\mathrm{H}(12 \mathrm{~B}) & 109.0 \\ \mathrm{C}(8)-\mathrm{C}(13)-\mathrm{C}(12) & 106.3(3) \\ \mathrm{C}(8)-\mathrm{C}(13)-\mathrm{H}(13 \mathrm{~A}) & 110.5 \\ \mathrm{C}(12)-\mathrm{C}(13)-\mathrm{H}(13 \mathrm{~A}) & 110.5 \\ \mathrm{C}(8)-\mathrm{C}(13)-\mathrm{H}(13 \mathrm{~B}) & 110.5 \\ \mathrm{C}(12)-\mathrm{C}(13)-\mathrm{H}(13 \mathrm{~B}) & 110.5 \\ \mathrm{H}(13 \mathrm{~A})-\mathrm{C}(13)-\mathrm{H}(13 \mathrm{~B}) & 108.7\end{array}$

Symmetry transformations used to generate equivalent atoms:

Table S-crystal-4. Anisotropic displacement parameters $\left(\AA^{2} \times 10^{3}\right)$ for Y. The anisotropic displacement factor exponent takes the form: $\quad-2 \pi^{2}\left[\mathrm{~h}^{2} \mathrm{a}^{* 2} \mathrm{U}^{11}+\ldots+2 \mathrm{~h} \mathrm{k} \mathrm{a}^{*} \mathrm{~b}^{*} \mathrm{U}^{12}\right]$

\begin{tabular}{|c|c|c|c|c|c|c|}
\hline & $\mathrm{U}^{11}$ & $\mathrm{U}^{22}$ & $\mathrm{U}^{33}$ & $\mathrm{U}^{23}$ & $\mathrm{U}^{13}$ & $\mathrm{U}^{12}$ \\
\hline $\operatorname{Br}(1)$ & $45(1)$ & $64(1)$ & $44(1)$ & $8(1)$ & $3(1)$ & $18(1)$ \\
\hline $\mathrm{F}(1)$ & $105(2)$ & $89(2)$ & $45(2)$ & $4(2)$ & $-7(2)$ & $16(2)$ \\
\hline $\mathrm{F}(2)$ & $58(2)$ & $120(3)$ & 142(3) & $47(3)$ & $49(2)$ & $41(2)$ \\
\hline $\mathrm{O}(1)$ & $56(2)$ & $42(2)$ & $99(3)$ & $-9(2)$ & $-27(2)$ & $-2(2)$ \\
\hline $\mathrm{N}(2)$ & $36(2)$ & $26(1)$ & $38(2)$ & $-2(1)$ & $7(1)$ & $2(1)$ \\
\hline $\mathrm{C}(1)$ & $31(2)$ & $44(2)$ & $36(2)$ & $6(2)$ & $7(1)$ & $4(2)$ \\
\hline$C(2)$ & $46(2)$ & $54(2)$ & $33(2)$ & $-5(2)$ & $1(2)$ & $7(2)$ \\
\hline $\mathrm{C}(3)$ & $50(2)$ & $40(2)$ & $38(2)$ & $-8(2)$ & $8(2)$ & $8(2)$ \\
\hline$C(4)$ & $28(2)$ & $31(2)$ & $34(2)$ & $2(1)$ & $6(1)$ & $1(1)$ \\
\hline$C(5)$ & $32(2)$ & $33(2)$ & $35(2)$ & $-5(1)$ & $3(1)$ & $-2(1)$ \\
\hline$C(6)$ & $32(2)$ & $33(2)$ & $47(2)$ & $-4(2)$ & $9(2)$ & $2(1)$ \\
\hline$C(7)$ & $31(2)$ & $31(2)$ & $38(2)$ & $5(1)$ & $7(1)$ & $4(1)$ \\
\hline $\mathrm{C}(8)$ & $52(2)$ & $28(2)$ & $80(3)$ & $5(2)$ & $-10(2)$ & $10(2)$ \\
\hline $\mathrm{C}(9)$ & $97(4)$ & $43(3)$ & $83(4)$ & $10(3)$ & $-31(3)$ & $11(3)$ \\
\hline$C(10)$ & $40(2)$ & $49(2)$ & $41(2)$ & $4(2)$ & $-2(2)$ & $9(2)$ \\
\hline \multirow[t]{2}{*}{$C(11)$} & $32(2)$ & $37(2)$ & $54(2)$ & $2(2)$ & $2(2)$ & $2(2)$ \\
\hline & & & & $\mathrm{SI}-38$ & & \\
\hline
\end{tabular}




$\begin{array}{lllllll}\mathrm{N}(1) & 32(2) & 30(2) & 45(2) & 3(1) & 0(1) & 2(1) \\ \mathrm{C}(12) & 56(2) & 25(2) & 61(3) & -7(2) & 6(2) & 2(2) \\ \mathrm{C}(13) & 82(3) & 29(2) & 56(3) & 3(2) & 0(2) & 6(2)\end{array}$

Table S-crystal-5. Hydrogen coordinates ( x 104) and isotropic displacement parameters $\left(\AA^{2} \times 10^{3}\right)$ for $\mathrm{Y}$.

\begin{tabular}{|c|c|c|c|c|}
\hline & $\mathrm{x}$ & $\mathrm{y}$ & $\mathrm{z}$ & $\mathrm{U}(\mathrm{eq})$ \\
\hline $\mathrm{H}(2)$ & -151 & 3790 & 5577 & 56 \\
\hline $\mathrm{H}(3)$ & 1625 & 1996 & 6325 & 53 \\
\hline $\mathrm{H}(5)$ & 2233 & 5647 & 8080 & 42 \\
\hline $\mathrm{H}(6)$ & 485 & 7458 & 7323 & 45 \\
\hline $\mathrm{H}(9 \mathrm{~A})$ & 6607 & 438 & 9472 & 103 \\
\hline $\mathrm{H}(9 \mathrm{~B})$ & 5384 & 1231 & 9742 & 103 \\
\hline $\mathrm{H}(12 \mathrm{~A})$ & 3161 & 33 & 7145 & 60 \\
\hline $\mathrm{H}(12 \mathrm{~B})$ & 2087 & 8 & 7633 & 60 \\
\hline $\mathrm{H}(13 \mathrm{~A})$ & 3661 & -759 & 8715 & 72 \\
\hline $\mathrm{H}(13 \mathrm{~B})$ & 4540 & -1376 & 8161 & 72 \\
\hline
\end{tabular}


X. ${ }^{1} \mathrm{H}$ and ${ }^{13} \mathrm{C}$ NMR Spectral Copies 


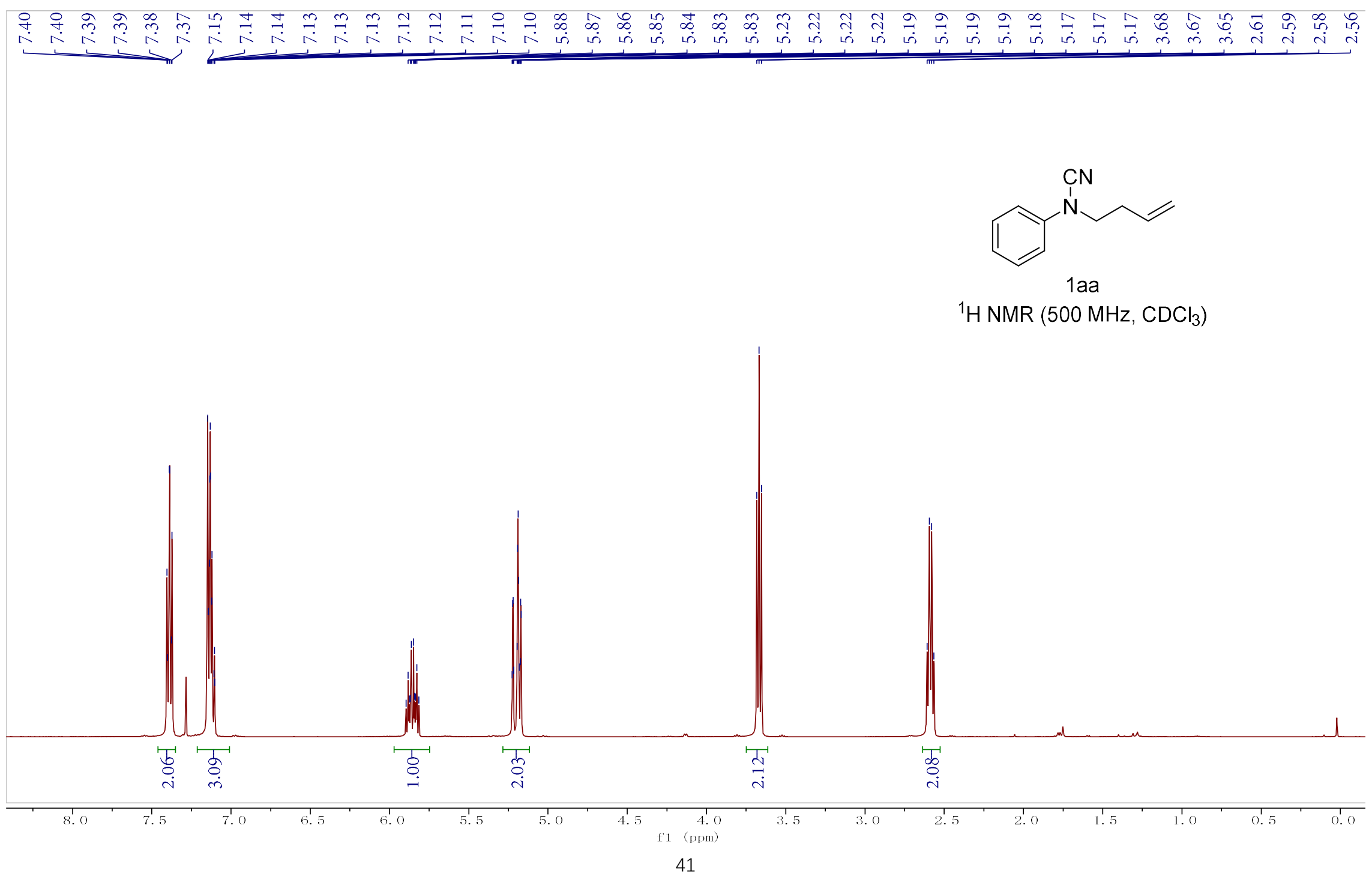




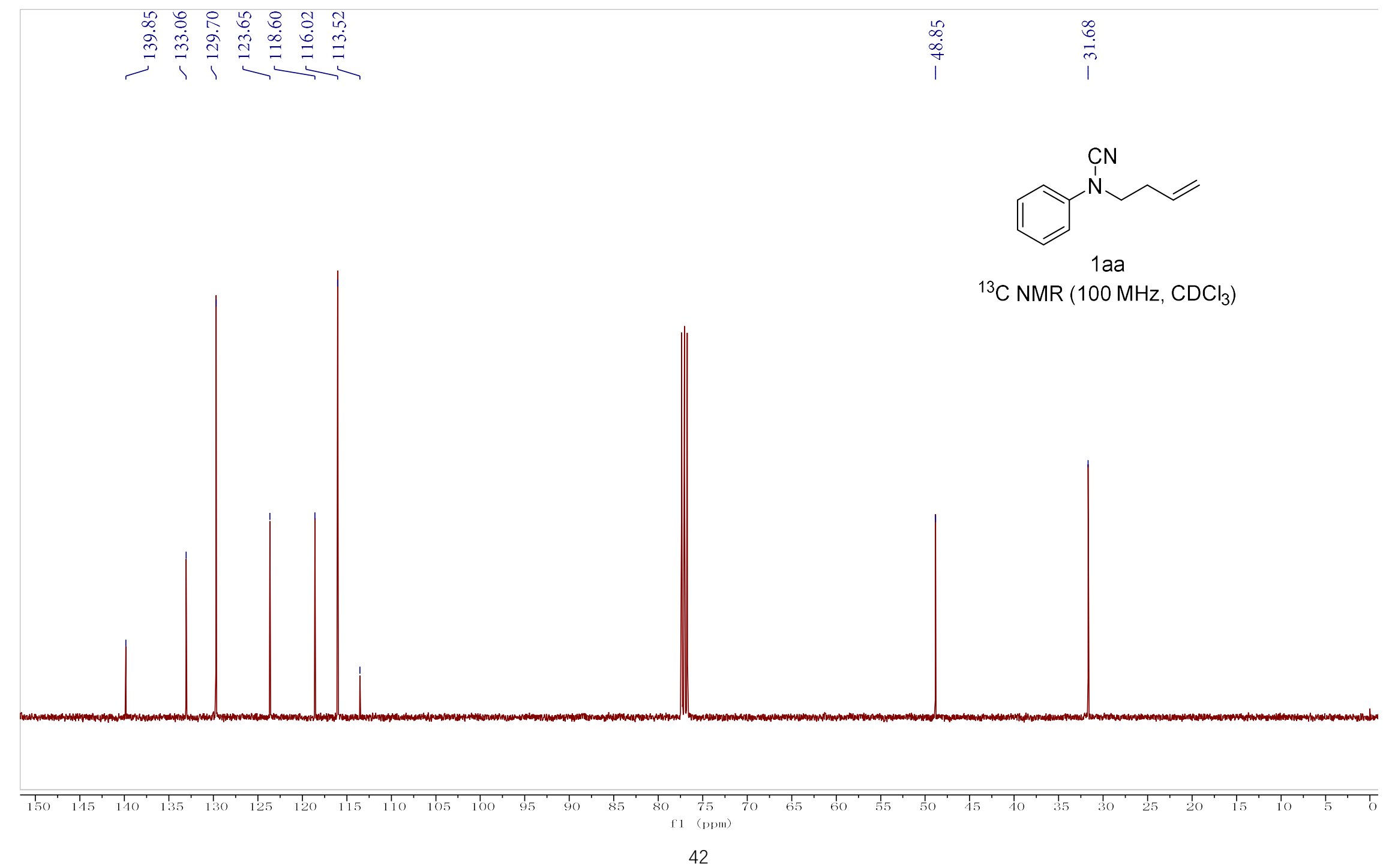




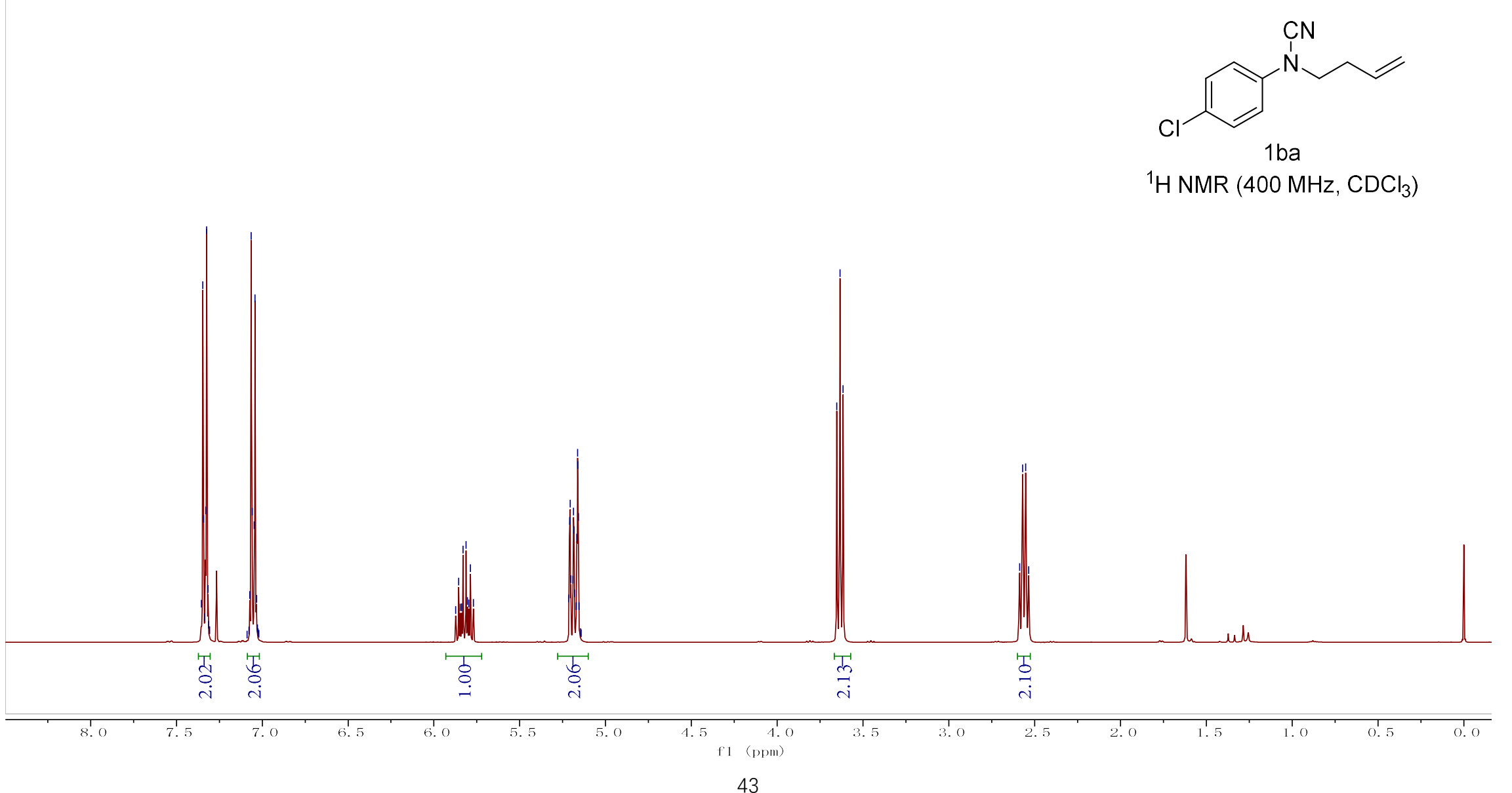




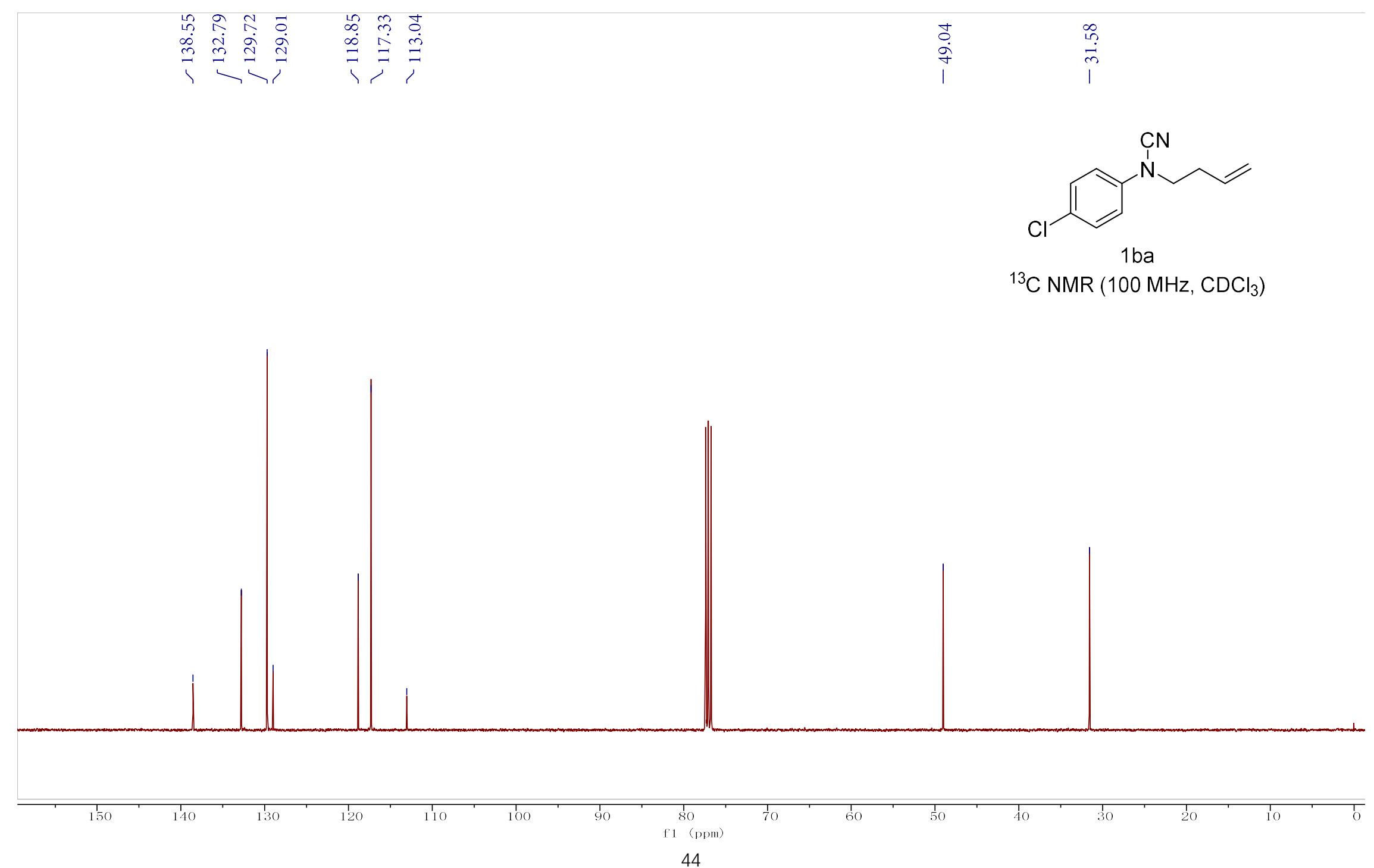




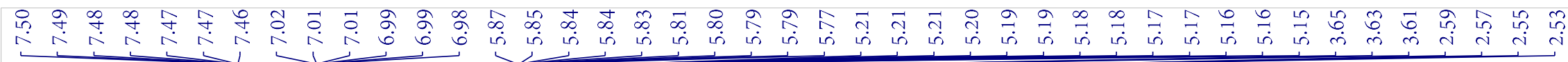
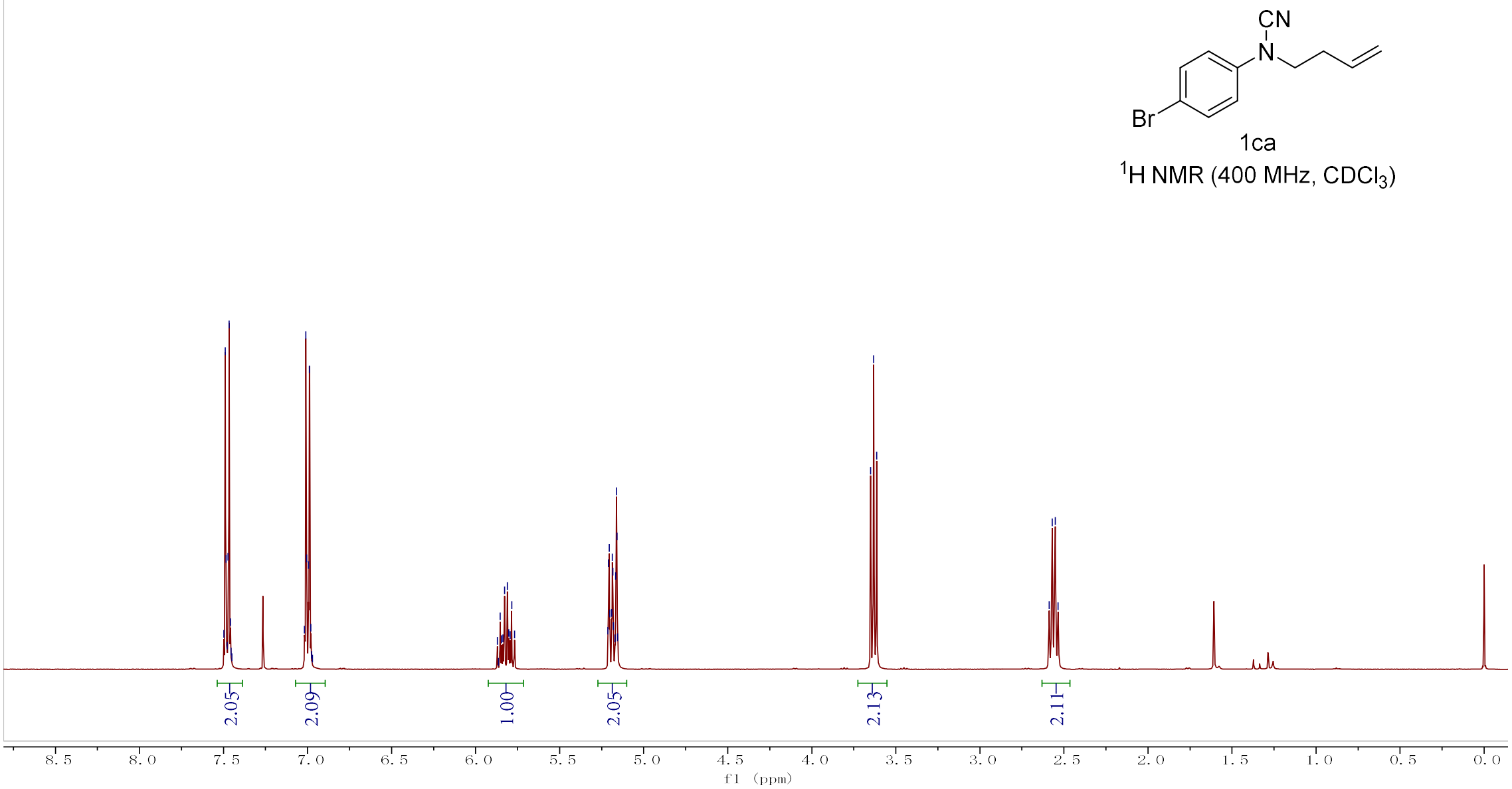


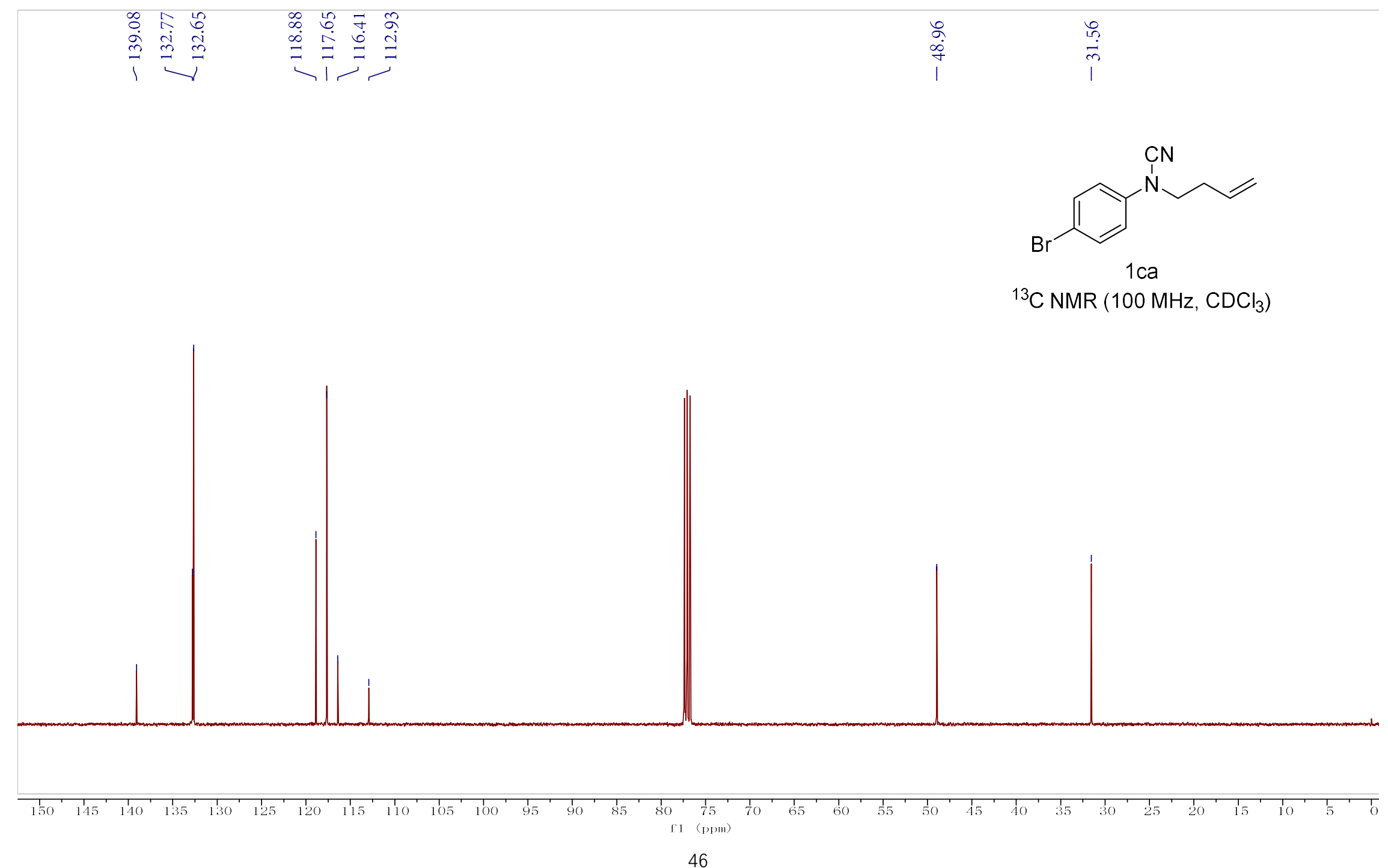




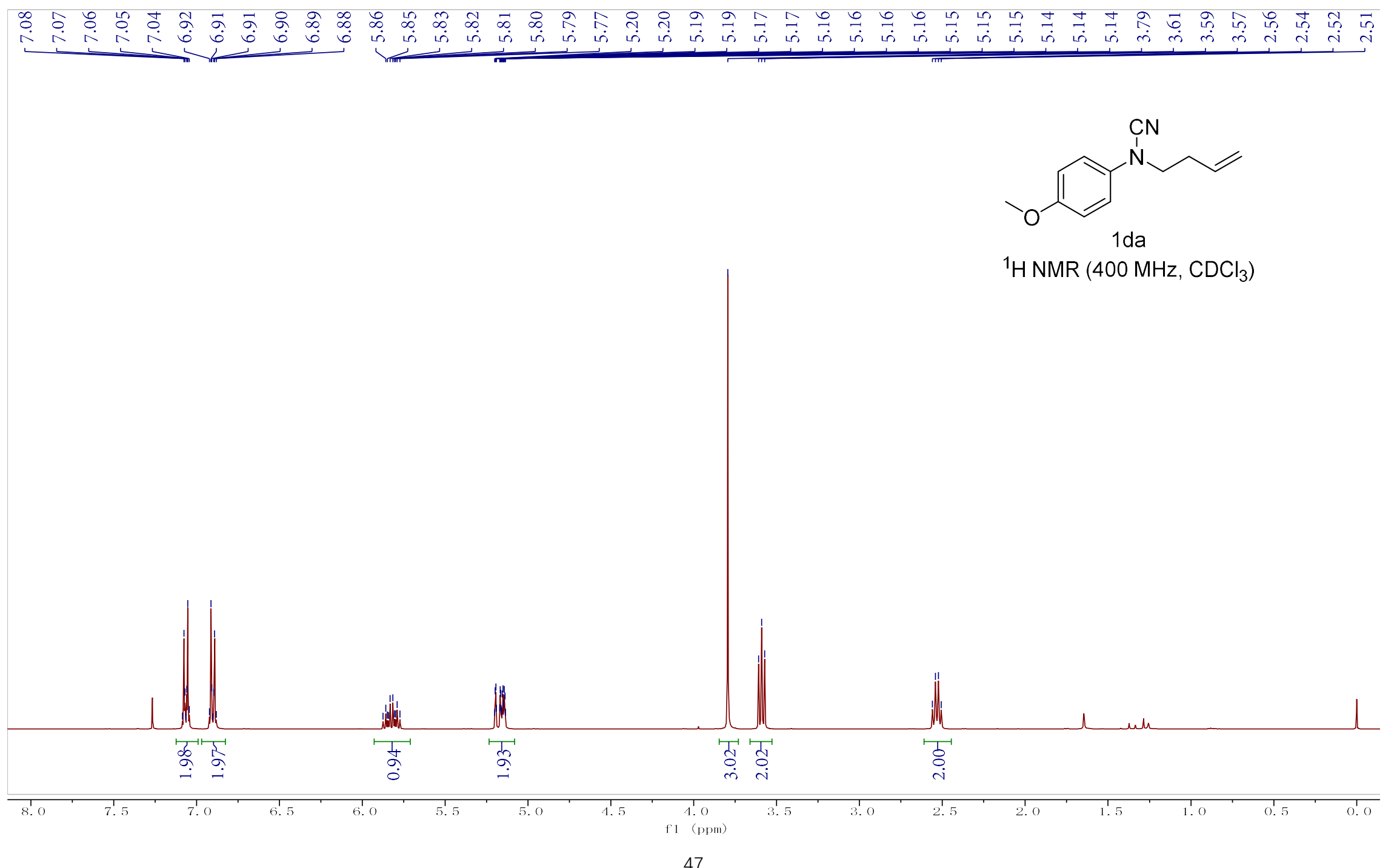




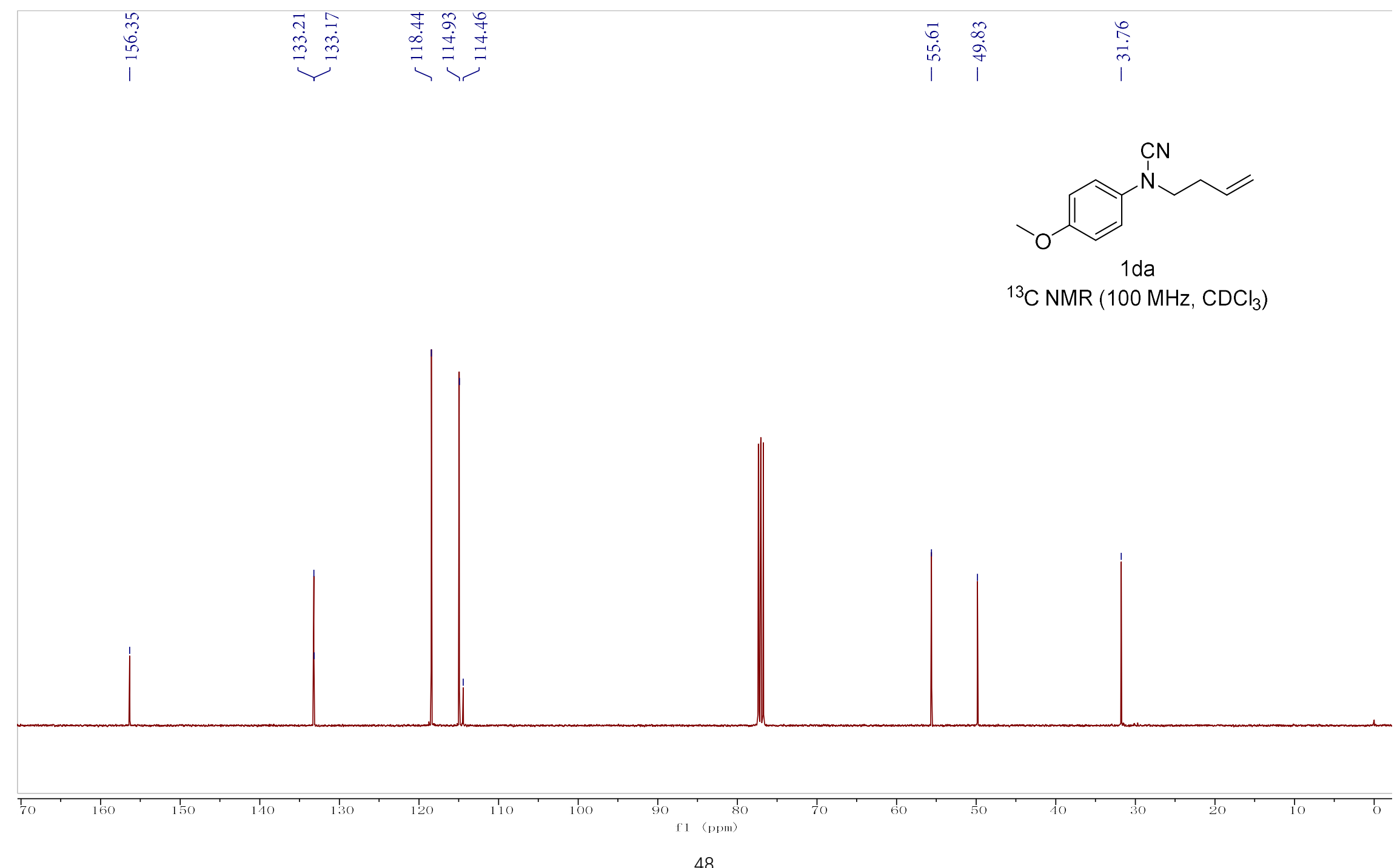




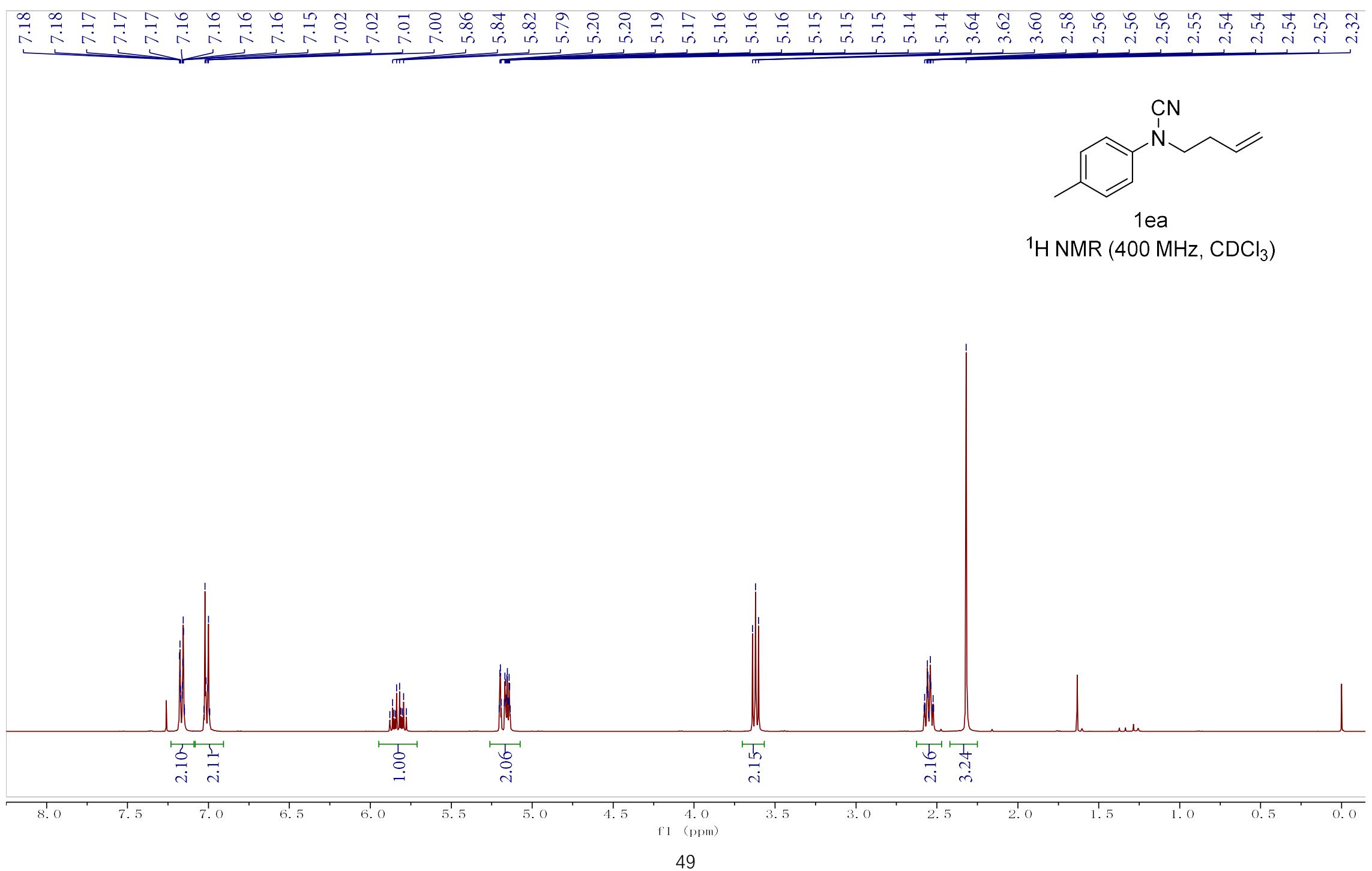




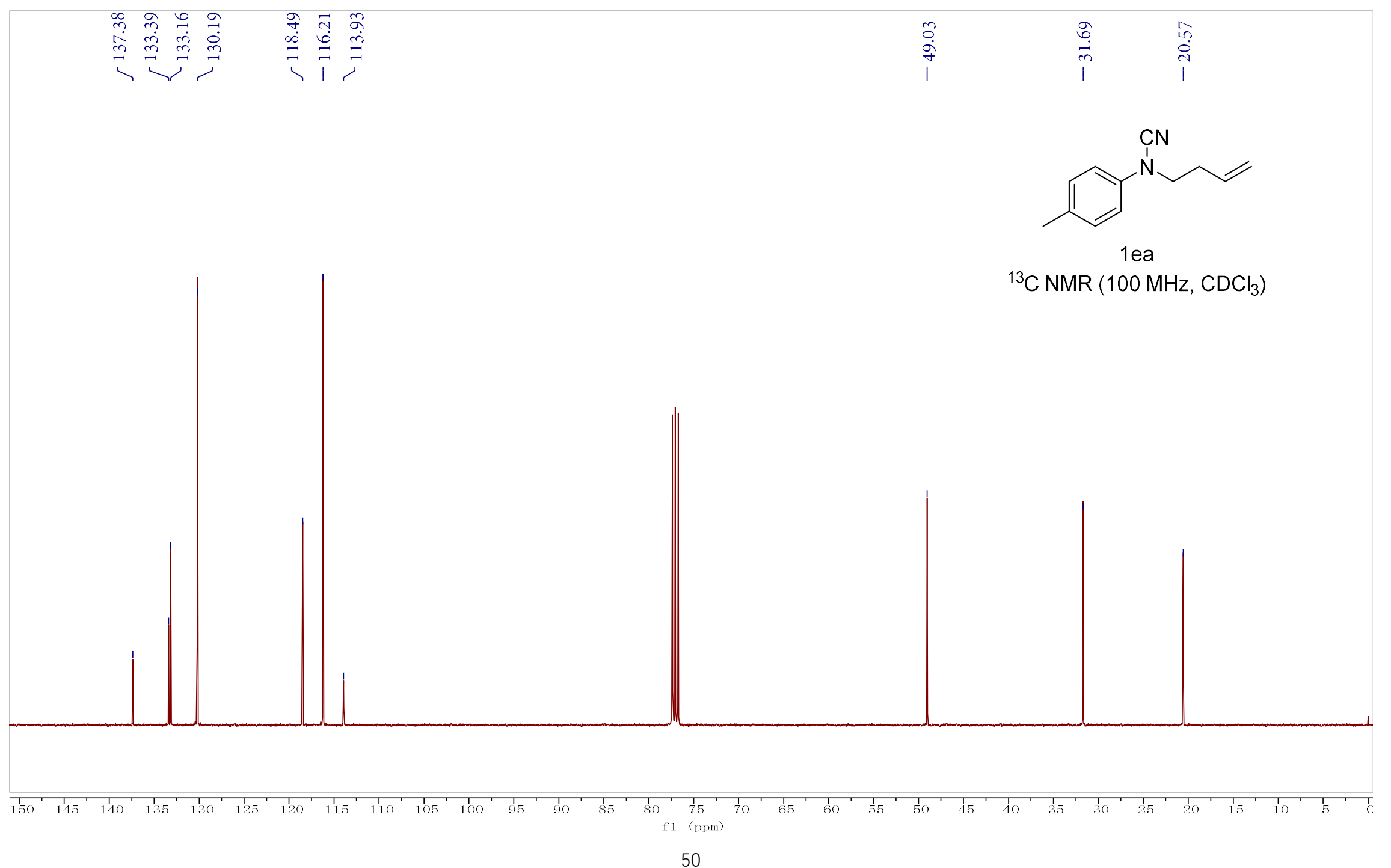




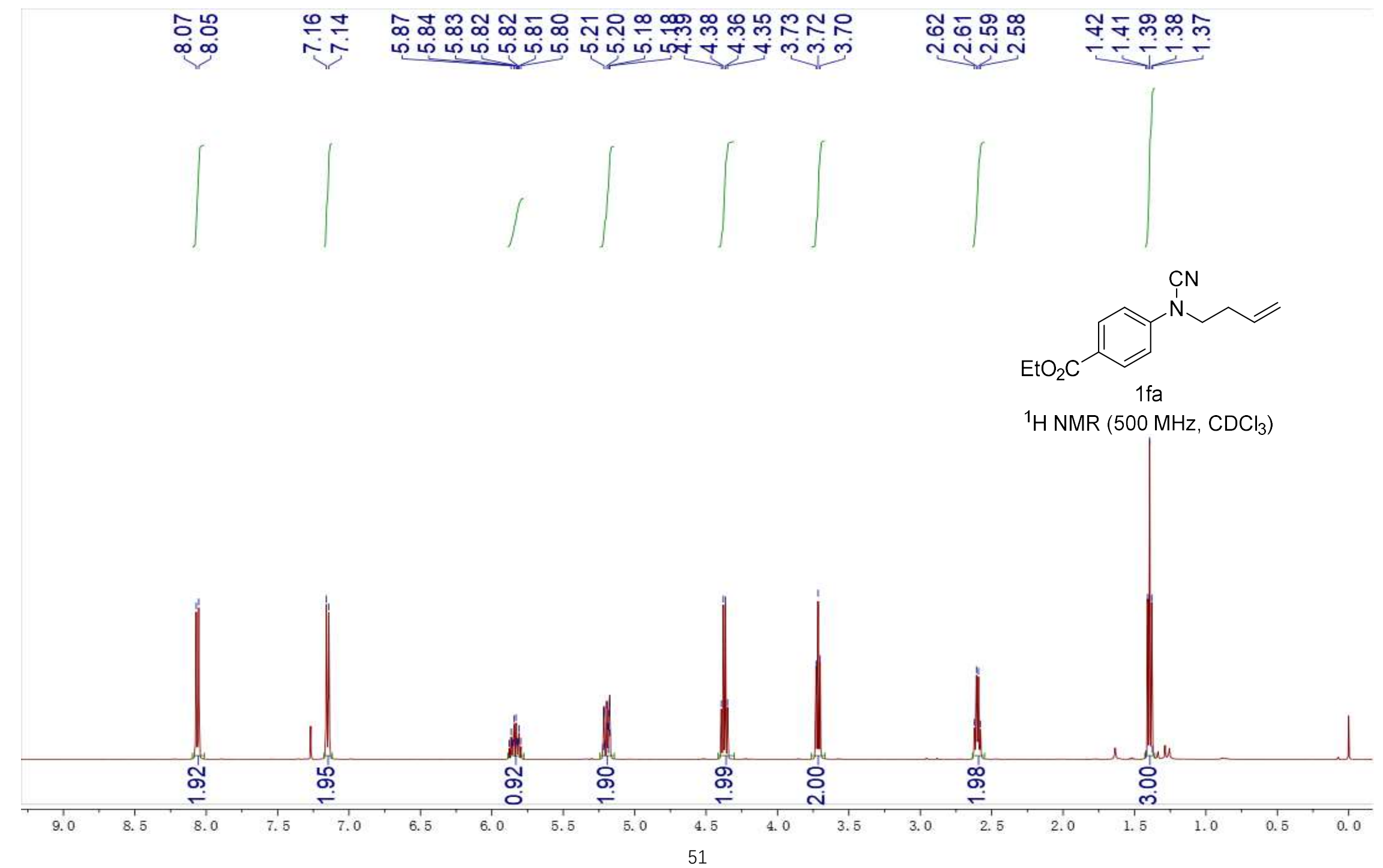




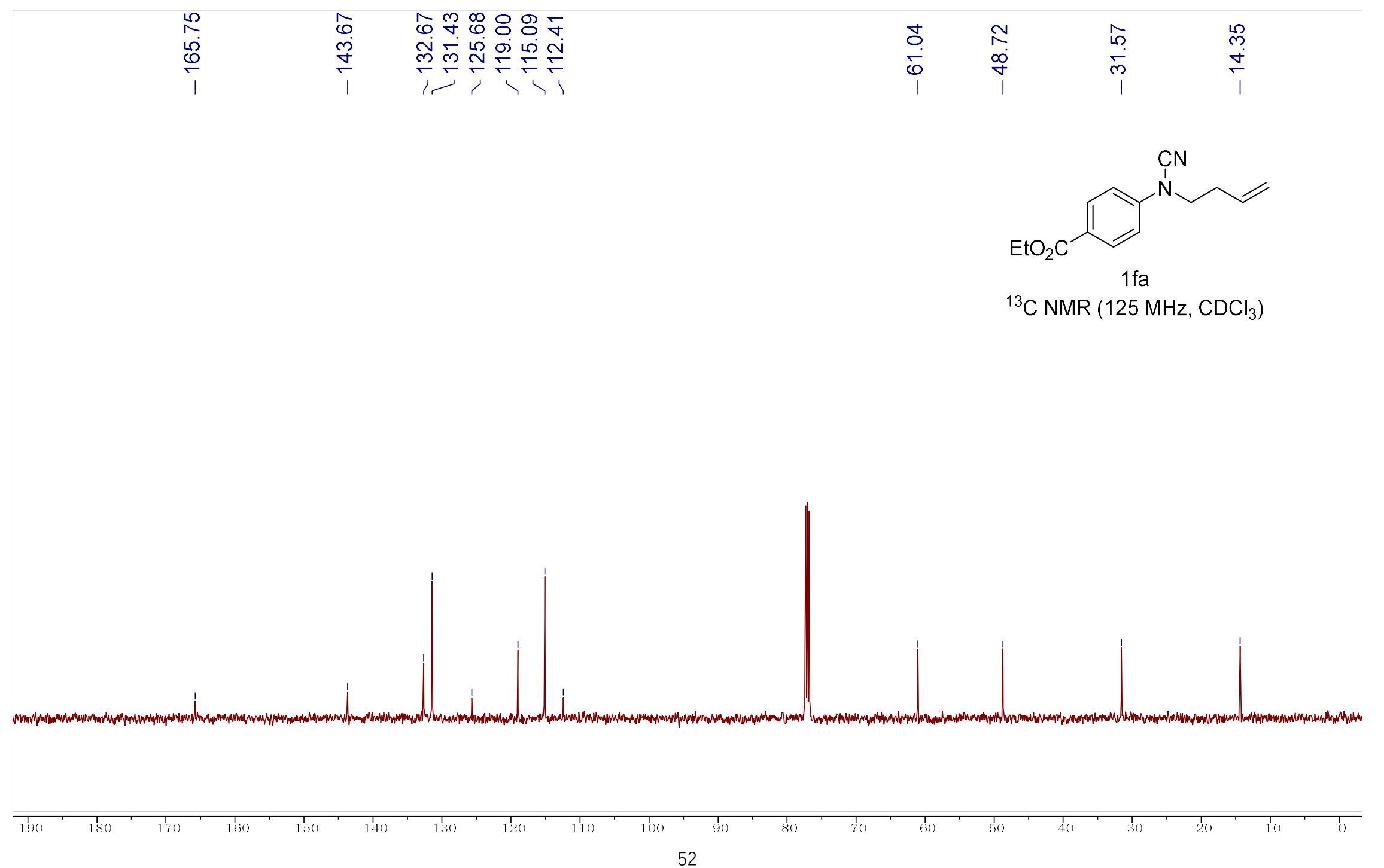




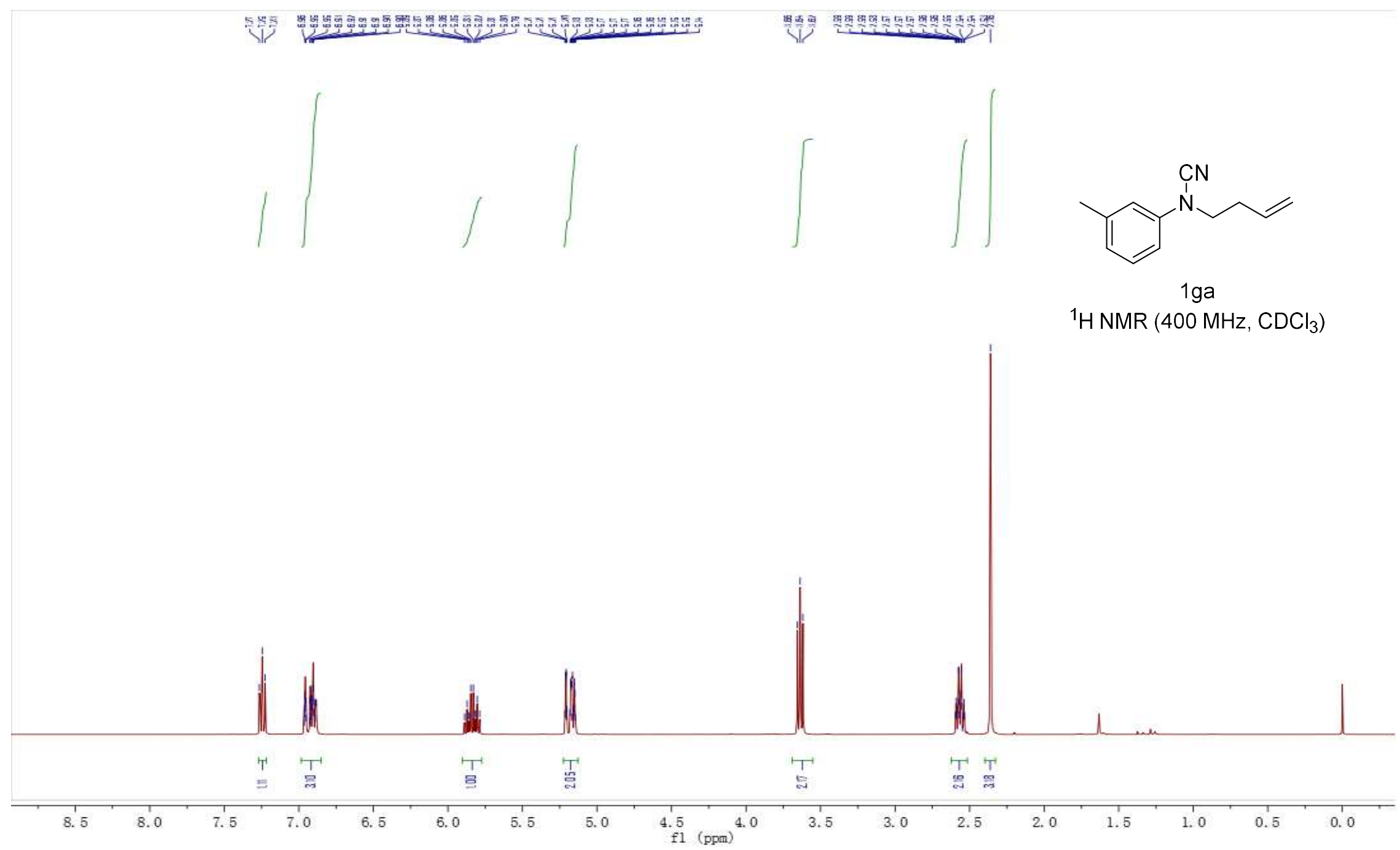




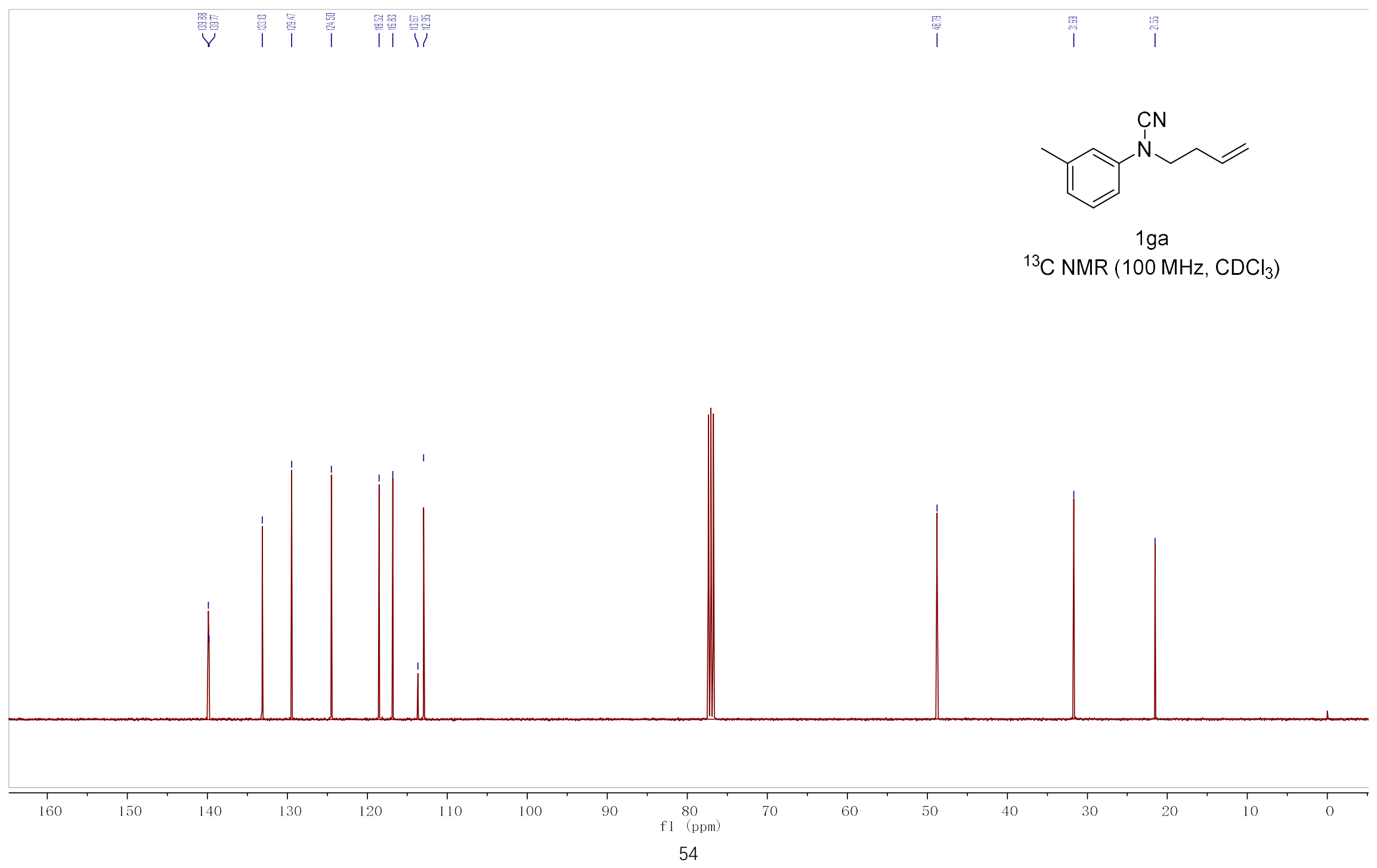




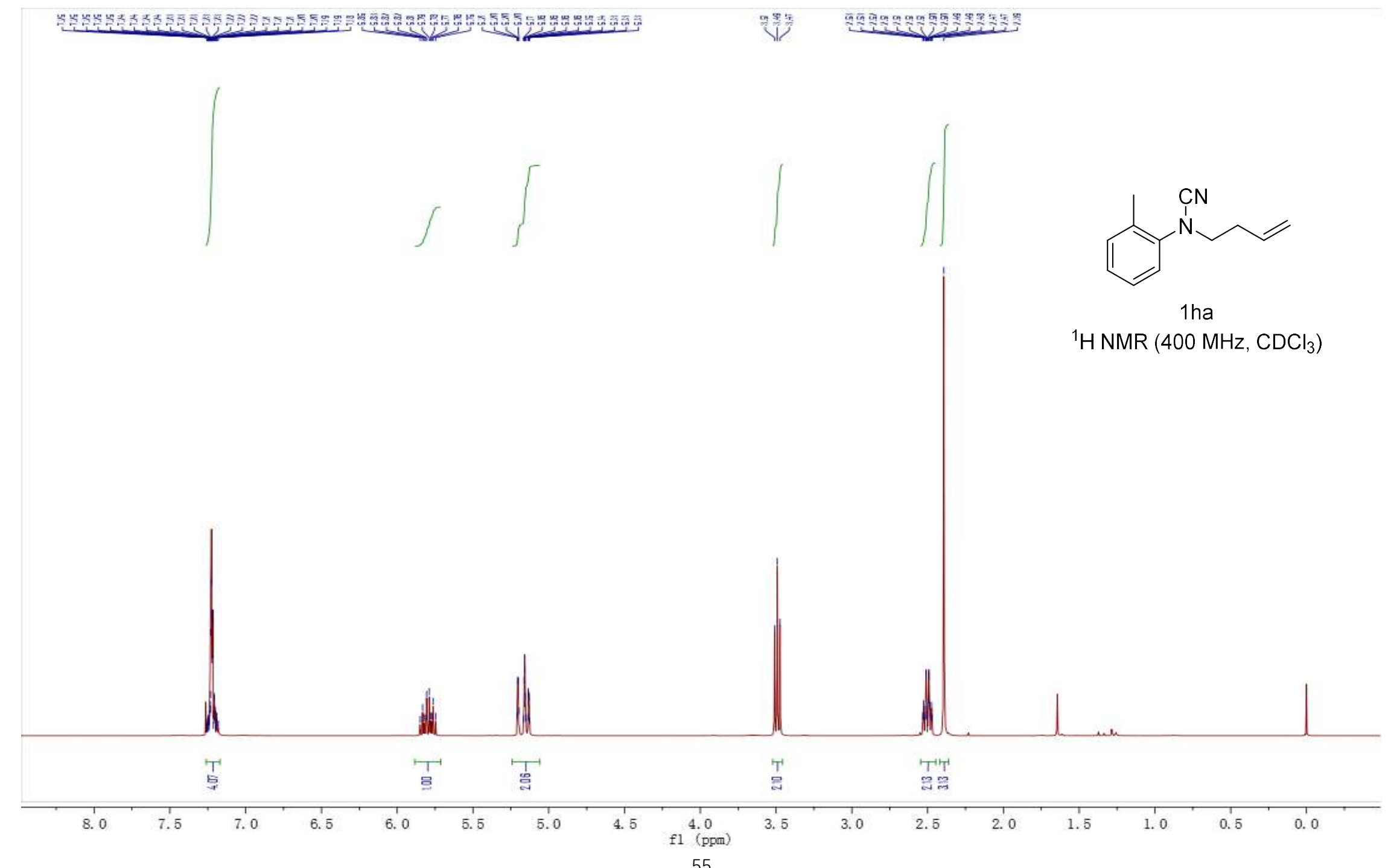




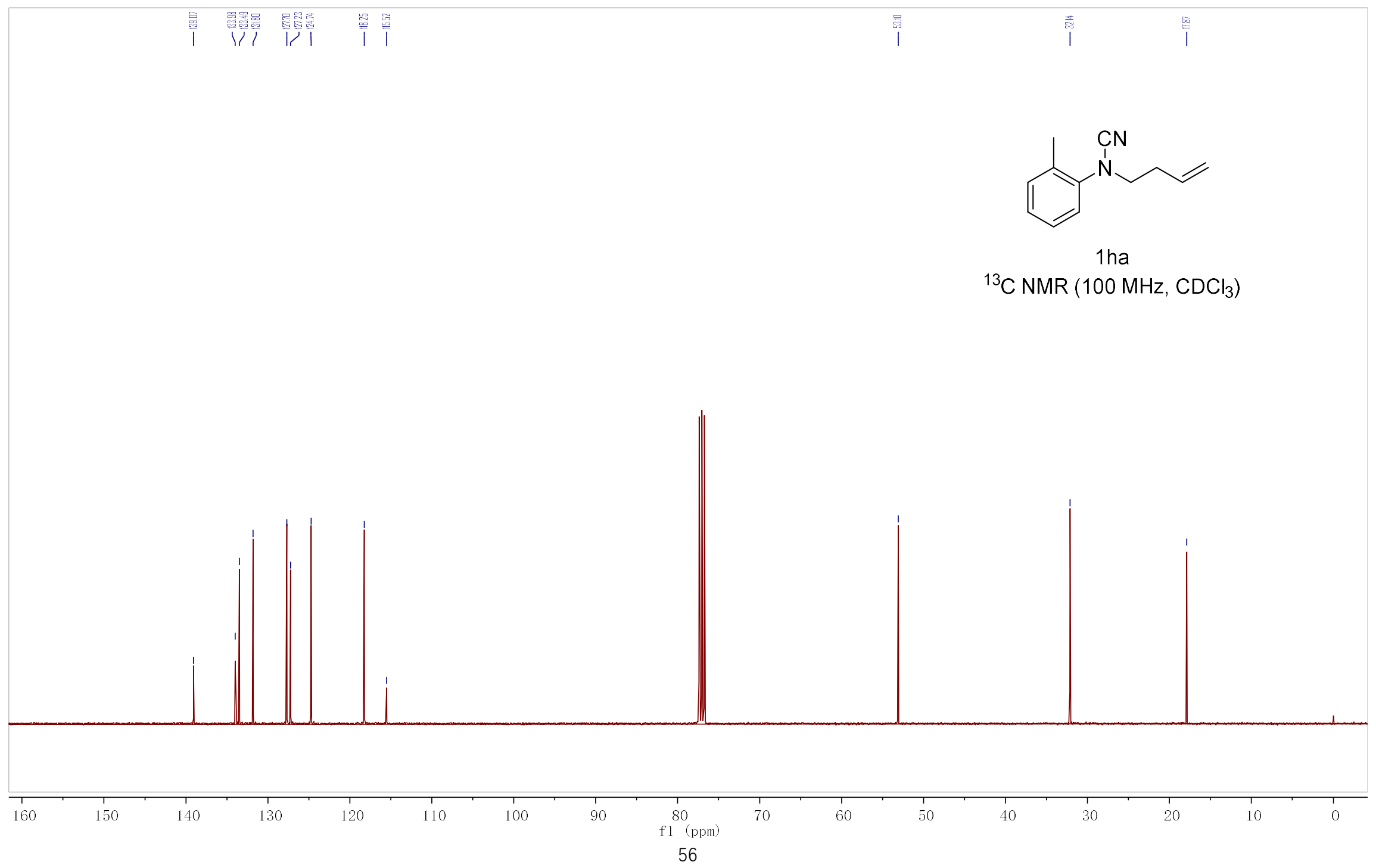




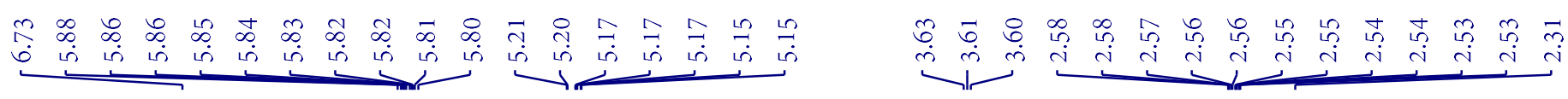

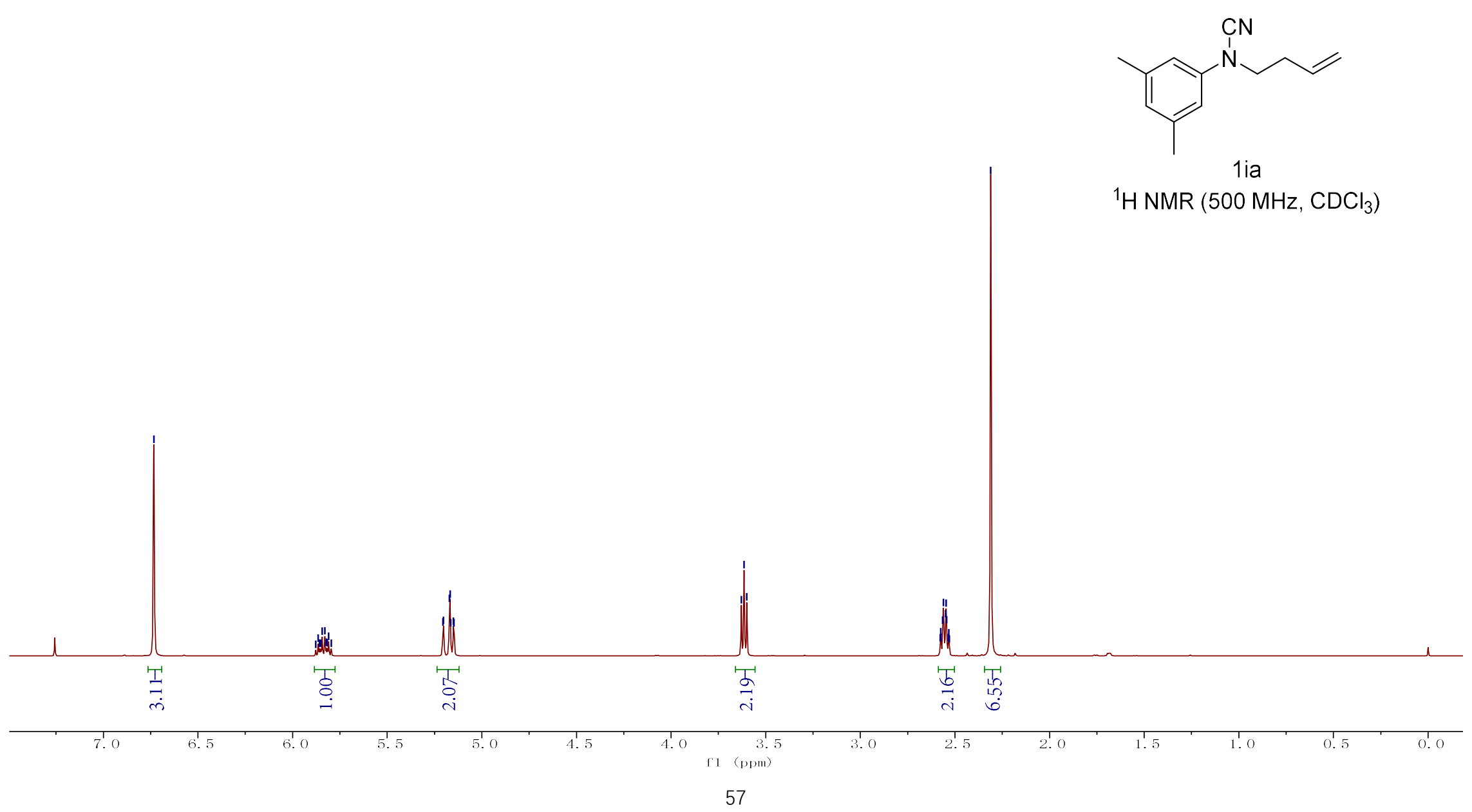




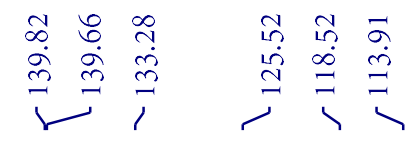

ind

$T$

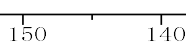

$140-130$

$\frac{1}{120}+110$

100

90

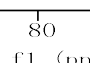

$80(\mathrm{ppm})$

60

50

40

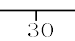

20 
$\underbrace{2}$

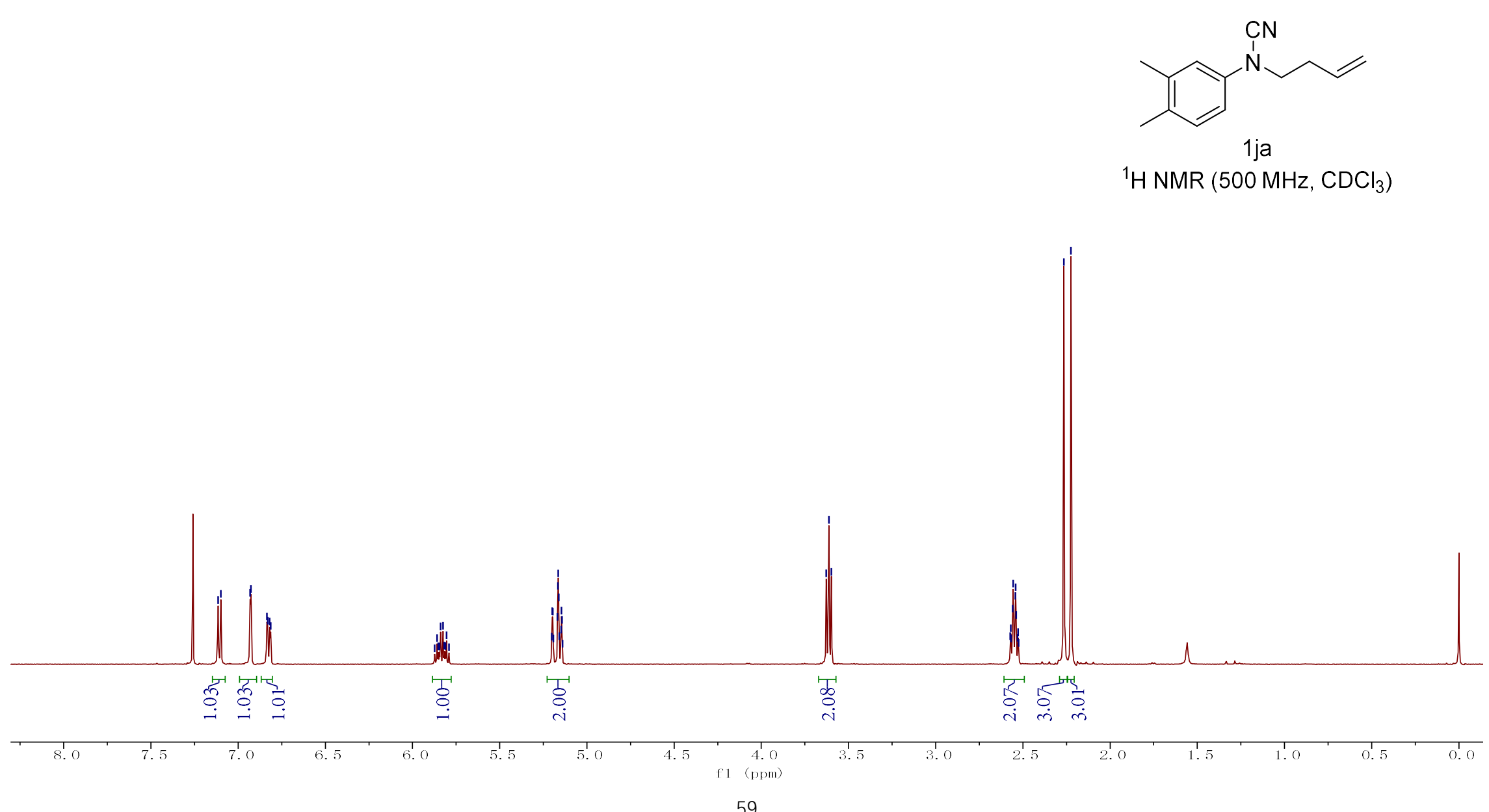




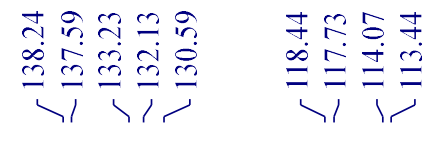

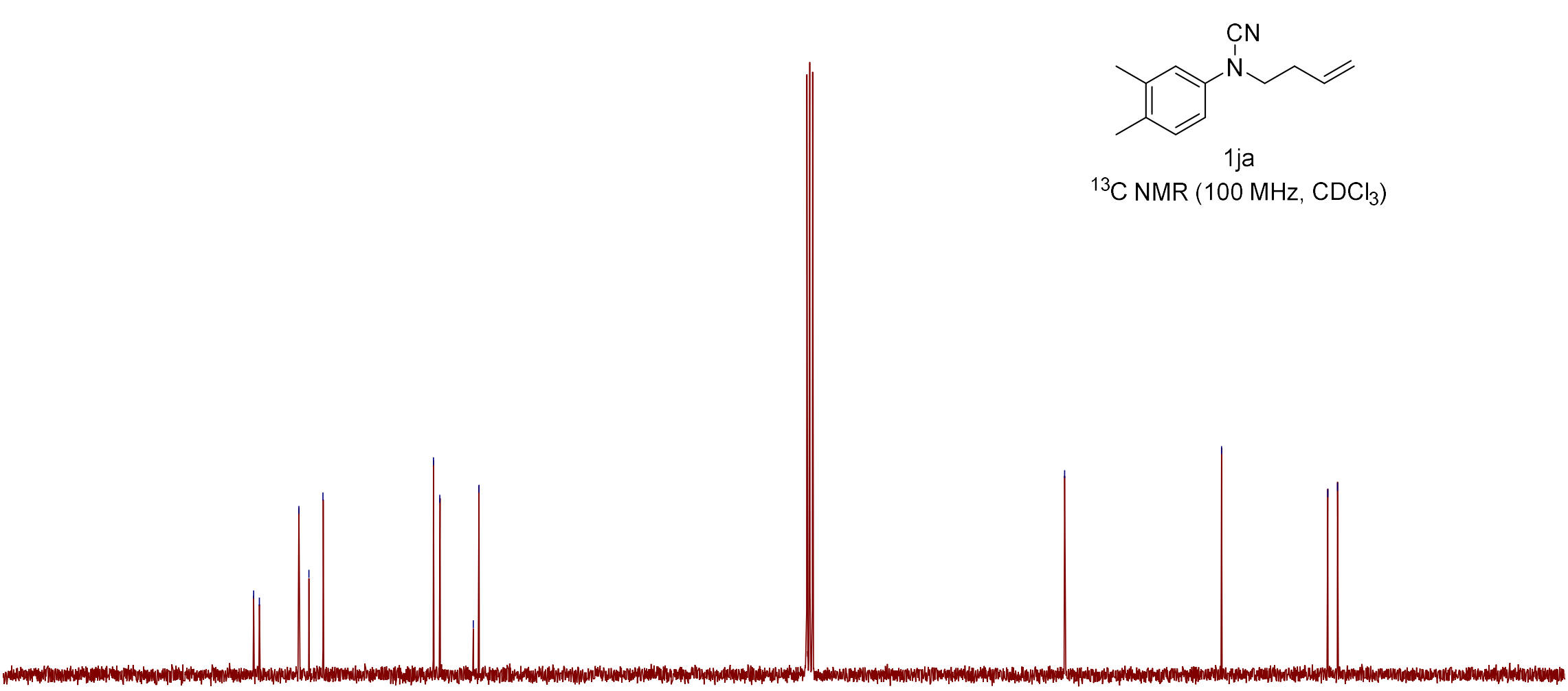

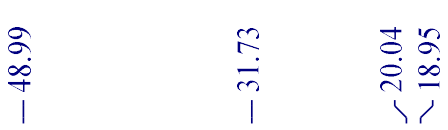

CN

1ja

${ }^{13} \mathrm{C}$ NMR $\left(100 \mathrm{MHz}, \mathrm{CDCl}_{3}\right)$
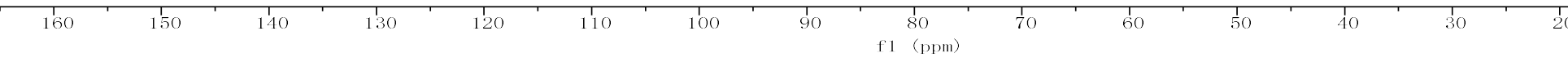

20 


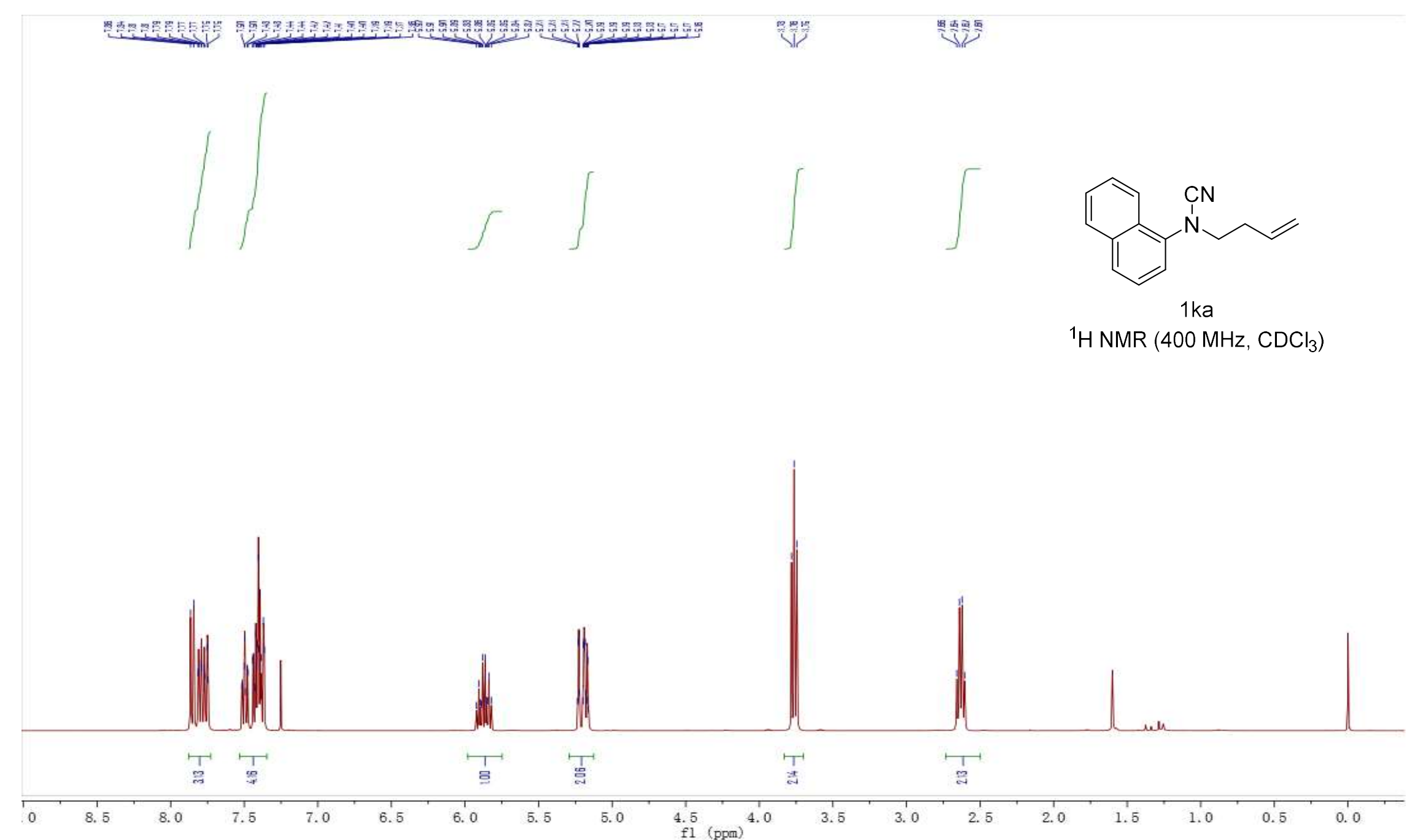




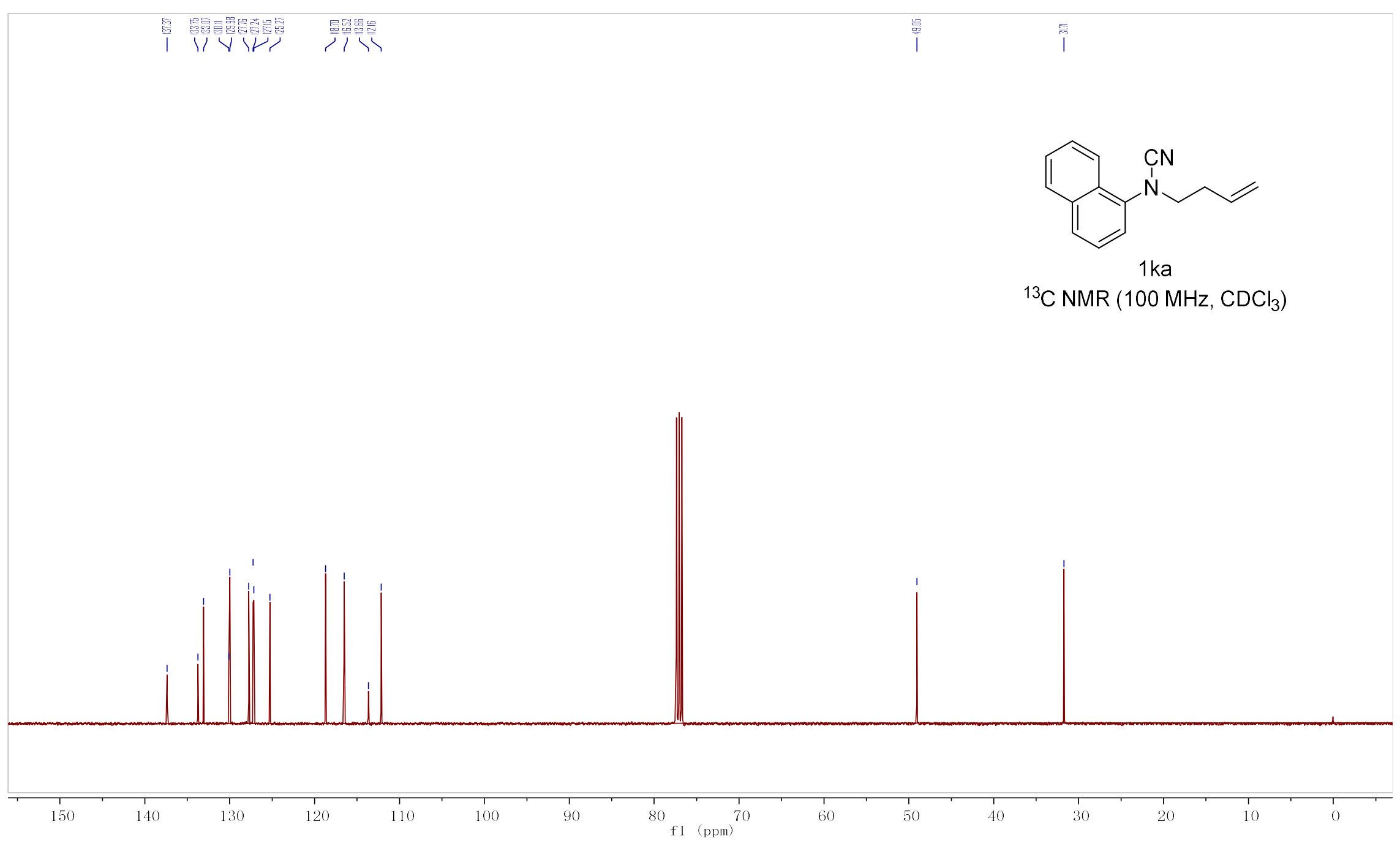


ప r.r.

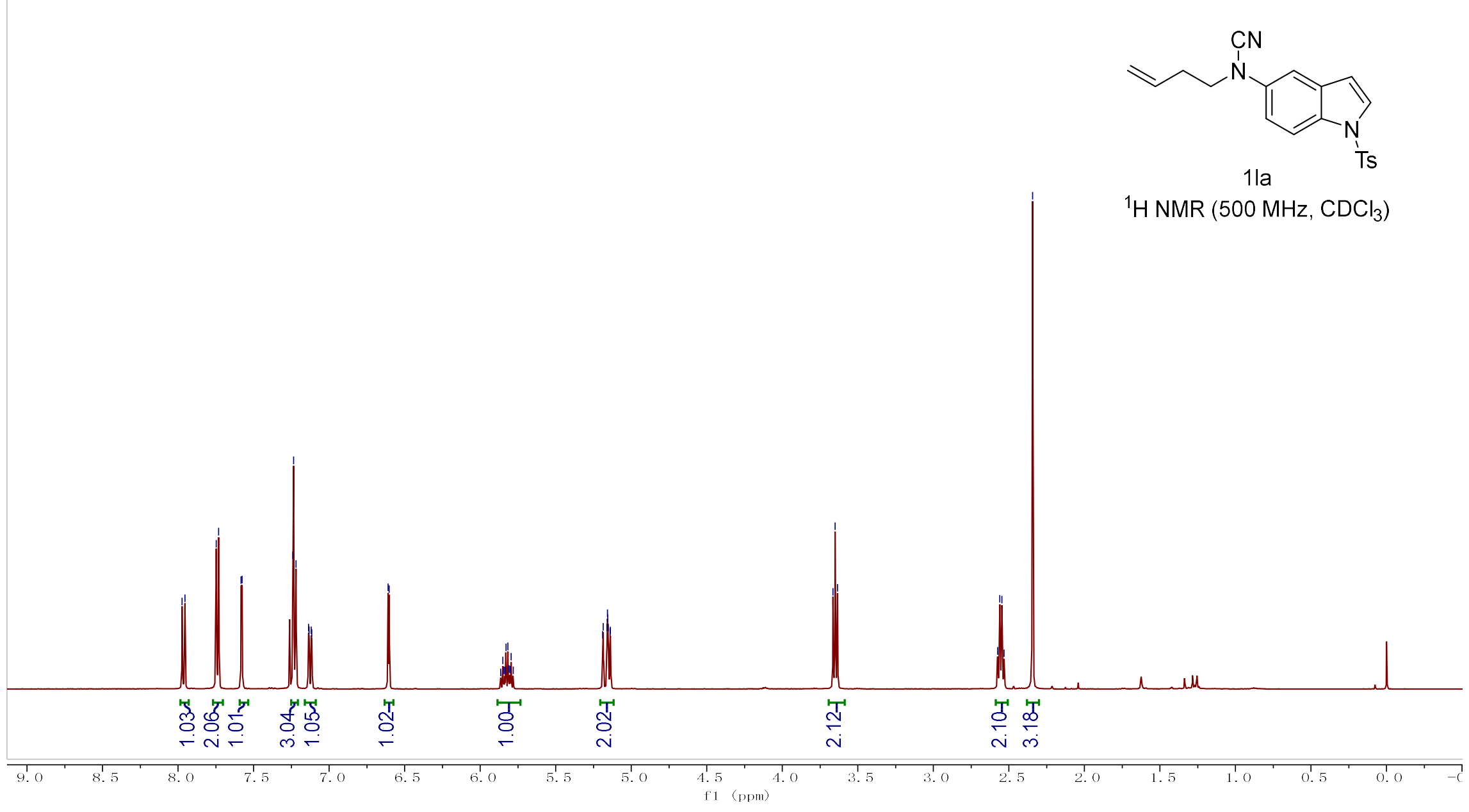




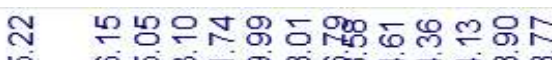

告 인

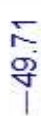

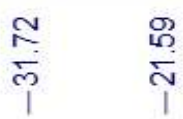

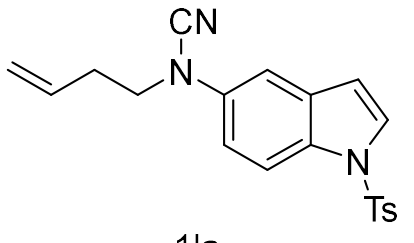

1 la

${ }^{13} \mathrm{C} \mathrm{NMR}\left(125 \mathrm{MHz}, \mathrm{CDCl}_{3}\right)$
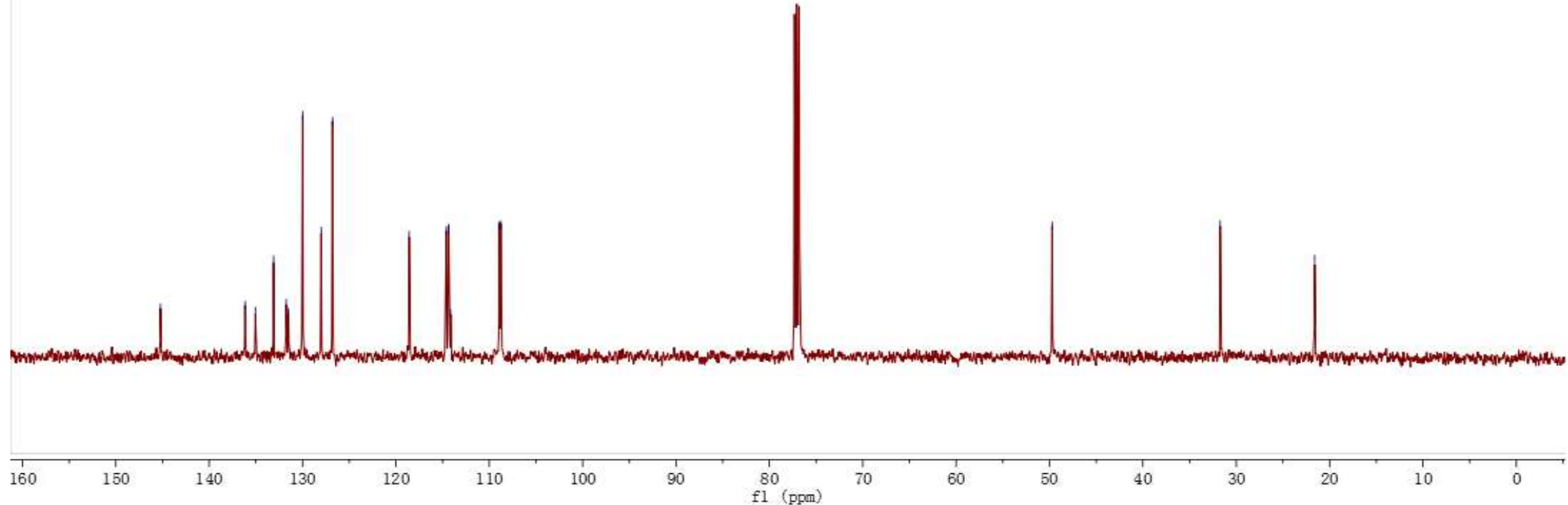


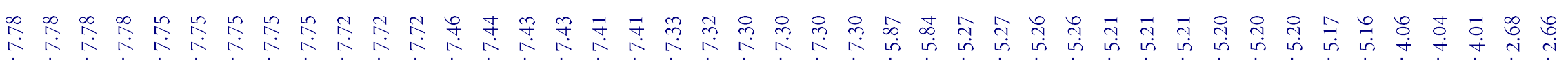
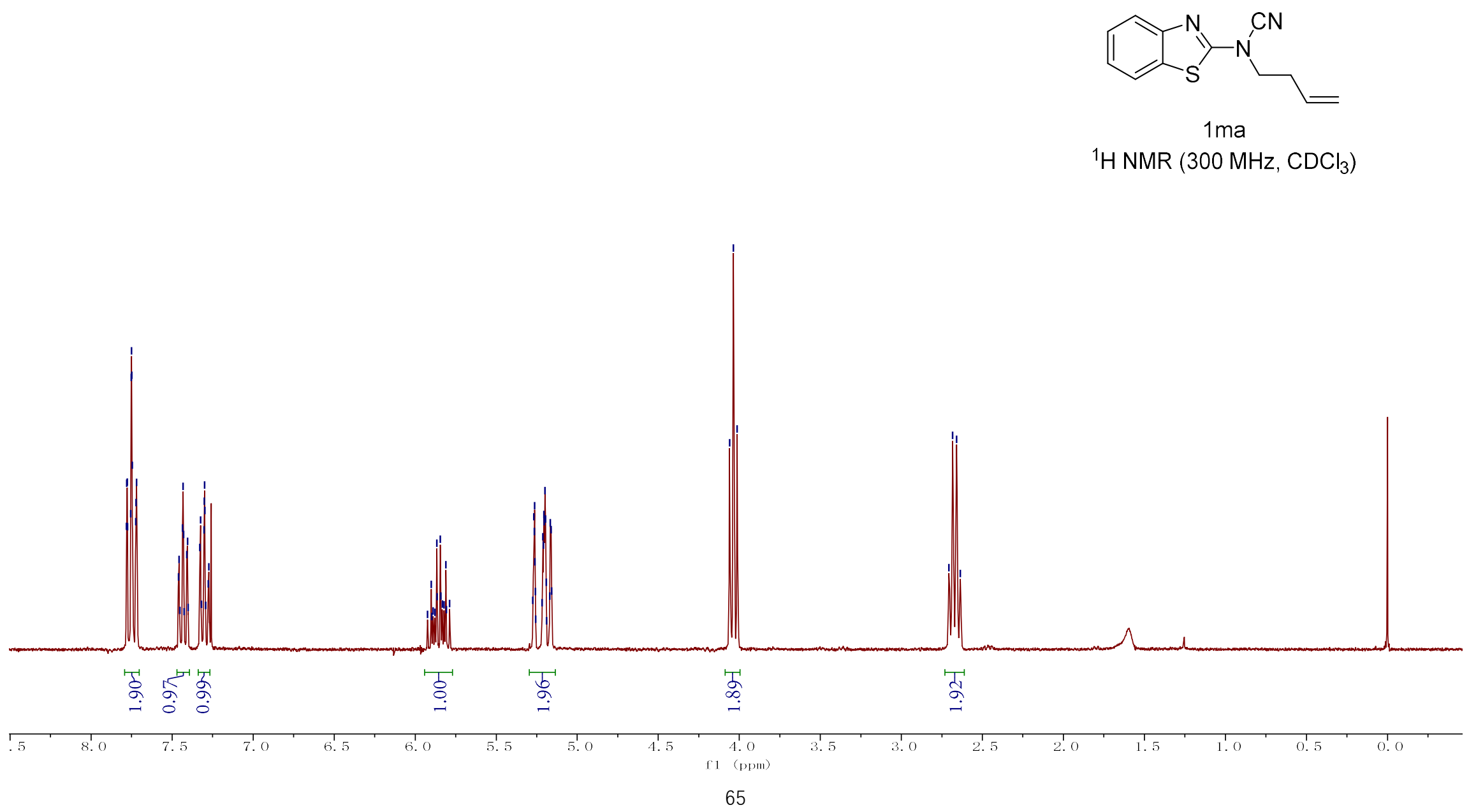


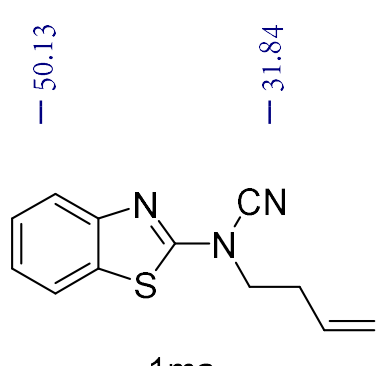

$1 \mathrm{ma}$

${ }^{13} \mathrm{C}$ NMR (125 MHz, $\mathrm{CDCl}_{3}$ )

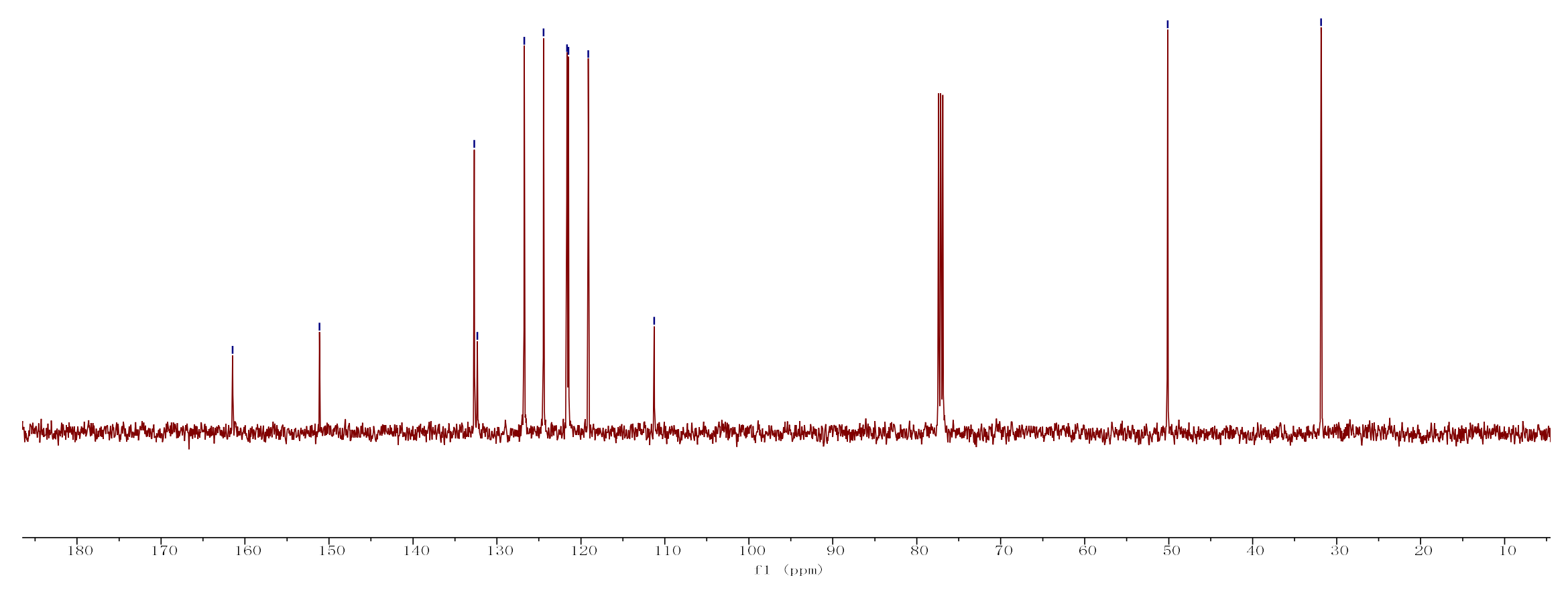




$$
\bar{a}
$$




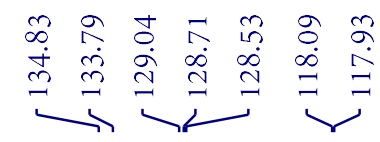

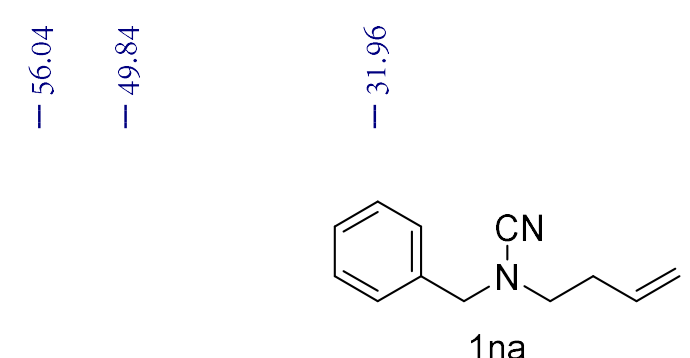

$\left.{ }^{13} \mathrm{C} \mathrm{NMR} \mathrm{(125} \mathrm{MHz,} \mathrm{CDCl}_{3}\right)$
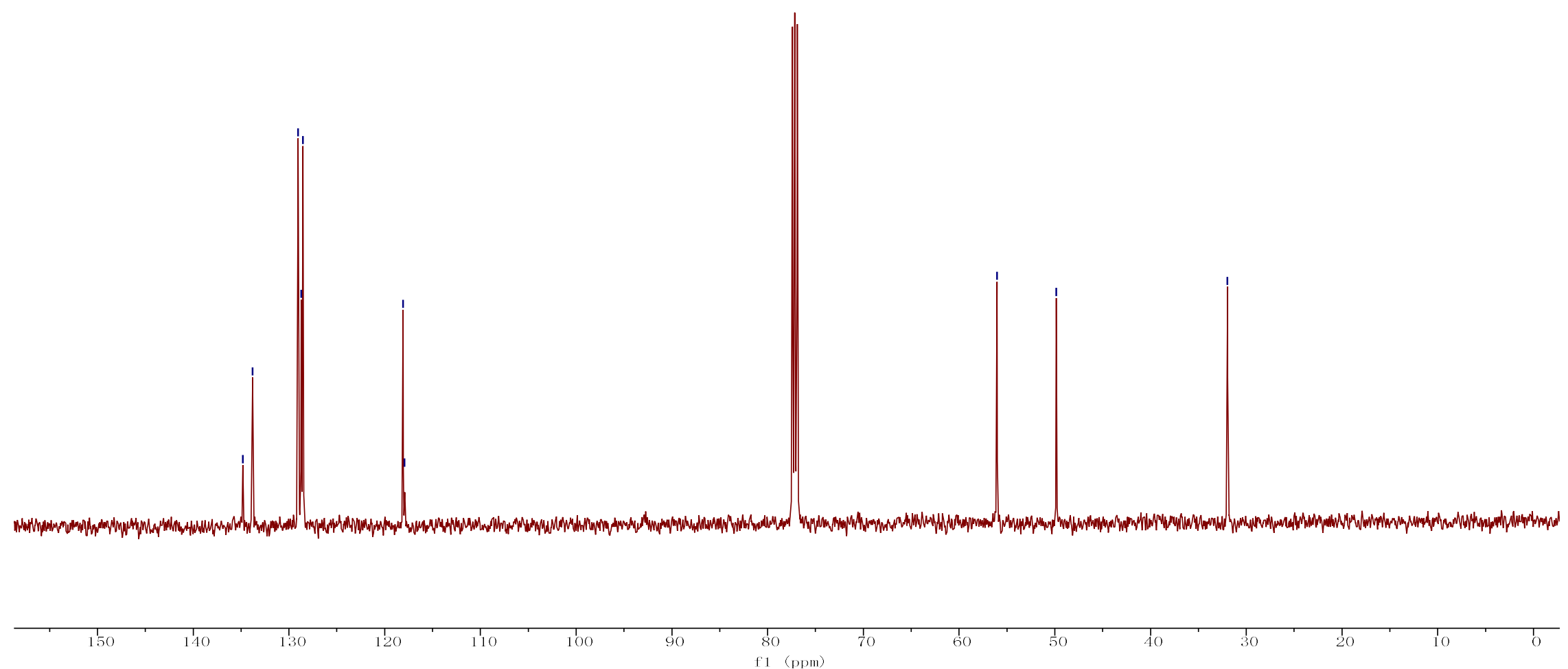


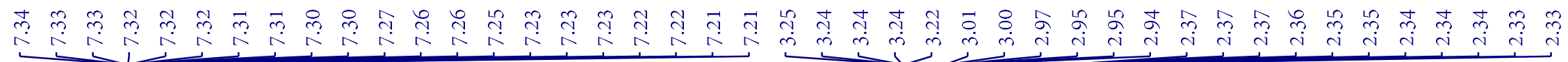

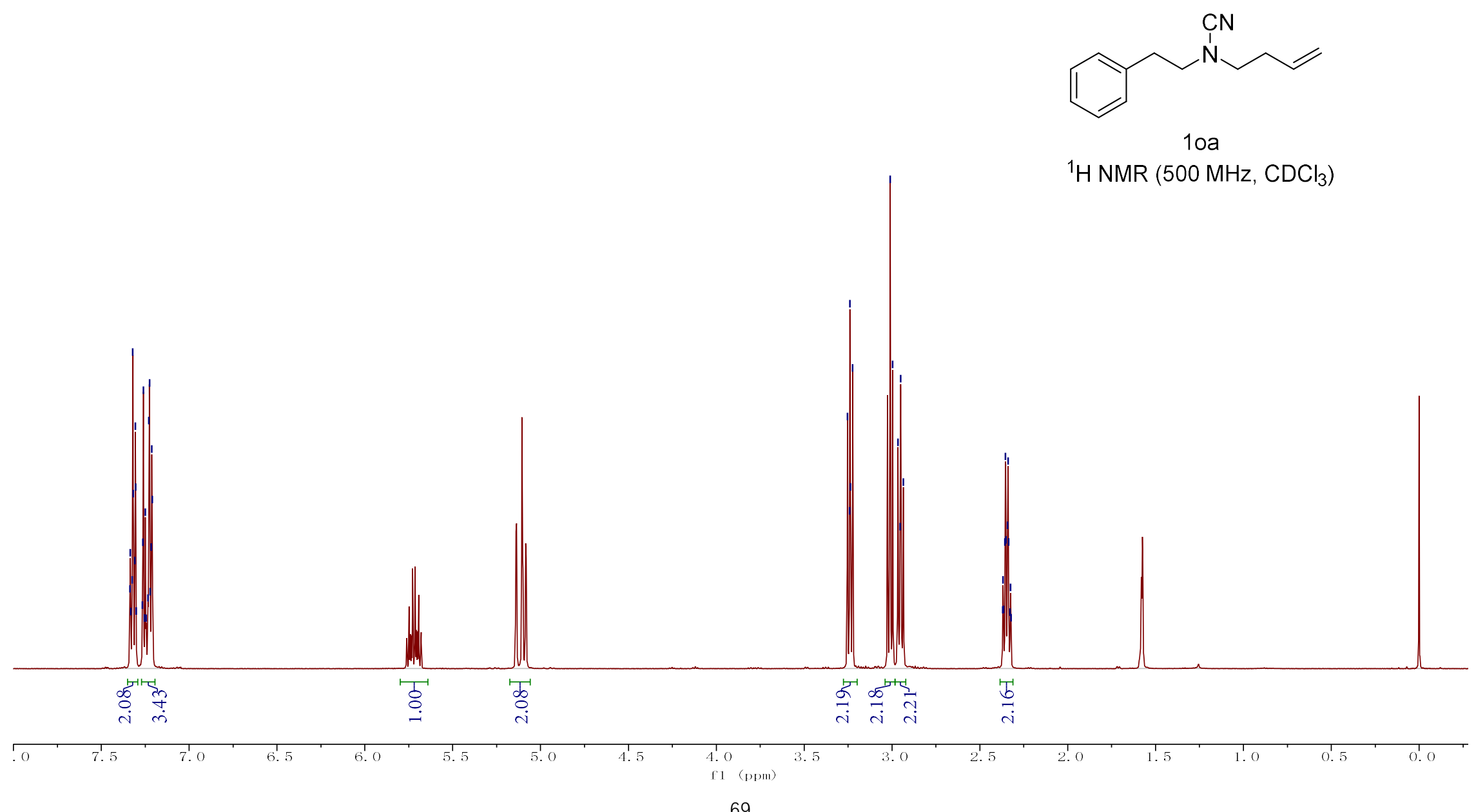




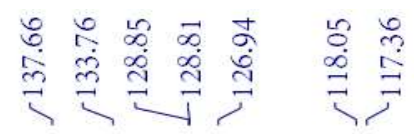

लै?

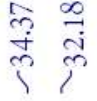

CN
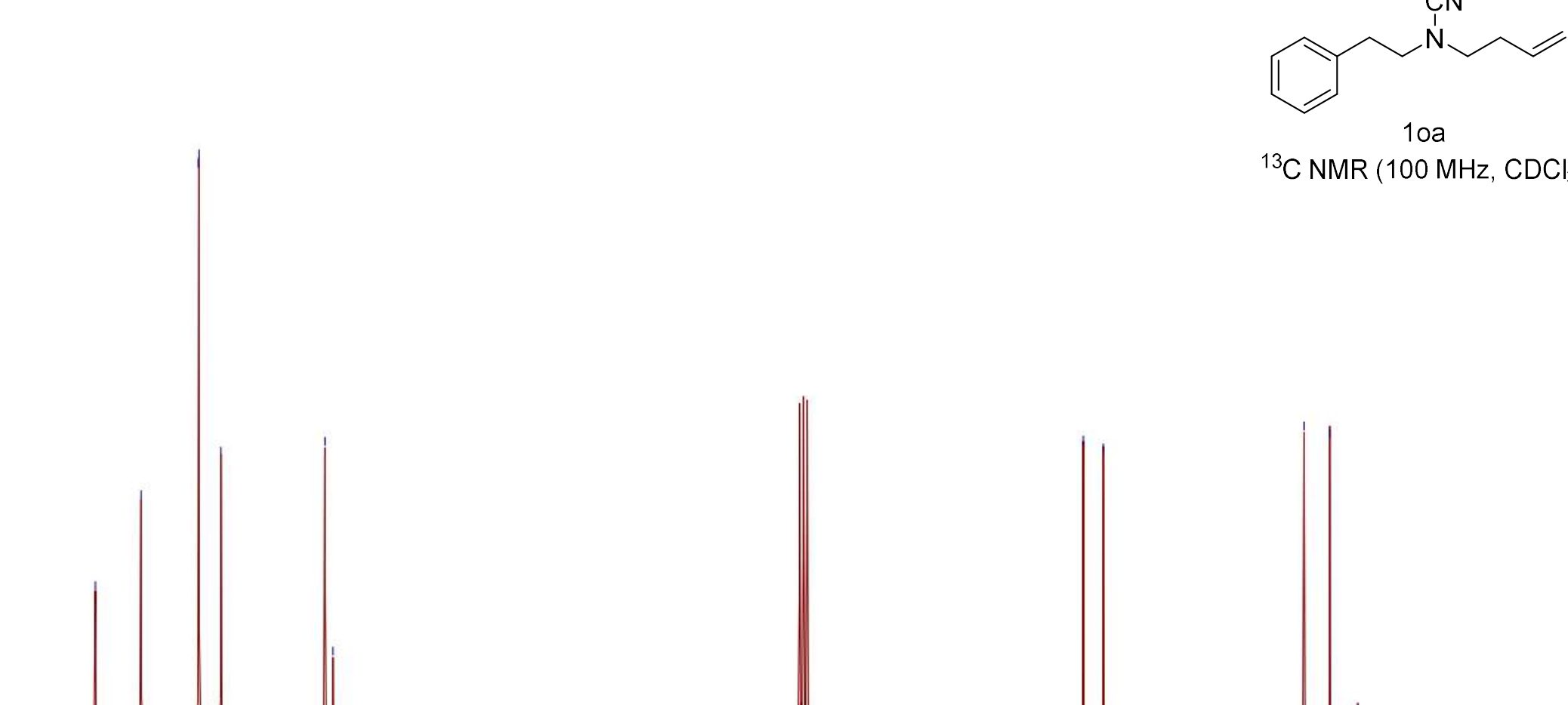

${ }^{13} \mathrm{C} \mathrm{NMR}\left(100 \mathrm{MHz}, \mathrm{CDCl}_{3}\right)$

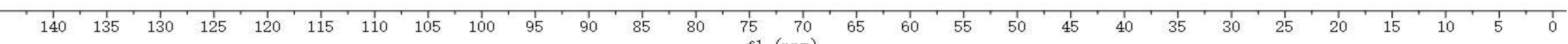




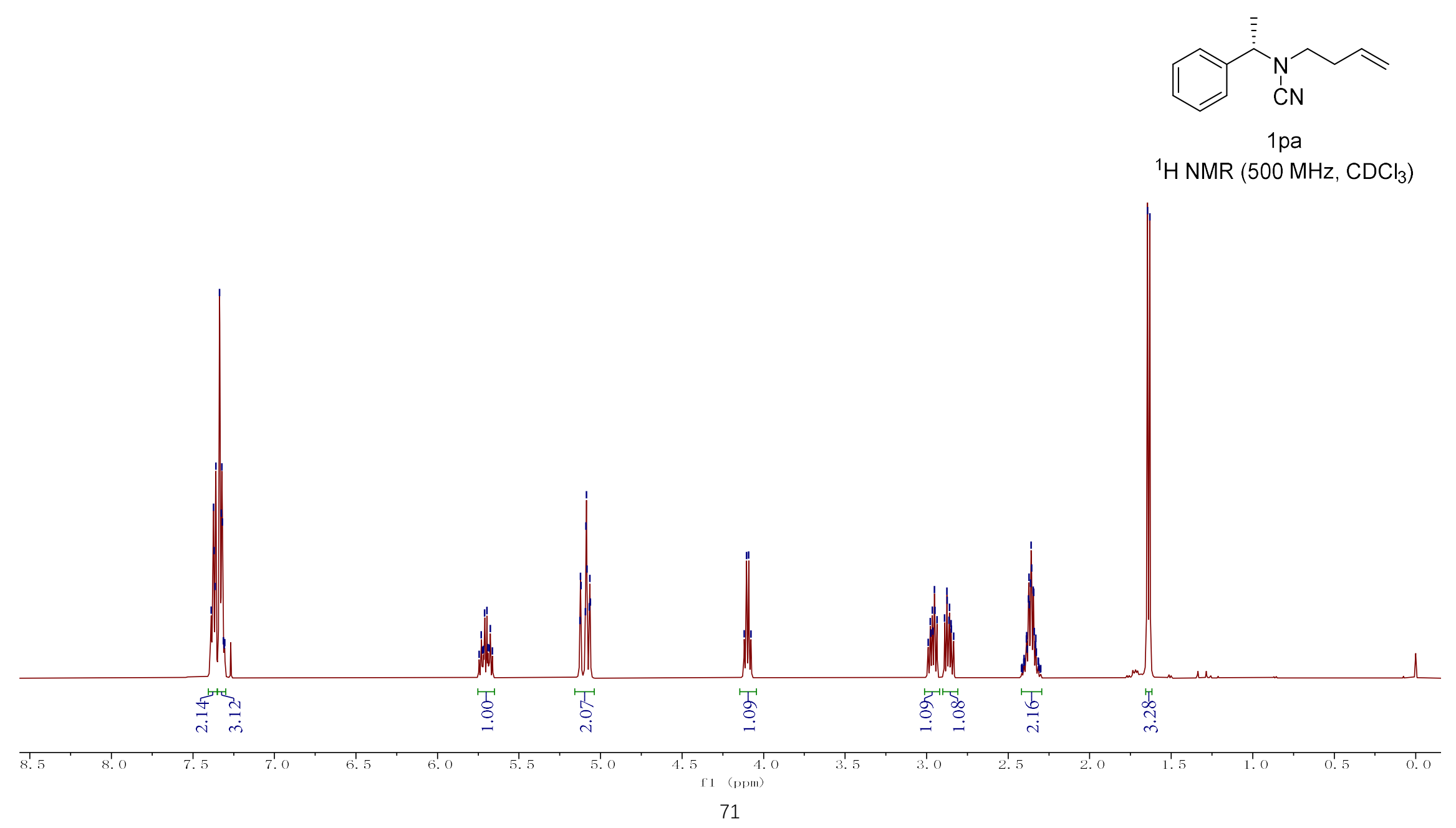




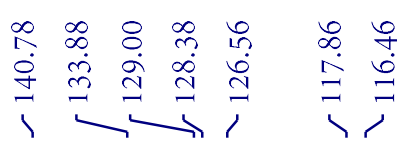

$\begin{array}{ll}\vec{j} & m \\ i & \stackrel{n}{i} \\ 1 & 1\end{array}$

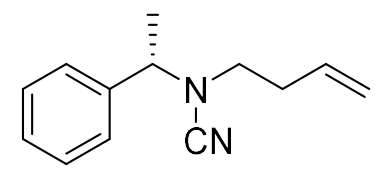

1 pa

${ }^{13} \mathrm{C} \mathrm{NMR}\left(125 \mathrm{MHz}, \mathrm{CDCl}_{3}\right)$
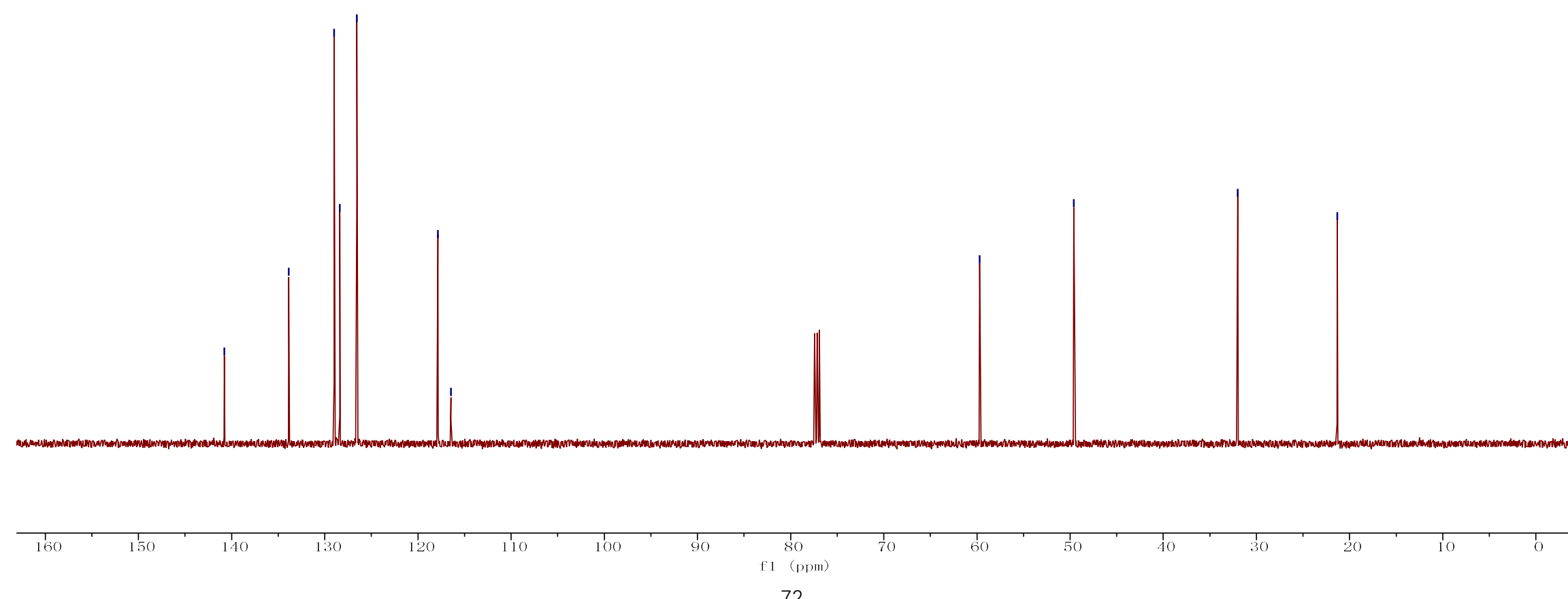


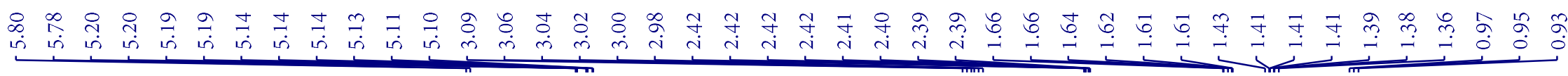

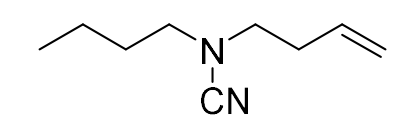

${ }^{1} \mathrm{H} \mathrm{NMR}\left(300 \mathrm{MHz}, \mathrm{CDCl}_{3}\right)$

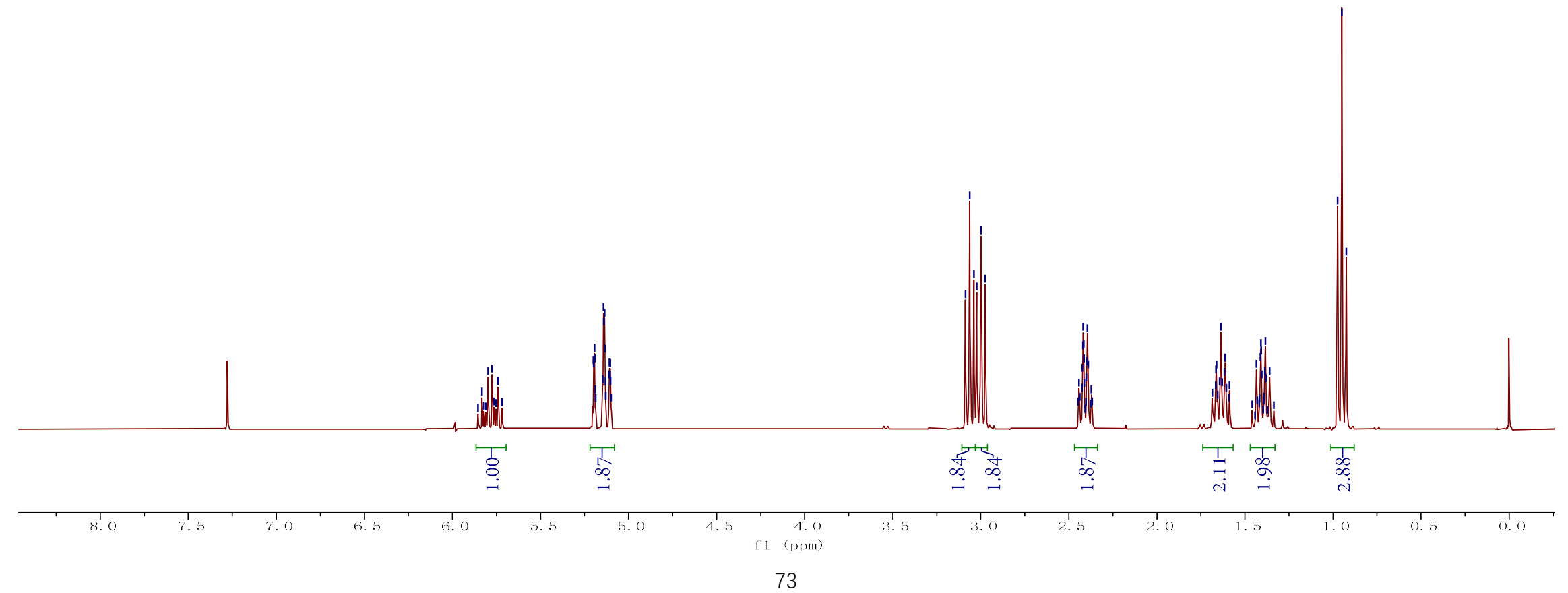




$$
\text { 些, }
$$




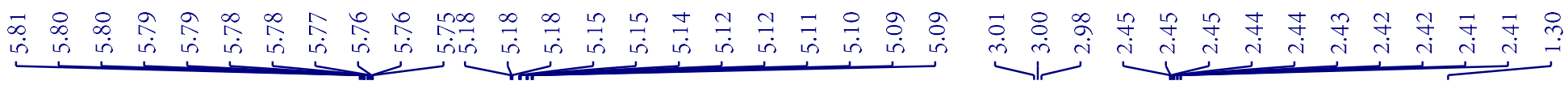

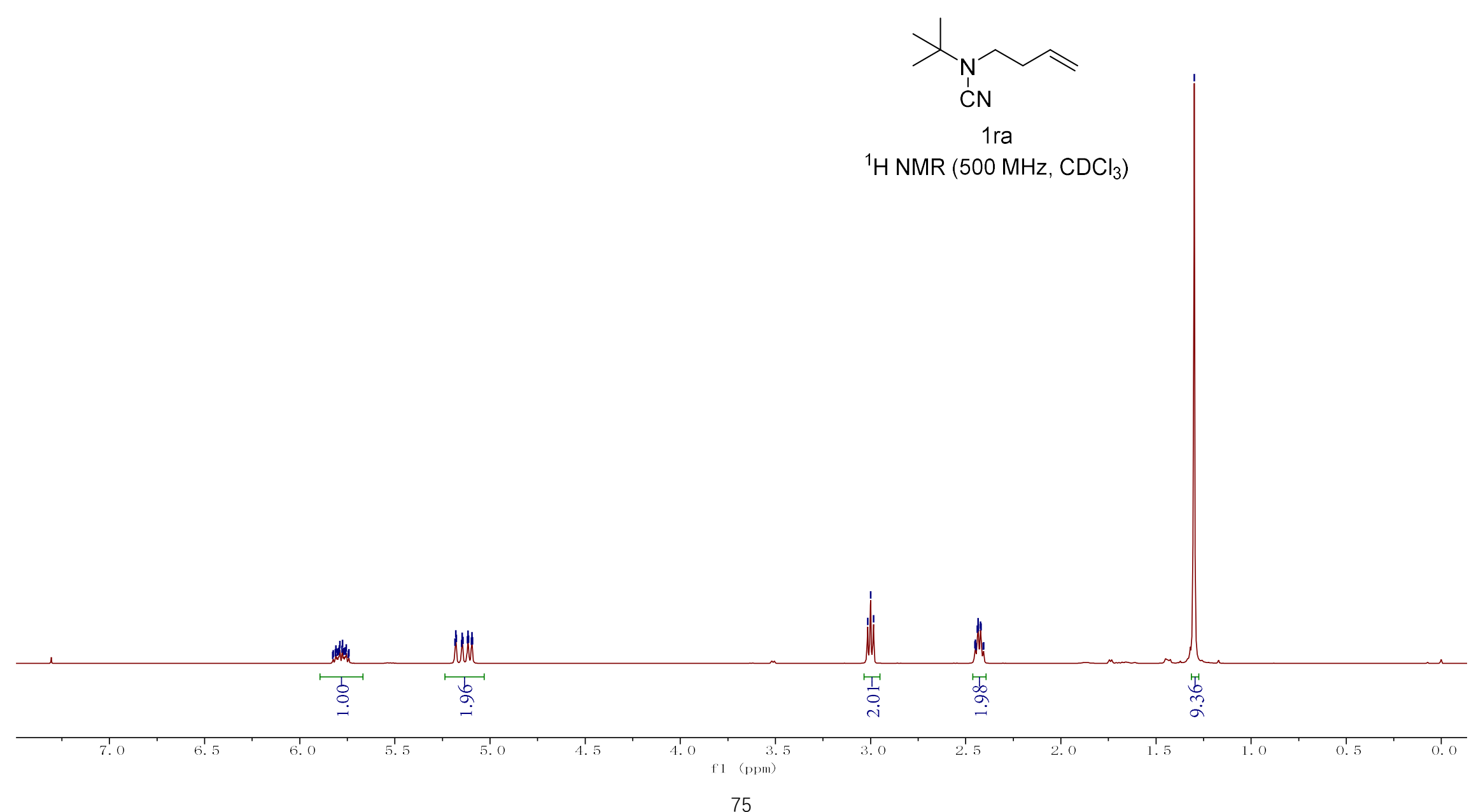




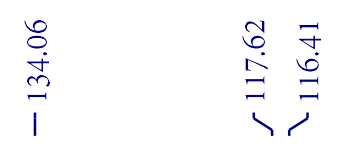

$\begin{array}{llll}3 & 0 & 2 & 8 \\ 0 & \infty & 0 & 0 \\ i & j & 0 & 0 \\ 1 & 1 & 1 & 1\end{array}$

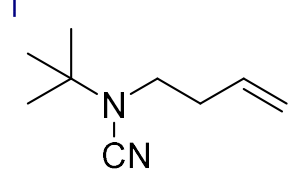

1 ra

${ }^{13} \mathrm{C}$ NMR (125 MHz, $\mathrm{CDCl}_{3}$ )
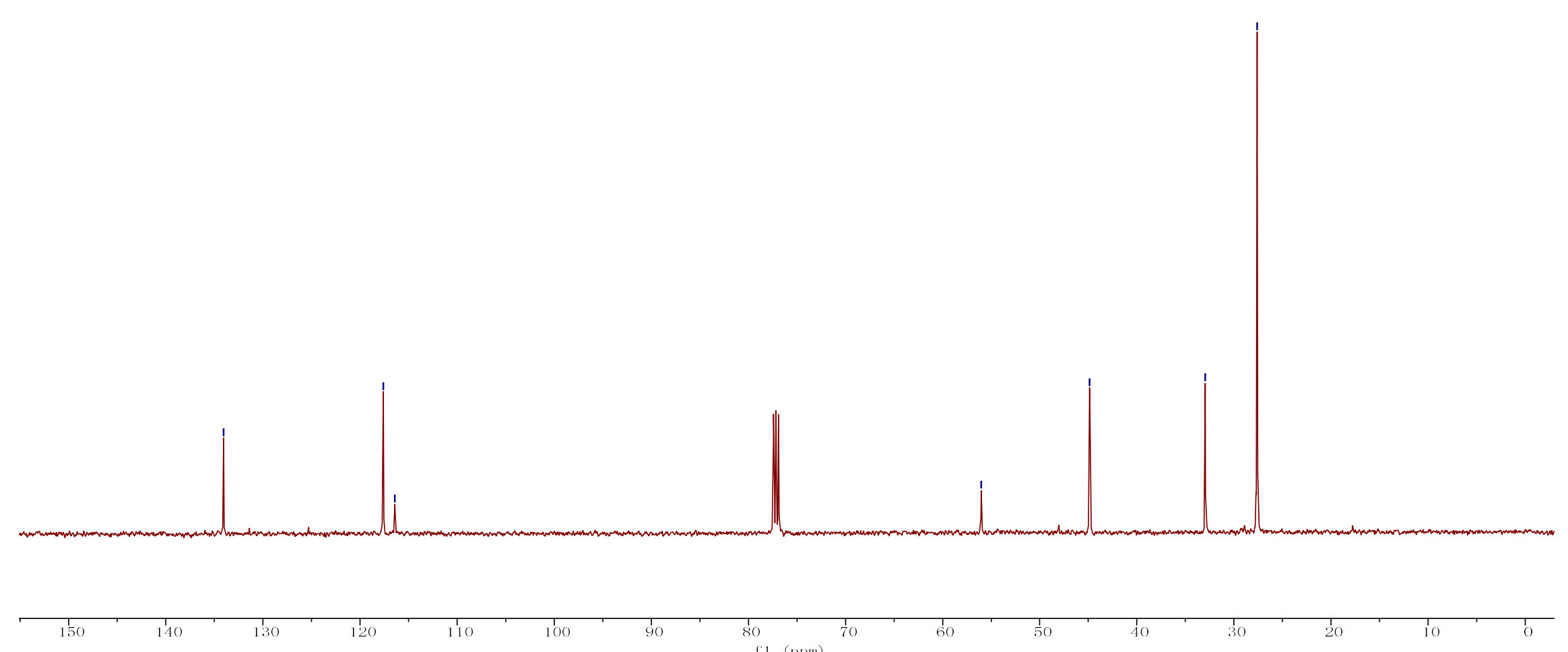

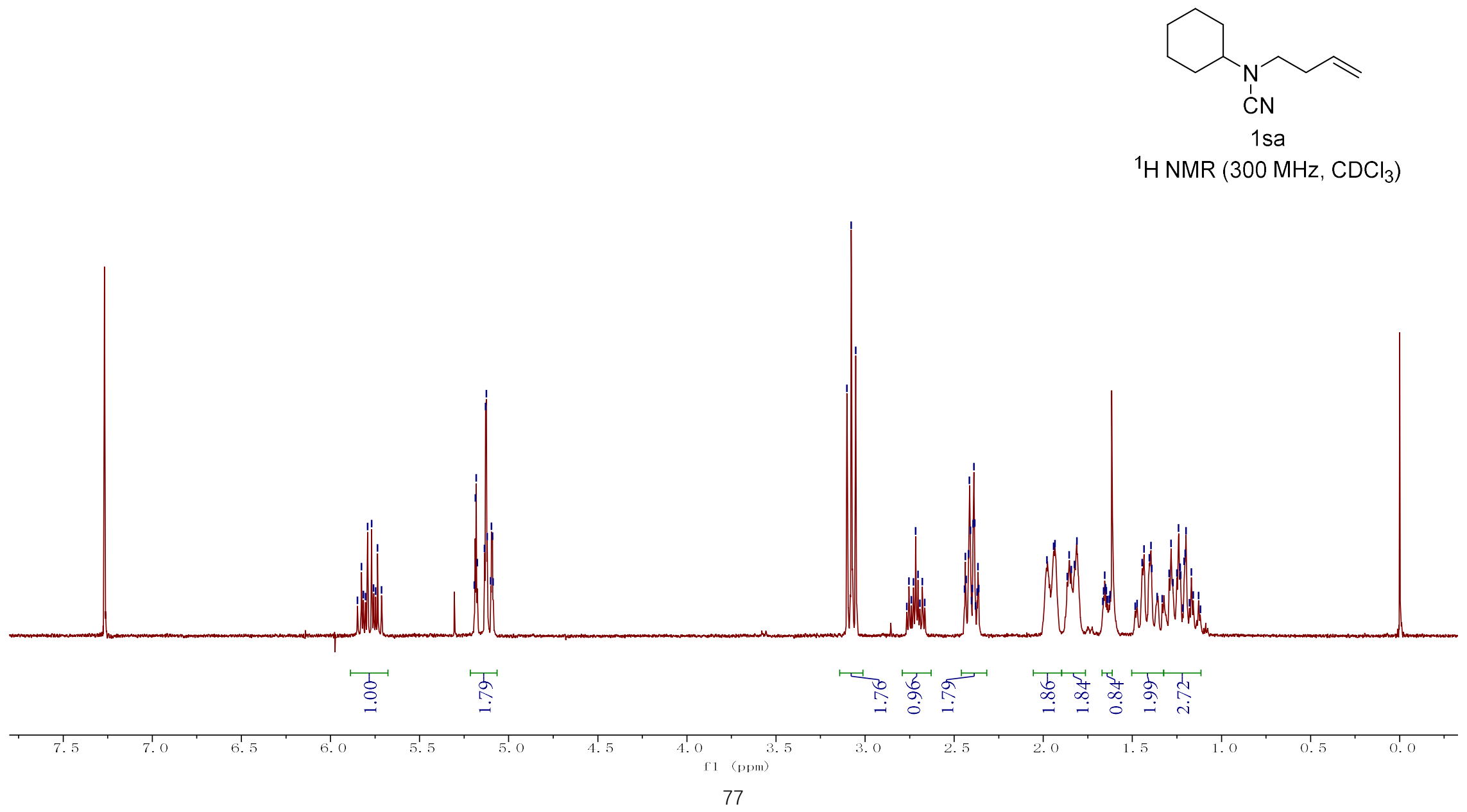

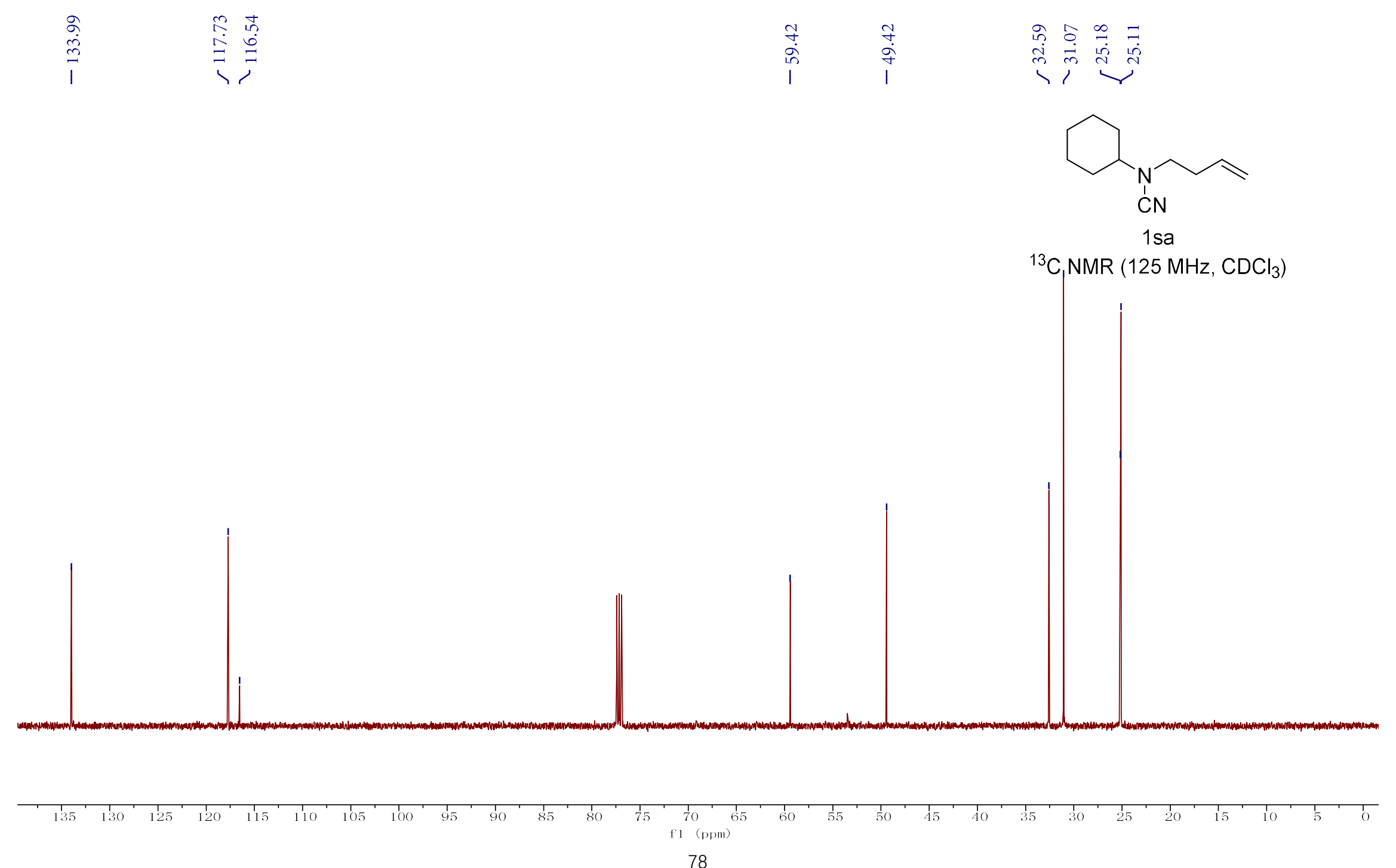


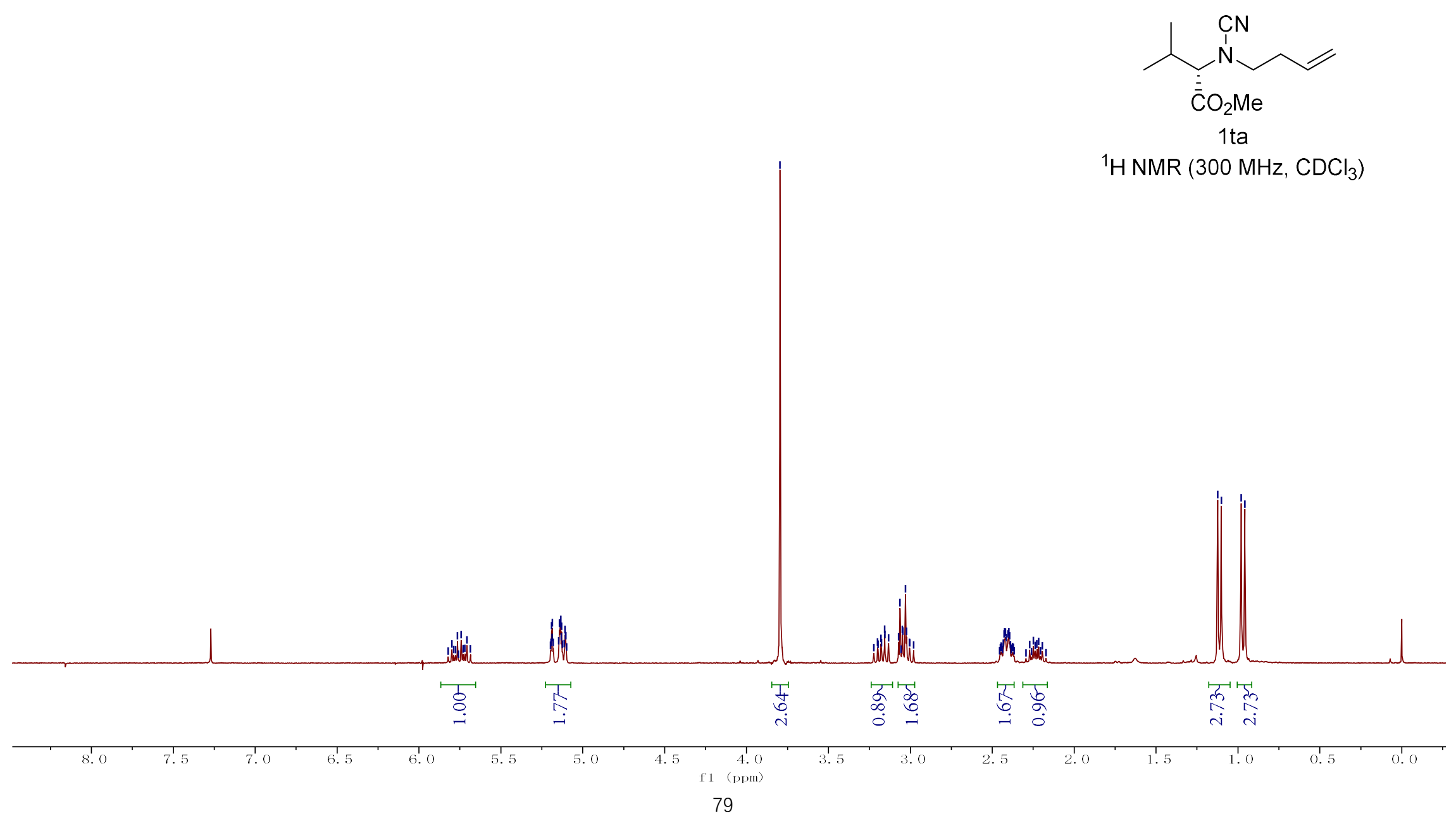




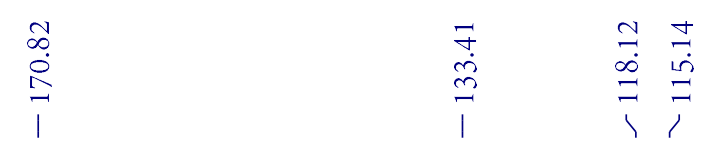

î

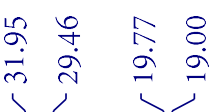

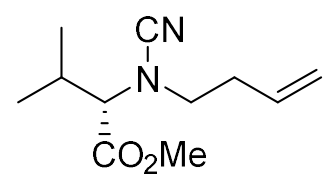

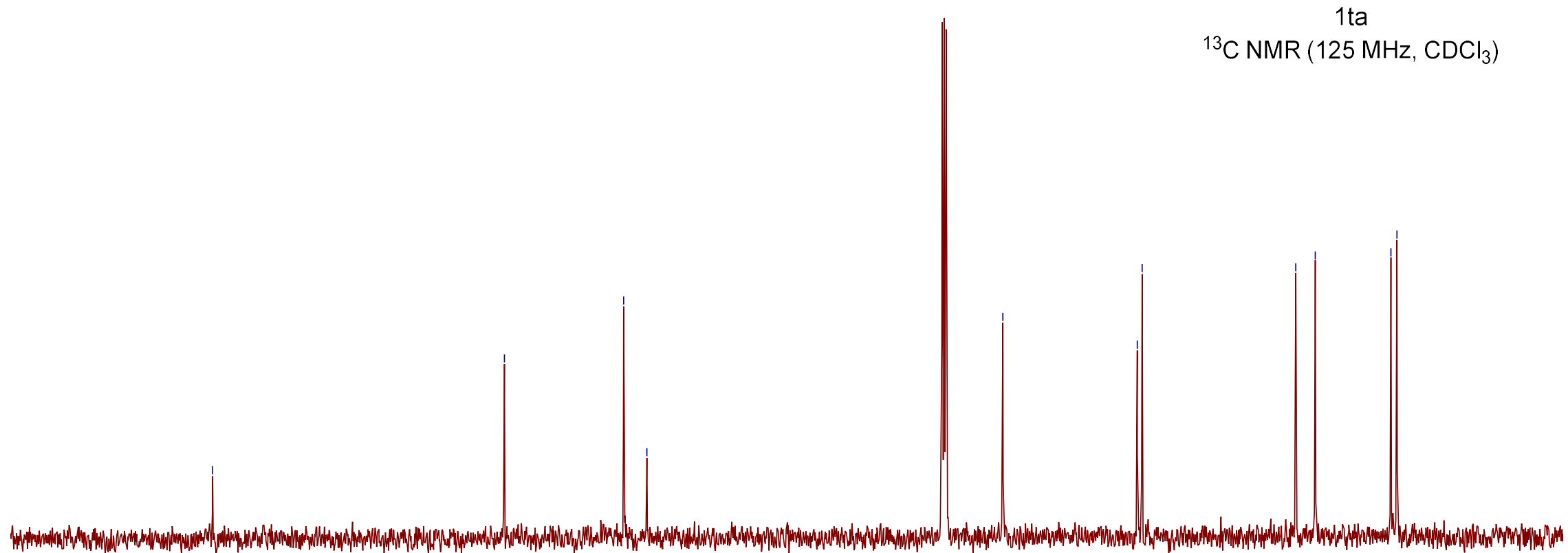

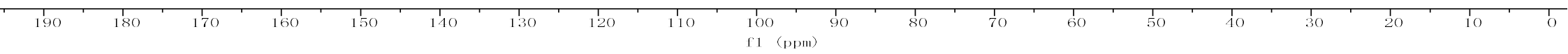




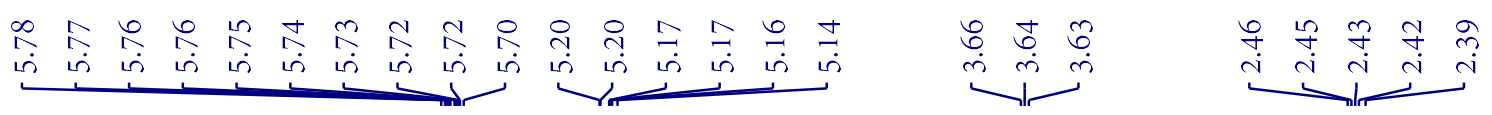

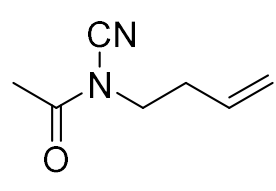

${ }^{1} \mathrm{H}$ NMR $\left(500 \mathrm{MHz}, \mathrm{CDCl}_{3}\right)$

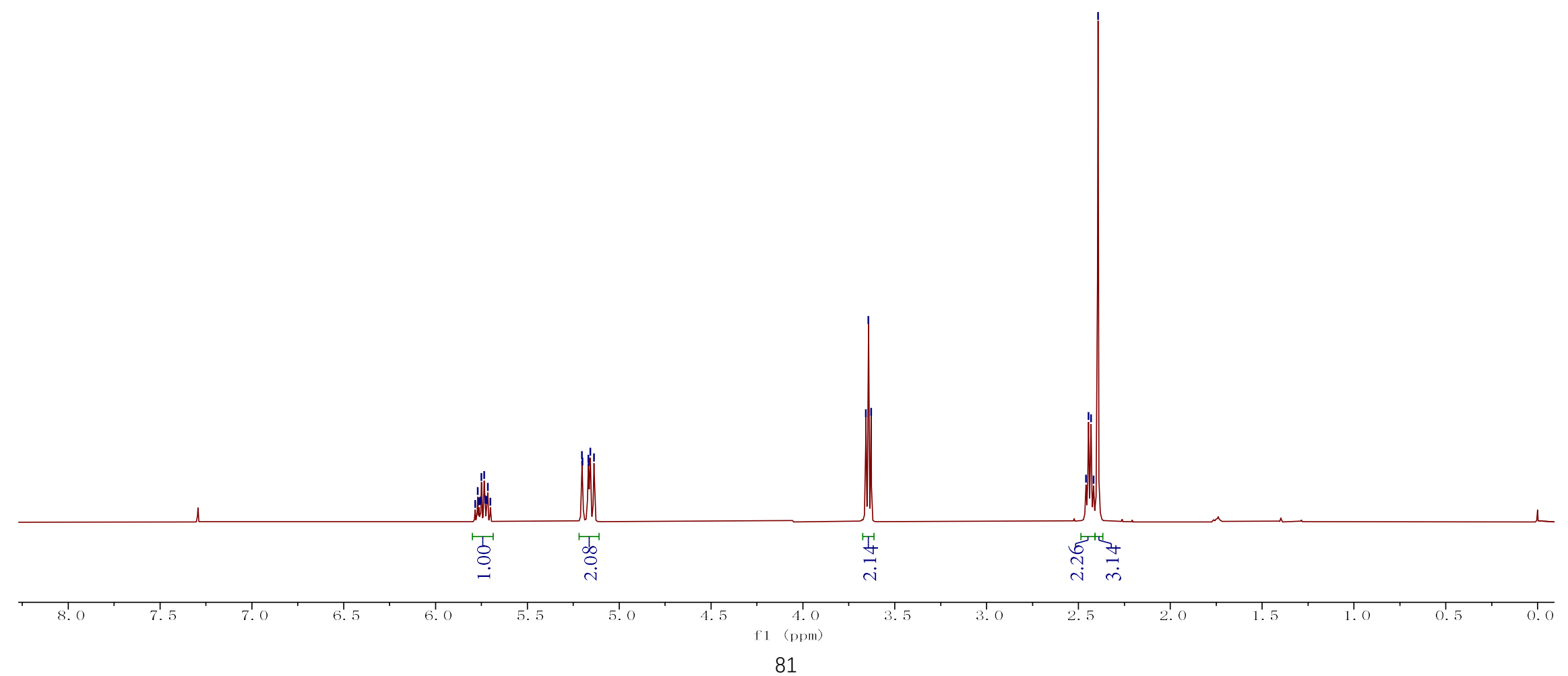




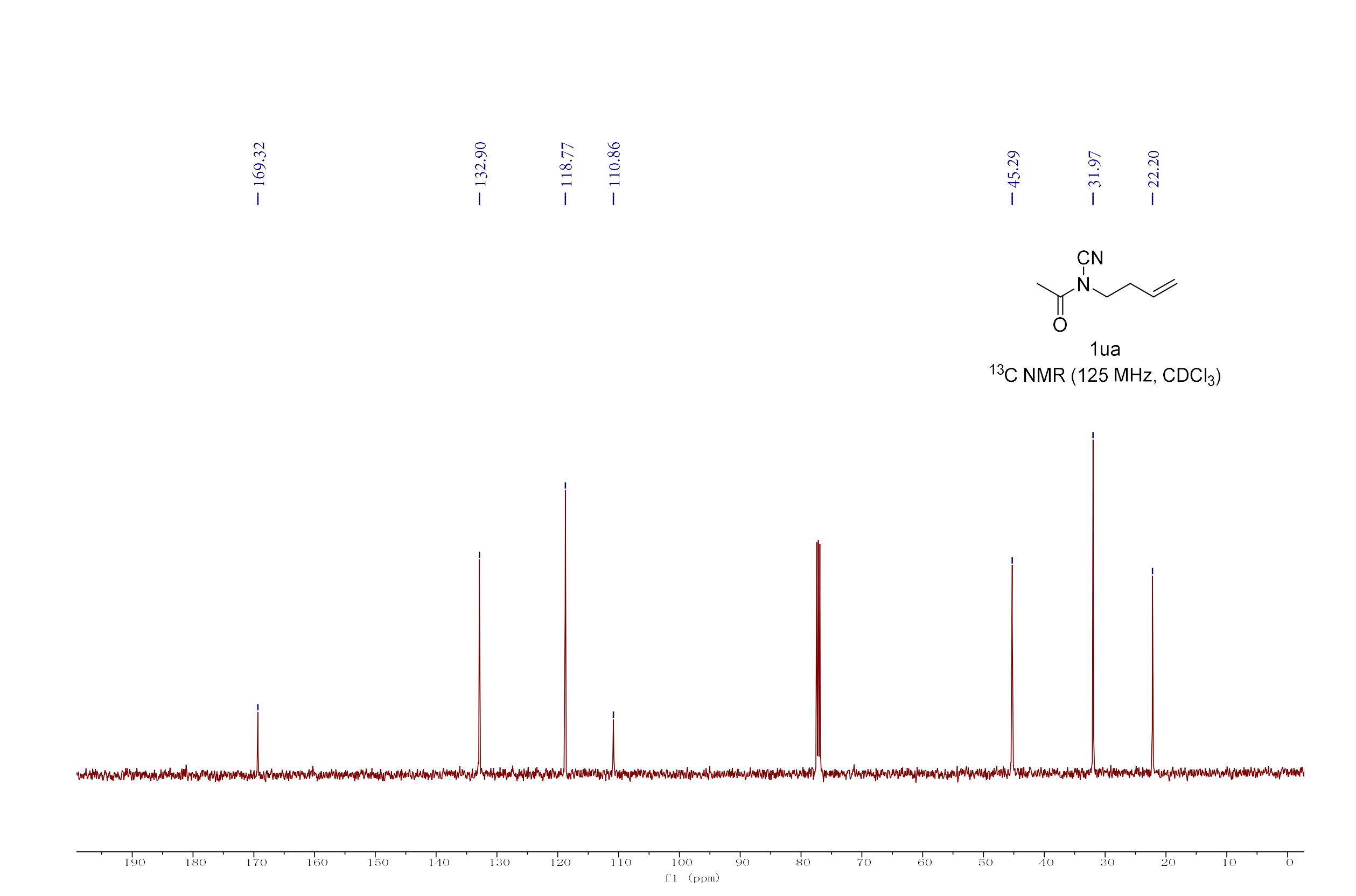




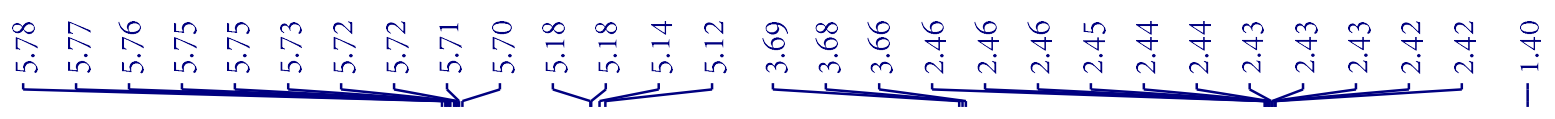

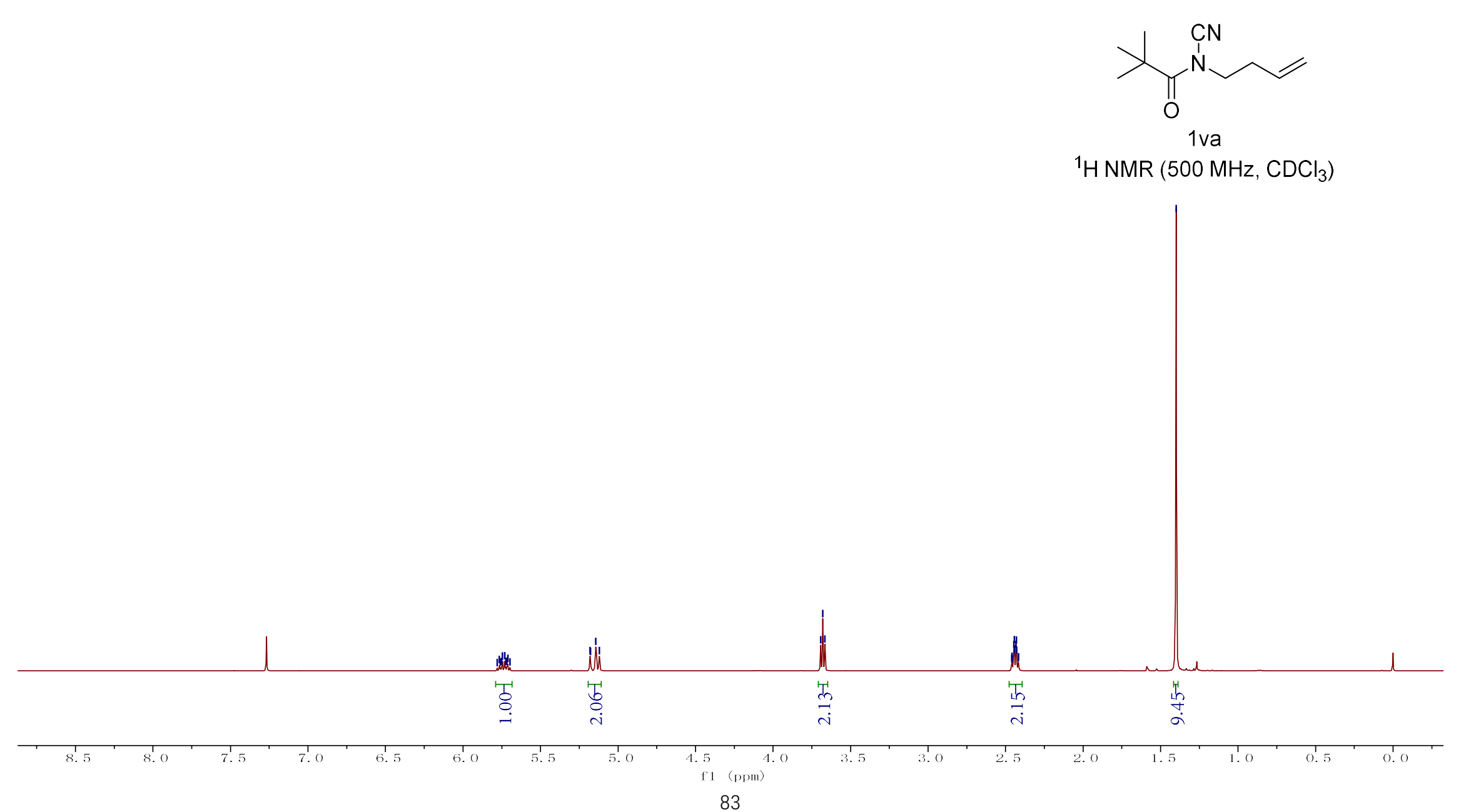




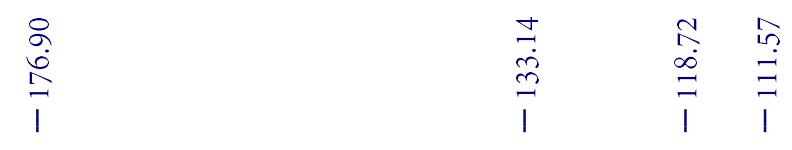

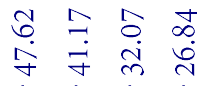

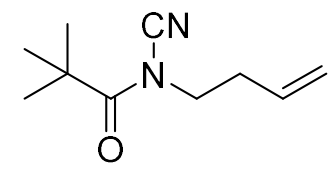

$1 \mathrm{va}$

${ }^{13} \mathrm{C} \mathrm{NMR}\left(125 \mathrm{MHz}, \mathrm{CDCl}_{3}\right)$

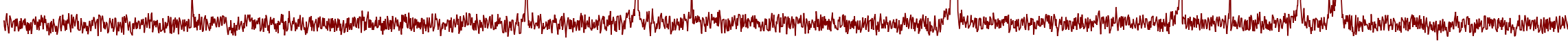

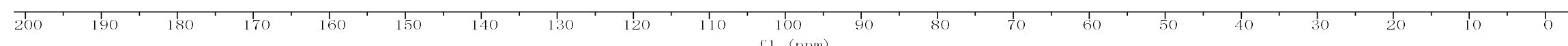




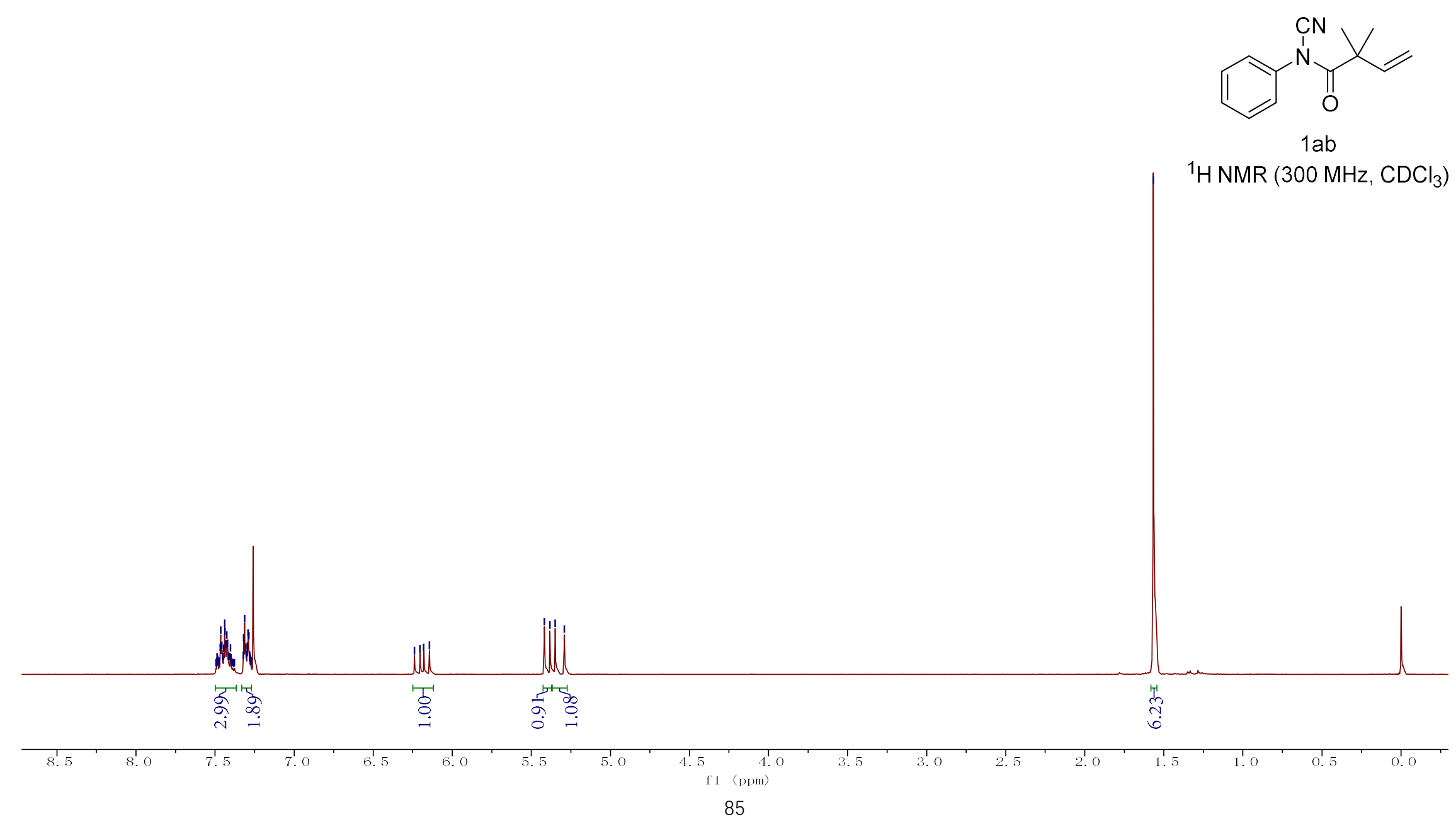




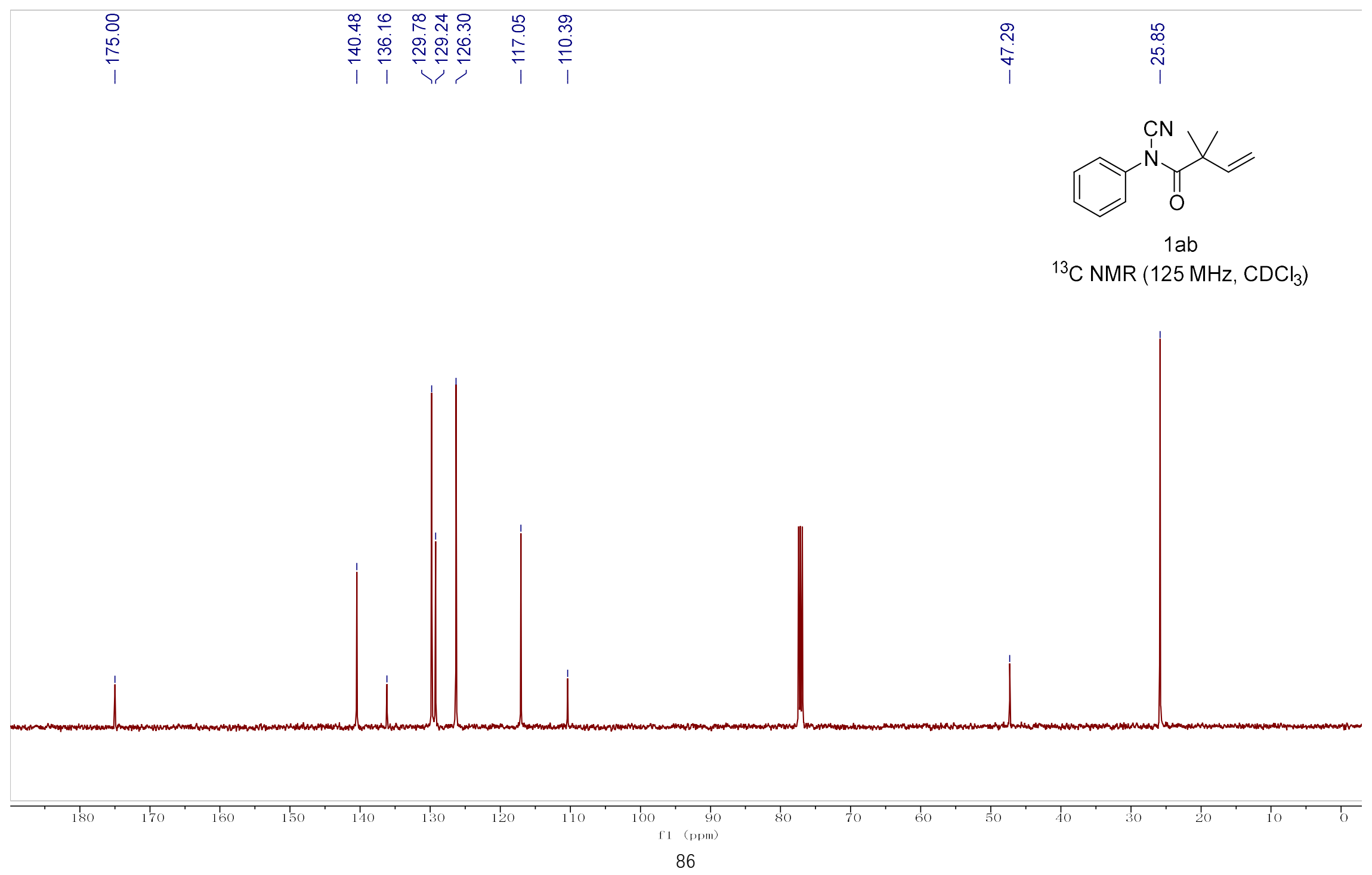




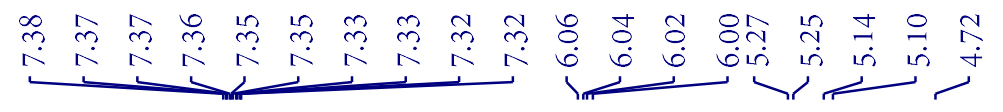

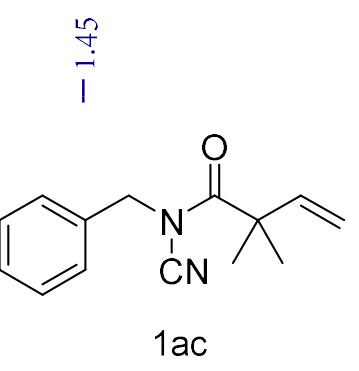

${ }^{1} \mathrm{H} \mathrm{NMR}\left(500 \mathrm{MHz}, \mathrm{CDCl}_{3}\right)$

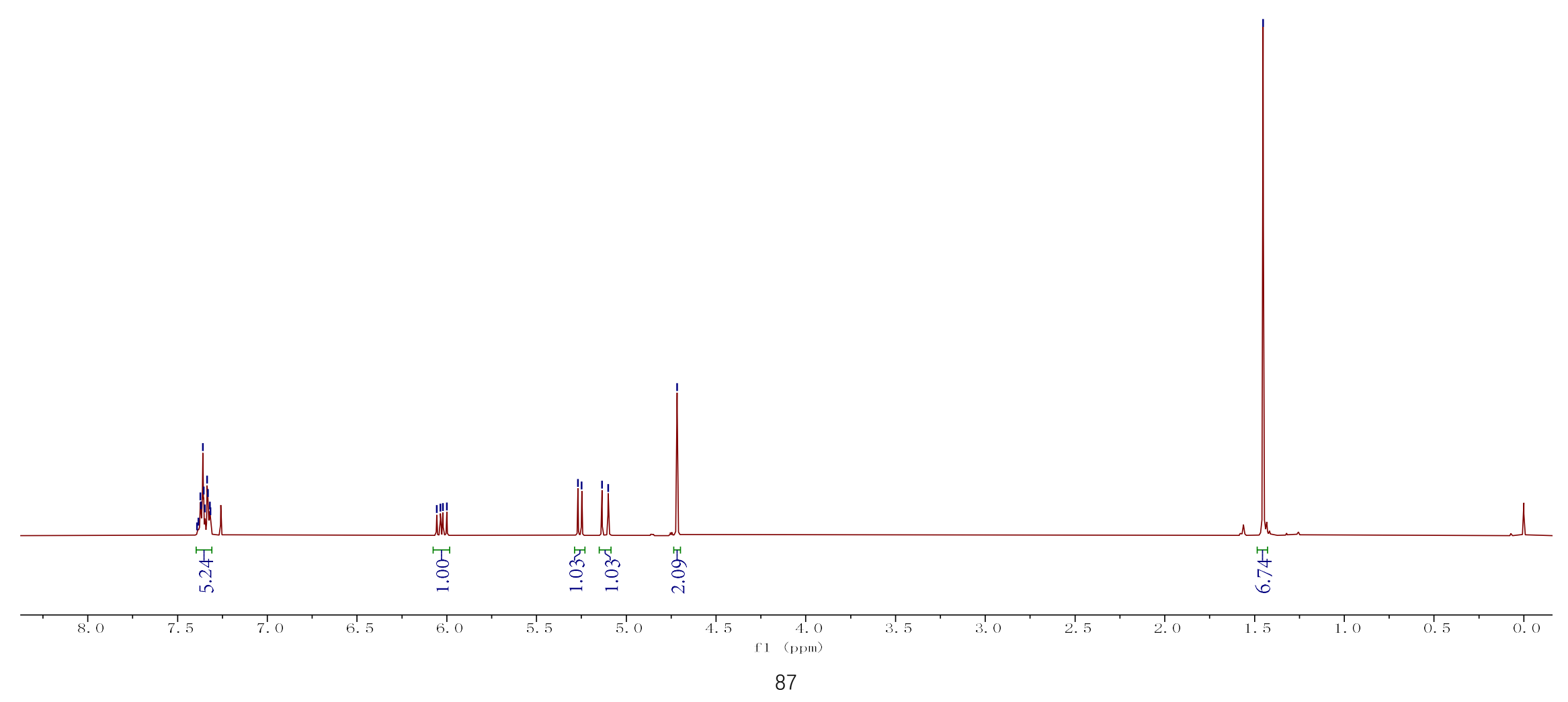




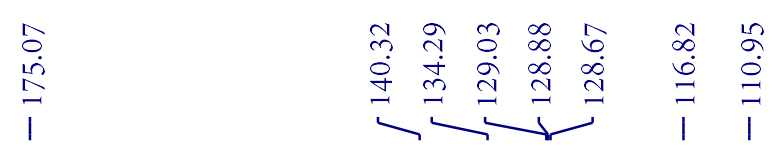

$\sigma$
iे
in
1

离

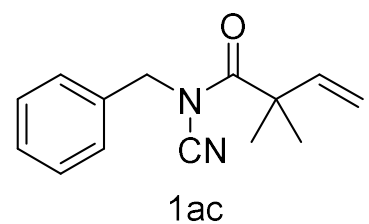

${ }^{13} \mathrm{C} \mathrm{NMR}\left(12,5 \mathrm{MHz}, \mathrm{CDCl}_{3}\right)$
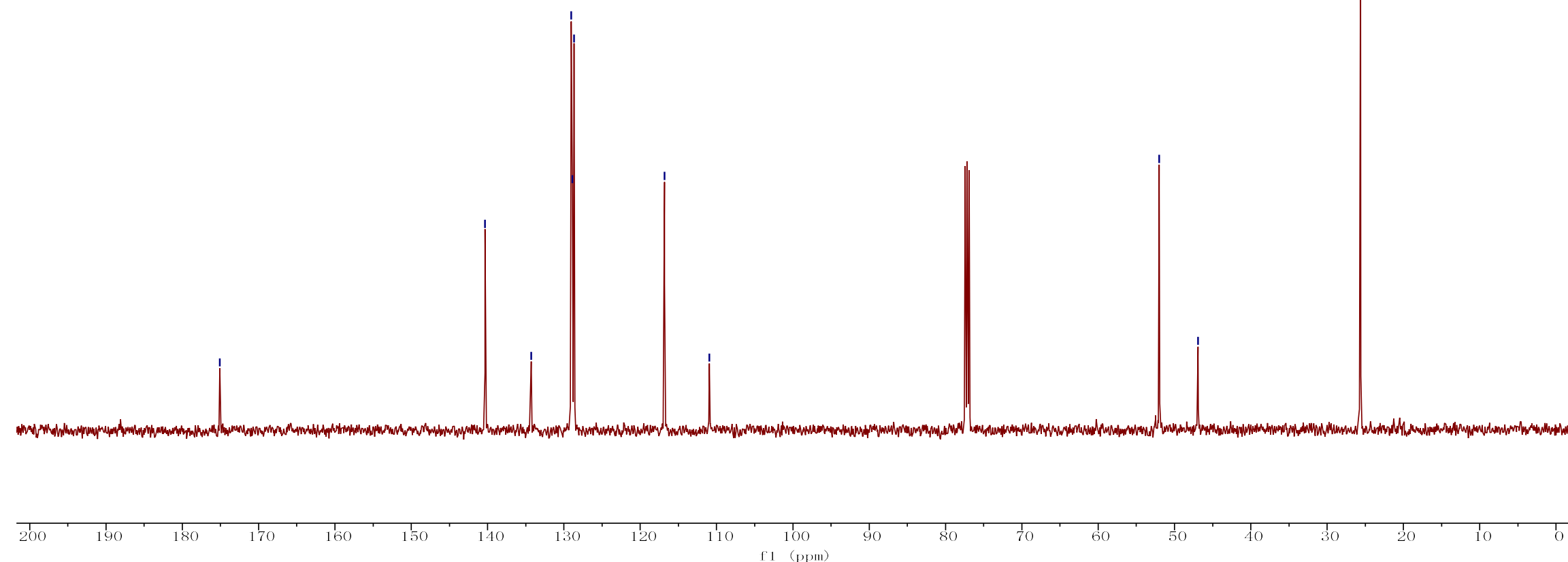


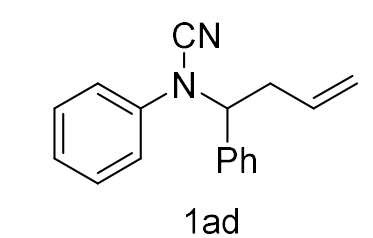

${ }^{1} \mathrm{H} \mathrm{NMR}\left(500 \mathrm{MHz}, \mathrm{CDCl}_{3}\right)$

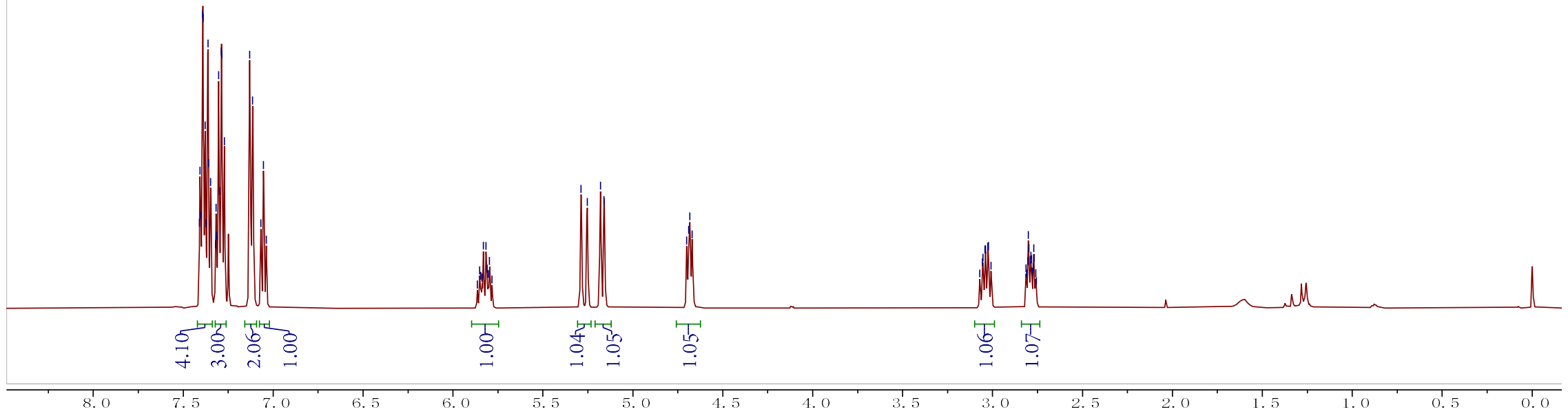



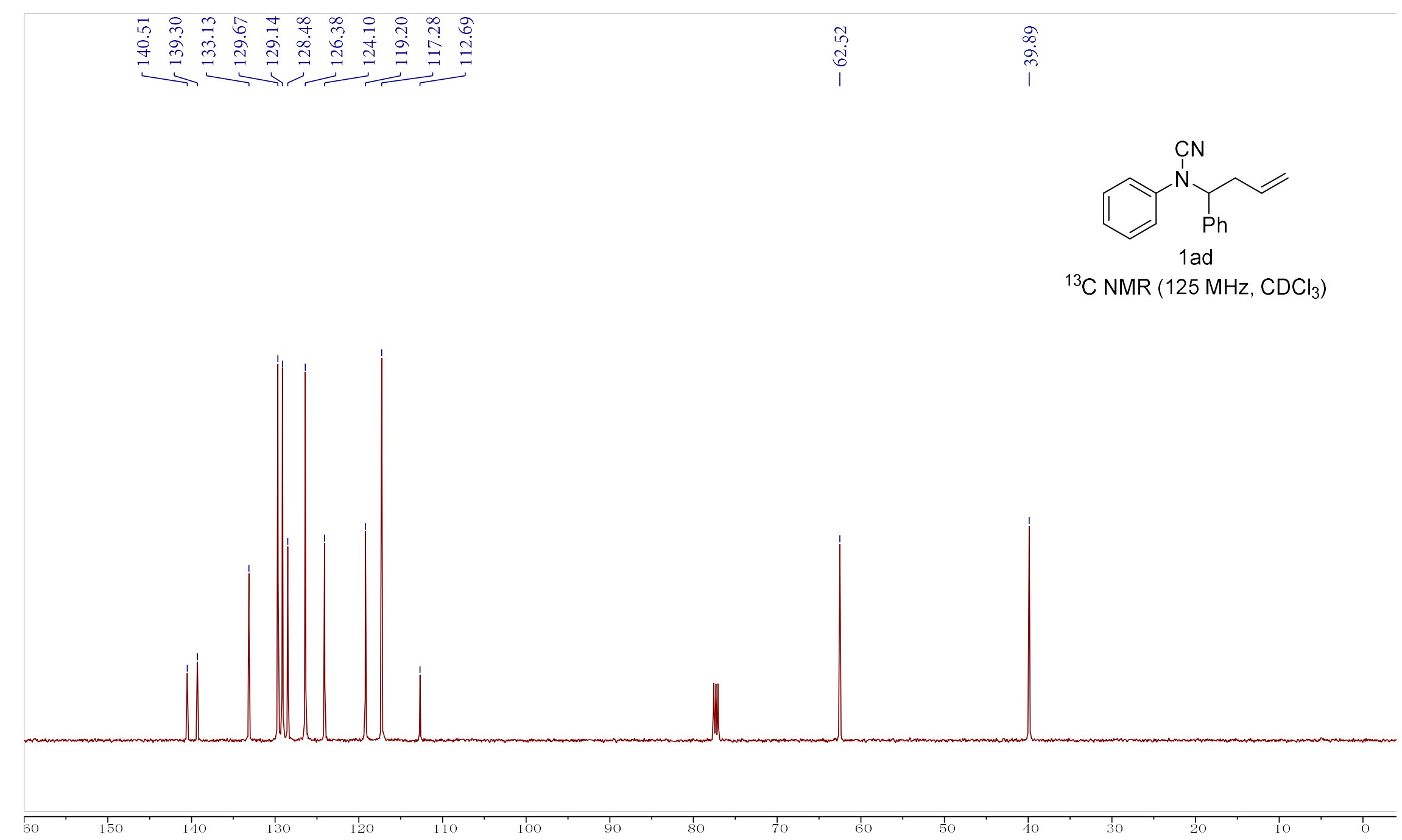


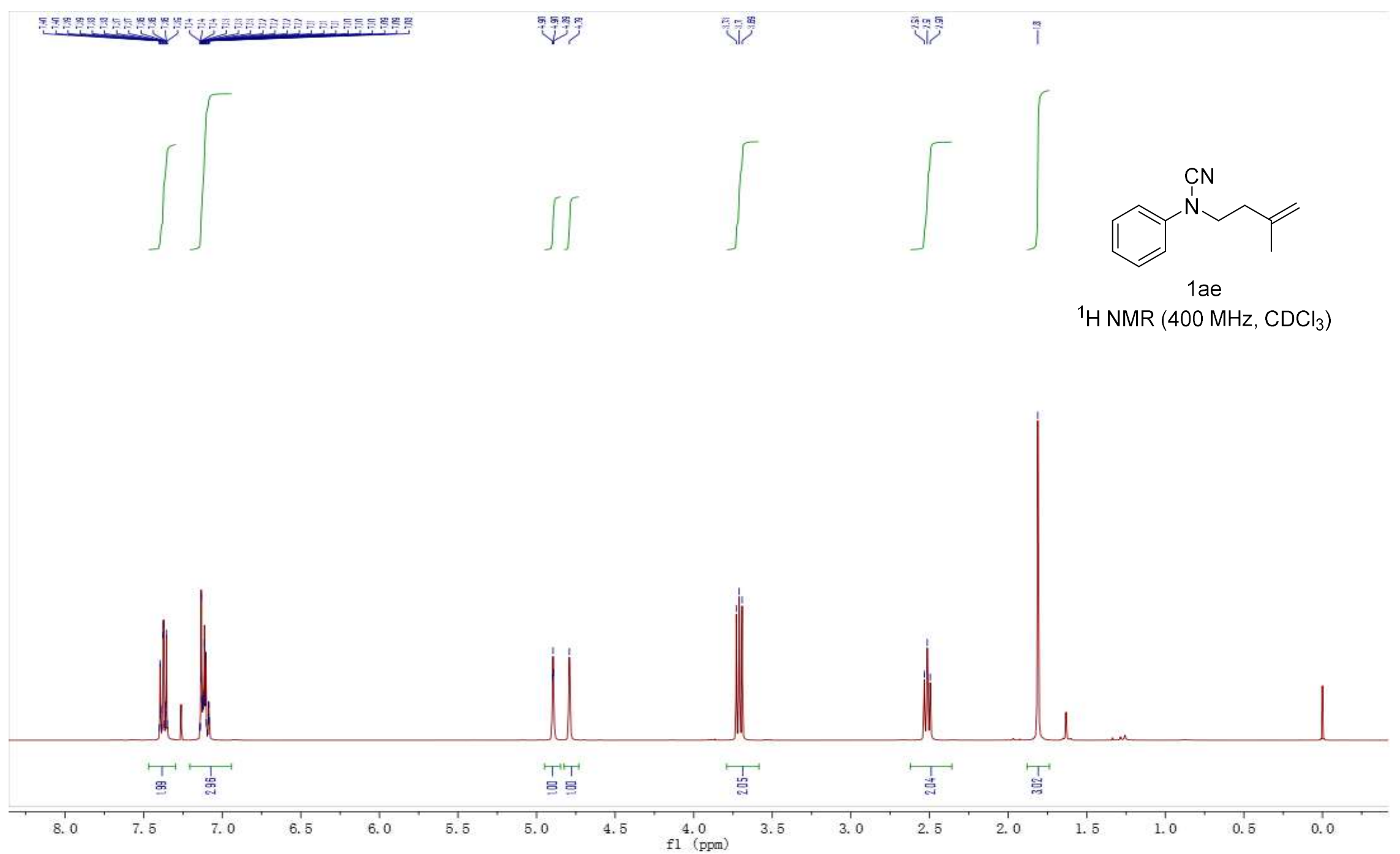

91 


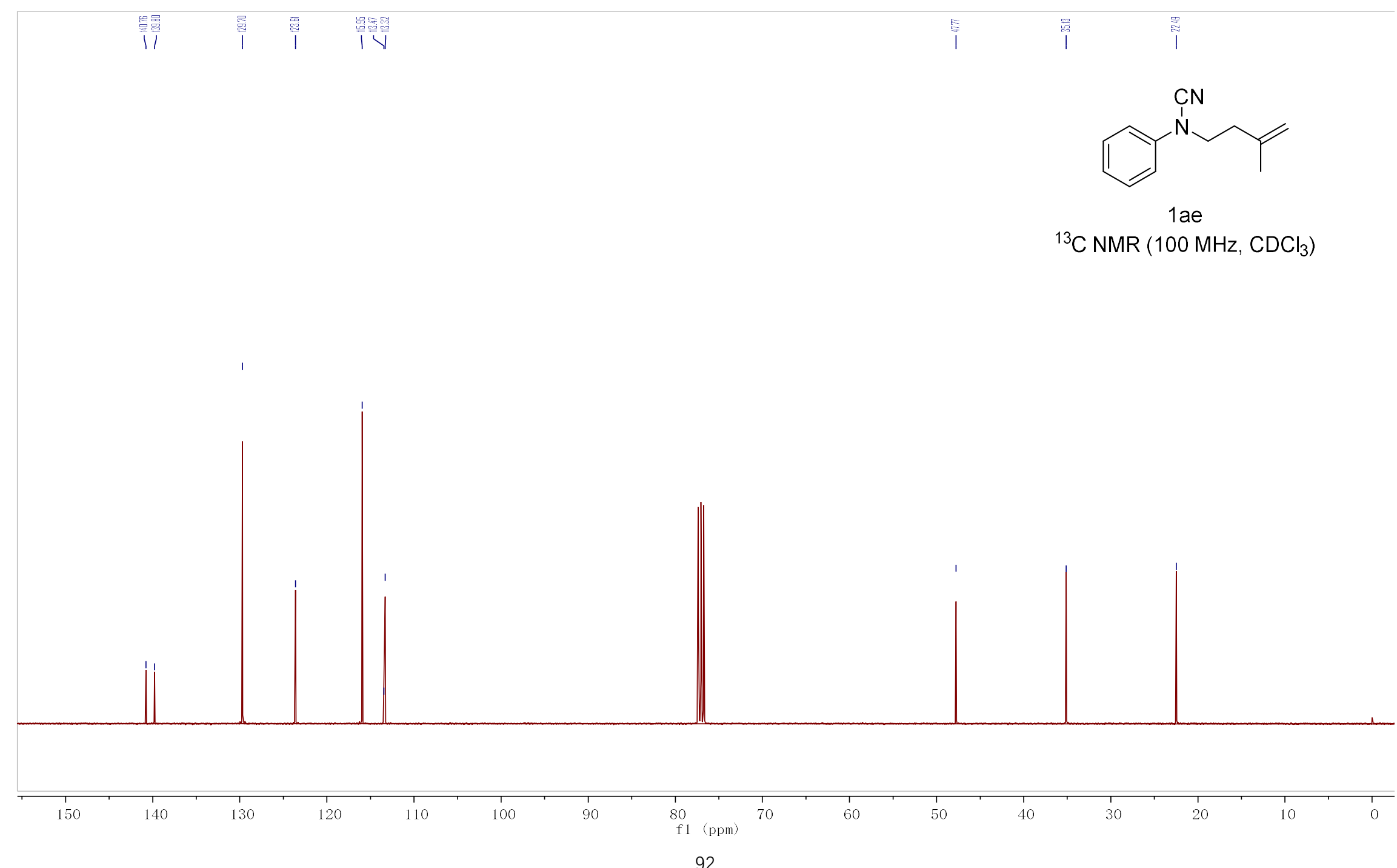




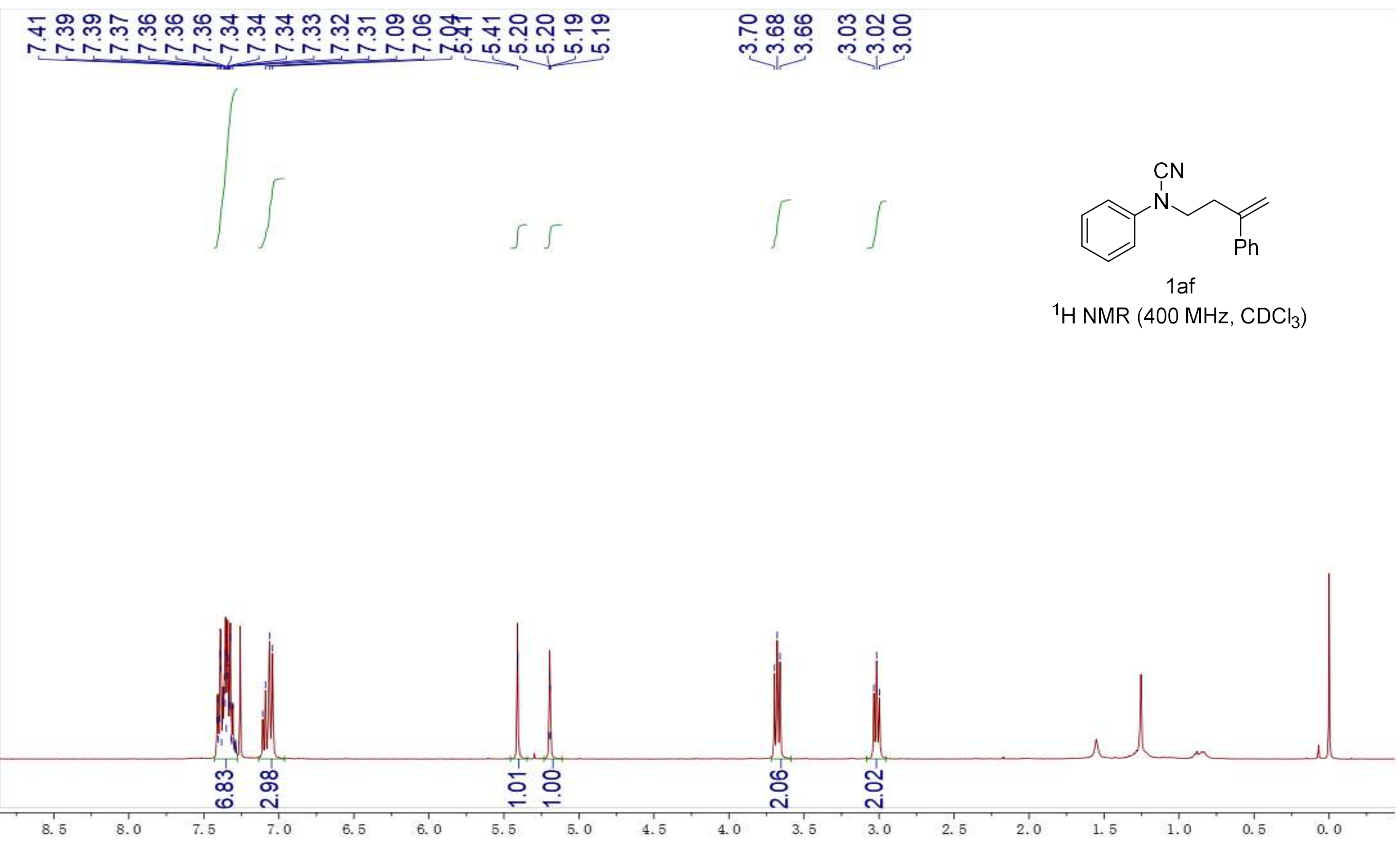

93 


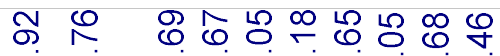

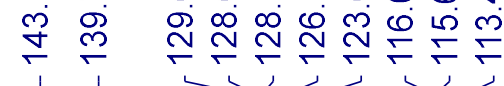

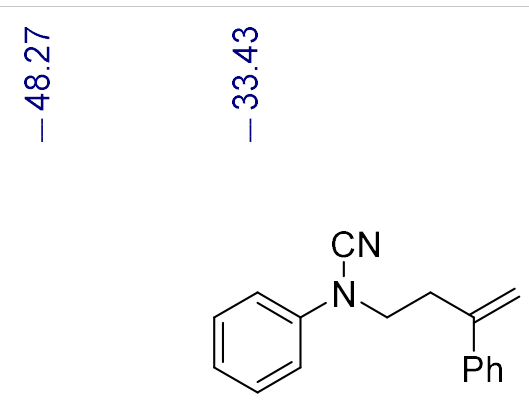

1 af

${ }^{13} \mathrm{C} \mathrm{NMR}\left(125 \mathrm{MHz}, \mathrm{CDCl}_{3}\right)$

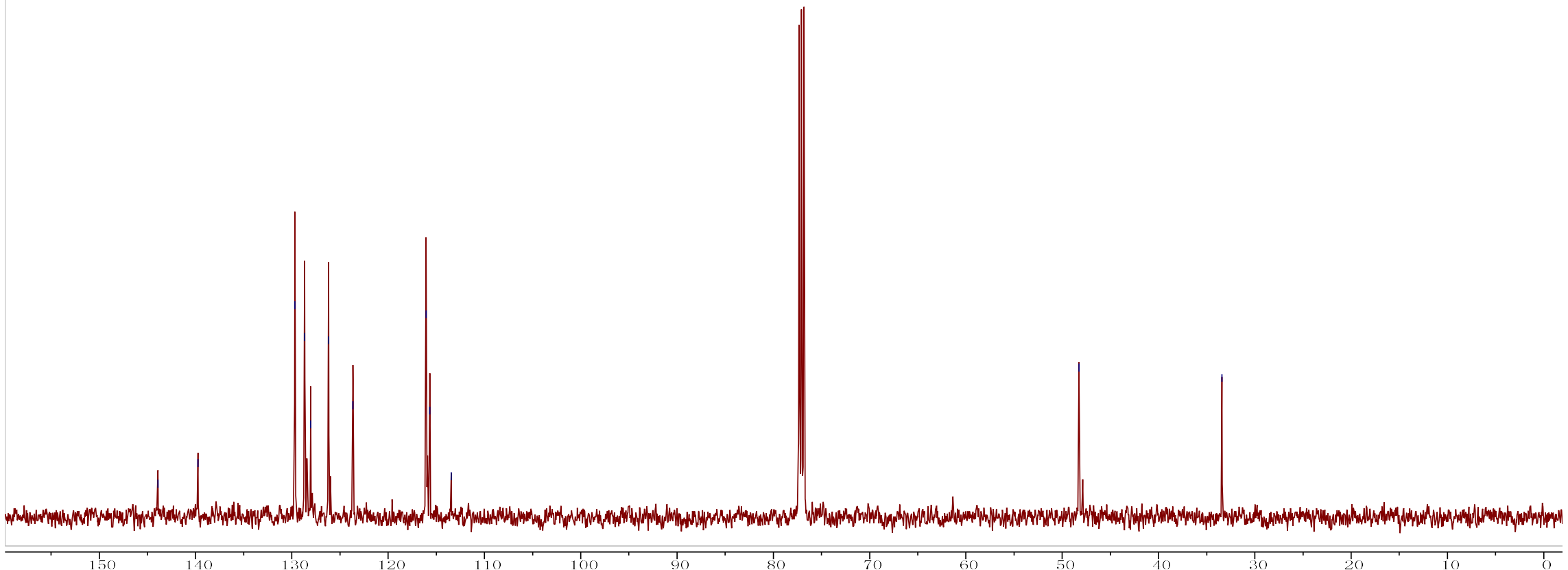




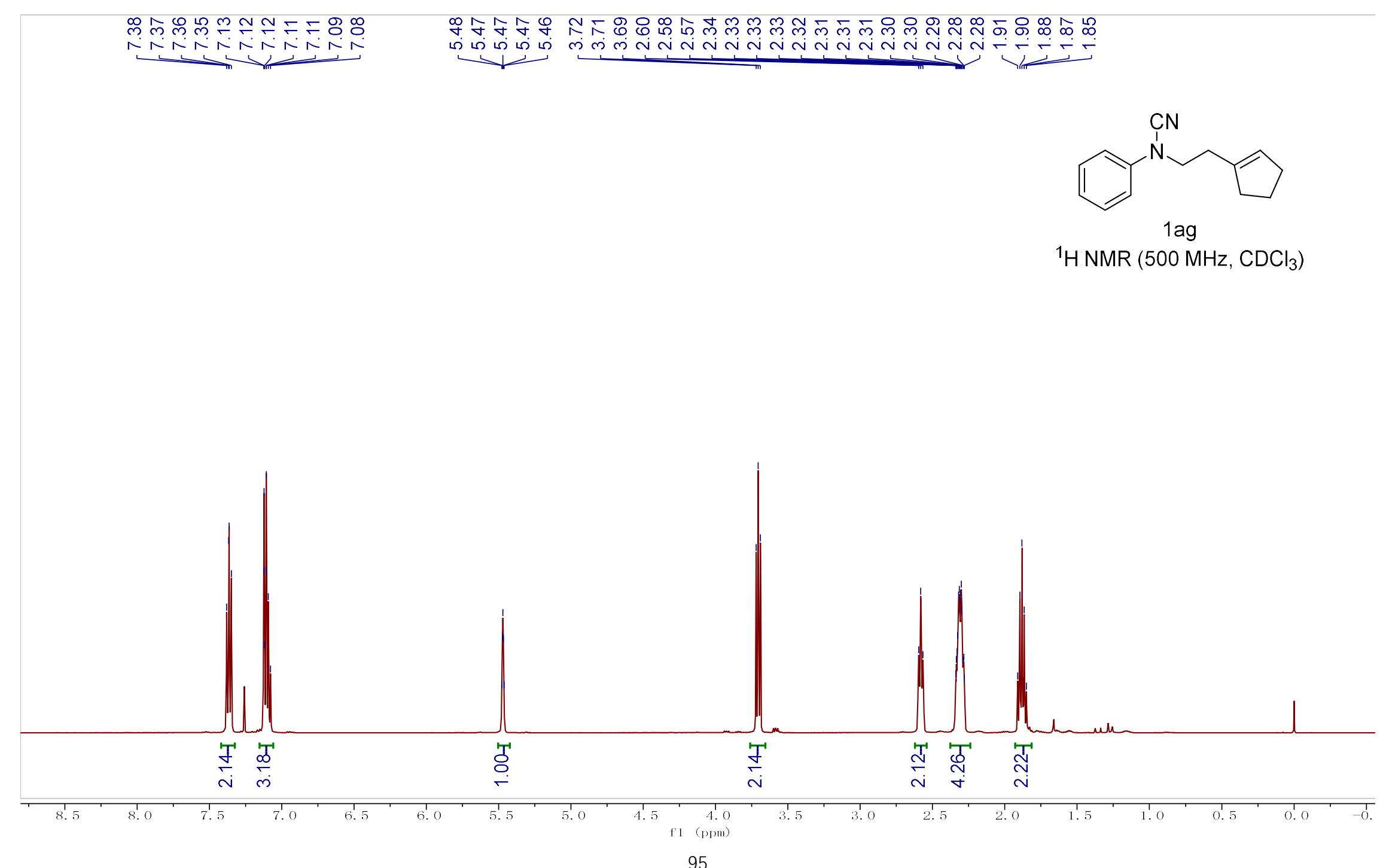



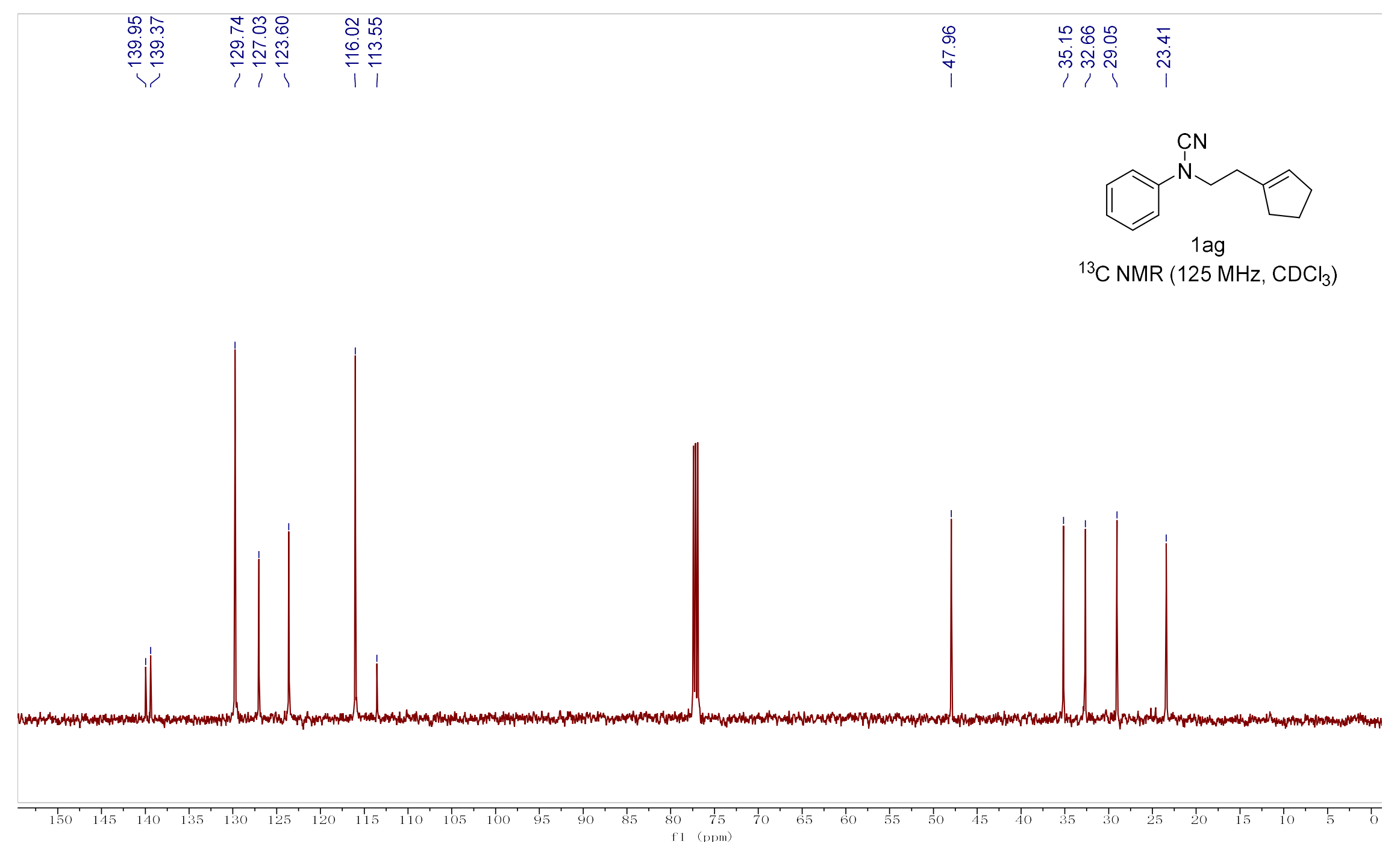


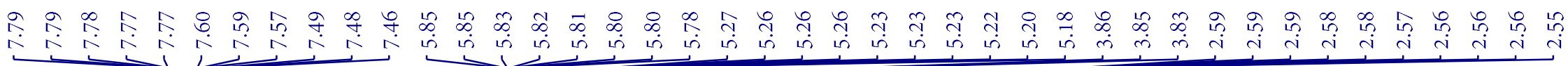

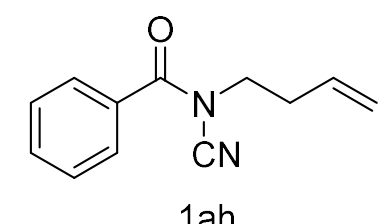

${ }^{1} \mathrm{H} \mathrm{NMR} \mathrm{(500} \mathrm{MHz,} \mathrm{CDCl}_{3}$ )

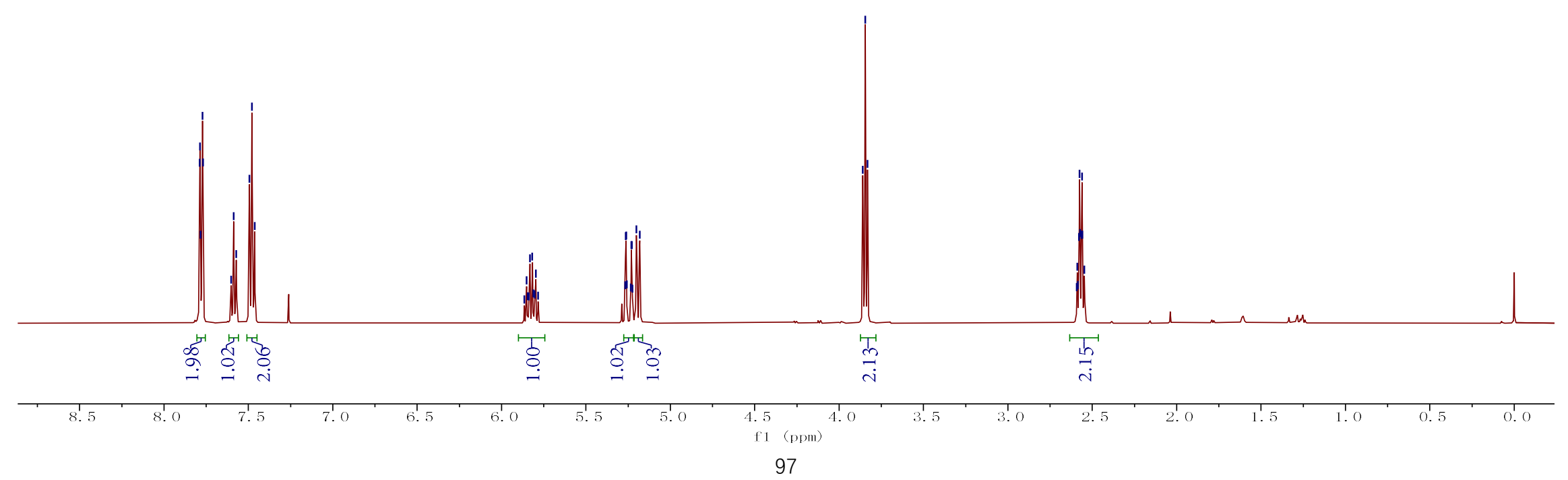




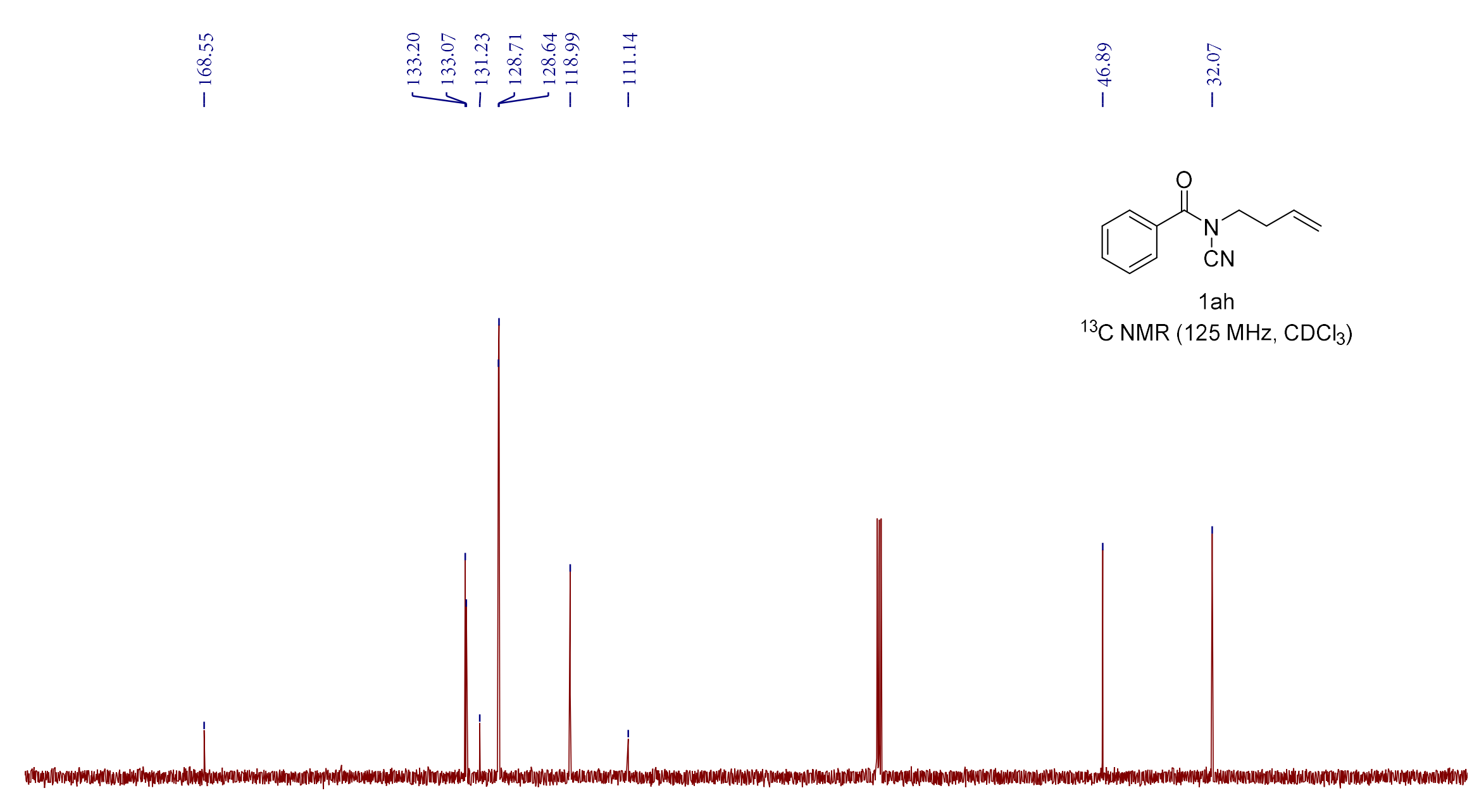




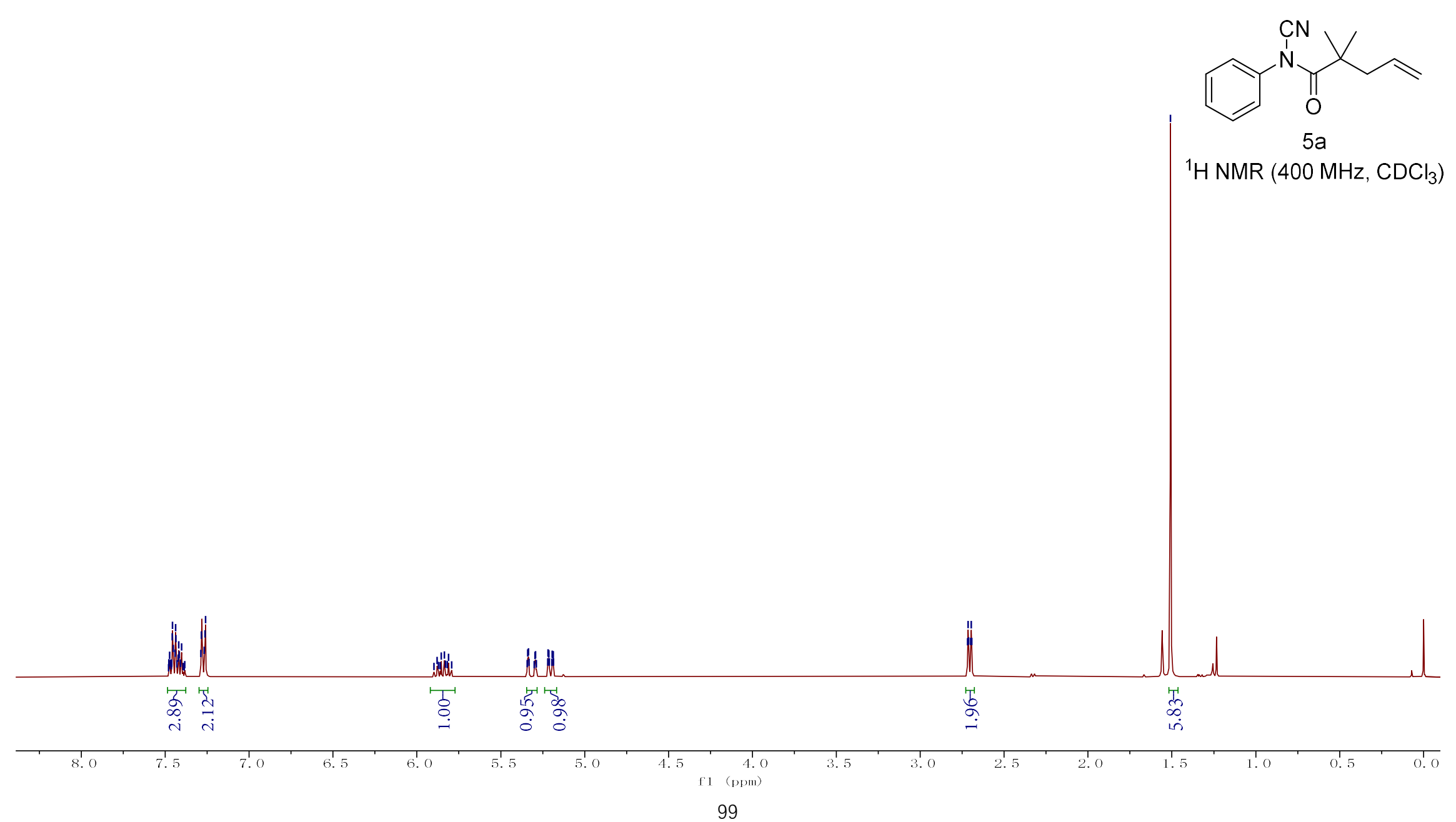




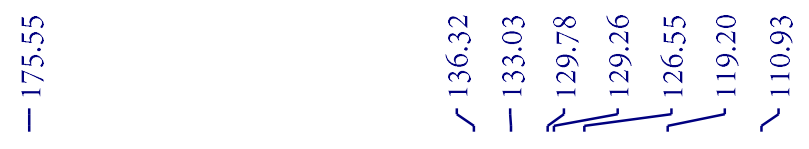

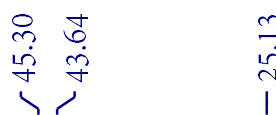

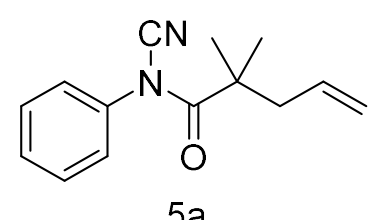

${ }^{13} \mathrm{CNMR}\left(125 \mathrm{MHz}, \mathrm{CDCl}_{3}\right)$
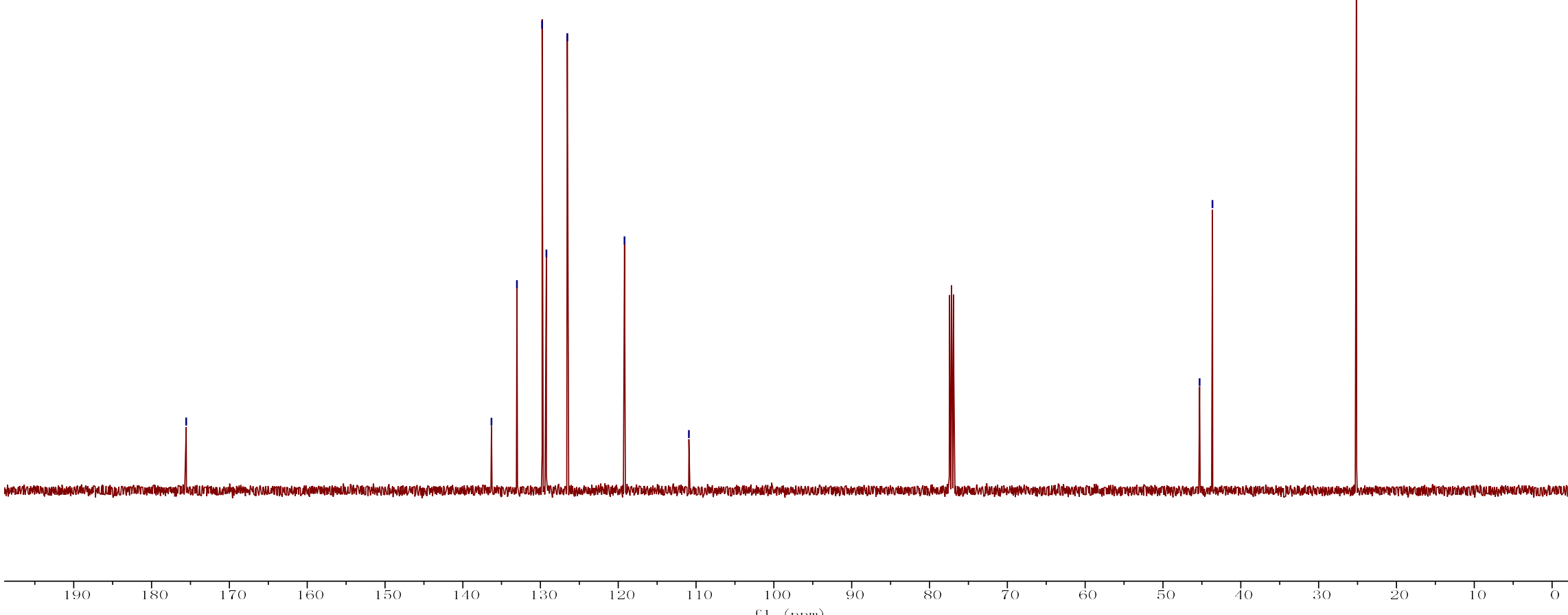


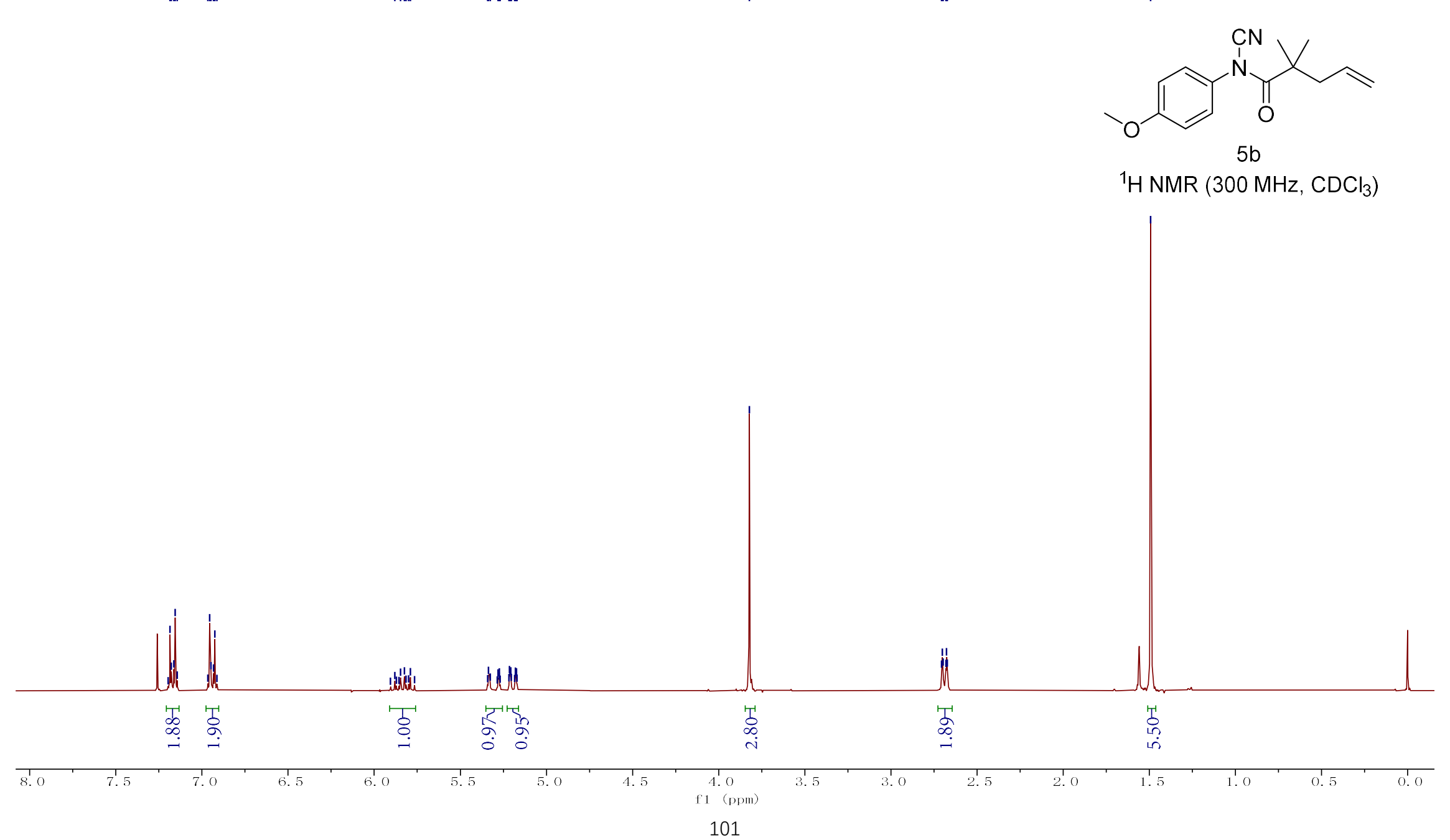




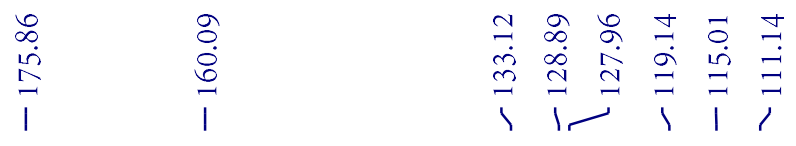

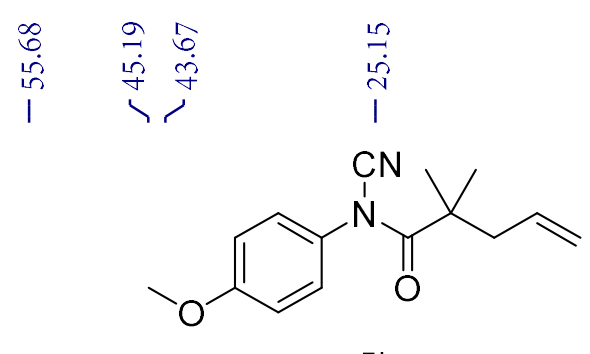

$5 b$

${ }^{13} \mathrm{C}$ NMR $\left(125 \mathrm{MHz}, \mathrm{CDCl}_{3}\right)$

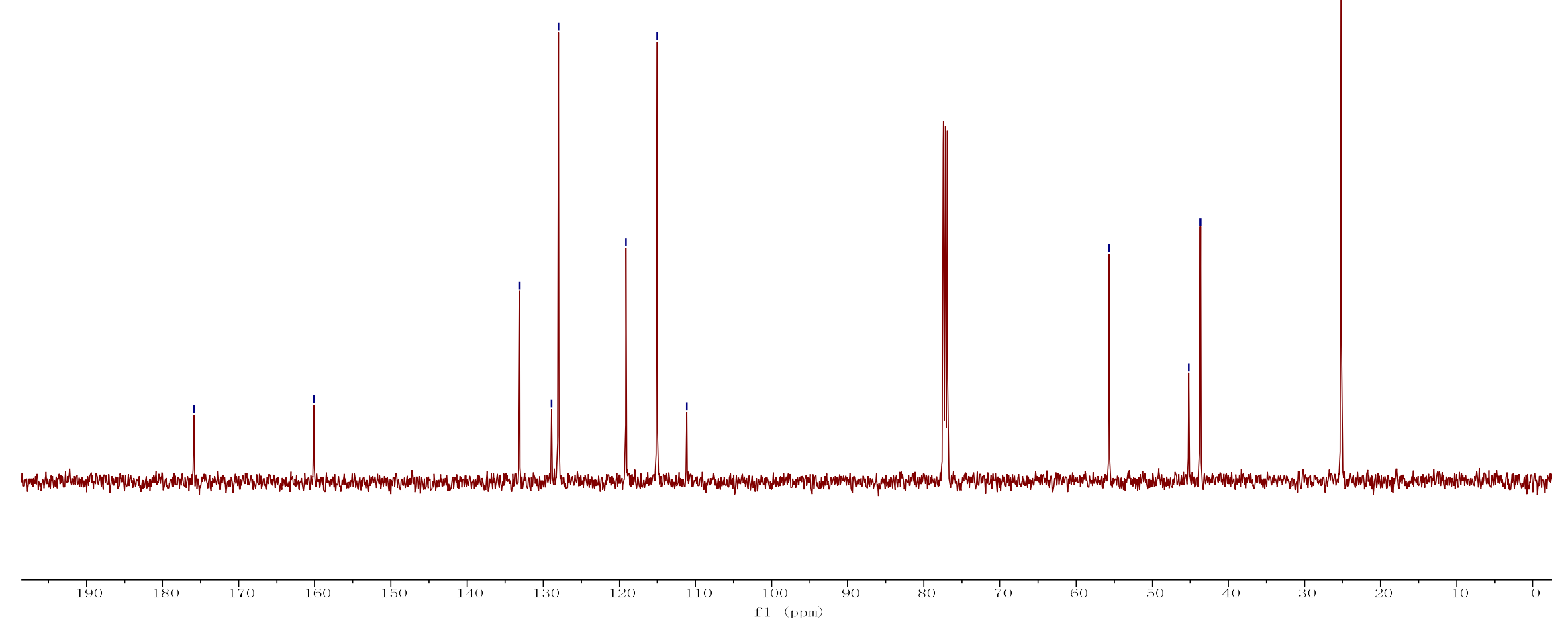




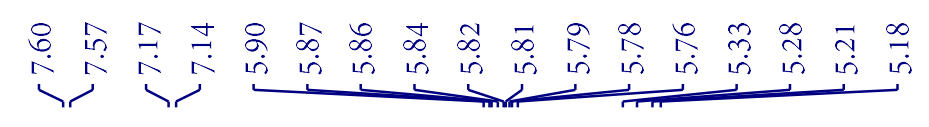

$\underbrace{\infty}_{\substack{R \\ i}}$

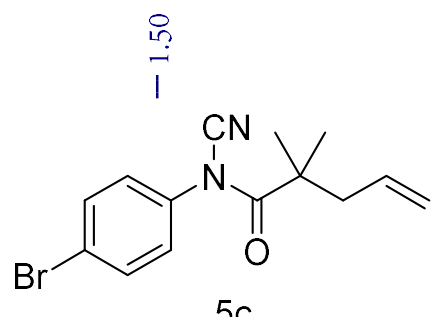

${ }^{1} \mathrm{H}$ NMR $\left(300 \mathrm{MHz}, \mathrm{CDCl}_{3}\right.$ )

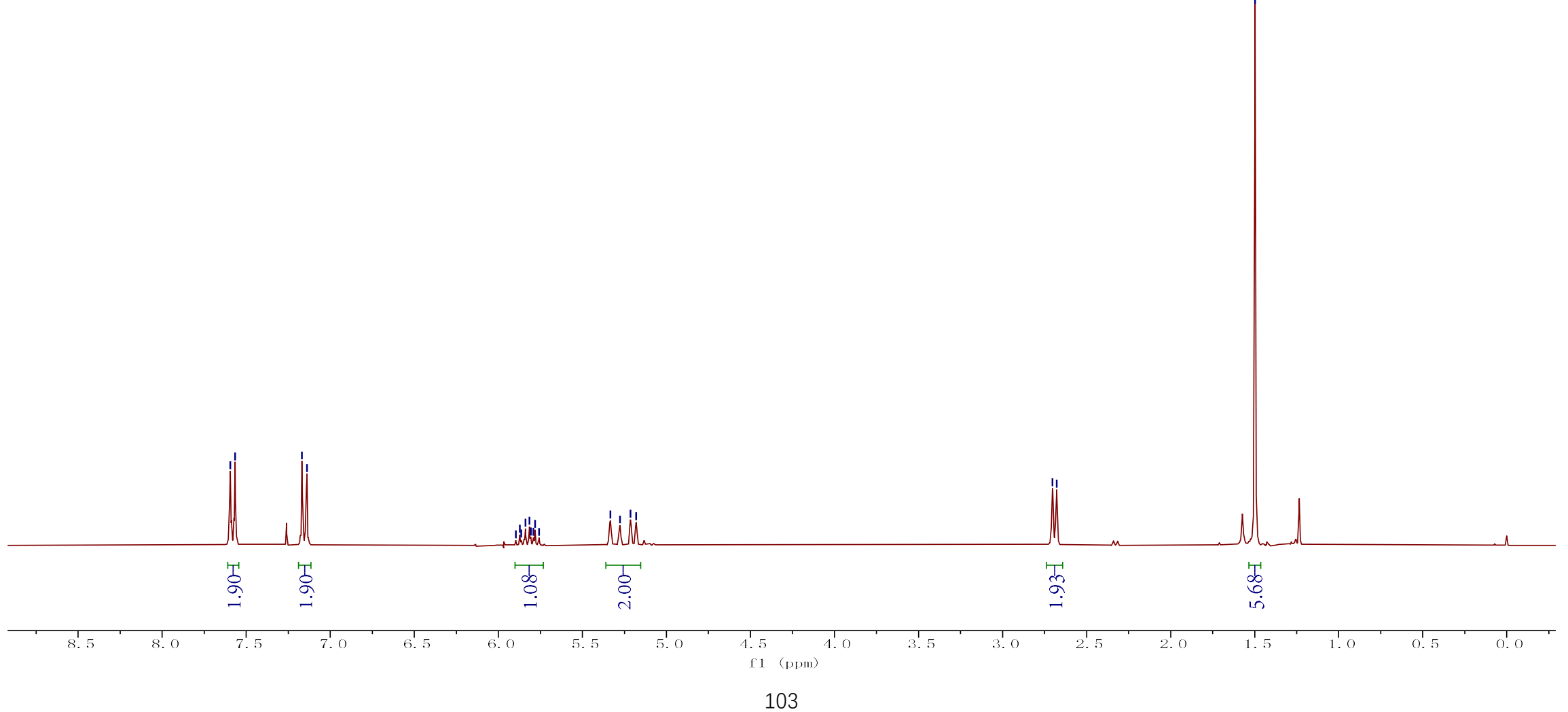




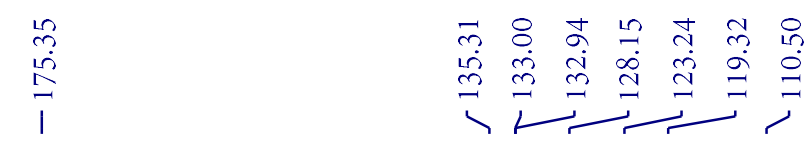

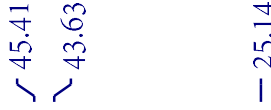

$\mathrm{CN}$

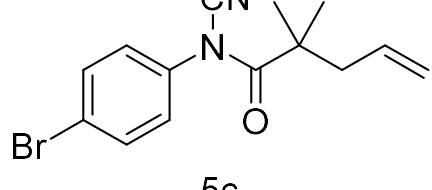

$5 \mathrm{c}$

${ }^{13} \mathrm{C} \mathrm{NMR}\left(125 \mathrm{MHz}, \mathrm{CDCl}_{3}\right)$
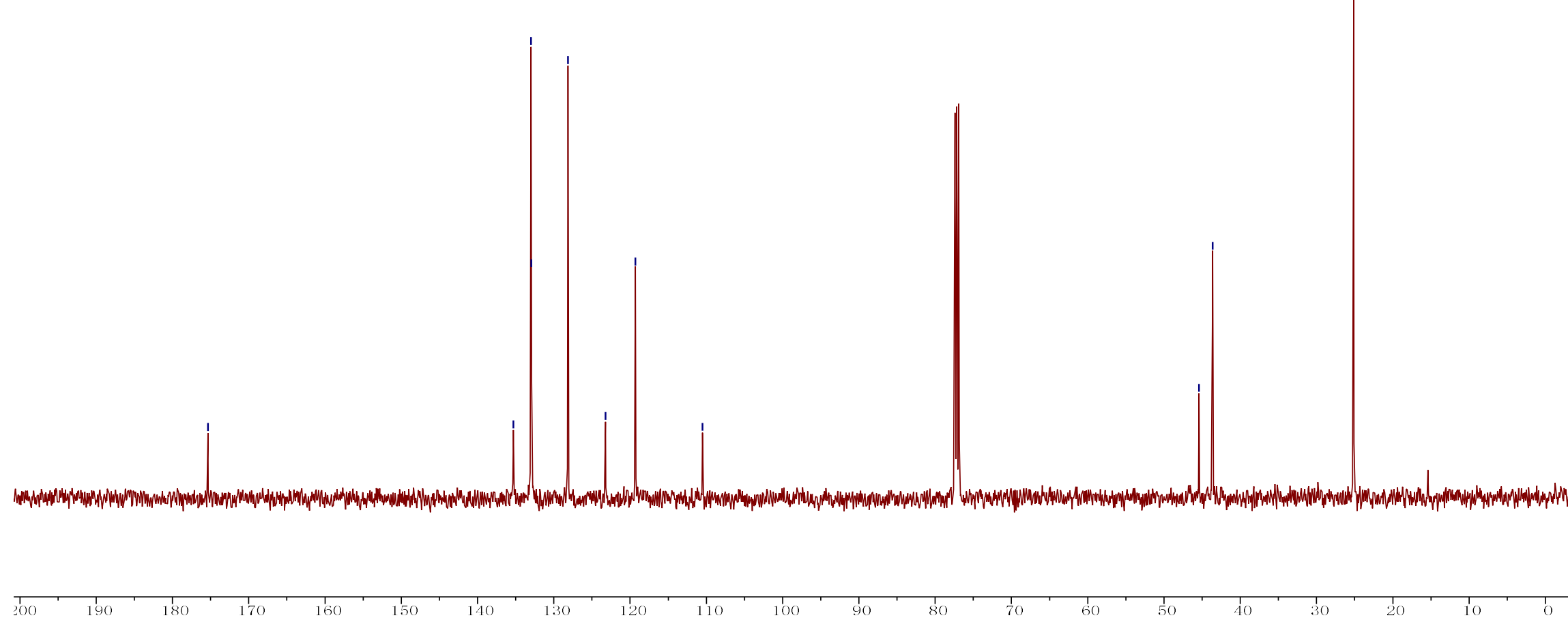


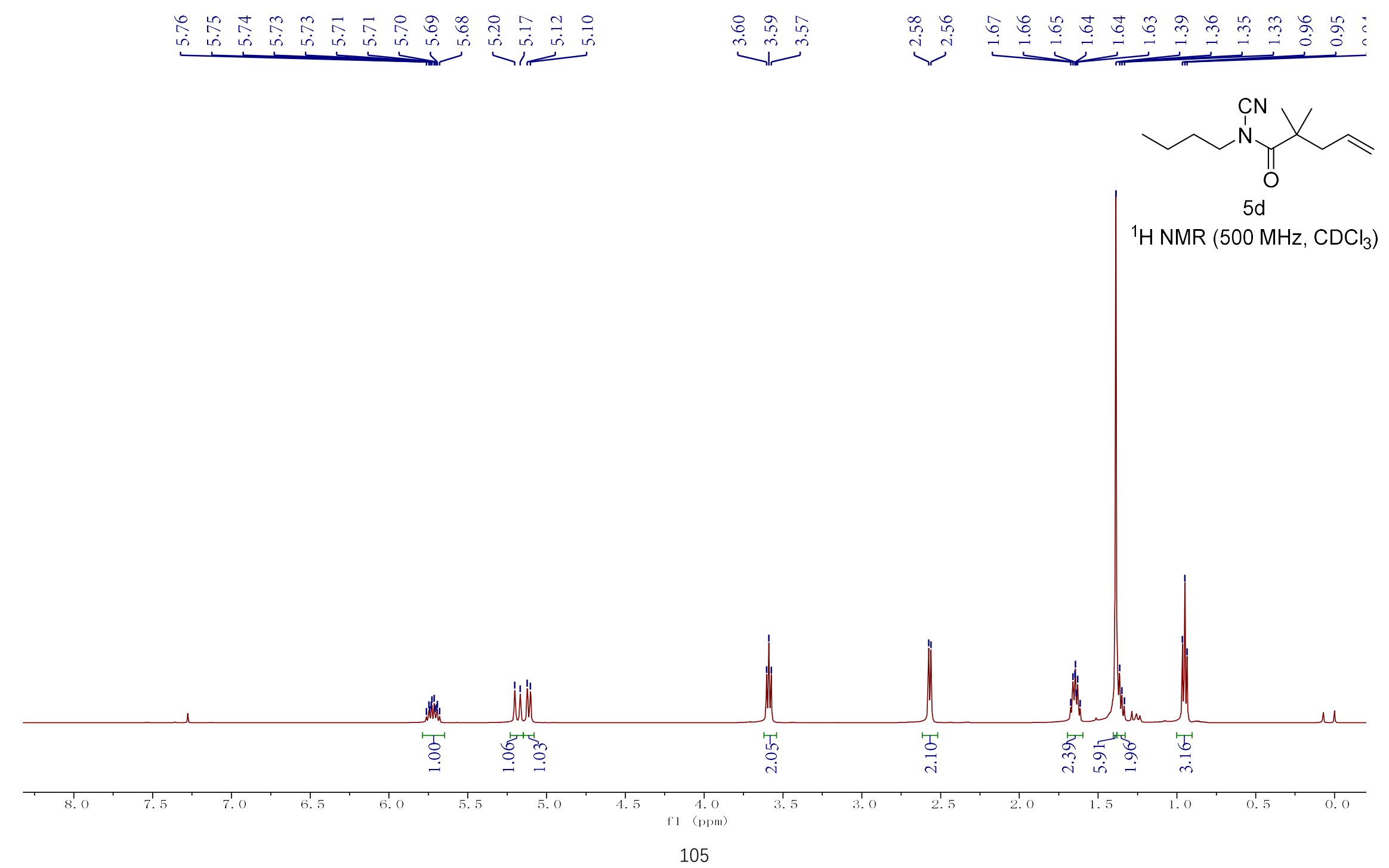




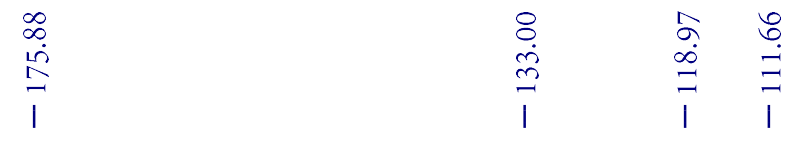

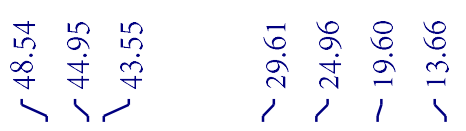

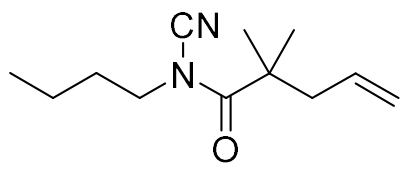

$5 d$

${ }^{13} \mathrm{C}$ NMR $\left(125 \mathrm{MHz}, \mathrm{CDCl}_{3}\right)$
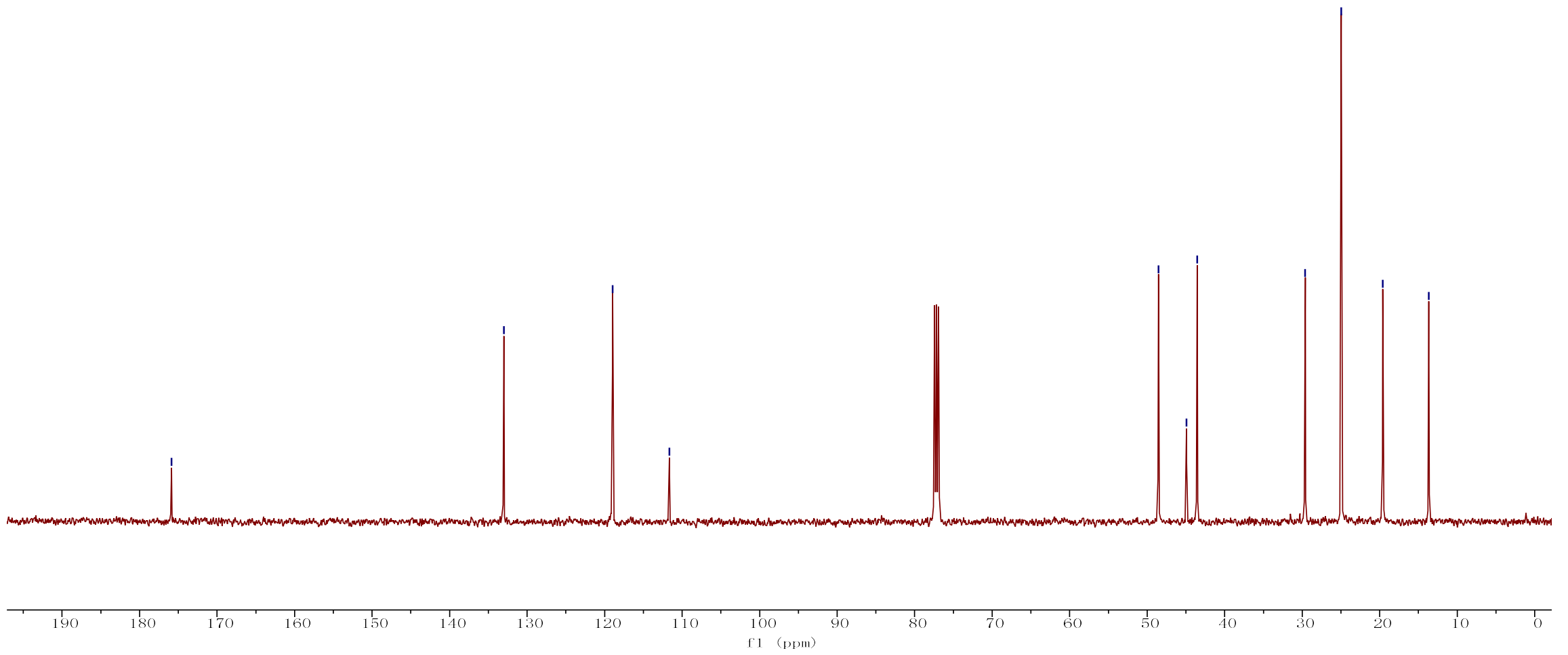


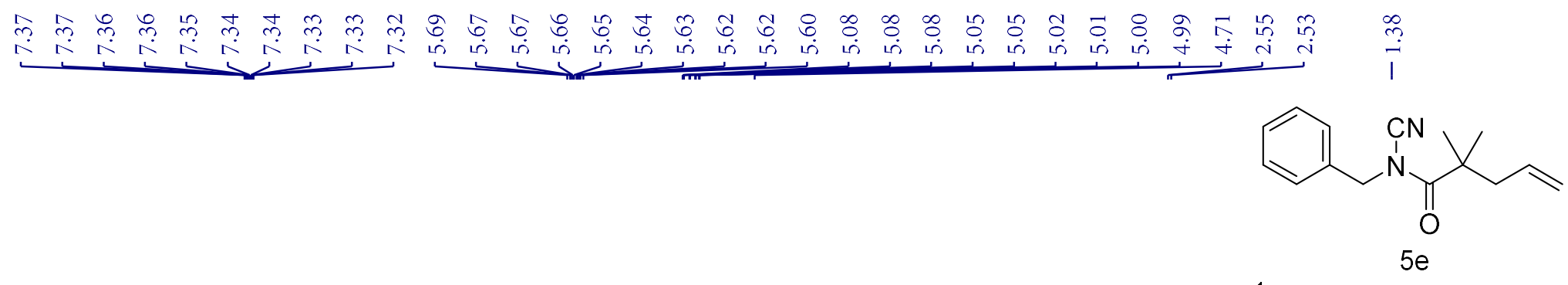

${ }^{1} \mathrm{H} \mathrm{NMR}\left(500 \mathrm{MHz}, \mathrm{CDCl}_{3}\right)$

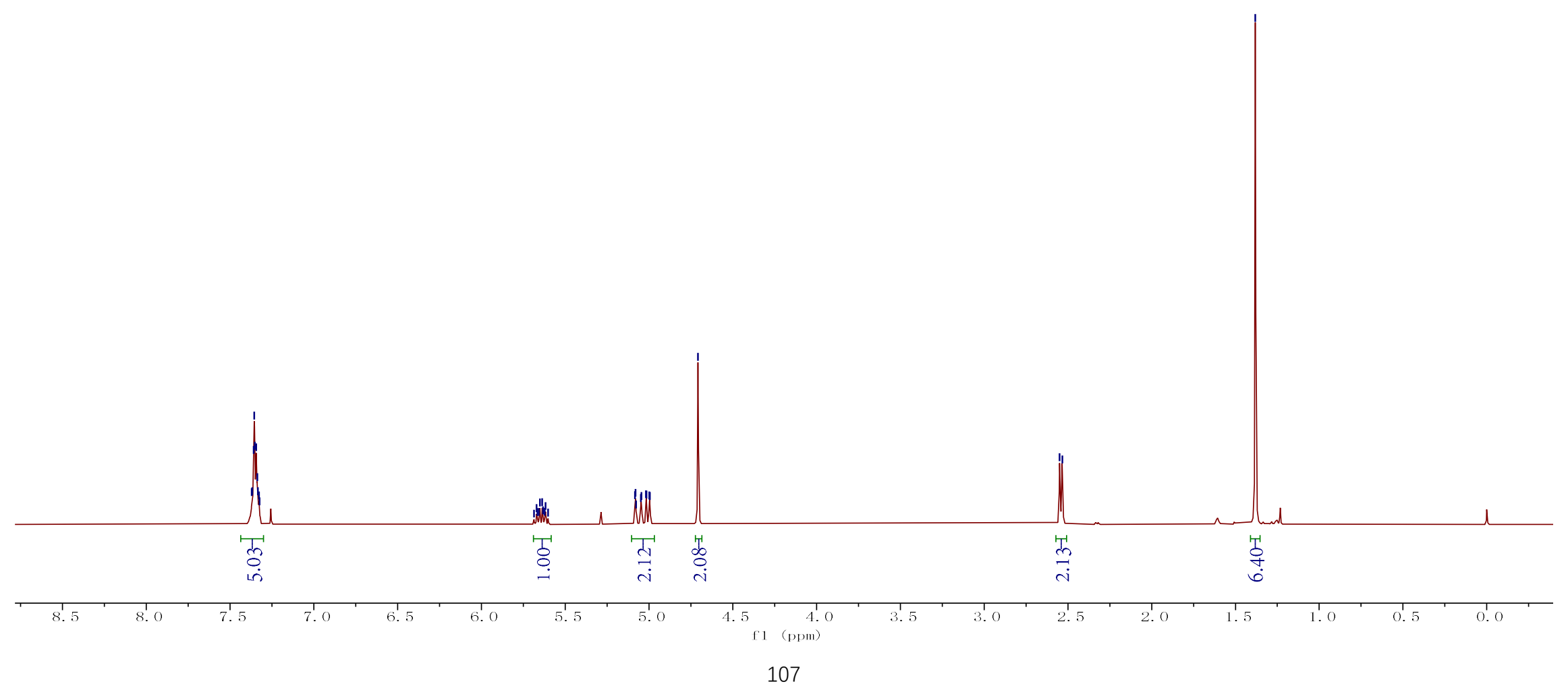




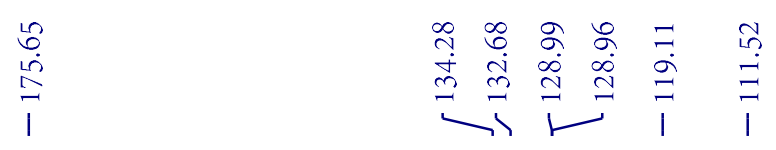

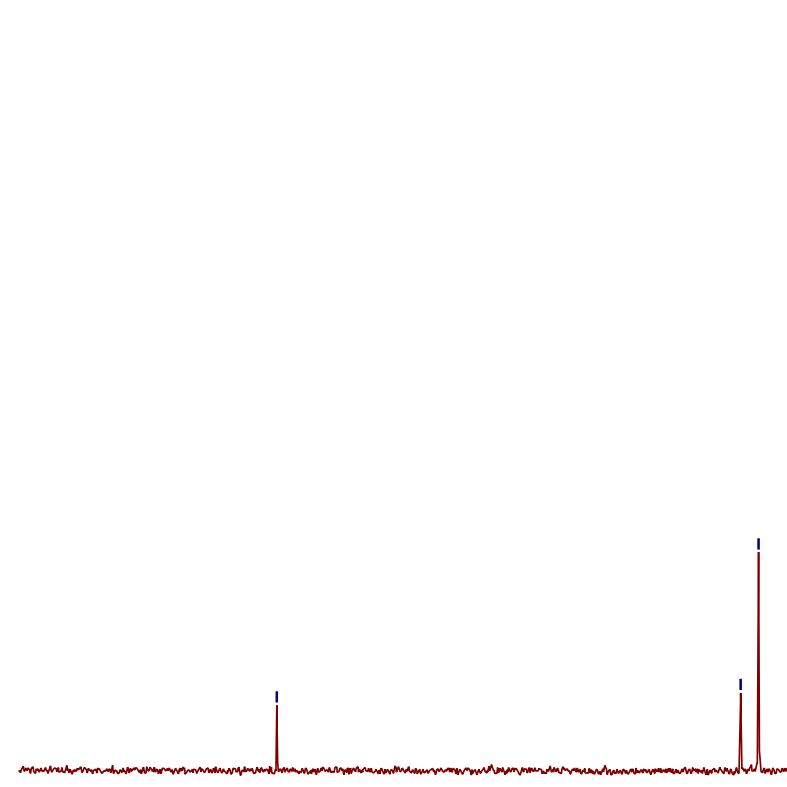

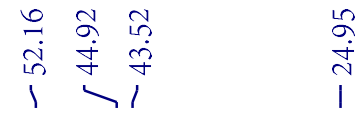

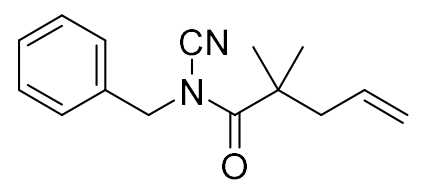

$5 e$

${ }^{13} \mathrm{C} \mathrm{NMR}\left(125 \mathrm{MHz}, \mathrm{CDCl}_{3}\right)$

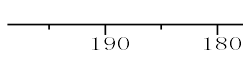

160

150

140

120

110

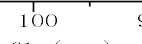




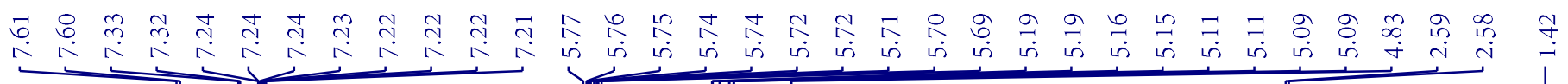

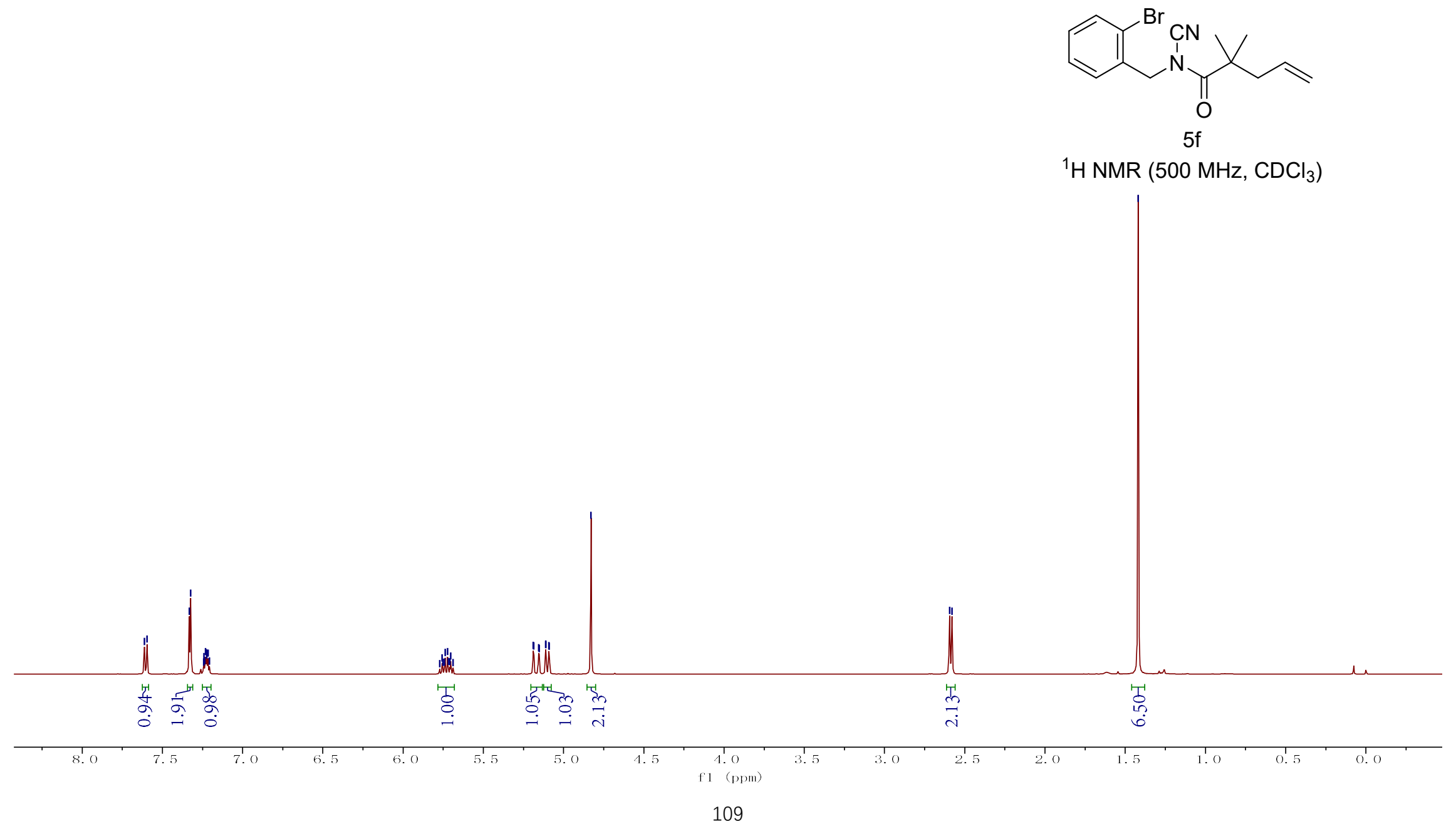



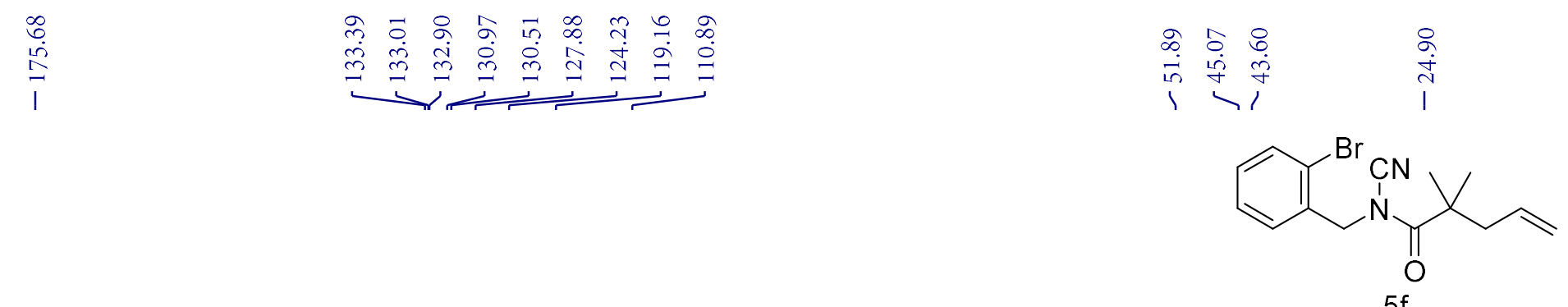

${ }^{13} \mathrm{C}$ NMR $\left(125 \mathrm{MHz}, \mathrm{CDCl}_{3}\right)$

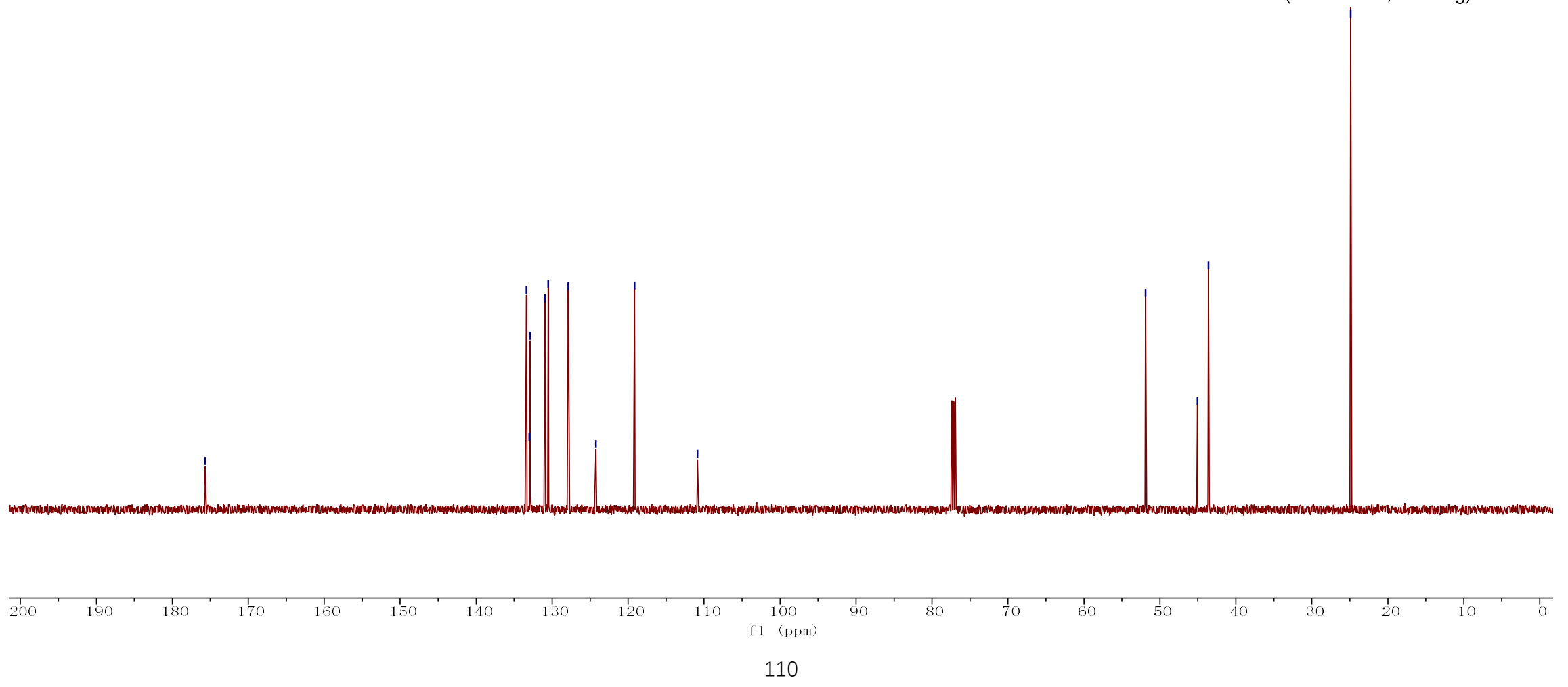




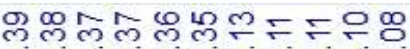
UNMNTNRNA

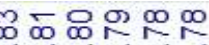
من

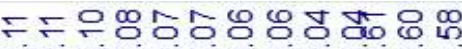

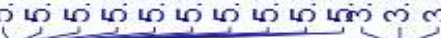

ஜํํㅇำ -

CN

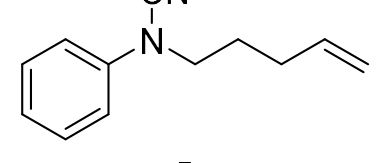

$5 \mathrm{~g}$

${ }^{1} \mathrm{H}$ NMR $\left(500 \mathrm{MHz}, \mathrm{CDCl}_{3}\right)$

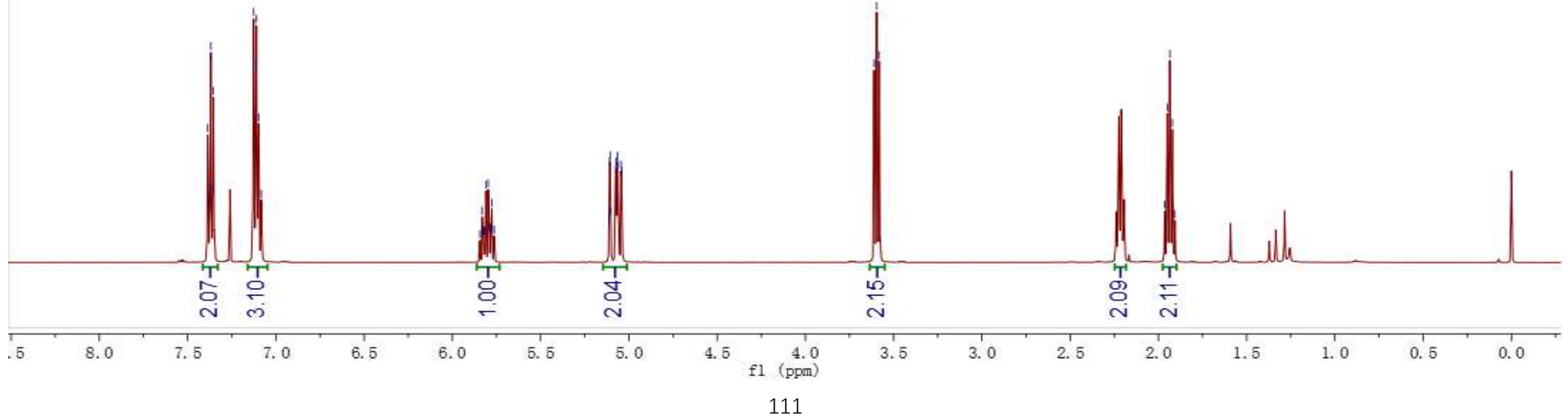




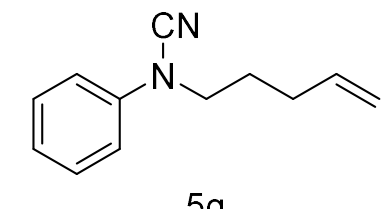

${ }^{13} \mathrm{C} \mathrm{NMR}\left(125 \mathrm{MHz}, \mathrm{CDCl}_{3}\right)$
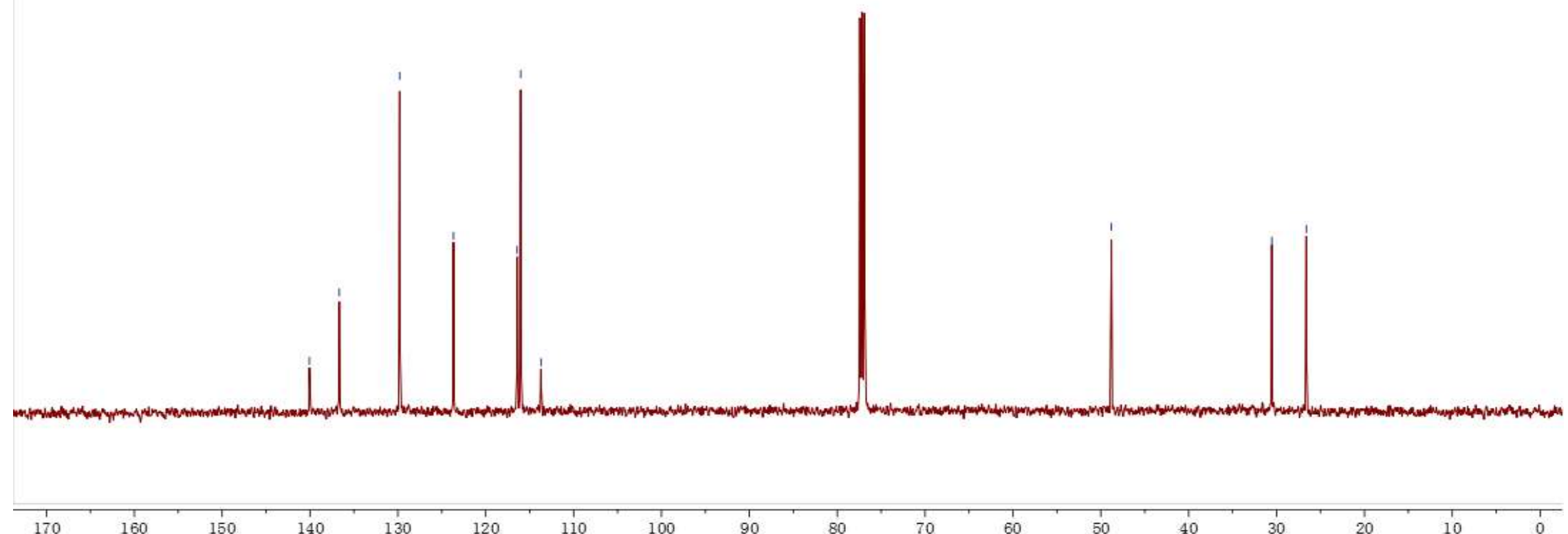

140

130 


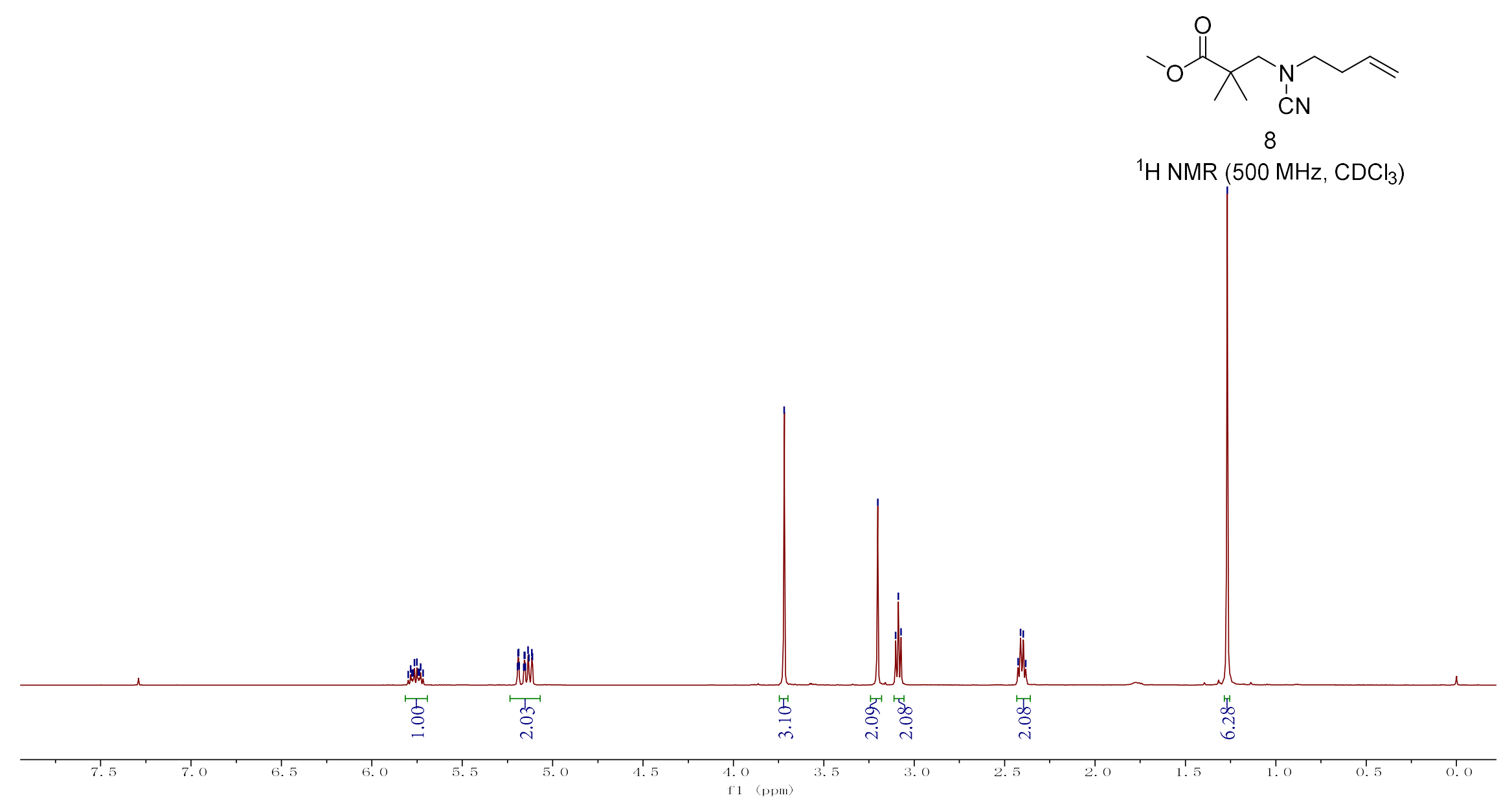



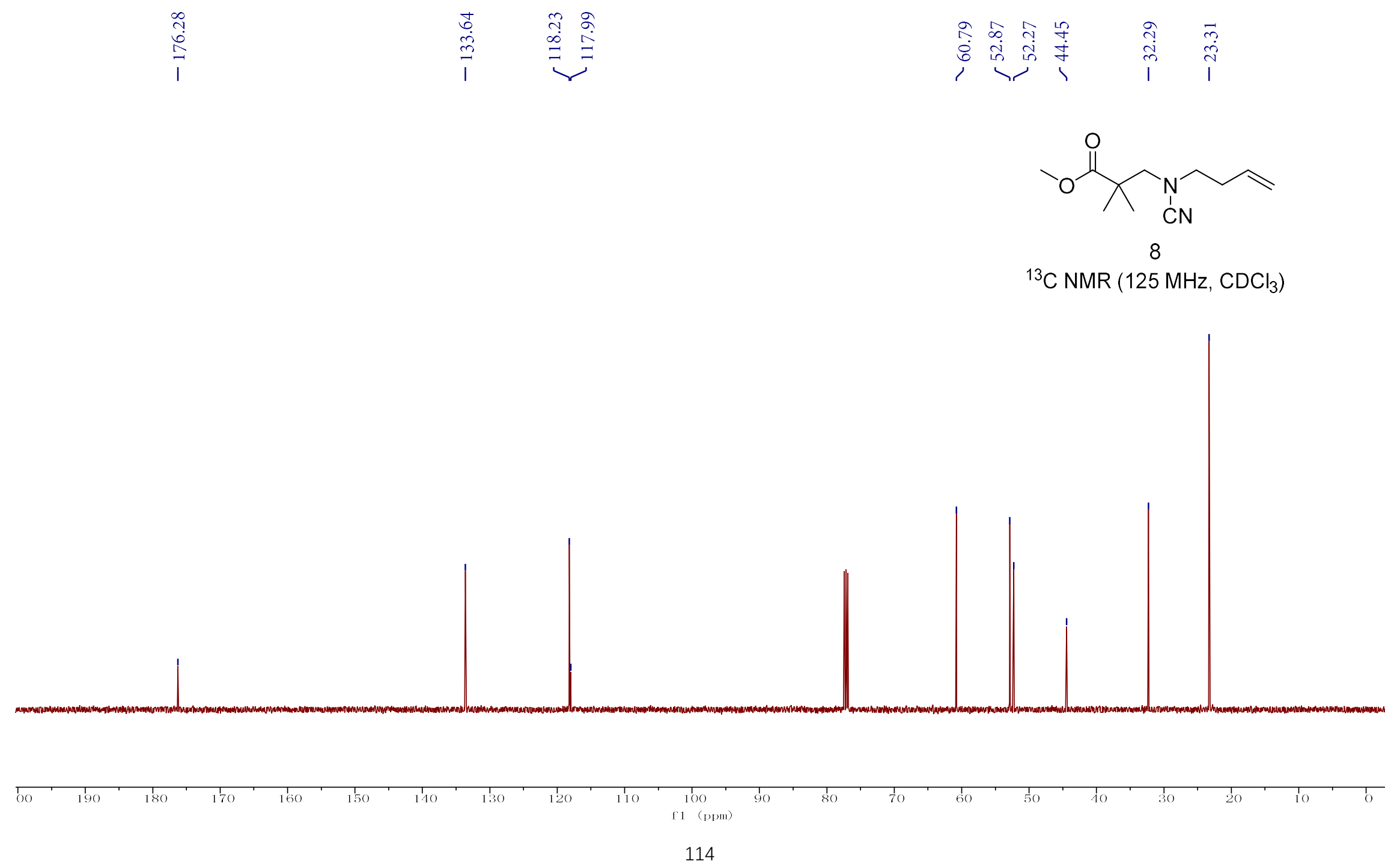


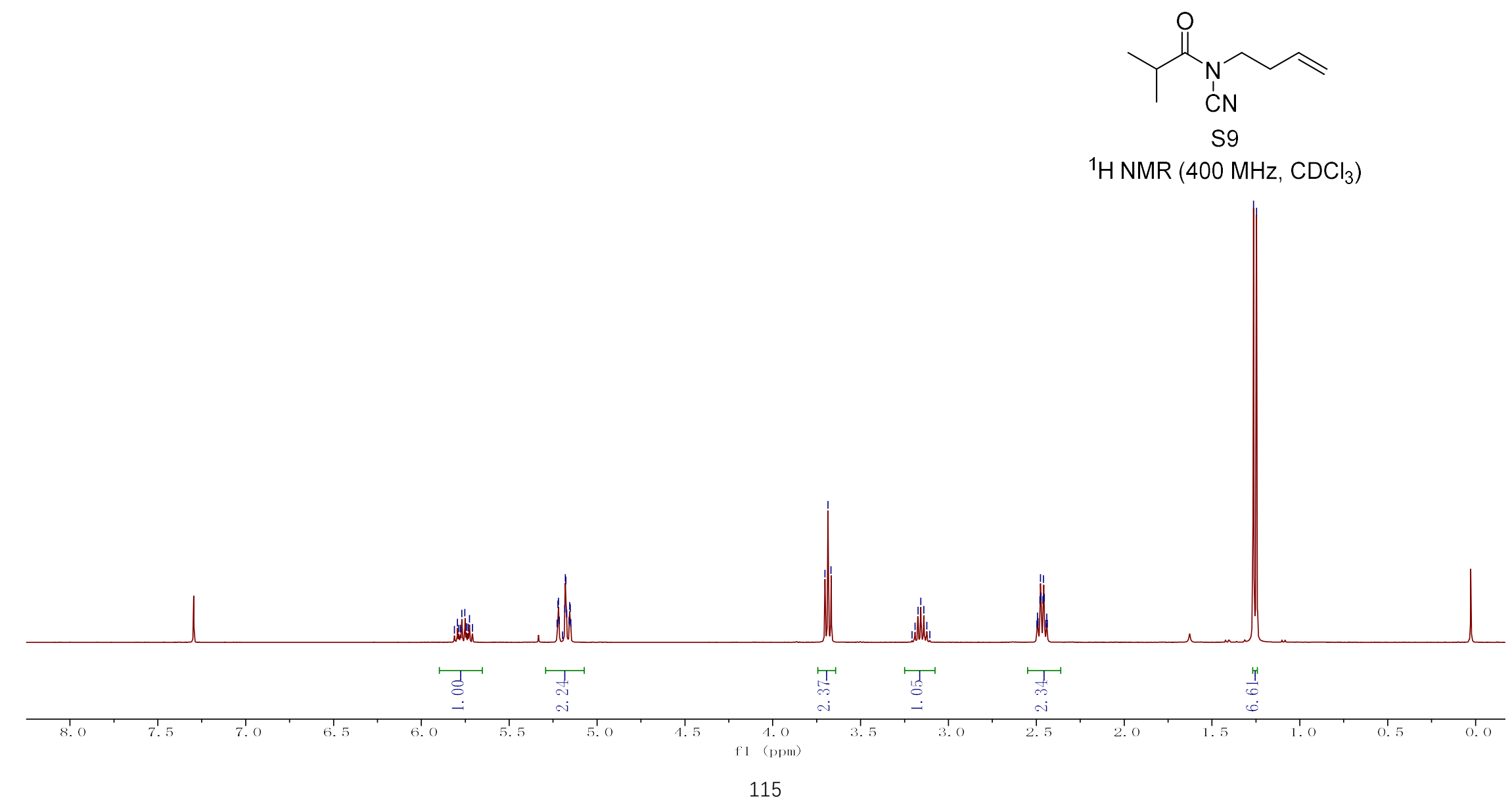




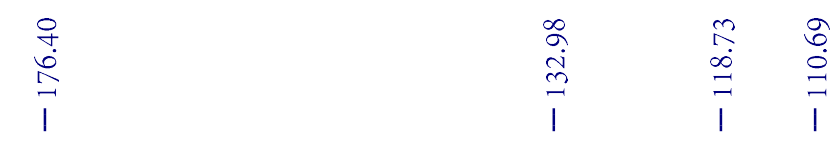

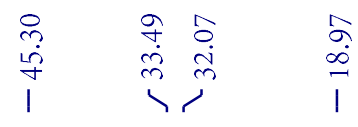

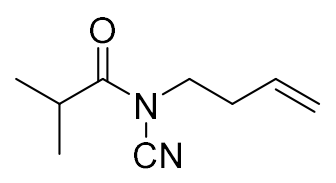

S9

${ }^{13} \mathrm{C}$ NMR $\left(125 \mathrm{MHz}, \mathrm{CDCl}_{3}\right)$

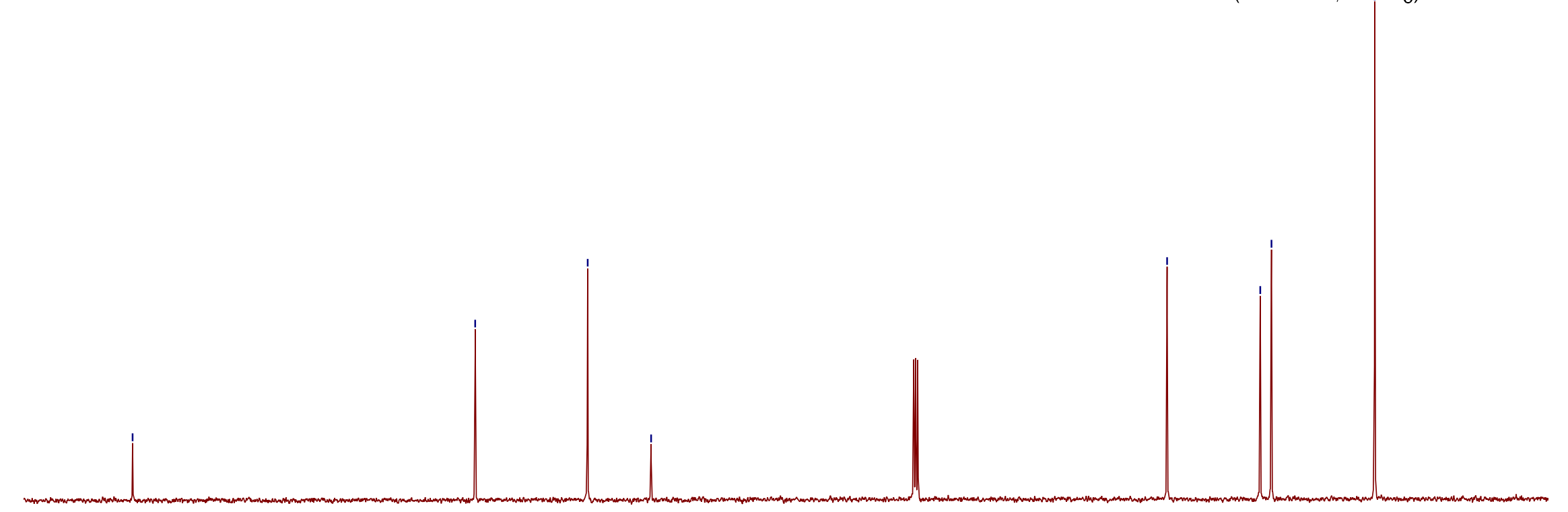

$90 \quad 180$

140

120

fl 90 


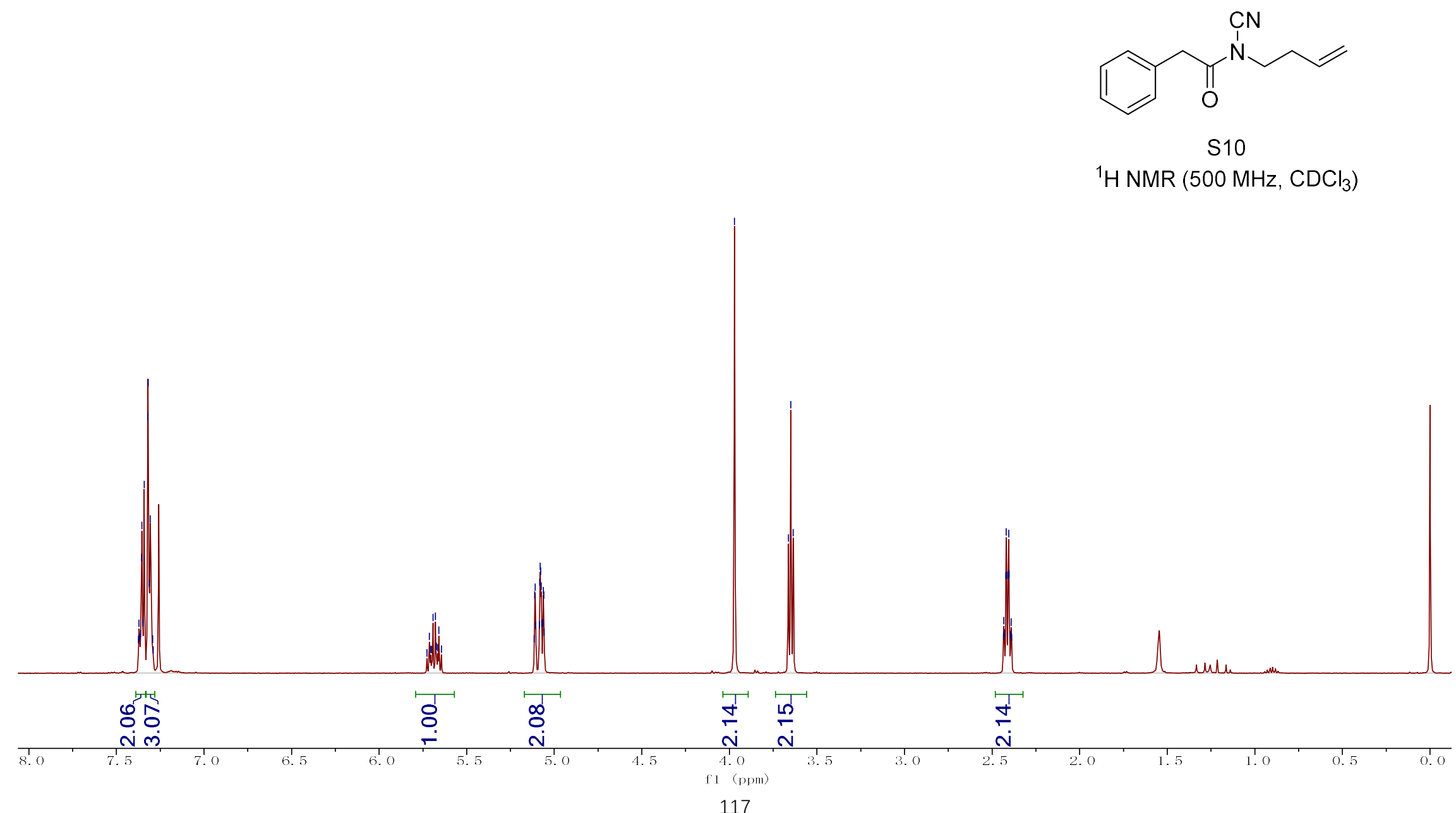



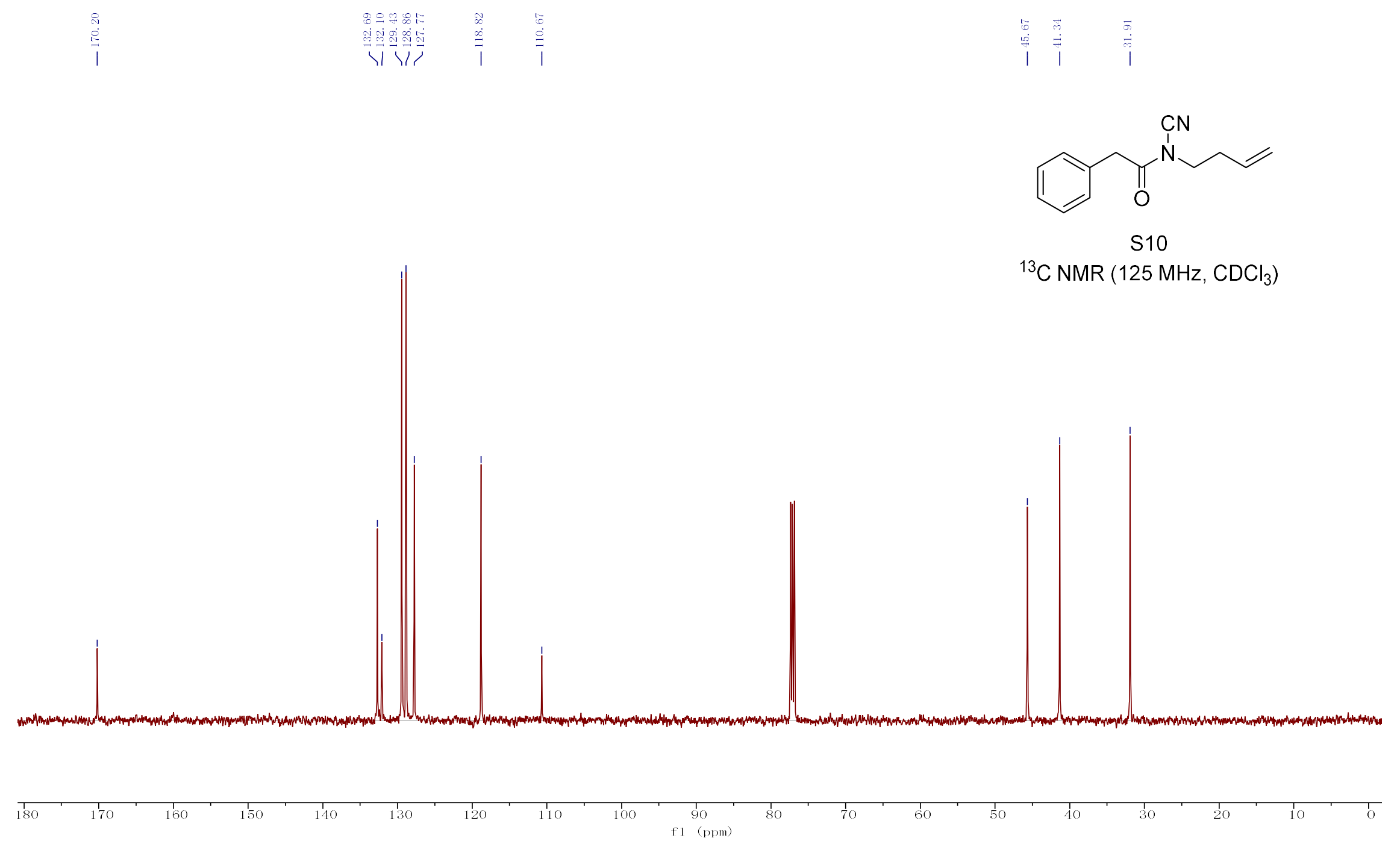


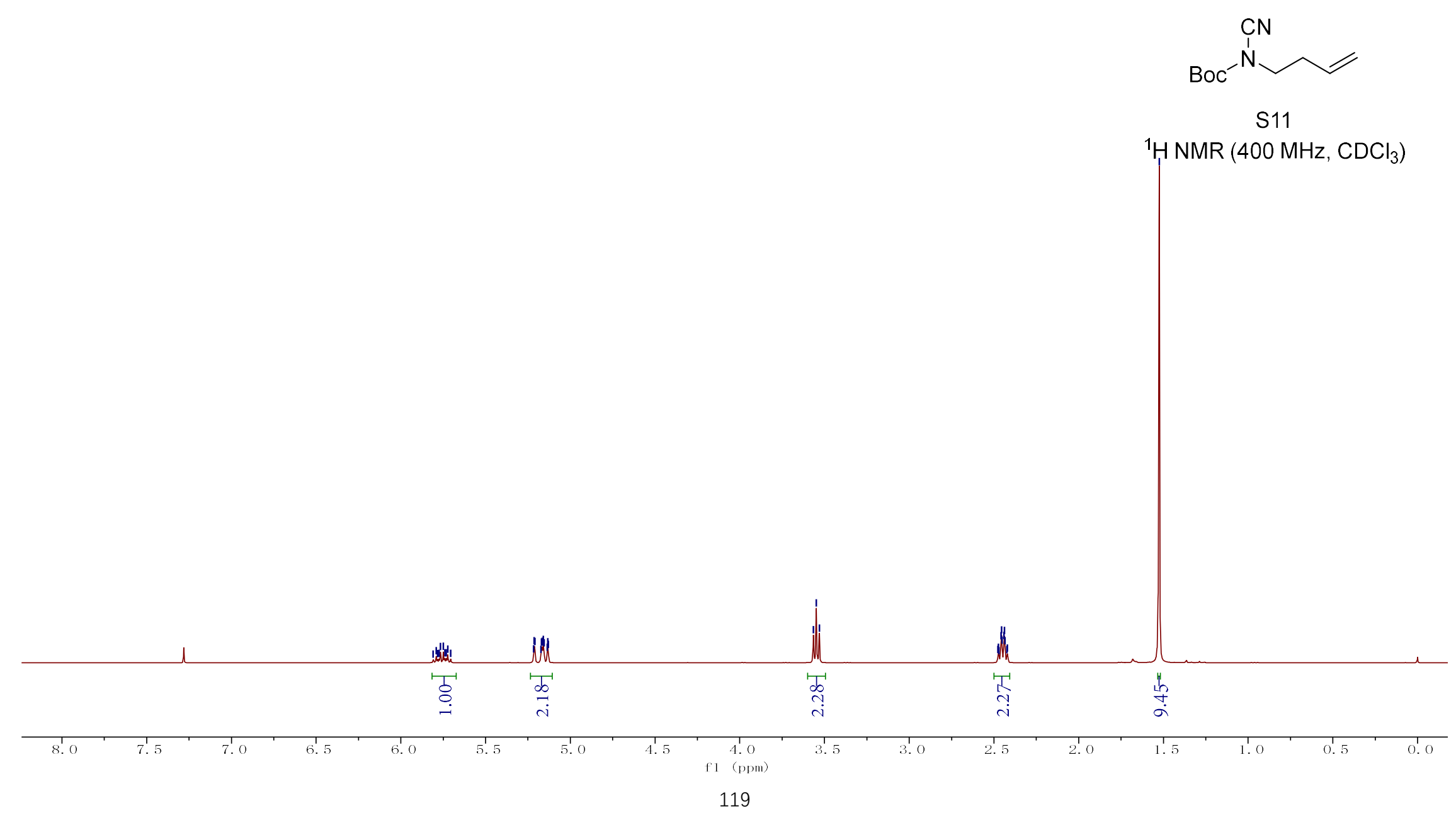


$\underset{\substack{n \\ \text { 1 }}}{\stackrel{8}{1}}$

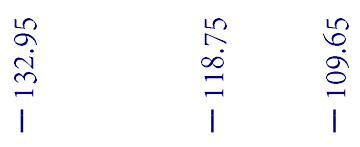

$\ln$
$\stackrel{2}{10}$
10
$\infty$
1

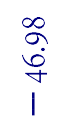

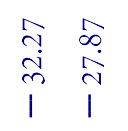

$\mathrm{Boc}^{-\mathrm{CN}}$

S11

${ }^{13} \mathrm{C}$ NMR $\left(100 \mathrm{MHz}, \mathrm{CDCl}_{3}\right)$
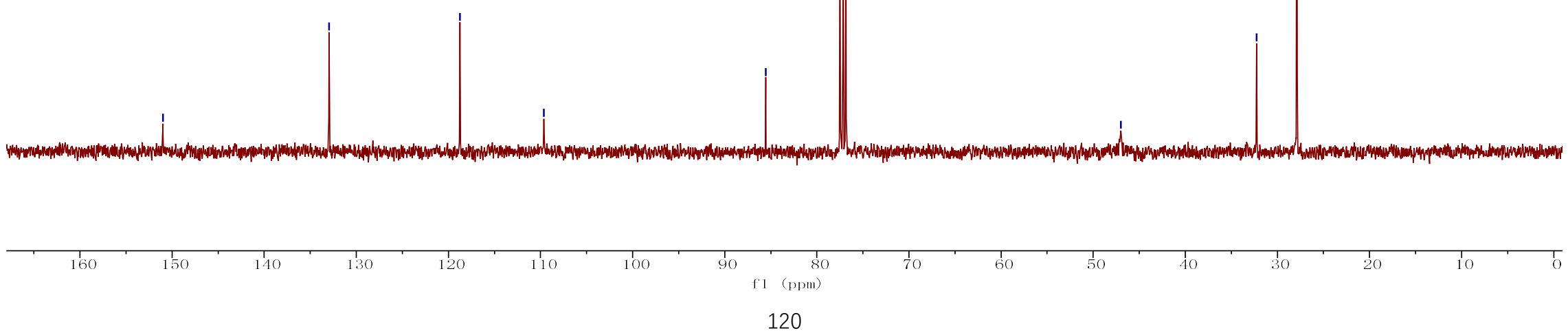


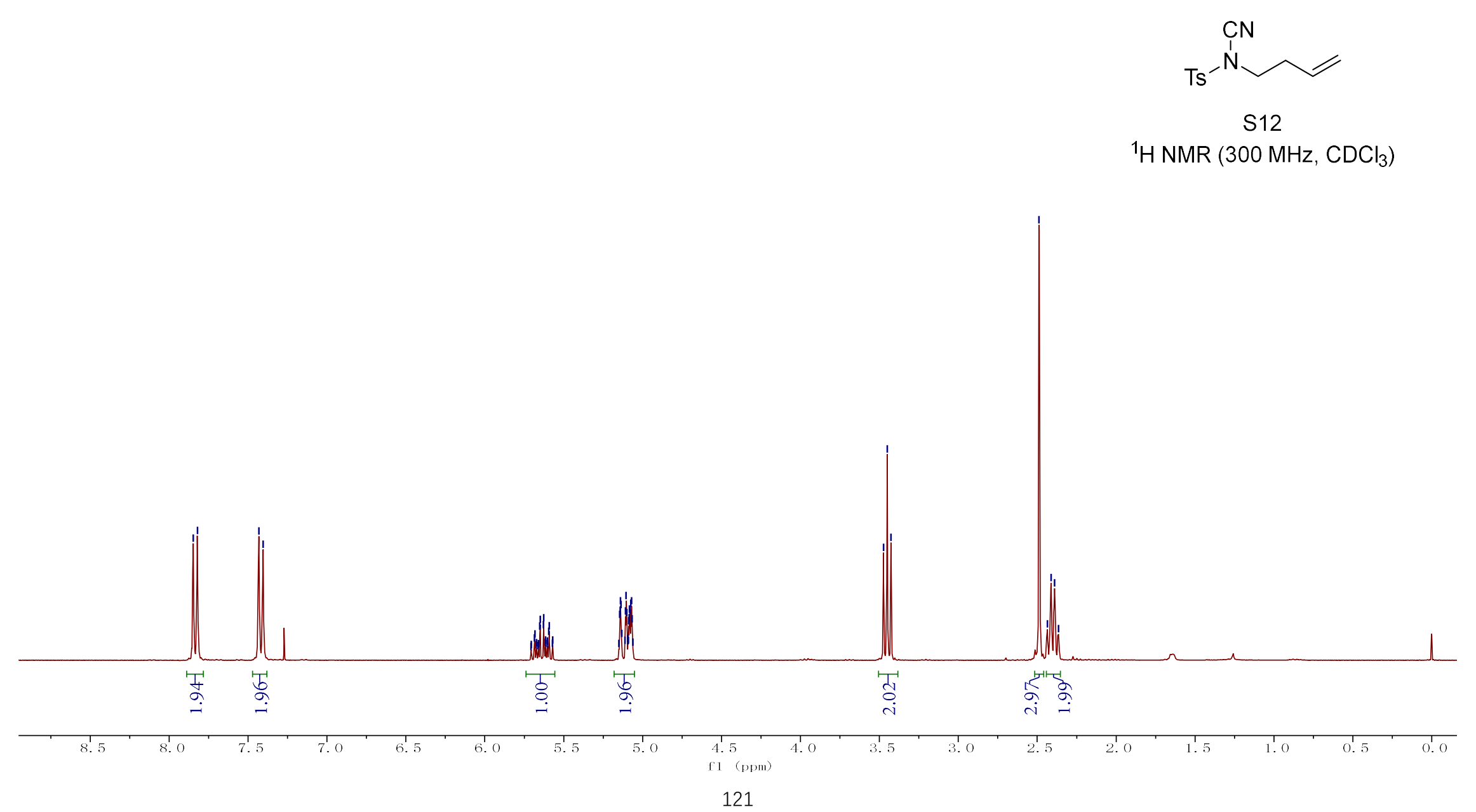




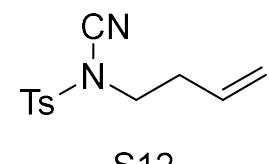

${ }^{13} \mathrm{C}$ NMR $\left(125 \mathrm{MHz}, \mathrm{CDCl}_{3}\right)$
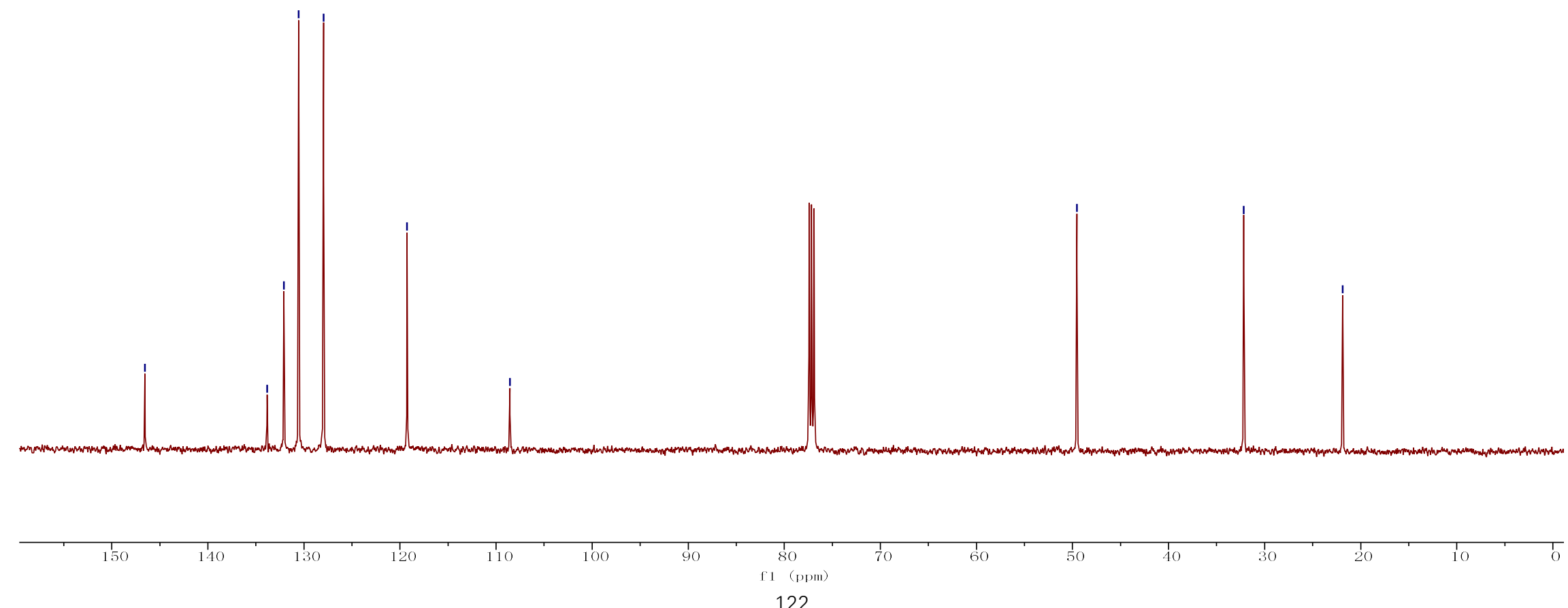


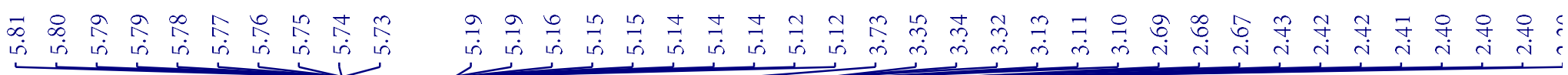

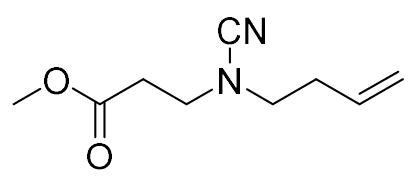

$S 13$

${ }^{1} \mathrm{H} \mathrm{NMR}\left(500 \mathrm{MHz}, \mathrm{CDCl}_{3}\right)$

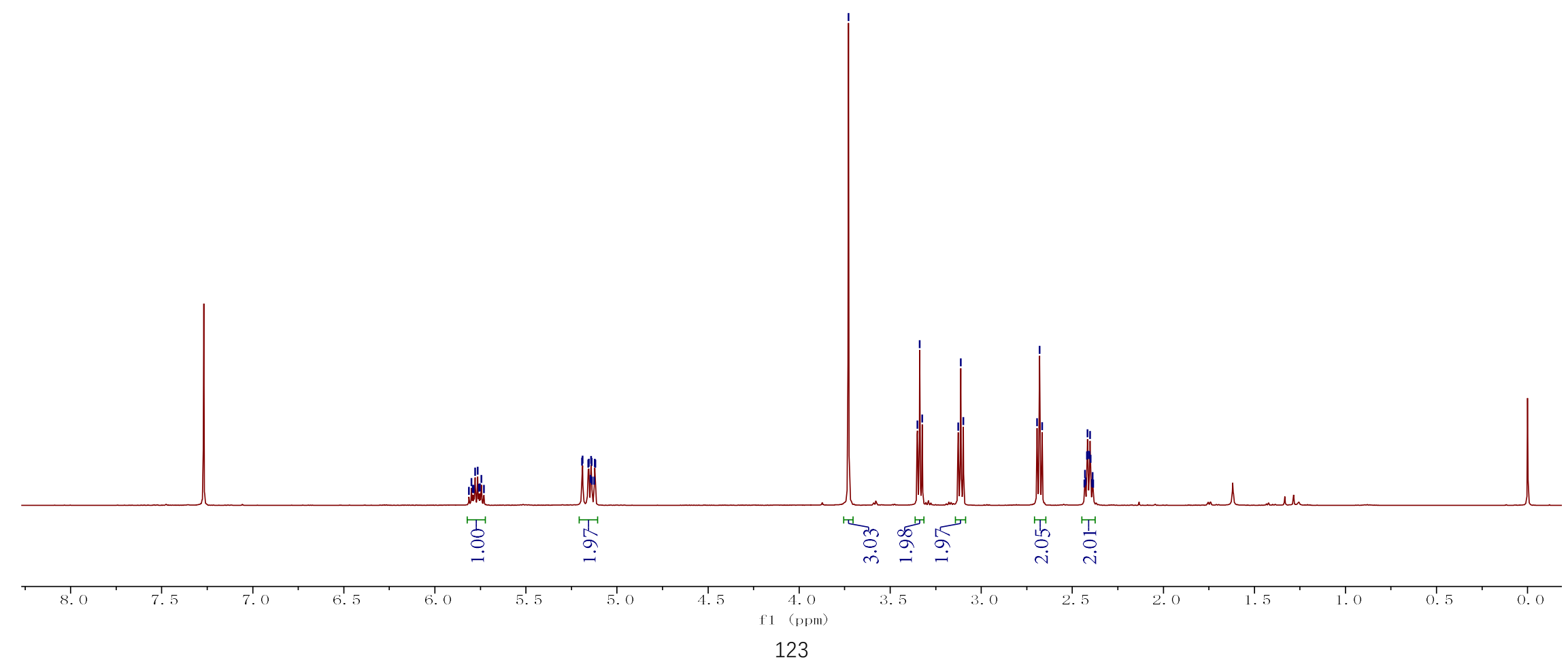




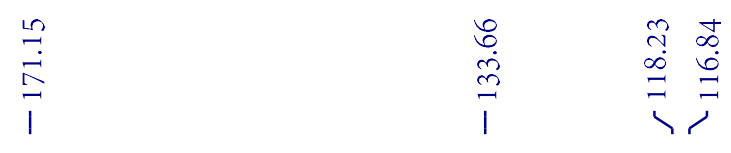

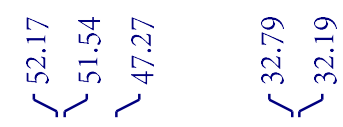

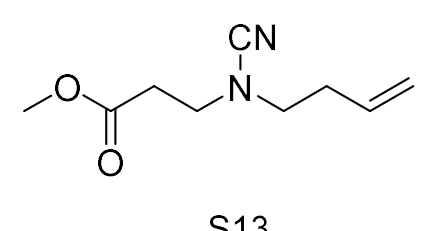

${ }^{13} \mathrm{C} \mathrm{NMR}\left(100 \mathrm{MHz}, \mathrm{CDCl}_{3}\right)$
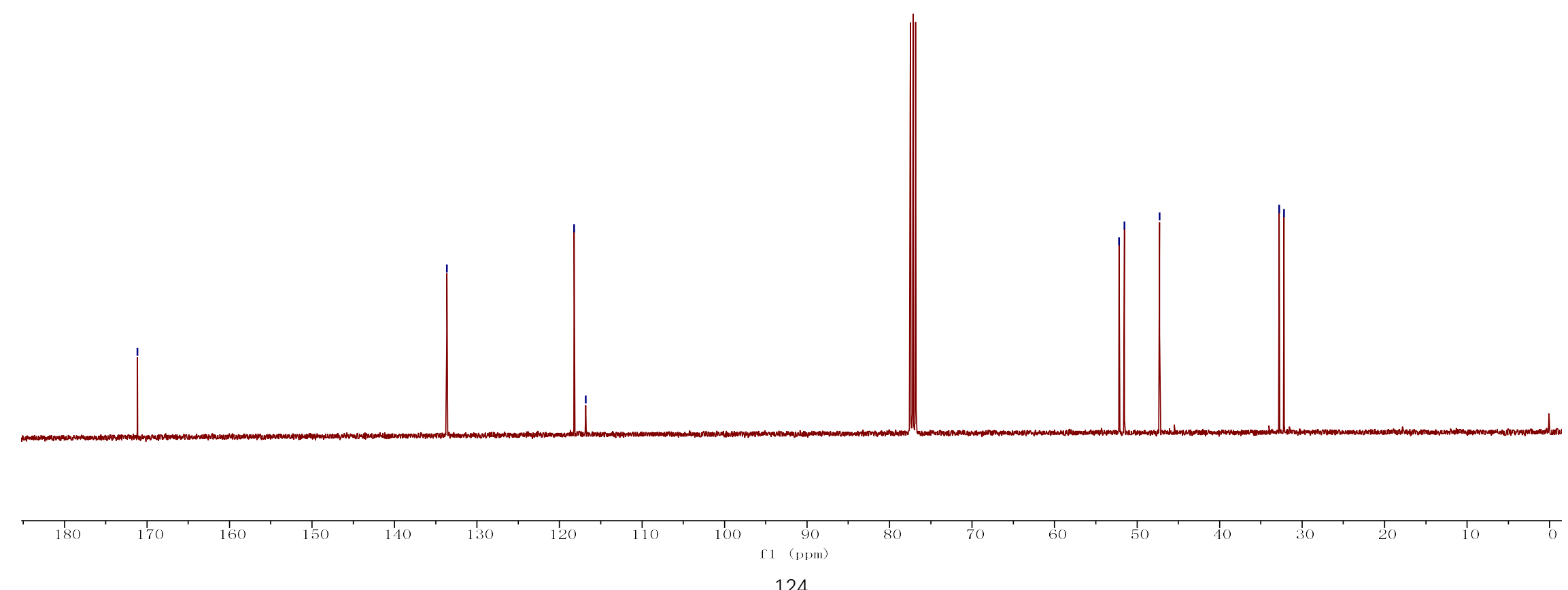


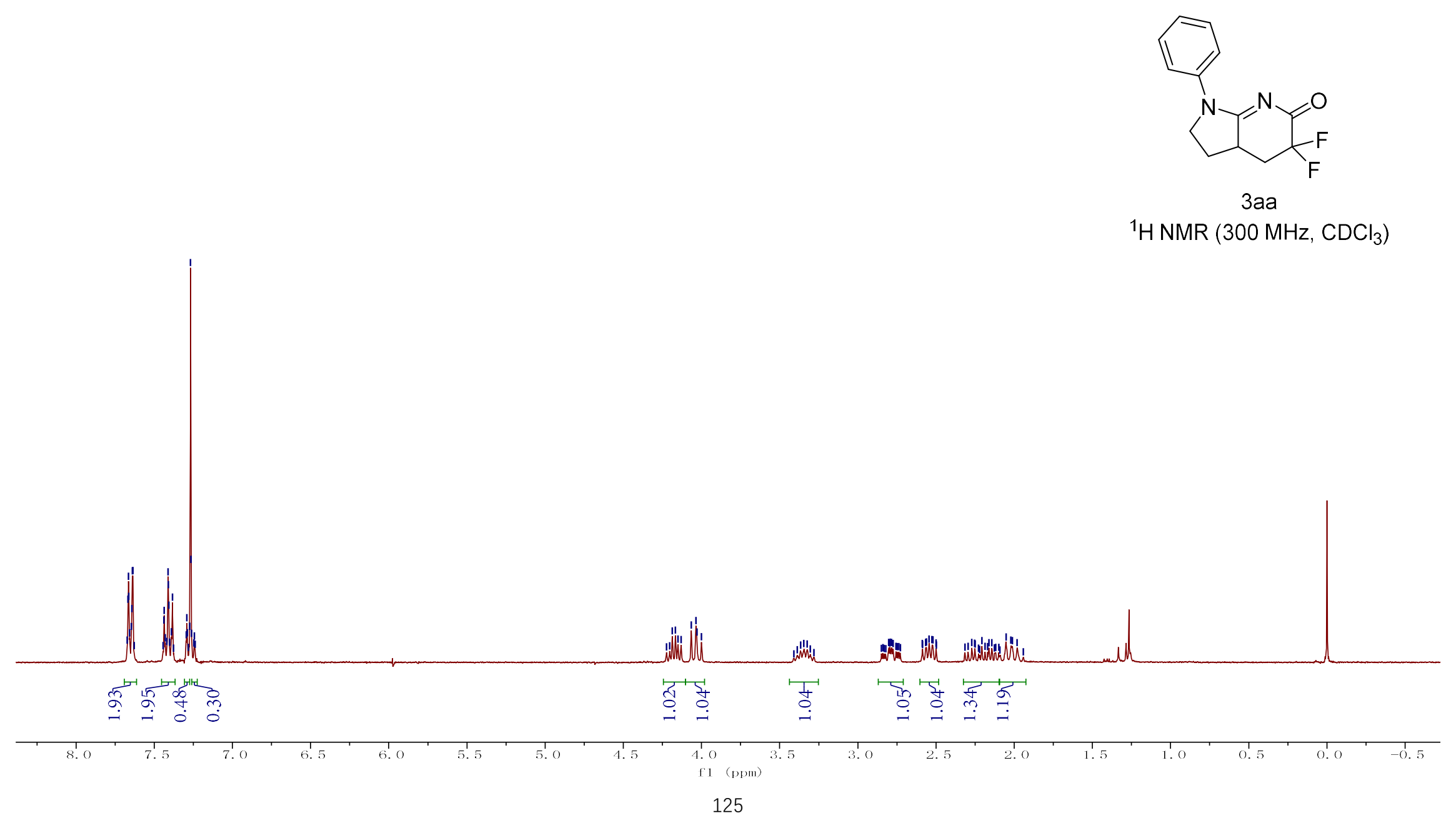



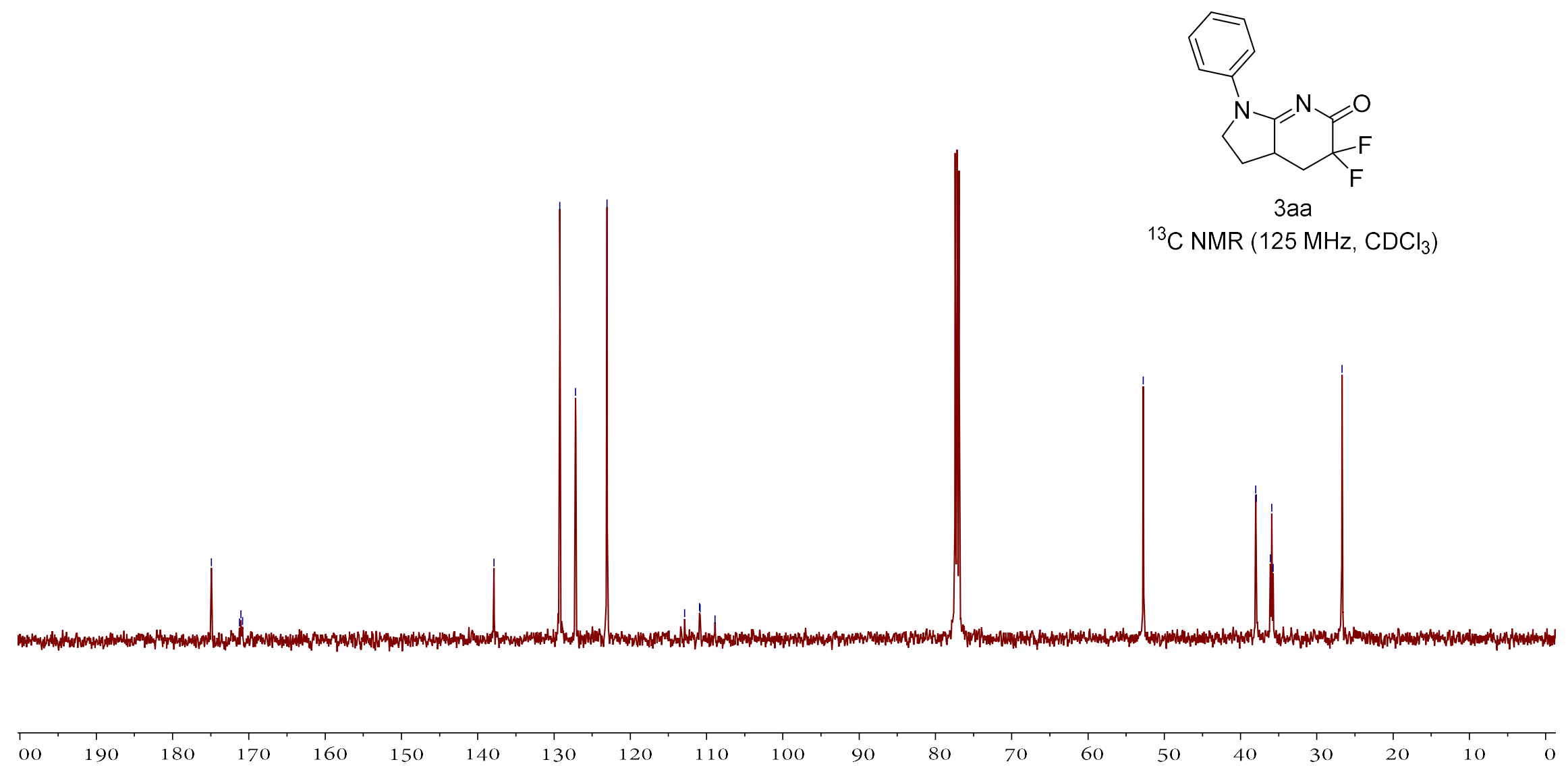
$110 \quad 100 \quad 90$ 80 $70 \quad 60$ $50 \quad 40$ $30 \quad 20$ 


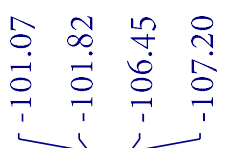

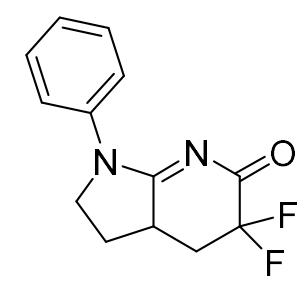

$3 a a$

${ }^{19} \mathrm{~F} \mathrm{NMR}\left(376 \mathrm{MHz}, \mathrm{CDCl}_{3}\right)$

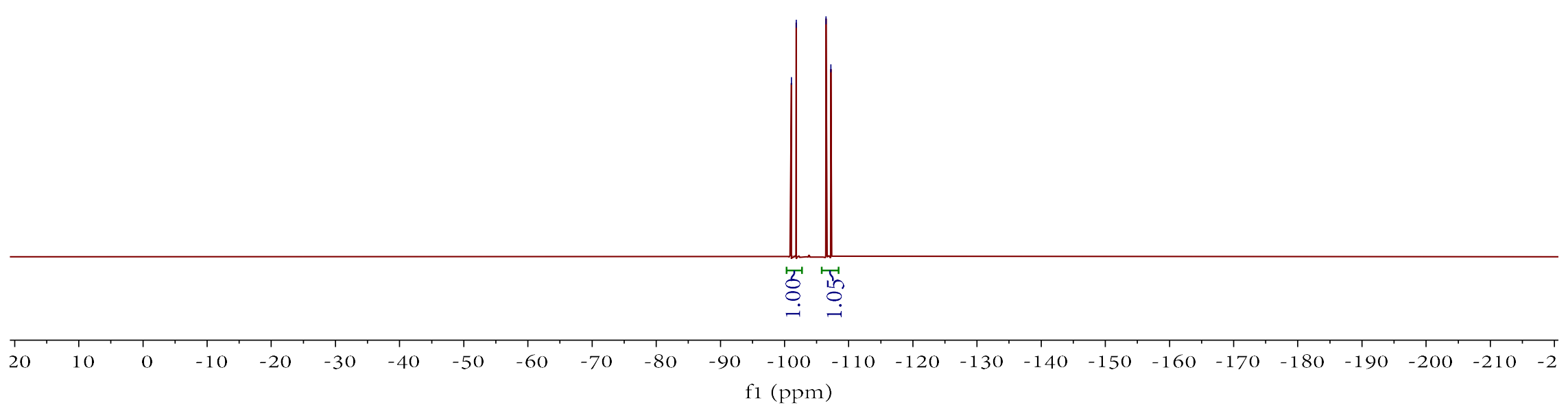




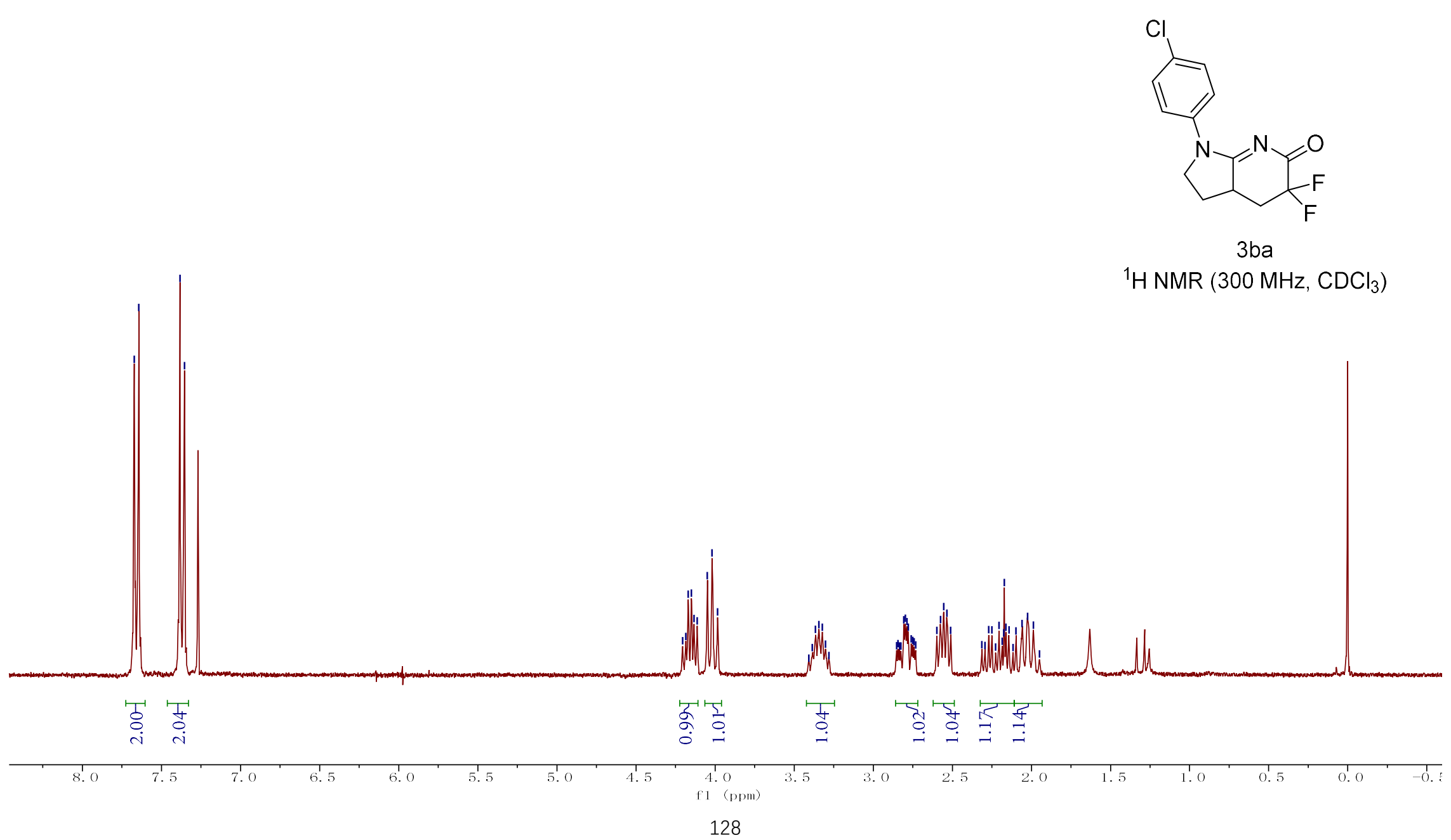




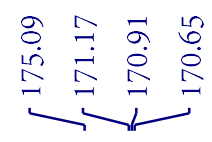

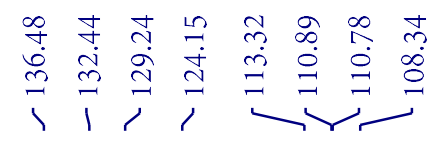

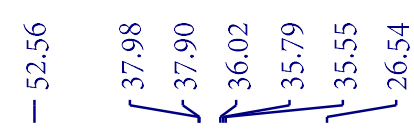

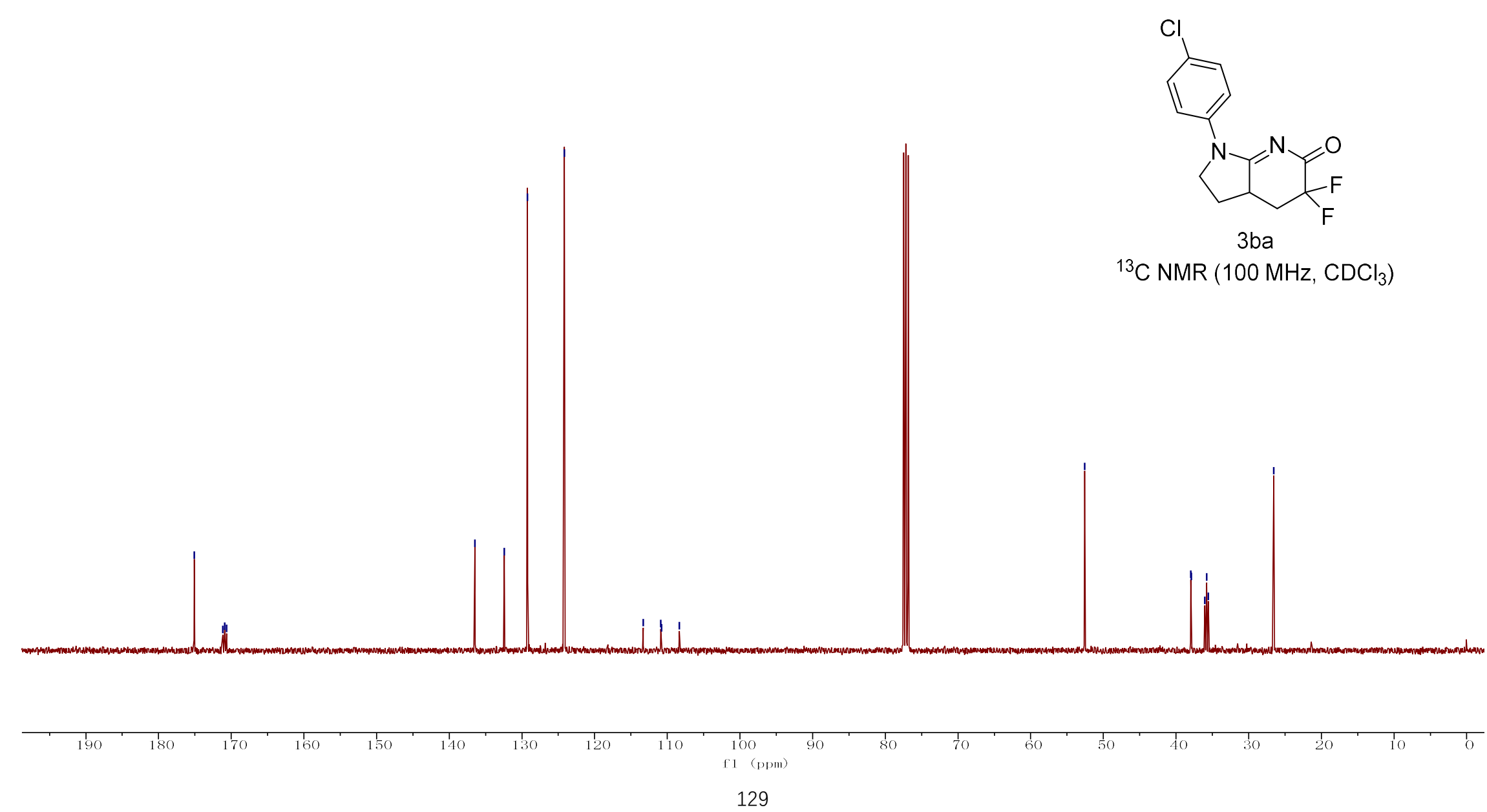




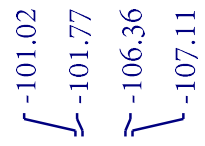

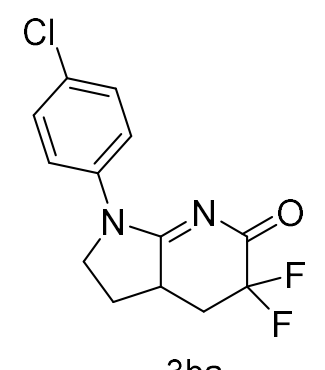

${ }^{19} \mathrm{~F} \mathrm{NMR}\left(376 \mathrm{MHz}, \mathrm{CDCl}_{3}\right)$

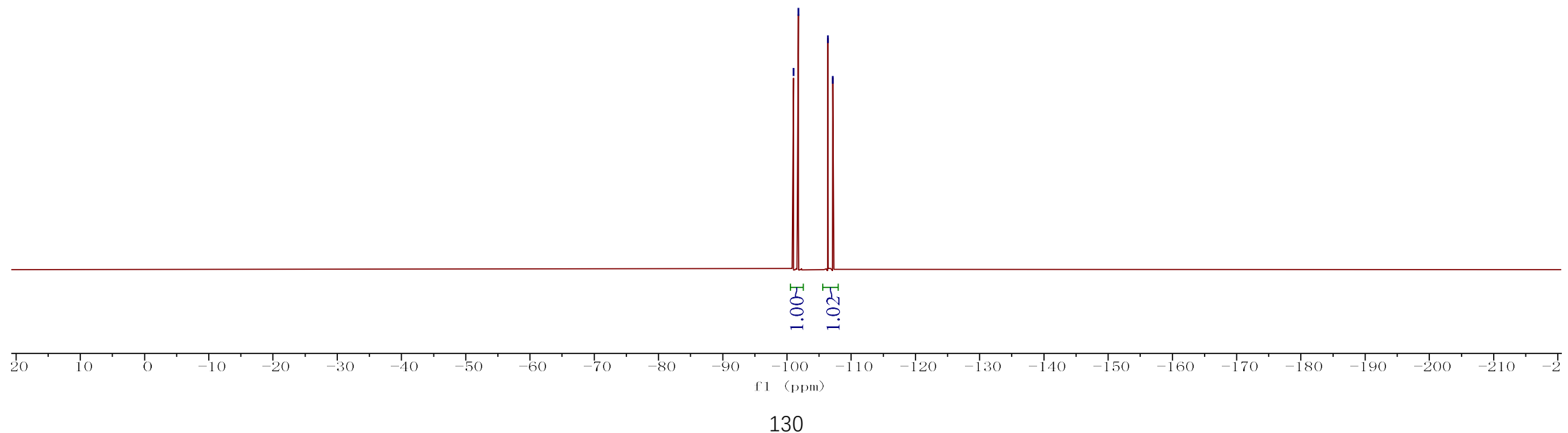




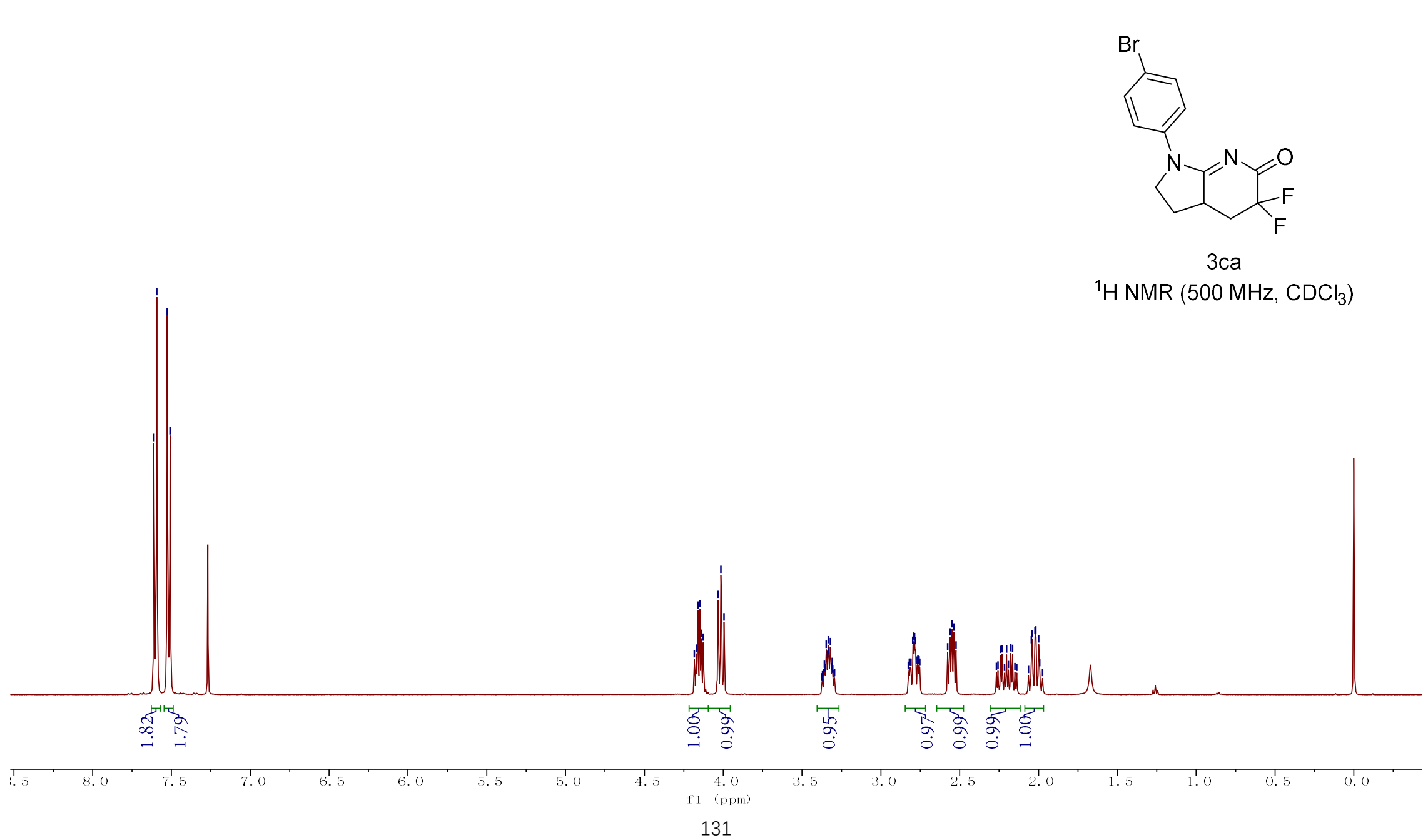




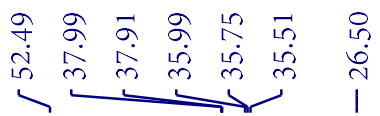

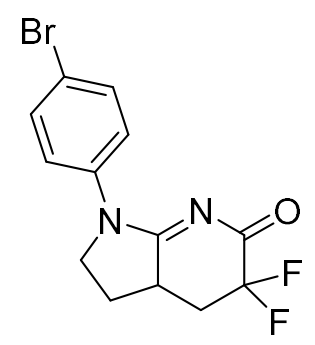

3ca

${ }^{13} \mathrm{C} \mathrm{NMR}\left(100 \mathrm{MHz}, \mathrm{CDCl}_{3}\right)$
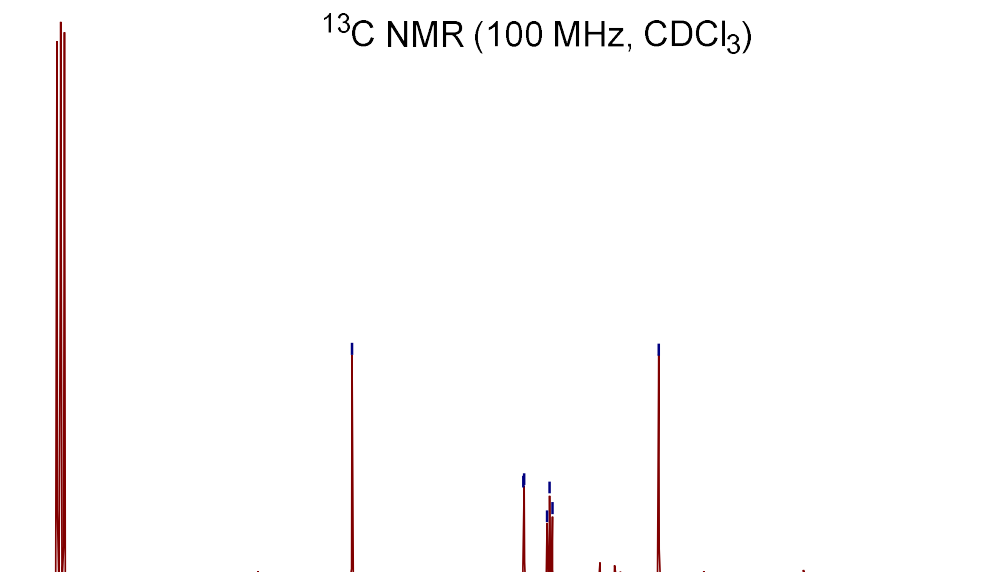

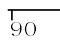

90
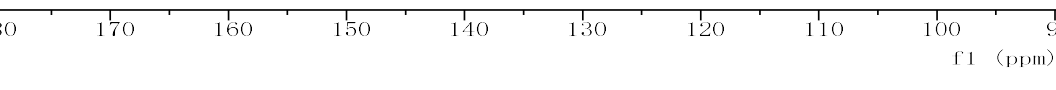


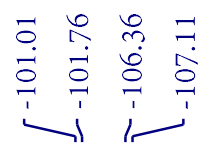

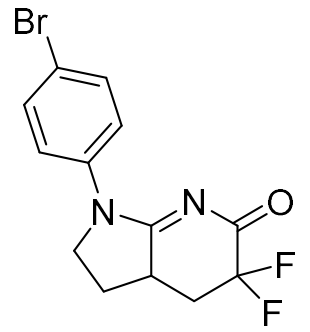

$3 \mathrm{ca}$

${ }^{19} \mathrm{~F} \mathrm{NMR} \mathrm{(376} \mathrm{MHz,} \mathrm{CDCl}_{3}$ )

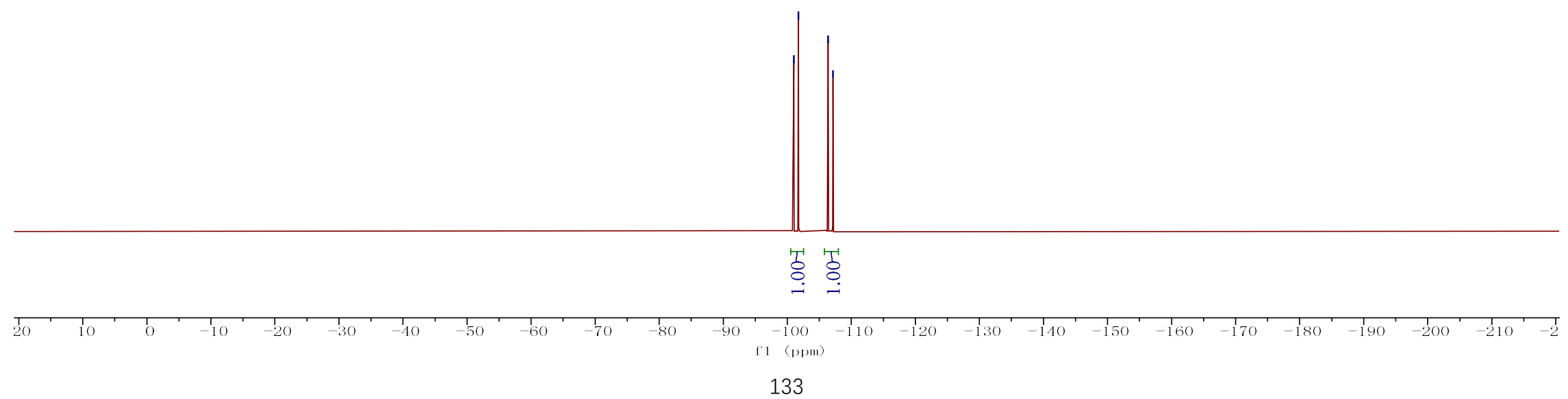




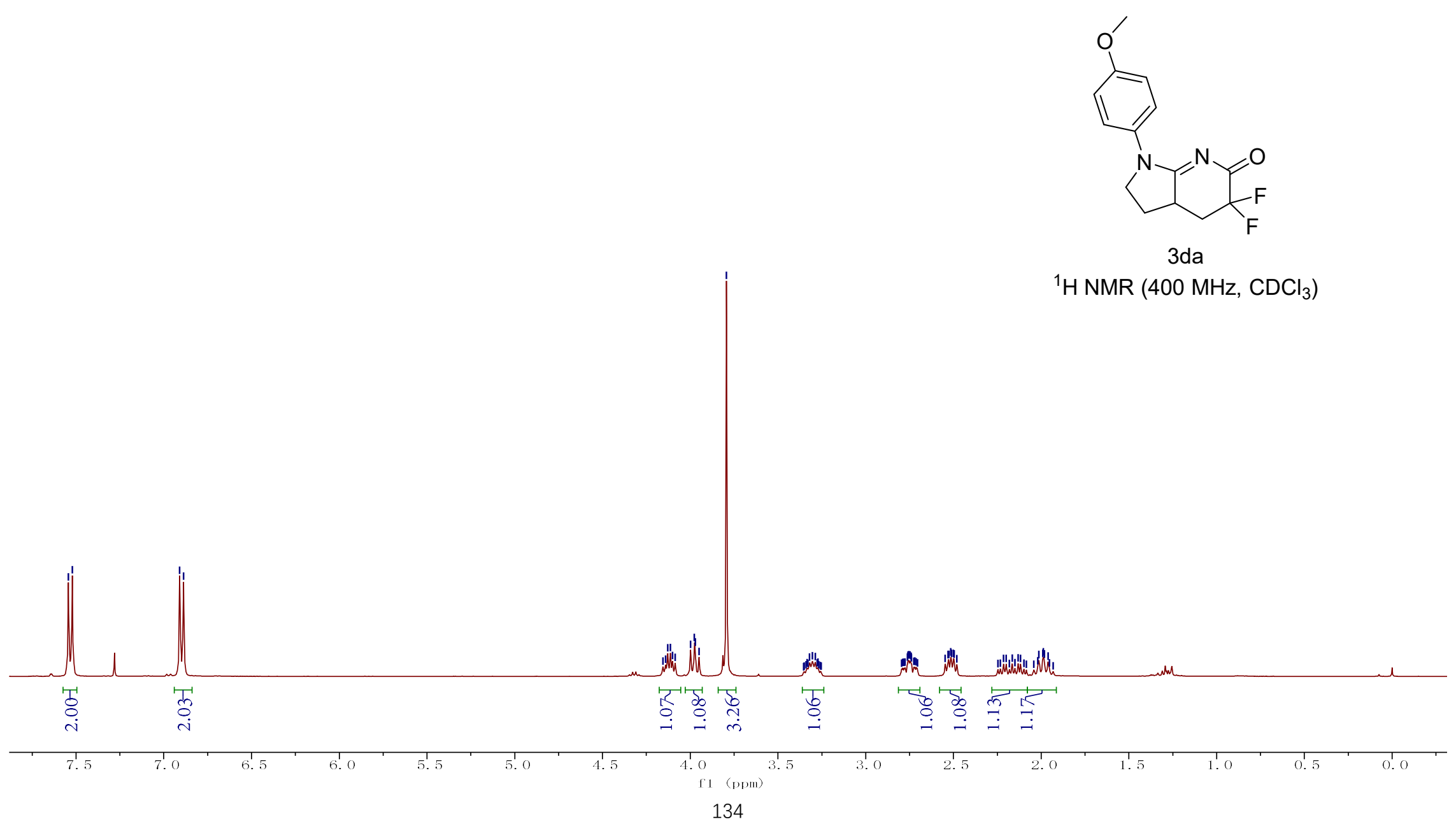




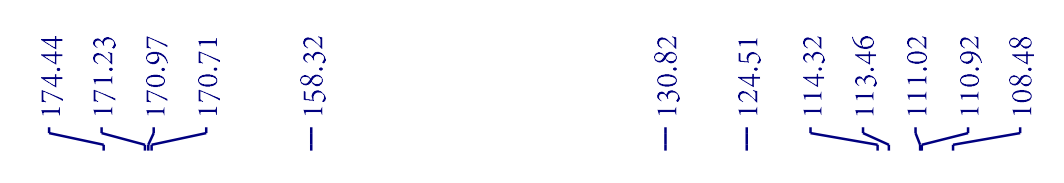

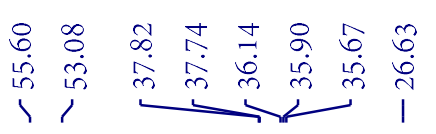

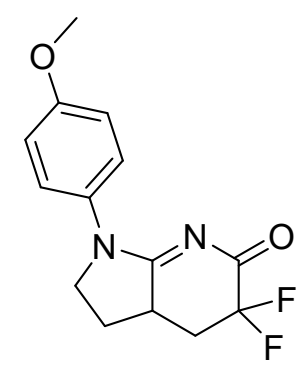

3da

${ }^{13} \mathrm{C}$ NMR $\left(100 \mathrm{MHz}, \mathrm{CDCl}_{3}\right)$
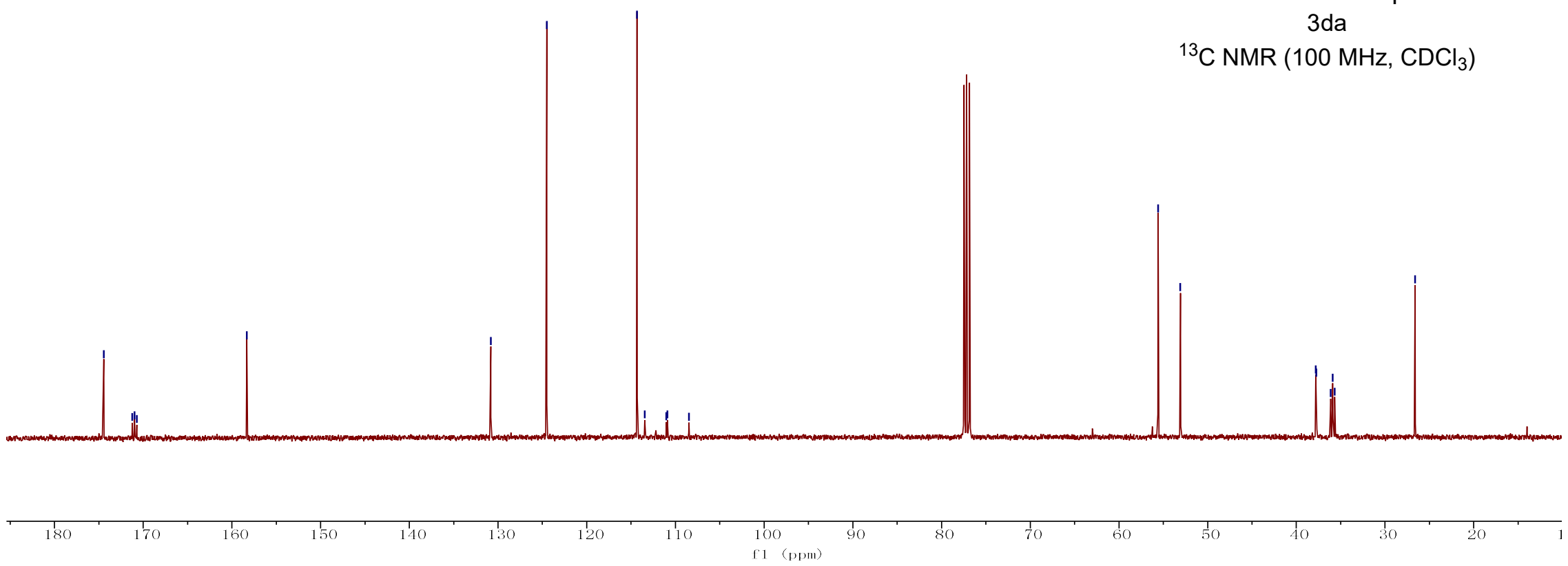


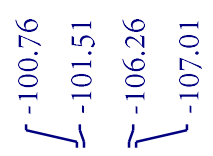

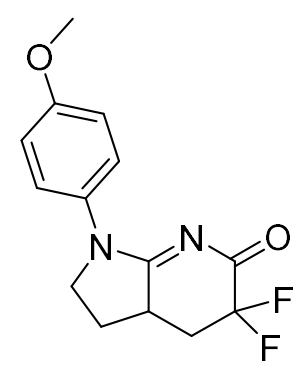

$3 \mathrm{da}$

${ }^{19} \mathrm{~F} \mathrm{NMR}\left(376 \mathrm{MHz}, \mathrm{CDCl}_{3}\right)$

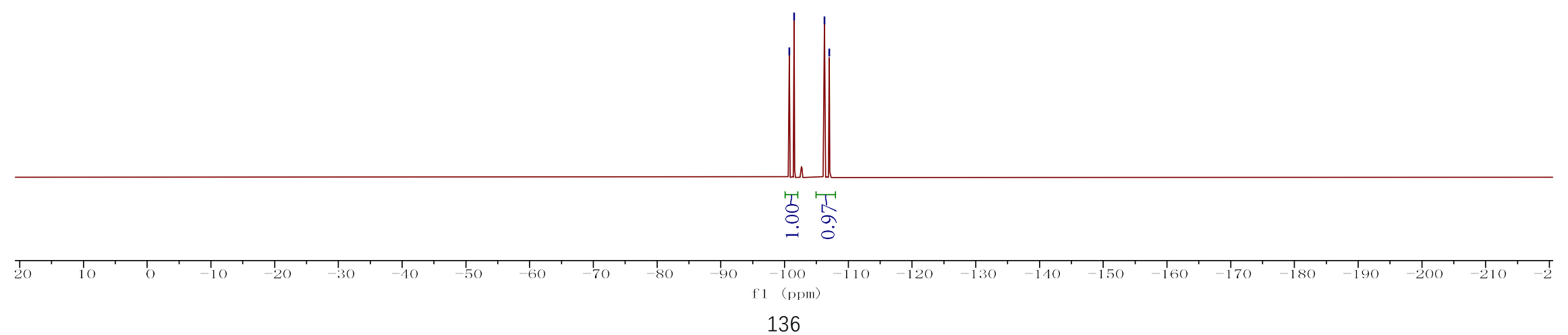




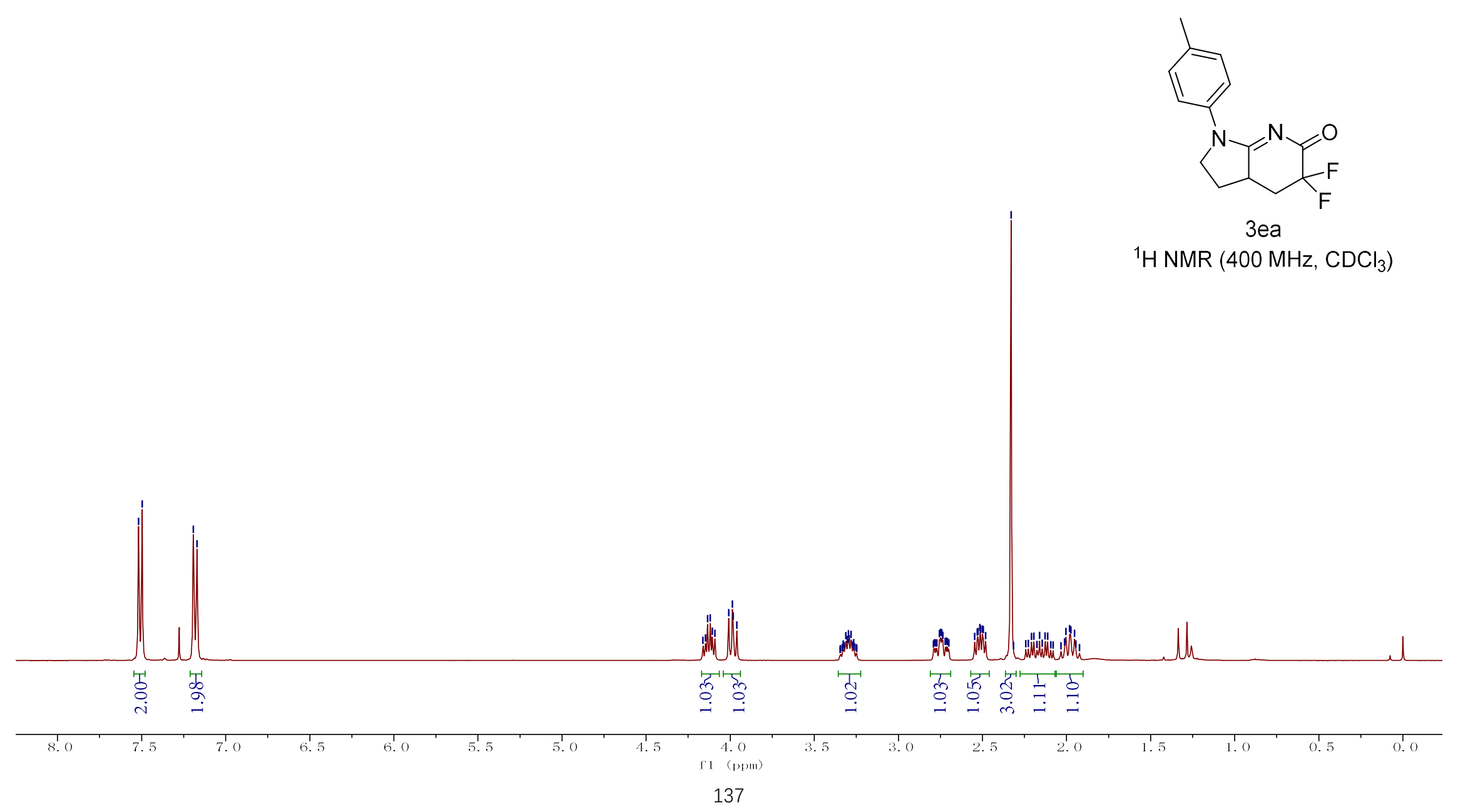




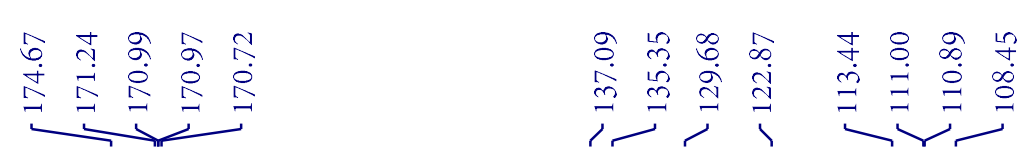
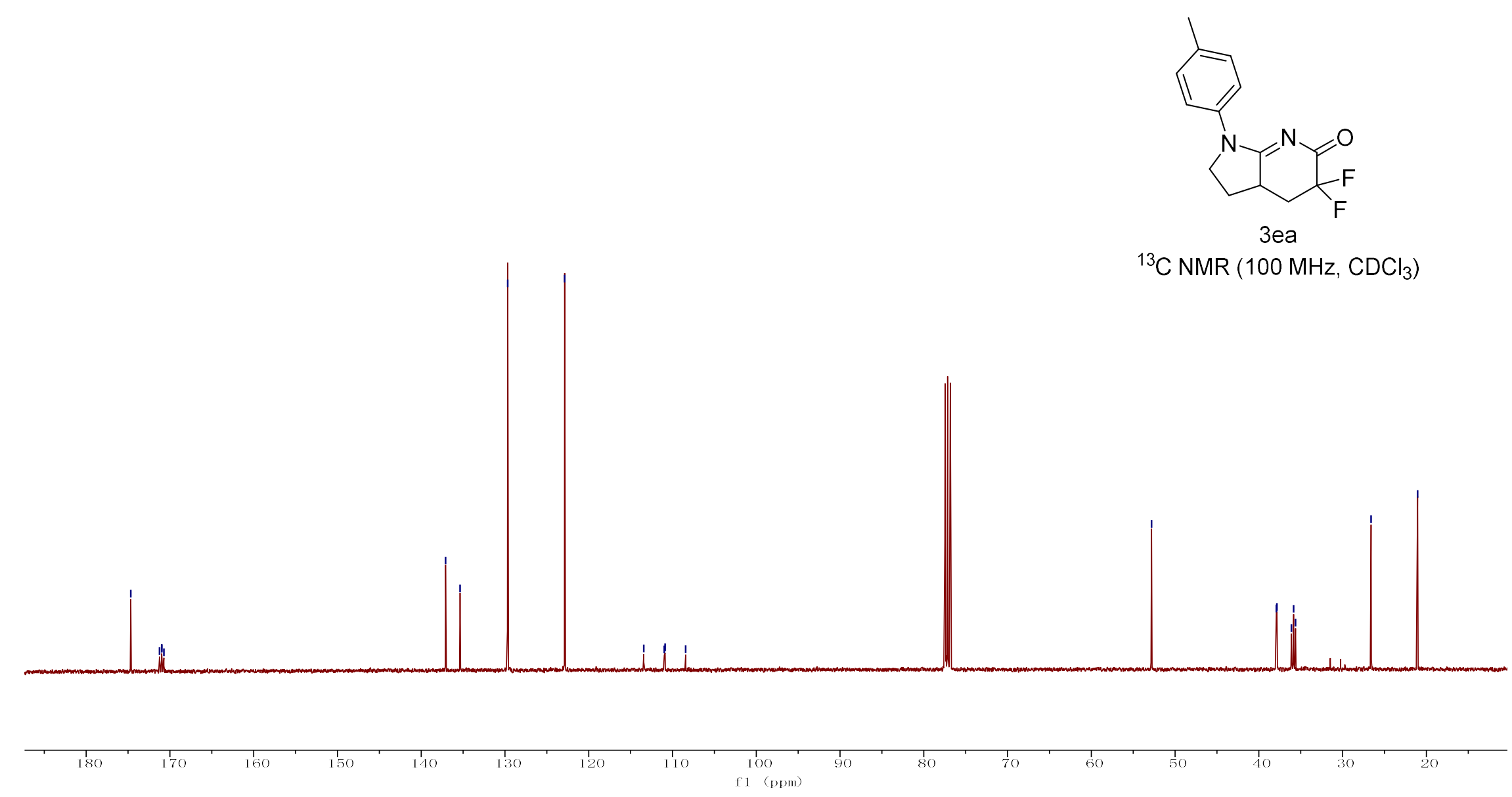

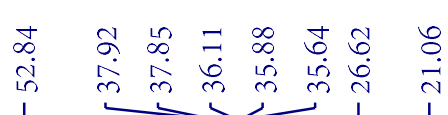

${ }^{13} \mathrm{C} \mathrm{NMR}\left(100 \mathrm{MHz}, \mathrm{CDCl}_{3}\right)$ 
$\begin{array}{llll}0 & - & 0 & \infty \\ \infty & 0 & \Omega \\ 0 & \dot{0} & 0 & \dot{0} \\ 0 & 0 & 0 & 0 \\ 1 & 1 & 1 & 1\end{array}$

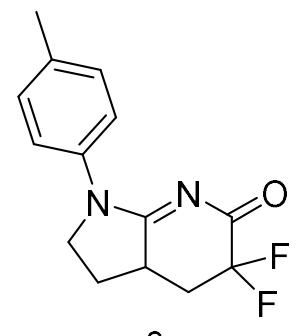

3 ea

${ }^{19} \mathrm{~F} \mathrm{NMR} \mathrm{(376} \mathrm{MHz}, \mathrm{CDCl}_{3}$ )

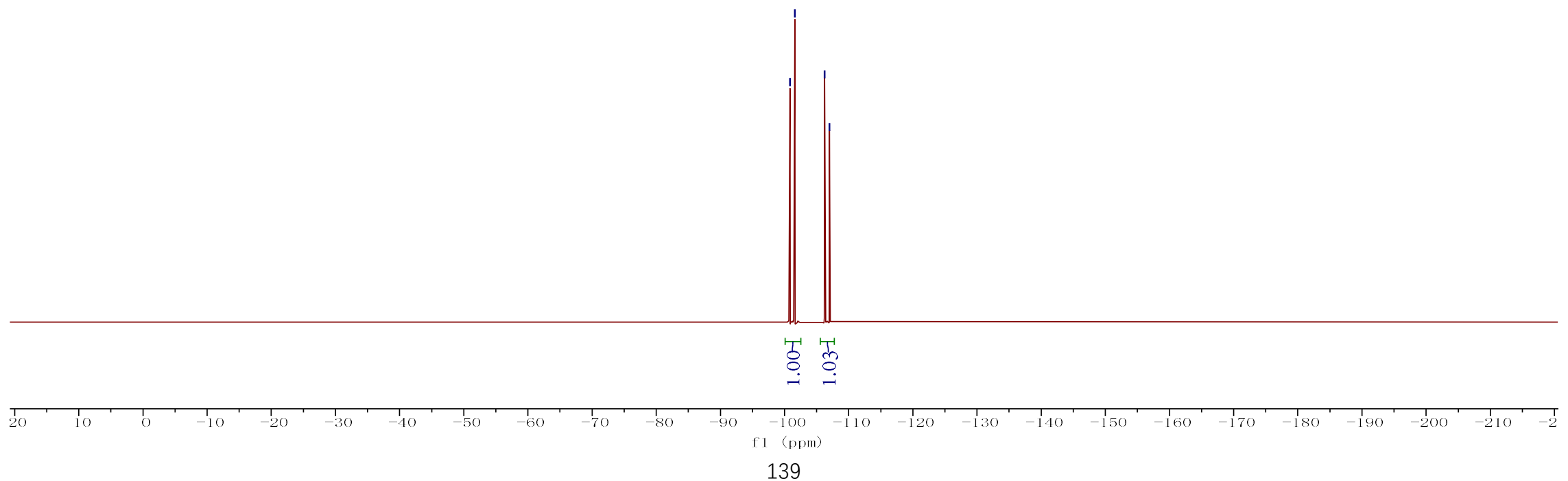




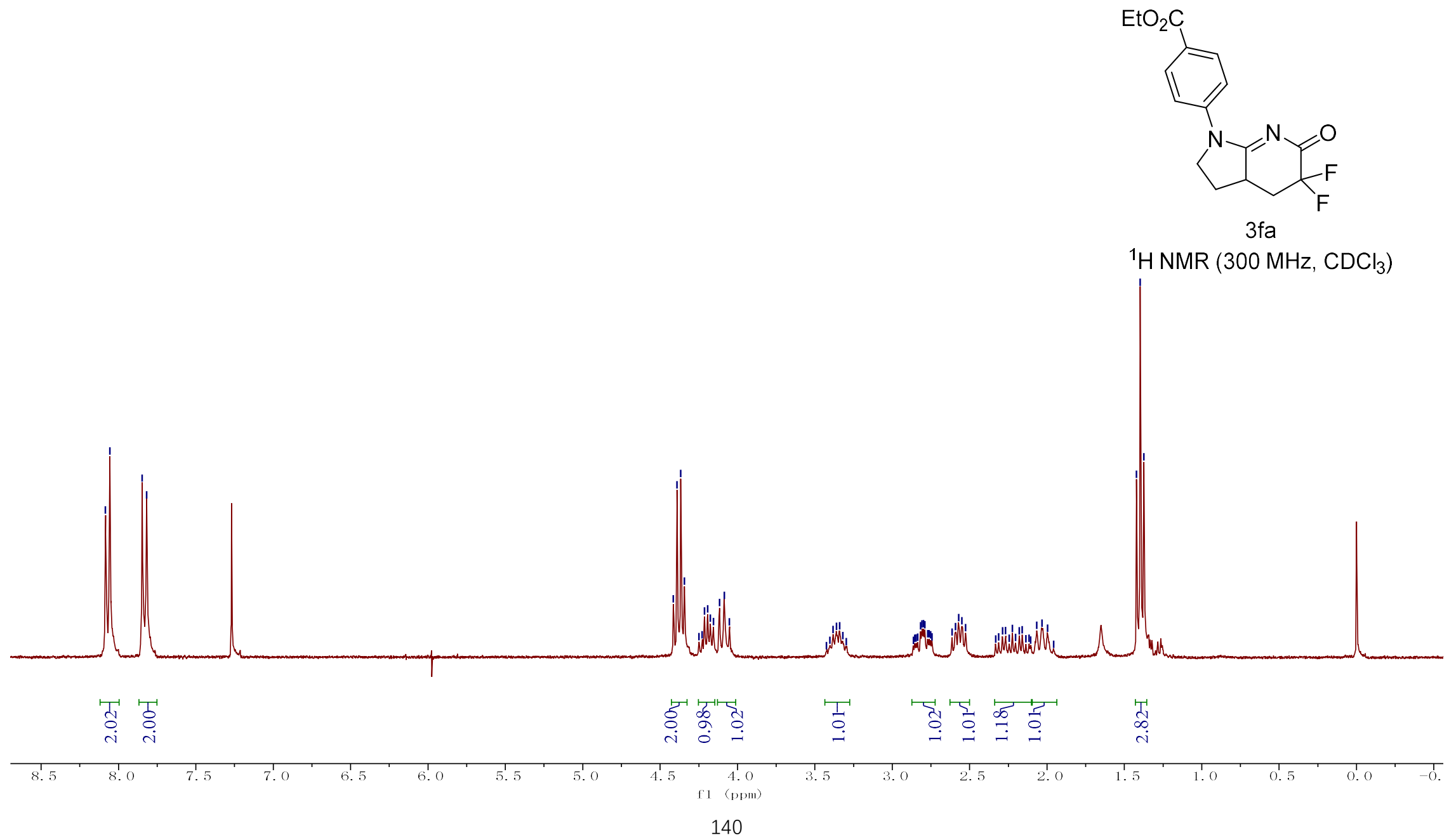


$\mathrm{EtO}_{2} \mathrm{C}$

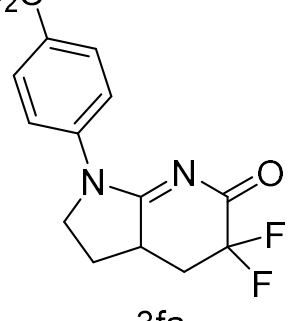

$3 \mathrm{fa}$

${ }^{13} \mathrm{C} \mathrm{NMR}\left(100 \mathrm{MHz}, \mathrm{CDCl}_{3}\right)$
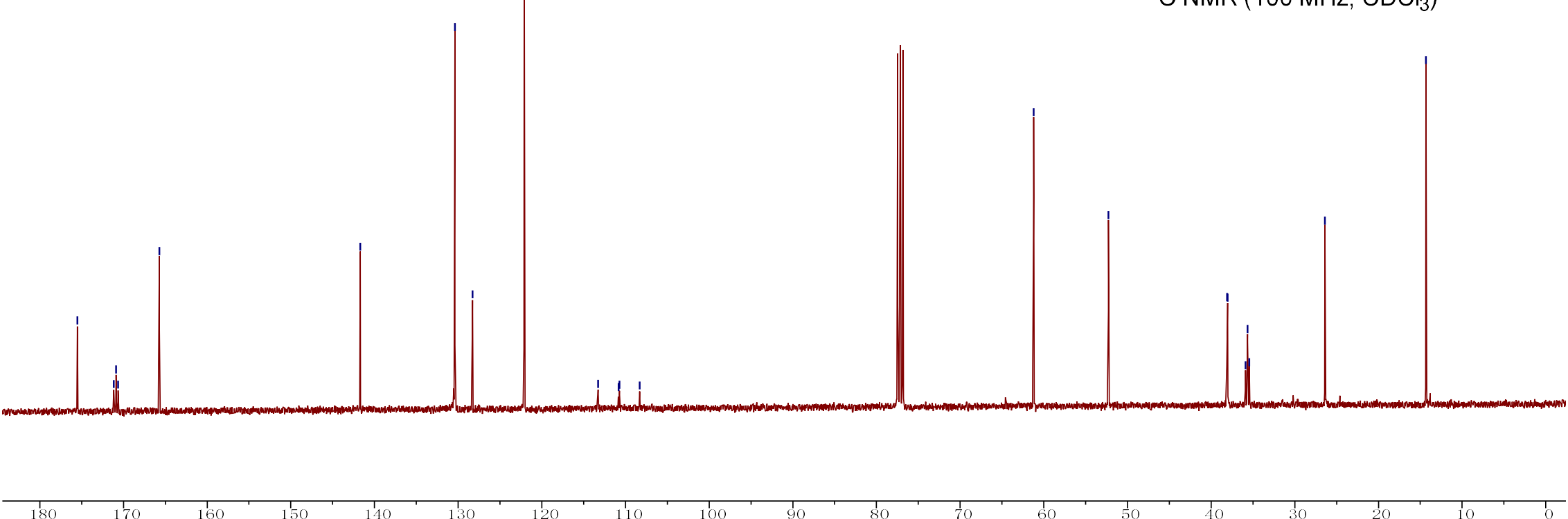

170
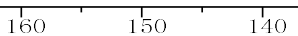

130

120

110

80

30 


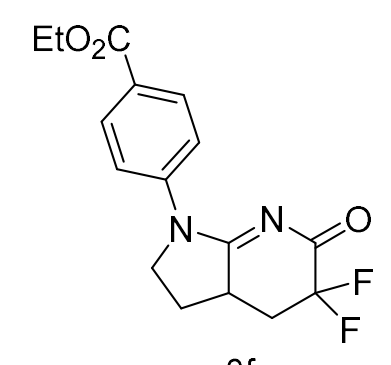

${ }^{19} \mathrm{~F} \mathrm{NMR}\left(376 \mathrm{MHz}, \mathrm{CDCl}_{3}\right)$

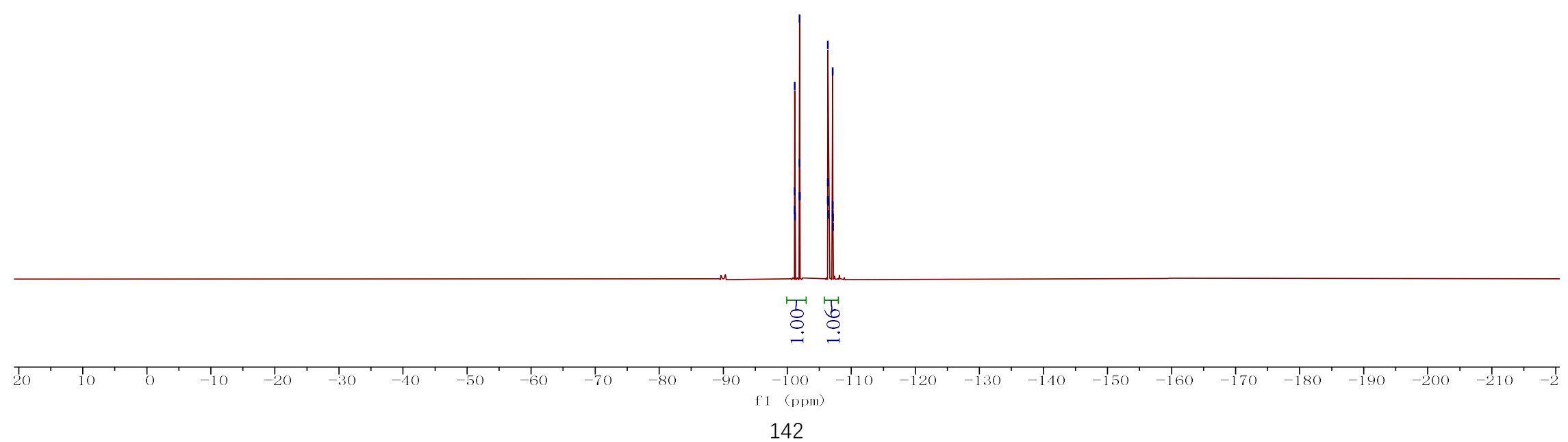




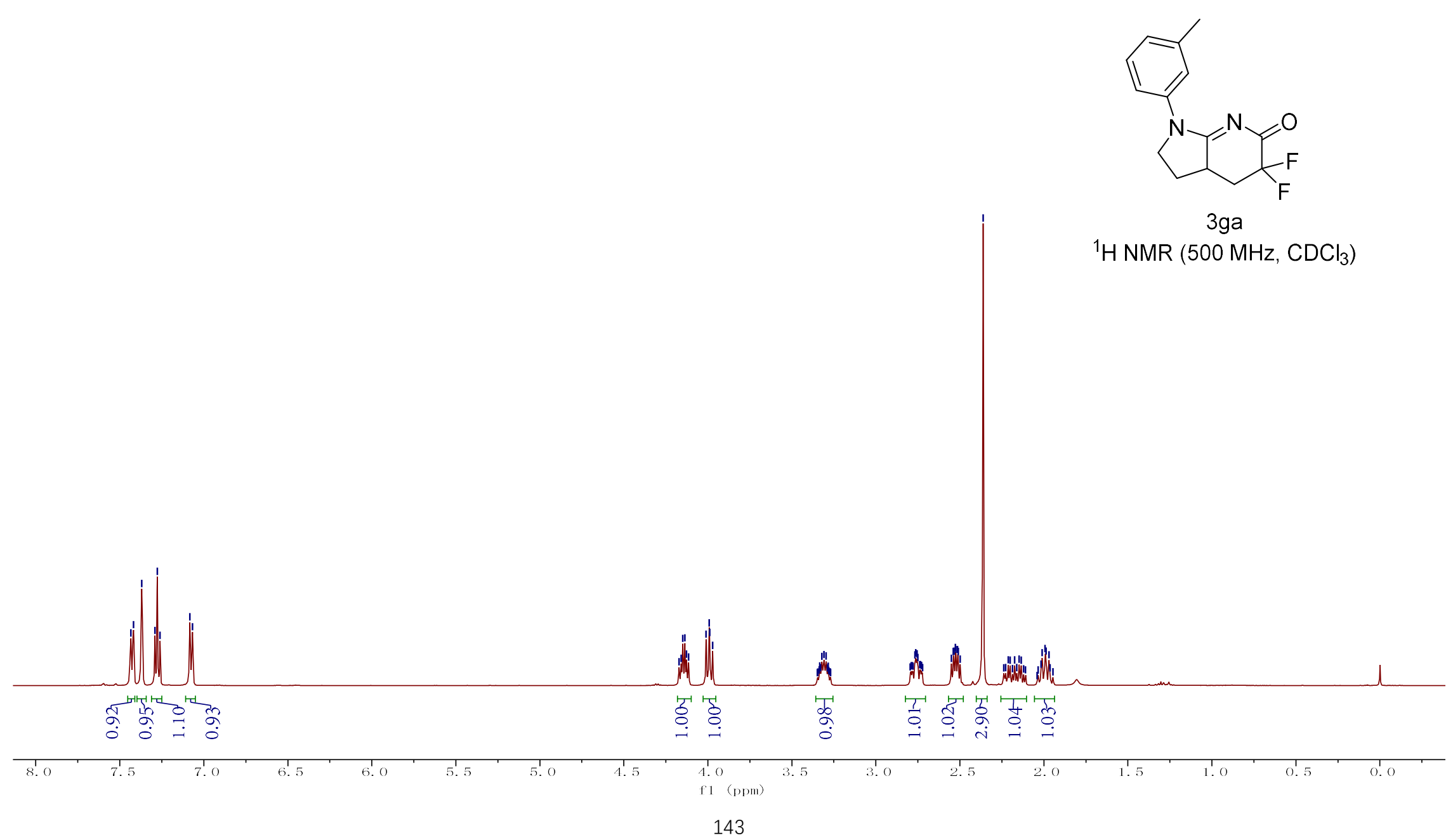



椱望置

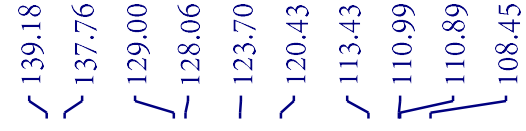

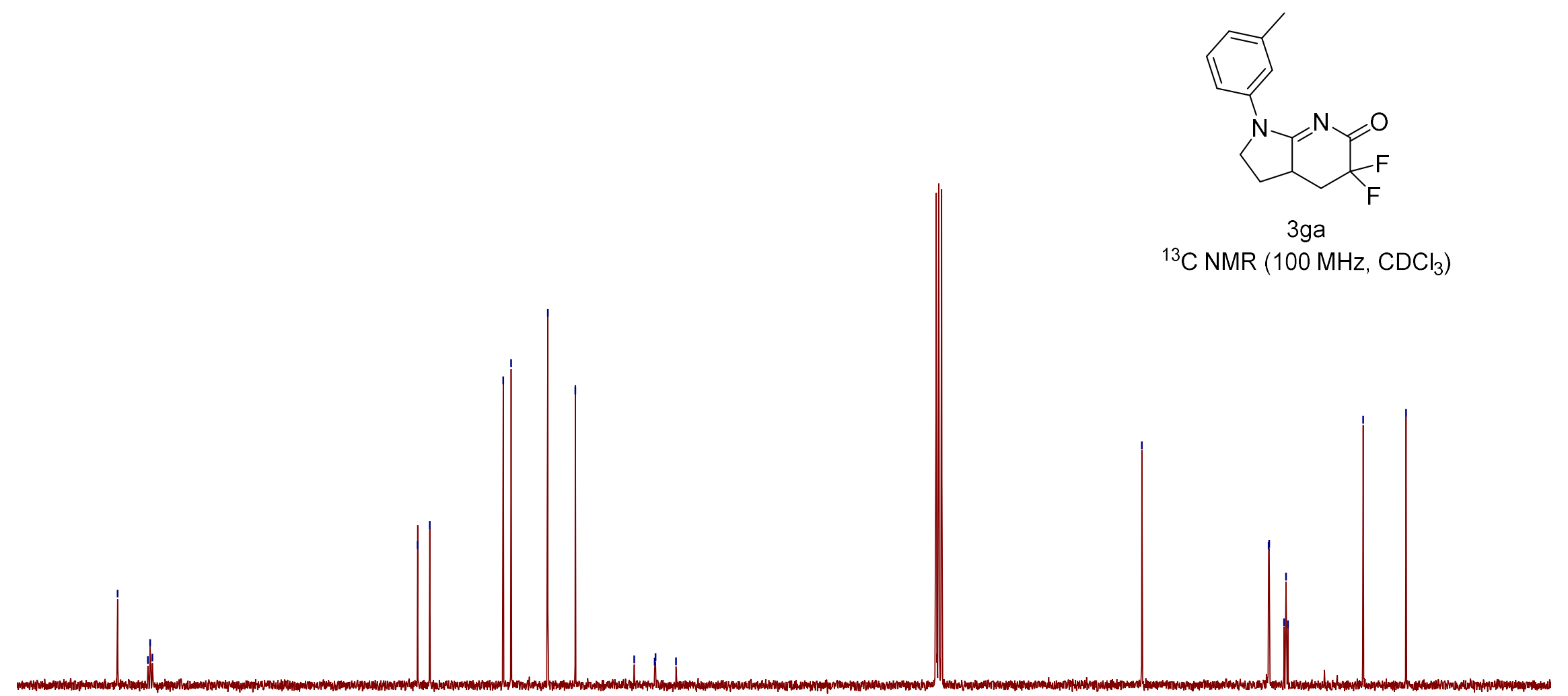

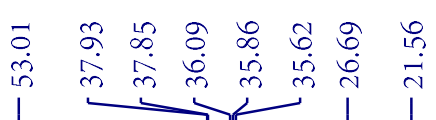

$$
3 g a
$$

${ }^{13} \mathrm{C} \mathrm{NMR}\left(100 \mathrm{MHz}_{\mathrm{CDC}}\right)$

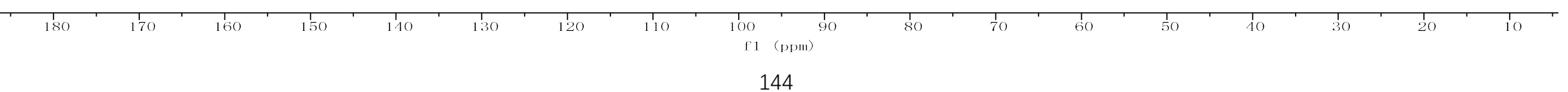




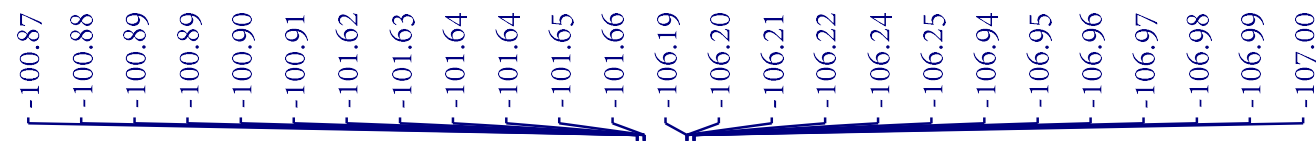

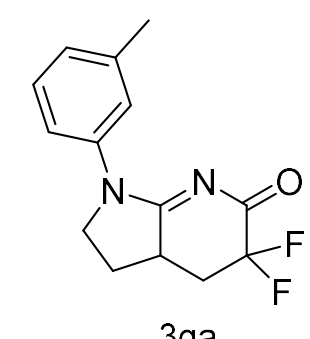

${ }^{19} \mathrm{~F}$ NMR (376 MHz, $\mathrm{CDCl}_{3}$ )

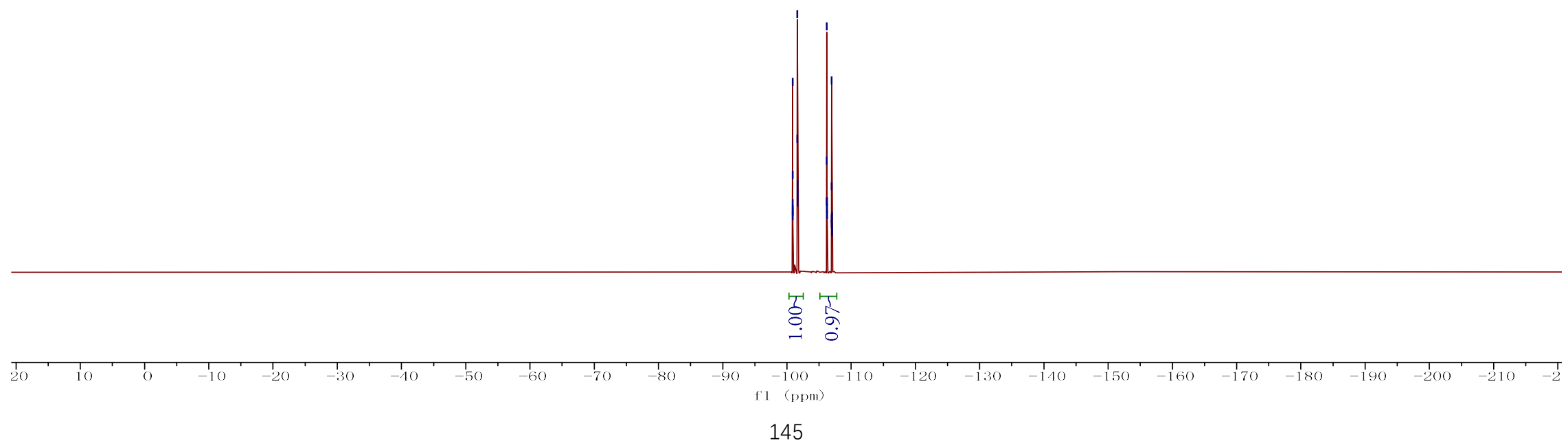




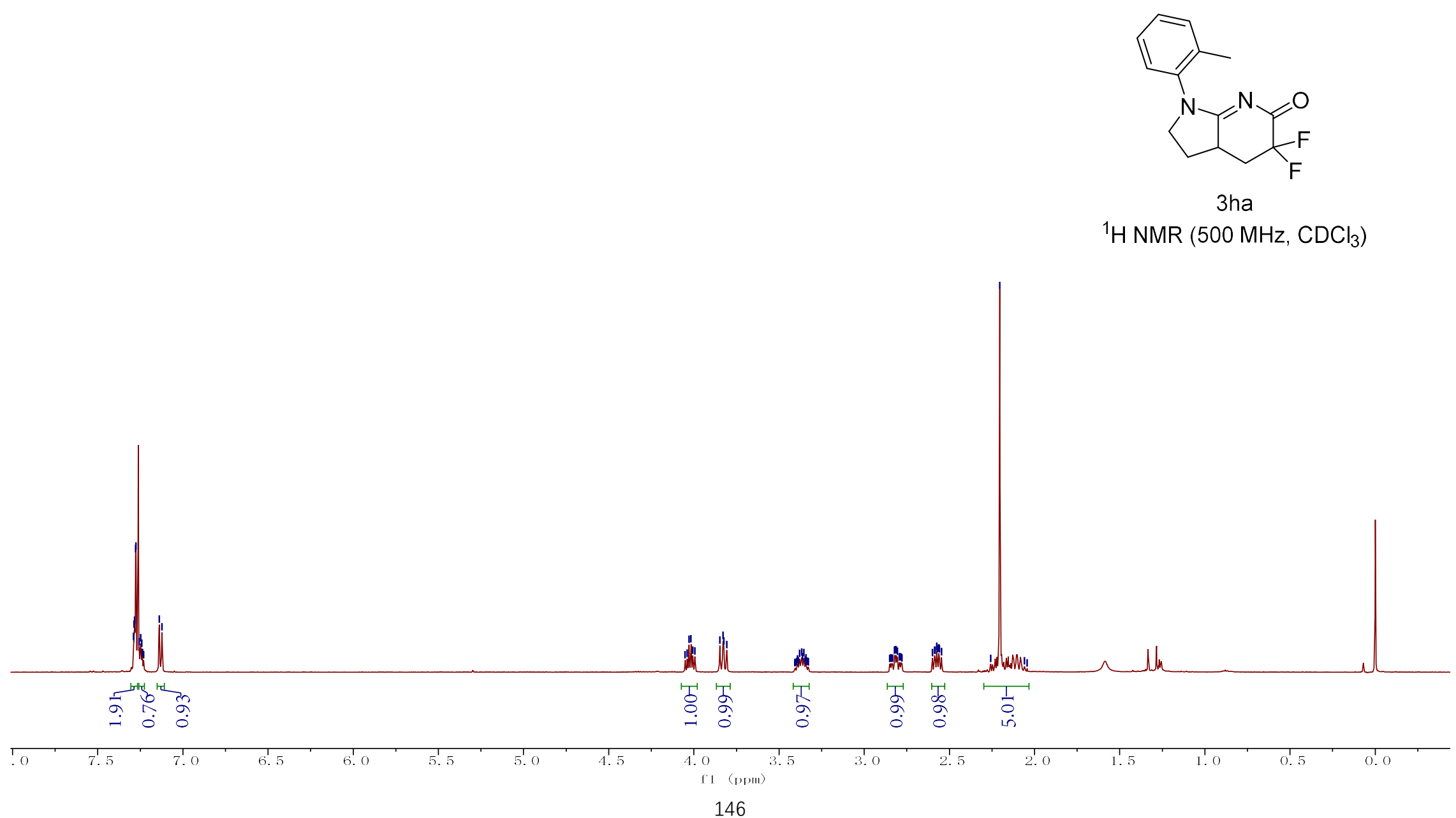




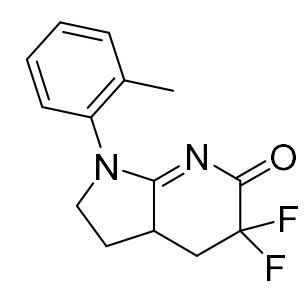

3ha

${ }^{13} \mathrm{C} \mathrm{NMR}\left(100 \mathrm{MHz}, \mathrm{CDCl}_{3}\right)$
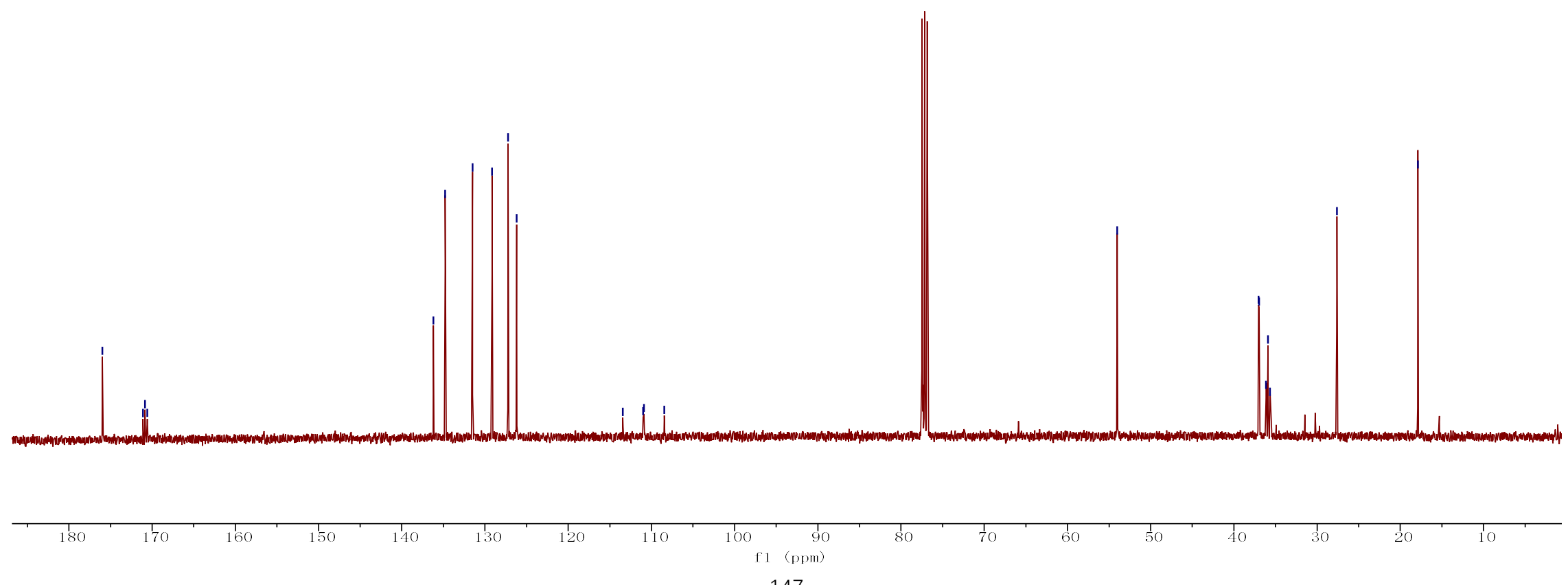

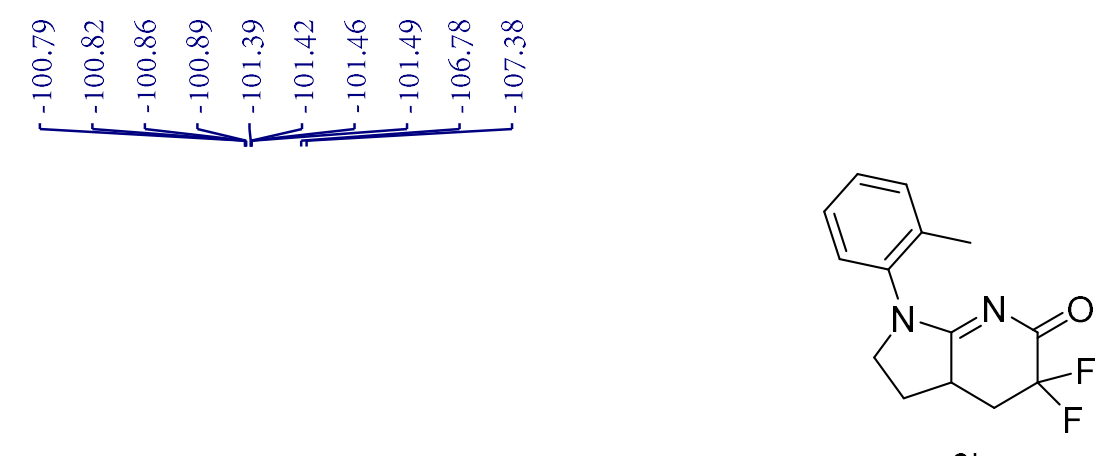

3ha

${ }^{19} \mathrm{~F} \mathrm{NMR}\left(470 \mathrm{MHz}, \mathrm{CDCl}_{3}\right)$

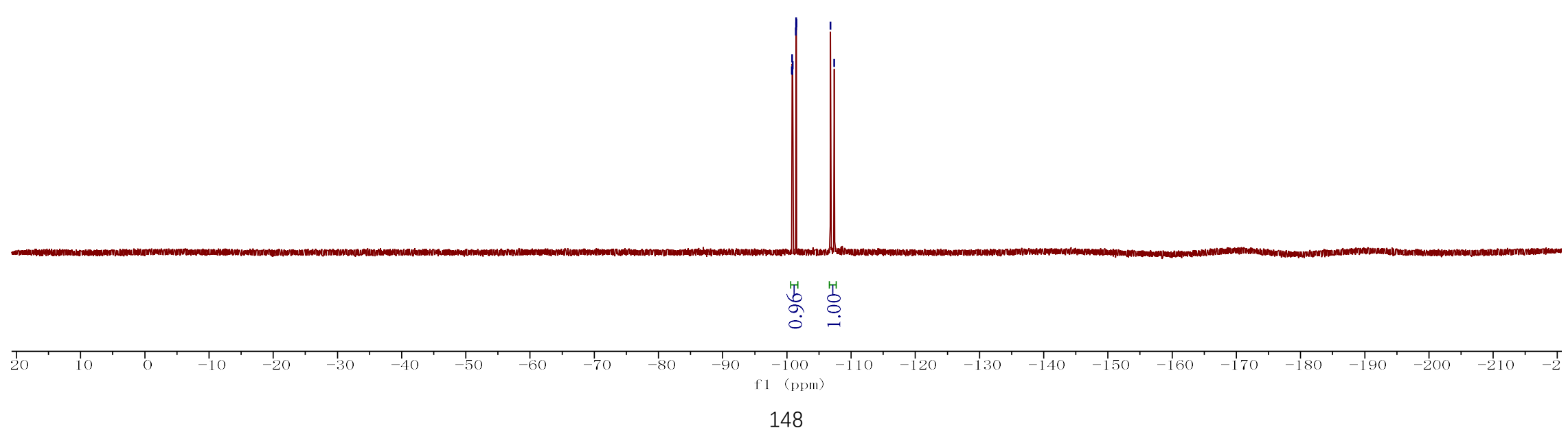




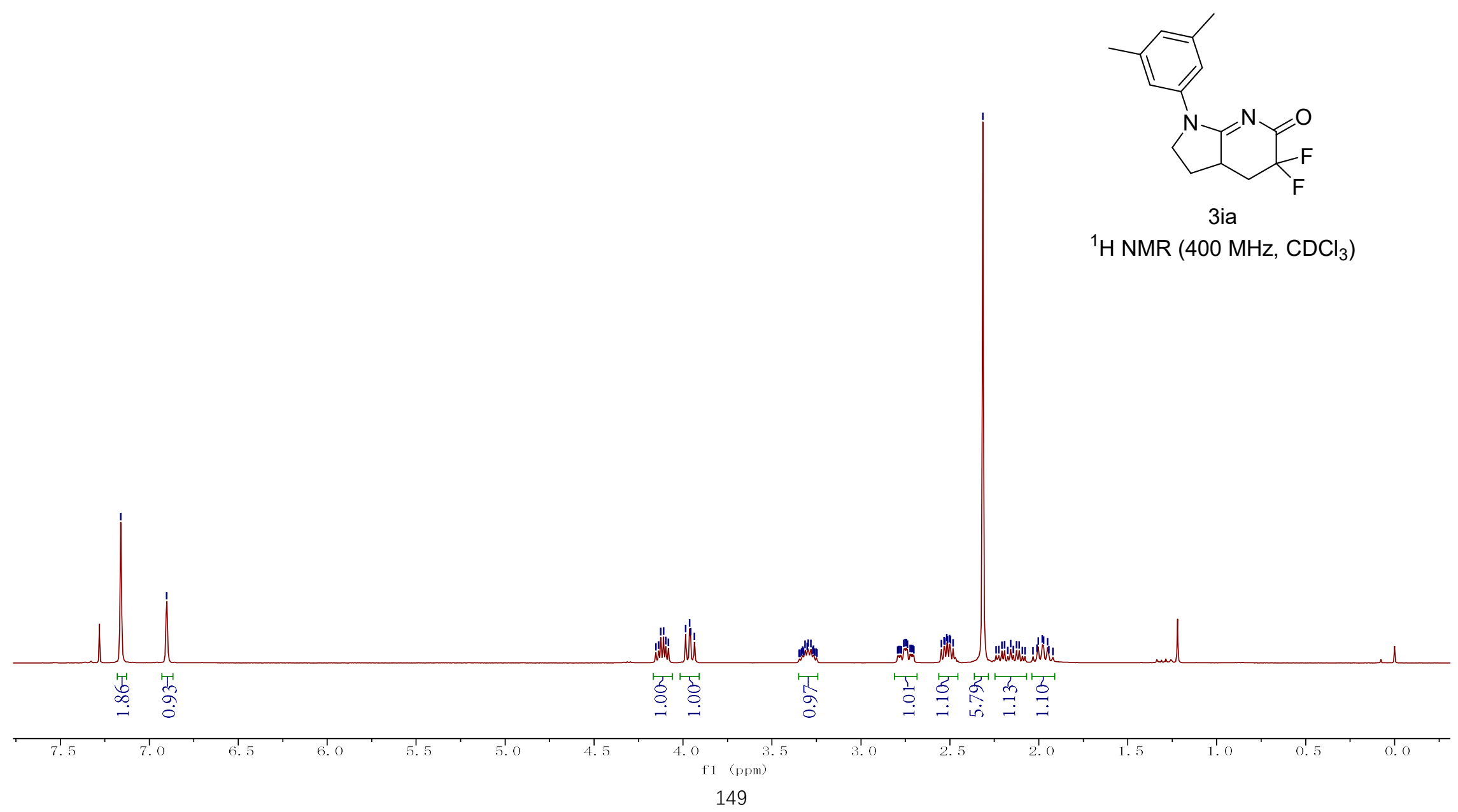



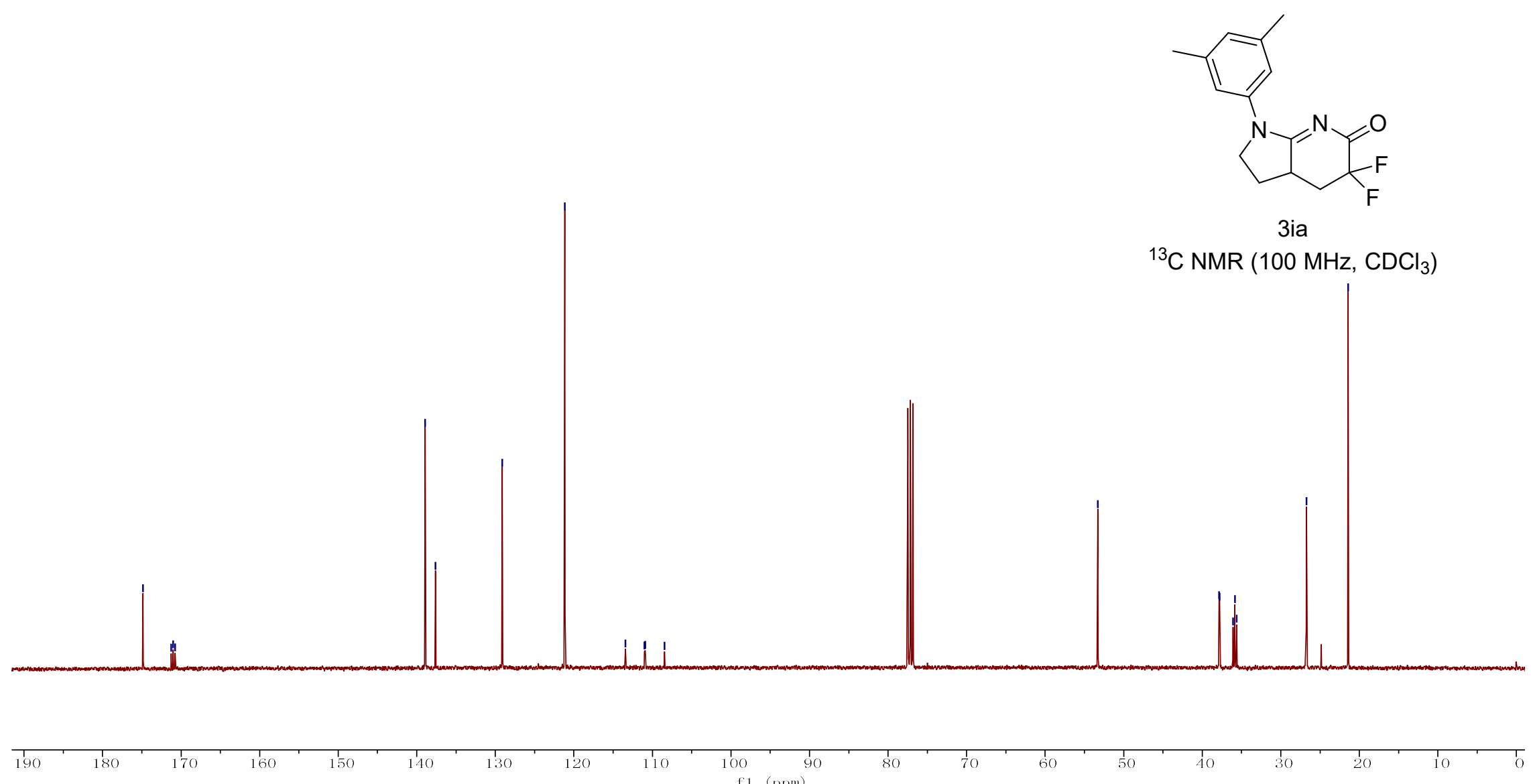


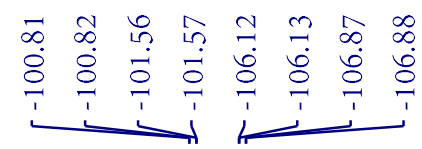

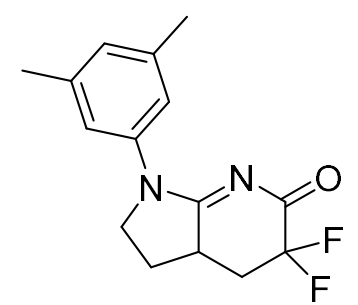

3ia

${ }^{19} \mathrm{~F}$ NMR (376 MHz, CDCl $\left.\right|_{3}$ )

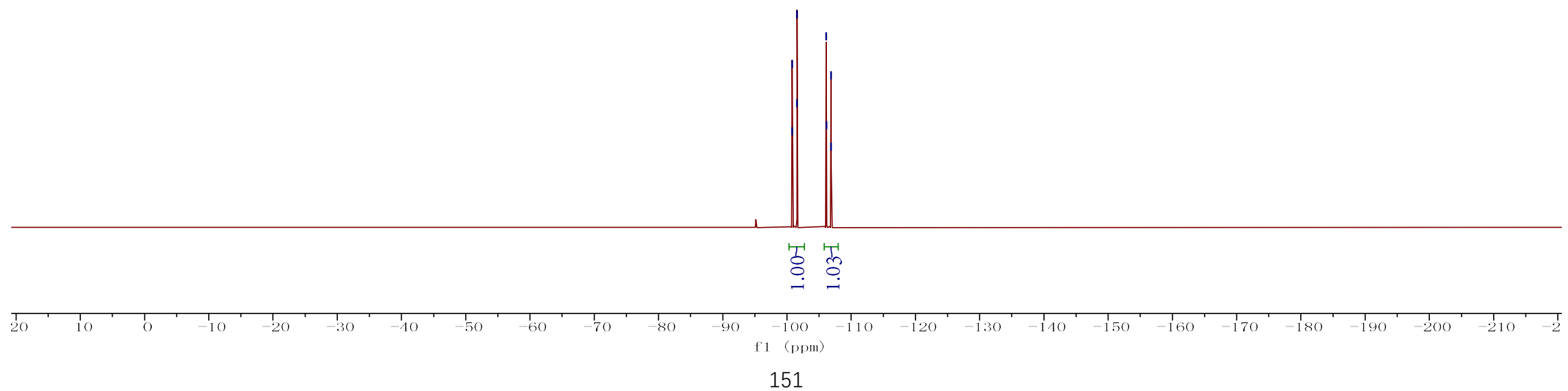




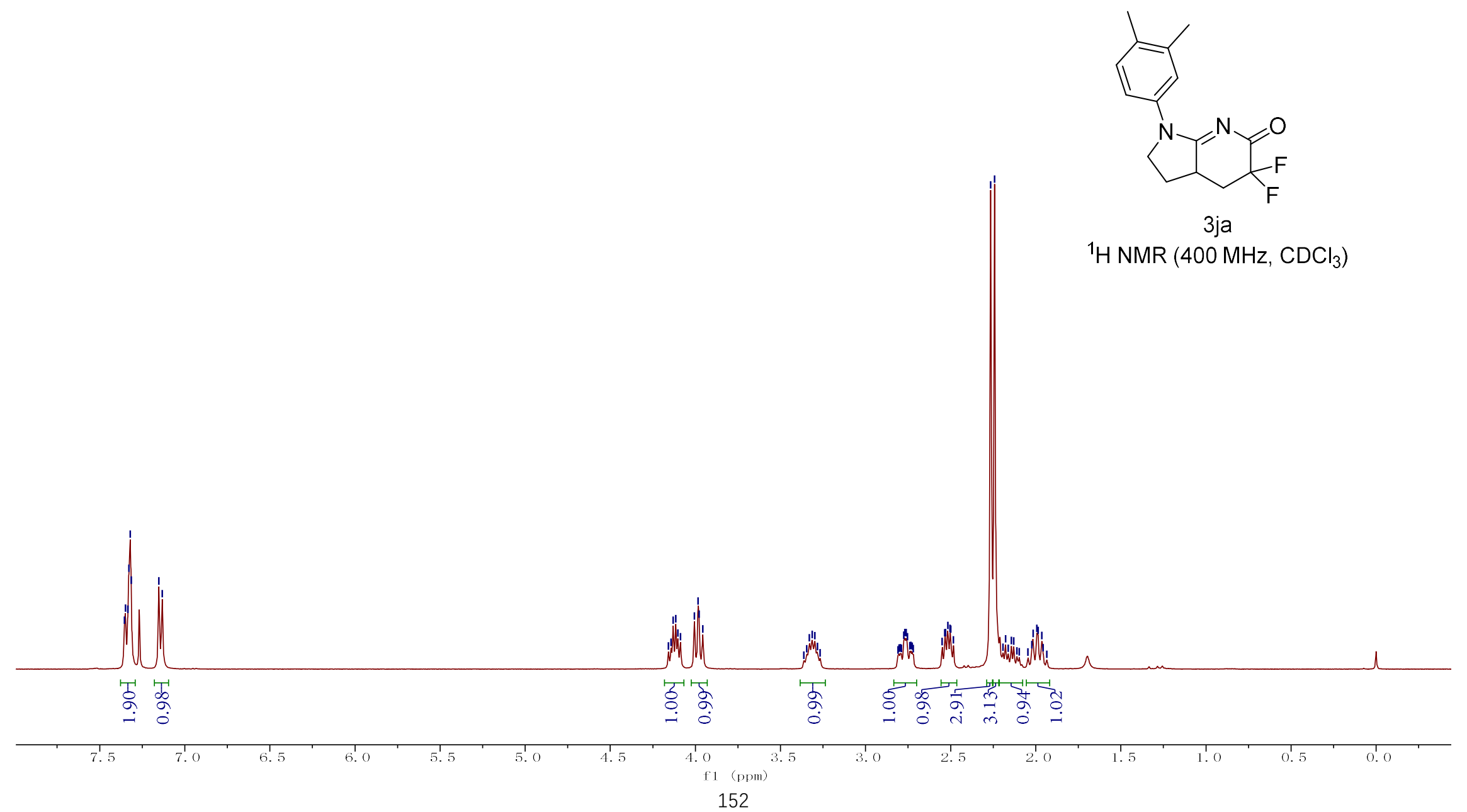




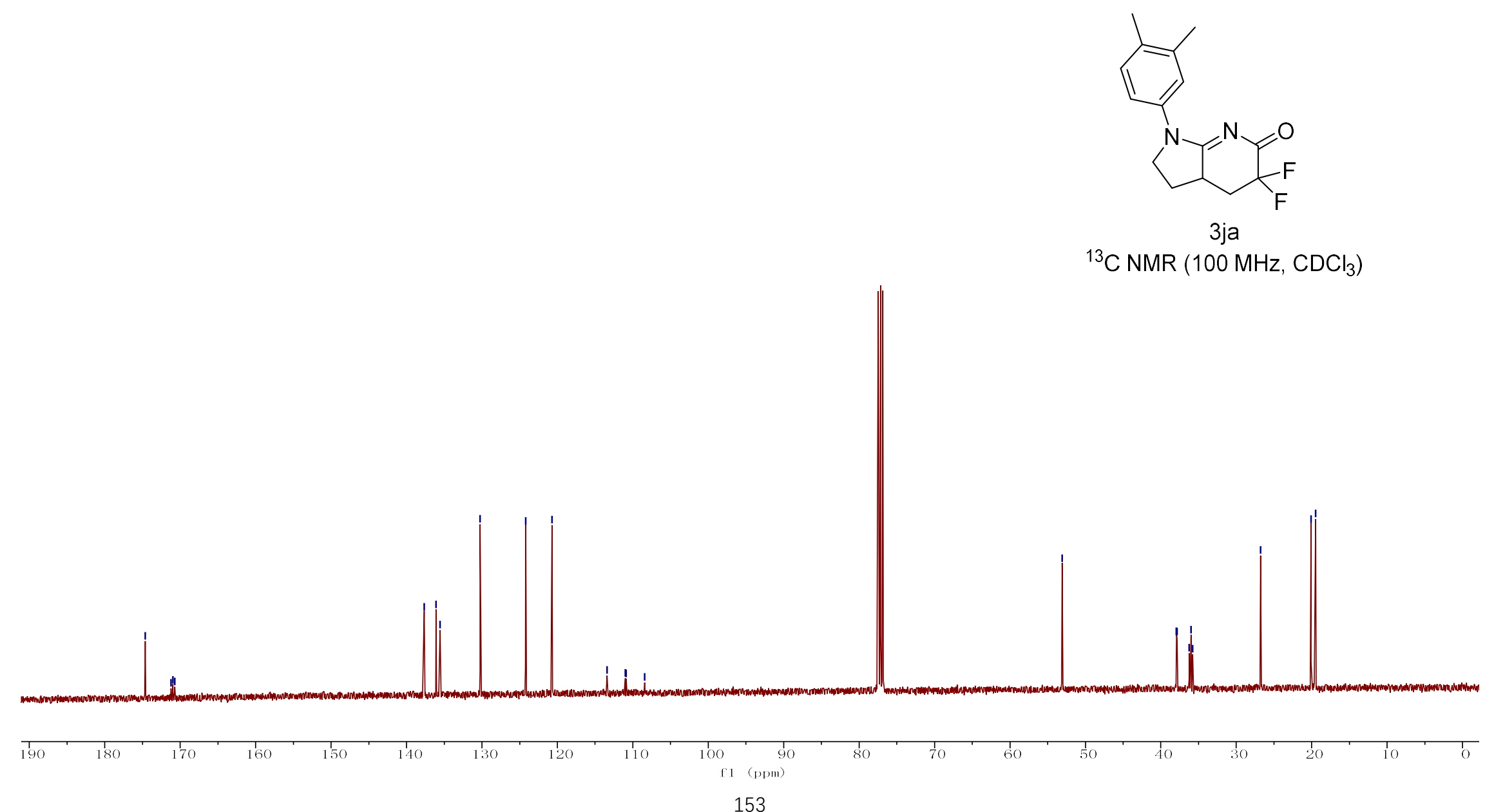


$\begin{array}{llll}\infty & 0 & 0 & 0 \\ 0 & 0 & 0 & 0 \\ 0 & 0 & 0 & 0 \\ 0 & 0 & 0 & 0 \\ 1 & 1 & 1 & 1\end{array}$

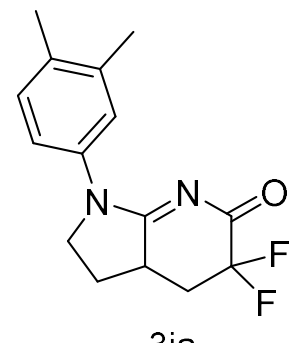

3ja

${ }^{19} \mathrm{~F} \mathrm{NMR}\left(376 \mathrm{MHz}, \mathrm{CDCl}_{3}\right)$

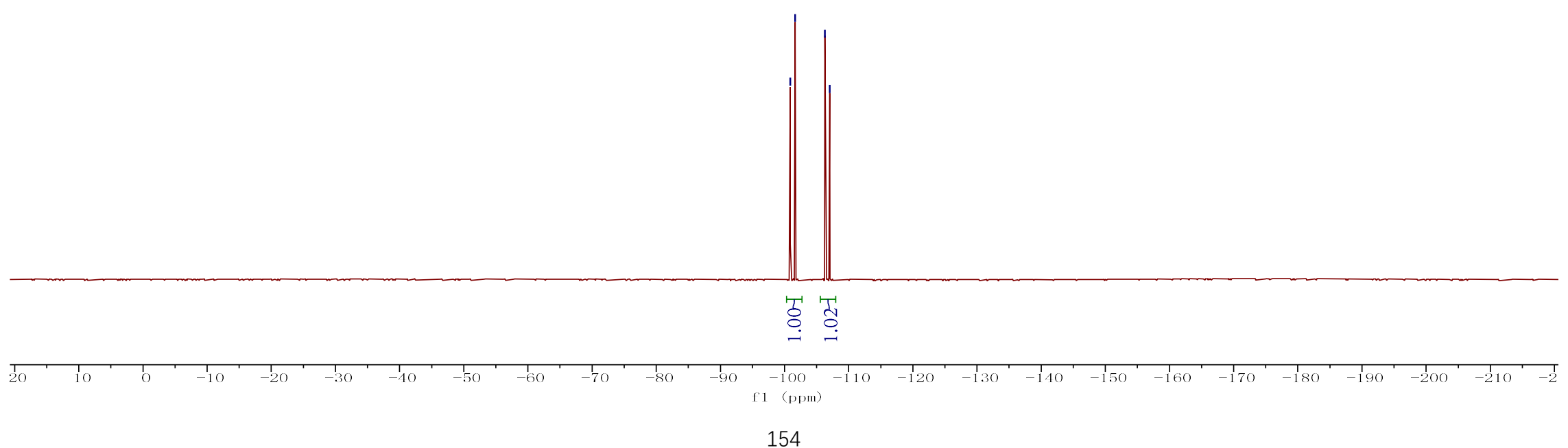




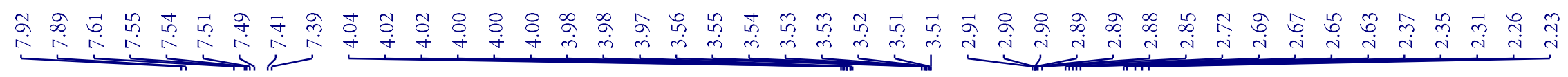

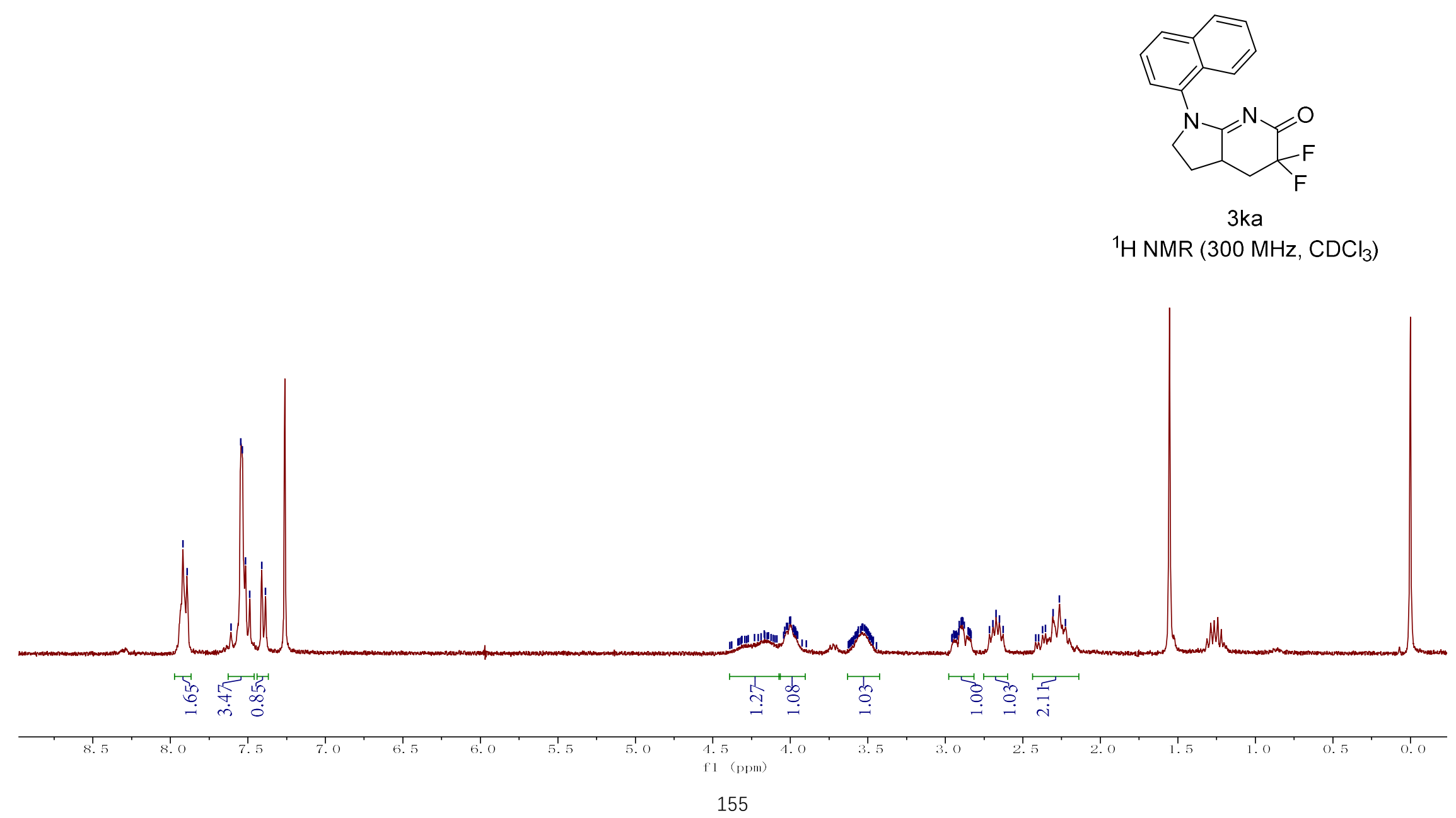



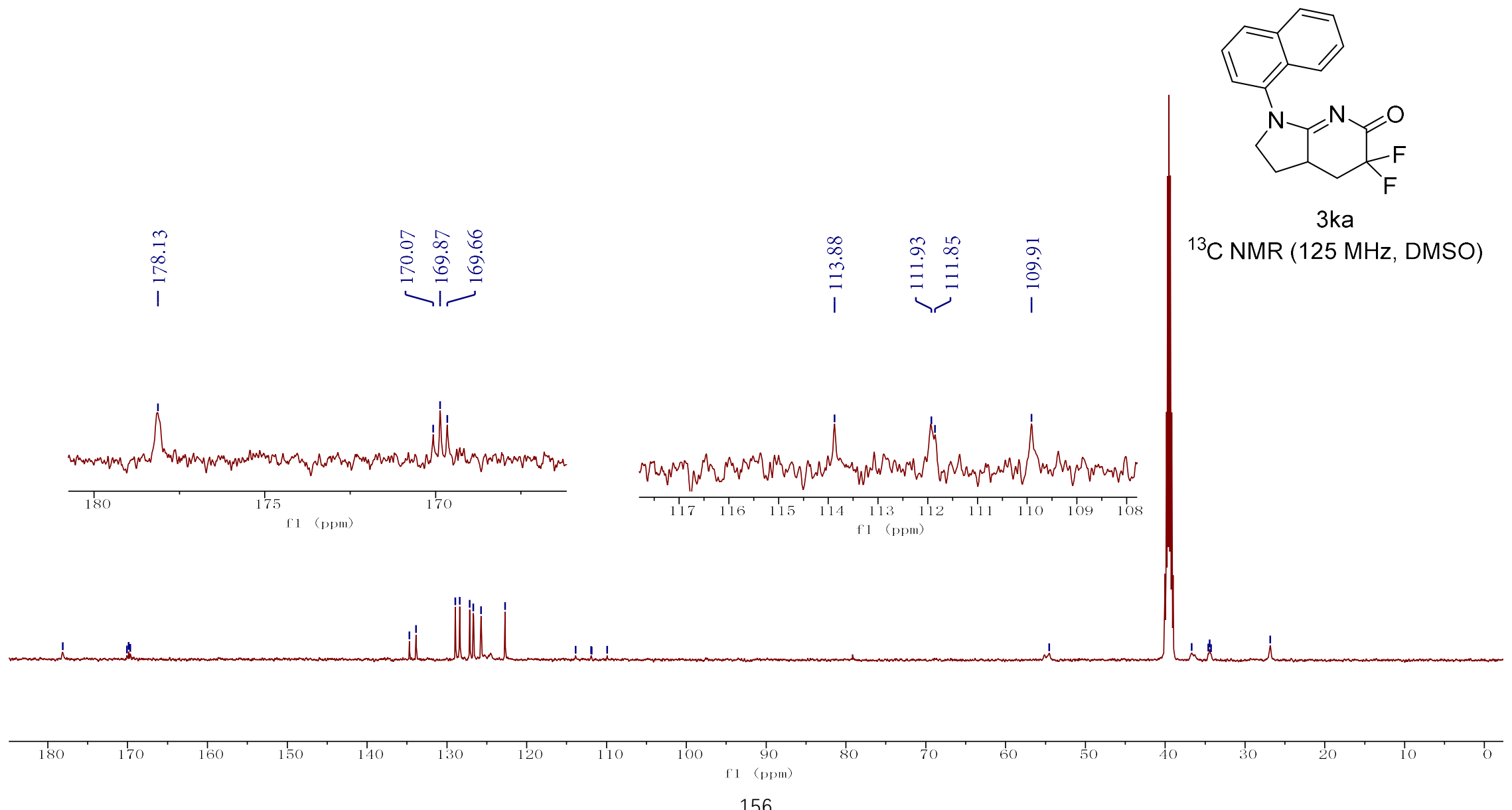


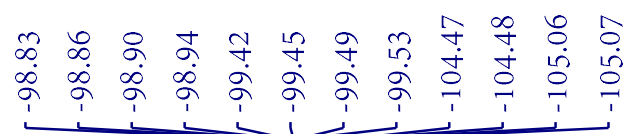

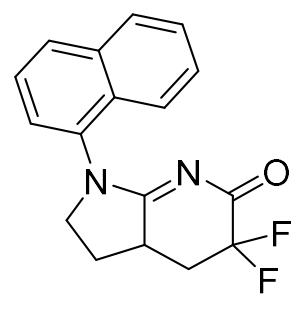

$3 \mathrm{ka}$

${ }^{19} \mathrm{~F} \mathrm{NMR}(470 \mathrm{MHz}, \mathrm{DMSO})$

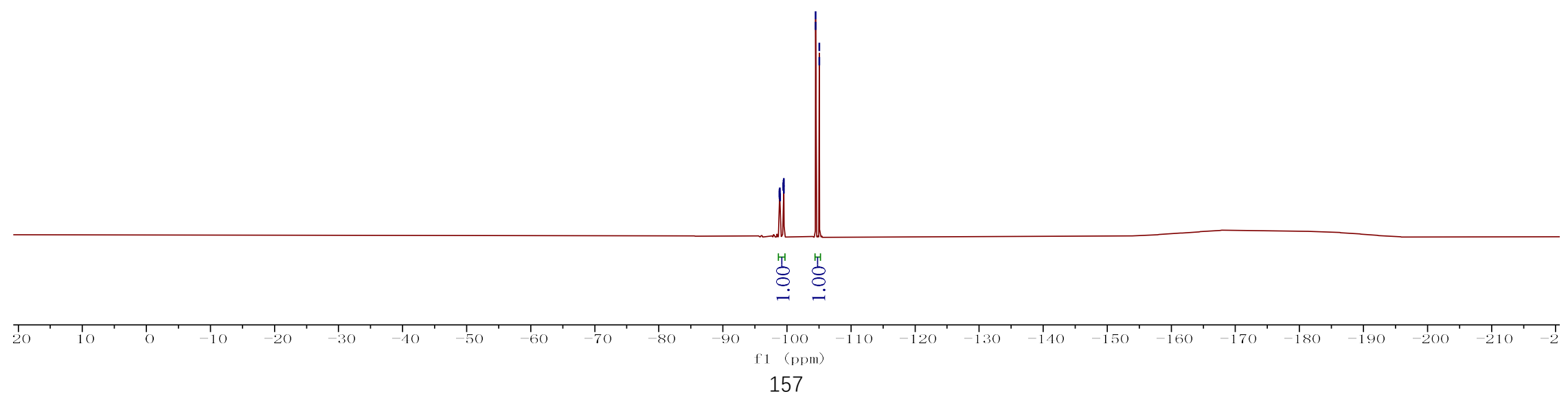




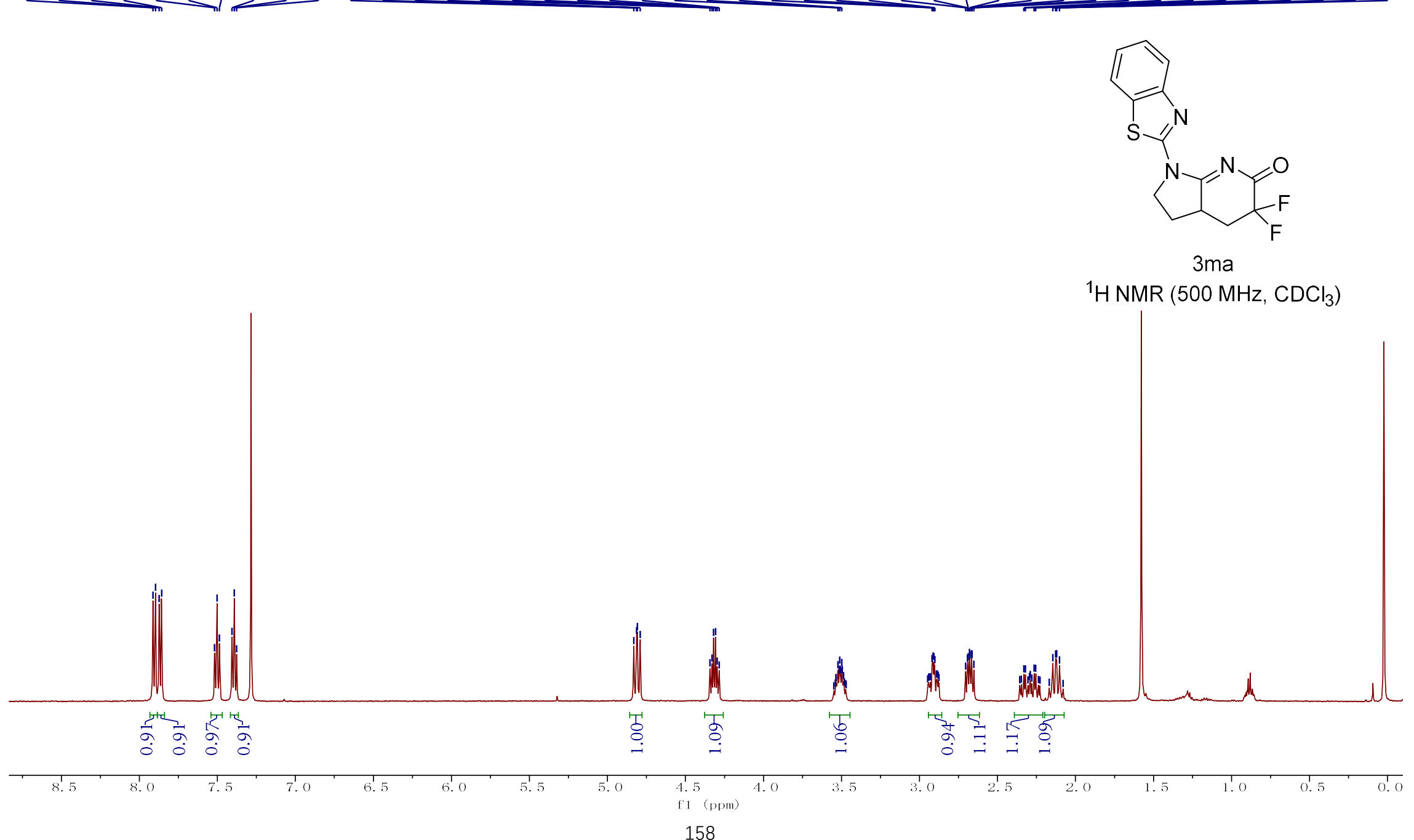



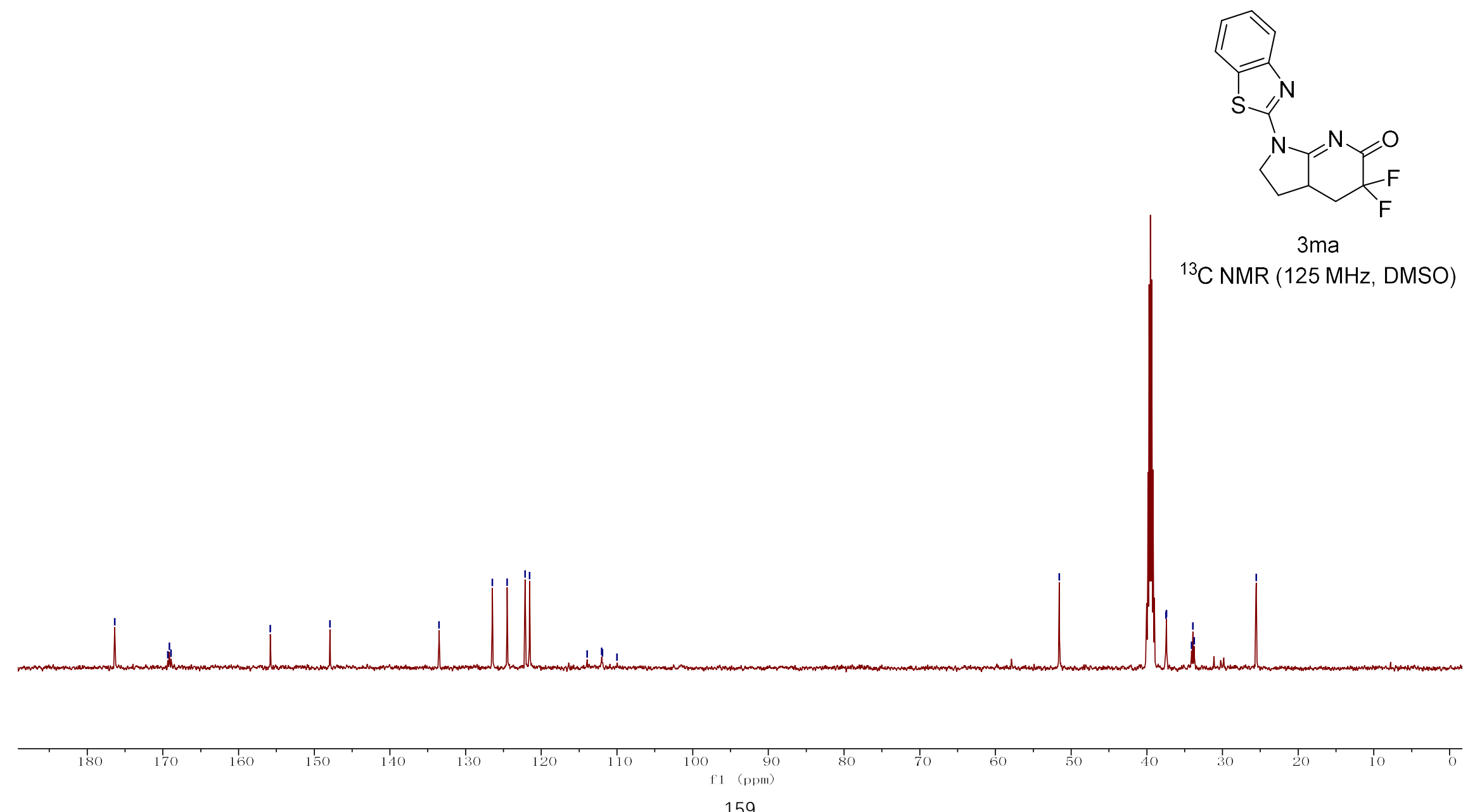


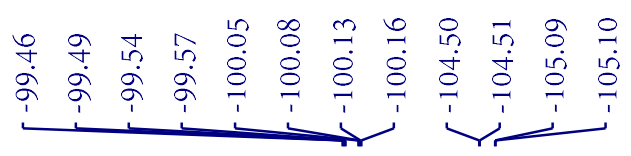

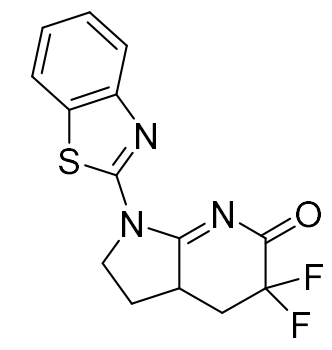

$3 \mathrm{ma}$

${ }^{13} \mathrm{C}$ NMR (470 MHz, DMSO)

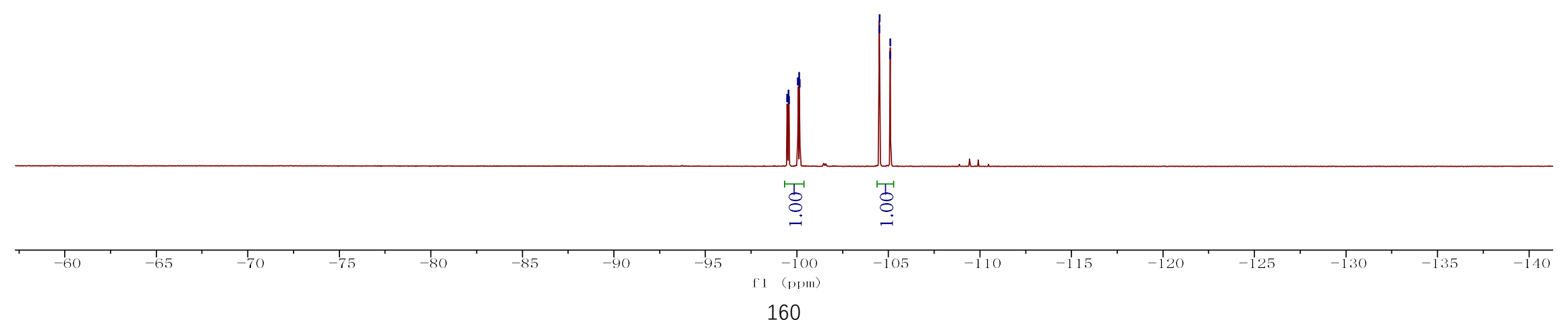




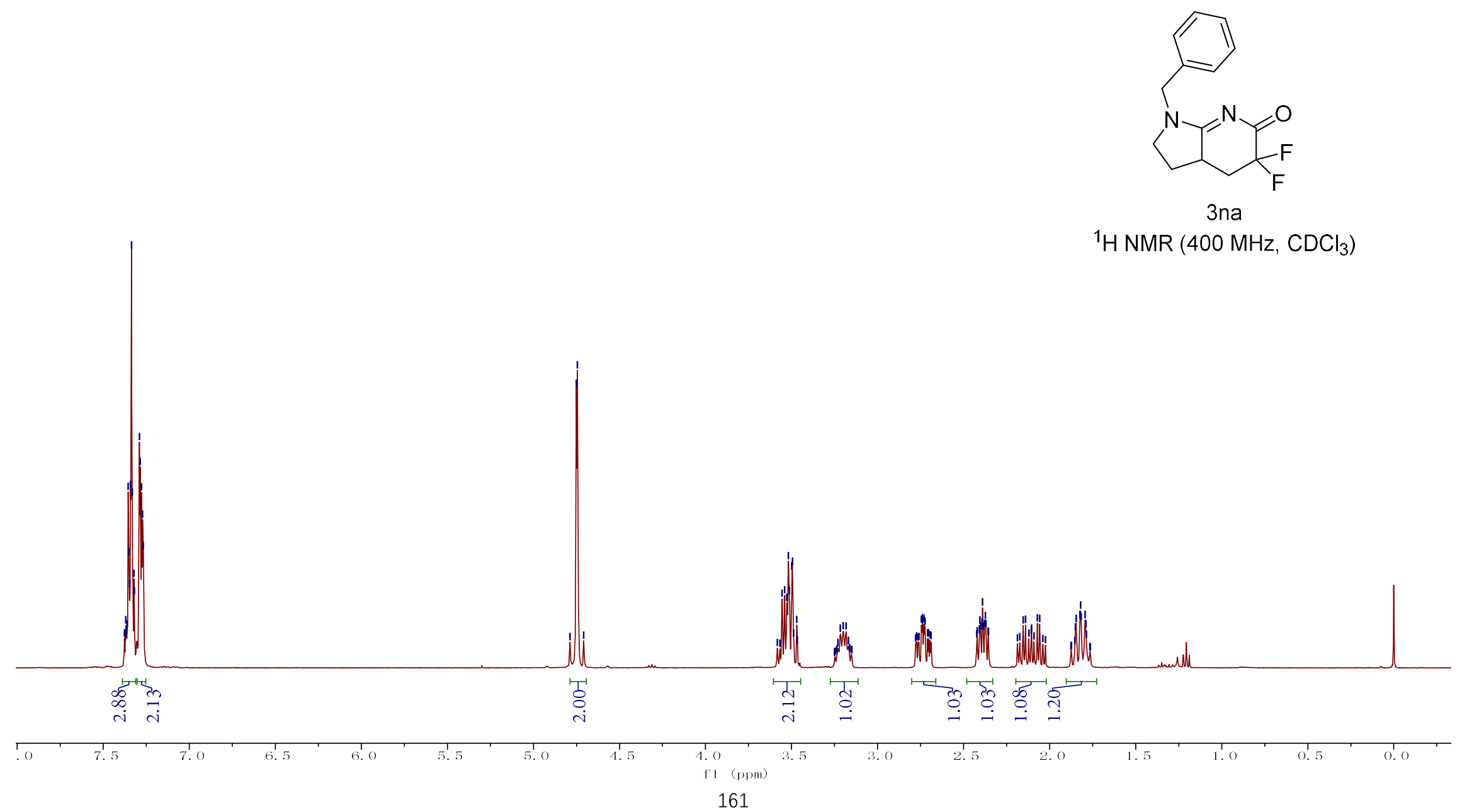




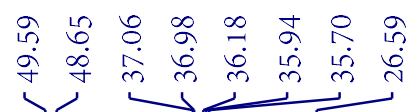

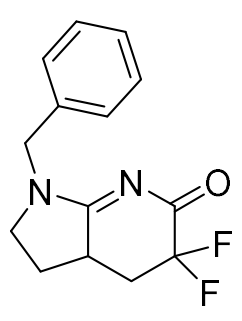

3na

${ }^{13} \mathrm{C}$ NMR $\left(100 \mathrm{MHz}, \mathrm{CDCl}_{3}\right)$
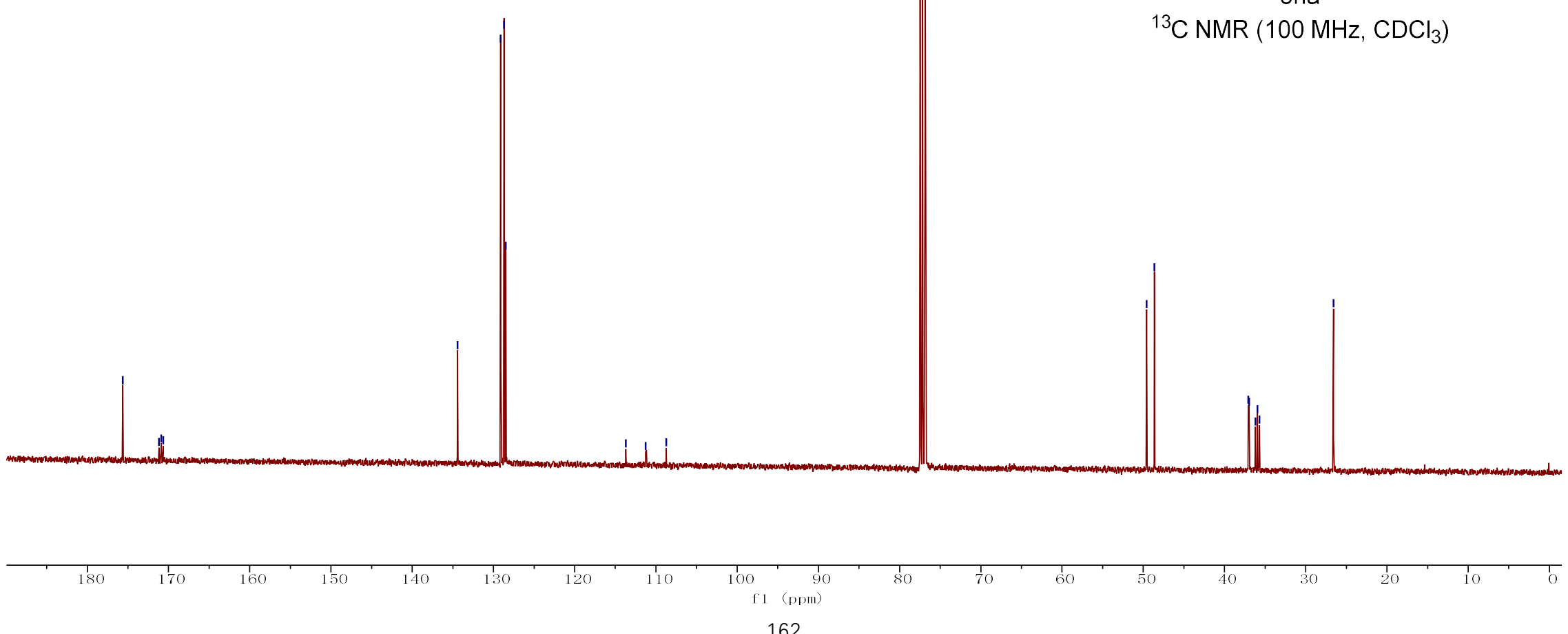


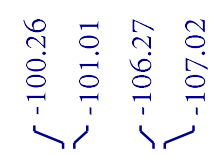

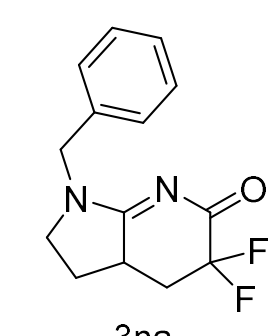

$\left.{ }^{19} \mathrm{~F} \mathrm{NMR} \mathrm{(376} \mathrm{MHz,} \mathrm{CDCl}_{3}\right)$

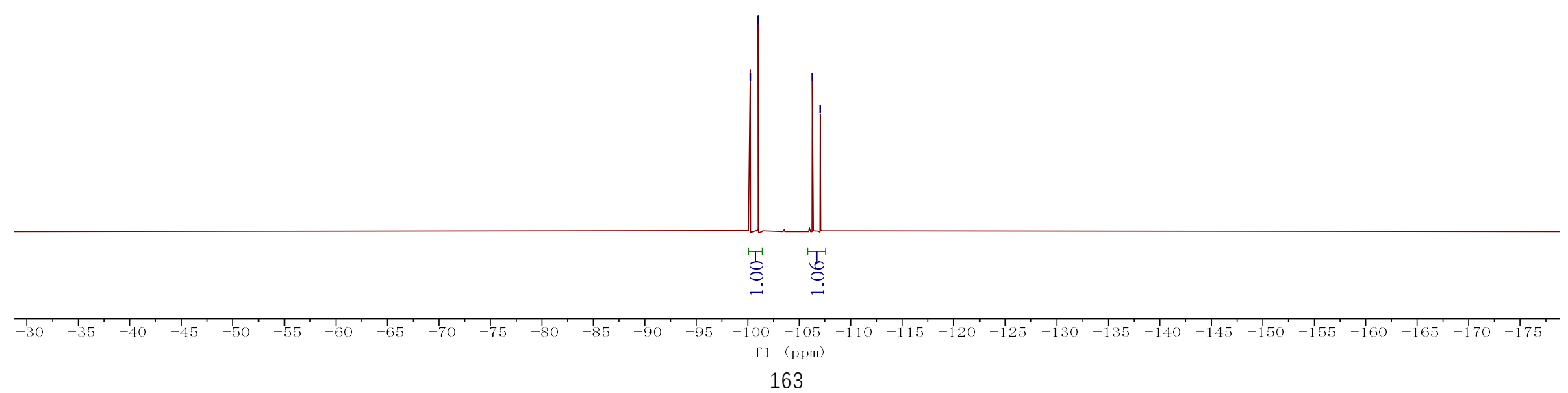




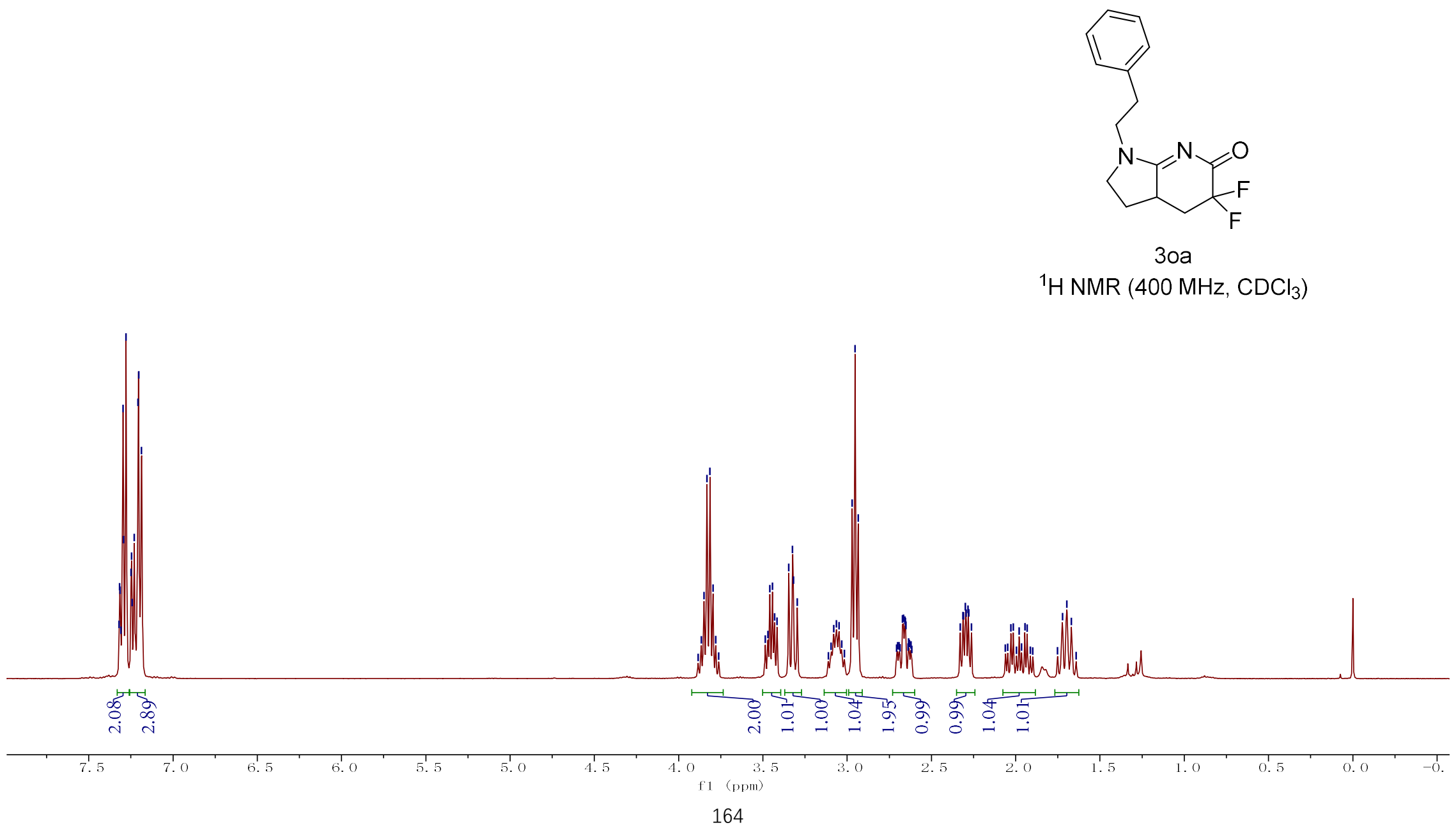



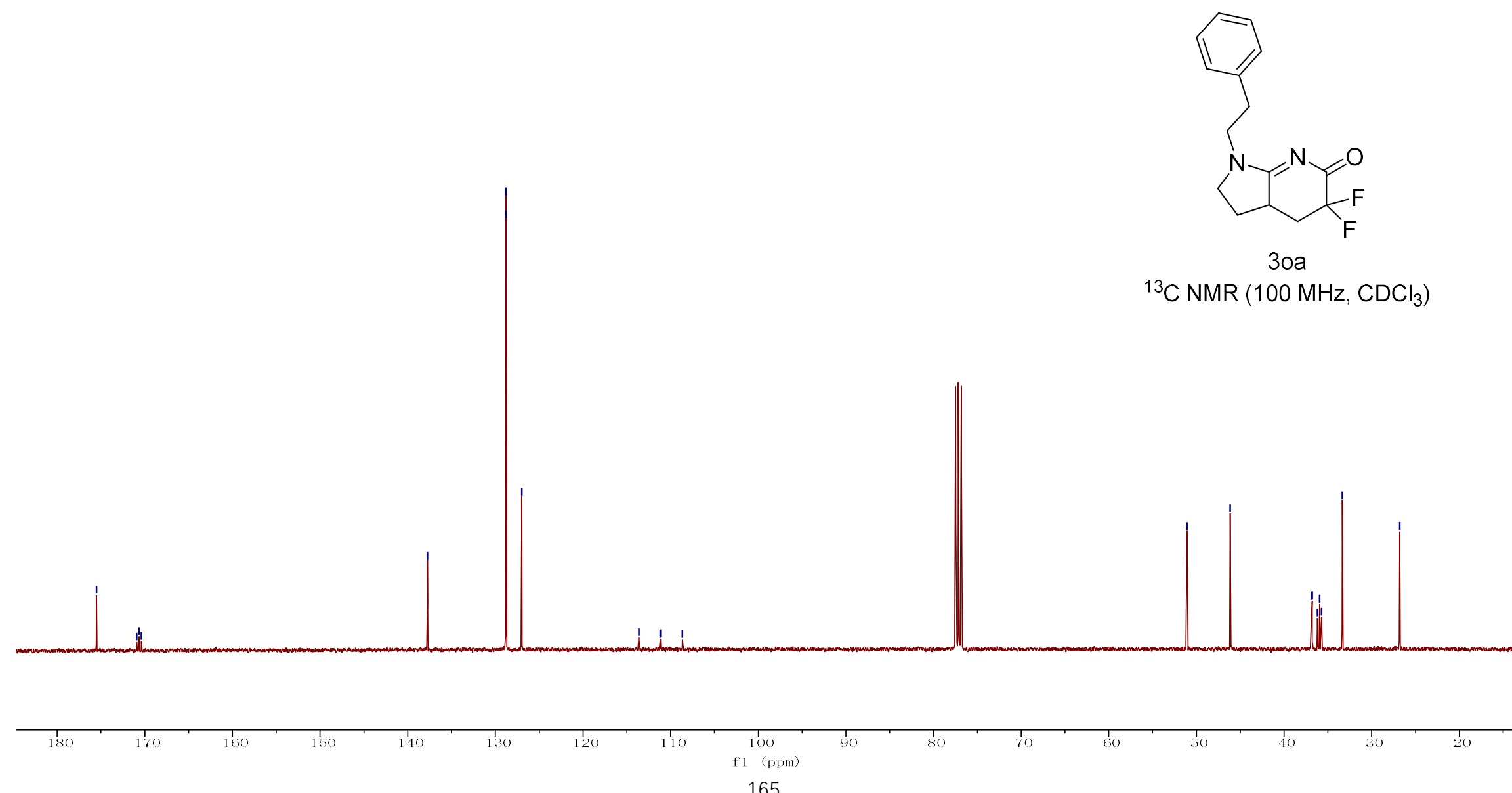


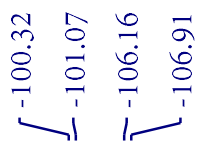

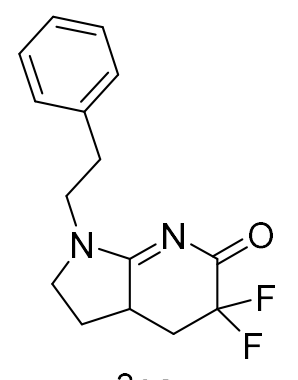

$30 a$

${ }^{19} \mathrm{~F}$ NMR (376 MHz, $\mathrm{CDCl}_{3}$ )

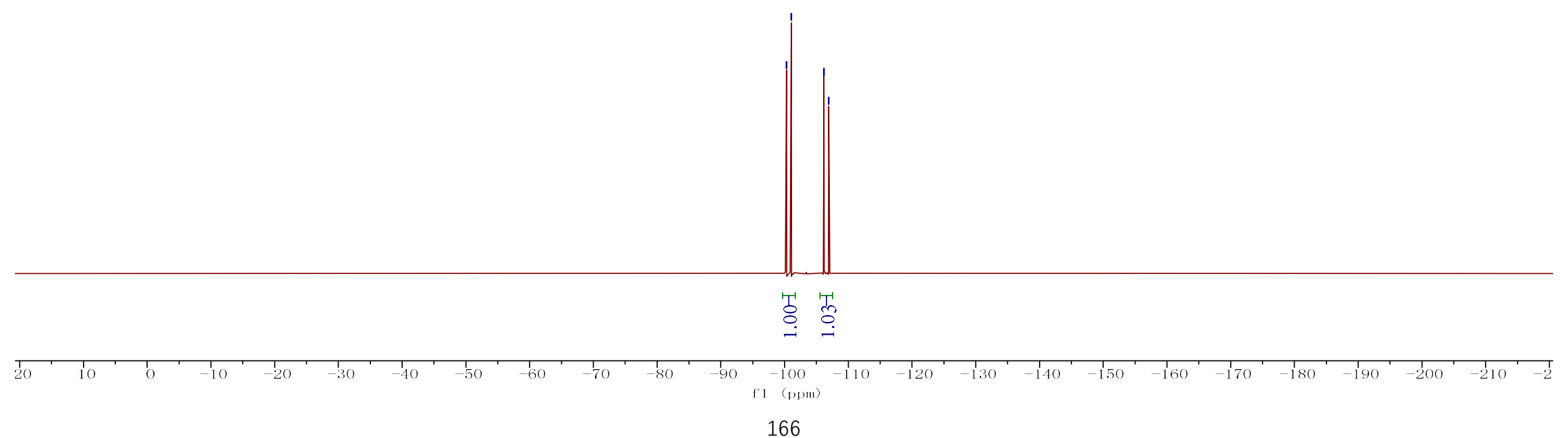




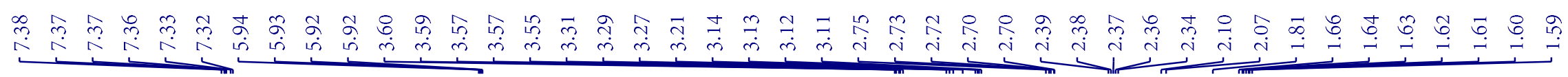

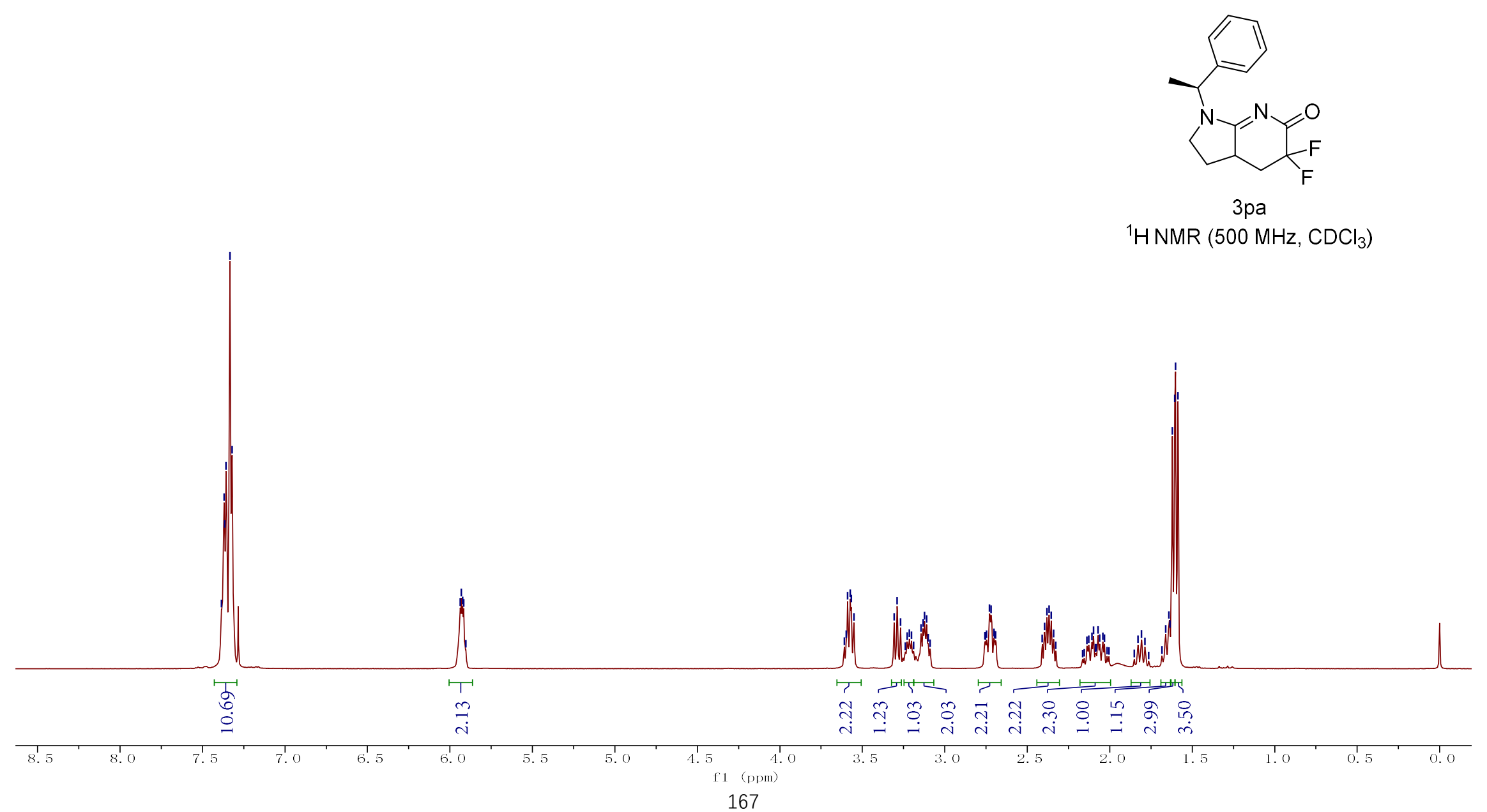



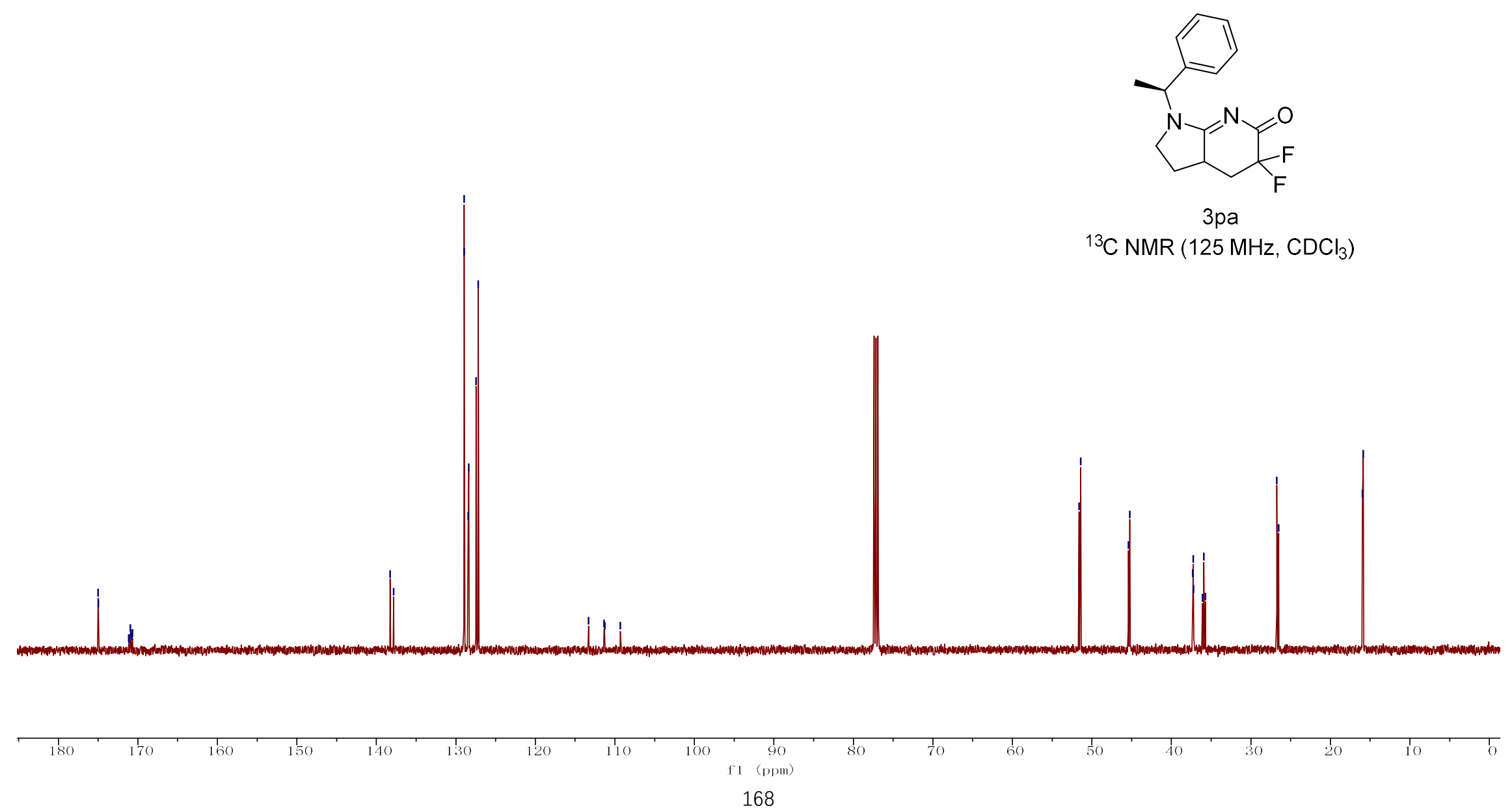


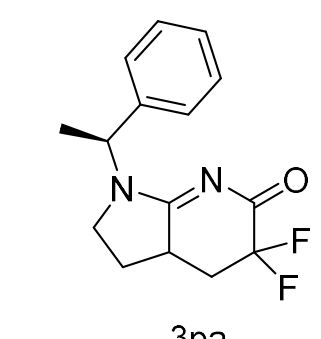

${ }^{19} \mathrm{~F} \mathrm{NMR}\left(470 \mathrm{MHz}, \mathrm{CDCl}_{3}\right)$

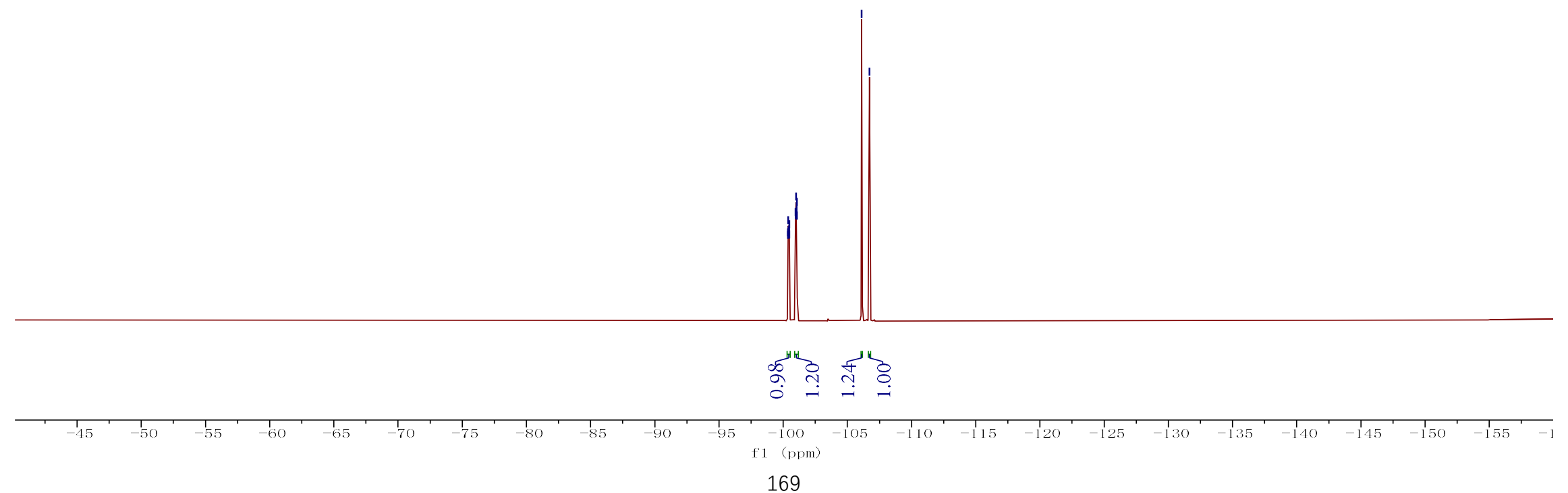




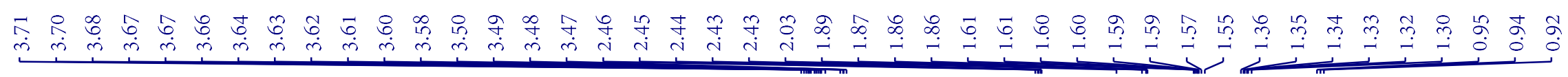

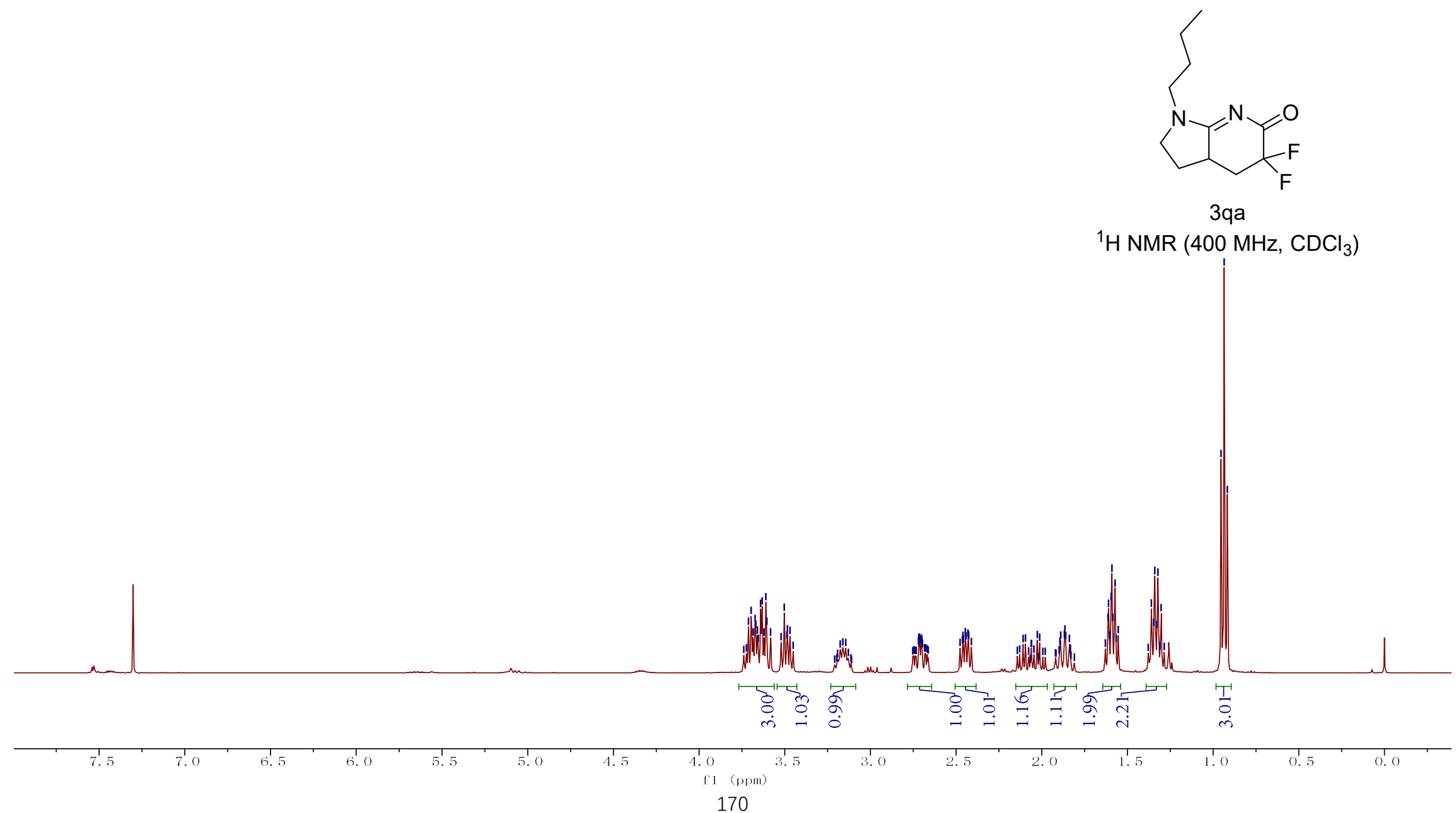




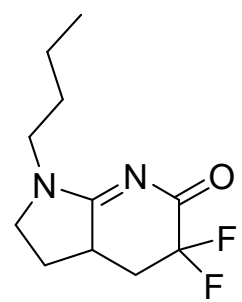

3qa

${ }^{13} \mathrm{C}$ NMR $\left(100 \mathrm{MHz}, \mathrm{CDCl}_{3}\right)$

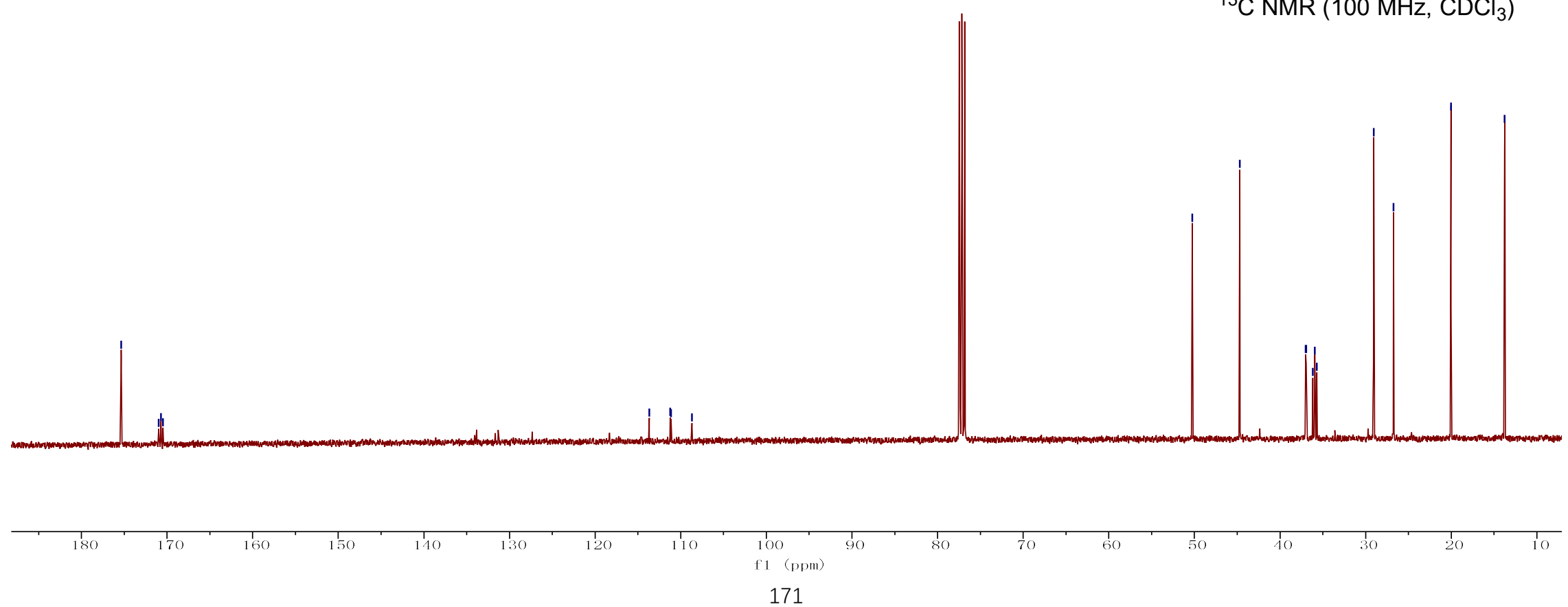




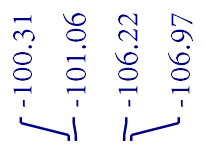

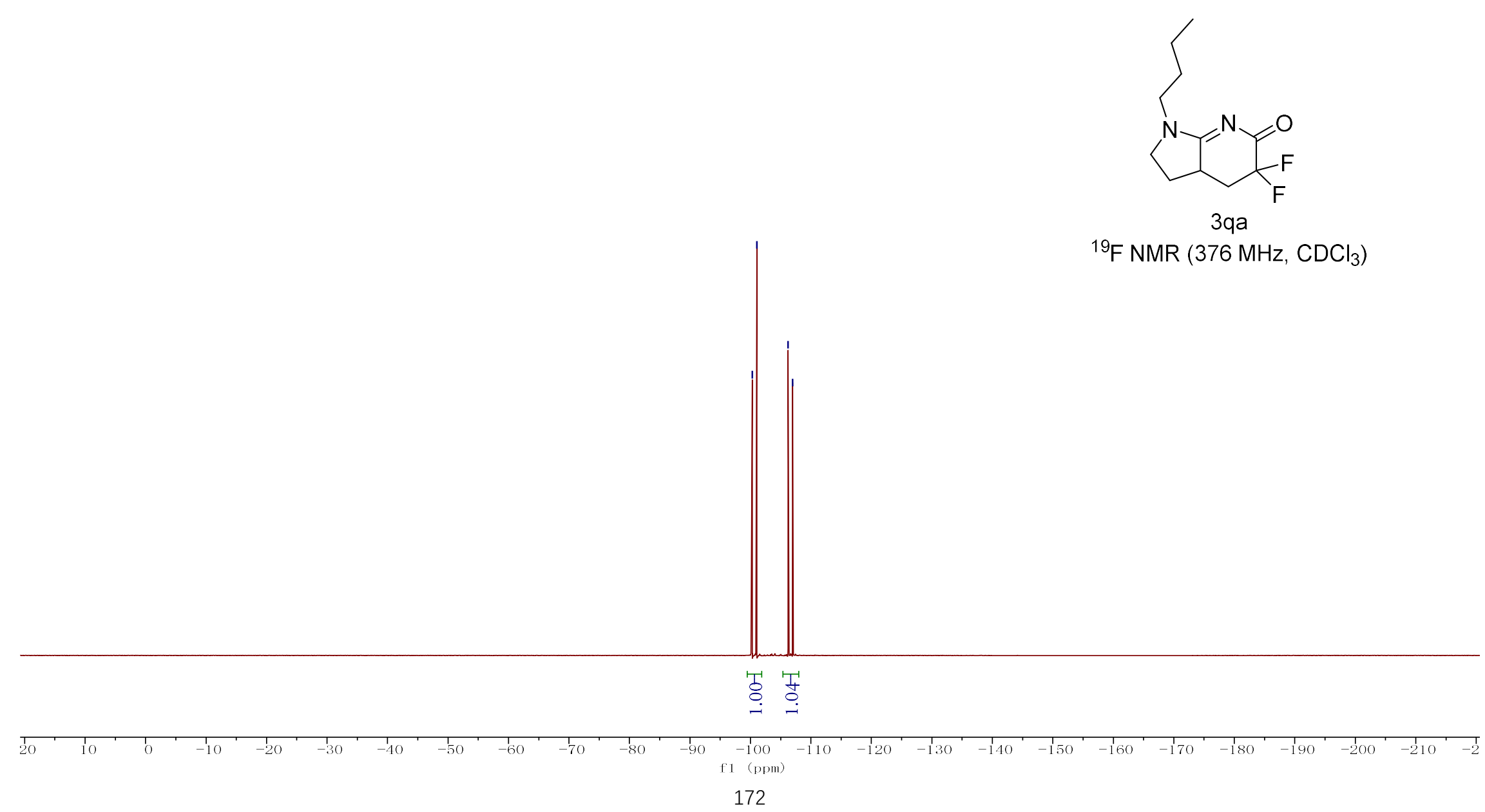




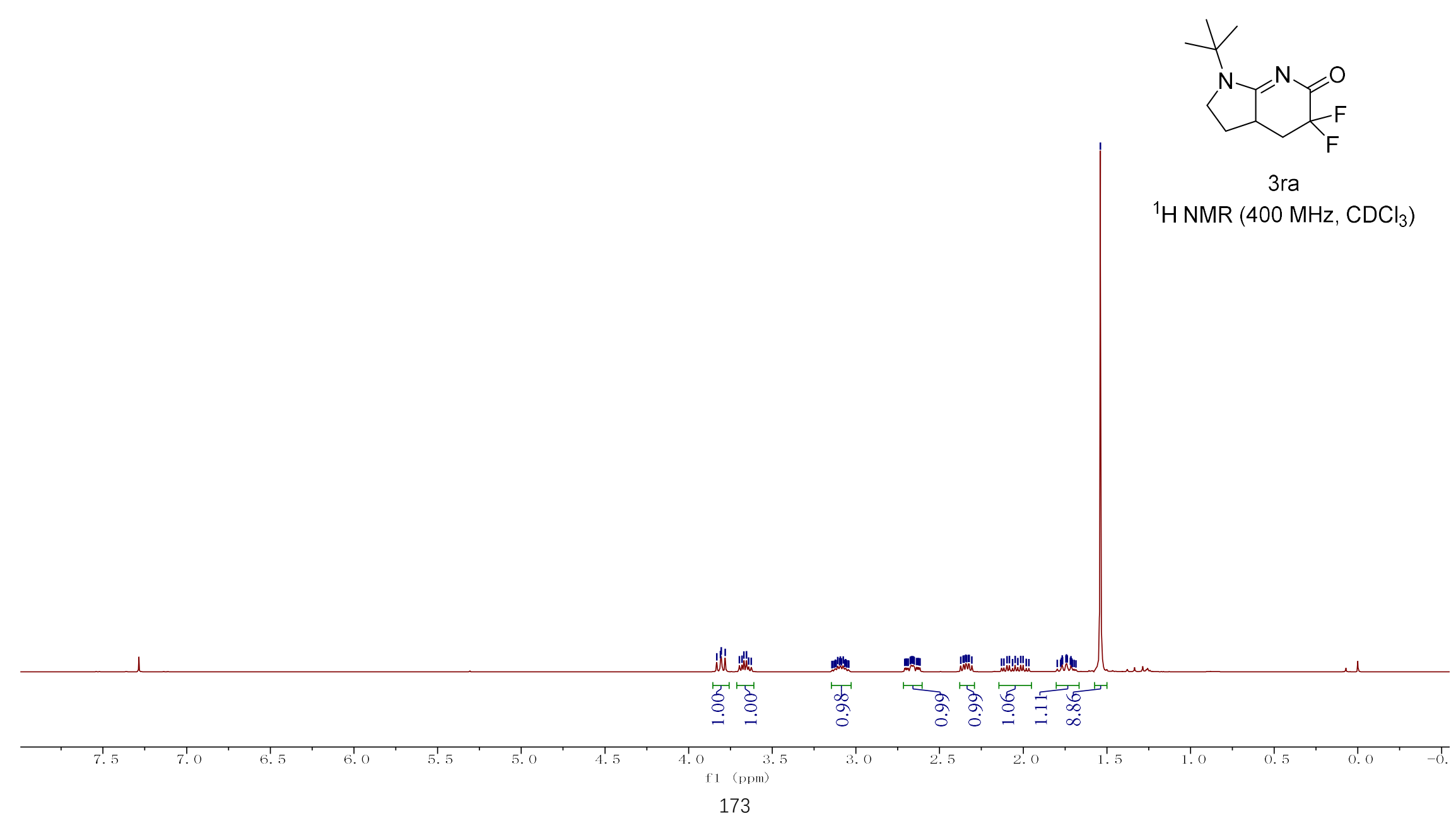




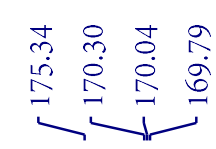

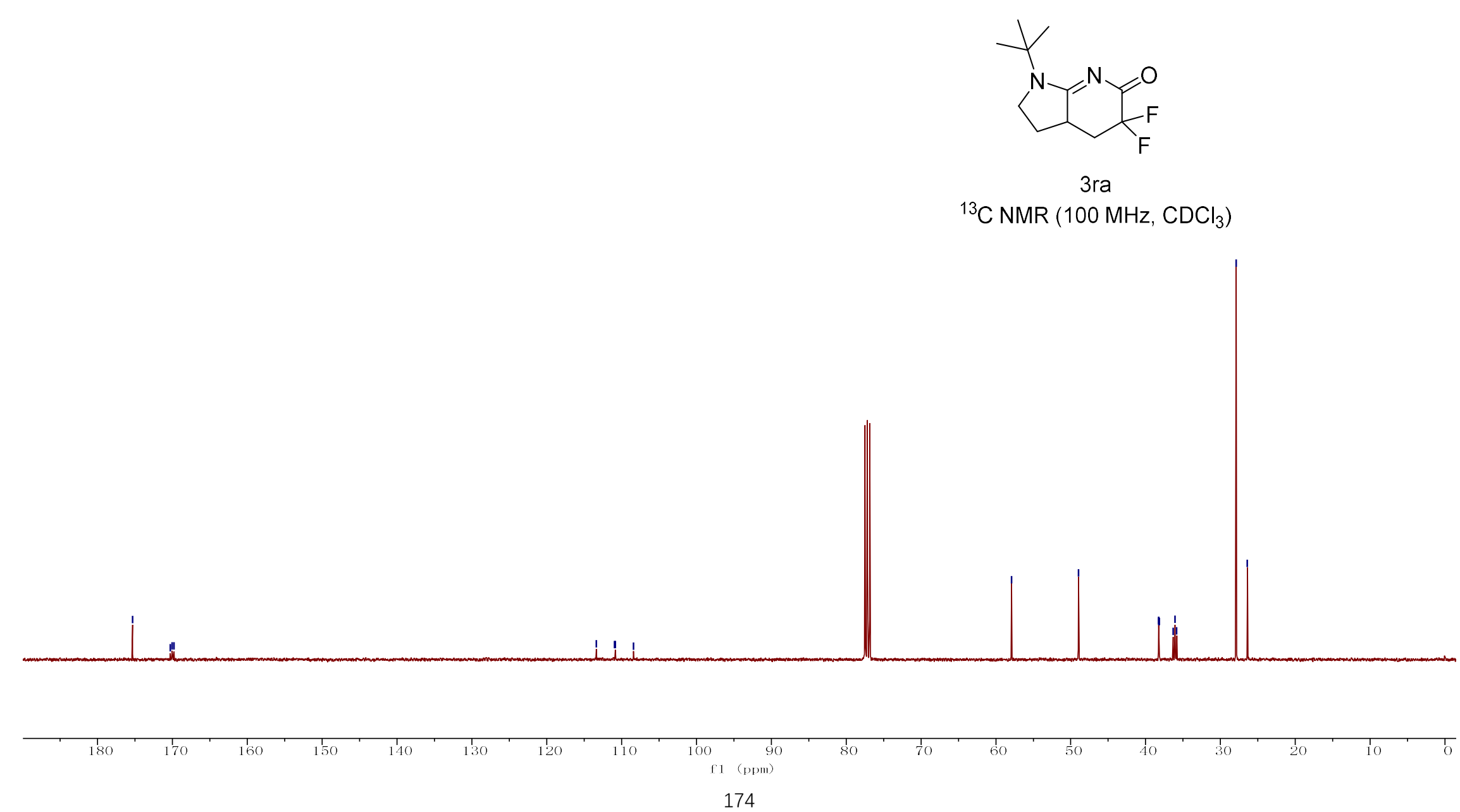

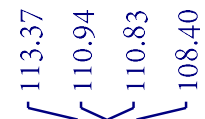

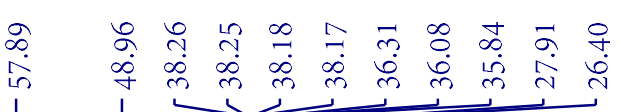




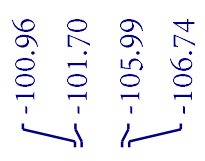

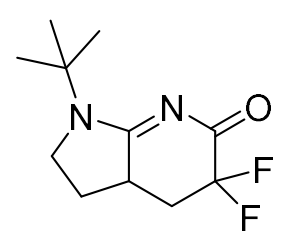

3 ra

${ }^{19} \mathrm{~F} \mathrm{NMR}\left(376 \mathrm{MHz}, \mathrm{CDCl}_{3}\right)$

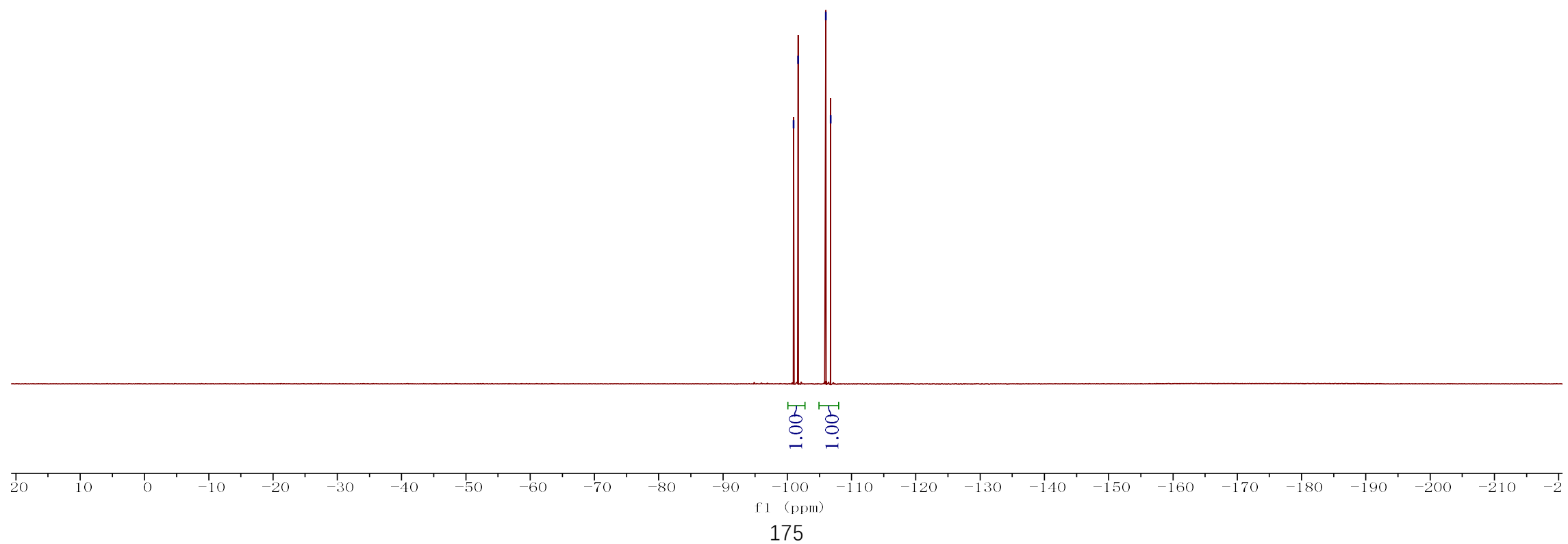




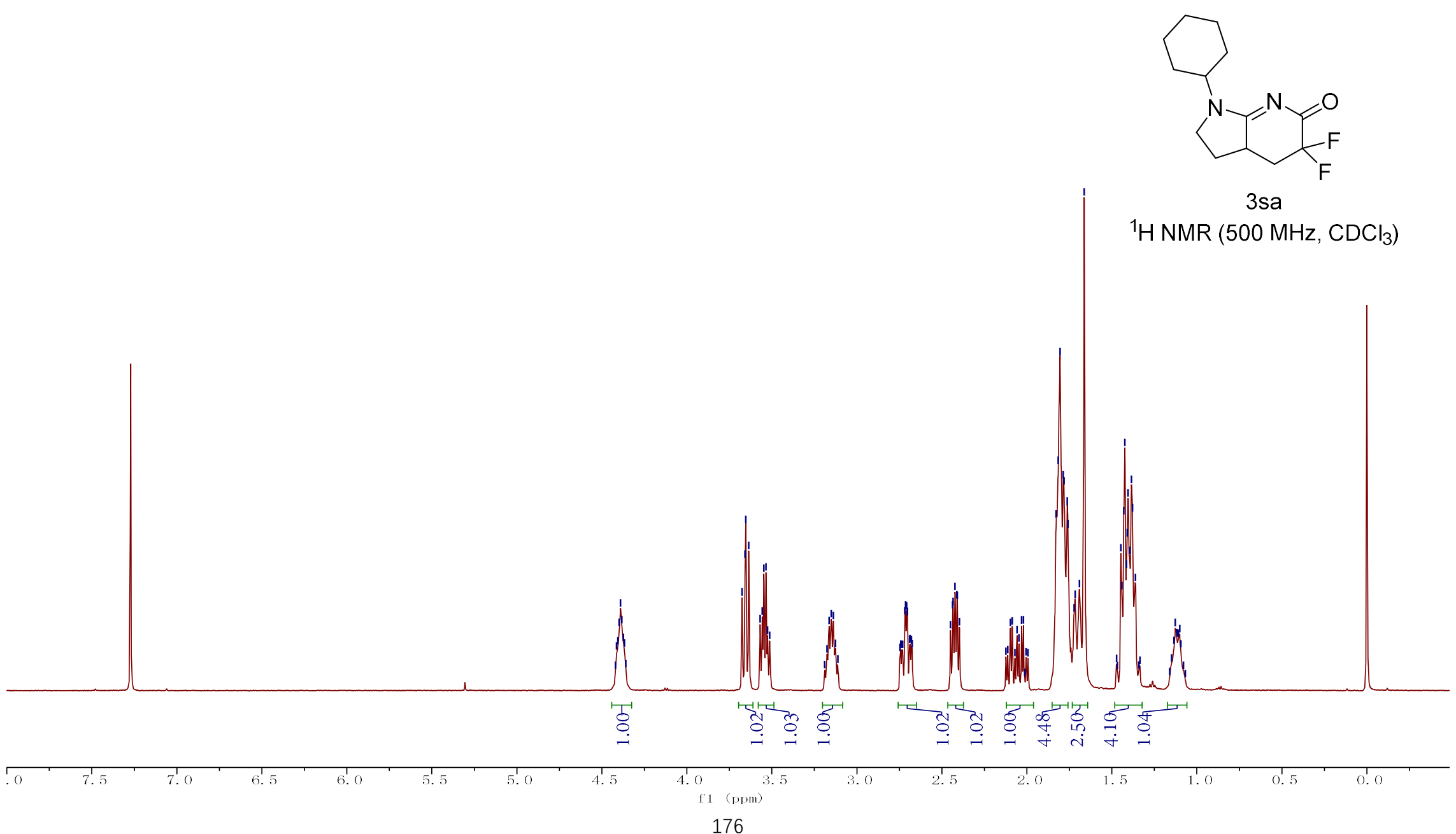



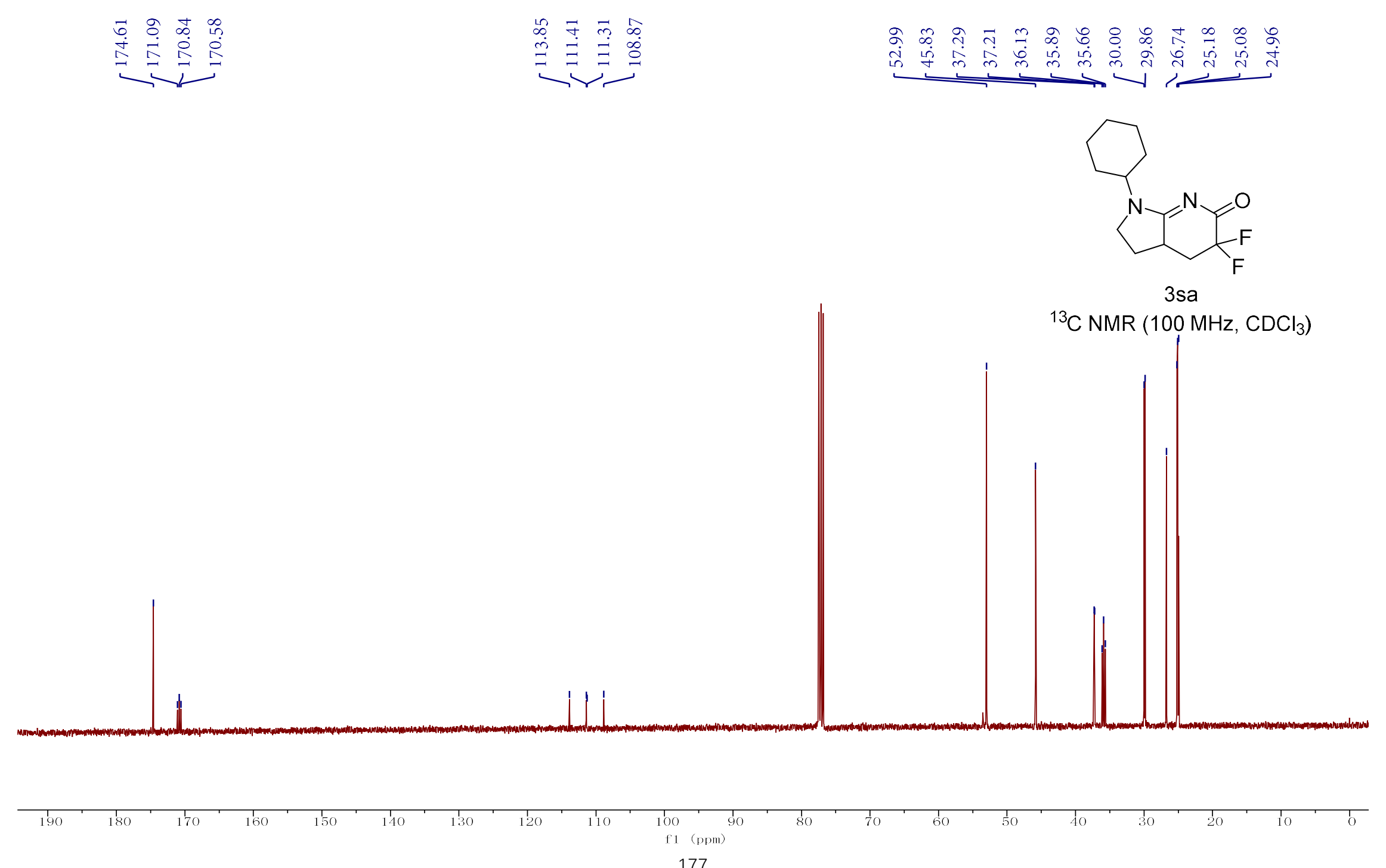

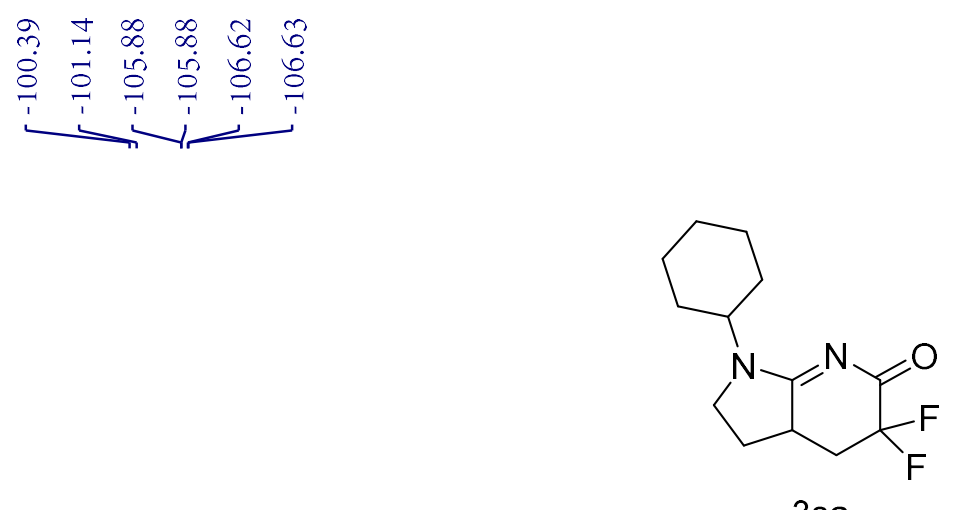

${ }^{19} \mathrm{~F} \mathrm{NMR}\left(376 \mathrm{MHz}, \mathrm{CDCl}_{3}\right)$

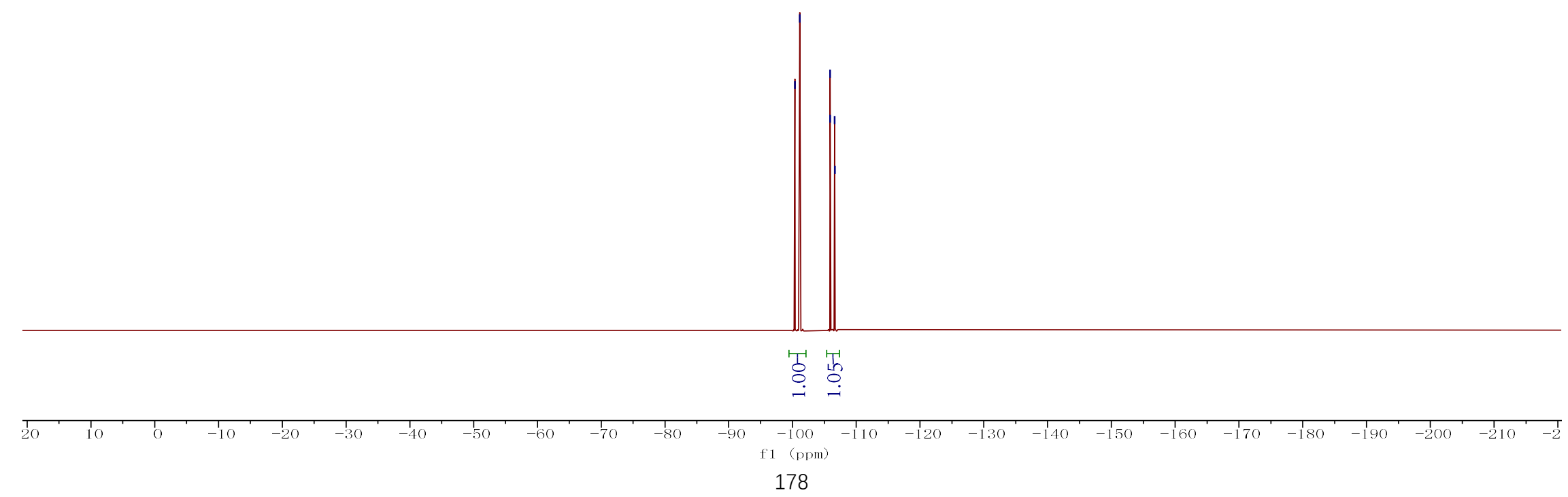




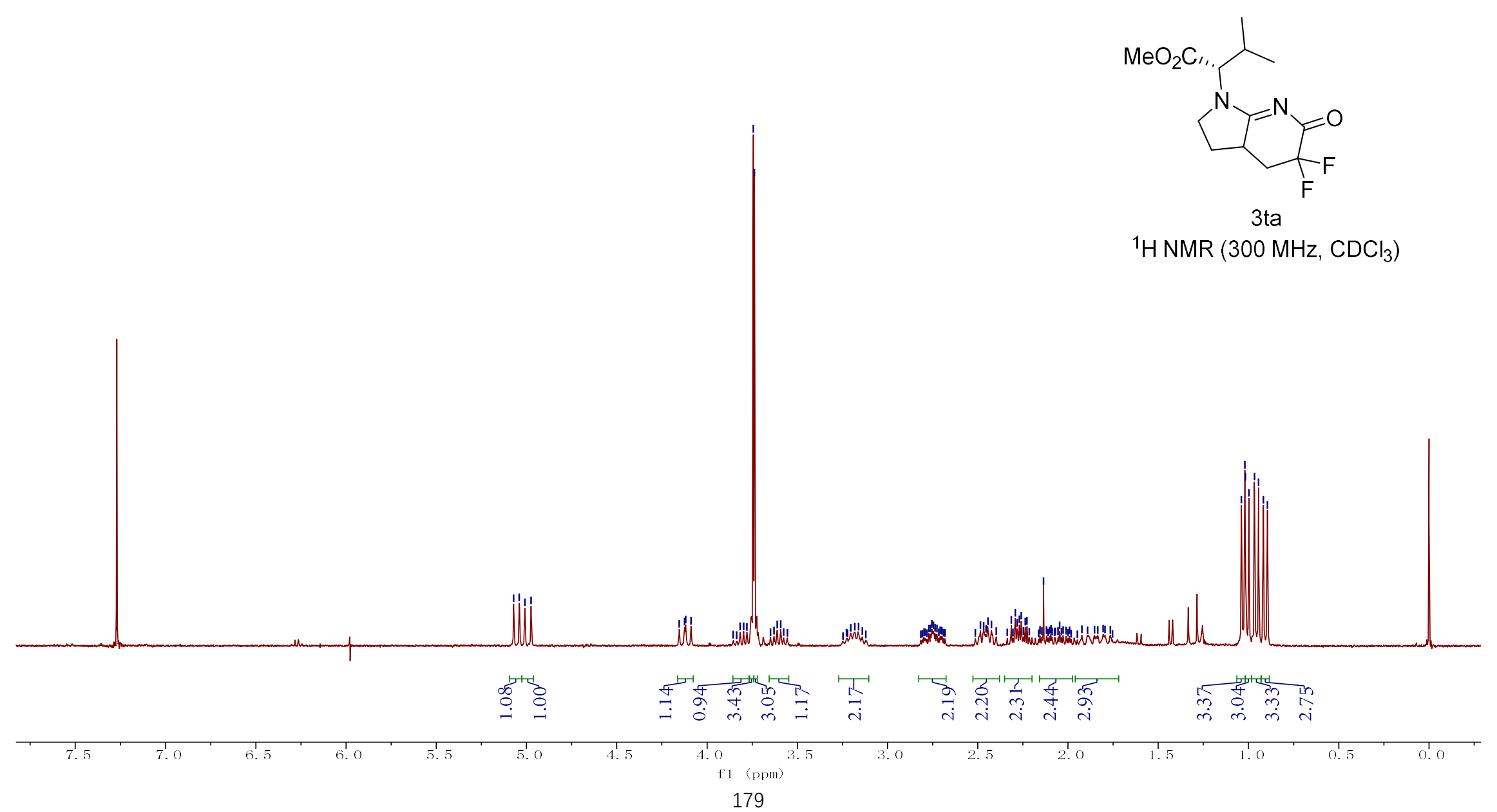



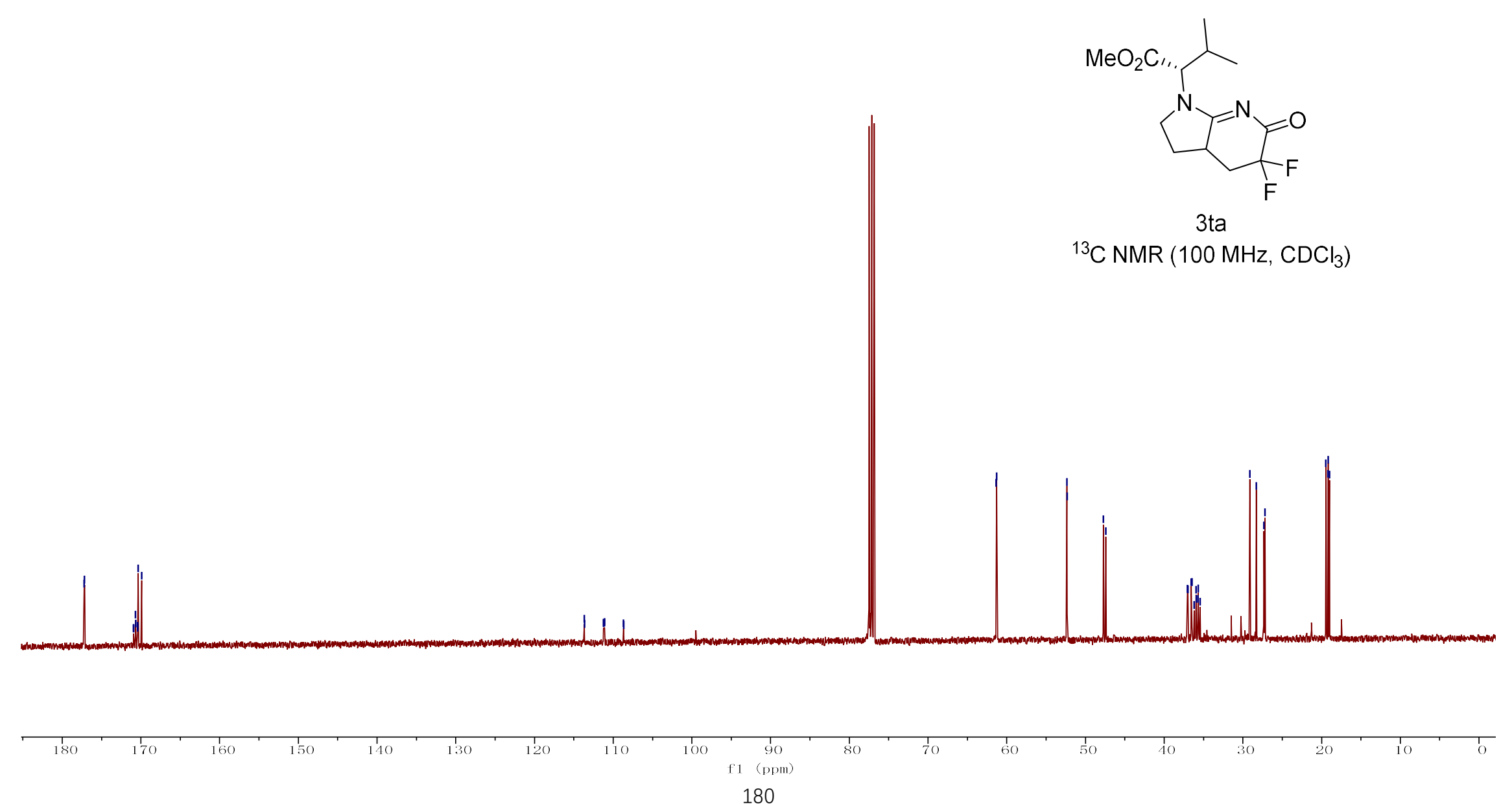


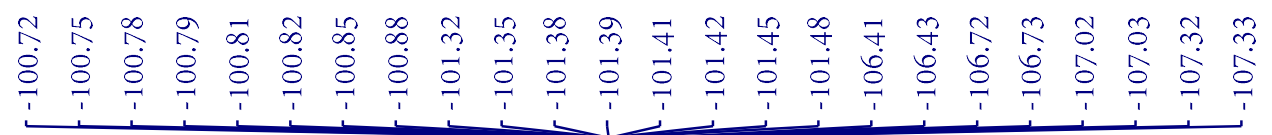

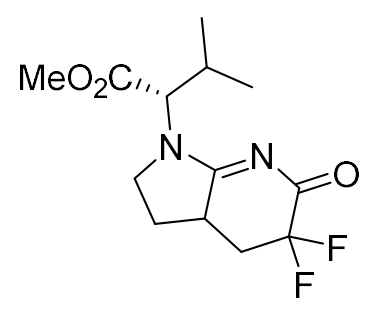

3 ta

${ }^{19} \mathrm{~F} \mathrm{NMR}\left(470 \mathrm{MHz}, \mathrm{CDCl}_{3}\right)$

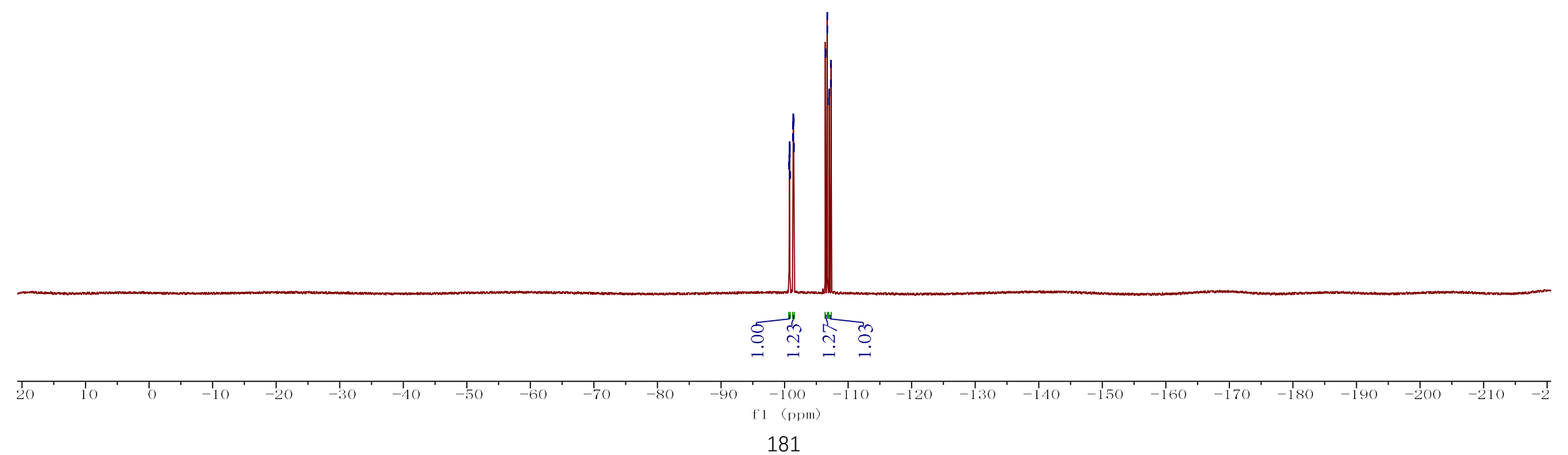




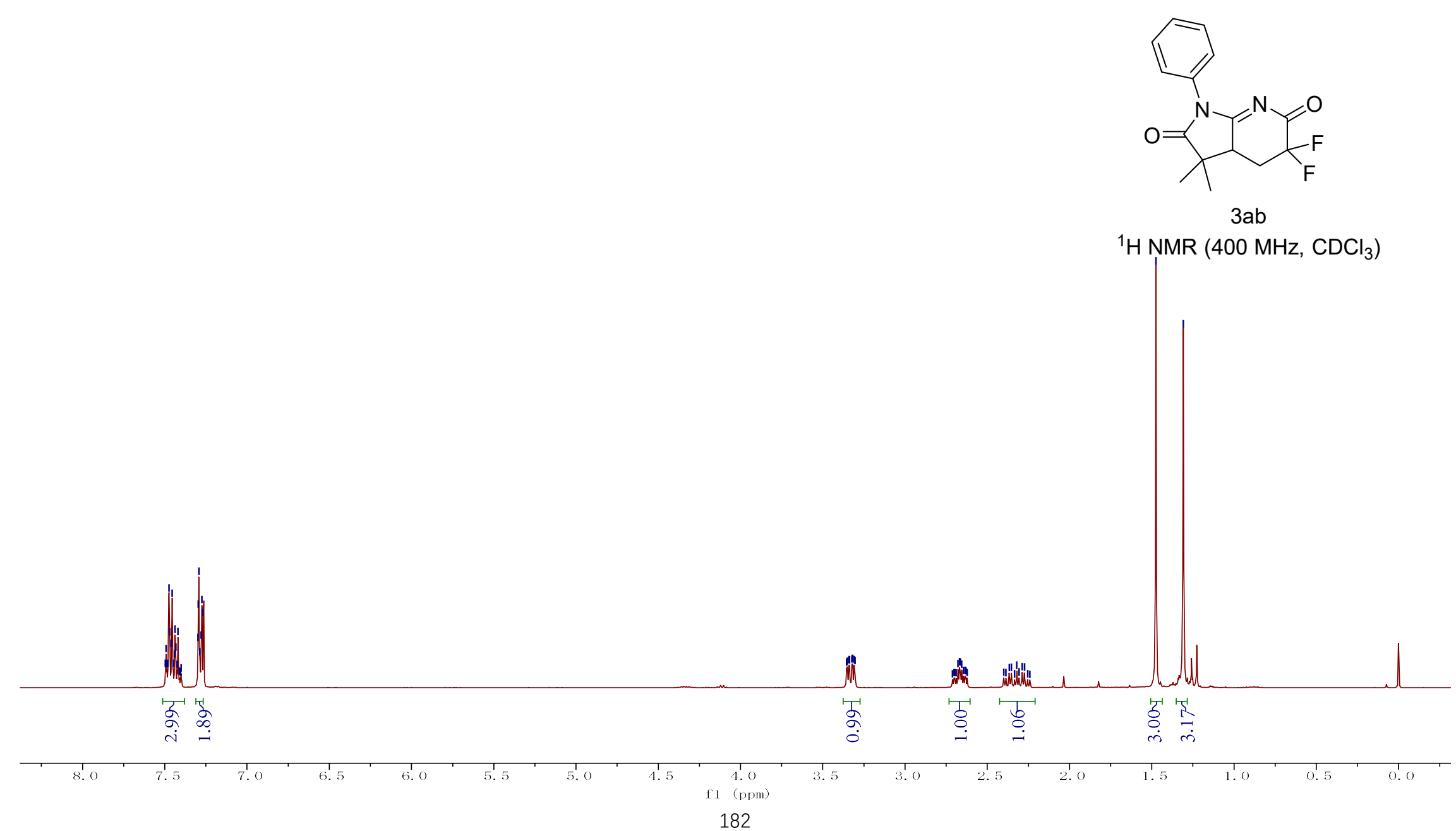



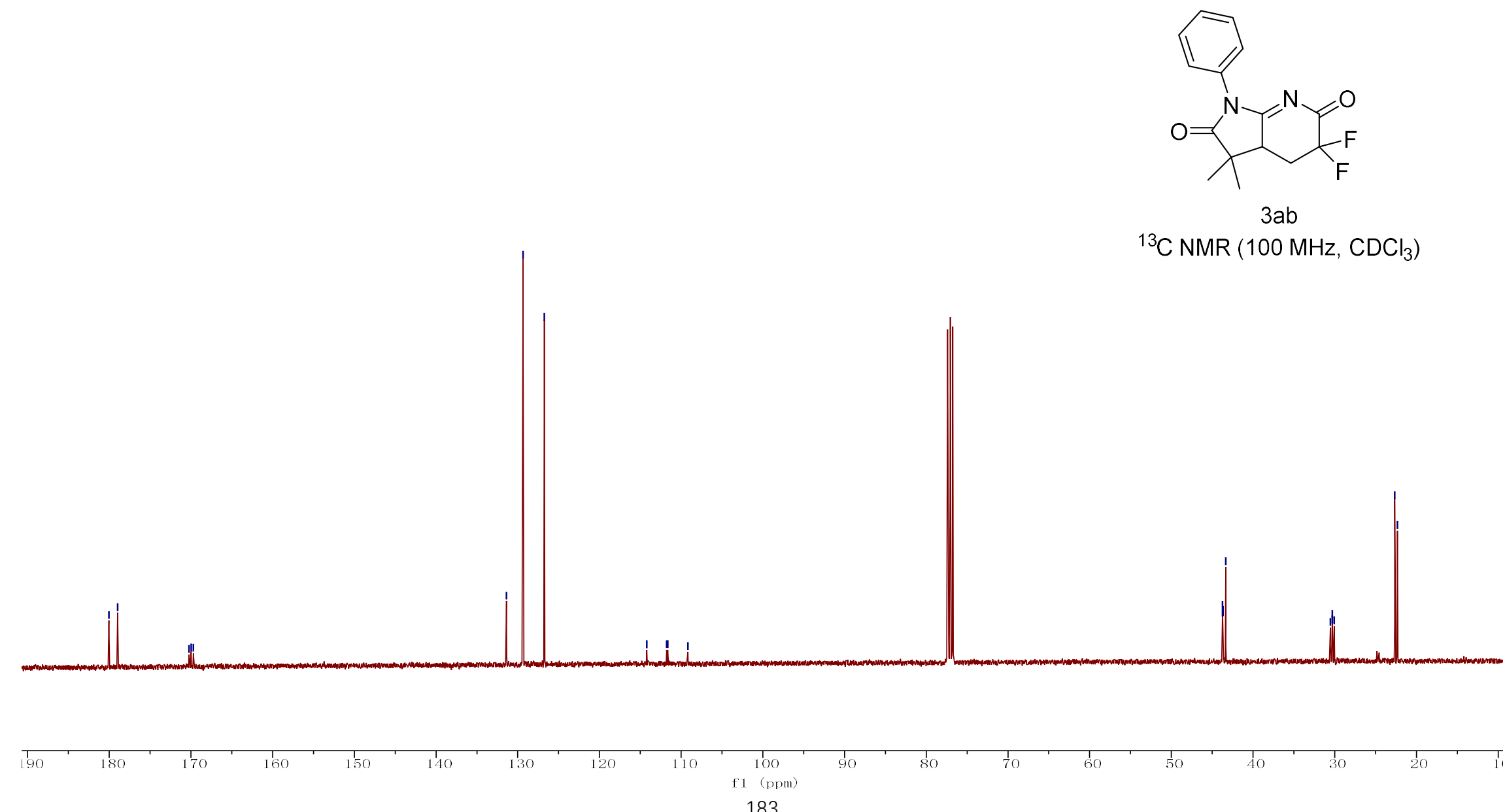


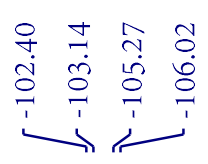

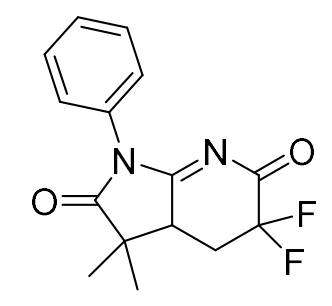

$3 a b$

${ }^{19} \mathrm{~F}$ NMR (376 $\mathrm{MHz}, \mathrm{CDCl}_{3}$ )

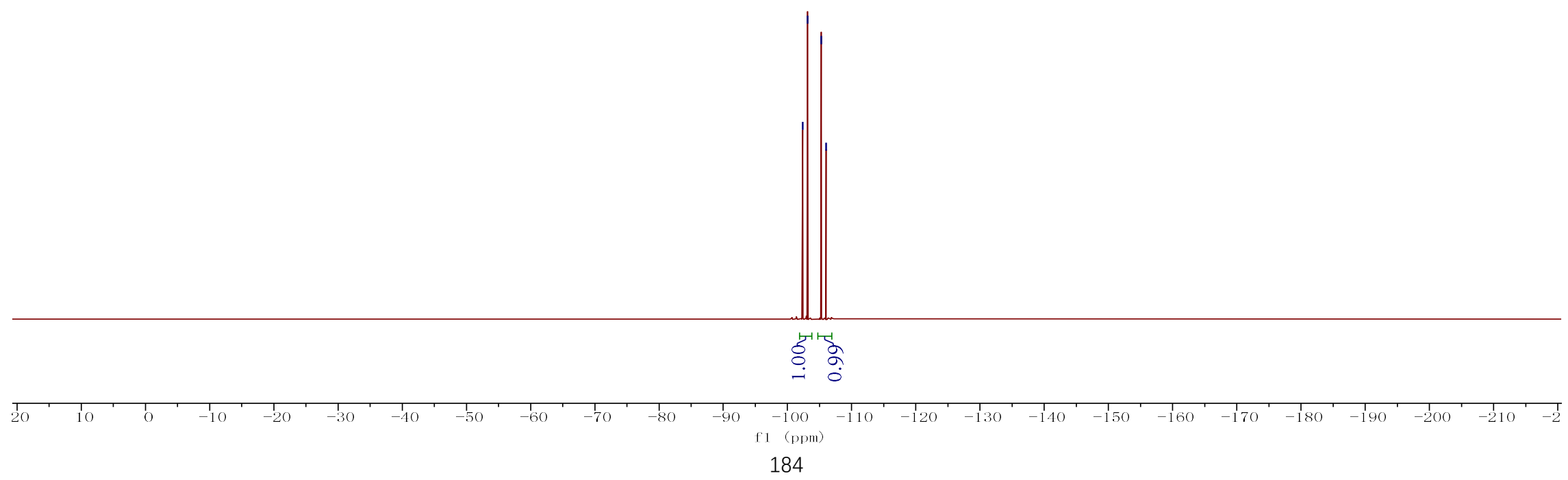




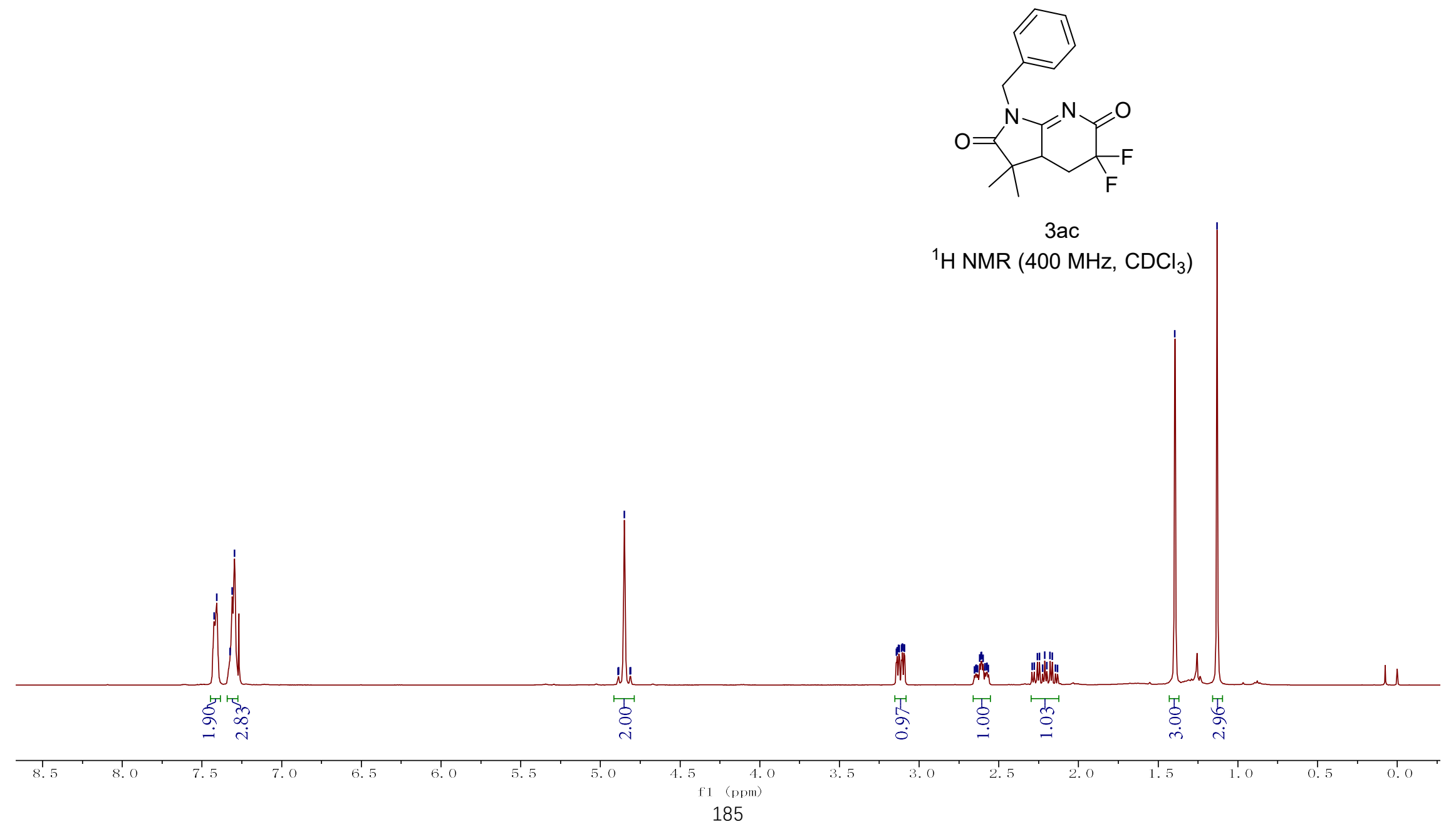




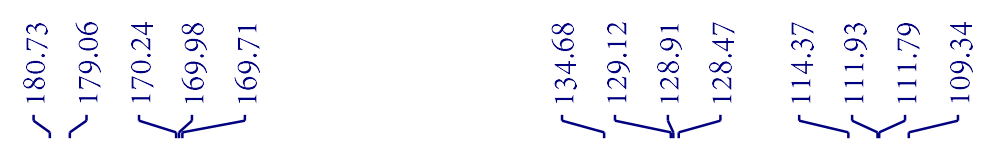

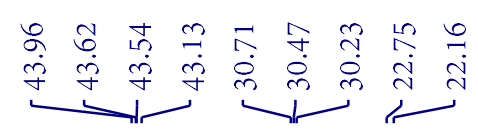

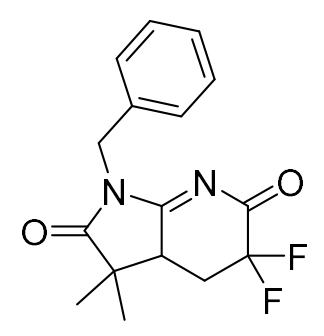

$3 a c$

${ }^{13} \mathrm{C} \mathrm{NMR}\left(100 \mathrm{MHz}, \mathrm{CDCl}_{3}\right)$
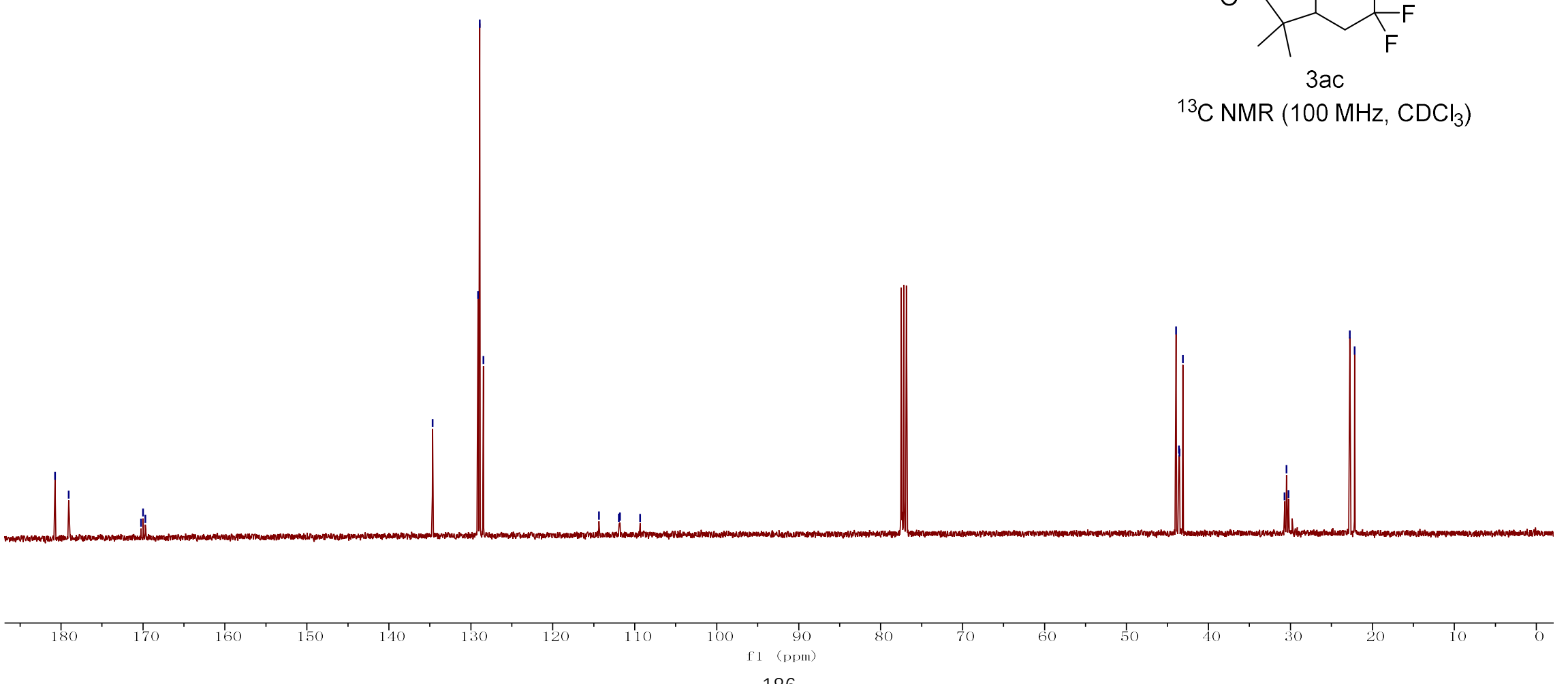


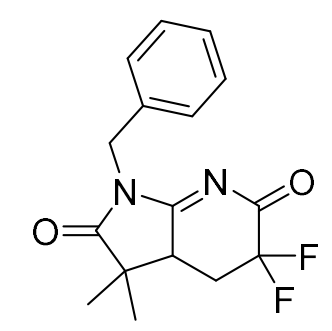

$3 a c$

${ }^{19} \mathrm{~F} \mathrm{NMR}\left(470 \mathrm{MHz}, \mathrm{CDCl}_{3}\right)$

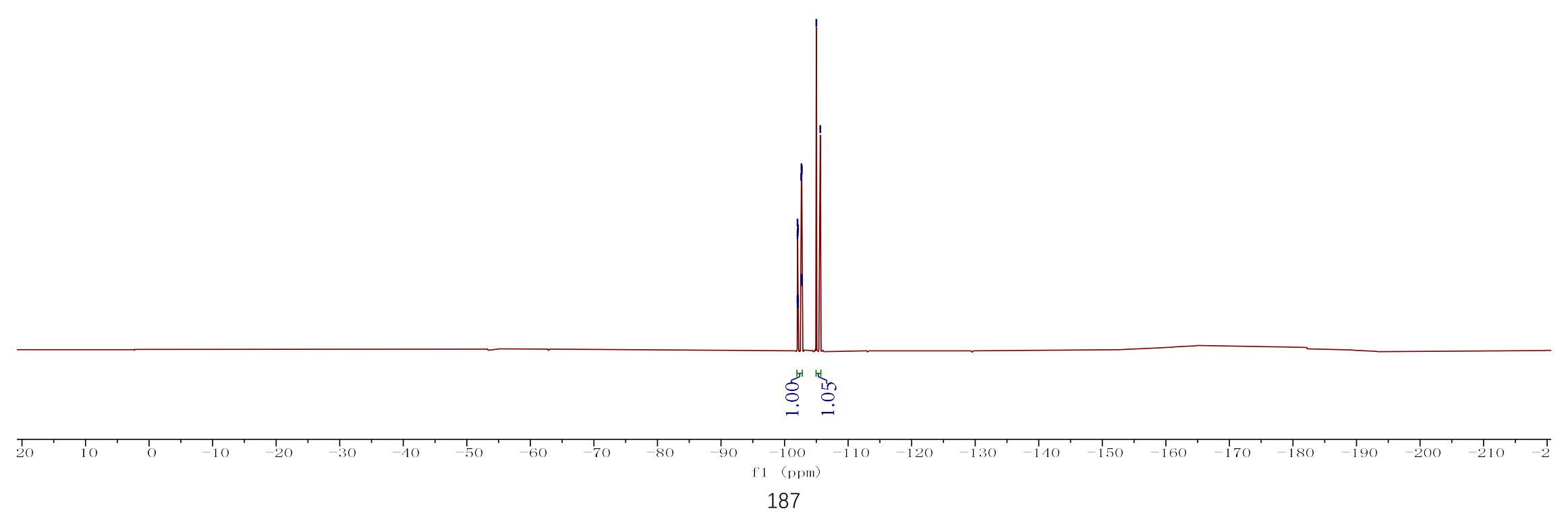




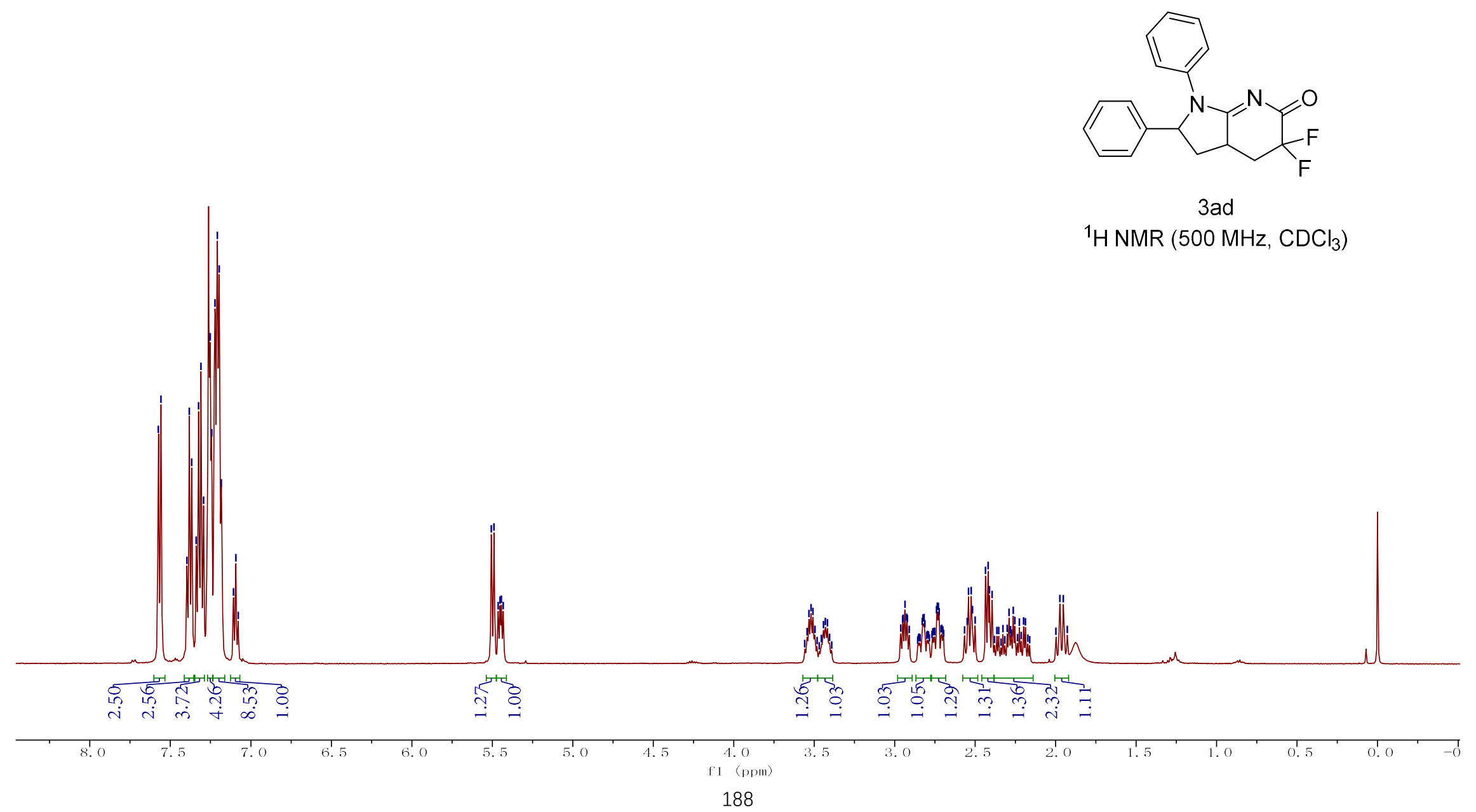




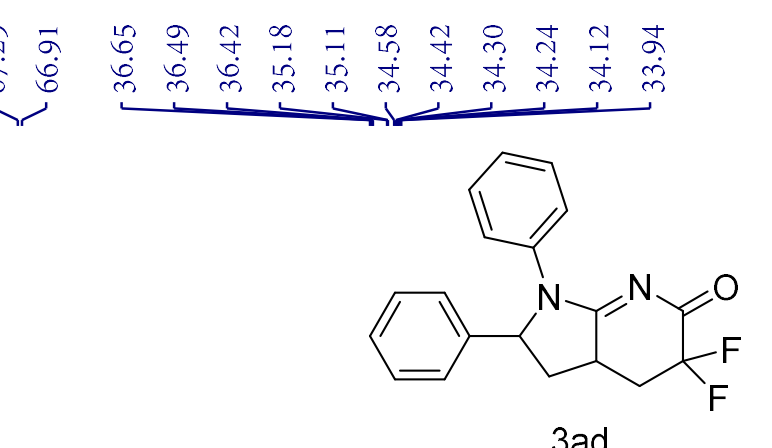

${ }^{13} \mathrm{C}$ NMR (125 MHz, DMSO)
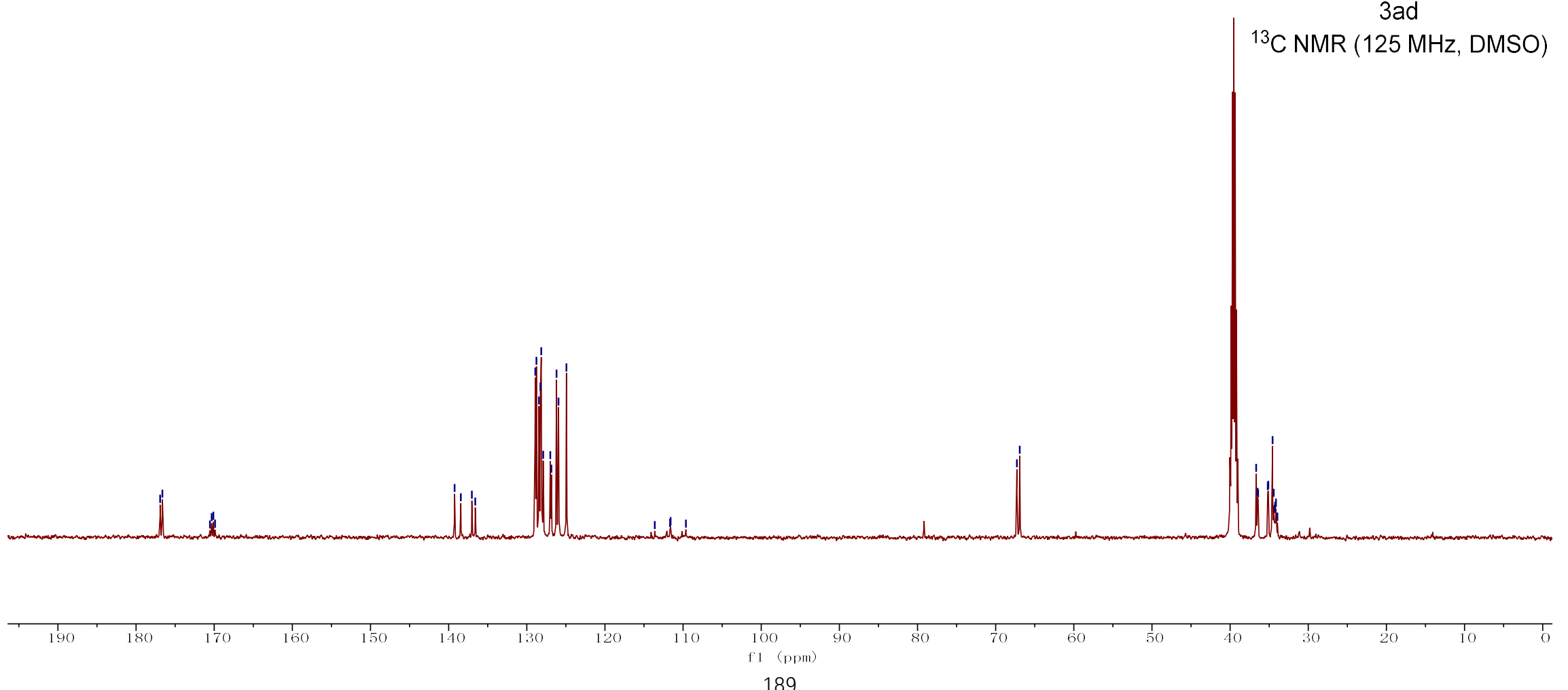

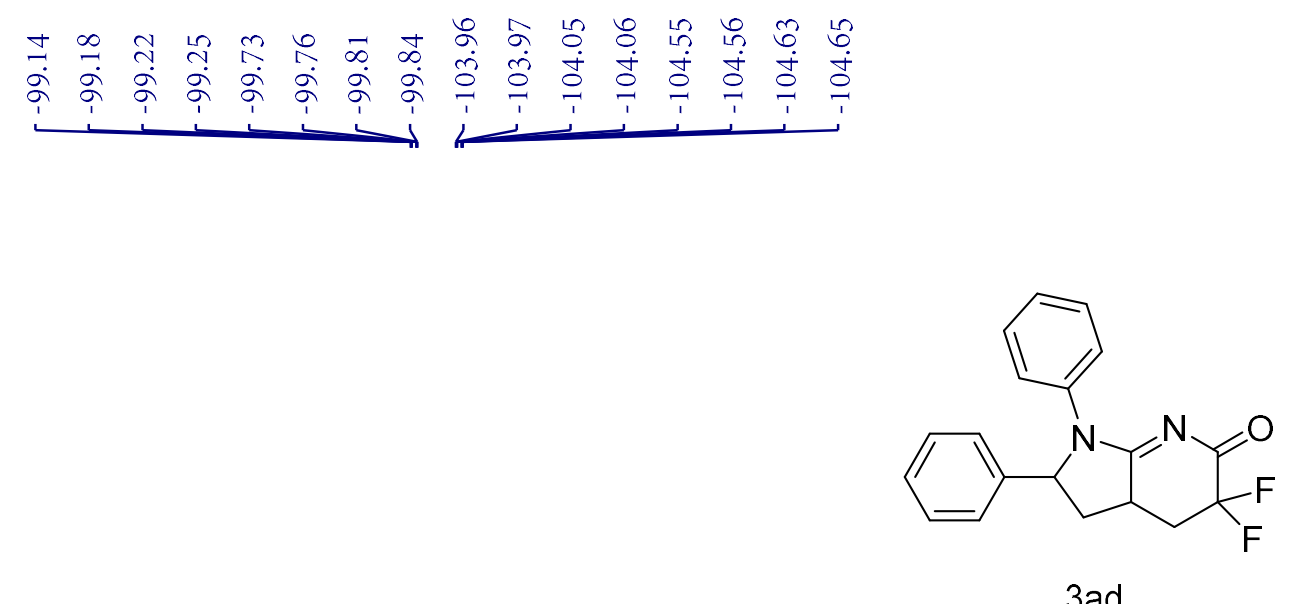

${ }^{19} \mathrm{~F}$ NMR (470 MHz, DMSO)

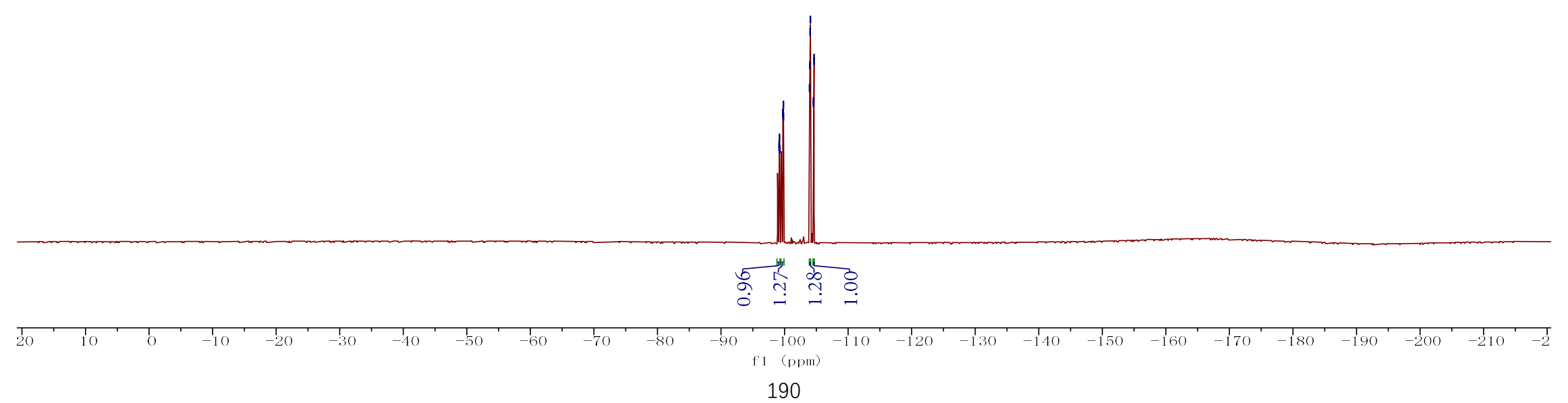




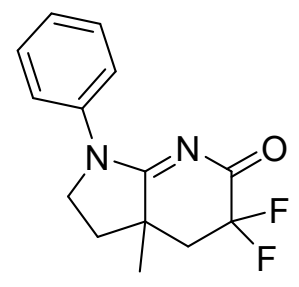

3ae

${ }^{1} \mathrm{H}$ NMR $\left(400 \mathrm{MHz}, \mathrm{CDCl}_{3}\right)$

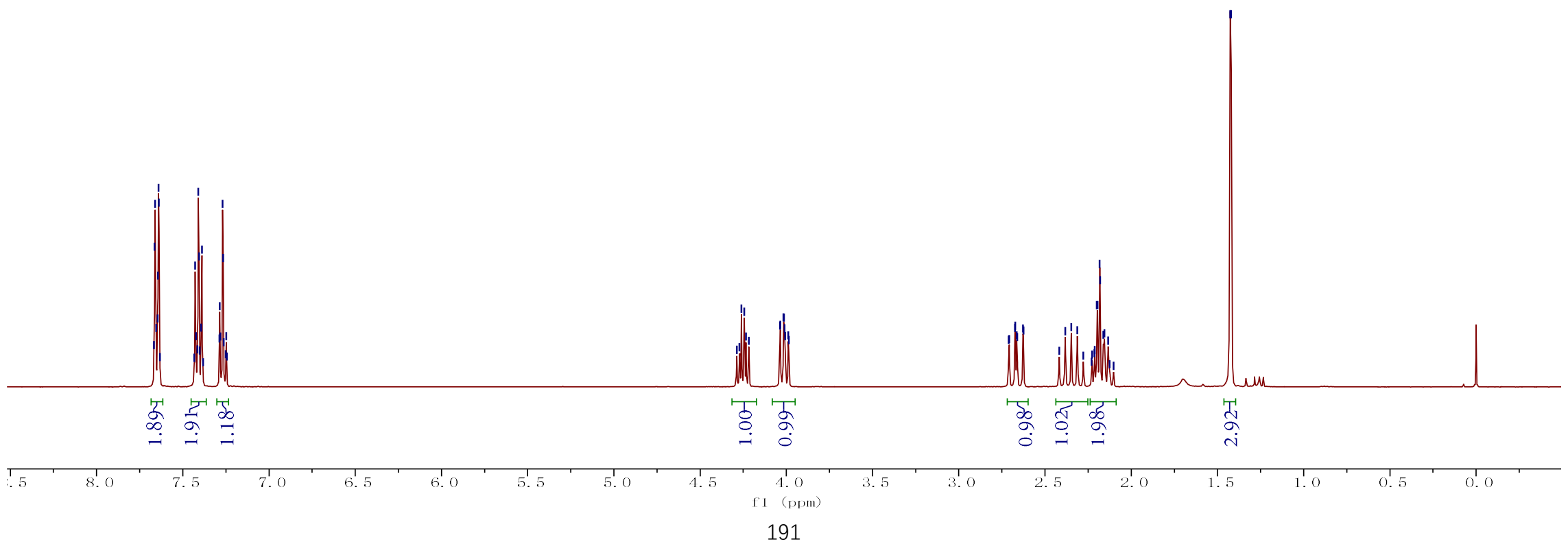




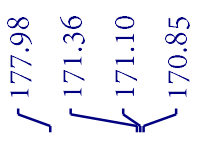

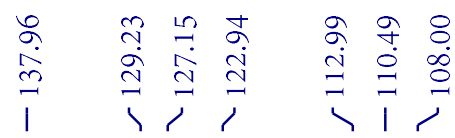

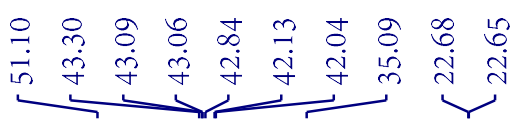

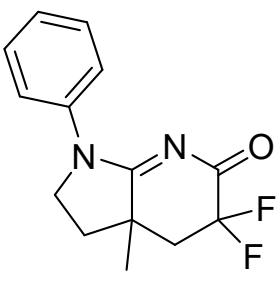

3ae

${ }^{13} \mathrm{C}$ NMR $\left(100 \mathrm{MHz}, \mathrm{CDCl}_{3}\right)$
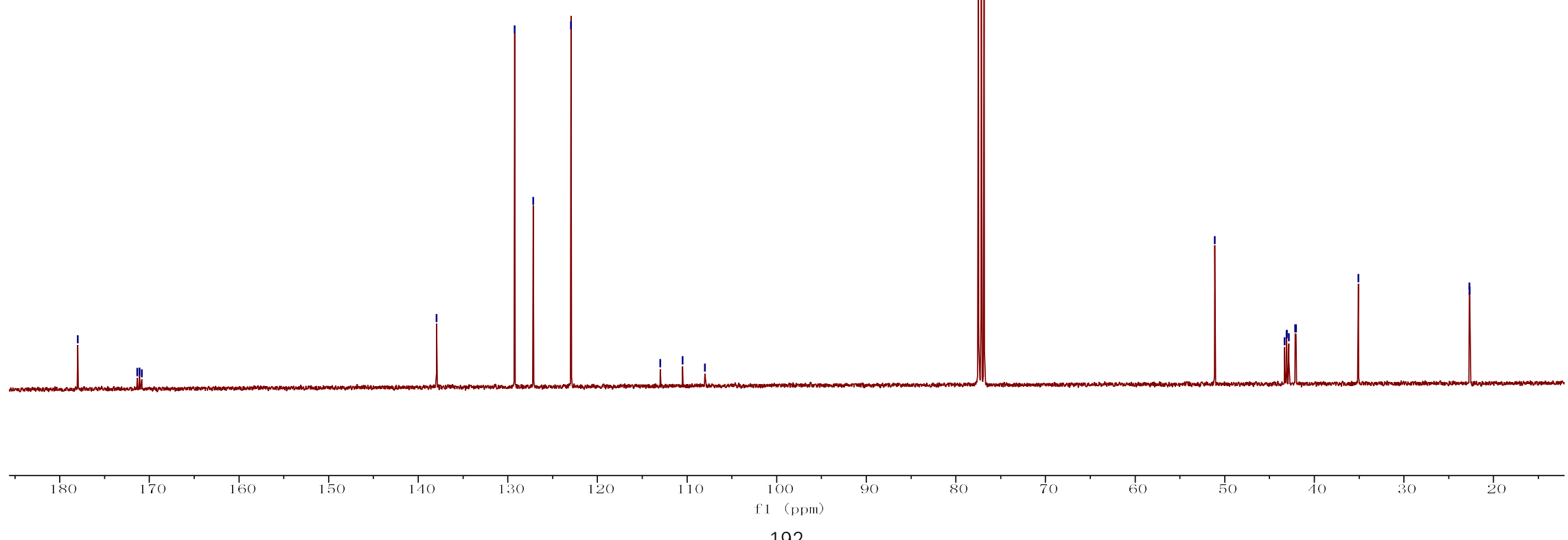


$$
!^{\text {ing }}
$$




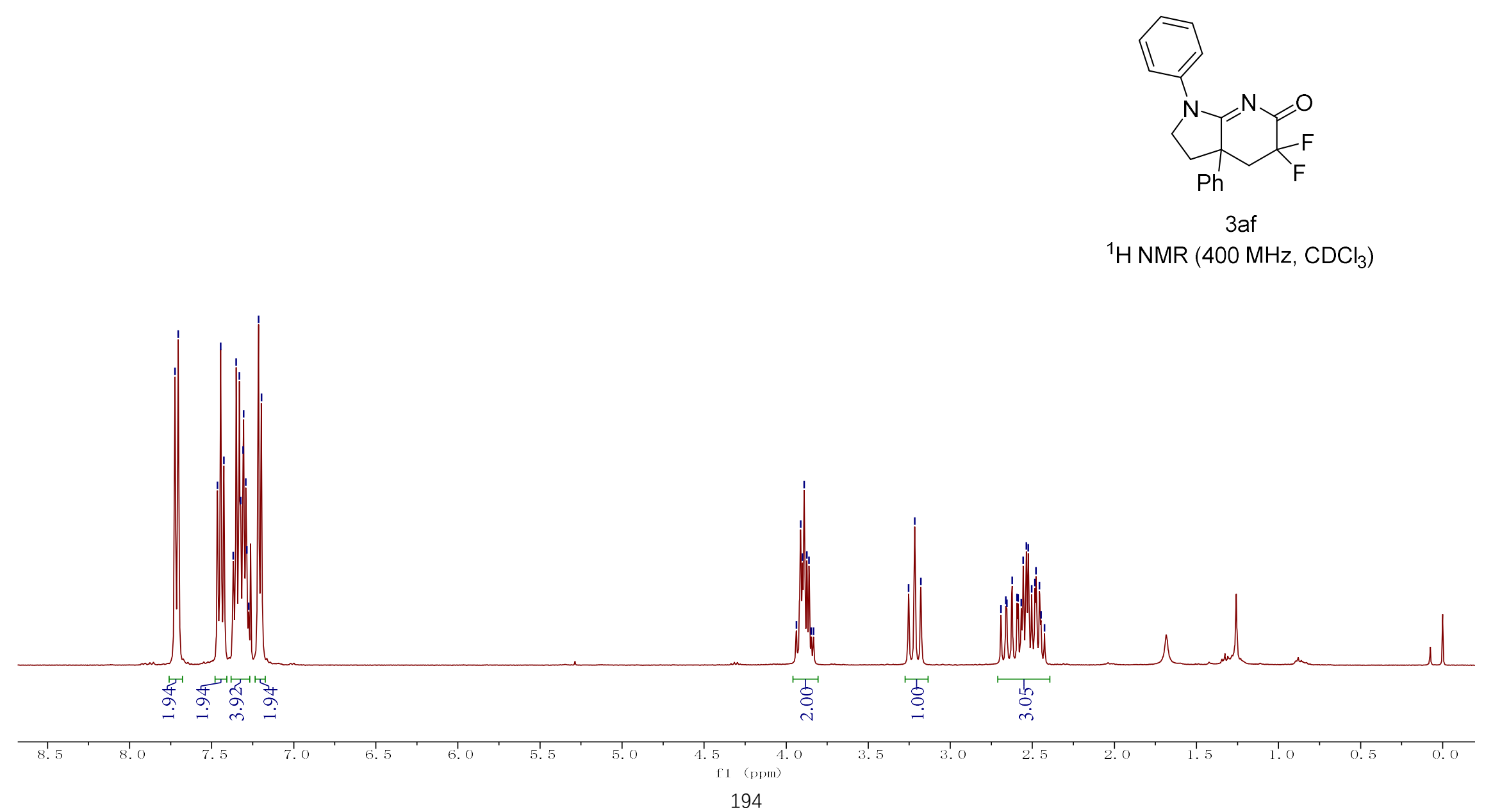




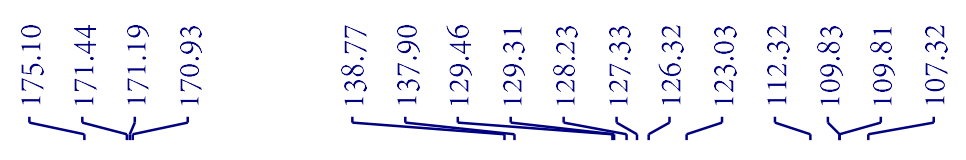

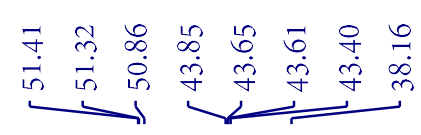
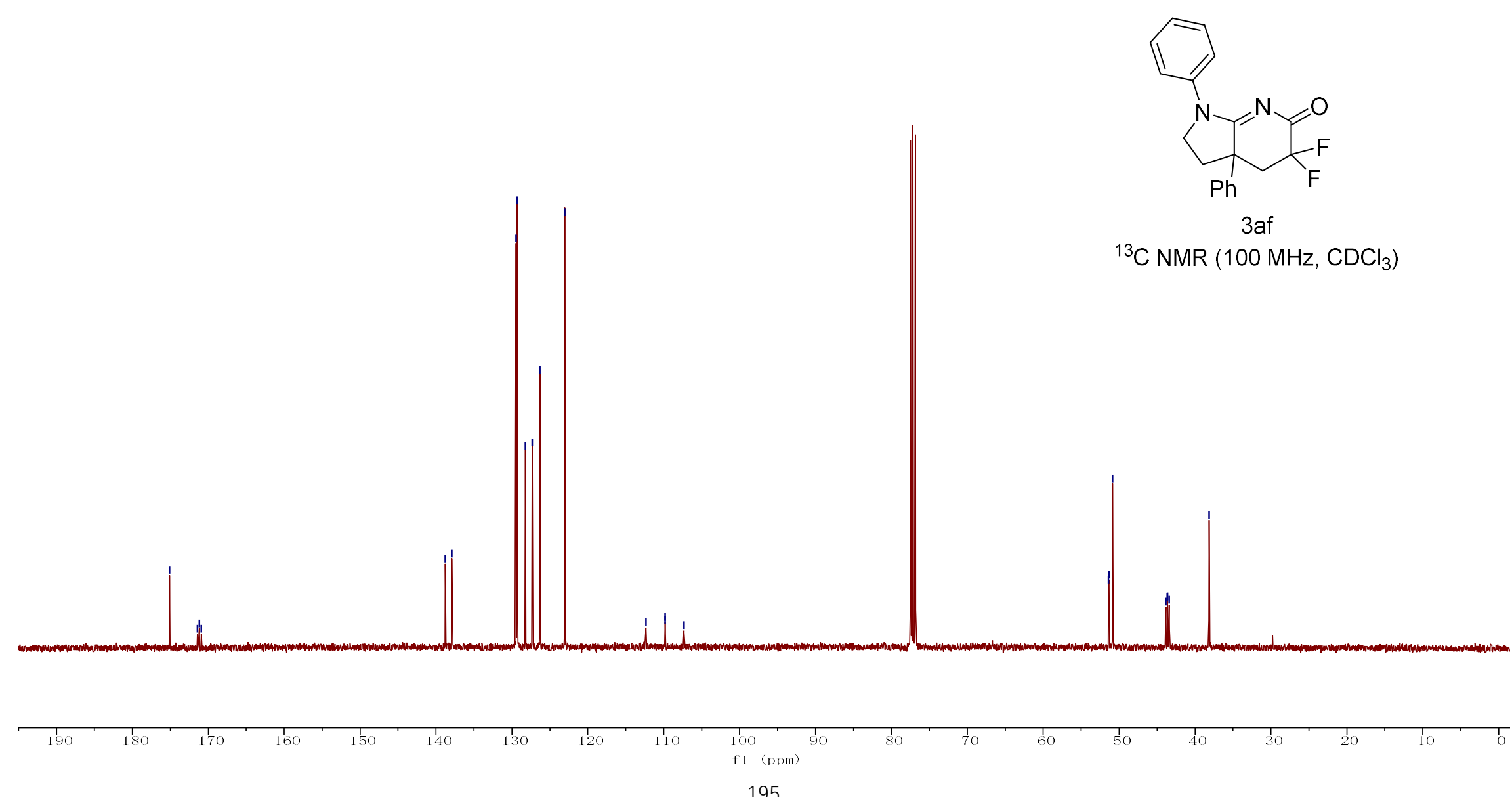


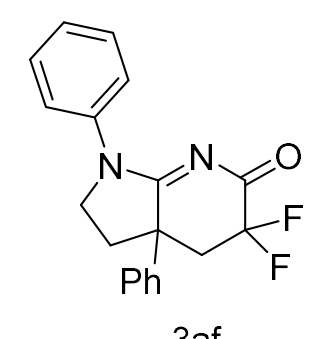

${ }^{19} \mathrm{~F} \mathrm{NMR}\left(376 \mathrm{MHz}, \mathrm{CDCl}_{3}\right)$

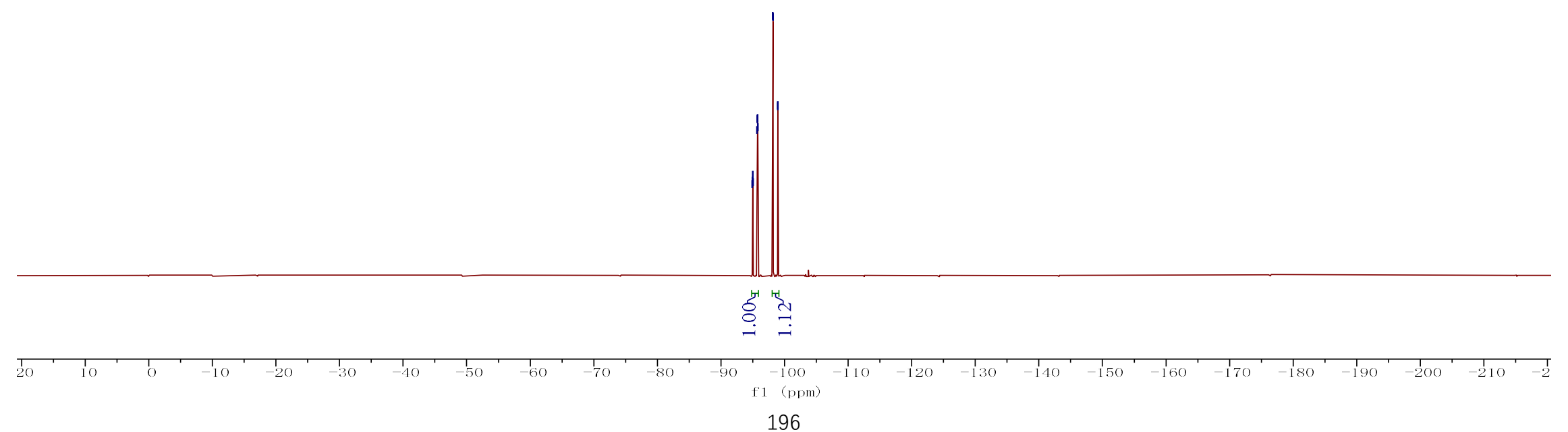




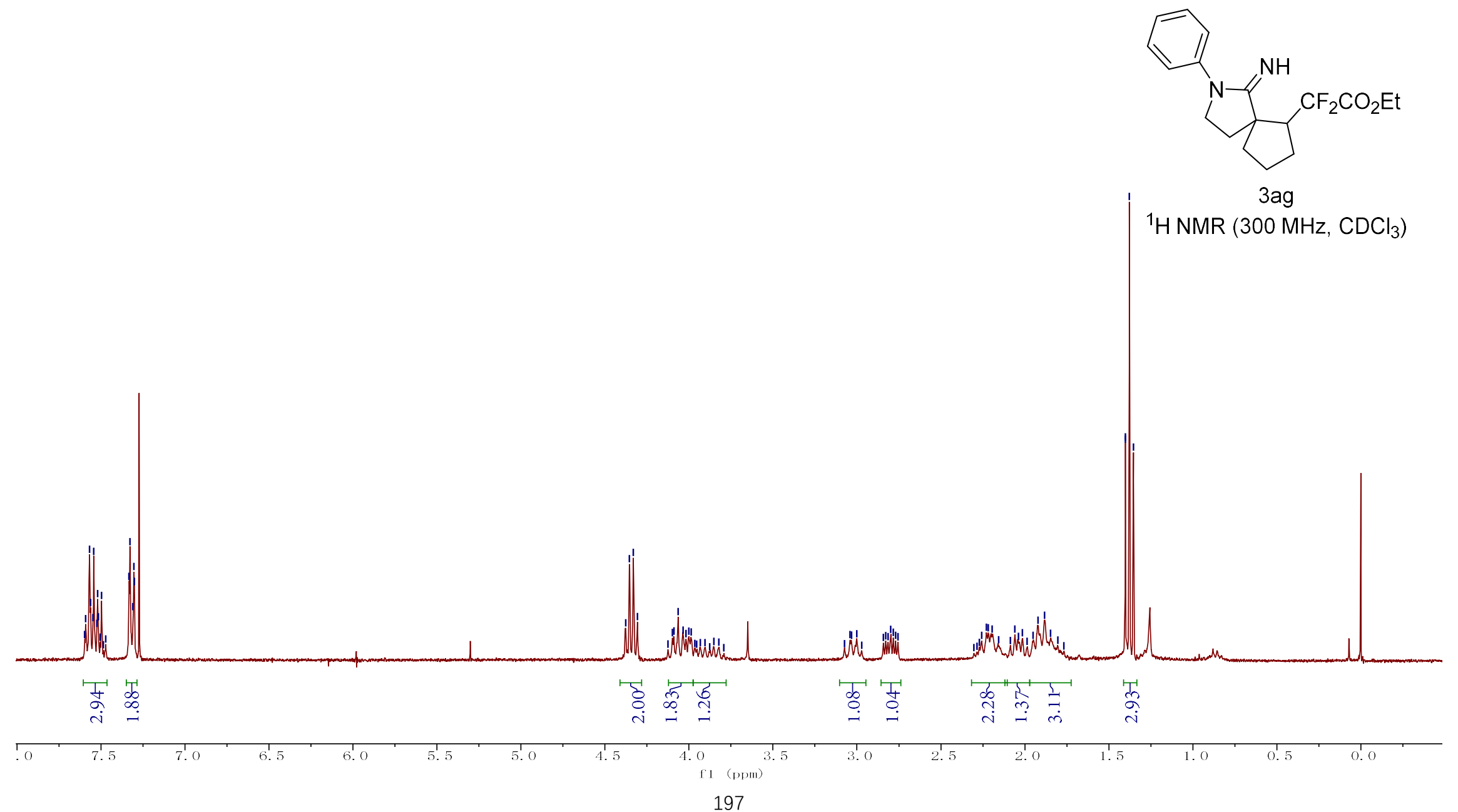



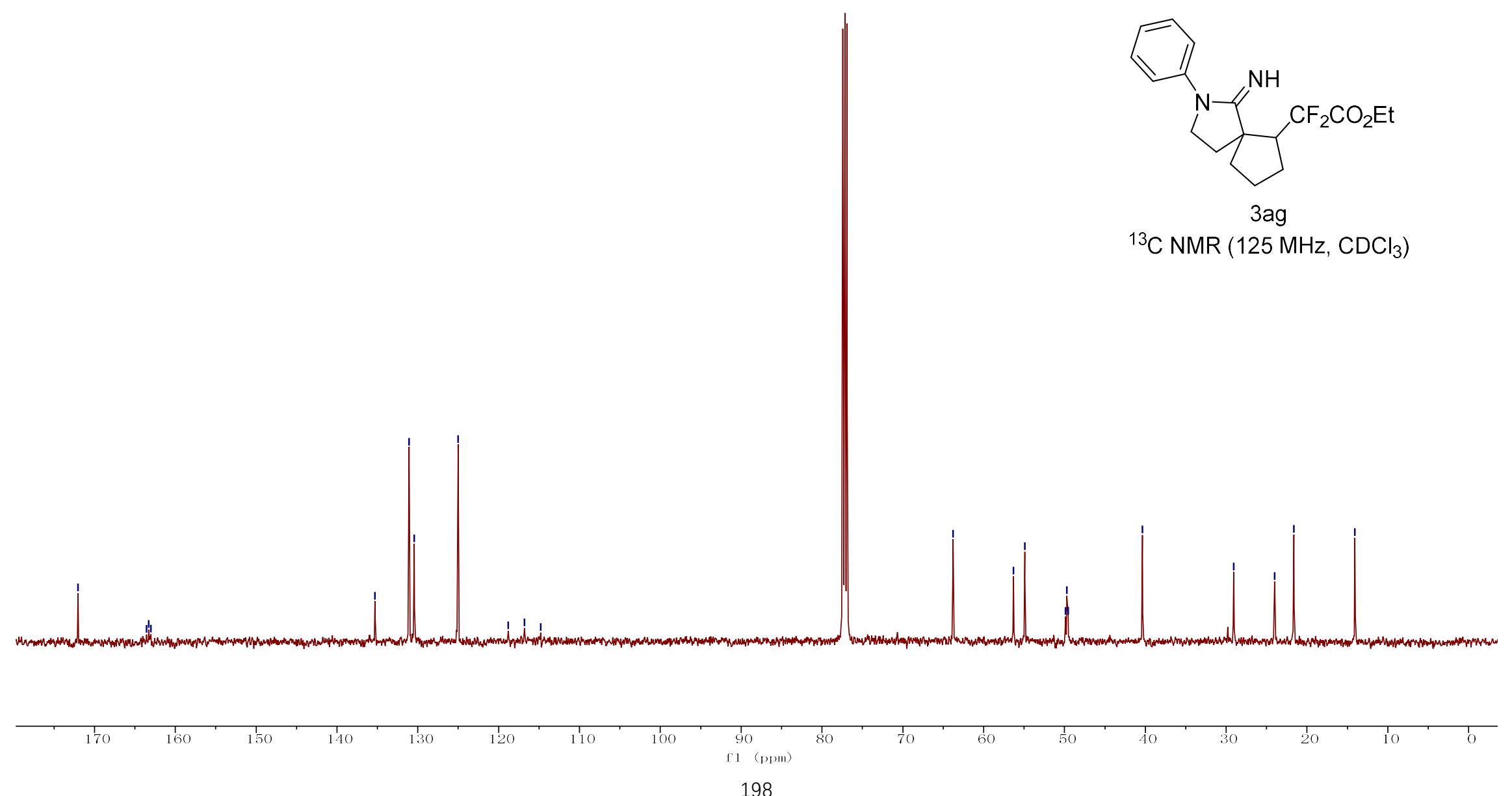


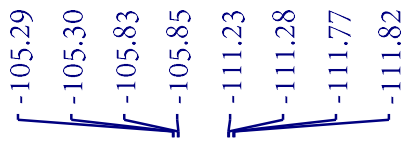

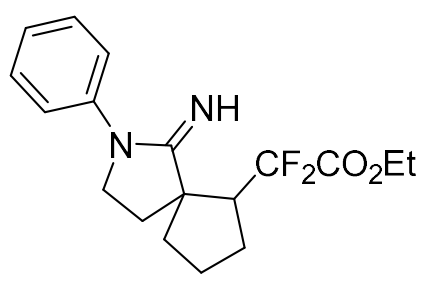

$3 a g$

${ }^{19} \mathrm{~F} \mathrm{NMR}\left(470 \mathrm{MHz}, \mathrm{CDCl}_{3}\right)$

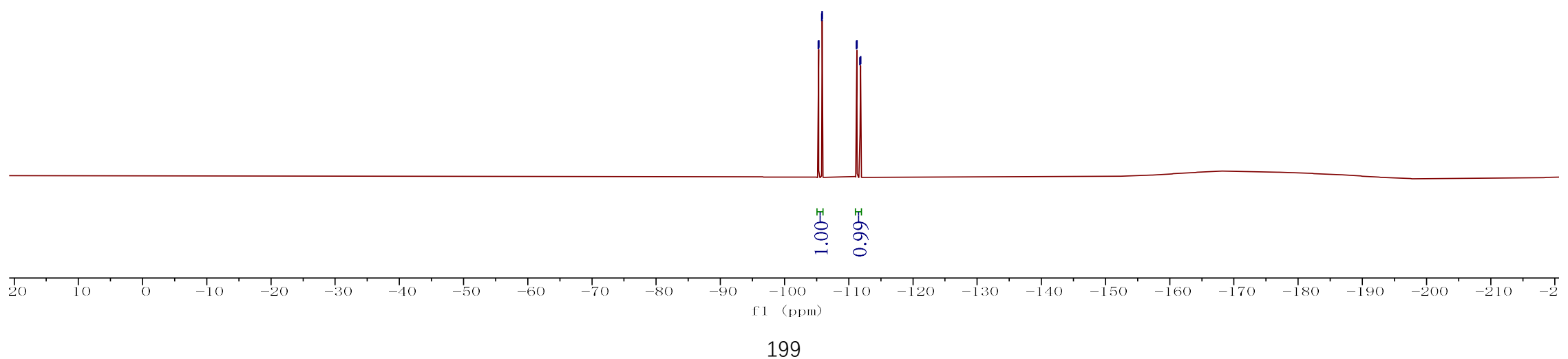




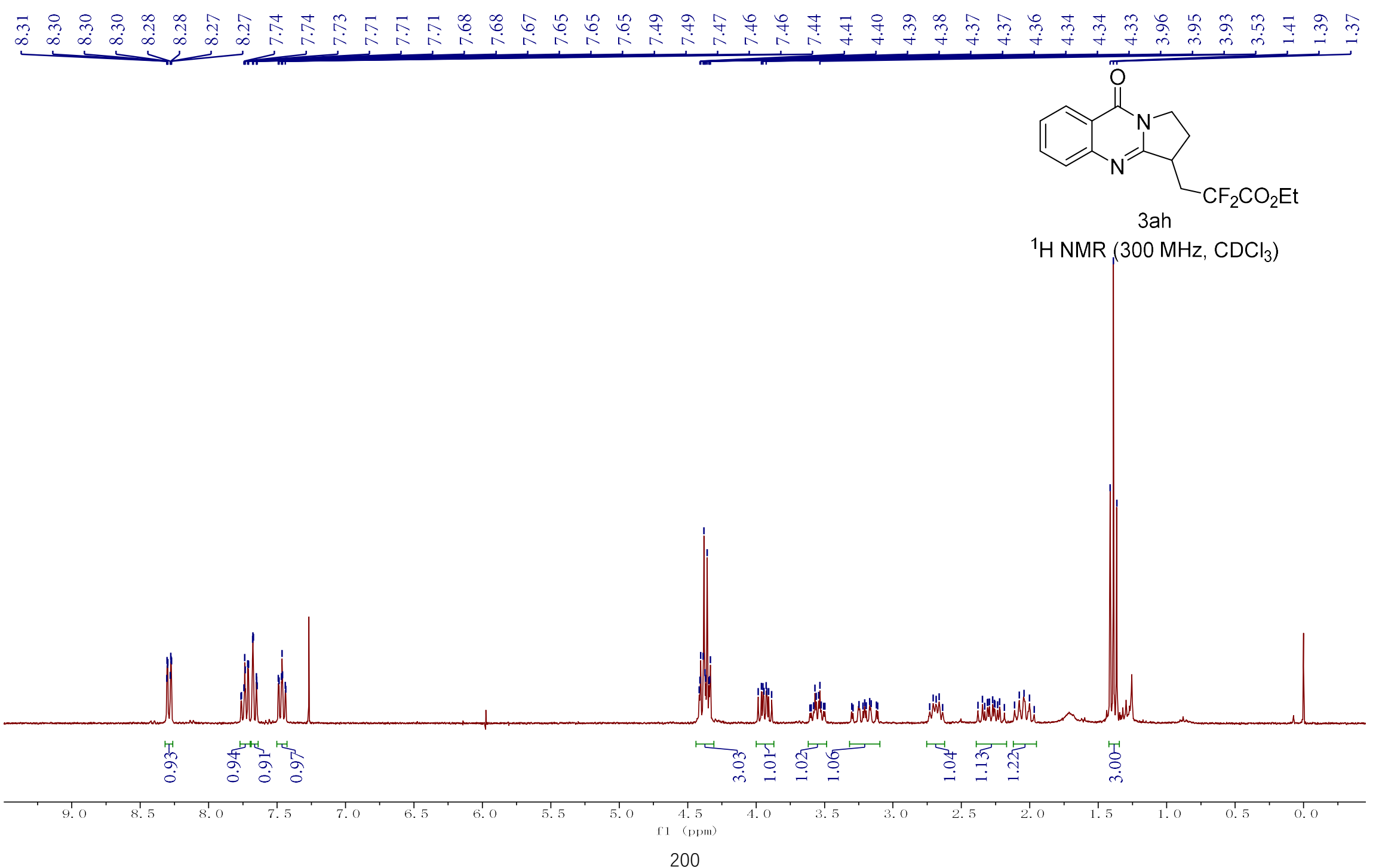




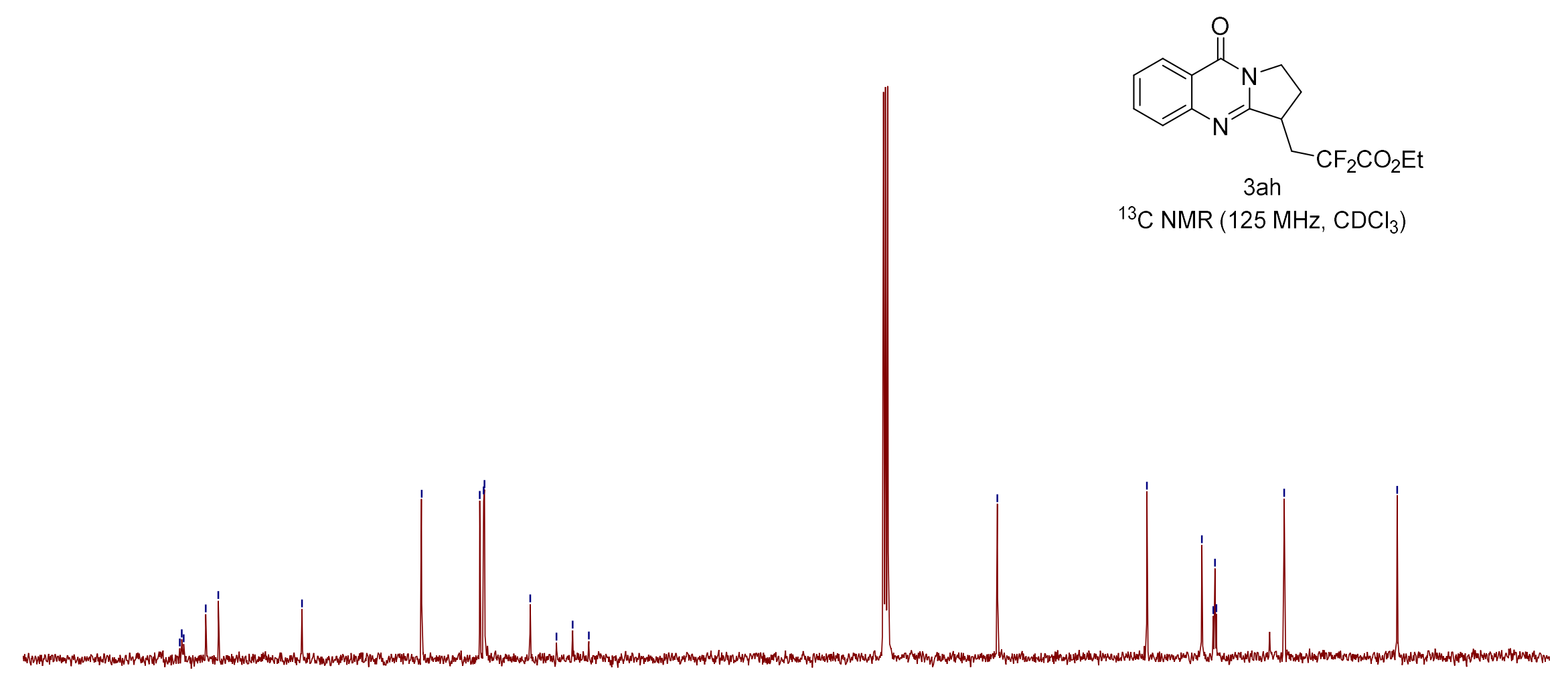

${ }^{13} \mathrm{C} \mathrm{NMR}\left(125 \mathrm{MHz}, \mathrm{CDCl}_{3}\right)$

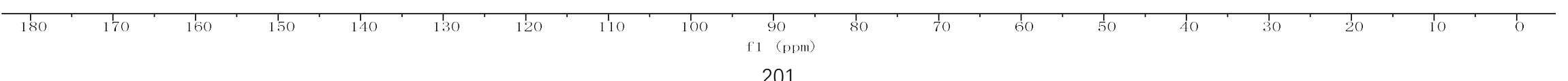



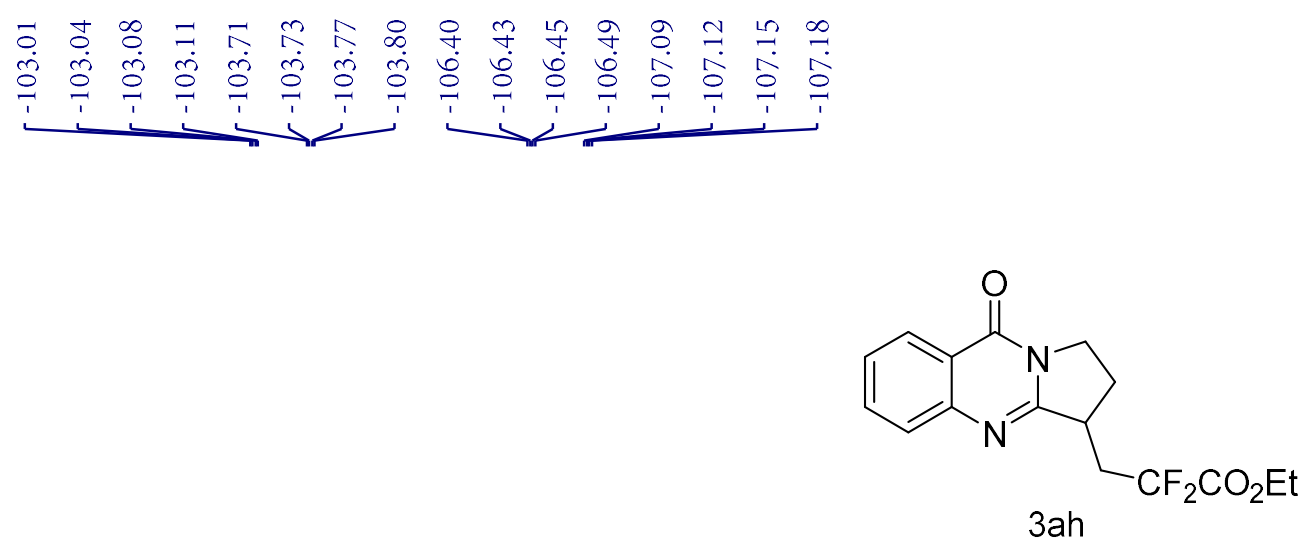

${ }^{19} \mathrm{~F} \mathrm{NMR} \mathrm{(376} \mathrm{MHz,} \mathrm{CDCl}_{3}$ )

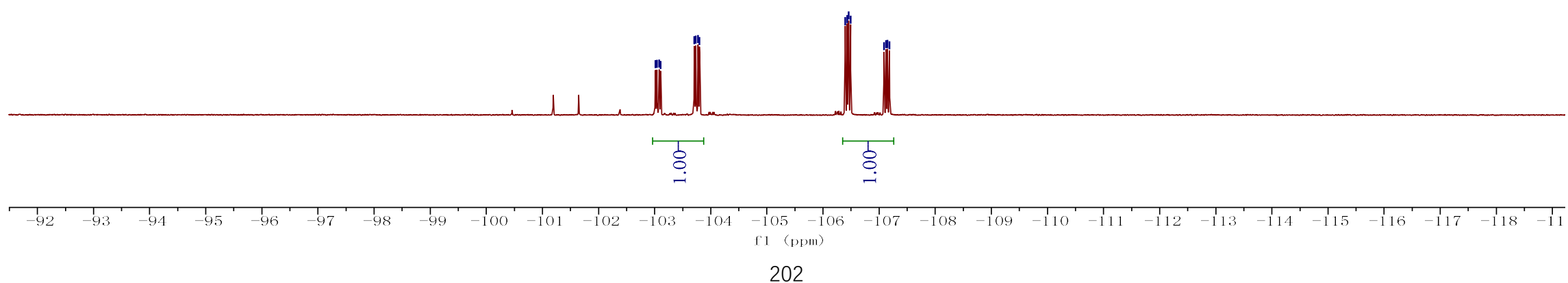

202 


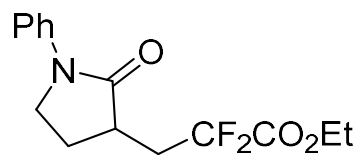

$4 a$

${ }^{1} \mathrm{H} \mathrm{NMR} \mathrm{(400} \mathrm{MHz,} \mathrm{CDCl} 3$ )

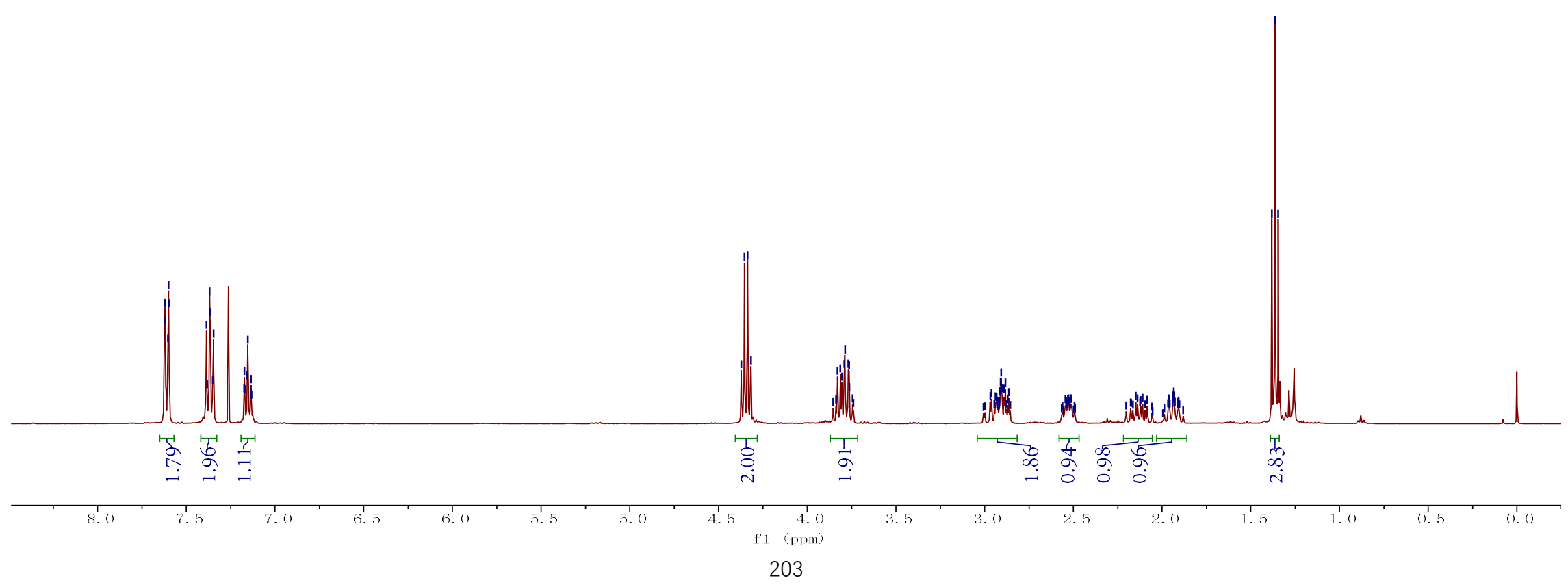




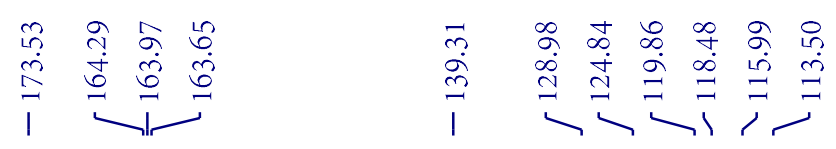

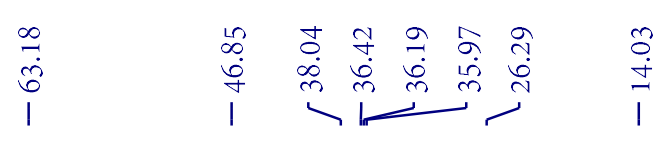

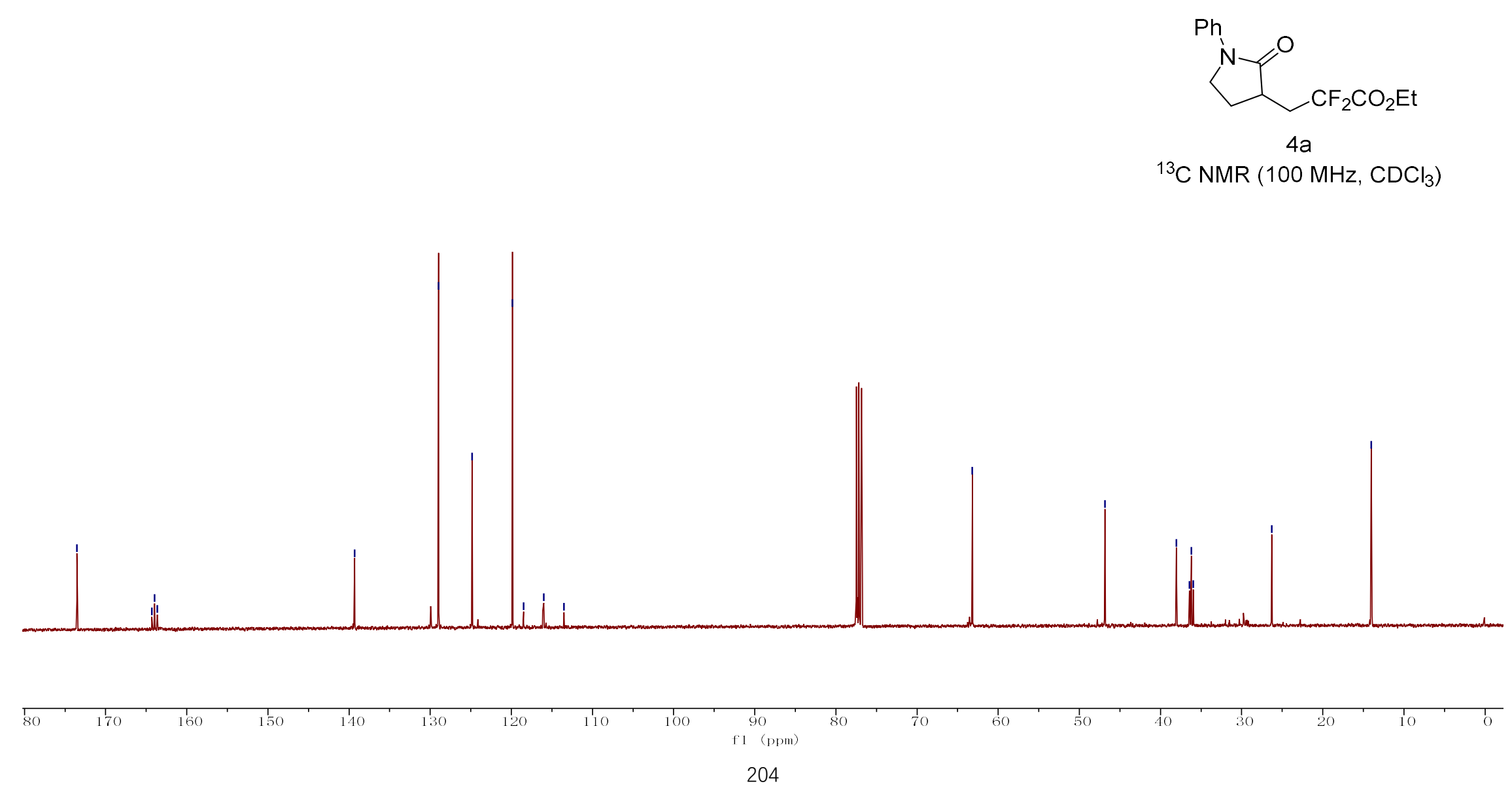




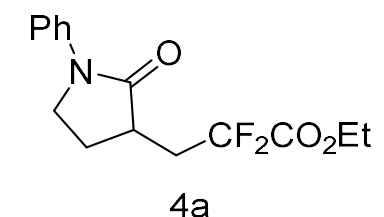

${ }^{19} \mathrm{~F} \mathrm{NMR}\left(470 \mathrm{MHz}, \mathrm{CDCl}_{3}\right)$

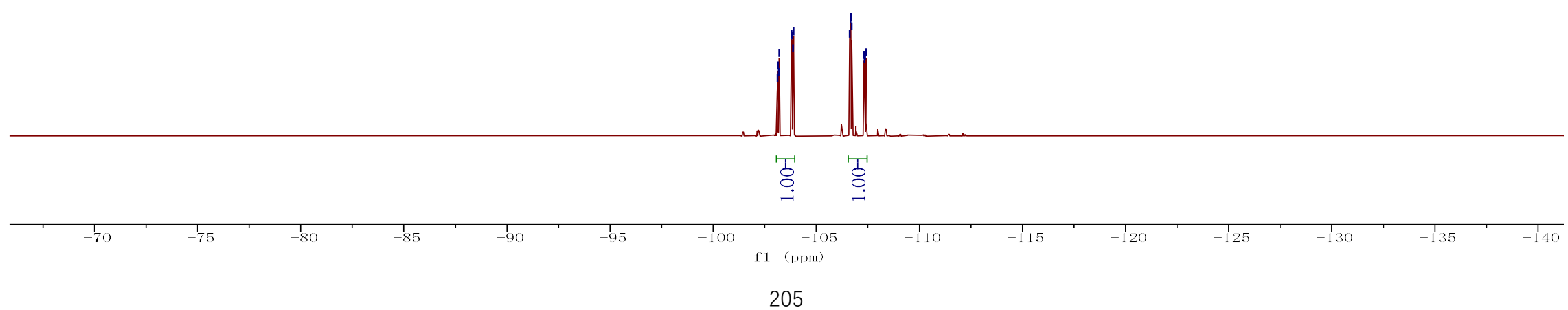




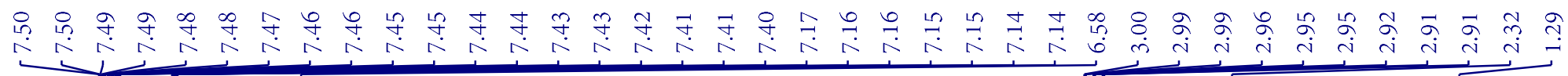

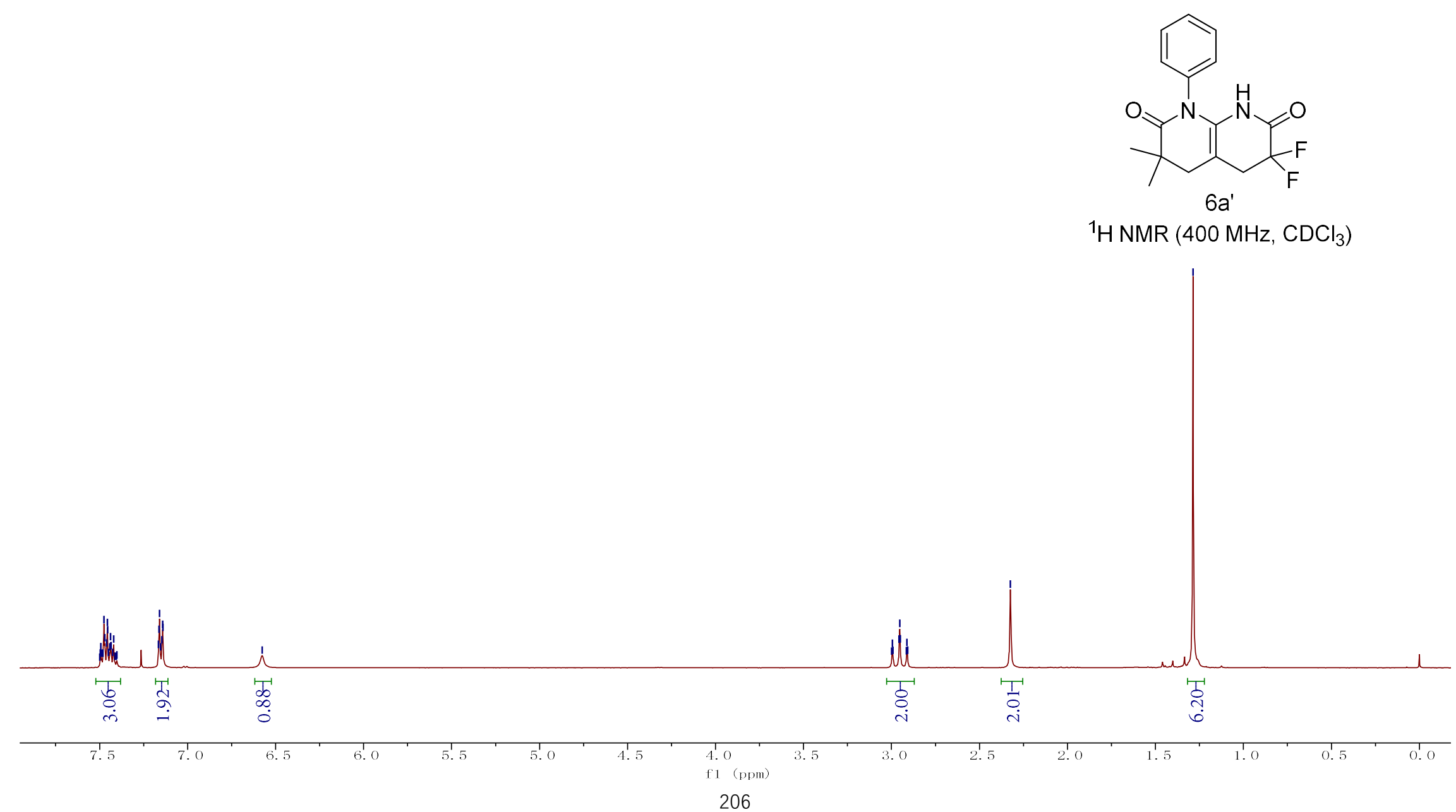




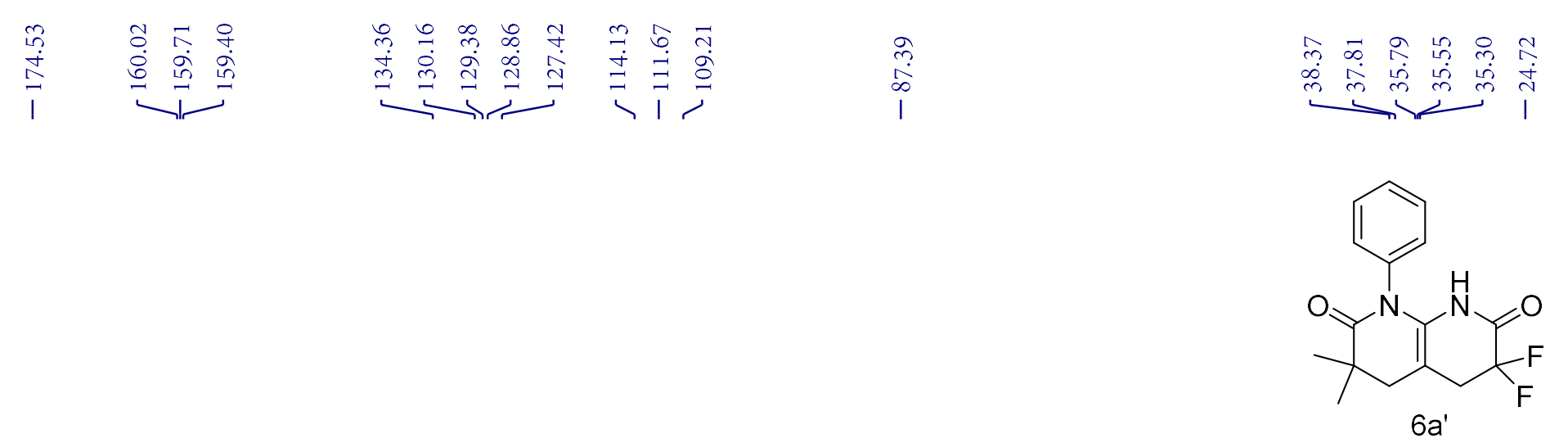

${ }^{13} \mathrm{C}$ NMR $\left(100 \mathrm{MHz}, \mathrm{CDCl}_{3}\right)$
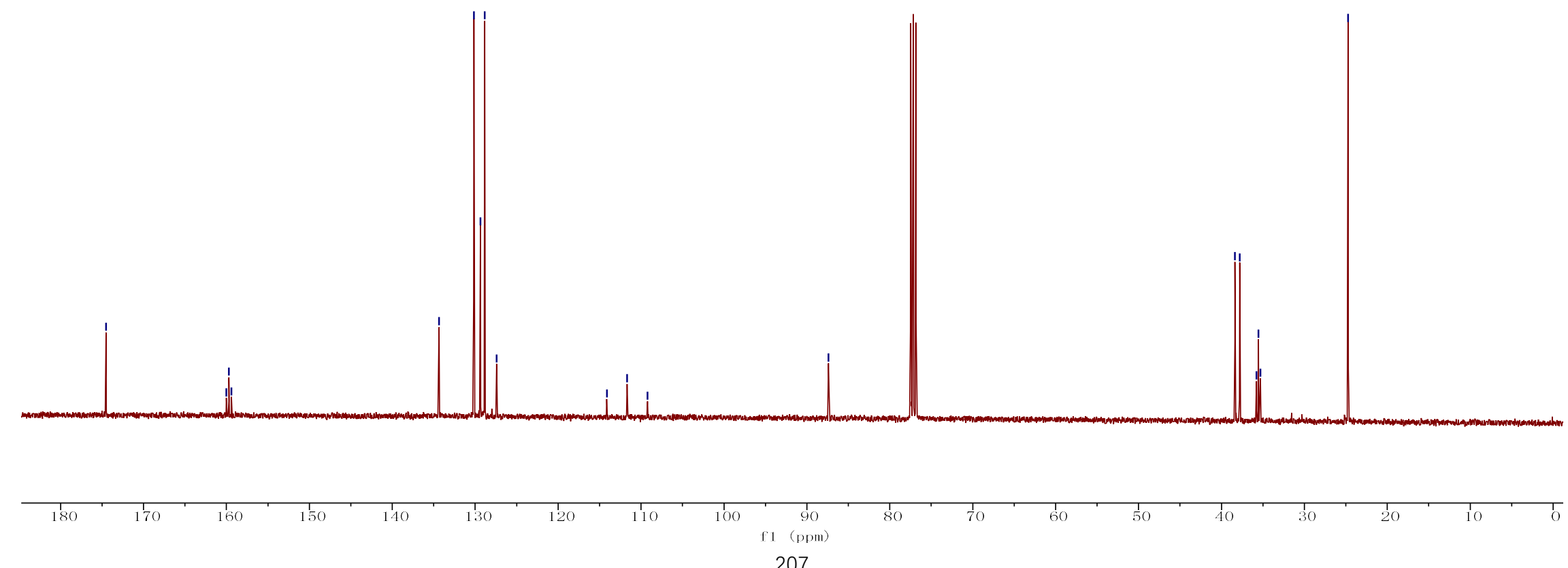


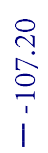

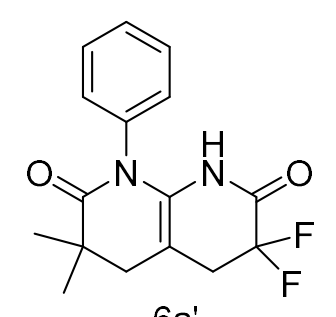

$\left.{ }^{19} \mathrm{~F} \mathrm{NMR} \mathrm{(376} \mathrm{MHz,} \mathrm{CDCl}_{3}\right)$

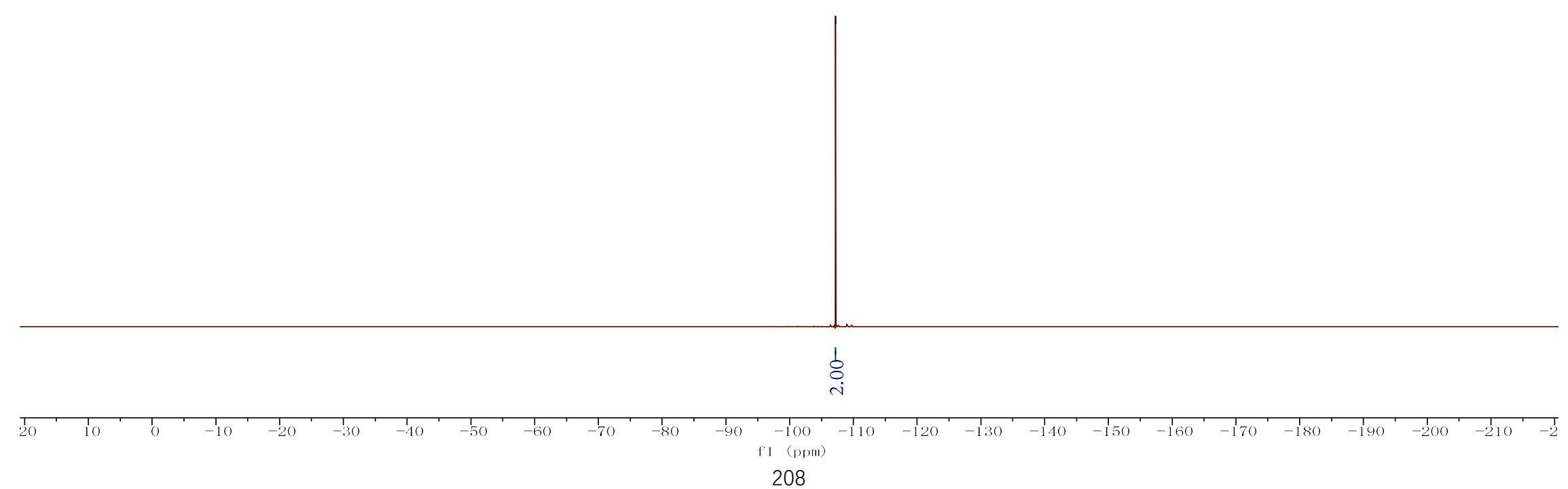




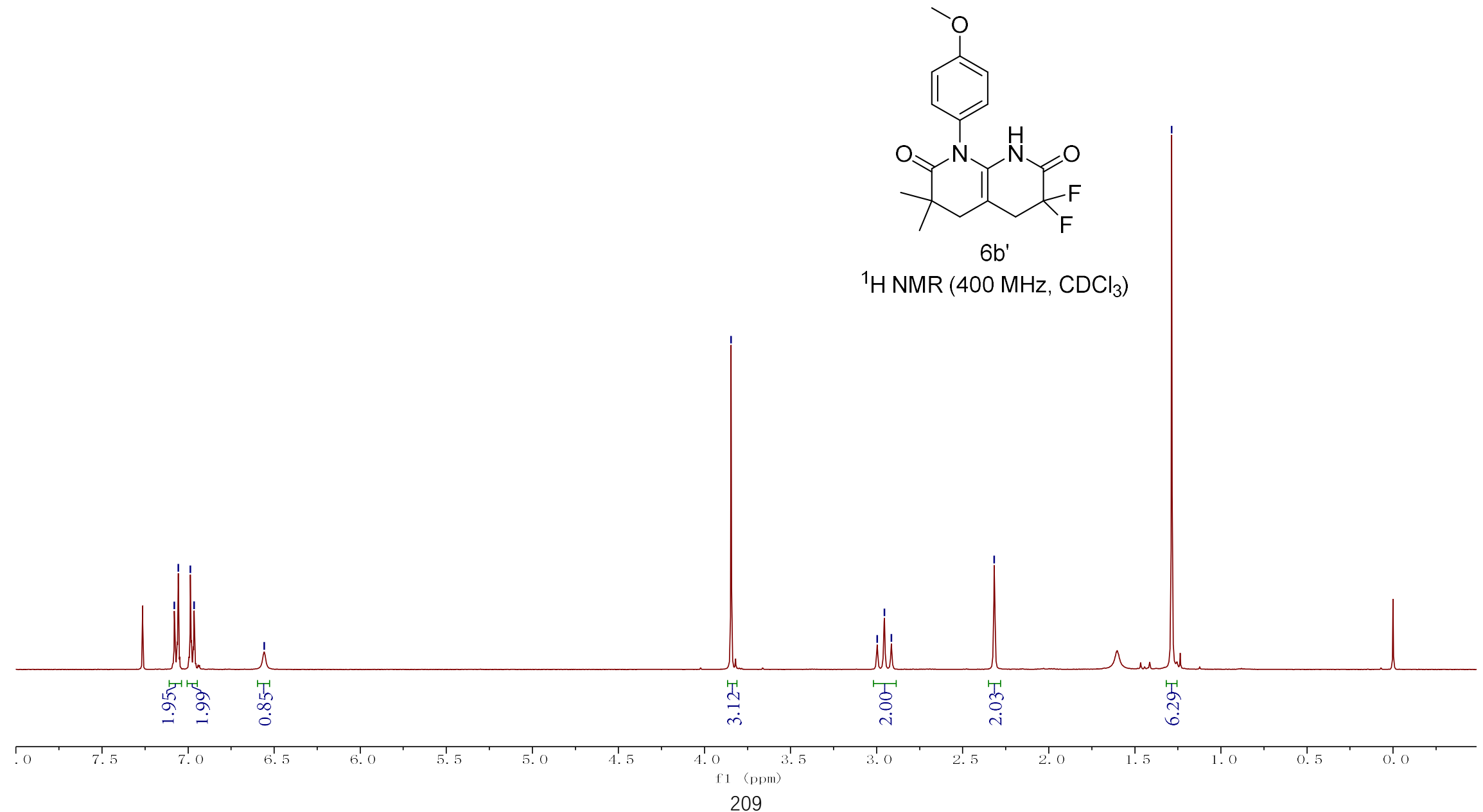



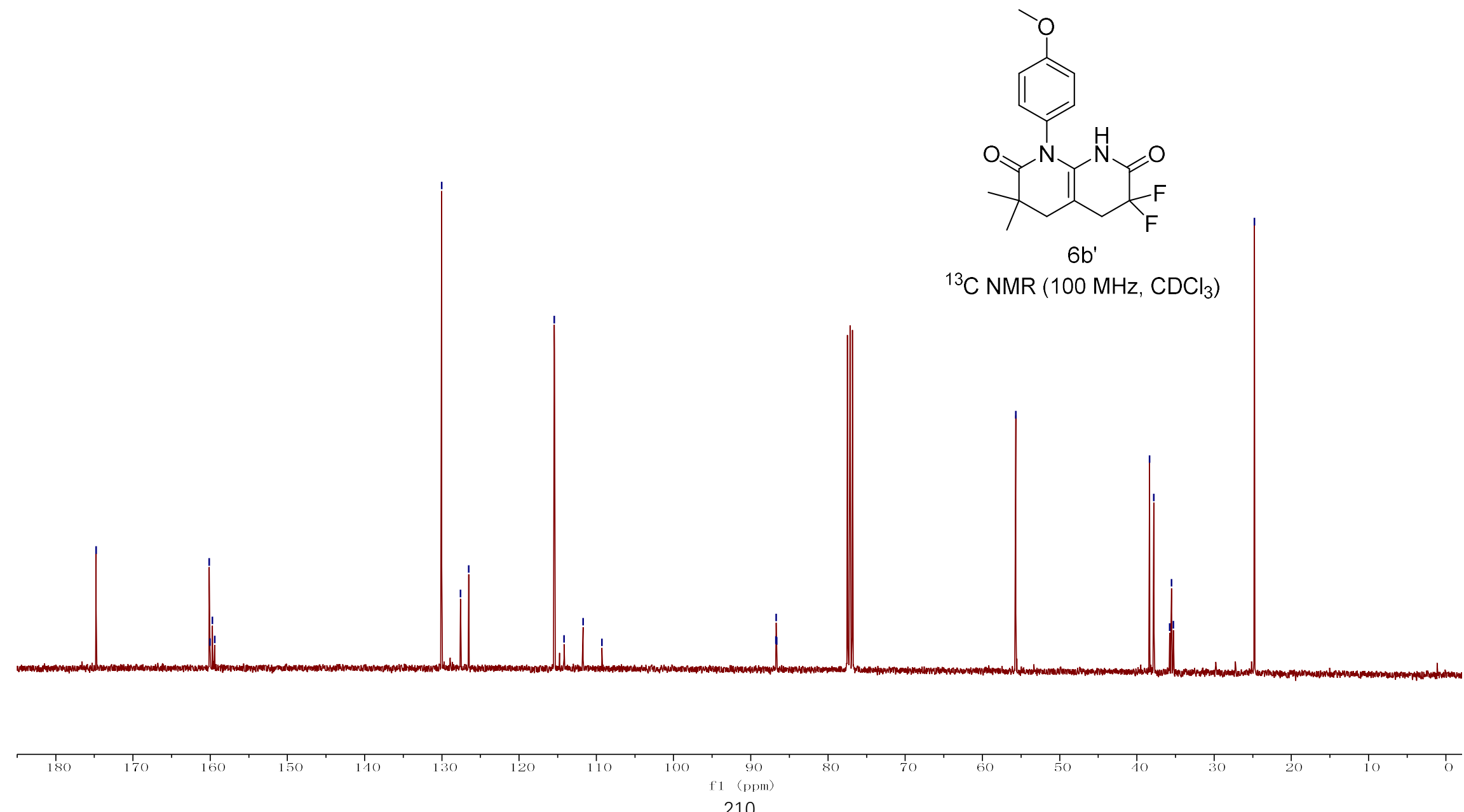
$\begin{array}{lll}3 & 0 & 0 \\ 0 & 0 \\ 0 & 0 & 0 \\ 0 & 0 & 1 \\ 1 & 1\end{array}$

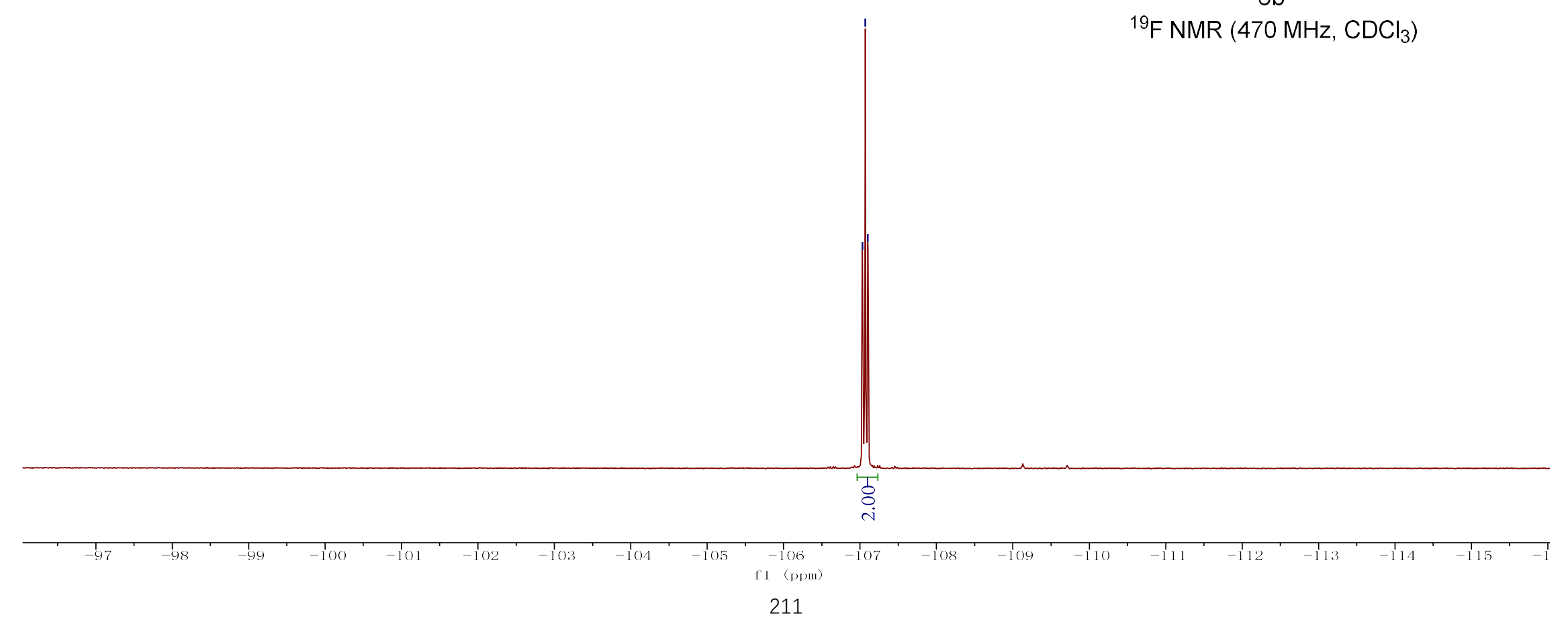

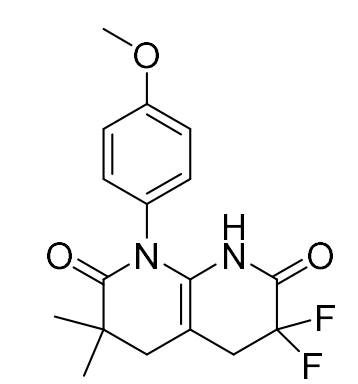

$6 b^{\prime}$

${ }^{19} \mathrm{~F} \mathrm{NMR}\left(470 \mathrm{MHz}, \mathrm{CDCl}_{3}\right)$ 


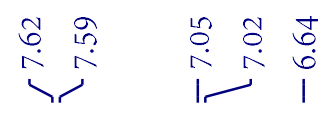

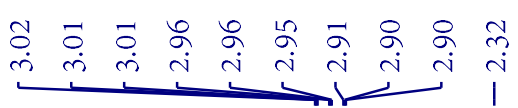

$\stackrel{\infty}{\stackrel{\infty}{\longrightarrow}}$

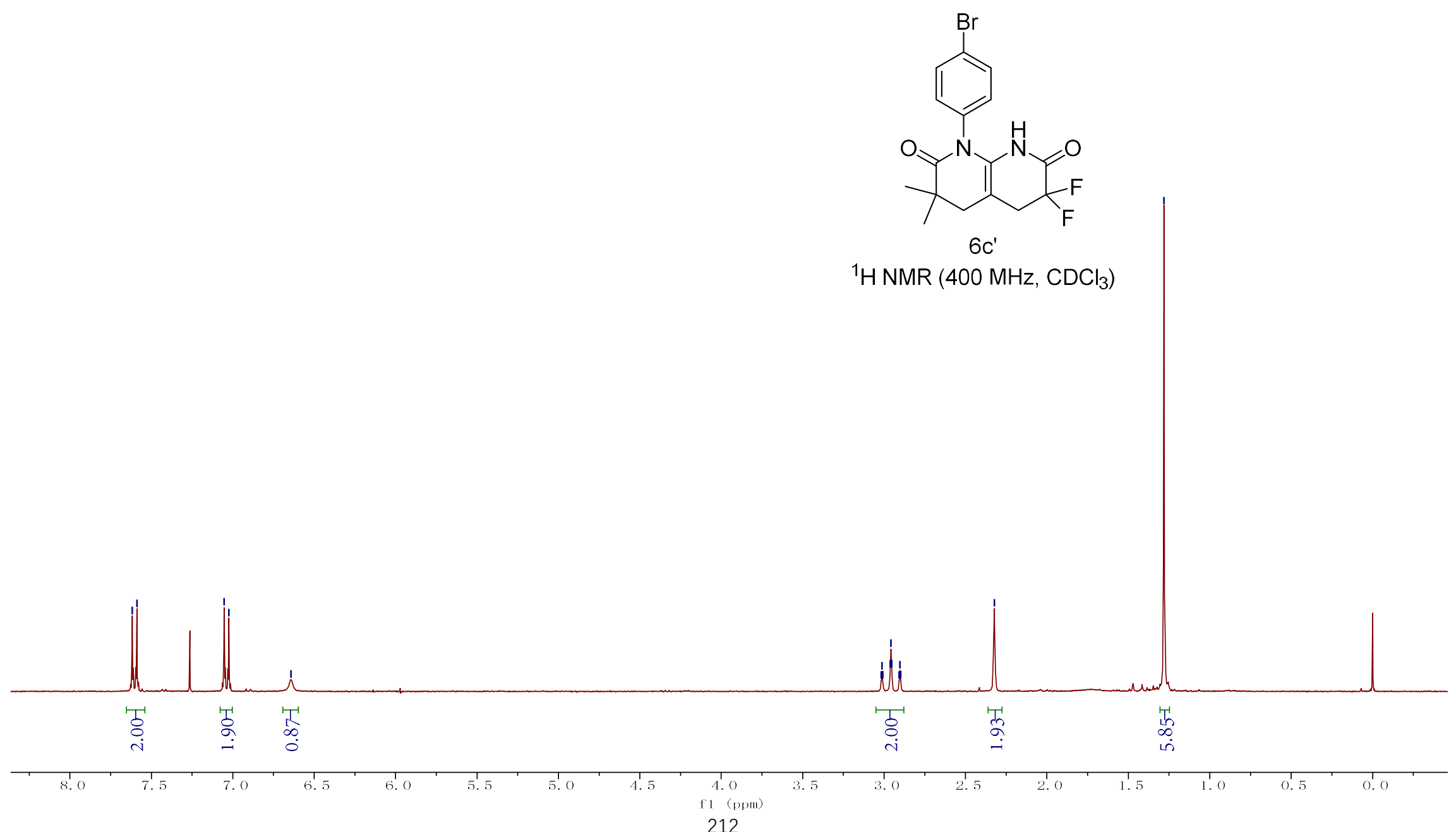




$$
1 \mid
$$




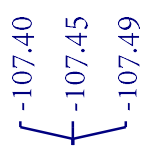

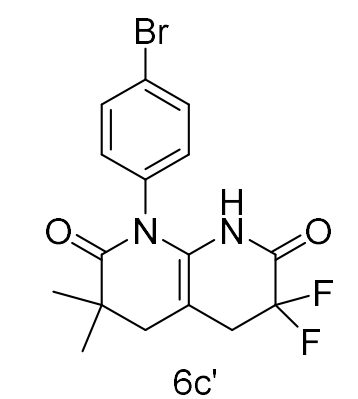

${ }^{19} \mathrm{~F} \mathrm{NMR}\left(376 \mathrm{MHz}, \mathrm{CDCl}_{3}\right)$

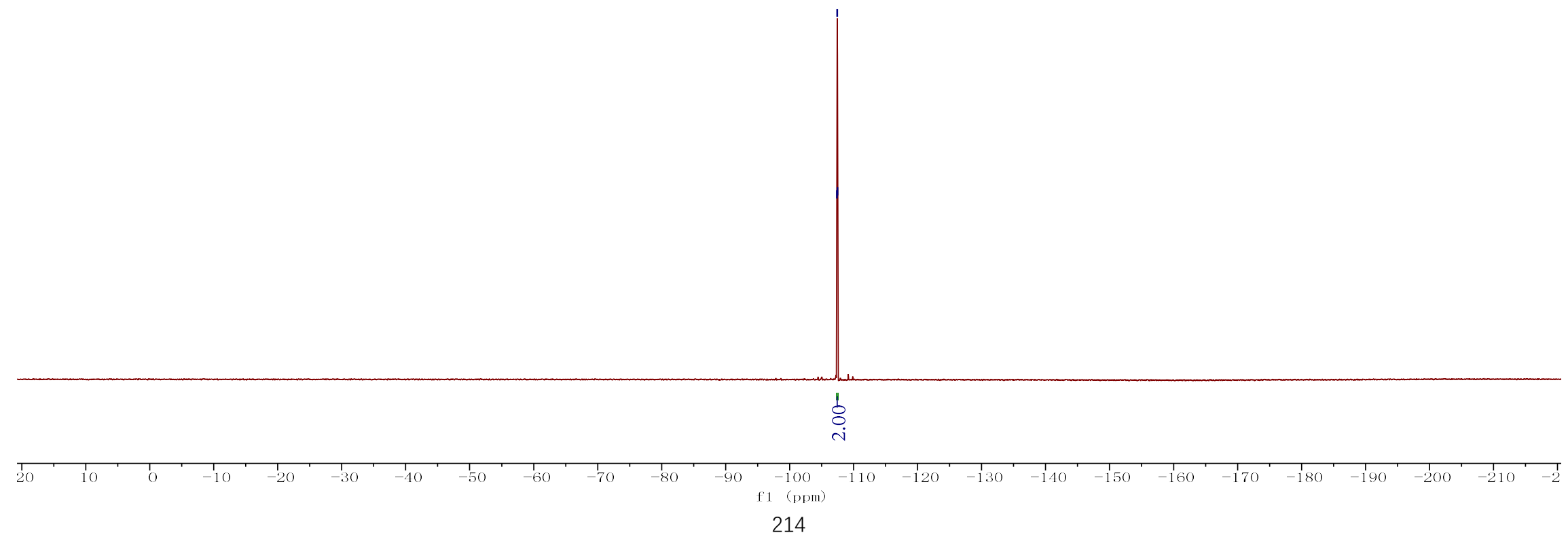




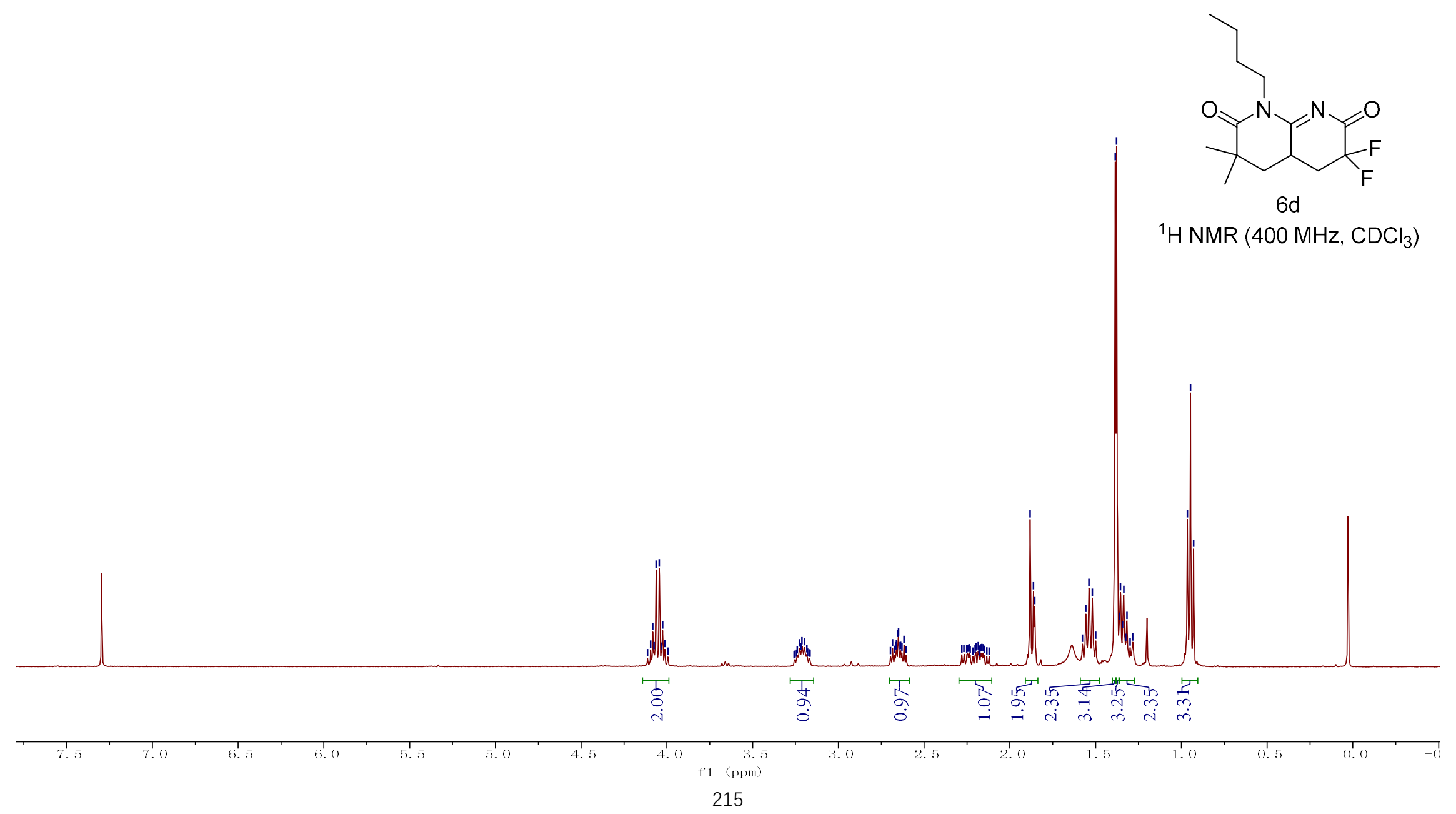




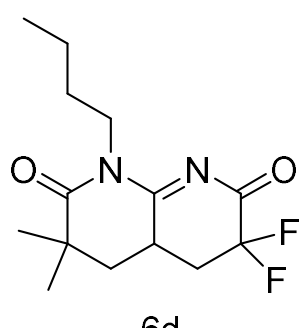

${ }^{13} \mathrm{C}$ NMR $\left(125 \mathrm{MHz}, \mathrm{CDCl}_{3}\right)$

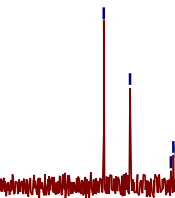

\section{|}

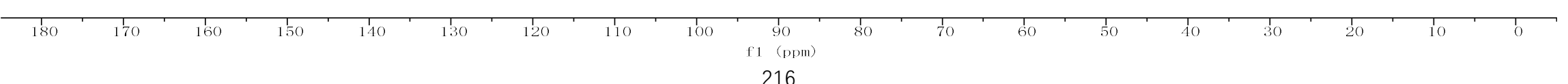




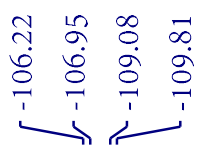

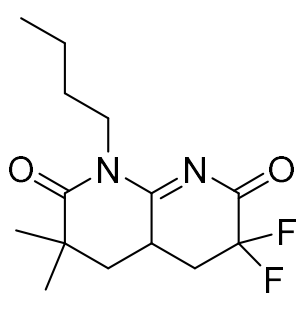

$6 d$

$\left.{ }^{19} \mathrm{~F} \mathrm{NMR} \mathrm{(376} \mathrm{MHz,} \mathrm{CDCl}_{3}\right)$

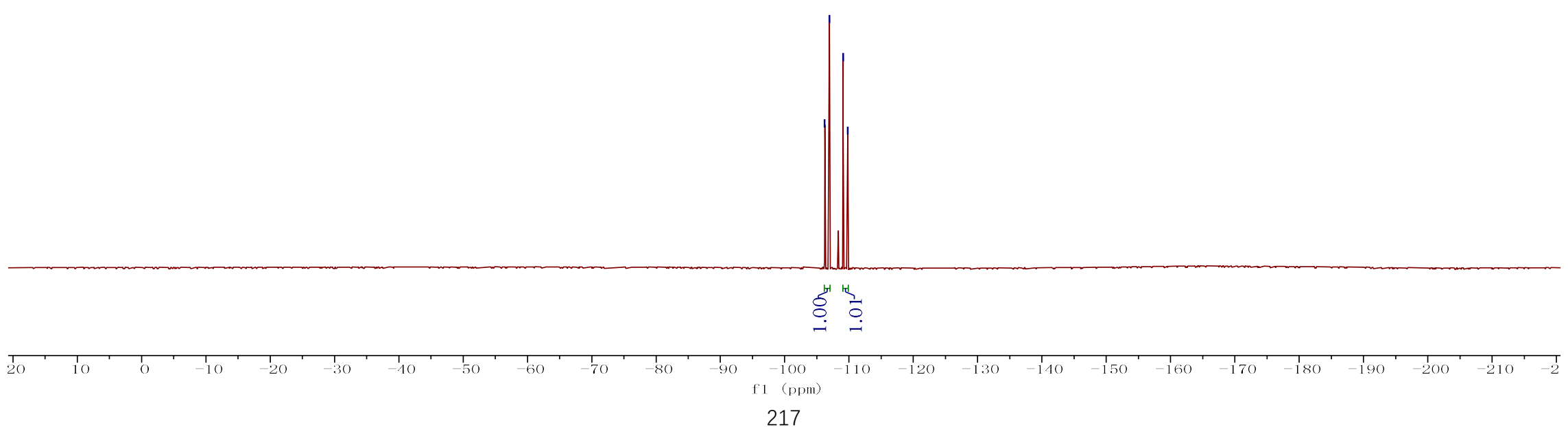




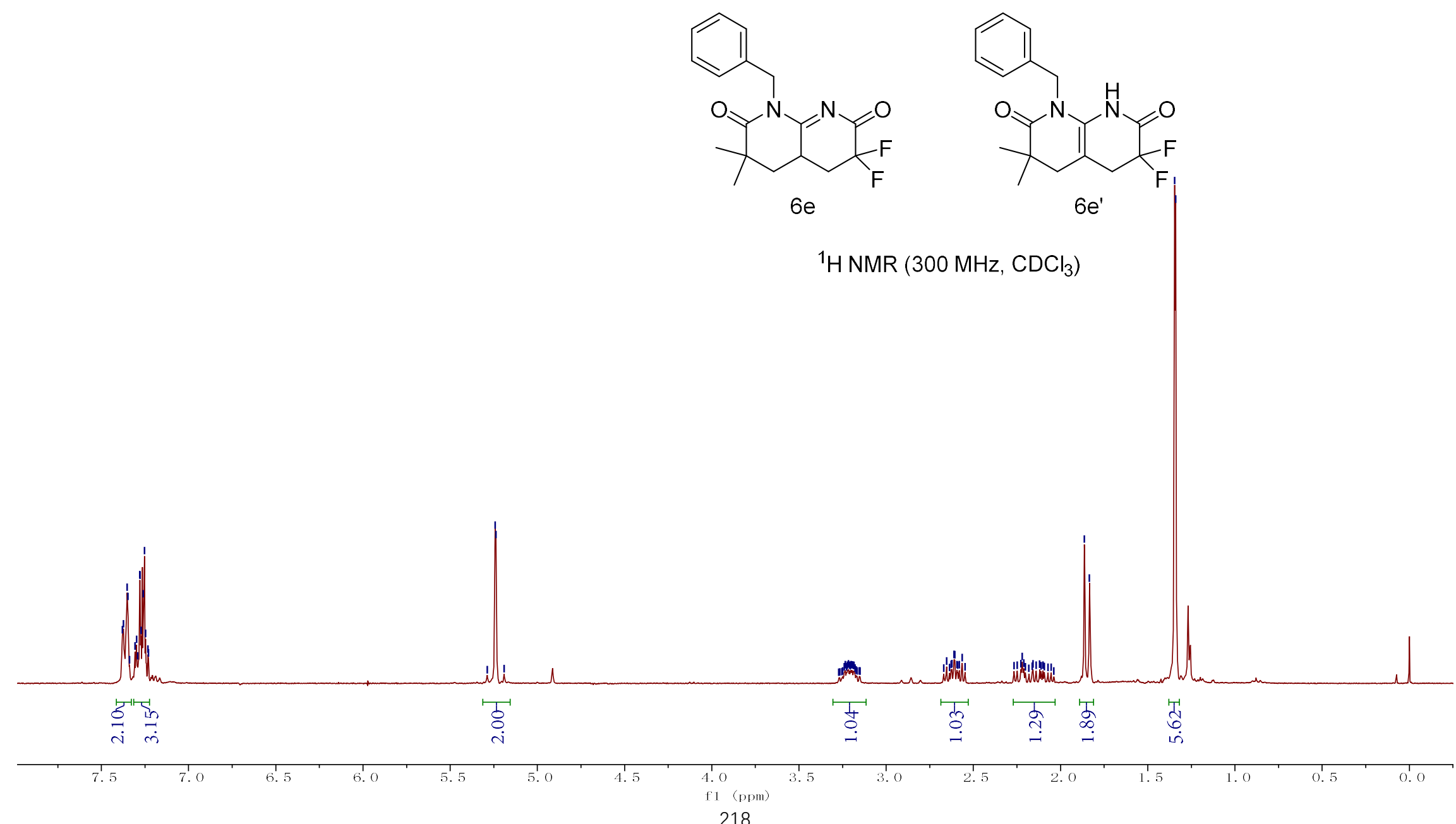




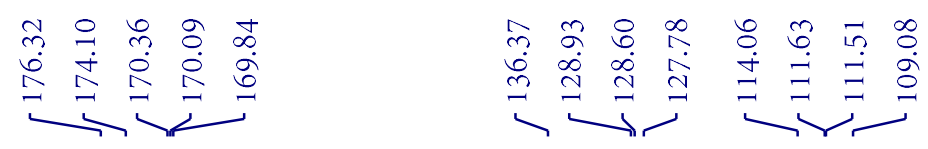

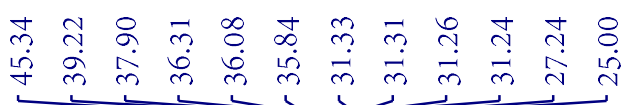

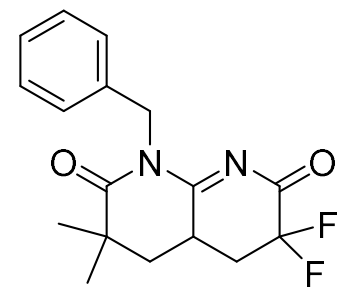

$6 e$

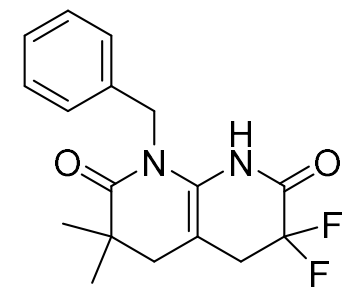

${ }^{13} \mathrm{C} \mathrm{NMR}\left(100 \mathrm{MHz}, \mathrm{CDCl}_{3}\right)$
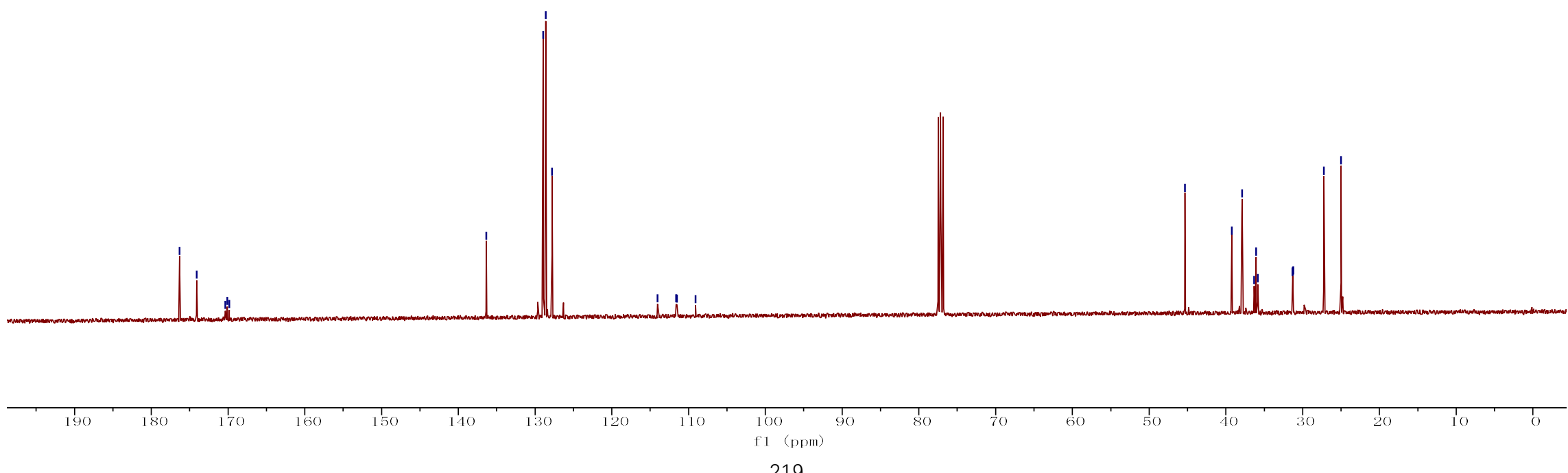


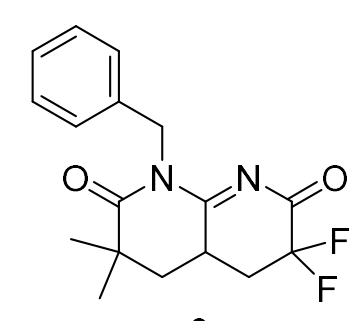

$6 e$

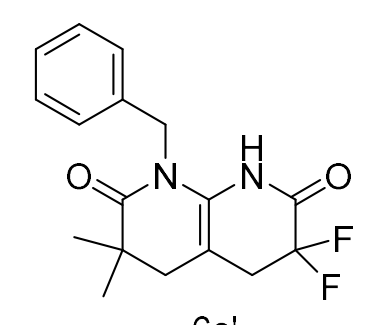

$6 e^{\prime}$

${ }^{19} \mathrm{~F} \mathrm{NMR}\left(376 \mathrm{MHz}, \mathrm{CDCl}_{3}\right)$

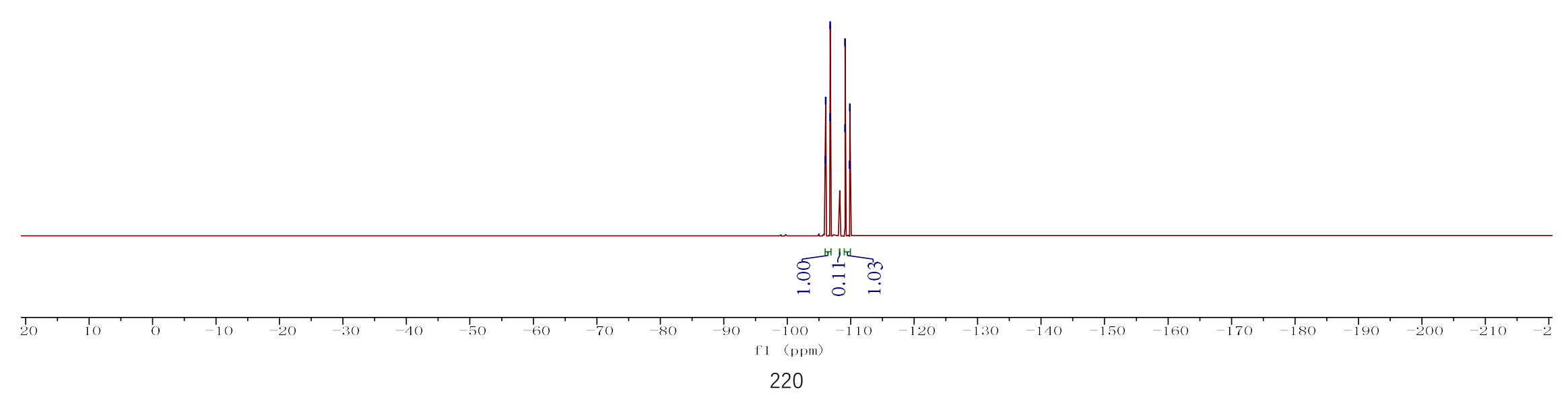



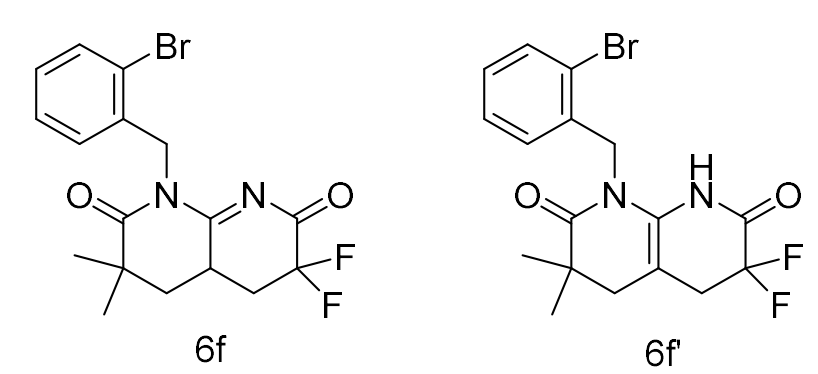

${ }^{1} \mathrm{HNMR}\left(400 \mathrm{MHz}_{\mathrm{CDCl}}\right)$

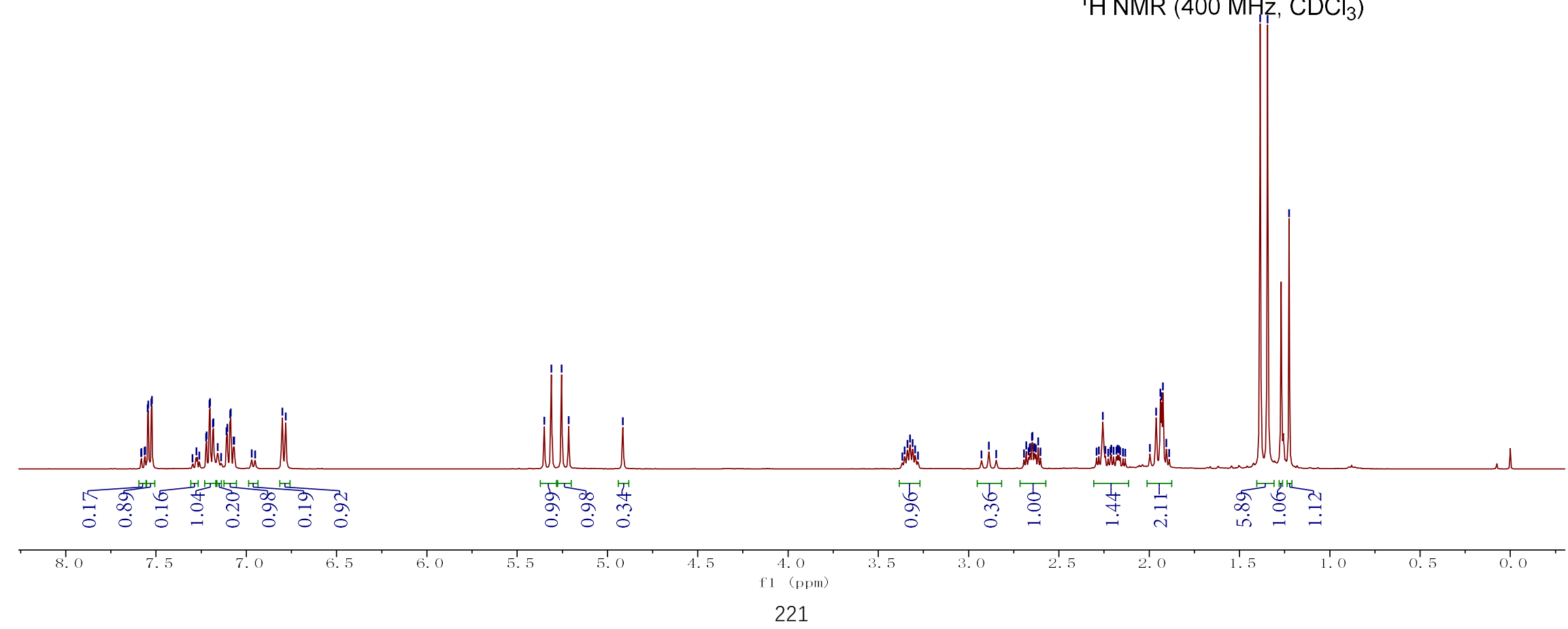




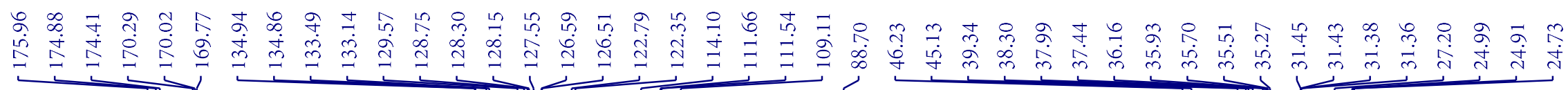

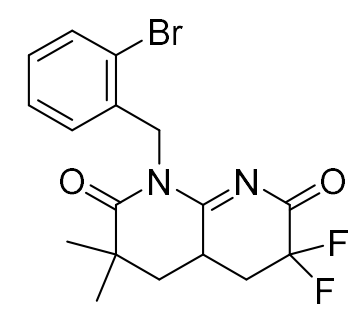

$6 f$

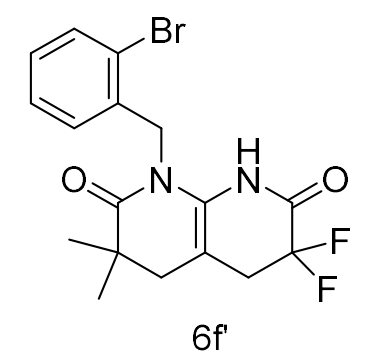

$6 f^{\prime}$

${ }^{13} \mathrm{C} \mathrm{NMR}\left(100 \mathrm{MHz}, \mathrm{CDCl}_{3}\right)$
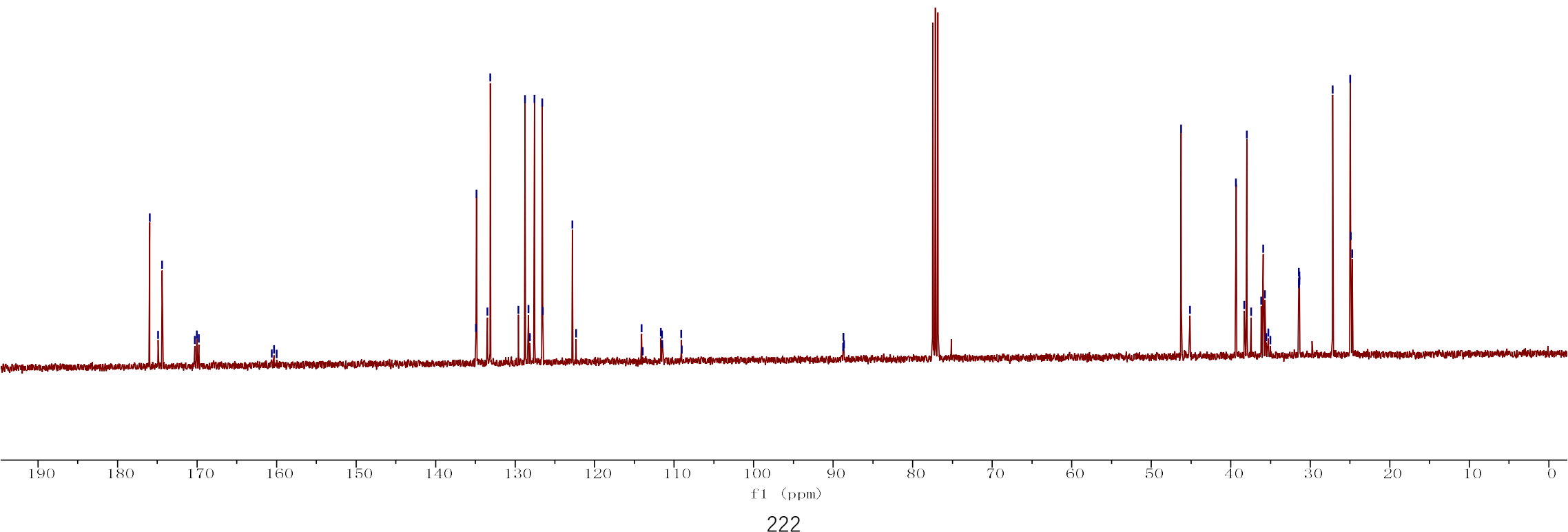

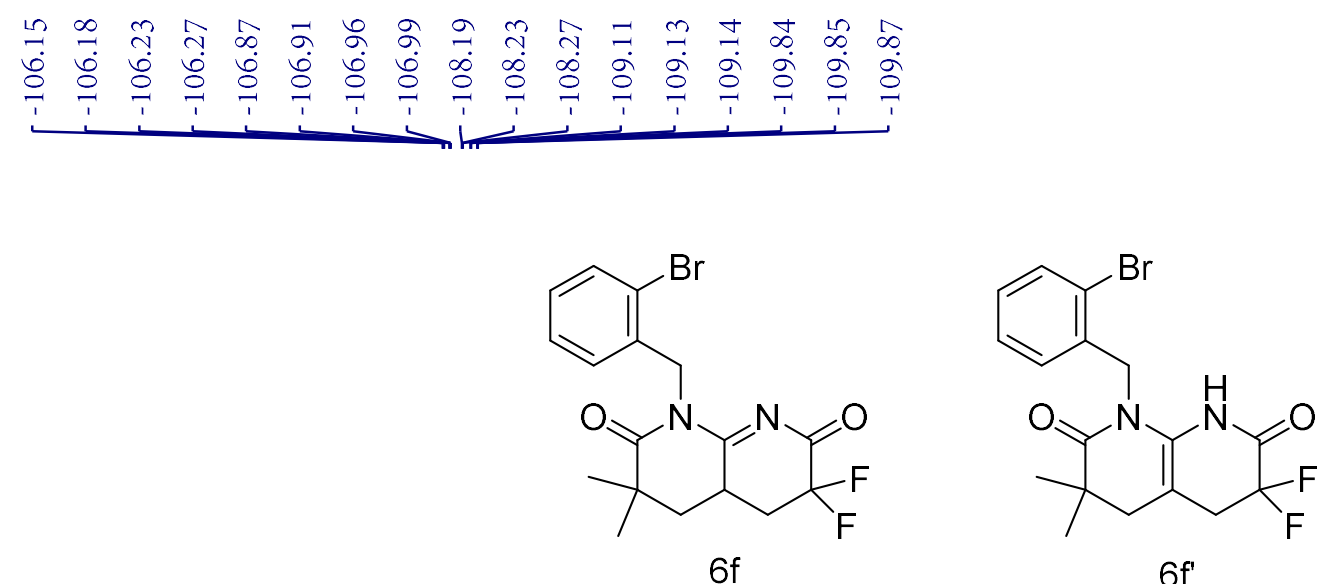

${ }^{19} \mathrm{~F} \mathrm{NMR} \mathrm{(376} \mathrm{MHz,} \mathrm{CDCl}_{3}$ )

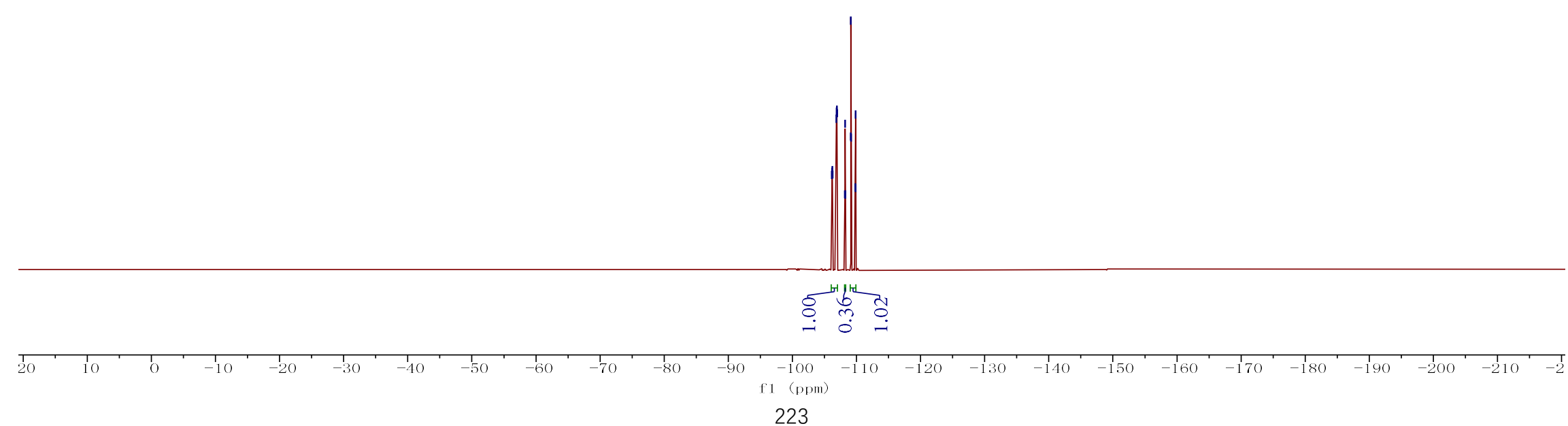



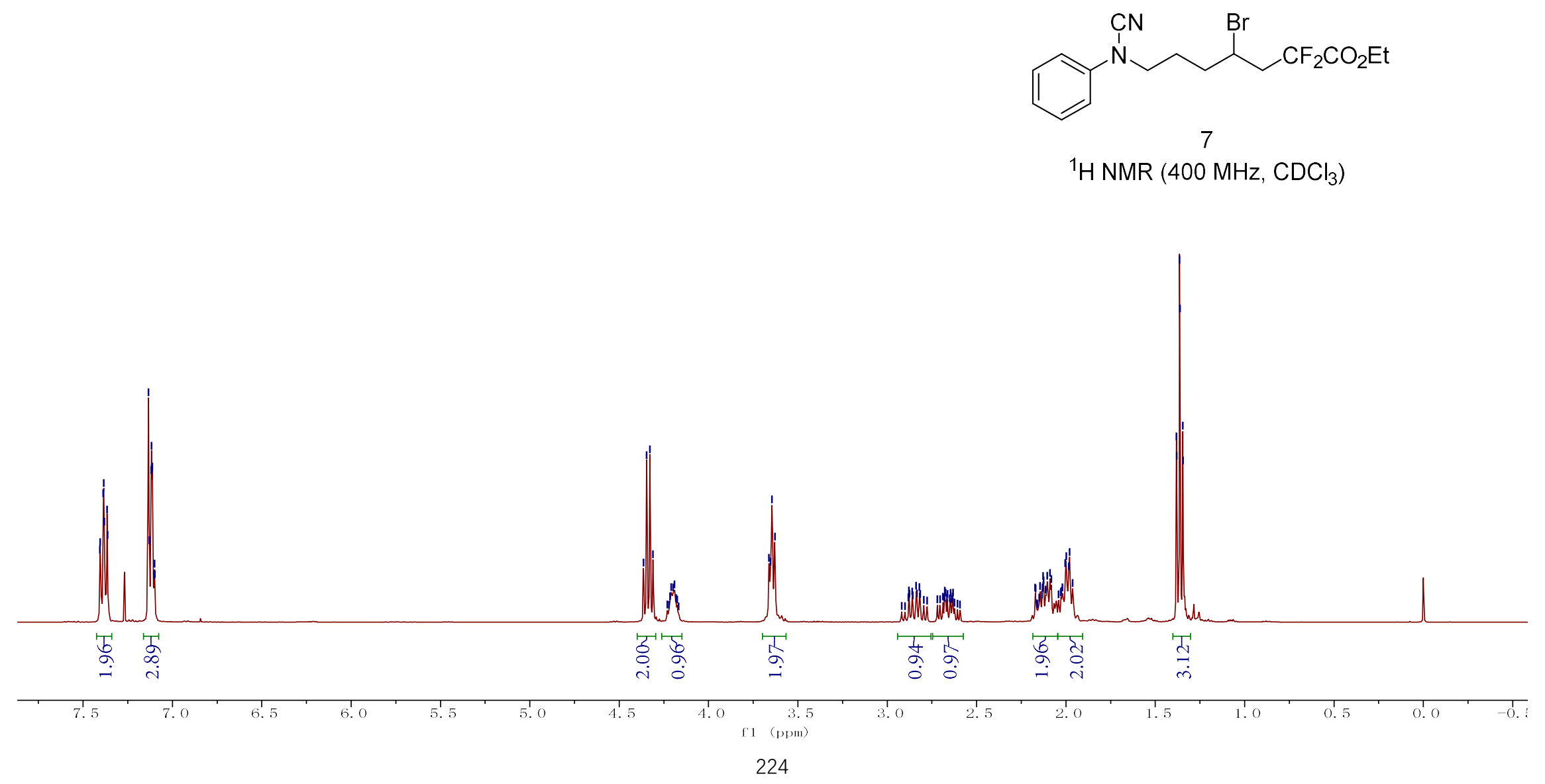


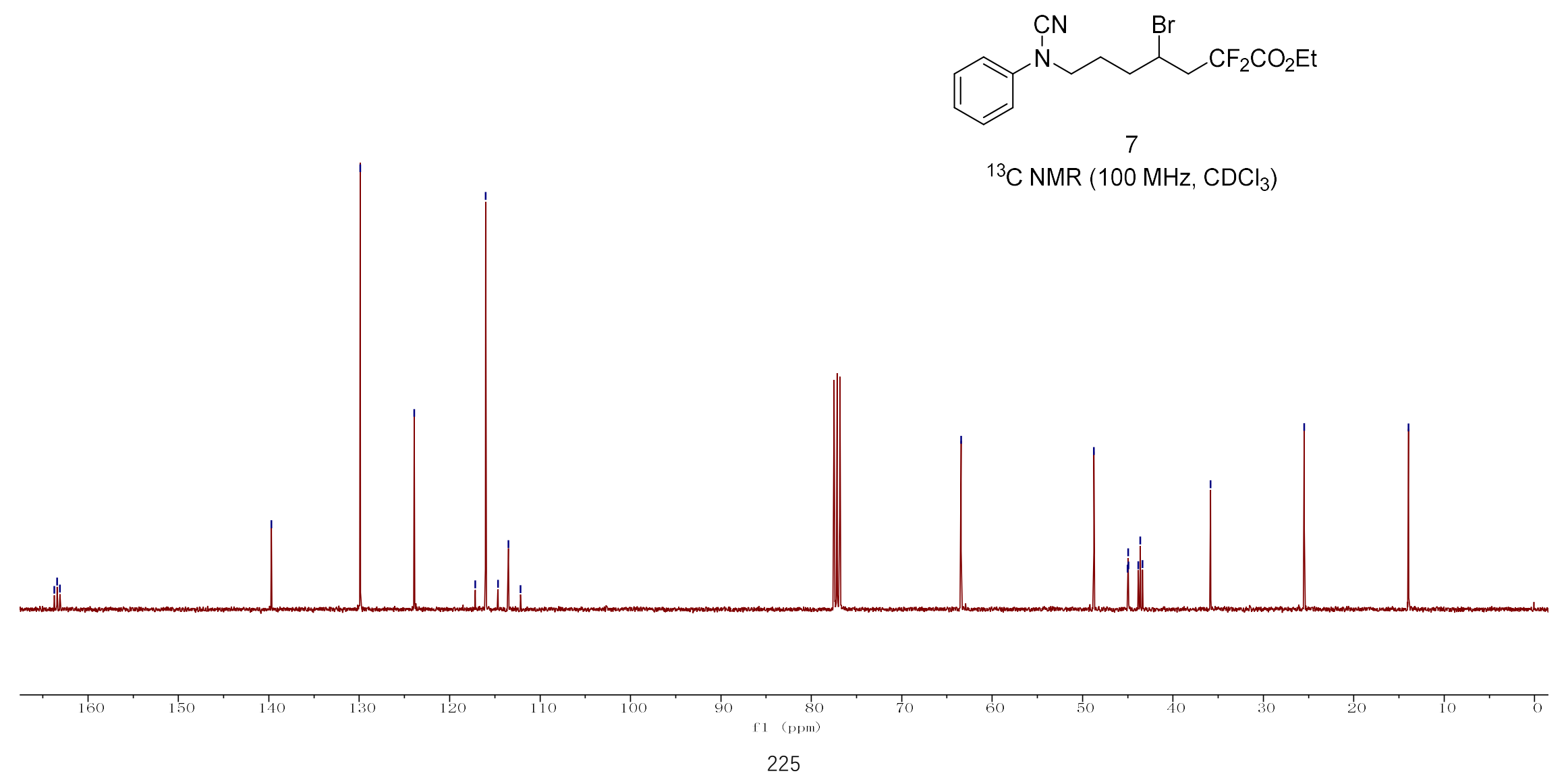




$$
\begin{aligned}
& \text { ำ ㅇำ }
\end{aligned}
$$

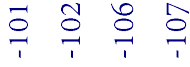

$$
\begin{aligned}
& \rightarrow 1
\end{aligned}
$$

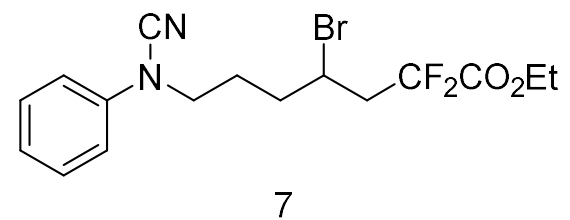

${ }^{19} \mathrm{~F} \mathrm{NMR} \mathrm{(376} \mathrm{MHz,} \mathrm{CDCl}_{3}$ )

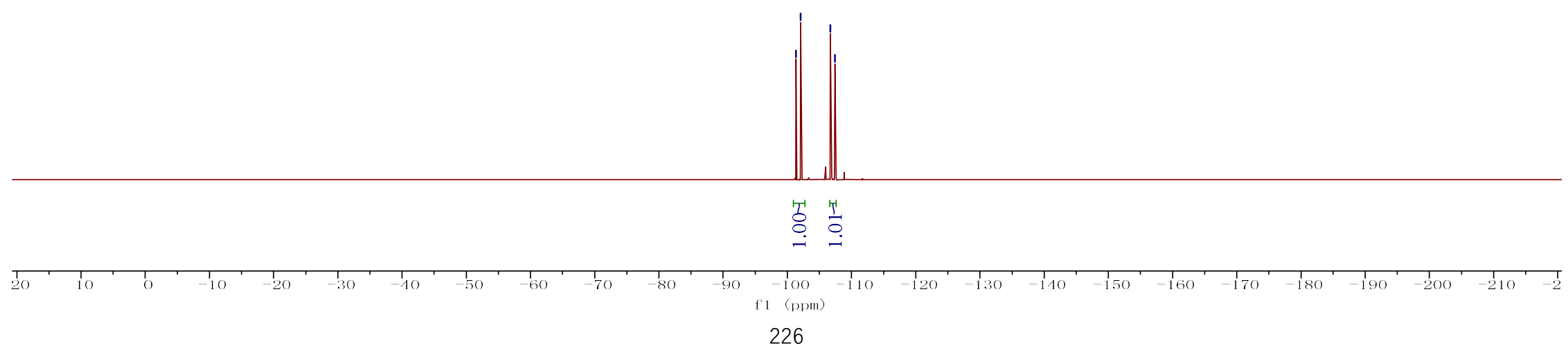




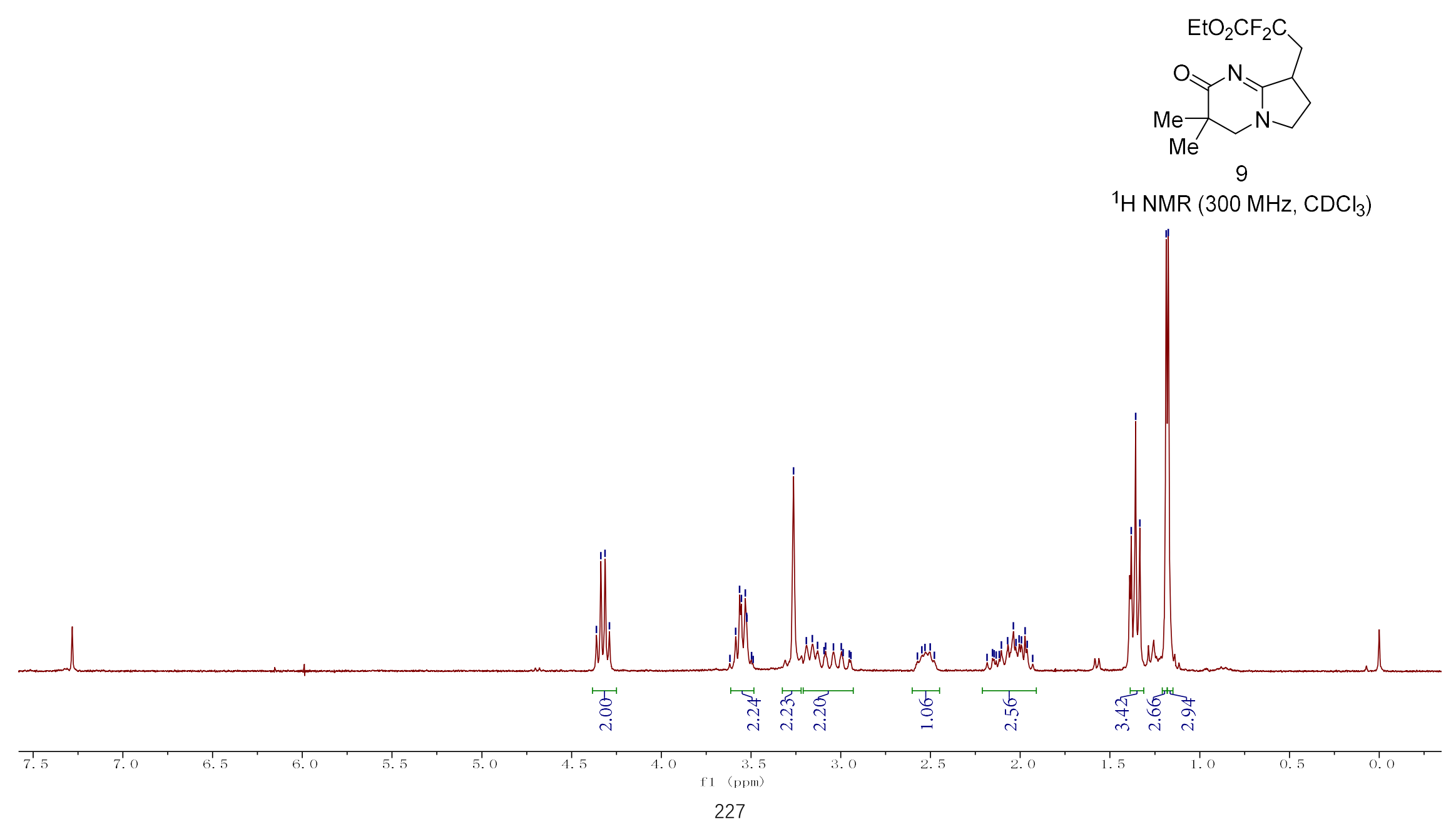




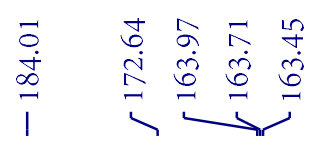

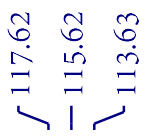

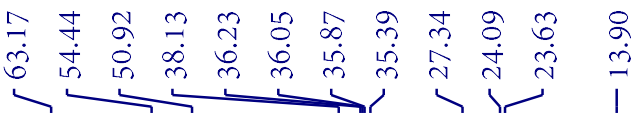

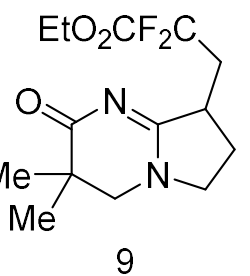

${ }^{3} \mathrm{C}$ NMR $\left(125 \mathrm{MHz}, \mathrm{CDCl}_{3}\right)$

$\mid$

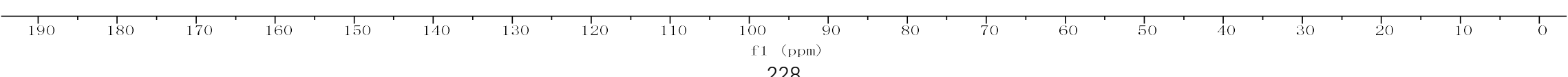




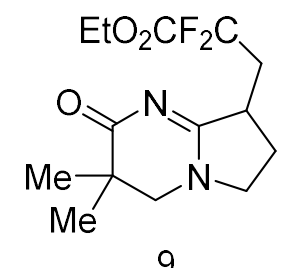

${ }^{19} \mathrm{~F} \mathrm{NMR}\left(470 \mathrm{MHz}, \mathrm{CDCl}_{3}\right)$

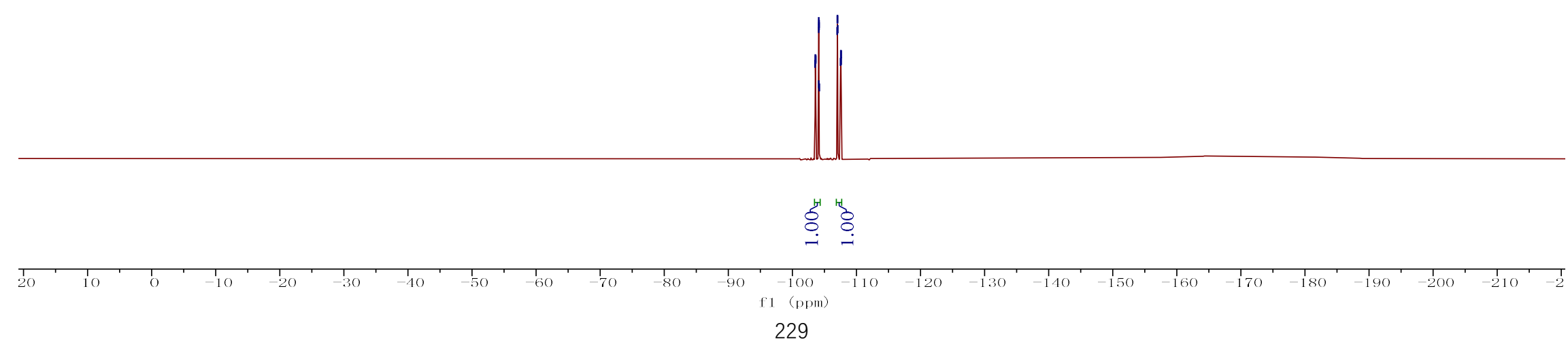




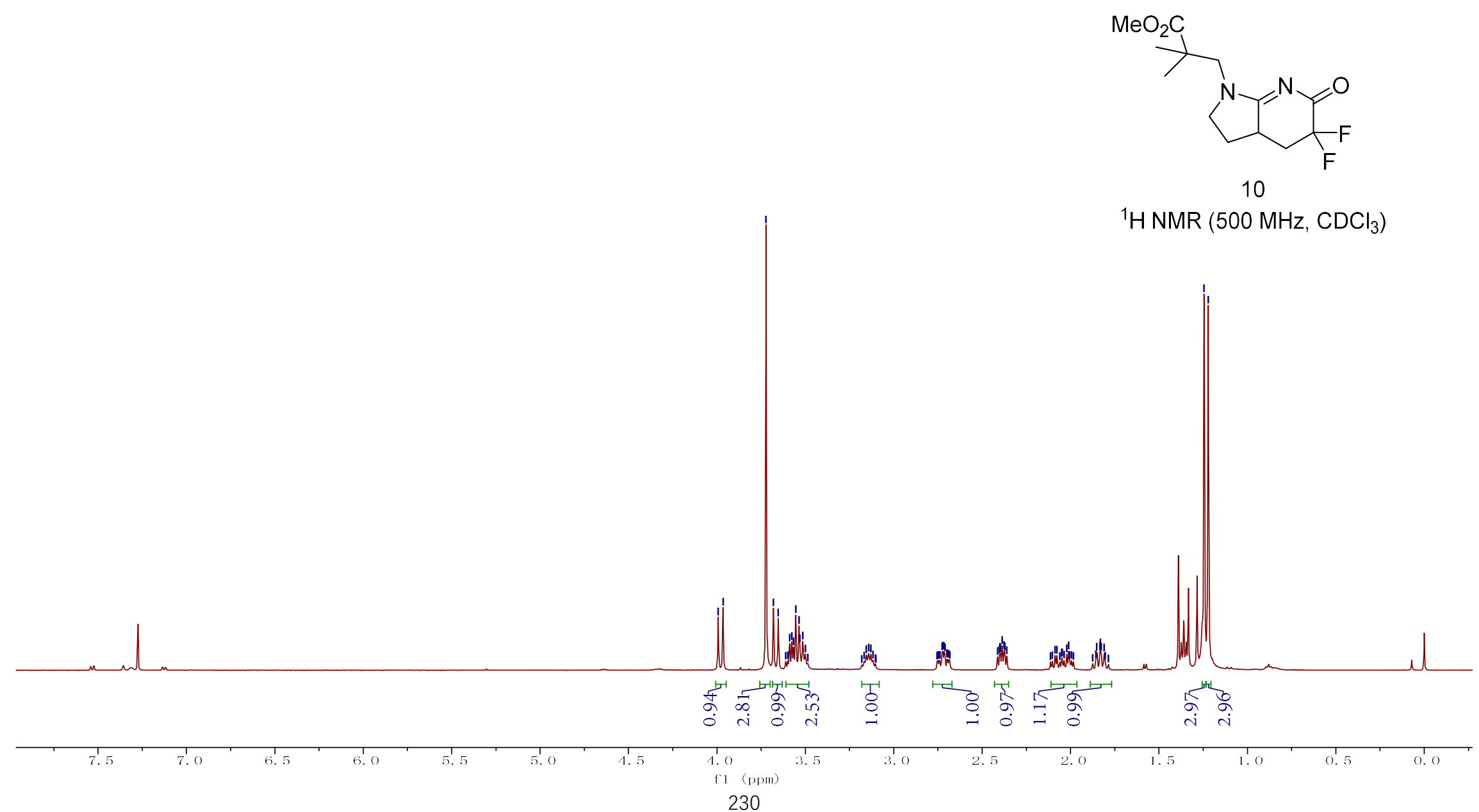


算望

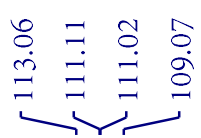

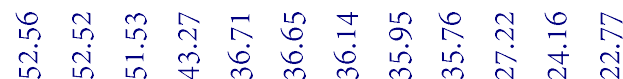

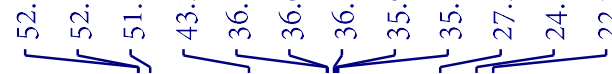

$\mathrm{MeO}_{2} \mathrm{C}$

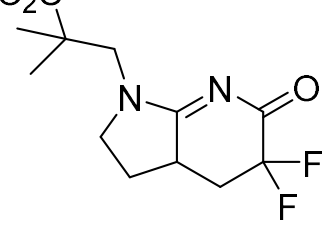

10

${ }^{13} \mathrm{C} \mathrm{NMR}\left(125 \mathrm{MHz}, \mathrm{CDCl}_{3}\right)$

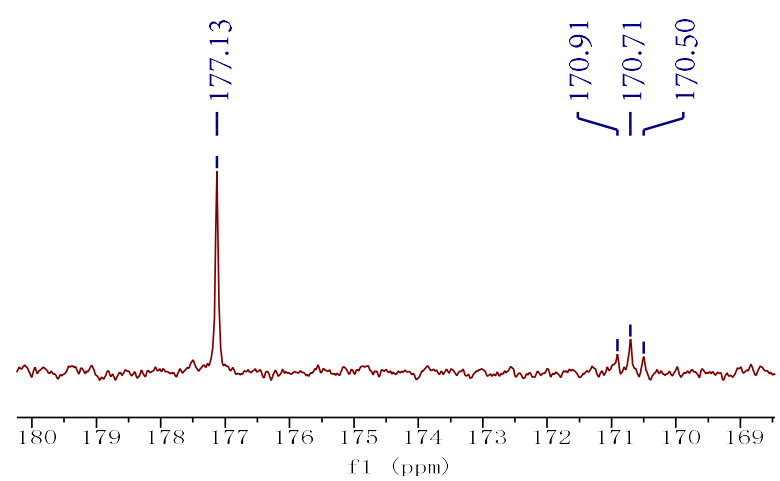

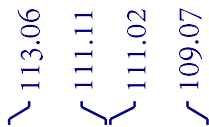

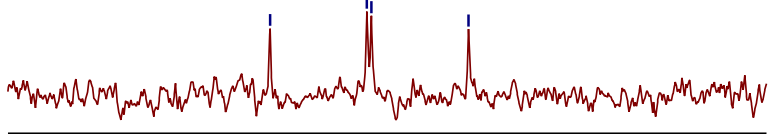
110
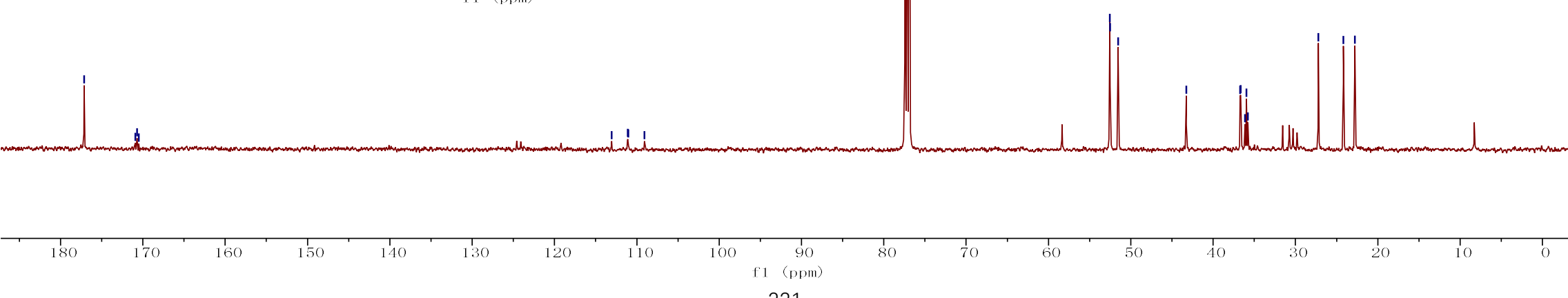


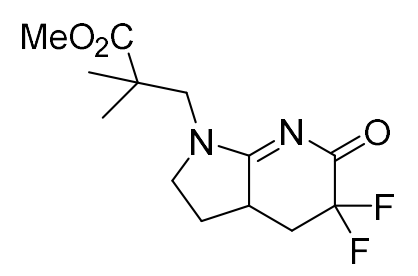

${ }^{19} \mathrm{~F} \mathrm{NMR}\left(470 \mathrm{MHz}, \mathrm{CDCl}_{3}\right)$

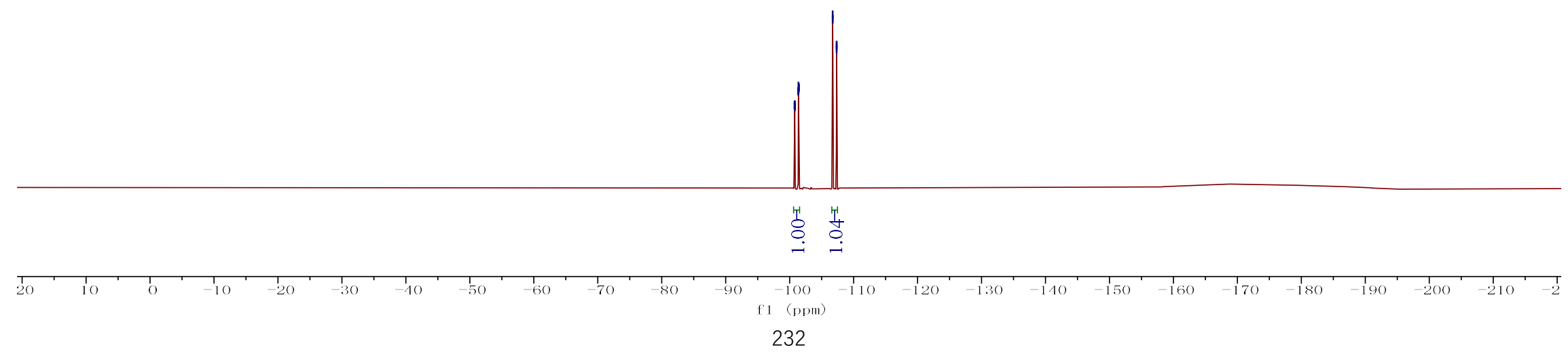




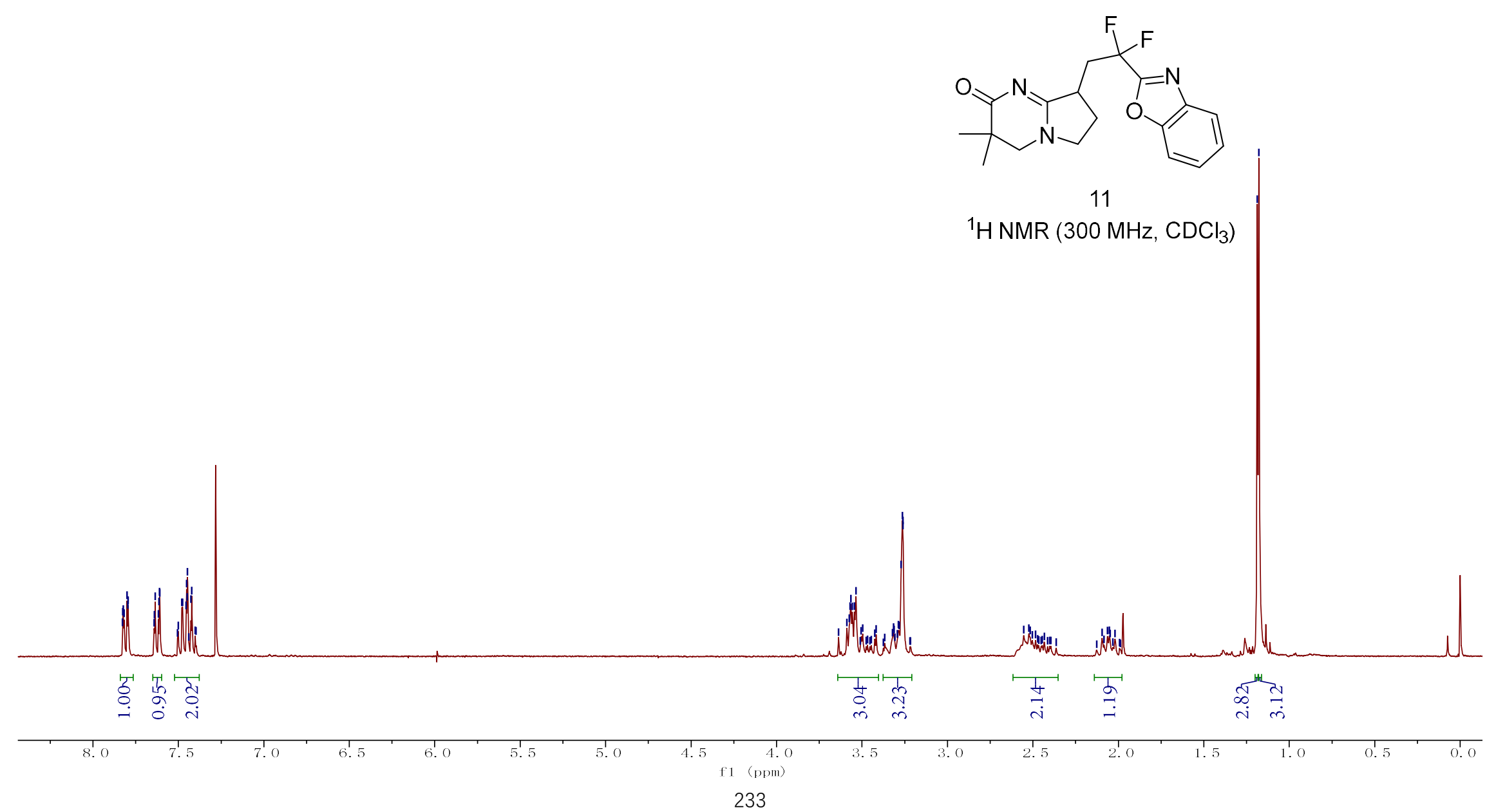




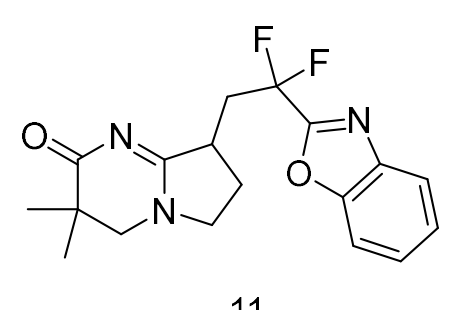

${ }^{13} \mathrm{C}$ NMR $\left(100 \mathrm{MHz}, \mathrm{CDCl}_{3}\right)$
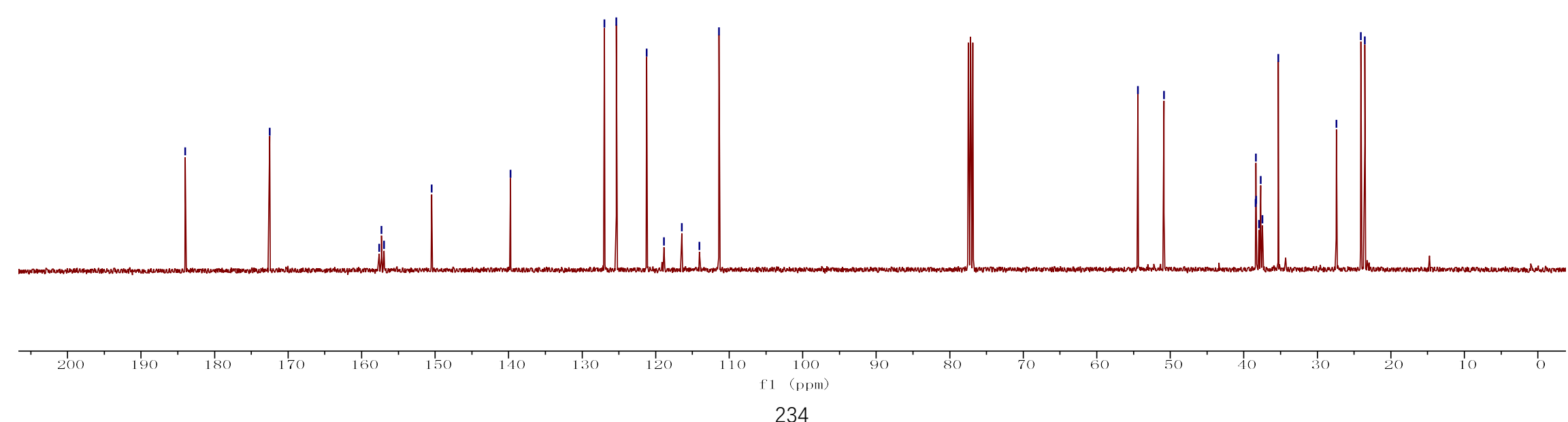


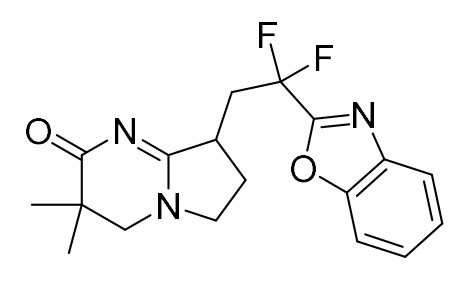

${ }^{19} \mathrm{~F}$ NMR $\left(376 \mathrm{MHz}, \mathrm{CDCl}_{3}\right)$

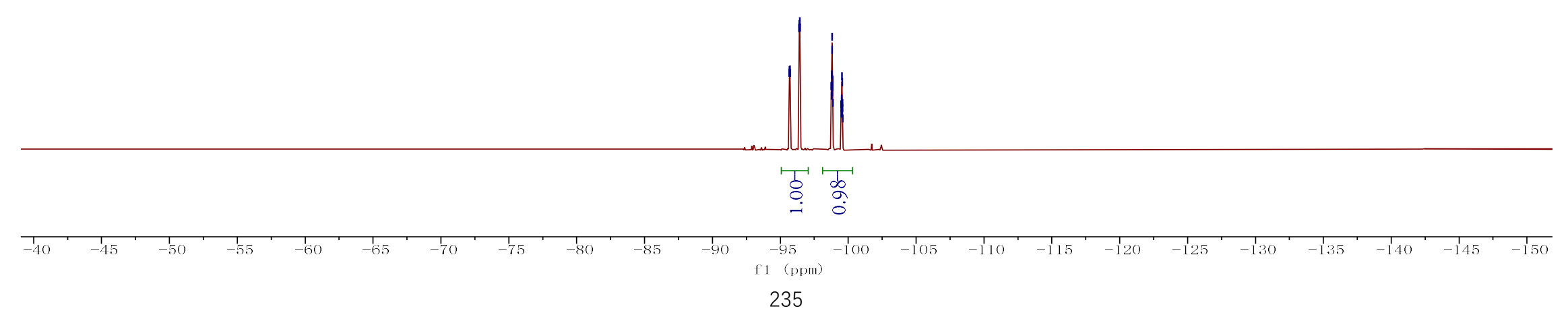




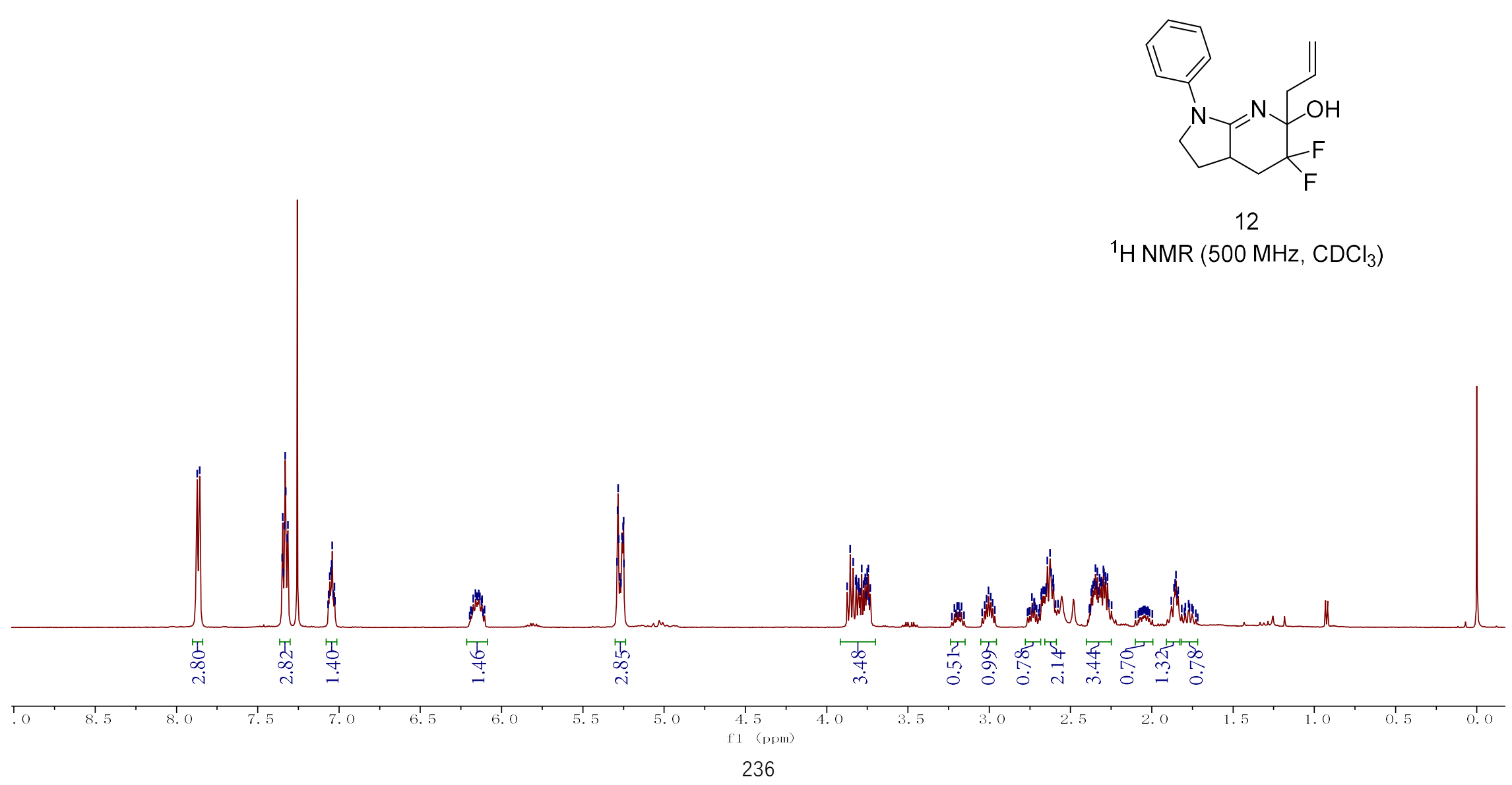



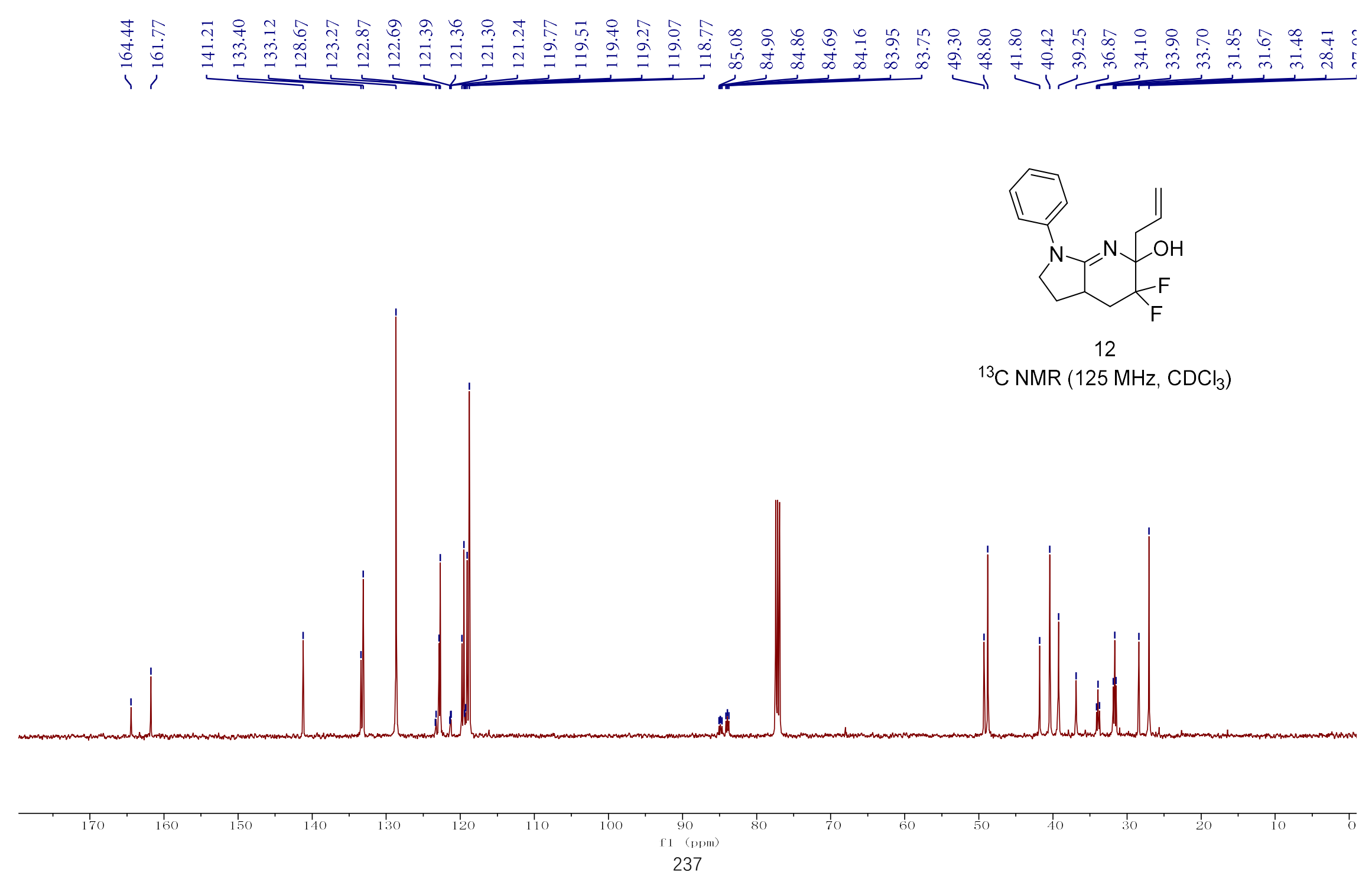


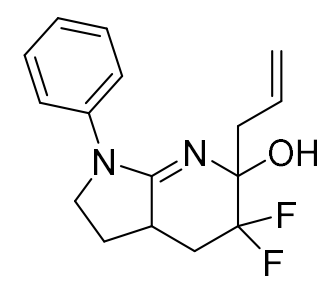

12

${ }^{19} \mathrm{~F} \mathrm{NMR}\left(470 \mathrm{MHz}, \mathrm{CDCl}_{3}\right)$

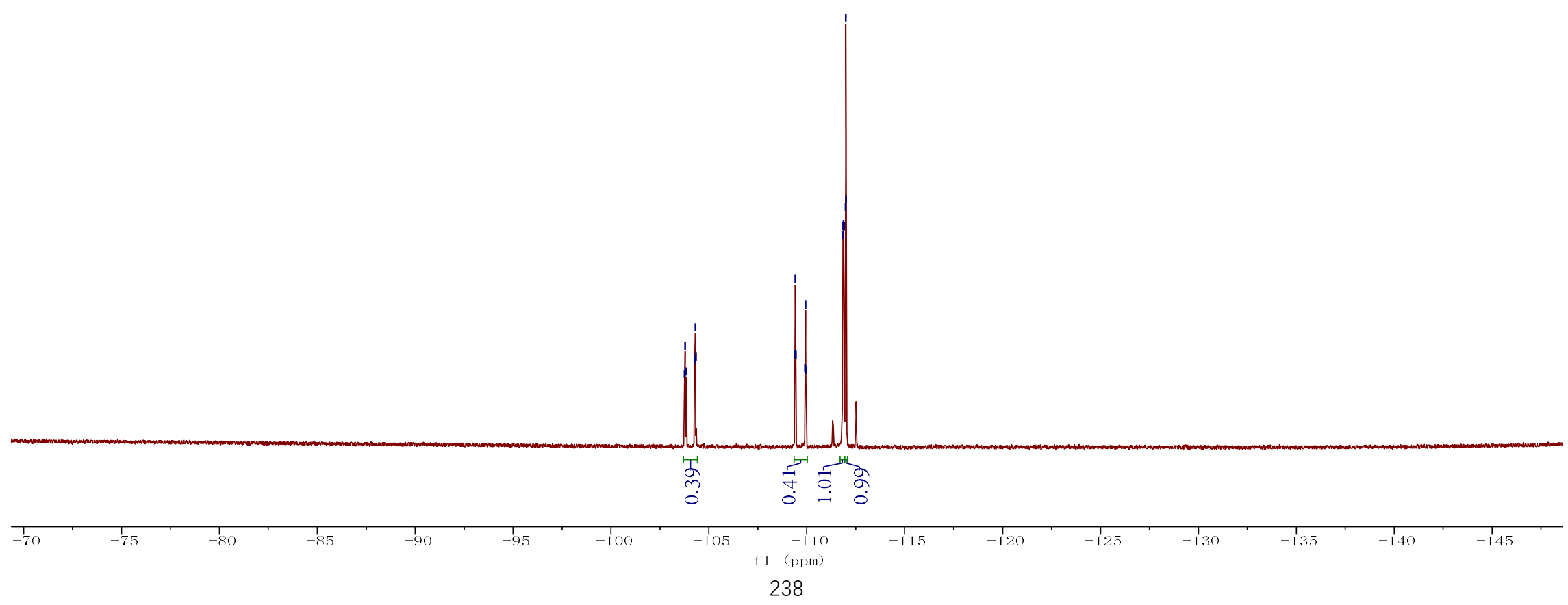




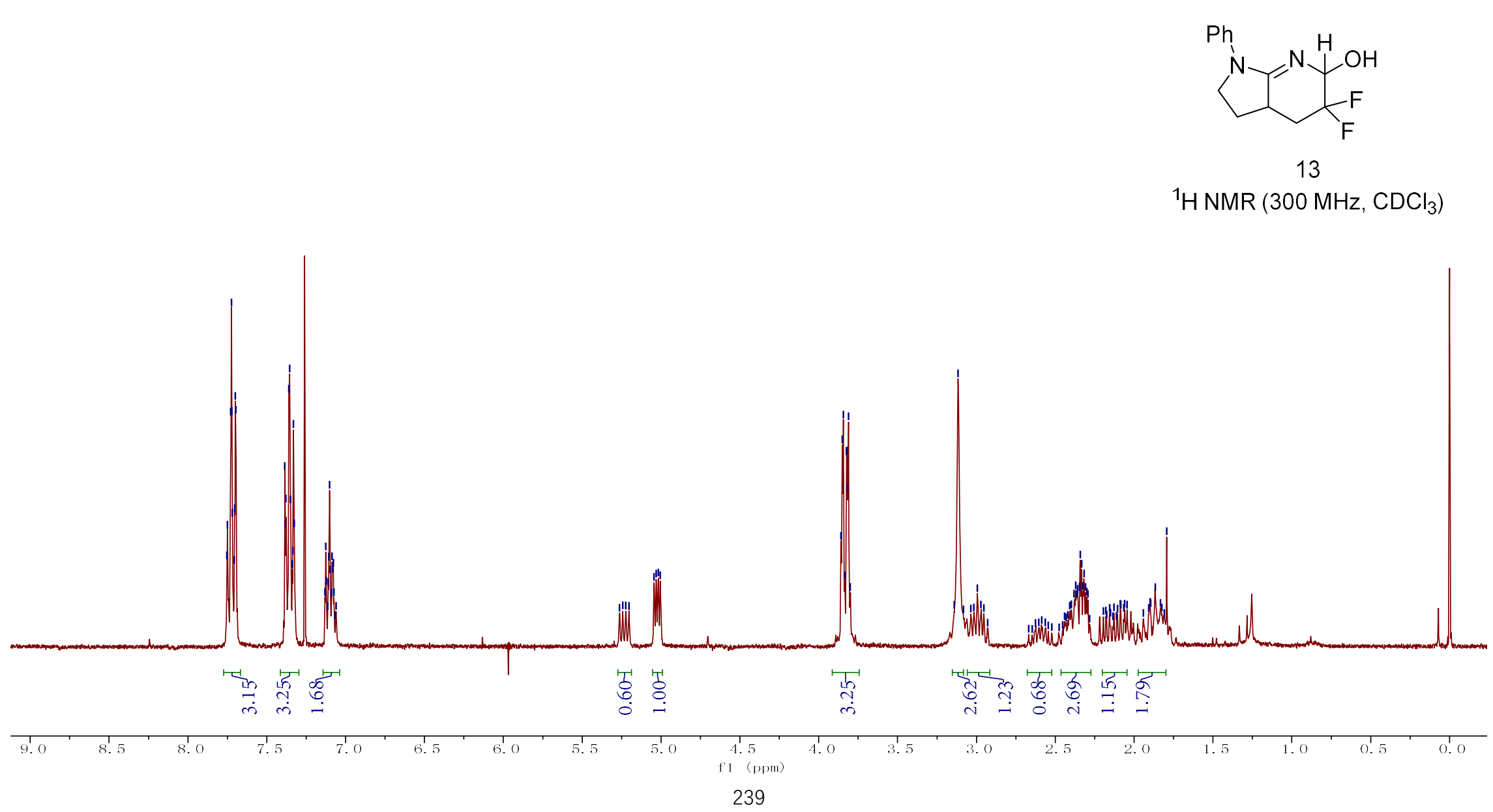




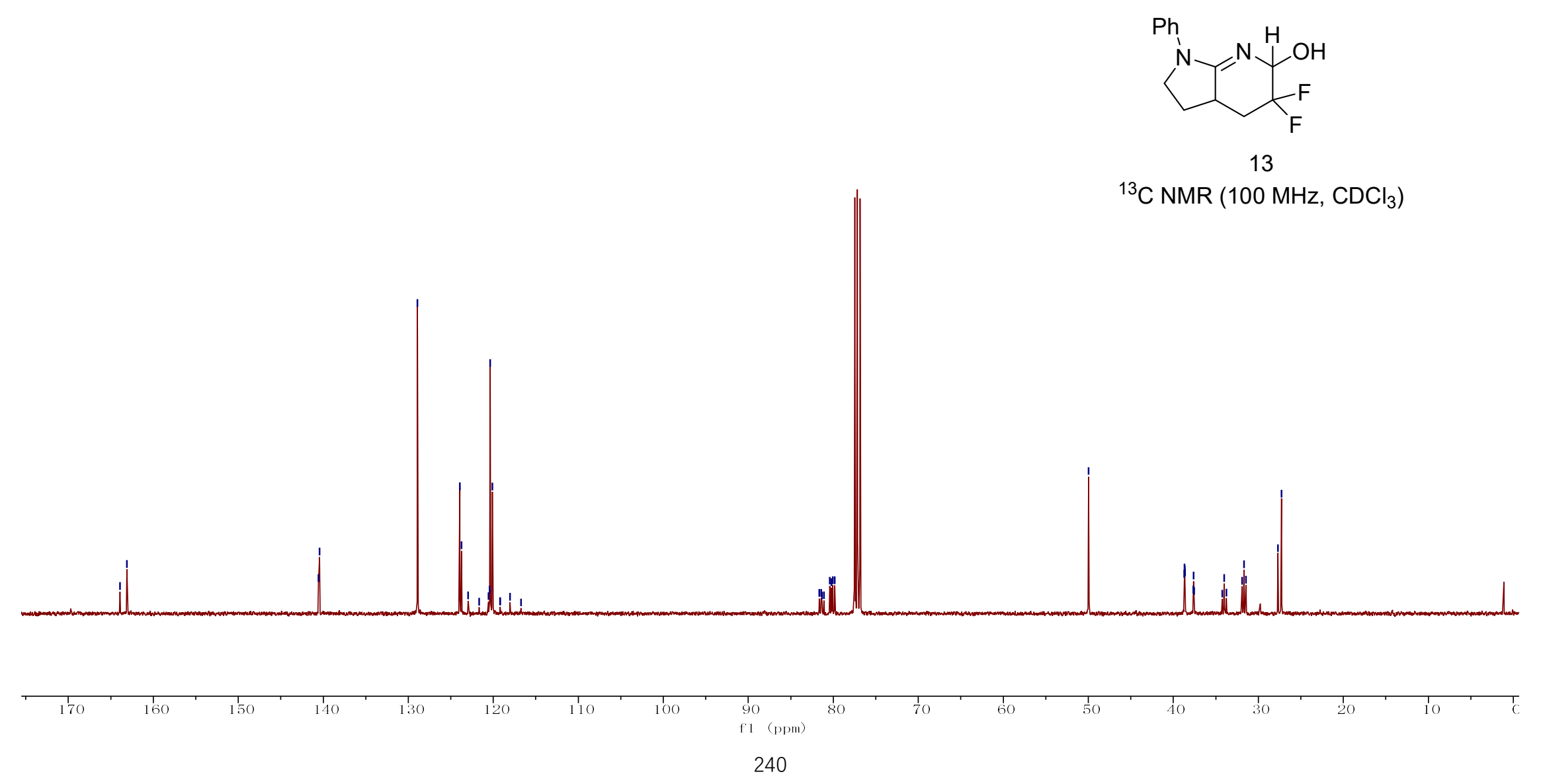




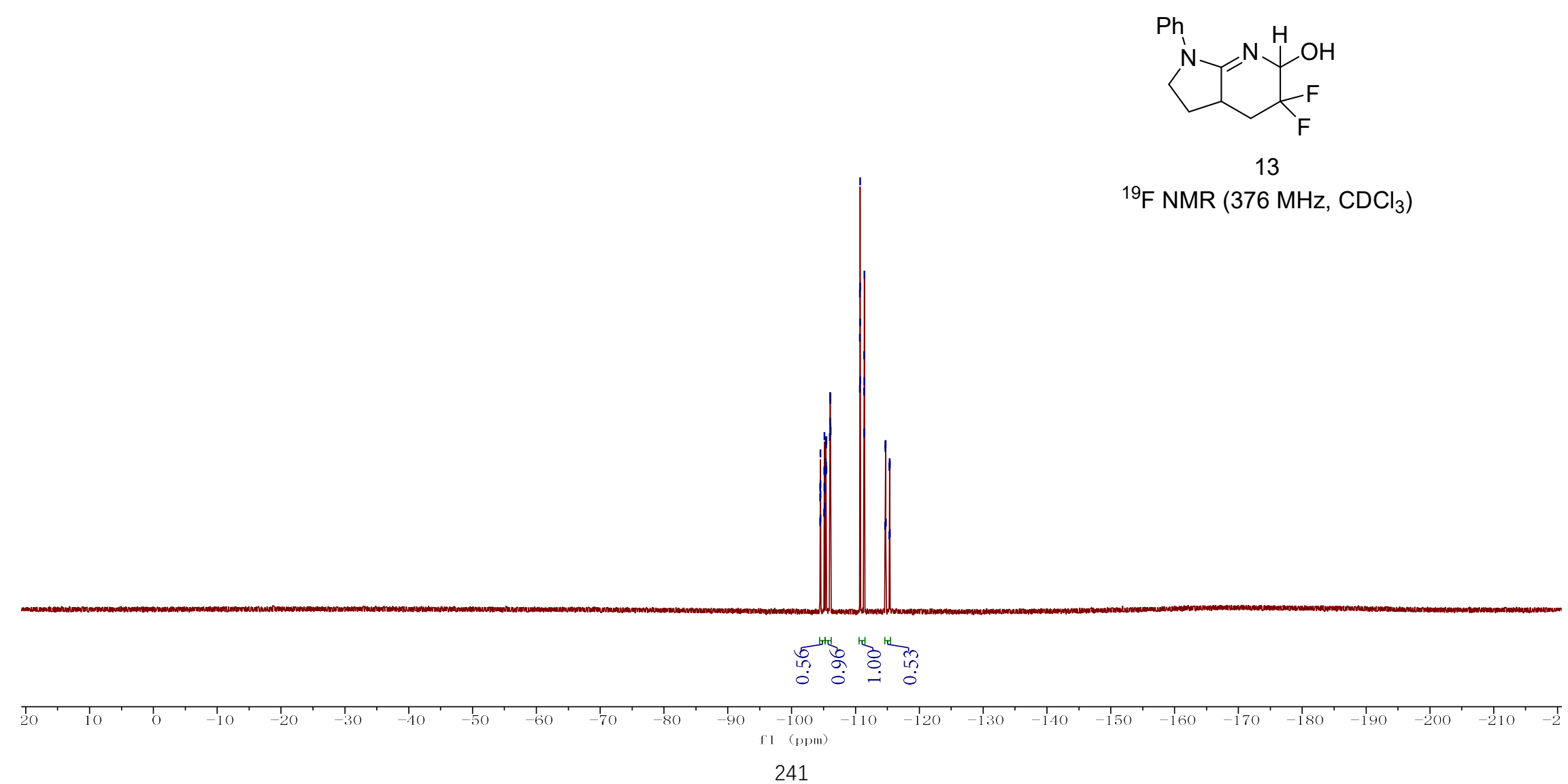




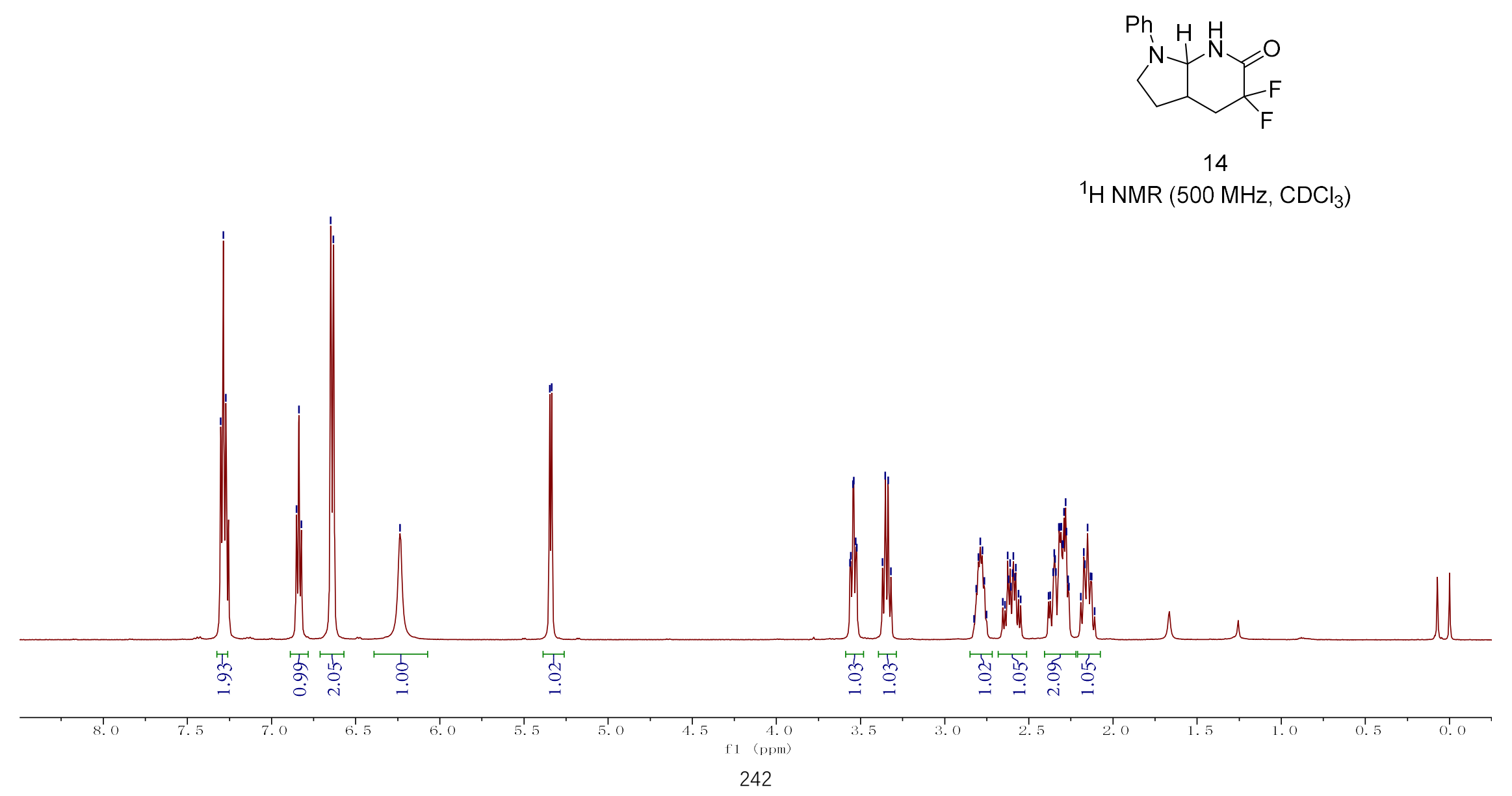




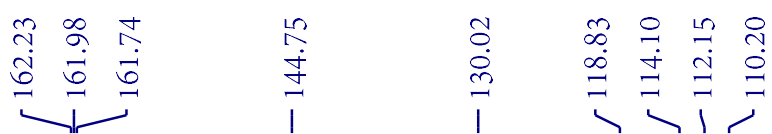

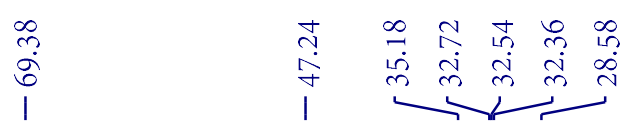

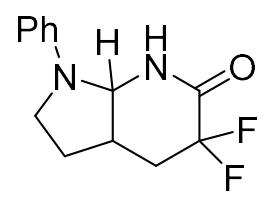

14
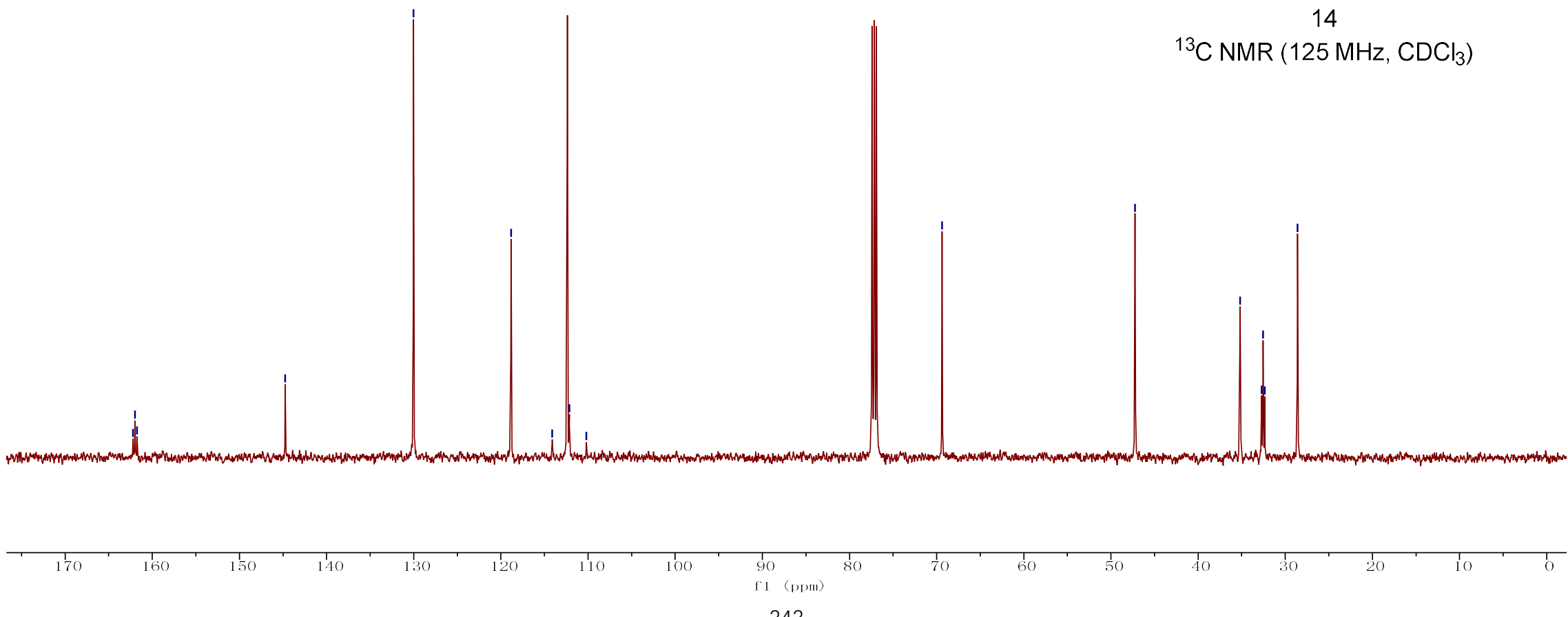

243 


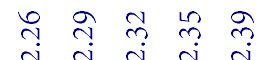

î.

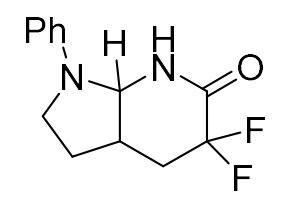

14

${ }^{19} \mathrm{~F} \mathrm{NMR}\left(470 \mathrm{MHz}, \mathrm{CDCl}_{3}\right)$

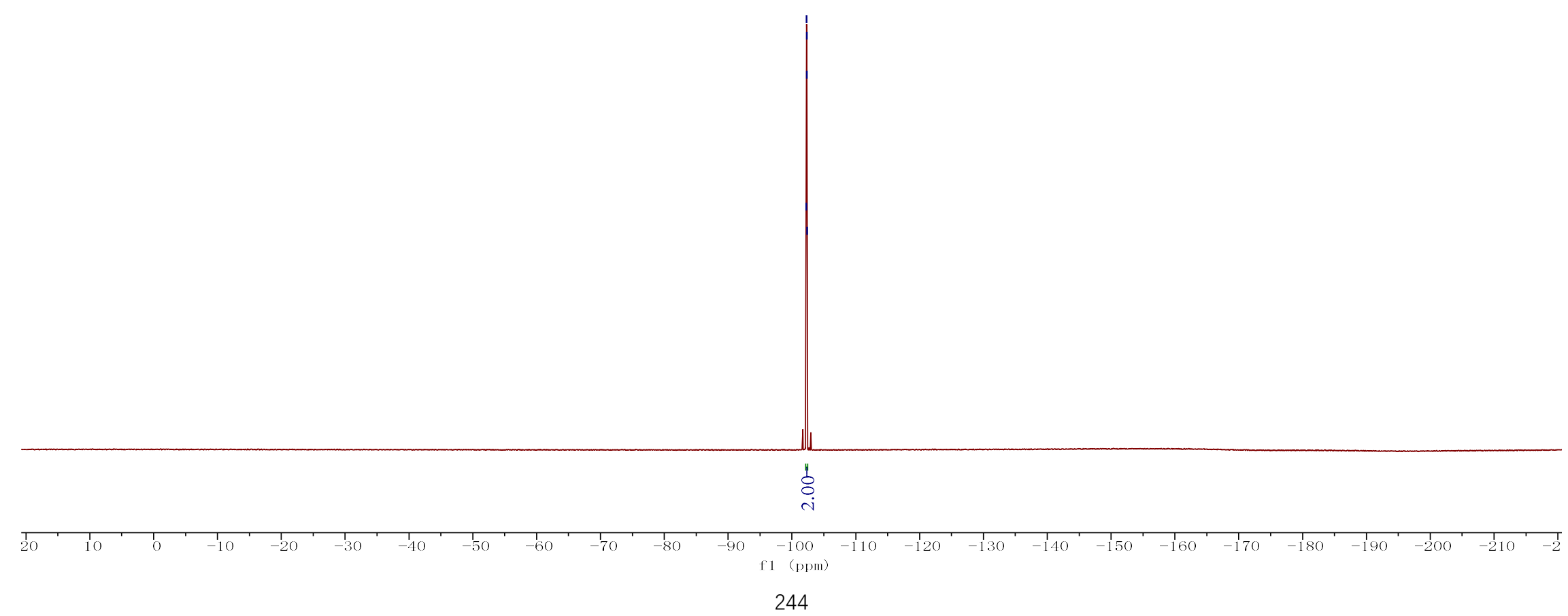




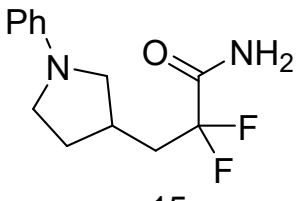

15

${ }^{1} \mathrm{H} \mathrm{NMR}\left(300 \mathrm{MHz}, \mathrm{CDCl}_{3}\right)$

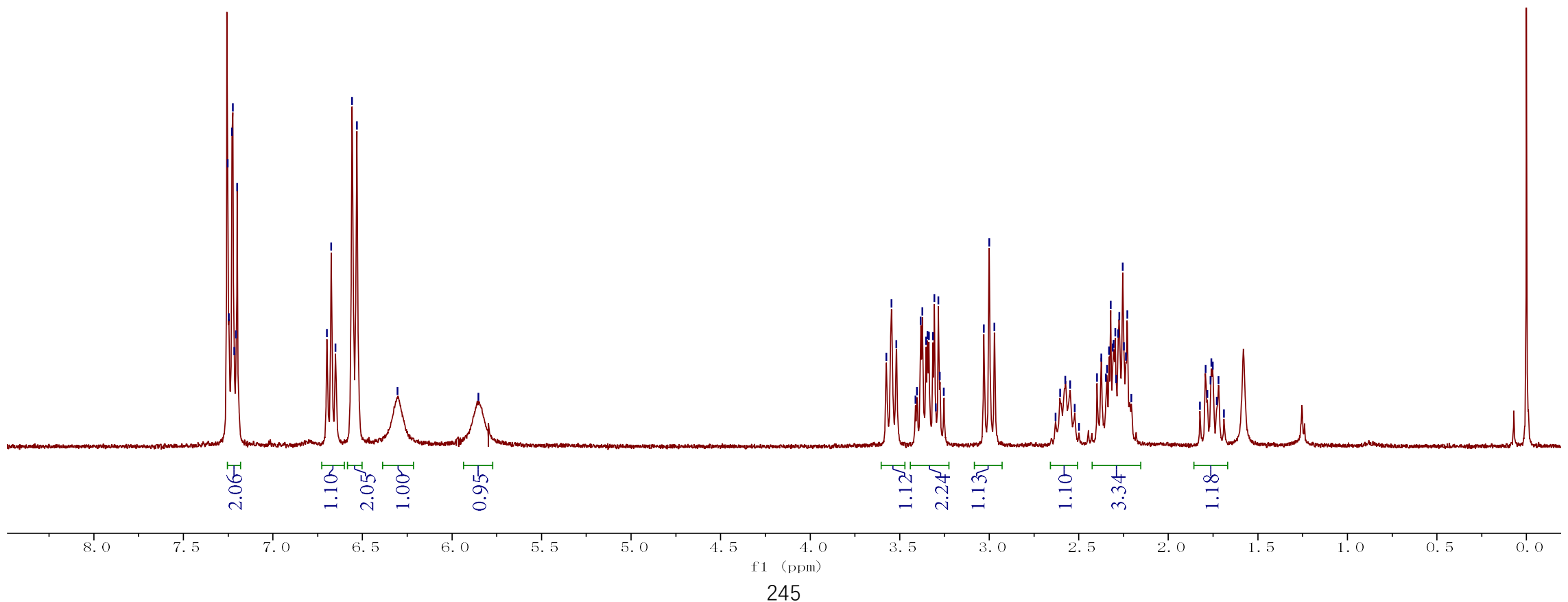




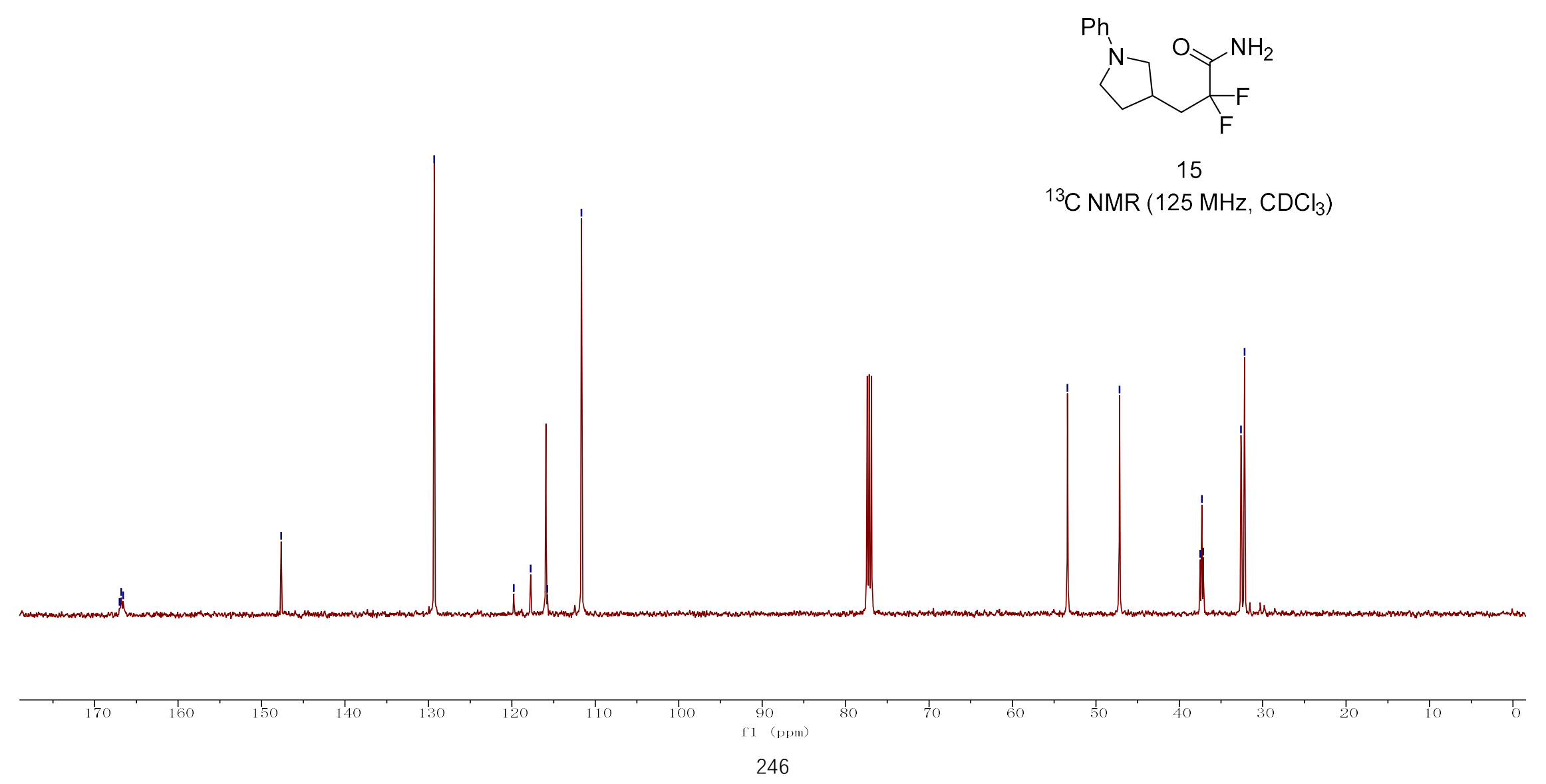




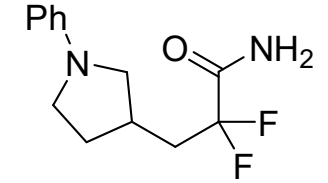

15

${ }^{19} \mathrm{~F} \mathrm{NMR}\left(470 \mathrm{MHz}, \mathrm{CDCl}_{3}\right)$

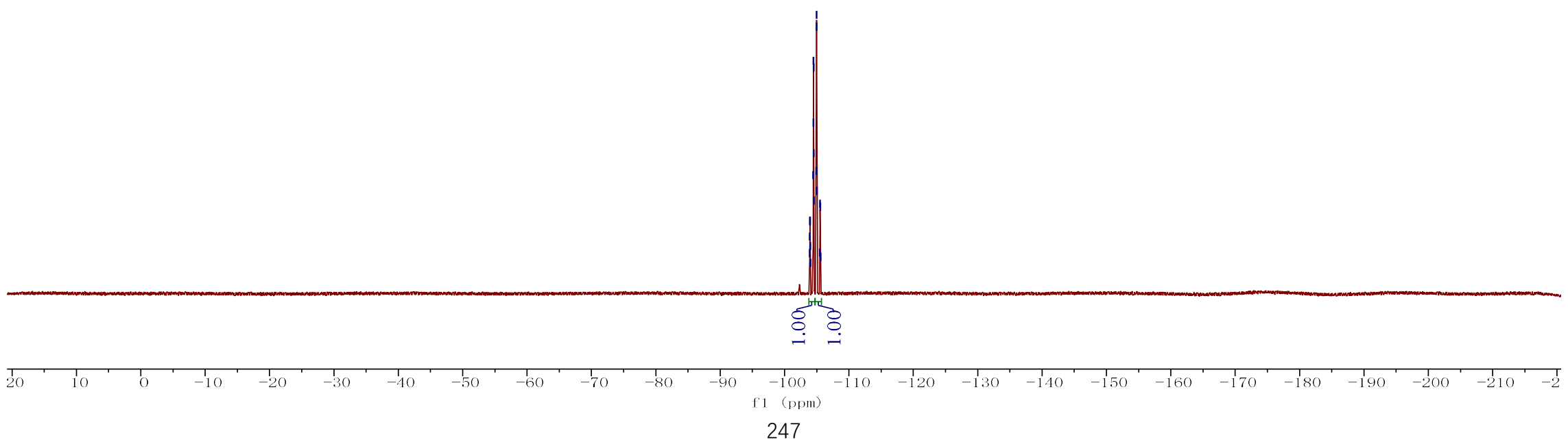




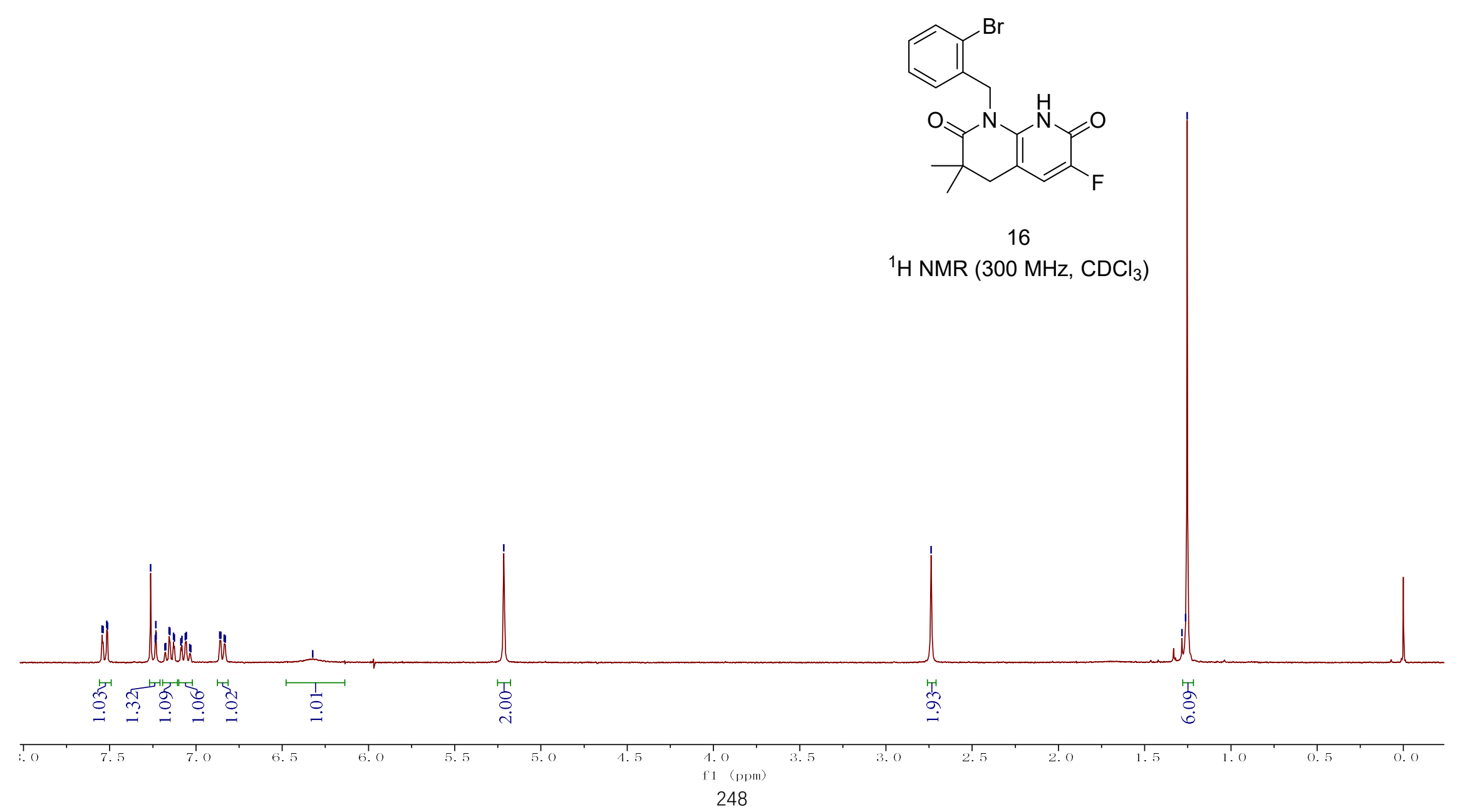




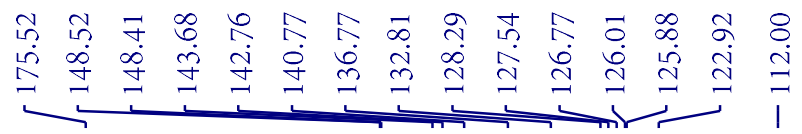

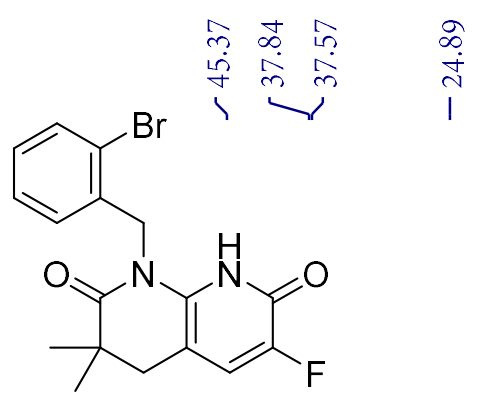

16

${ }^{13} \mathrm{C} \mathrm{NMR}\left(125 \mathrm{MHz}, \mathrm{CDCl}_{3}\right)$
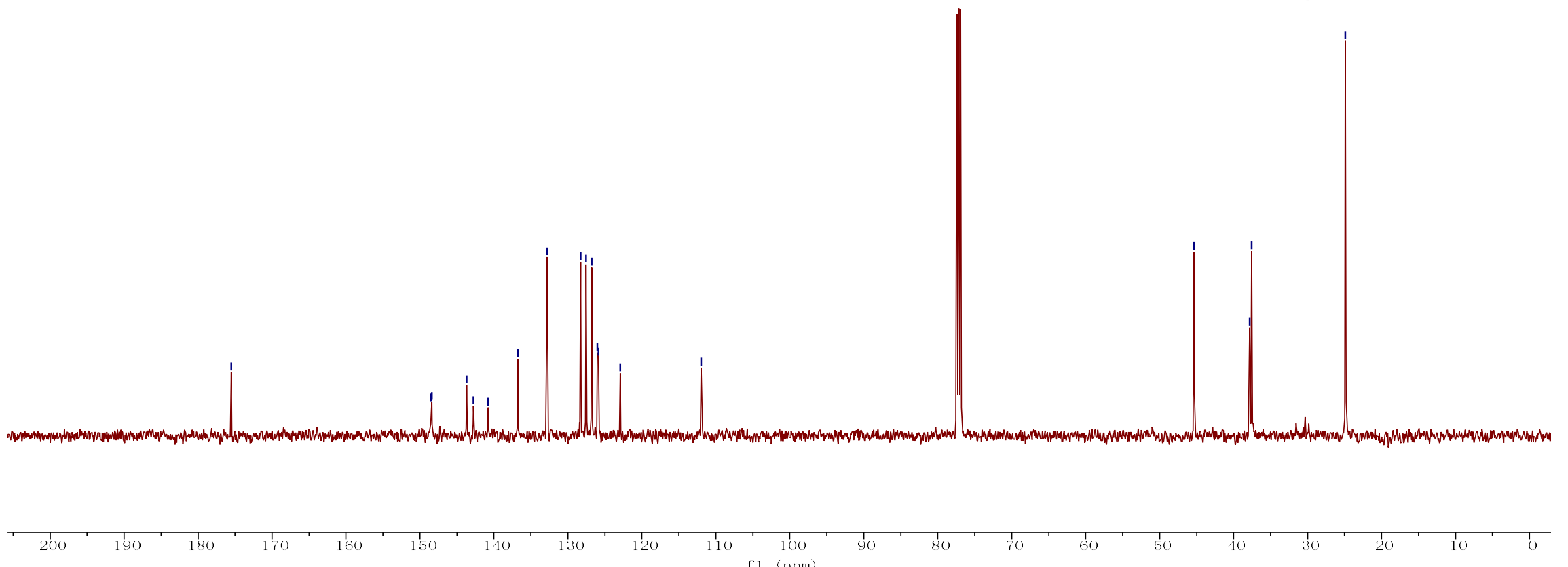
量量

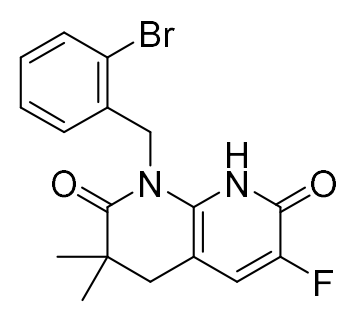

16

${ }^{19} \mathrm{~F} \mathrm{NMR}\left(470 \mathrm{MHz}, \mathrm{CDCl}_{3}\right)$

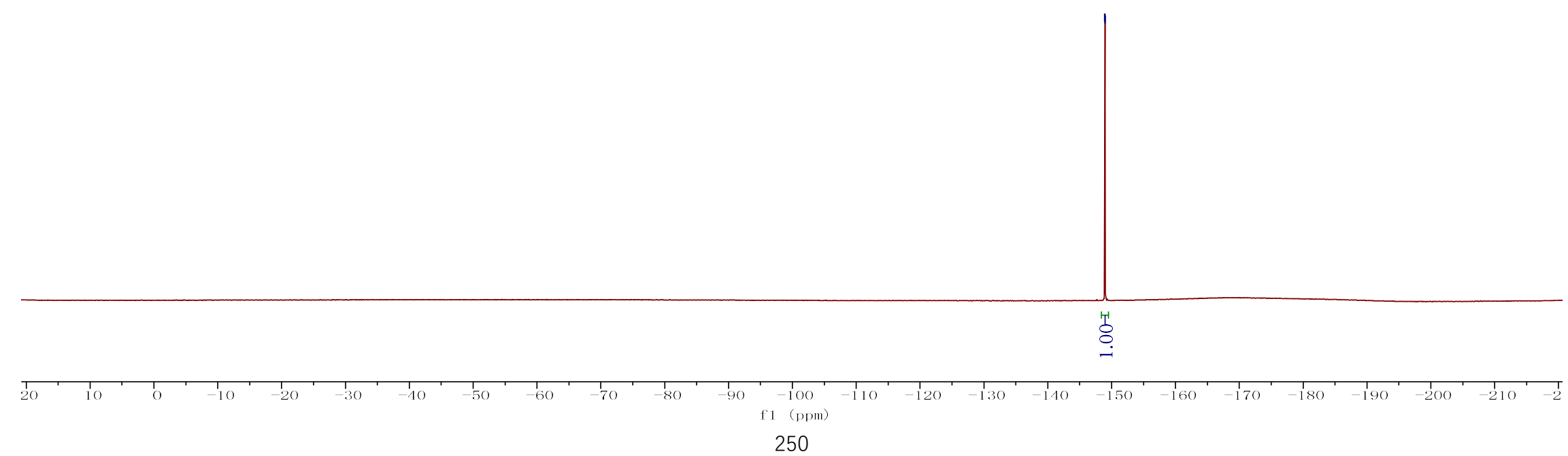

